\title{
Acmella ciliata (H.B.K.) Cassini
}

\author{
Phytochemische und enzymatische \\ Untersuchungen, \\ galenische Präformulierungen
}

Dissertation zur Erlangung des akademischen Grades des

Doktors der Naturwissenschaften (Dr. rer. nat.)

eingereicht im Fachbereich Biologie, Chemie, Pharmazie der Freien Universität Berlin

vorgelegt von

RONALD KEIPERT

aus Berlin

März, 2009 

Die Arbeit wurde angefertigt in den Jahren 2003 - 2009 unter Leitung von Herrn Prof. Dr. M. F. Melzig.

1. Gutachter: Prof. Dr. Melzig

2. Gutachter: PD Dr. Jenett-Siems

Disputation am: 24.06.2009 

Meinen Eltern 



\section{Danksagung}

Mein besonderer Dank gilt Herrn Prof. Dr. M. F. Melzig* für die Überlassung dieses vielseitigen Themas, seine stete Unterstützung der Arbeit und Bereitschaft zur Diskussion. Außerdem bin ich ihm dankbar für die Freiräume, die Arbeit den Erfordernissen entsprechend anpassen und erweitern zu können.

Frau PD Dr. K. Jenett-Siems* danke ich für die große Hilfe bei der Strukturaufklärung, insbesondere bei der Auswertung von NMR-Spektren, sowie für wertvolle phytochemische Tipps.

Mein Dank gilt weiter Frau Dr. R. Schenk und Mitarbeitern, Landwirtschaftlich-Gärtnerische Fakultät der Humboldt-Universität, Berlin, die durch Aufzucht und Anbau von Acmella ciliata die Arbeit primär ermöglichten.

Bei Herrn Dr. W. Mehnert** möchte ich mich herzlich für die Mitarbeit und Unterstützung bei den galenischen Themen und statistischen Auswertungen bedanken.

Frau Dr. K. Wölkart, Mathematisch-Naturwissenschaftliche Fakultät der Karl-FranzensUniversität, Graz, danke ich ganz besonders für die HPLC-MS-Analysen zweier Acmella ciliata-Fraktionen und für die Anregungen zu deren Auswertung.

Frau Dr. B. Rennert ${ }^{(*)}$ danke ich für die Einführung in die Elastaseanalytik.

Weiterhin danke ich den Diplomandinnen Frau F. Gollesch* und Frau K. Henke* für die Messung von Acmella-Proben im Trypsin- bzw. Thrombin-Assay.

Den Studierenden Frau J. Stratmann, Frau A. Reibert, Frau C. Behrends, Herr C. Dehne gilt mein Dank für ihr Mitwirken im Rahmen ihrer Wahlpflichtarbeit, Frau Dr. J. LazarSchurreit* für die Hilfe bei der mikroskopischen Auswertung, Frau P. Schlupp** für die Herstellung der Nanoemulsion, Herrn Dr. B. Schwabe** für die Mitarbeit bei der Tablettenherstellung, Herrn Prof. Dr. B. Müller und Mitarbeitern, Christian-AlbrechtsUniversität, Kiel, für die Herstellung der $\mathrm{CO}_{2}$-Extrakte, Herrn Dr. K. Siems, AnalytiCon AG, Potsdam, für die Hilfe bei NMR-Problemen, Herrn Dr. M. Fengler für wertvolle Statistiktipps und meinem Bruder T. Keipert für die Hilfe in Gestaltungsfragen der Arbeit.

Schließlich gilt mein Dank den Mitarbeitern des Arbeitskreises von Prof. Melzig für ihre Kollegialität und besonders Frau M. Mayer und Frau S. Ott für wertvolle Tipps im Laboralltag.

Mein herzlichster Dank gebührt meinen Eltern, meiner Lebensgefährtin Jeannette und meinem Sohn Manuel, die mir stets geistige und moralische Unterstützung bei der Durchführung und Fertigstellung meiner Arbeit gewährten und viel Geduld aufbrachten.

* Institut für Pharmazie, Pharmazeutische Biologie im Fachbereich Biologie, Chemie, Pharmazie der Freien Universität, Berlin

** Institut für Pharmazie, Pharmazeutische Technologie im Fachbereich Biologie, Chemie, Pharmazie der Freien Universität, Berlin 



\section{Inhaltsverzeichnis}

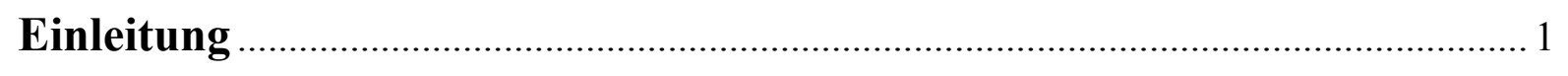

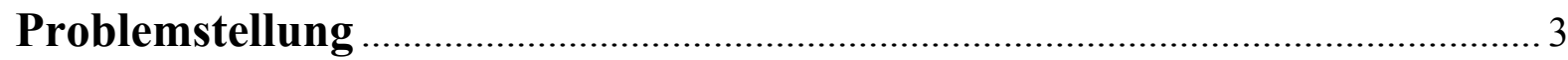

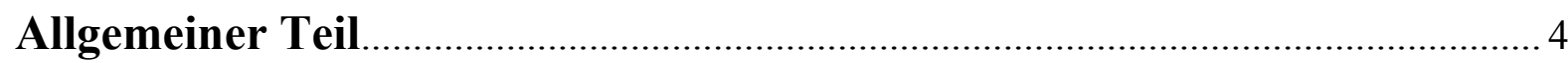

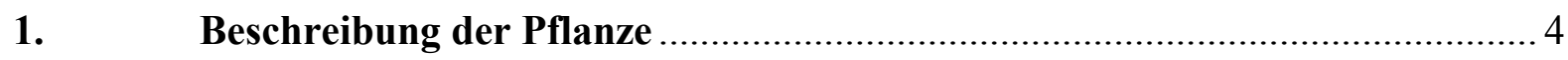

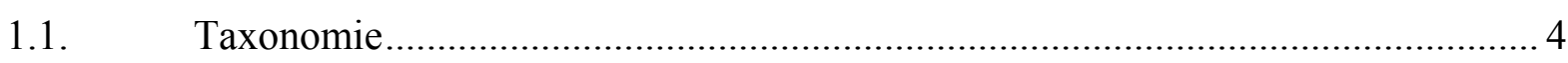

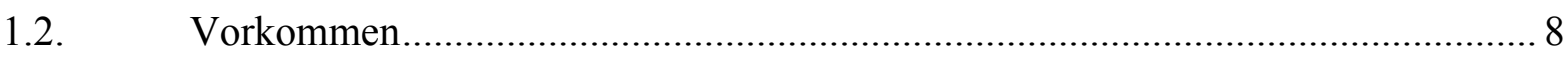

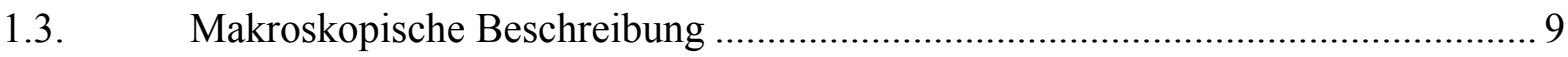

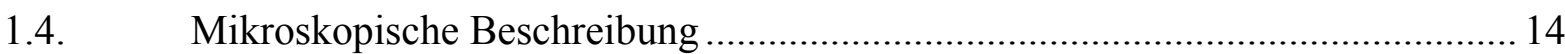

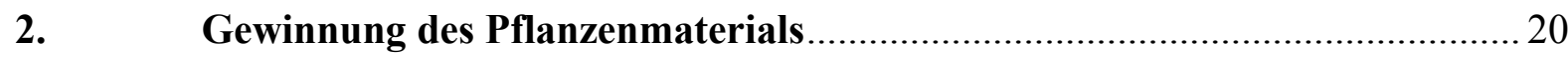

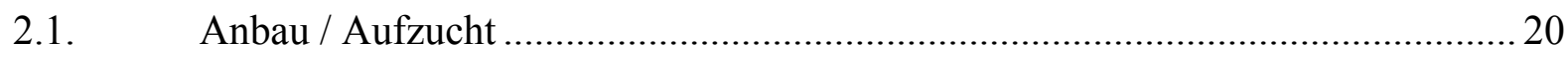

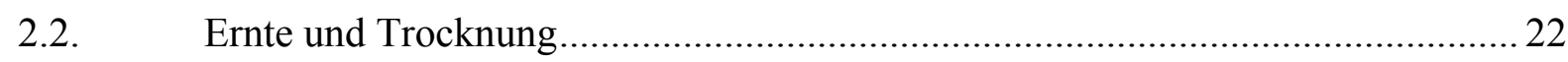

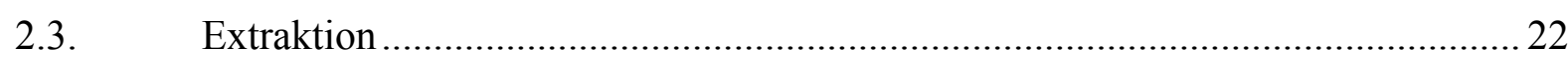

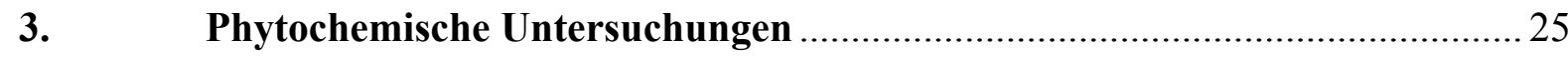

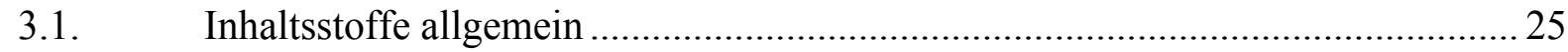

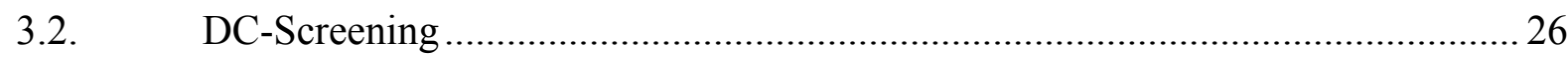

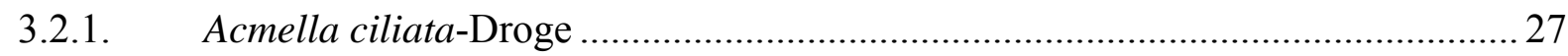

3.2.2. Acmella ciliata / Acmella oleracea-Vergleich - Frischpflanzen ......................... 31

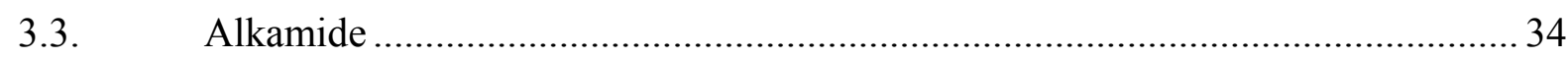

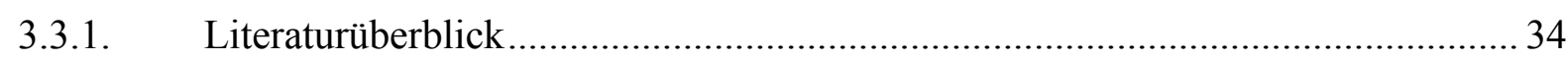

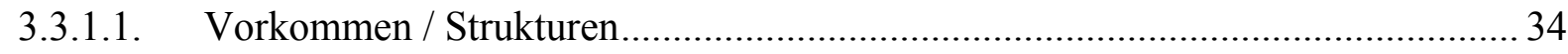

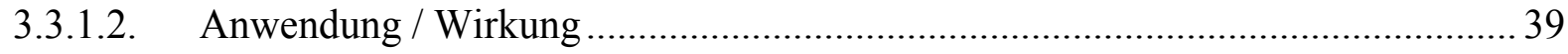

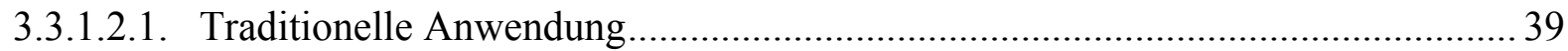

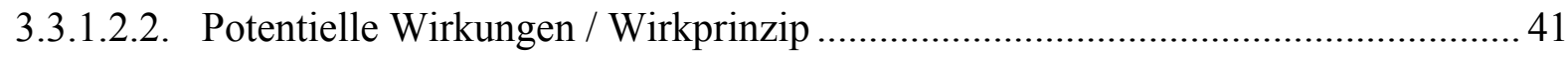

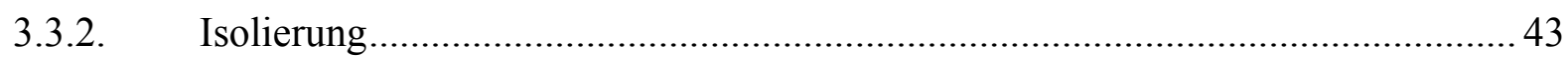

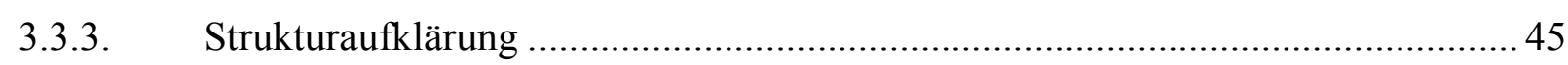

3.3.3.1. +ESI-MS-Fragmentierung der Amidreste .................................................. 50

3.3.3.2. Undeca-2E,4E-dien-8,10-diinsäure-isobutylamid (Verbindung $\boldsymbol{A 1}$ ) ....................52

3.3.3.3. Nona-2E-en-6,8-diinsäure-phenylethylamid (Verbindung $A 2)$........................... 56

3.3.3.4. 7-Hydroxy-dodeca-2E,4E,8Z,10E-tetraensäure-isobutylamid (Verbindung A3) . 59

3.3.3.5. 2,4-Dihydroxy-nona-6,8-diinsäure-phenylethylamid (Verbindung A4) .............. 61 


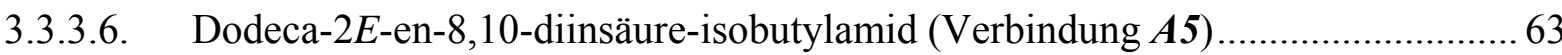

3.3.3.7. 2-Hydroxy-undeca-7Z,9E-diensäure-isobutylamid (Verbindung $\boldsymbol{A 6}_{\boldsymbol{1}}$ )

Undeca-2E,7Z,9E-triensäure-isobutylamid (Verbindung $\boldsymbol{A 6}_{2}$ ) 64

3.3.3.8. Nona-2Z-en-6,8-diinsäure-isobutylamid (Verbindung $A$ 7) und 2,3(c)-Epoxy-nona-6,8-diinsäure-phenylethylamid (Verbindung $\boldsymbol{A 8}$ )...................66

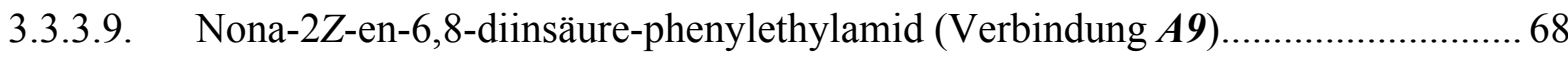

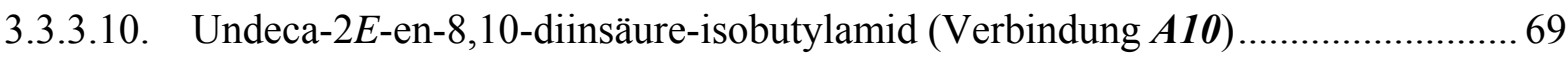

3.3.3.11. Undeca-2E,4E-dien-8,10-diinsäure-methylbutylamid (Verbindung A11)........... 71

3.3.3.12. Weitere Verbindungen aus Sammelfraktion 2 (Retentionszeiten 20,95; 21,67

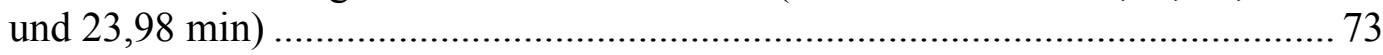

3.3.3.13. 2,5-Dihydroxy-deca-6Z,8E-diensäure-isobutylamid (2,5-Dihydroxyhydro-

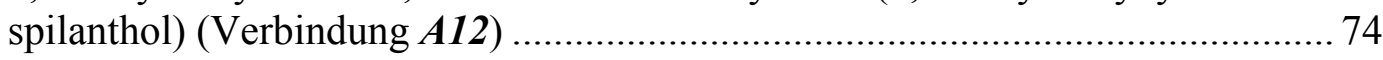

3.3.3.14. Spilanthol (Deca-2E,6Z,8E-triensäure-isobutylamid) (Verbindung $\boldsymbol{A 1 3}$ ) ........... 76

3.3.3.15. Trideca-5Z-en-10,12-diinsäure-isobutylamid (Verbindung A14) ........................ 78

3.3.3.16. Hydrospilanthol (Deca-6Z,8E-diensäure-isobutylamid) (Verbindung $\boldsymbol{A 1 5}$ )......... 79

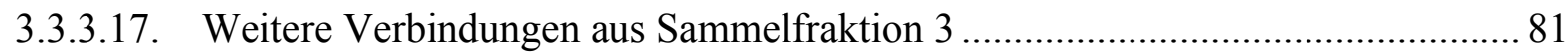

3.3.3.18. Dodeca-2E,4E,8Z,10E-tetraensäure-isobutylamid (Verbindung A16) .................. 82

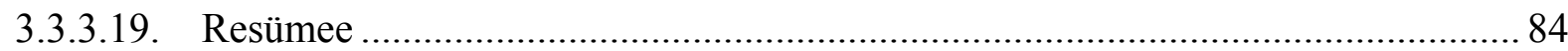

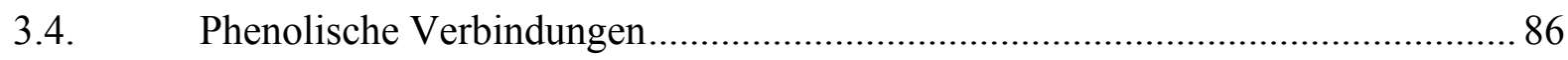

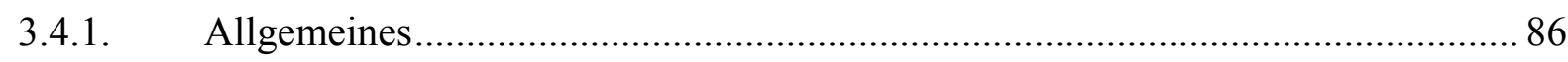

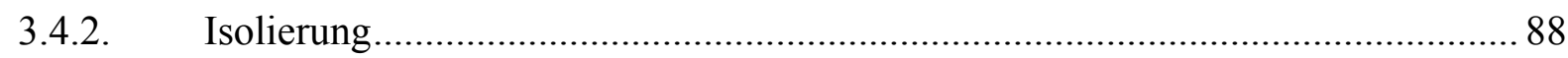

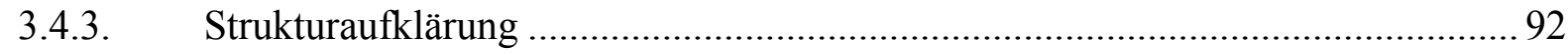

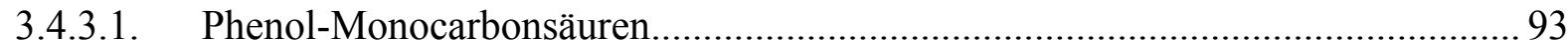

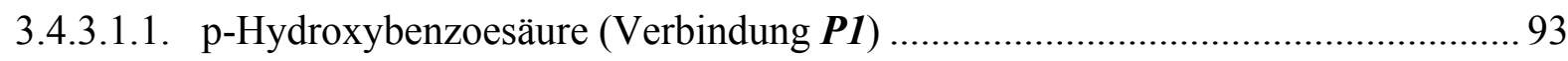

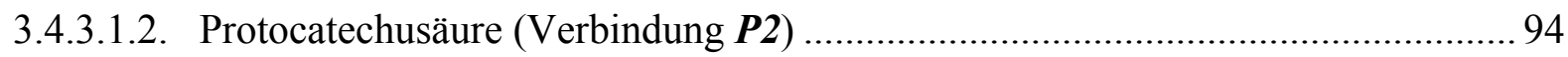

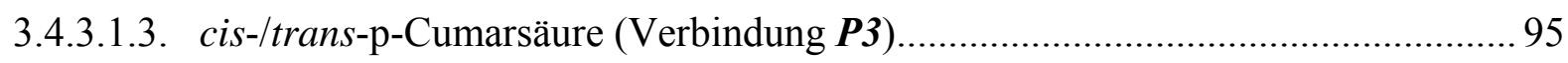

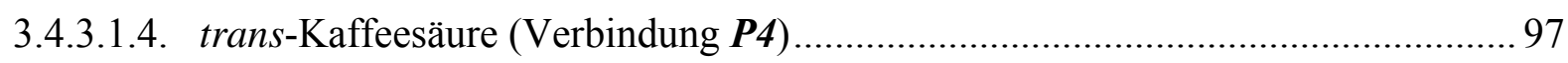

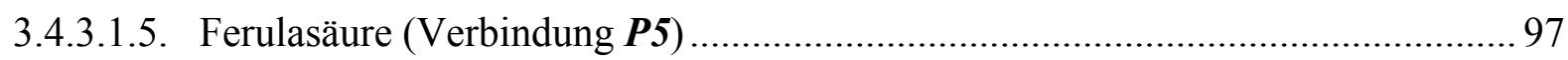

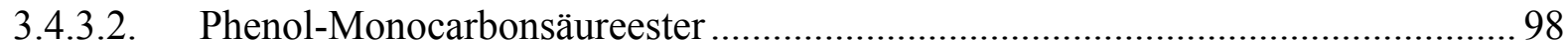

3.4.3.2.1. cis-/trans-p-Cumaroyläpfelsäureester (Verbindung P6) .................................... 98

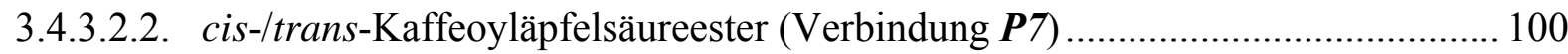

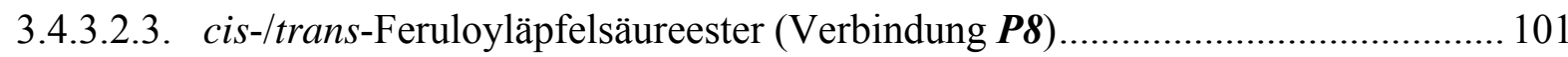

3.4.3.2.4. trans-Kaffeoyläpfelsäure-1-methylester / trans-Kaffeoyläpfelsäure-4-methylester (Verbindungen $\boldsymbol{P 9}$ und $\boldsymbol{P 1 0}$ )............... 104

3.4.3.2.5. cis-/trans-Chlorogensäure (cis-/trans-5-Kaffeoylchinasäure) (Verbindung $\boldsymbol{P 1 1}$ ) 


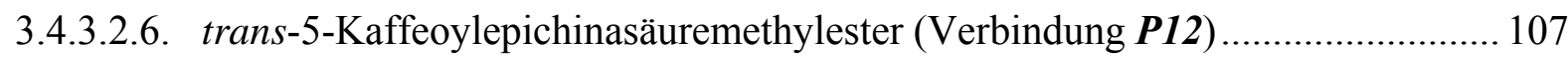

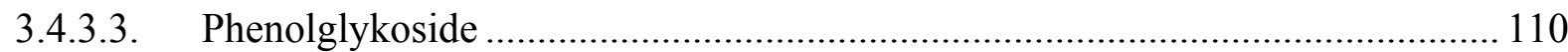

3.4.3.3.1. Isoquercitrin (Quercetin-3-O- $\beta$-D-glucosid) (Verbindung P13) ......................... 110

3.4.3.3.2. Rutosid (Quercetin-3-O-(6-O- $\alpha$-L-rhamnosyl)- $\beta$-D-glucosid)

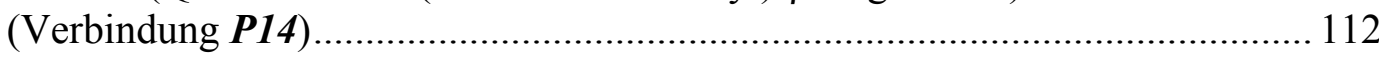

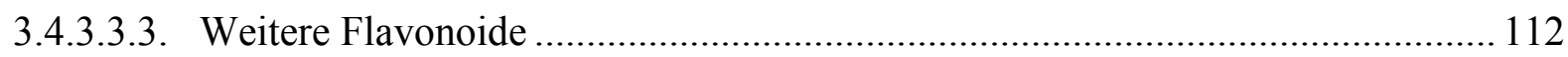

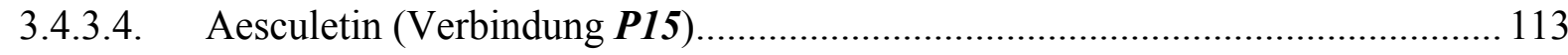

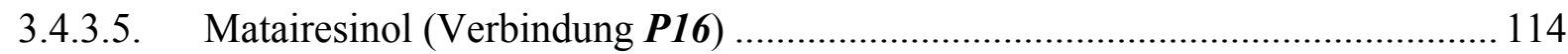

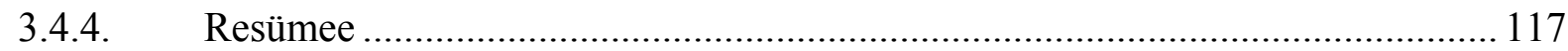

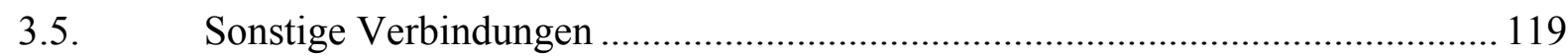

3.5.1. Verbindungen aus Dichlormethanextrakt................................................... 119

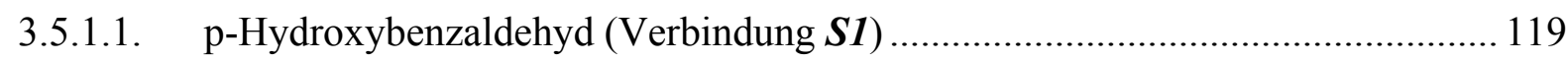

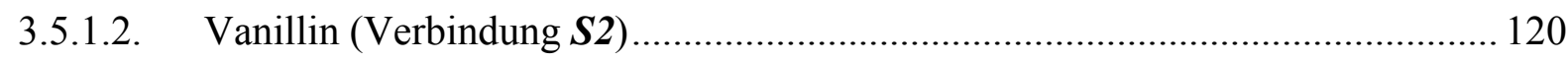

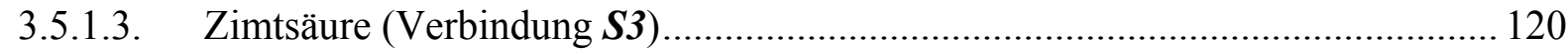

3.5.1.4. Spilanthessäure (Deca-2E,6Z,8E-triensäure) (Verbindung $S 4$ ) ........................... 121

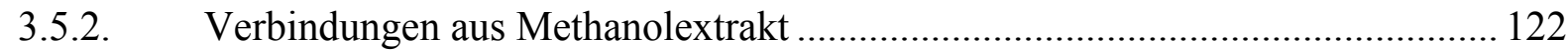

3.5.2.1. Fumarsäure (trans-Butendisäure) (Verbindung S5) ......................................... 122

3.5.2.2. Fumaroylisobutylamid ((E)-4-[(2-methylpropyl)amino]-4-oxo-

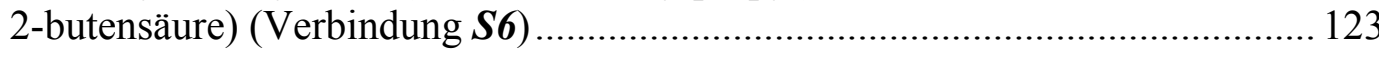

3.5.2.3. 4E-Hexenoylisobutylamid ((E)-6-[(2-methylpropyl)amino]-6-oxo-

3.5.2.4. Äpfelsäure (Monohydroxydicarbonsäure) (Verbindung S8) ............................. 126

3.5.2.5. Chinasäure (1,3,4,5-Tetrahydroxy-1-cyclohexen-1-carbonsäure

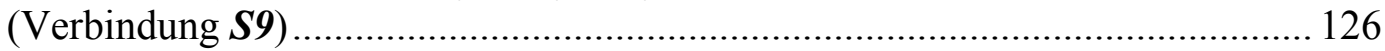

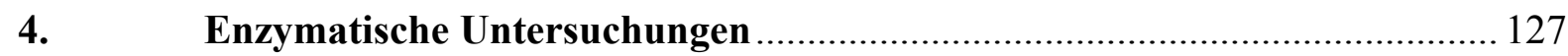

4.1. Hemmung der humanen Neutrophilen-Elastase............................................. 127

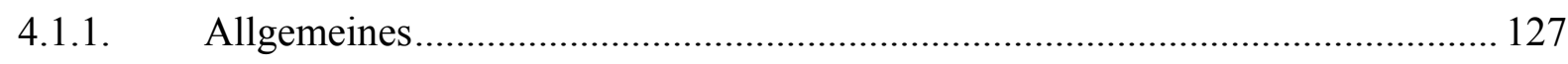

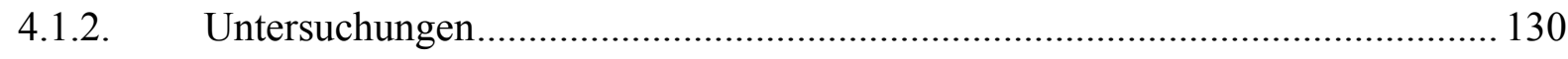

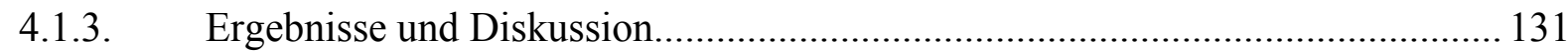

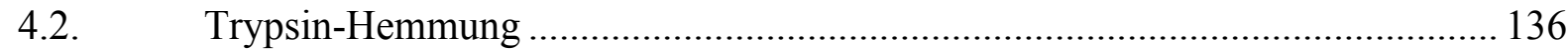

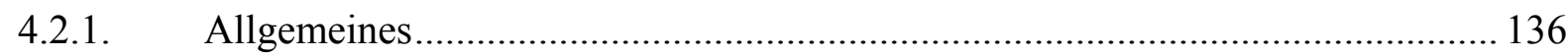

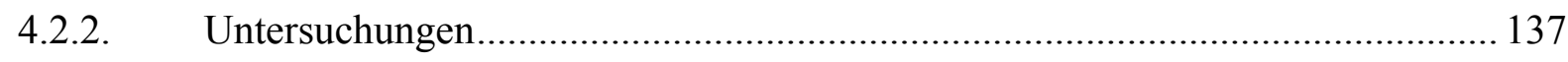

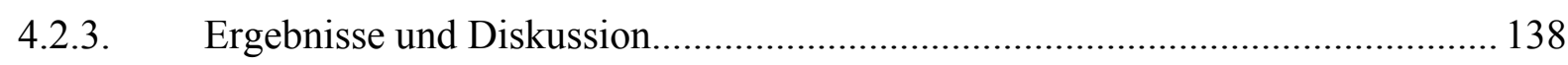

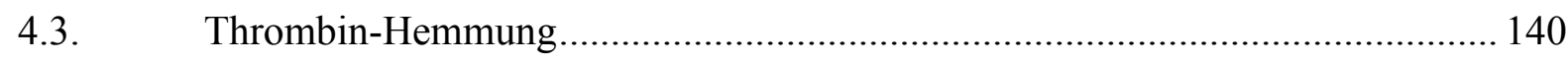

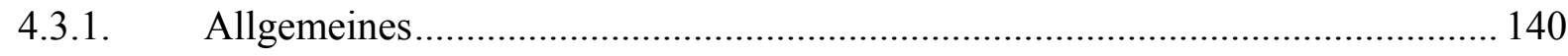




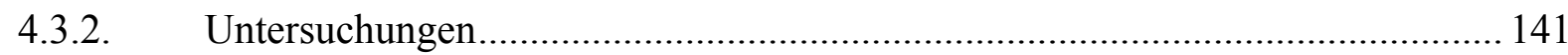

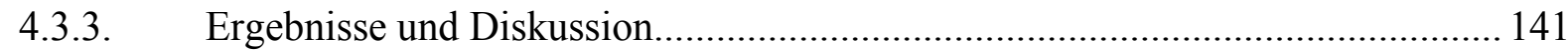

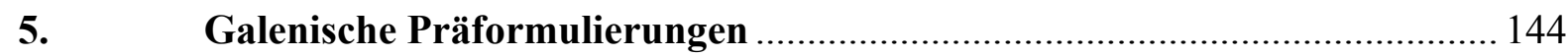

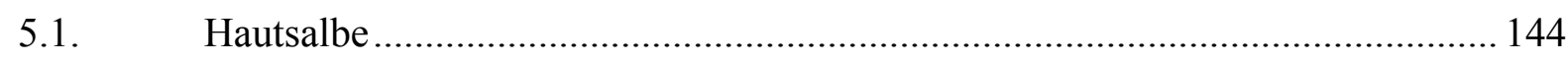

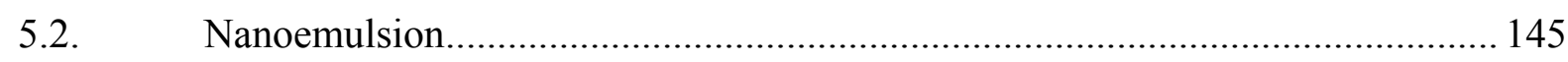

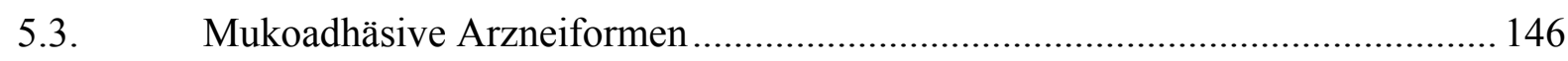

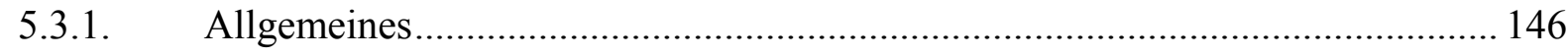

5.3.2. Haftpaste

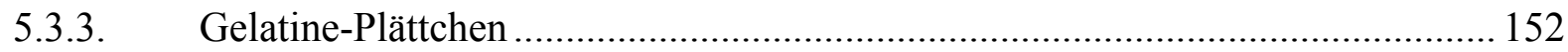

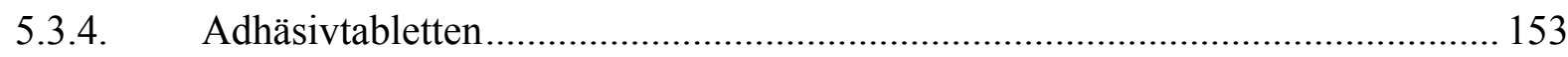

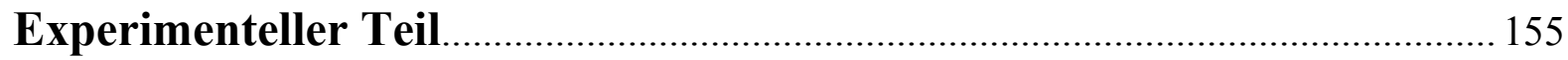

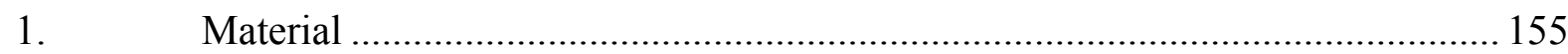

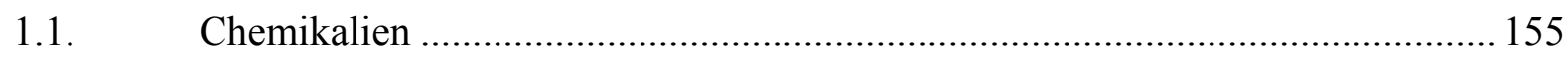

1.1.1. Lösungs-/ Extraktions-/ Fließmittel ............................................................. 155

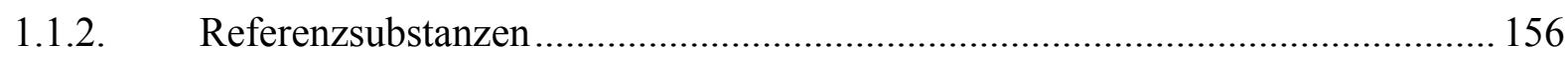

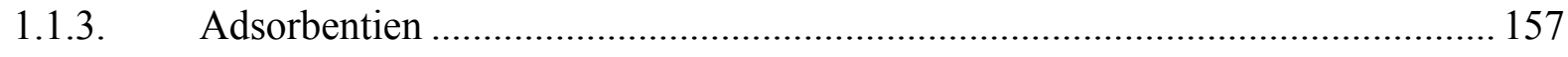

1.1.4. Reagenzien DC...................................................................................... 157

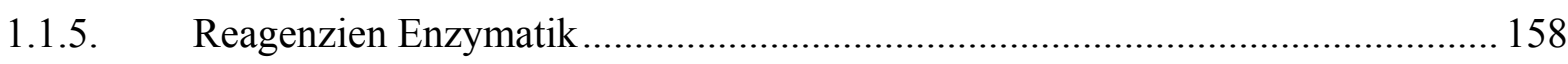

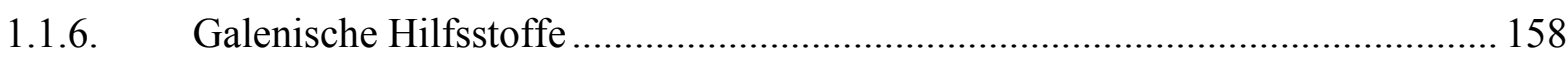

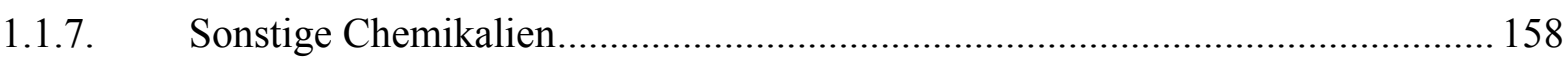

1.2. Geräte und sonstiges Zubehör .................................................................. 159

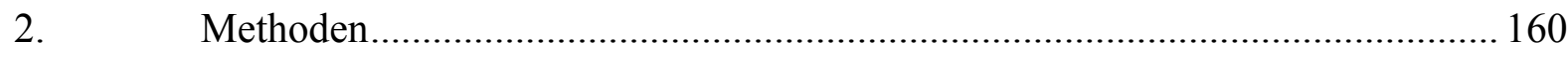

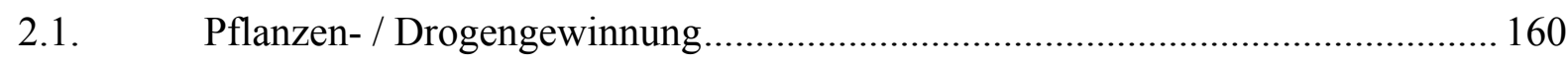

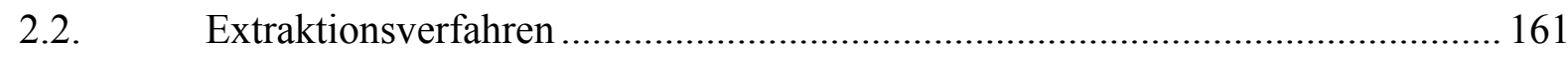

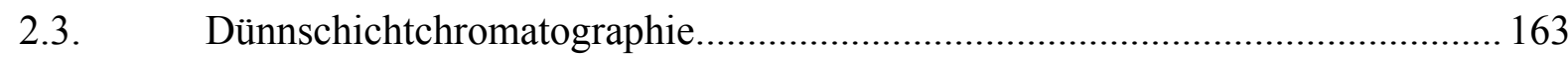

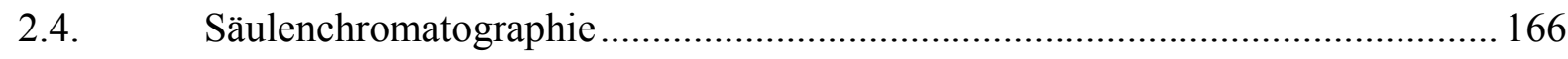

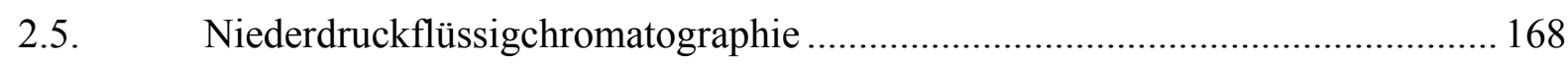

2.6. Hochleistungsflüssigchromatographie ....................................................... 170

2.7. Spektroskopische/- metrische Methoden ………………………………....... 175

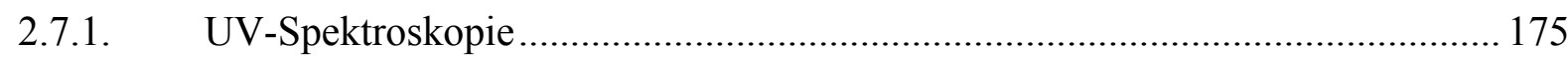

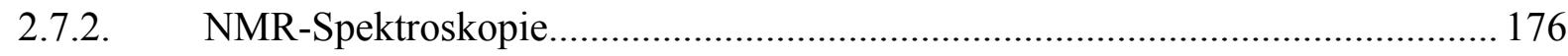

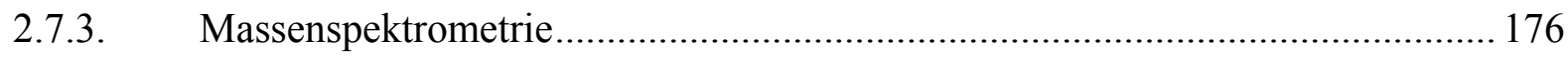

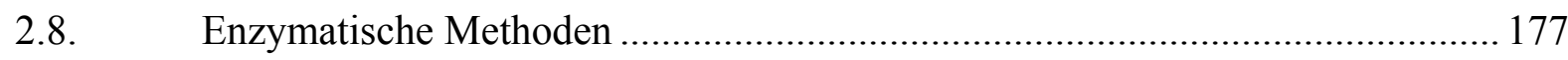


2.8.1. Hemmung der Aktivität der humanen Neutrophilen-Elastase ........................... 177

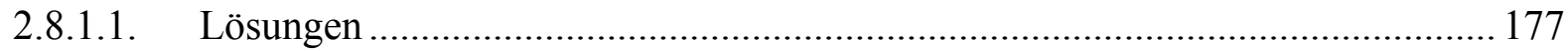

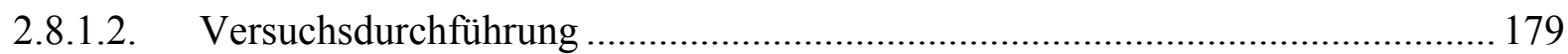

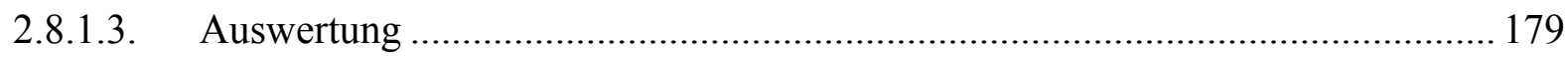

2.8.2. Hemmung der Trypsin-Aktivität ............................................................... 180

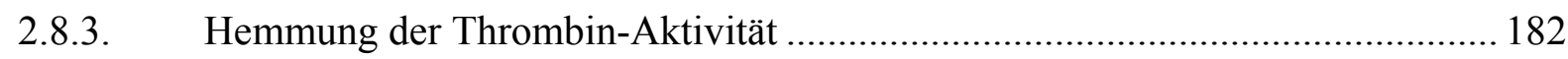

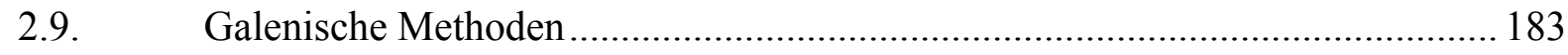

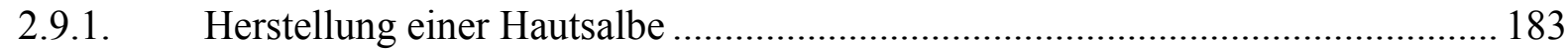

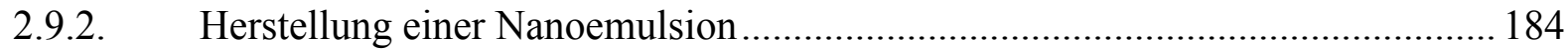

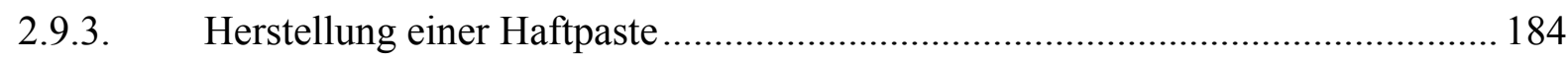

2.9.4. Herstellung von Glycerol-Gelatine-Plättchen ................................................ 185

2.9.5. Herstellung von Adhäsivtabletten ............................................................... 185

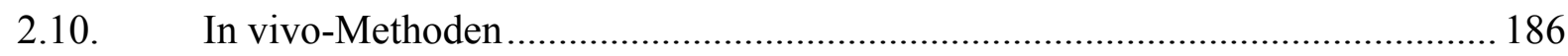

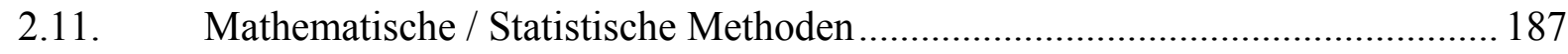

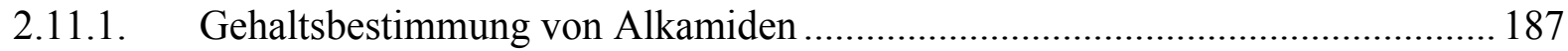

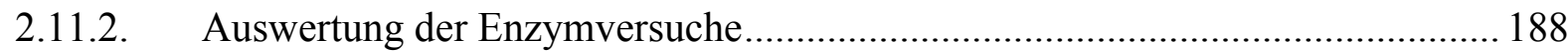

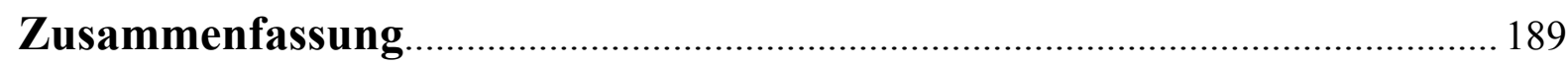

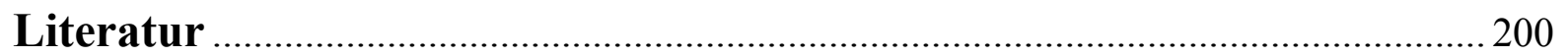





\section{Abkürzungsverzeichnis}

C5a

DC

dc

EI

ESI

FAB

FM

fMLP

GM-CSF

HPLC

IC

IL-6, IL-8

KG

LM

LPLC

LPS

MCP-1

MS

NMR

PAF

PMA

PAR

$\mathrm{R}$

$\mathrm{R}_{\mathrm{f}}$

RT

sh

SC

sc

SLPI

TNF- $\alpha$
Spaltprodukt von Komplementfaktor C5 (ein Anaphylatoxin)

Dünnschichtchromatografie / Dünnschicht-Chromatogramm

dünnschichtchromatografisch

Elektronenstoß-Ionisation

Elektro-Spray-Ionisation

fast atom bombardment

Fließmittel

$\mathrm{N}$-formyl-methionyl-leucyl-phenylalanin

granulocyte macrophage colony stimulating factor

high performance liquid chromatography

(Hochleistungsflüssigchromatografie)

inhibitory concentration

Interkeukin-6, Interleukin-8

Kieselgel

Lösungsmittel

low pressure liquid chromatographie (Niederdruckchromatographie)

Lipopolysaccharid

monocyte chemotactic protein-1

Massenspektrometrie

nuclear magnetic resonance (Kern-, Protonenresonanzspektroskopie)

platelet-activating factor

Phorbol-12-Myristat-13-Acetat

Protease-aktivierter Rezeptor

Reagenz

retention factor

retention time (Retentionszeit)

Schulter im UV-Spektrum

Säulenchromatografie

säulenchromatografisch

secretory leukocyte proteinase inhibitor

Tumor-Nekrose-Faktor 



\section{Einleitung}

Pflanzliche Arzneistoffe spielen eine wichtige Rolle in der Arzneitherapie. Am europäischen Phytopharmakamarkt hat Deutschland mit 38 \% (gefolgt von Frankreich mit $22 \%$ ) den größten Anteil (Quelle: IMS International 2005). Jedoch ist die Verordnungsfähigkeit dieser Präparate auf Grund der derzeit von den deutschen Krankenkassen praktizierten eingeschränkten Erstattungsfähigkeit, insbesondere auf dem Sektor der Phytotherapeutika, stark limitiert. Ein Grund dafür ist der häufig fehlende, eindeutige Wirksamkeitsnachweis. Die Anwendung vieler Phytopharmaka beruht auf volksmedizinischen Erkenntnissen, jedoch zumeist auf reichen, positiven Erfahrungen.

Das Problem ihrer Standardisierung erklärt sich aus dem Vielstoffgemisch, das z. B. in Tees oder auf Basis von Pflanzenextrakten hergestellten Arzneiformen vorliegt und eine komplexe Wirkung entfaltet. Selbst die Festlegung auf einen Hauptwirkstoff würde hier nur bedingt eine definierte Aussage zur Gesamtwirkung zulassen.

Intensive Forschungsarbeiten auf dem Phytopharmakagebiet auf nationaler und internationaler Ebene haben jedoch, dank der modernen Analysenverfahren und pharmakologischen Methoden, in den vergangenen Dezennien bedeutende Fortschritte erzielt und die Zulassung bzw. Registrierung entsprechender Arzneimittel bewirkt, sofern deren Qualität, Wirksamkeit und Unbedenklichkeit zu belegen waren. In den letzten Jahren vollzogen sich diesbezüglich bedeutende regulatorische Entwicklungen auf europäischer Ebene. In der Richtlinie 2004/24/EG wurde neben den durch ihren „,well established medical use“ (bibliographische Zulassung) charakterisierten Phytopharmaka mit den „traditionellen“ (Kriterien: ausreichende Qualität und Unbedenklichkeit, Wirkung bei geringfügigen Gesundheitsstörungen auf Grund mindestens 30-jähriger medizinischer Erfahrung plausibel) pflanzlichen Arzneimitteln eine weitere Gruppe pflanzlicher Zubereitungen im europäischen Arzneimittelrecht verankert.

Die große Pflanzenfamilie der Asteraceae (Korbblütler) umfasst allein im Tribus Heliantheae etwa 189 - 300 Gattungen mit ca. 2500 - 3000 Arten. Viele der heimischen Korbblütler haben Aufnahme in den Arzneischatz gefunden, z. B. Arnica montana L., Artemisia absinthium L., Echinacea-Arten, Solidago-Arten, Tussilago farfara L. und zahlreiche andere. Die meisten 
dieser Pflanzen-Präparate zeigen milde, aber volksmedizinisch außerordentlich geschätzte Wirkungen und sind zumeist nebenwirkungsarm.

Die Asteraceae gehören $\mathrm{zu}$ den wenigen Pflanzenfamilien, die als Sekundärstoffwechselprodukte die relativ selten auftretenden Alkamide - in bemerkenswerter struktureller Vielfalt - führen. Im Tribus Heliantheae, Subtribus Ecliptinae, findet sich neben der in letzter Zeit intensiv beforschten Gattung Echinacea die Gattung Acmella mit großem Artenreichtum, ursprünglich beheimatet in Südamerika, heute weit verbreitet in tropischen und subtropischen Regionen. Beide Gattungen zeigen chemotaxonomische Ähnlichkeiten, wobei besonders die Alkamide gegenwärtig für die vielfältigen und pharmakologisch relevanten Wirkungen als verantwortlich angesehen werden.

In der Roten Liste $[152,224]$ ist unter dem Namen Spolera ${ }^{\circledR}$ ein Präparat zur äußerlichen Anwendung aus Acmella ciliata (Humboldt, Bonpland \& Kunth) Cassini registriert. Die hiesige medizinische Anwendung geht aber noch weiter zurück, was die Registrierung im DAB 1 von 1872 von Spilanthes oleracea Linnaeus, eine veraltete Nomenklatur für eine Acmella-Art (Acmella oleracea (Linnaeus) R. K. Jansen), verdeutlicht. Hagers Handbuch der Pharmazeutischen Praxis von 1927 führt Herba Spilanthis oleracea und Tinctura Spilanthis (oleracea) composita (mit Bertramwurzel) zusammen auf.

Diese offensichtliche Bedeutung von Vertretern der Gattung Acmella als potente Pharmaka veranlasste uns zur phytochemischen Bearbeitung der etwas weniger bekannten Art Acmella ciliata (H. B. K.) Cass. und ihren Anwendungsmöglichkeiten. 


\section{Problemstellung}

Auf Grund der seit 1985 [81] gültigen botanischen Klassifikation, nach der Acmella und Spilanthes eigenständige Gattungen und nicht wie vorher nur Sektionen bilden, war es zunächst Anliegen dieser Arbeit, die Merkmale der beiden Arten Acmella ciliata (Humboldt, Bonpland \& Kunth) Cassini und Acmella oleracea (L.) Jansen (vorher: Spilanthes oleracea L.) nach paralleler Aufzucht detailliert makroskopisch und mikroskopisch zu charakterisieren, um weitere Verwechslungen weitgehend auszuschließen.

Auch in das anschließende DC-Screening zur Erfassung der Hauptinhaltsstoffklassen wurden beide Acmella-Arten (Acmella oleracea in zwei Varietäten) einbezogen. Die nachfolgenden Untersuchungen beschränkten sich dann vorwiegend auf Acmella ciliata.

Im Vordergrund der phytochemischen Studien stand in Erweiterung der Arbeiten von MARTIN [110 - 112], der die Blütenköpfchen hinsichtlich ihres Amidspektrums untersuchte, die Erschließung der Gesamtpflanze durch apolare und polare Extraktion mit Konzentration einmal auf die Alkamide und zum anderen auf die phenolischen Inhaltsstoffe, von denen bisher wenig Kenntnis existiert.

Weitere Zielstellung der Arbeit war die Untersuchung ausgewählter Acmella-Extrakte hinsichtlich ihrer Hemmwirkung gegenüber Enzymen (Elastase, Trypsin, Thrombin), auf die die in der traditionellen Anwendung dominierende Entzündungshemmung maßgeblich zurückzuführen sein dürfte.

Schließlich sollten Arzneiformen entwickelt werden, die sich für orientierende in vivoTestungen eigneten. Diese galenischen Präformulierungen waren u. a. auf Basis moderner Prinzipien der Mukoadhäsion zwecks bukkaler bzw. sublingualer Applikation zu konzipieren. 


\section{Allgemeiner Teil}

\section{Beschreibung der Pflanze(n)}

\subsection{Taxonomie}

Die Taxonomie soll der Arbeit vorangestellt werden, da die botanische Einordnung von Acmella ciliata (Humboldt, Bonpland \& Kunth) Cassini sowie ihrer Verwandten bis heute Probleme aufwirft und in der Literatur nicht einheitlich gehandhabt wird.

Lange Zeit wurden die Pflanzen der heutigen Gattung Acmella lediglich als eine eigene Sektion innerhalb der Gattung Spilanthes geführt [25]. Dadurch kam und kommt es zu Verwechslungen und Überschneidungen zwischen verschiedenen Acmella-Arten sowie mit Pflanzen, die früher als eigenständige Arten innerhalb der Gattung Spilanthes galten, nun seit JANSEN 1981/1985, [81, 82] - aber als Art der Gattung Acmella zugeordnet sind.

Diese Verwechslungen erstrecken sich darüber hinaus teilweise auf Spezies weiterer Gattungen desselben Subtribus (Ecliptinae), wie Blainvillea und Bidens. Auch die Zuordnung der Gattungen Acmella und Spilanthes zum entsprechenden Subtribus innerhalb der Heliantheae wurde immer wieder revidiert. Die hier dargestellte Zuordnung zu den Ecliptinae folgt der aktuellen Einteilung nach ROBINSON [149]. Nicht nur morphologische Merkmale, sondern auch ähnliche Inhaltsstoffspektren der verschiedenen Arten und sogar Gattungen waren und sind Ursache für diese Verwechslungen. Die Analogien bereiteten auch diversen Beschreibern Probleme, was sich in einer Vielzahl von Überschneidungen botanischer Namen, gehäufter Synonymbildungen und vielfältiger taxonomischer Revidierungen und Neueinordnungen einzelner Pflanzen in andere Taxa äußert. Weiterhin trugen die Überlieferungen von Eingeborenen, die verschiedene Pflanzen eines Lebensraums mit gleichen Eigenschaften und Wirkungen auch für gleiche Zwecke einsetzten, zu der Konfusion bei.

Spätere genetische, anatomisch-morphologische sowie phytochemische Untersuchungen zeigten, dass es tatsächlich enge verwandtschaftliche Beziehungen zwischen den genannten Genera gibt, besonders zwischen Acmella, Spilanthes und Salmea [82]. Auf Grund morphologischer Merkmale und an Hand von Chromosomenuntersuchungen ergaben sich 
sogar engere verwandtschaftliche Beziehungen zwischen den Gattungen Spilanthes und Salmea als zwischen Acmella und Spilanthes [81, 82].

Innerhalb des Subtribus Ecliptinae werden die Verwandtschaftsbeziehungen durch chemotaxonomische Gemeinsamkeiten untermauert. So kommt die Inhaltsstoffgruppe der Alkamide im gesamten Subtribus vor, wobei einige Alkamide nicht nur innerhalb einer Gattung, sondern auch innerhalb verschiedener Gattungen und Arten desselben Subtribus identisch sind (vgl. z. B. Acmella- und Echinacea-Alkamide (3.1.2.)).

Blickt man auf die botanische Geschichte, so wurde Acmella erstmalig 1807 von RICHARD [147] beschrieben als eine Gattung mit fünf radiären, nicht-pappösen Arten. 1822 verbesserte CASSINI [25] die Charakterisierung dieser Art und diskutierte die Verwandtschaft zu Spilanthes Jaquin. Spilanthes unterscheidet sich demnach durch scheibenförmige Blüten und einen begrannten Pappus von Acmella. Auf Grund der jedoch insgesamt geringen Unterschiede zwischen Acmella und Spilanthes entschied CASSINI, beide als gattungsverwandte Sektionen zu betrachten. DeCANDOLLE [32] folgte der Einteilung von CASSINI und beschrieb darüber hinaus mehrere neue Spezies in beiden Sektionen. Auch MoORE (1907) [126] folgte der vorausgegangenen Einteilung in Sektionen, unterteilte sie allerdings weiter in die Untersektionen Magnoradiatae und Parvoradiatae, basierend auf der relativen Länge der Strahlenblüten (Zungenblüten) und der Tegula (Hüllschuppen).

JANSEN und StUESSY (1980, [80]) und JANSEN (1981, [82]) entschieden sich dagegen auf Grund morphologischer und chromosomaler Belege für die Trennung von Acmella und Spilanthes in zwei eigenständige Gattungen. Die Parameter für die Trennung in zwei eigenständige Taxa waren: Art des Pappus, Morphologie und Anatomie der Achänen, die Art der Blattbefestigung am Stängel sowie die Beschaffenheit des Blütenkopfs und die Farbe der Kronblätter.

Die derzeit gültige taxonomische Beschreibung der Gattung Acmella stammt von JANSEN aus dem Jahr 1985 [81]. Danach besteht die Gattung aus 30 Arten und neun weiteren Varietäten. Es sind dies: Acmella radicans var. radicans, A. radicans var. debilis, A. darwinii, A. sodiroi, A. leucantha, A. alba var. ecuadorensis, A. alba var. alba, A. calva, A. pusilla, A. paniculata, A. uliginosa, A. oleracea, A. bellidioides, A. grandiflora var. discoidea, A. grandiflora var. brachyglossa, A. grandiflora var. grandiflora, A. caulirhiza, A. oppositifolia var. 
oppositifolia, A. oppsositifolia var. repens, A. ciliata, A. iodiscaea, A. brachyglossa, A. glaberrima, A. filipes var. cayensis, A. filipes var. parvifolia, A. filipes var. filipes, A. alpestris, A. psilocarpa, A. pilosa, A. poliolepidica, A. grisea, A. papposa var. papposa, A. papposa var. macrophylla, A. decumbens var. decumbens, A. decumbens var. affinis, $A$. ramosa, A. lundellii, A. leptophylla, A. serratifolia.

JANSEN [81] unterteilt die Gattung Acmella weiter in die drei Hauptgruppen oder Sektionen Acmella (mehrjährige), Annuae (einjährige) und Megaglottis (großblütige) und untermauert dies durch phylogenetische Studien. Die Pflanze Acmella ciliata (H. B. K.) Cassini ist der Sektion Acmella, die Pflanze Acmella oleracea (L.) Jansen hingegen der Sektion Annuae zuzuordnen. Hauptunterschied bei der botanischen Bestimmung bleibt der Aufbau des Blütenkopfs (strahlenförmig oder scheibenförmig, d. h., mit oder ohne Zungenblüten (s. 1.3.)).

Die Gattung Spilanthes hingegen besteht nach der 1981 veröffentlichten Arbeit von JANSEN [82] lediglich aus den sechs Spezies: Spilanthes leiocarpa, S. nervosa, S. paraguayensis, S. anactina, S. costata und S. urens.

Botanischer „Stammbaum“ von Acmella ciliata (Abb. 1): Ordnung: Asterales; Familie: Asteraceae; Unterfamilie: Asteroideae; Tribus: Heliantheae; Subtribus: Ecliptinae; Gattung: Acmella; Art: Acmella ciliata.

Bisher kam es ständig zu Verwechslungen von Acmella ciliata mit der bekannteren Art Acmella oleracea (Abb. 1), die im Gegensatz zu Acmella ciliata erst durch JANSEN 1985 [81] ihre eindeutige botanische Zuordnung erhielt.

Die Gefahr, Acmella ciliata und Acmella oleracea miteinander zu verwechseln, besteht hauptsächlich im getrockneten Zustand, bedingt durch Ausfallen der Zungenblüten von Acmella ciliata beim Trocknungsprozess (siehe auch Beschreibung der Pflanzen, 1.3.). Im frischen Zustand sind beide Pflanzen nebeneinander an Hand ihrer verschiedenen Blütenköpfe gut zu unterscheiden. Dennoch zeigt z. B. eine neuere Abbildung aus dem asiatischen Raum eindeutig Acmella ciliata, die aber mit Spilanthes oleracea, also Acmella oleracea Jansen, untertitelt ist [194]. Drei weitere Fotos zeigen Acmella ciliata mit der Benennung Spilanthes acmella ([200, 204, 211] und s. u.). 
Acmella oleracea taucht bis heute in der Literatur unter verschiedenen Bezeichnungen auf, wobei sie i. d. R. fälschlicherweise noch der Gattung Spilanthes zugerechnet wird, wie zeitweilig auch Acmella ciliata als Spilanthes ciliata (s. u.). Als veraltete Synonyme für Acmella oleracea finden sich: Spilanthes acmella DC. var. oleracea (L.) Hook. f., Spilanthes acmella Murr. var. oleracea Hook. f., Spilanthes acmella DC. var. oleracea (Jacq.) Baker, Spilanthes acmella DC. var. oleracea (L.) Zoll., Spilanthes acmella var. oleracea (Linnaeus) Clarke ex Hooker f., Spilanthes oleracea Jacq., Spilanthes oleracea L., Spilanthus oleracea L., Pyrethrum spilanthus Medikus, Bidens fervida Lamarck, Bidens oleracea (L.) Cav. ex Steud. [81, 212, 214, 219, 221].

Auch für Acmella ciliata existieren einige wenige Synonyme, wie Spilanthes ciliata Humboldt, Bonpland \& Kunth, Ceratocephalus ciliatus (H. B. K.) Kuntze sowie manchmal Spilanthes acmella (L.) J. A. Murray [81, 196, 214, 217]. Dieser letztgenannte Name findet sich jedoch auch als Synonym für weitere Acmella-Arten, woraus ein großes Verwechslungspotential mit anderen Pflanzen resultiert. Da die Bezeichnung Spilanthes acmella aber oft zusammen mit dem Zusatz einer bestimmten Varietät geführt wird, erleichtert sich die Zuordnung. So steht z. B. Spilanthes acmella var. oleracea für Acmella oleracea (L.) Jansen. In den meisten Veröffentlichungen dürfte allerdings mit dem Namen Spilanthes acmella tatsächlich Blainvillea acmella (L.) Philipson gemeint sein, also ein Vertreter einer anderen Gattung innerhalb des Subtribus Ecliptinae (s. [81, 220, 221]).

Nach JANSEN [81] handelt es sich bei Acmella oleracea um eine Zuchtform, die aus der in Zentral-Peru heimischen Acmella alba (L' Heritier) Jansen hervorging und teilweise ausgewildert ist. Diese Zuchtform wird inzwischen im Handel in einer roten und einer gelben Variante angeboten. Beide werden unter den Namen Parakresse (Vorkommen u. a. im heutigen brasilianischen Bundesstaat Para), Husarenknopf (siehe Beschreibung, 1.3.), Augapfelpflanze, Prickelknöpfchen oder Zahnwehpflanze (engl.: toothache plant) geführt bzw. unter der veralteten botanischen Bezeichnung Spilanthes oleracea im Pflanzenfachhandel und in Baumärkten vertrieben. So wurden im Laufe dieser Arbeit Samen von „Spilanthes oleracea“ der Firma Floraself/Hornbach ausgesät. Das Foto auf der Samentüte zeigte eindeutig die rote Variante, heraus kam aber die gelbe.

Da es bisher keine vergleichenden Beschreibungen und auch keine genetischen Untersuchungen der beiden Varianten gibt, offensichtlich aber eine große Verwandtschaft 
zwischen beiden Pflanzen besteht, soll im Folgenden von der gelben und roten Varietät von Acmella oleracea gesprochen werden (s. auch 1.3. und [222]). Im Laufe dieser Arbeit wurden eine vergleichende makroskopische und mikroskopische Beschreibung sowie erste vergleichende phytochemische Untersuchungen durchgeführt (s. 1.3., 1.4., 3.2.2.).

Zur Benennung „Zahnwehpflanze“ ist anzumerken, dass diese Bezeichnung auf die Anwendung südamerikanischer Ureinwohner zurückgeht, Blätter der Pflanze bei Zahnschmerzen zu kauen [81]. Zutreffend dürfte dieser Name jedoch wohl eher für Acmella ciliata und weitere verwandte Arten sein, die in Südamerika ursprünglich natürlich vorkamen, nicht für die Zuchtvarianten.

Die geschilderten taxonomischen Probleme verdeutlichen, wie schwierig es zu beurteilen ist, mit welcher Pflanze in der Literatur dargestellte Untersuchungen tatsächlich durchgeführt wurden, sofern nicht eindeutig die heute gültige Nomenklatur, d. h., im konkreten Fall der Name Acmella ciliata genutzt wird. Wenn man allerdings davon ausgeht, dass die Inhaltsstoffe innerhalb der heutigen Gattung Acmella sowie anderer Gattungen der Ecliptinae qualitativ sehr ähnlich bis gleich sind, erscheint es opportun, Literatur heranzuziehen, die heute als veraltet geltende Namen benutzt bzw. sich auf eng verwandte Arten bezieht, zumindest um Hinweise auf Inhaltsstoffe und Vorgehensweisen zu erhalten.

Anmerkung: Im Laufe der Arbeit werden Pflanzennamen aus zitierter Literatur mit definitiv veralteter Nomenklatur in Anführungszeichen gesetzt; z. B. steht „Spilanthes oleracea“ L. für Acmella oleracea (L.) Jansen.

\subsection{Vorkommen}

Die Heimat von Acmella ciliata ist das tropische Südamerika, wo diese Pflanze vom Meeresspiegelniveau bis in Höhen von $2600 \mathrm{~m}$ vorkommt. Fundorte liegen in den Ländern Bolivien, Brasilien, Kolumbien, Ecuador, Peru, Venezuela, Argentinien. Eingeschleppt wurde die Pflanze auch in Panama, den Galapagos Inseln (zu Ecuador), Indien und Teilen Südostasiens (Thailand, Laos, Malaysia, Indonesien, Japan, Taiwan). Das Vorkommen in Laos wird vom Verfasser selbst hier erstmalig beschrieben, der ein Exemplar in der laotischen Hauptstadt Vientiane entdeckte. Acmella ciliata bevorzugt feuchte, verunkrautete Lebensräume und ist meist entlang von Flüssen, Straßen und Feldern anzutreffen [28, 81, 192, 195 $197,202,203,205,207,211,213,215,217,225,227,228]$. 
Acmella oleracea ist ausgewildert ebenfalls in verunkrauteten Habitaten in folgenden Ländern Südamerikas anzutreffen: Bolivien, Brasilien, Ecuador, Peru sowie auf Martinique (Karibik), in Afrika (Madagaskar, Tansania und Kenia) und in Asien in Indien und Nepal [69, 81, 198, $199,216,218,223,226,228]$.

\subsection{Makroskopische Beschreibung}

Da es möglich war, neben Acmella ciliata auch die gelbe Varietät von Acmella oleracea als Samen und die rote Varietät als Pflanze zu erhalten, wurden die Pflanzenbeschreibung sowie einige grundlegende phytochemische Untersuchungen auf alle drei (Abb. 1) ausgedehnt, um Gemeinsamkeiten und Unterschiede herauszuarbeiten (s. auch 1.4. und 3.2.2.).

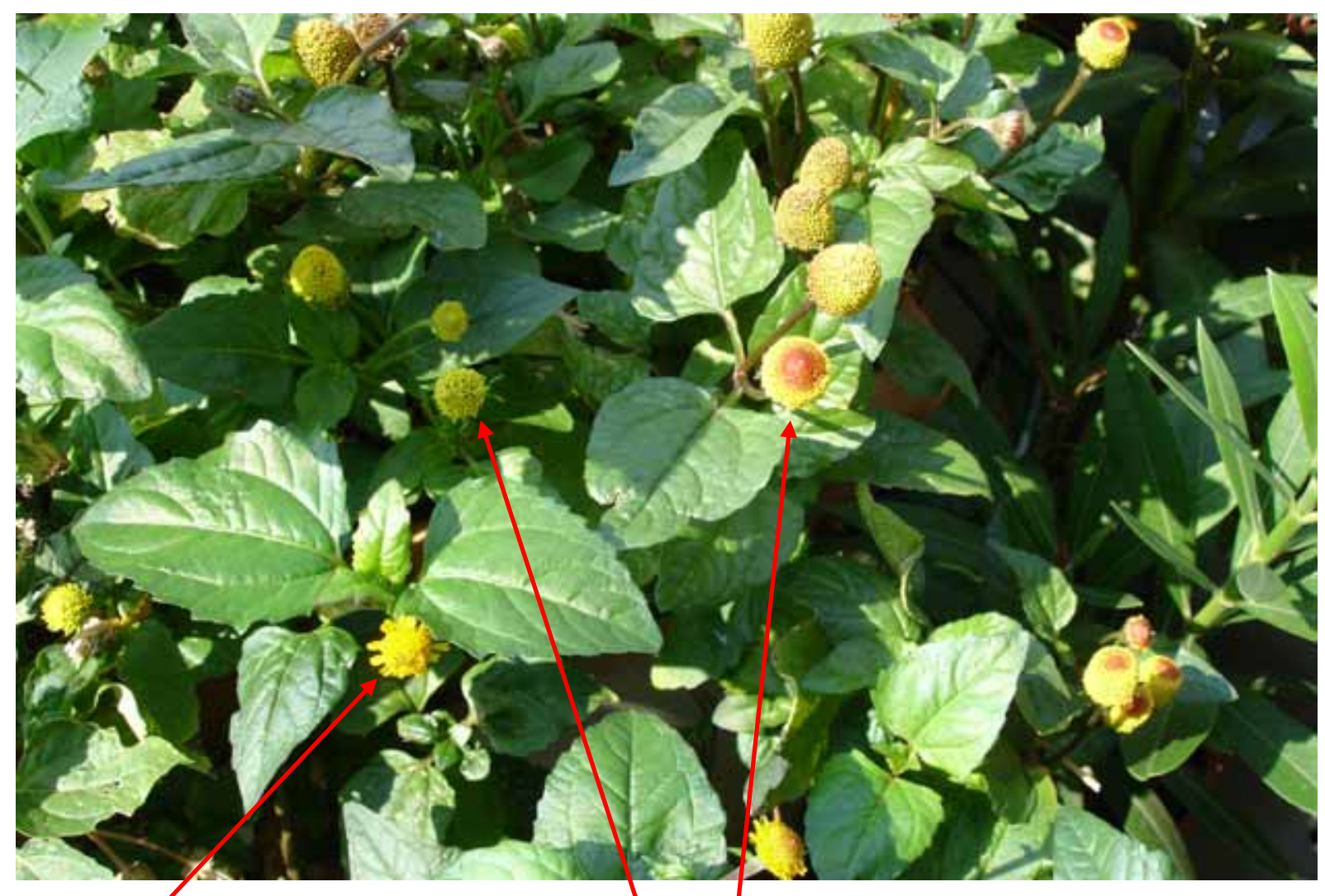

Abb. 1: Acmella ciliata neben Acmella oleracea (gelbe und rote Varietät)

\section{Acmella ciliata}

Es handelt sich um eine in der Herkunftsgegend mehrjährige, krautige Pflanze. Zunächst erscheint ein Primärspross in vertikaler Richtung. Mit zunehmendem Alter und bei entsprechendem Platz entwickelt die Pflanze mehrere horizontale, oberirdische Triebe mit langen Internodien, die sich an den Nodien wiederum verzweigen und dort auch neue 
sprossbürtige Wurzeln sowie neue Triebe entwickeln können. Dies führt zur raschen Besiedlung großer Flächen. Die vertikalen Triebe sind nach kurzer Zeit niederliegend, so dass die Pflanze in der Höhe kaum mehr als $30 \mathrm{~cm}$ erreicht.

Die Bewurzelung der Pflanze ist generell allorhiz. Die einzelnen Wurzeltriebe weisen einen Durchmesser von 1-5 mm auf und bilden dichte Geflechte. Der Wurzelstock kann in Tiefe und Breite ca. $30 \mathrm{~cm}$ erreichen.

Die vertikalen, zunächst monopodialen Triebe entwickeln gegenständig angeordnete, gestielte Laubblätter von zugespitzt herzförmiger Gestalt mit gesägtem Blattrand (Abb. 1). Die Blätter sind von grüner Farbe, die unteren Blattadern und Blattränder älterer Blätter, ebenso wie ältere Haupttriebe, eher dunkelrot-violett. Die Farbintensität nimmt bei vermehrter Sonneneinstrahlung zu. Spross, Blattstiel und Blätter zeigen eine schwache bis mittelstarke Behaarung.

Der Primärspross schließt mit einem terminalen, langgestielten Blütenstand ab, dessen Stiel die Länge der Blattstiele übertrifft. Der Endblütenstand wird von zwei weiteren, im spitzen Winkel angeordneten, blattachselständigen Blütenständen flankiert, die oft den Hauptblütenstand überragen und die Anfänge neuer Seitentriebe darstellen. Die Seitentriebe entwickeln sich generell blattachselständig. Somit wandelt sich der Habitus von anfänglich monopodialer zu monochasialer Gestalt.

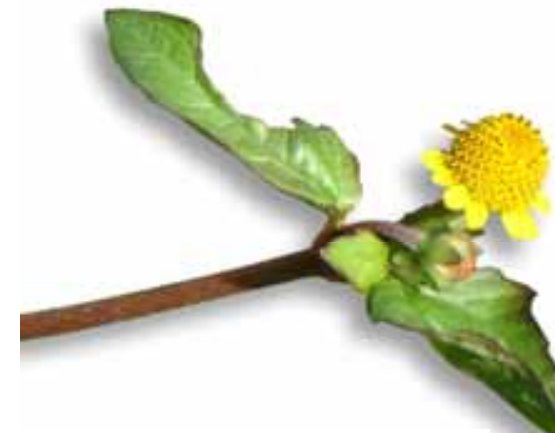

Abb. 1a: Blütenkopf, (Blätter, Stängel) von $A$. ciliata
Die Blütenköpfe - zunächst die Gesamtheit der Röhrenblüten ohne Zungenblüten betrachtet - haben radialen Querschnitt, sind anfänglich fast flach, nehmen aber mit zunehmendem Alter eine rundlichkegelförmige Gestalt an (Abb. 1 und 1a), wobei das Ende erst spitz zulaufend, ausgereift später sanft abgerundet ist. Die Farbe ist anfänglich gelb und in der Mitte ungleichmäßig schwach rötlich, voll erblüht dann gelb-orange. Die Farbe der kurzen Zungenblüten, die nur wenig die Hüllblatter (Hüllschuppen) überragen, ist stets gelb, der Hüllkelch, einschließlich der Spreublätter, grün. Ein einfacher Kranz von aus drei miteinander verwachsenen Kronblättern bestehenden Zungenblüten umgibt die in eben erwähnter Gestalt vereinigten Röhrenblüten. Die Röhrenblüten bestehen aus fünf miteinander kreisrund 
verwachsenen Kronblättern. Der als Pappus entwickelte Kelch fehlt meist bei Zungen- und Röhrenblüten oder besteht selten aus zwei ungleichen Federn.

Als Früchte sind bei Zungen- und Röhrenblüten die bei den Asteraceen typischen einsamigen Achänen anzutreffen. Diese sind mäßig bis dicht bewimpert bzw. behaart (Zwillingshaare) und von schwarzer Farbe, haben keine Schultern, aber einen korkähnlichen Rand. Hier ist, wie für die Gattung Acmella insgesamt, ein Dimorphismus zu beobachten, indem die Achänen der äußersten Reihen der Röhrenblüten drei-flügelig mit drei Gefäßsträngen sind, die inneren nur zwei-flügelig mit zwei Gefäßsträngen.

Die gametische Chromosomenzahl beträgt $\mathrm{n}=39$ [81], und die Anzahl somatischer Chromosomen in der mitotischen Metaphase ist 2n $=78$ [28].

Acmella oleracea (gelbe und rote Varietät)

RAMSEWAK et al. [144] sprechen bereits von zwei Varietäten und geben der gelben Varietät den Namen Spilanthes acmella L. var. oleracea, der roten Varietät den Namen Spilanthes acmella L., wobei sie sich auf die Beschreibung von NAKATANI et al. [134] beziehen. In Herbalistics, einem Internet-Samenvertrieb, werden die rote Variante als Spilanthes oleracea, die gelbe jedoch als Spilanthes acmella angeboten, für beide aber diesselben Eigenschaften und Inhaltsstoffe benannt [209]. Obwohl diese Angaben erst aus den 1990er Jahren stammen, dürften sie bereits durch die nunmehr verbindliche Nomenklatur von JANSEN [81], spätestens seit 1985, obsolet sein, wonach der „Parakresse“ der Gattung Acmella, Sektion Annuae, die Bezeichnung Acmella oleracea zukommt. JANSEN [81] schreibt allerdings in seinem Grundsatzwerk über Acmella lediglich von einer Art und keiner weiteren Varietät. Seiner Ausführung ist zu entnehmen, dass es sich um die hier als Acmella oleracea (rot) bezeichnete Pflanze handeln muss.

Da sich die Merkmale dieser einjährigen Pflanze deutlich von denen anderer Arten unterscheiden, erhielt Acmella oleracea den Status einer eigenen Art (s. v.). Als ursprüngliche Zuchtsorte, die später auswilderte, ist sie jedoch am bekanntesten und wird häufiger als Acmella ciliata global angebaut und angewendet.

Der Aufbau von Spross und Wurzel sowie der generelle Habitus sind Acmella ciliata sehr ähnlich. Es fällt aber auf, dass die Bildung oberirdischer Ausläufer vermindert ist, ebenso die 
Länge der Internodien, was vor allem für die gelbe Varietät zutrifft und sie dadurch besonders buschig und kompakt erscheinen lässt (Abb. 2).

Die Art der Bewurzelung lässt, ebenso wie die Behaarung von Pflanzenteilen, keinen Unterschied zwischen Acmella oleracea und Acmella ciliata erkennen. Allerdings kommt es bei Acmella oleracea nicht zur Sekundärbewurzelung an den Nodien. Dies wiederum scheint eine Voraussetzung für die Mehrjährigkeit von Acmella ciliata zu sein. Die Wuchshöhe ist bei gleichen Lichtverhältnissen auch in etwa identisch. (Weniger Licht führt $\mathrm{zu}$ größerer Wuchshöhe, bis zum Ausgeilen des Triebes; beste Wachstumsbedingungen für Acmella ciliata im Halbschatten als Regenwaldpflanze.)

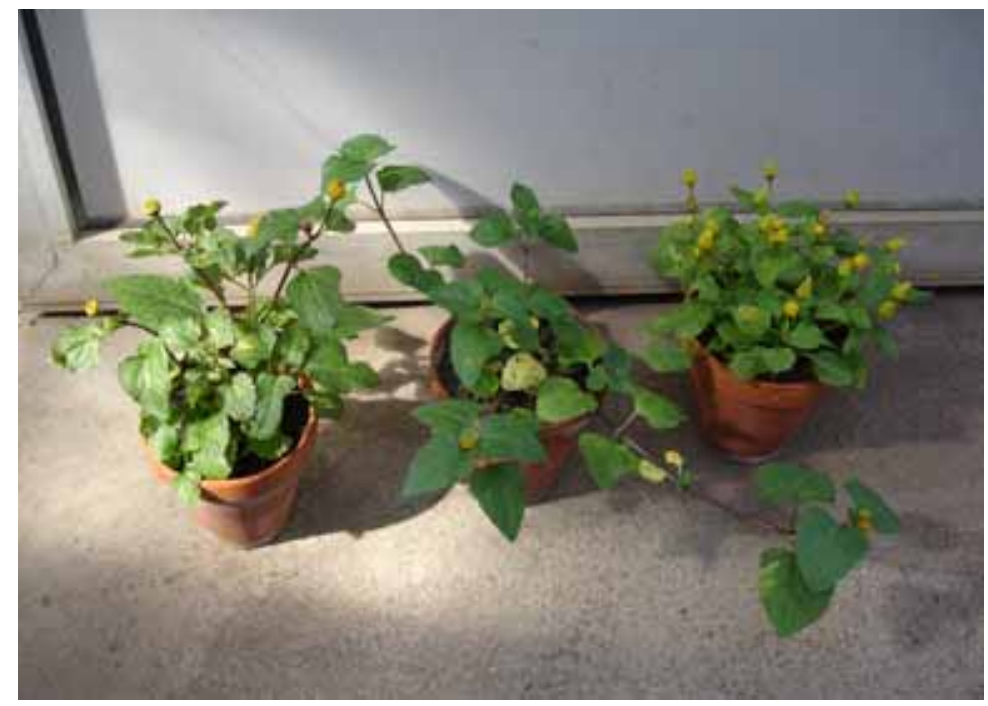

Abb 2: Vergleich des Habitus der drei Acmella Arten/ Varietäten; (von links nach rechts: A. oleracea (rot), A. ciliata, A. oleracea (gelb)
Beide Acmella-Arten weisen die gleiche gestielte Anheftung der Blätter und identische Blattstellung auf. Der Blattrand erscheint bei Acmella oleracea weniger stark und gleichmäßig gesägt, die Blattform ist weniger stark zugespitzt herzförmig als bei Acmella ciliata, und die Blätter sind teilweise kleiner, besonders die der roten Varietät. Bei letzterer entwickeln sich andererseits weitaus längere Blütenköpfchen als bei Acmella ciliata und der gelben Varietät von Acmella oleracea, deren Blütenköpfchen wiederum größer sind als die von Acmella ciliata (Abb. 1). Die Breite der Blütenköpfchen der Acmella oleracea-Varietäten entspricht in etwa der Breite der Blütenköpfe von Acmella ciliata, letztere gemessen mit Zungenblüten. Bei allen drei Acmella-Arten sind die Blütenköpfchen lang gestielt und entwickeln sich, mit Ausnahme der Terminalblüte des Haupttriebs, ebenfalls blattachselständig (vgl. Abb. 1a+c).

Diese feinen Differenzierungen in Größe und Ausprägung einzelner Pflanzenteile lassen sich nur ermitteln, wenn alle drei Pflanzen nebeneinander betrachtet werden können und sind zudem von den Standort- und Zuchtbedingungen abhängig. Als Hauptunterschied und sicherstes Merkmal zur Unterscheidung der Arten/Varietäten dienen die Farbe und besonders 
die Form der Blütenköpfe. Beide Varietäten von Acmella oleracea weisen scheibenförmige Blütenköpfe ohne Zungenblüten auf, der Blütenkopf ist länger als bei

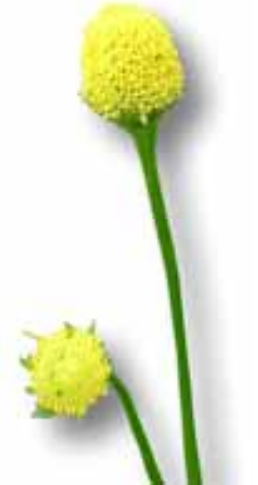

Abb. 1b: Blütenköpfe, (Stängel) von A. oleracea (gelb) Acmella ciliata und von konischer Form, an der Spitze mehr abgeplattet. Der Blütenkopf der „roten“ Varietät ist auf Grund unterschiedlich gefärbter Röhrenblüten außen gelb mit einem roten Punkt in der Mitte (Abb. 1c). Daher stammt wohl auch der deutsche Name Husarenknopf ${ }^{1)}$. Im älteren, voll ausgereiften Stadium, kurz vor dem

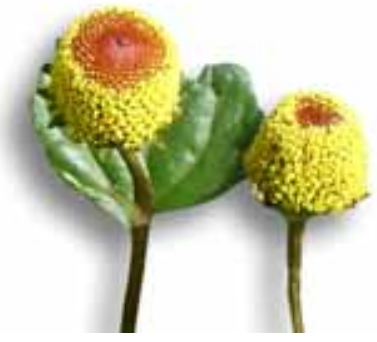

Abb. 1c: Blütenköpfe, (Blatt, Stängel) von A. oleracea (rot)

Abblühen, wandelt sich die Blütenfarbe in reingelb, und das Ende des Blütenköpfchens ist mehr rundlich gewölbt, ähnlich Acmella ciliata.

Die Blütenkopffarbe der gelben Varietät (Abb. 1b) ist zu jeder Zeit reingelb. Der Hüllkelch mit Hüllblättern, die Stängel, Blätter und Blattnerven dieser Varietät sind komplett grün, während die rote Varietät, ebenso wie Acmella ciliata, violett-rote Stängel, Hauptblattnerven sowie Hochblätter des Hüllkelchs (Involucrum) aufweist. Hier wirken auch die Blätter teilweise violettstichig. Ein Pappus ist, im Gegensatz zu Acmella ciliata, bei Acmella oleracea stets vorhanden, bestehend aus zwei ungleich langen Federn. Die Früchte (Achänen) sind länger und breiter als bei Acmella ciliata, ebenfalls mäßig bis dicht bewimpert bzw. behaart und haben keine Schultern. Sie besitzen aber auch keinen korkähnlich gestalteten Rand.

Die Anzahl somatischer Chromosomen in der mitotischen Metaphase beträgt 2n $=52$ [28].

1) Es sei angemerkt, dass die sieben Arten der Gattung Sanvitalia, die auch den Ecliptinae angehören, ebenfalls die Bezeichnung Husarenknöpfe führen. 


\subsection{Mikroskopische Beschreibung}

Wie schon bei der makroskopischen Beschreibung (1.3.) bezieht sich die mikroskopische Beschreibung ebenfalls nicht nur auf Acmella ciliata, sondern stellt ihr beide Varietäten von Acmella oleracea gegenüber. Zur besseren Übersicht werden alle drei Formen direkt unterbzw. nebeneinander abgebildet (Abb. 3-12). Die Untersuchungen und Vergleiche wurden mit den Röhren- und Zungenblüten, den Pollen, den Sprossachsen, Gliederhaaren und den Blättern durchgeführt.

\section{$\underline{\text { Vergleich der Röhrenblüten }}$}

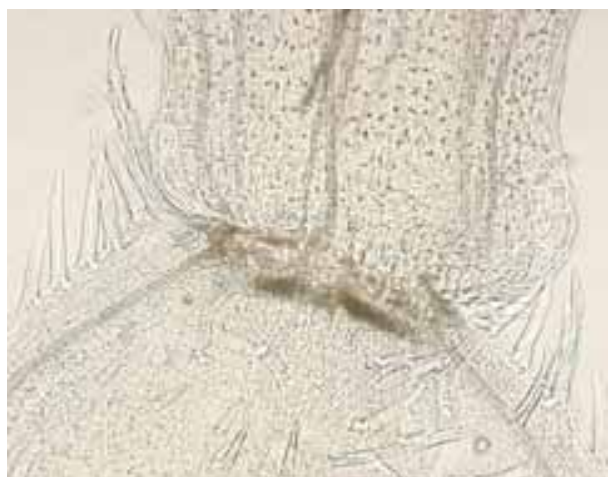

Abb. 3a: Fruchtknotenbauch mit Zwillingshaaren von $A$. ciliata

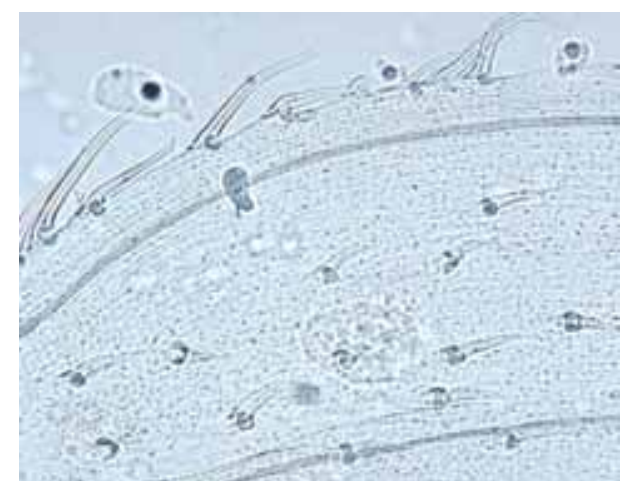

Abb. 3b: Fruchtknotenbauch mit Zwillingshaaren von $A$. oleracea (gelb)

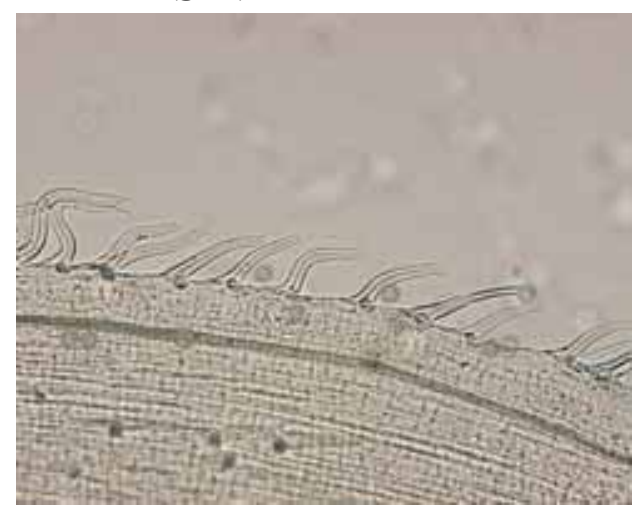

Abb. 3c: Fruchtknotenbauch mit Zwillingshaaren von $A$. oleracea (rot)

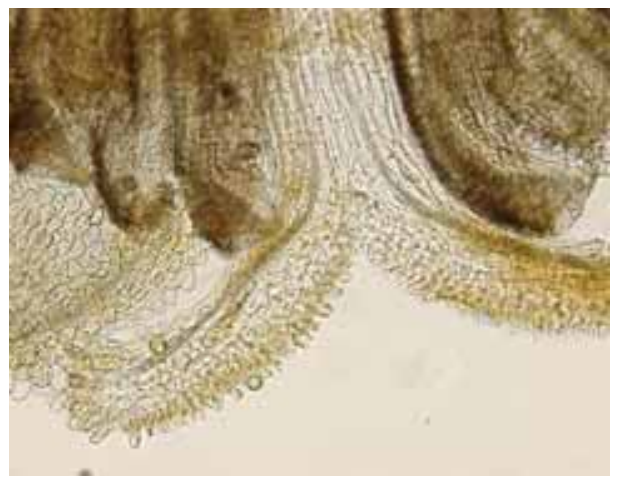

Abb. 4a: Narbe mit Papillen von A. ciliata

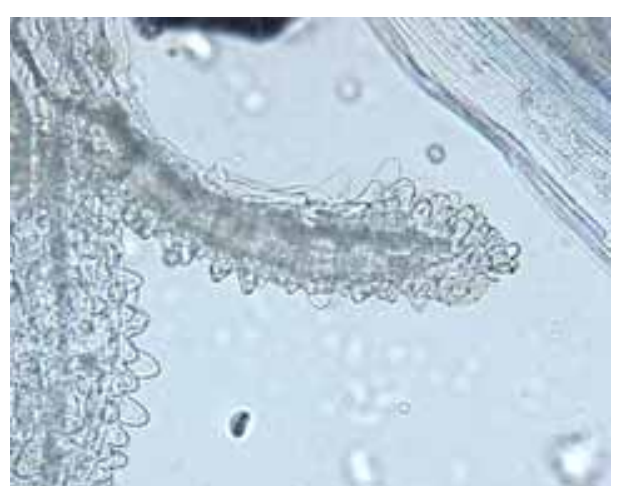

Abb. 4b: Narbe mit Papillen von A. oleracea (gelb)

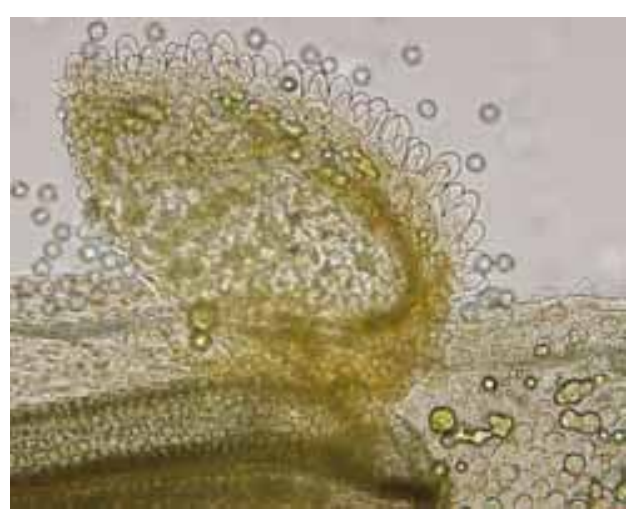

Abb. 4c: Narbe mit Papillen von A. oleracea (rot) 
Der Fruchtknoten trägt in allen drei Fällen Gliederhaare (Abb. 3a-c). Bei Acmella ciliata (Abb. 3a) ist der Übergang vom deutlich sichtbaren, unterständigen Fruchtknoten zum Blütenblatt erkennbar. Aus dem Fruchtknoten entwickelt sich als Frucht eine Achäne, die eine Sonderform der Nussfrüchte darstellt, bei welcher Fruchtwand und Samenschale miteinander verwachsen sind. Die Nussfrucht wiederum gehört zu den Schließfrüchten. Auch die Achäne ist mit Zwillingshaaren ausgestattet (nicht dargestellt).

Die Narbenschenkel aller drei Exemplare besitzen Papillen (rechte Bildfolge, Abb. 4a-c). Auch bei den Narben lässt sich mikroskopisch kein Unterschied feststellen.

Alle drei Pflanzen haben, wie für Vertreter der Asteraceae üblich, Drüsenschuppen (Abb. 5ac).

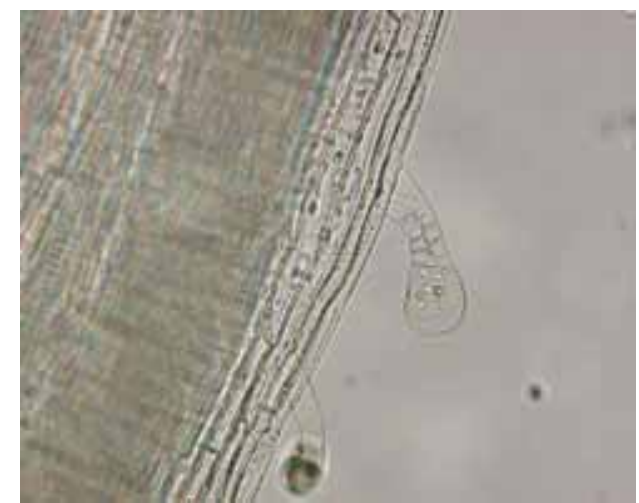

Abb. 5a: Drüsenschuppe von $A$. ciliata

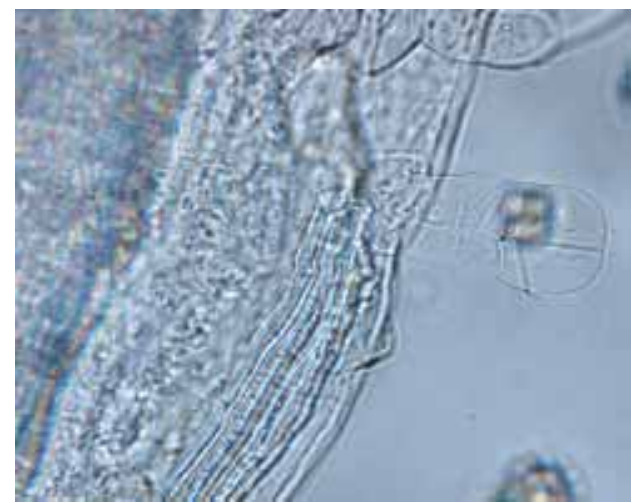

Abb. 5c: Drüsenschuppe von A. oleracea (rot)

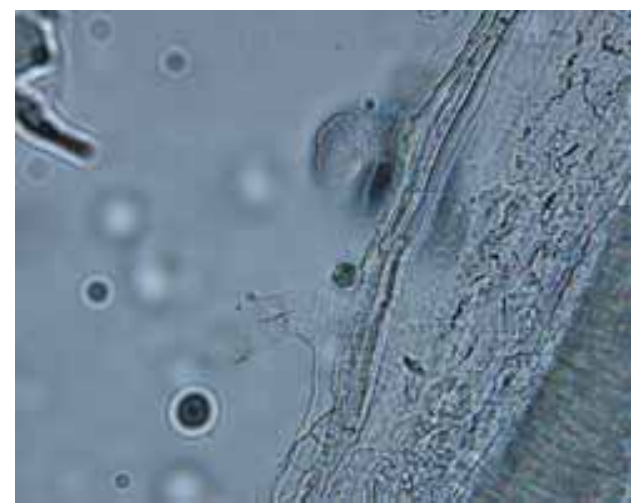

Abb. 5b: Drüsenschuppe von $A$. oleracea (gelb)

Acmella ciliata weist, wie oben beschrieben, als einzige Zungenblüten auf. Es folgen zwei Aufnahmen der Zungenblüten. 


\section{$\underline{\text { Zungenblüte von Acmella ciliata }}$}

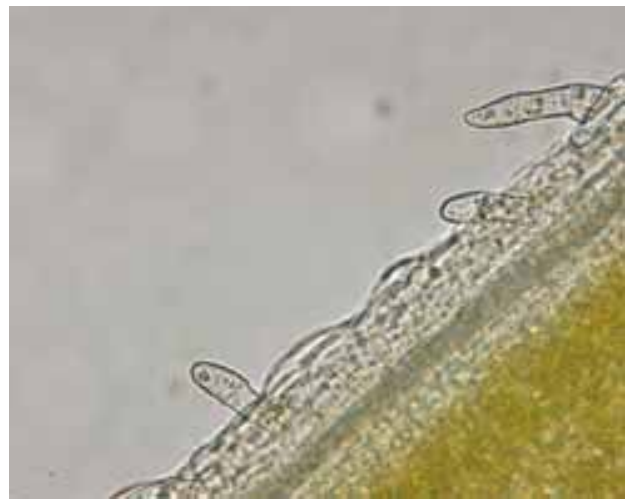

Abb. 6a: Drüsenhaare an Zungenblüte von $A$. ciliata

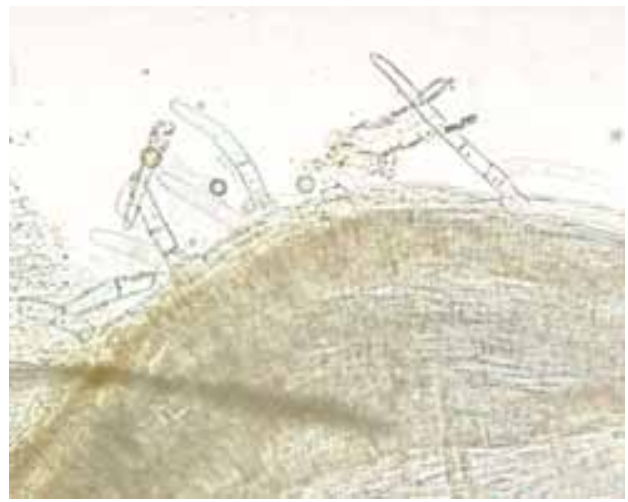

Abb 6b: Gliederhaare an Zungenblüte von $A$. ciliata

Wie aus den Abb. 6a und b ersichtlich, tragen die Zungenblüten deutlich in Zellen gegliederte Drüsenhaare.

\section{Vergleich der Pollen}

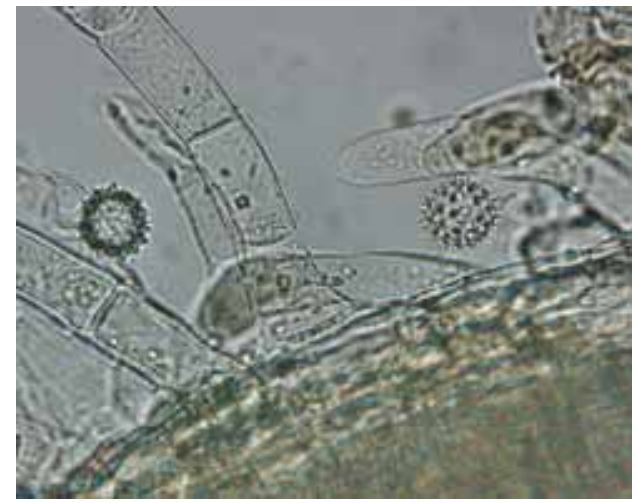

Abb. 7a: Polle von $A$. ciliata neben Gliederhaaren

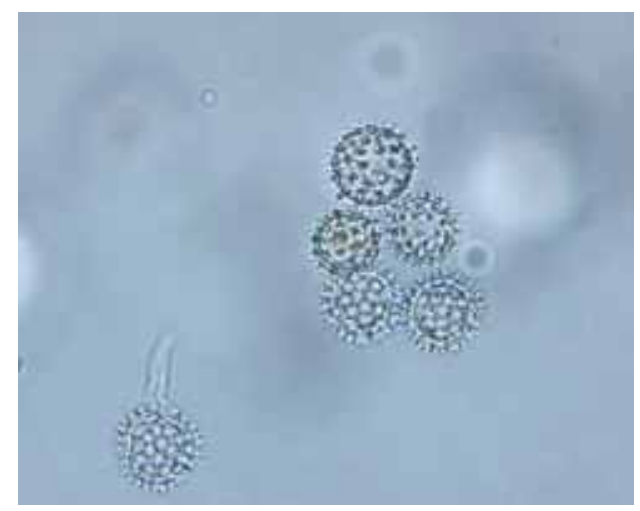

Abb. 7c: Polle von A. oleracea (rot)

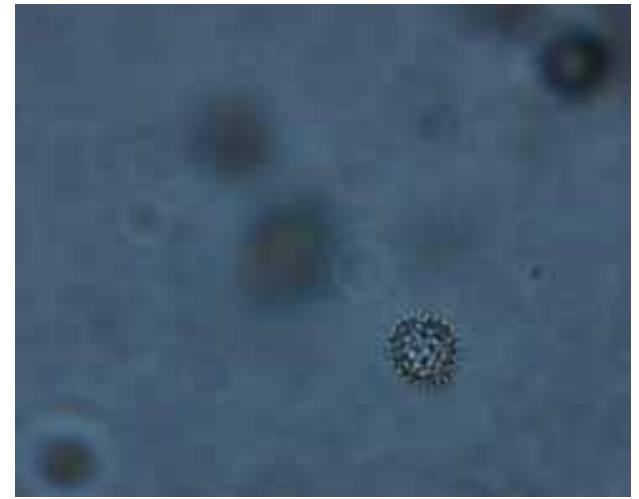

Abb. 7b: Polle von A. oleracea (gelb)

Alle Blütenpollen weisen eine stachelige Exine auf (Abb. 7a-c), was typisch für Asteraceen ist. 


\section{Vergleich der Sprossachsen}

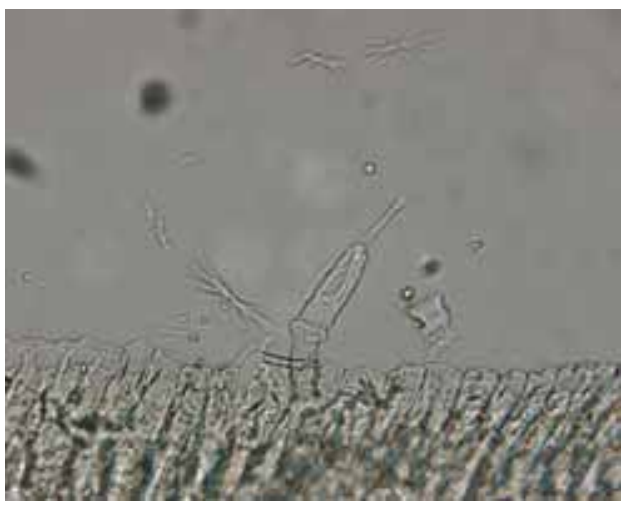

Abb. 8a: Gliederhaar von $A$. ciliata

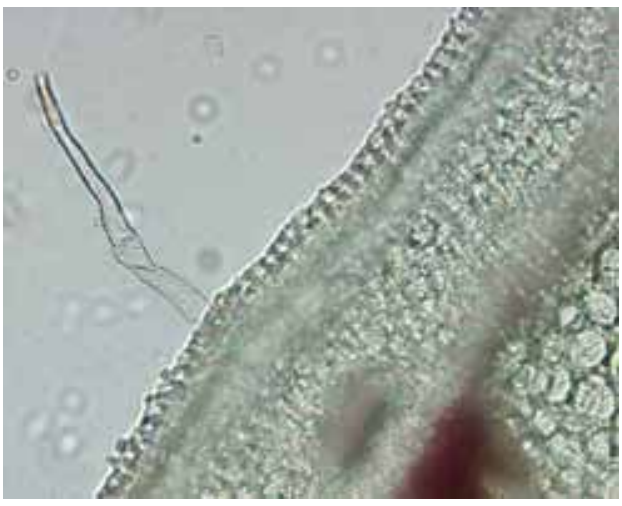

Abb. 8b: Gliederhaar von A. oleracea (gelb)

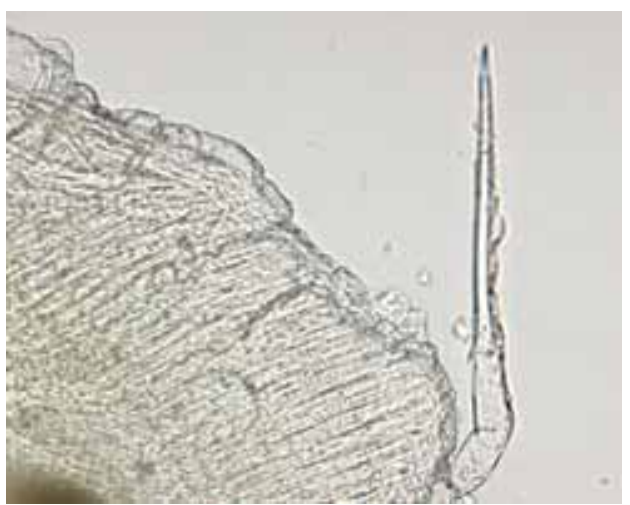

Abb. 8c: Gliederhaar von $A$. oleracea (rot)

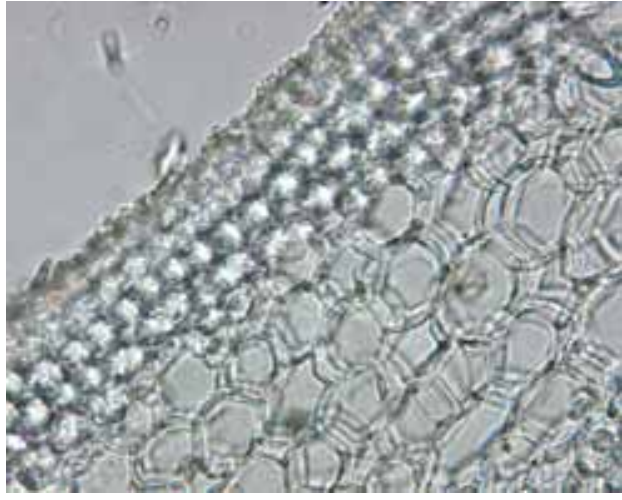

Abb. 9a: Sprossachsenquerschnitt von A. ciliata

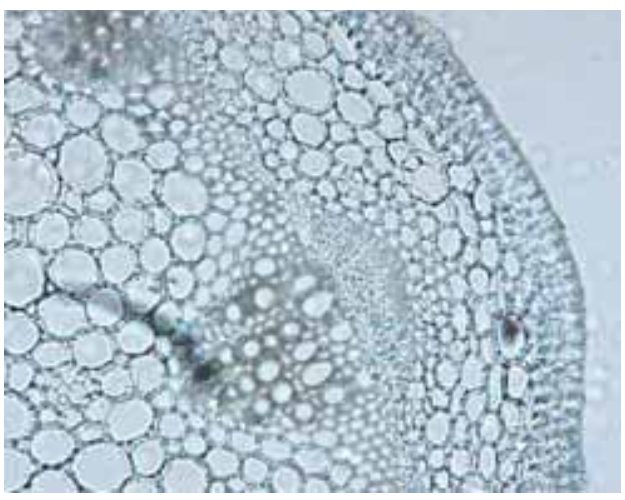

Abb. 9b: Sprossachsenquerschnitt von A. oleracea (gelb)

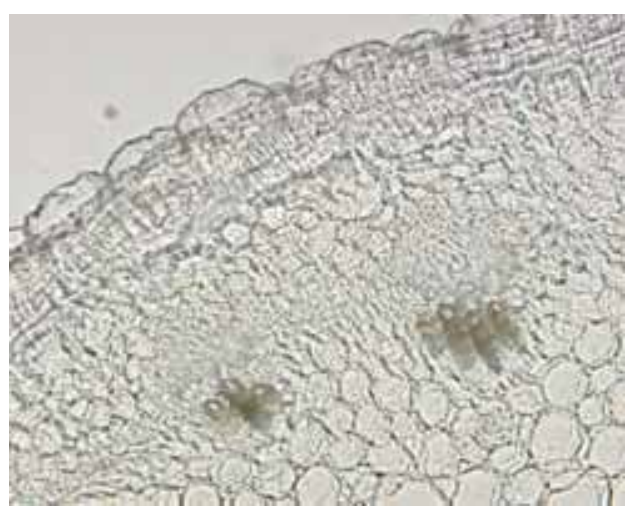

Abb. 9c: Sprossachsenquerschnitt von A. oleracea (rot)

In der linken Bildfolge sind Gliederhaare an der Sprossachse aller drei Pflanzen zu sehen. Im ersten Bild (Abb. 8a) sind bei Acmella ciliata außerdem gelbe Lipidtröpfchen erkennbar. Diese könnten auch Träger der lipohilen Alkamide sein. In Abb. 8b bei Acmella oleracea (gelb) ist das Leitbündel auffällig, welches mit Phloroglucin-Salzsäure angefärbt wurde und 
daher rot erscheint. Bei Acmella oleracea (rot) in Abb. 8c ist ein Gliederhaar an der Sprossachse zu erkennen.

In der rechten Bildfolge (Abb. 9a-c) ist der Querschnitt der Sprossachse aller drei Pflanzen dargestellt. Von außen nach innen sind folgende Gewebetypen zu erkennen: (gewellte) Kutikula, Epidermis, Kollenchym, Rindenparenchym. In Abb. 9b bei Acmella oleracea (gelb) fällt ein kleiner Zellhaufen neben dem Leitbündel auf. Bei Acmella oleracea (rot) ist eine Verdickung der Kutikula zu erkennen (Abb. 9c).

Die Gliederhaare und Zelltypen (Kutikula, Epidermis, Kollenchym und Parenchym) lassen sich mikroskopisch nicht voneinander unterscheiden. Minimale Unterschiede, wie etwa eine Kutikulaverdickung, sind vermutlich eher auf unterschiedliche Entwicklungsstadien der drei Sprossachsen zurückzuführen.

\section{Vergleich der Blätter}

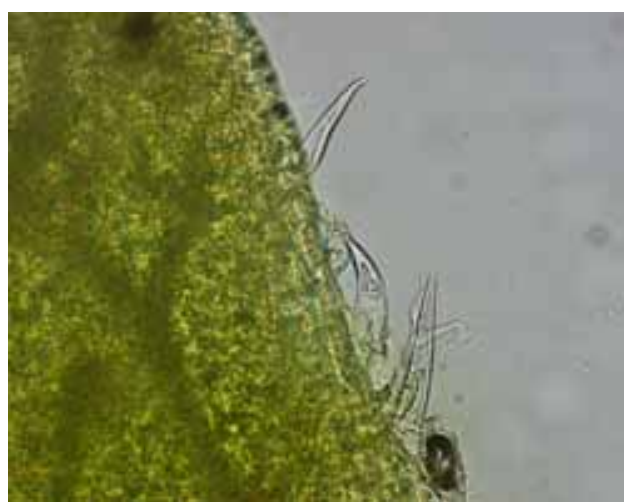

Abb. 10a: Blattunterseite mit Gliederhaaren von $A$. ciliata

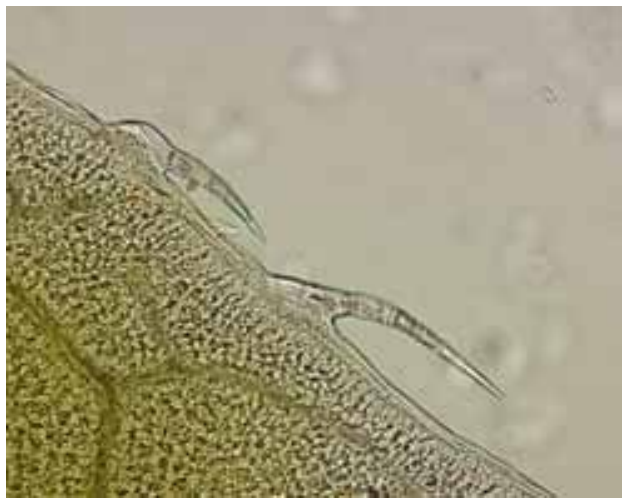

Abb. 10c: Blattunterseite mit Gliederhaaren von $A$. oleracea (rot)

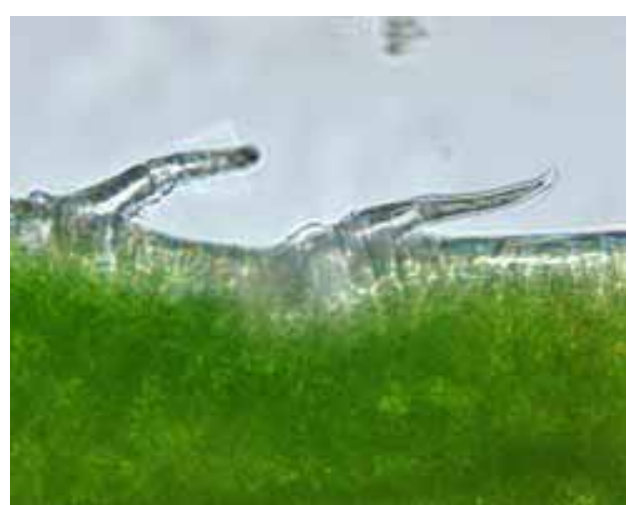

Abb. 10b:Blattunterseite mit Gliederhaaren von $A$. oleracea (gelb)

Die Blattunterseite zeigt in allen Fällen spitz zulaufende Gliederhaare (Abb. 10a-c). 


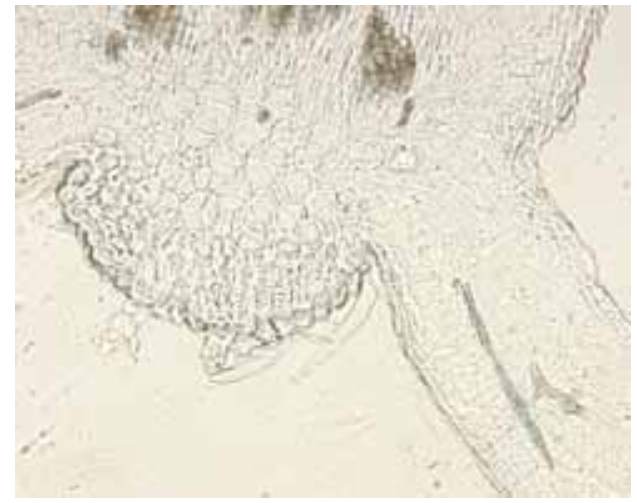

Abb. 11a: Mittelrippe mit Gliederhaaren von $A$. ciliata

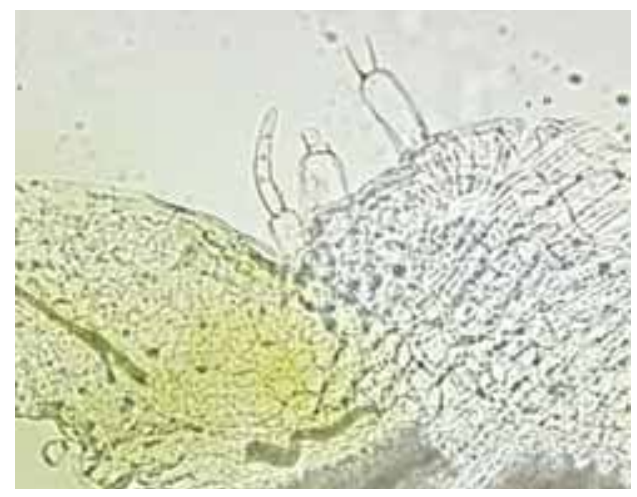

Abb. 11b:Mittelrippe mit Gliederhaaren von A. oleracea (gelb)

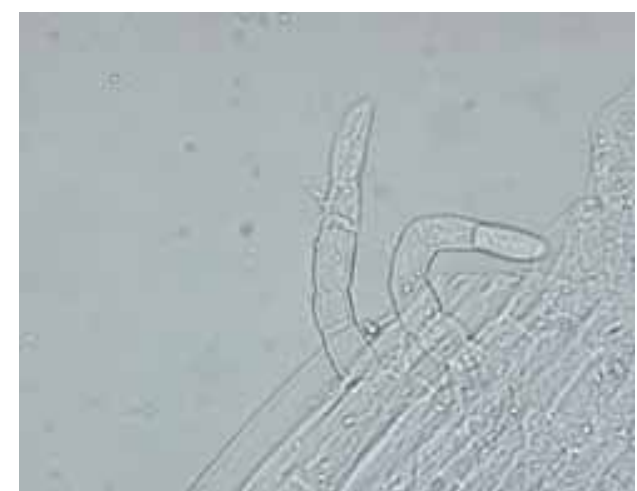

Abb. 11c: Mittelrippe mit Gliederhaaren von $A$. oleracea (rot)

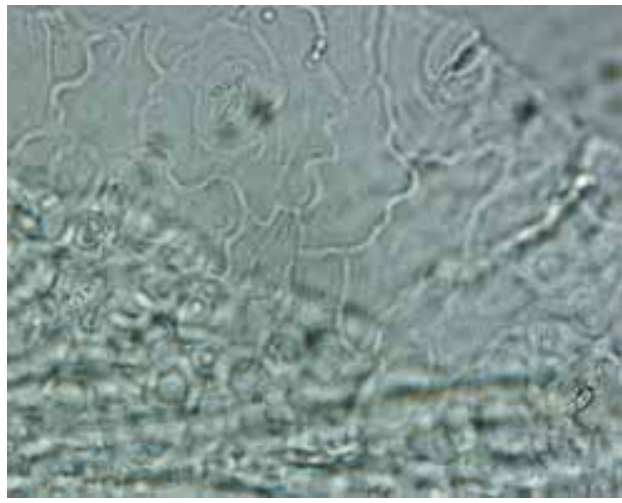

Abb. 12a: Spaltöffnungen von $A$. ciliata

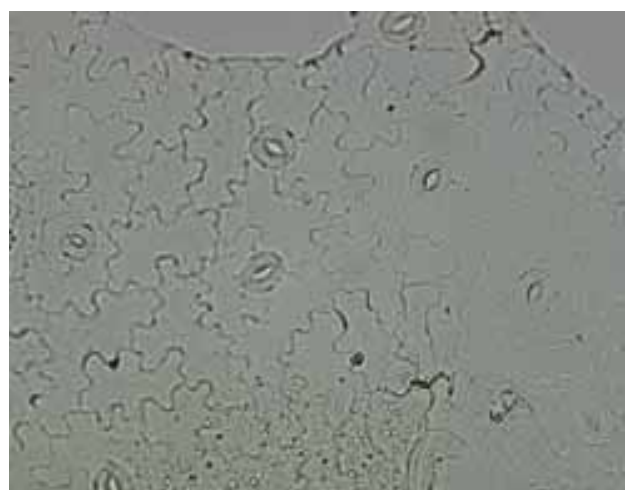

Abb. 12b:Spaltöffnungen von

A. oleracea (gelb)

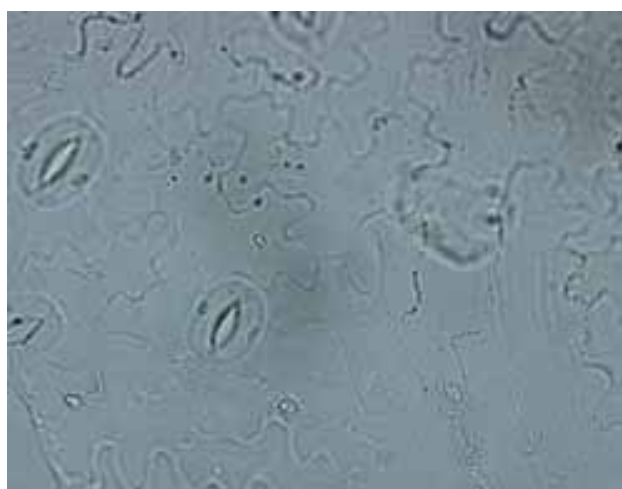

Abb. 12c: Spaltöffnungen von A. oleracea (rot)

Im obersten Bild der linken Bildfolge (Abb. 11a ) ist die Mittelrippe (Hauptnerv) von Acmella ciliata zu erkennen. Hier fallen besonders die drei Leitbündel nebeneinander auf. Außerdem sind Gliederhaare sichtbar.

Im zweiten Bild (Abb. 11b) ist die Mittelrippe (Hauptnerv) von Acmella oleracea (gelb) zu sehen. Auch hier sind Gliederhaare zu erkennen. Das unterste Glied der Haare ist verdickt, was allerdings auch bei den anderen beiden vorkam. Das Bild links unten (Abb. 11c) zeigt die Mittelrippe (Hauptnerv) von Acmella oleracea (rot), ebenfalls mit Gliederhaaren . 
Die Mittelrippe lässt auch keine mikroskopische Unterscheidung zu.

In der rechten Bildfolge (Abb. 12a-c) sind bei allen drei Exemplaren anomocytische Spaltöffnungen in den Blättern zu erkennen.

Bei den Blättern ist somit ebenfalls keine mikroskopische Unterscheidung möglich.

Zusammenfassend muss festgestellt werden, dass bei den mikroskopischen Betrachtungen zwischen den drei Arten bzw. Varietäten keine spezifischen Unterschiede zu konstatieren waren, was u. a. auf eine enge Verwandtschaft der drei Pflanzen schließen lässt. Der einzige, jedoch makroskopische Unterschied besteht im Vorhandensein von Zungenblüten bei Acmella ciliata, die Drüsenhaare aufweisen. Die gelbe und rote Varietät von Acmella oleracea können mikroskopisch auch nicht voneinander unterschieden werden. In allen drei Fällen sollte einer Bestimmung an Hand makroskopischer Merkmale, insbesondere der Blütenköpfchen (s. o.), der Vorrang gegeben werden.

\section{Gewinnung des Pflanzenmaterials}

\subsection{Anbau / Aufzucht}

Anbau bzw. Aufzucht von Acmella ciliata sowie Acmella oleracea sind grundsätzlich im Gewächshaus und eingeschränkt im Freiland möglich. Im Rahmen der vorliegenden Arbeit wurden beide Wege erprobt. Die Vor- und Nachteile beider Alternativen werden nachfolgend beschrieben.

Freilandanbau ist nur möglich während der absolut frostfreien Zeit. Als tropische Pflanze toleriert Acmella ciliata auch keine gelegentlich auftretenden Nachtfröste. So wurde die Pflanze zumeist im Gewächshaus oder am Fensterbrett ab Januar/Februar vorgesät, und die Jungpflanzen kamen erst ab Anfang/Mitte Mai aufs Feld. Blütenköpfe erscheinen dann ab einer bestimmten Pflanzengröße bis zum Ende der Vegetationsperiode (ca. Ende Oktober/Anfang November mit den ersten Nachtfrösten) kontinuierlich. 
Der Freilandanbau von Acmella ciliata erfolgte 2003 und 2004 auf Feldern mit lehmhaltigem Boden $^{1)} .2004$ wurde zu Testzwecken eine Stickstoffbedüngung vorgenommen (s. Exp. Teil). Proportional zur Düngerkonzentration ließ sich ein verstärktes Größenwachstum aller Pflanzenorgane konstatieren. Auf künstliche Bewässerung und Schädlingbekämpfungsmaßnahmen konnte verzichtet werden. Darüber hinaus wurden 2005 Acmella ciliata und Acmella oleracea (gelb) auf einem Grundstück anderer Bodenbeschaffenheit ${ }^{2)}$ angebaut. Sofern keine Voraussaat (s. o.) erfolgte, wurden die einsamigen Schließfrüchte (Achänen) breitwürfig ausgesät und nur mit sehr wenig Erde bedeckt (Nacktsamer). Die Keimdauer betrug zwischen 1-3 Wochen, je nachdem, ob mit oder ohne über den Keimschalen/Töpfen angebrachten Pflanzenlampen gearbeitet wurde.

Parallel erfolgte der Anbau von Acmella ciliata zwischen 2003 und 2007 durchgängig im temperierten Gewächshaus ${ }^{3)}$. 2005 - 2007 wurden zusätzlich Acmella oleracea (gelb) und 2006 auch Acmella oleracea (rot) im Gewächshaus aufgezogen. Unter diesen günstigen Bedingungen, insbesondere durch Nachahmen annähernd tropischer Bedingungen, wie das Herstellen von Tag- und Nachtgleiche, entsprechende Feuchtigkeit und ggf. mit Hilfe von Berieselungsanlagen etc., ist es möglich, Acmella ciliata zu überwintern, d. h., sie auch als mehrjährige Pflanze zu ziehen.

Als Problem erwies sich im Sommer die z. T. starke Überhitzung des Glases bei hohem Sonnenstand, was trotz Lüften und Schattierung nicht komplett zu verhindern war. Im Winter ist dagegen eher die geringe Beleuchtungsstärke, bedingt durch den niedrigen Sonnenstand, problematisch, was aber nur partiell durch Zusatzbeleuchtung mittels Licht mit hohem Blauanteil zu kompensieren war.

Außerdem sind die Pflanzen diversen Schädlingen, wie Weiße Fliege, Spinnmilben und Asseln, ausgesetzt, die sich durch die Treibhausbedingungen und mangels Fressfeinden wie im Freiland besonders rasant vermehren. An Acmella oleracea (rot) waren Schnecken zu finden. Infolgedessen mussten, im Gegensatz zum Freilandanbau, Schädlingbekämpfungsmaßnahmen durchgeführt werden.

\footnotetext{
1) Landwirtschaftlich-Gärtnerische Fakultät der Humboldt-Universität Berlin in Blumberg bei Berlin

${ }^{2)}$ Landwirtschaftlich-Gärtnerische Fakultät der Humboldt-Universität in Berlin Dahlem

3) Institut für Pharmazeutische Biologie der Freien Universität Berlin
} 
Zunächst wurde mit Gelbtafeln sowie mit ausgesetzten Fressfeinden, z. B. Raubmilben (Phytoseiulus persimilis) gegen Spinnmilben (Tetranychidae), als biologische Methode gearbeitet. Gelegentlich, d. h., bei zu starkem Befall, gelangten chemische Methoden (Zierpflanzenspray; s. Exp. Teil) zum Einsatz.

\subsection{Ernte und Trocknung}

Geerntet werden kann beim ertragreicheren Freilandanbau während der gesamten Vegetationsperiode. Es erfolgten mehrere Ernten von September bis Oktober. Bis zum Ende der Vegetationsperiode wurde nur die oberirdische Pflanze geerntet, um das Nachwachsen zu ermöglichen. Bei der letzten Ernte vor dem ersten Frost wurde die Gesamtpflanze mit Hauptwurzeln geerntet. Im Gewächshaus ist die Ernte weitgehend unabhängig von den Jahreszeiten.

Zur späteren Extraktgewinnung wurde das Pflanzenmaterial von anhaftender Erde befreit, grob zerkleinert und getrocknet. Sofern nicht die Gesamtpflanze zur weiteren Aufarbeitung vorgesehen war, wurde vor der Trocknung in einzelne Pflanzenteile (z. B. Blüten) zerlegt.

Die Trocknung erfolgte als Hordentrocknung im Trockenschrank bei $40^{\circ}$ oder $60^{\circ} \mathrm{C}$. Als Kriterium für hinreichende Trocknung (keine Bestimmung der Restfeuchte) diente z. B. die leichte Durchknickbarkeit der Stängel, da sie dem Pflanzenorgan mit dem höchsten Wassergehalt entsprachen (s. Tab. 32). Die durchschnittliche Trockenzeit betrug vier Tage. Das Verhältnis Droge zu Frischpflanze(nteile) lag bei etwa 1:5, weitgehend unabhängig von der Trocknungstemperatur (s. Tab. 32).

\subsection{Extraktion}

Zur Extration des getrockneten und zerkleinerten/gemahlenen Pflanzenmaterials (s. Exp. Teil) gelangten verschiedene Lösungsmittel bzw. -gemische zum Einsatz (Tab. 1). Deren Auswahl richtete sich zum einen nach den zu extrahierenden Pflanzenteilen und zum anderen nach dem Polaritätscharakter der zu isolierenden Inhaltsstoffe. So wurde zur Extraktion der vorwiegend lipophilen Alkamide (3.3.2.) das apolare Dichlormethan (Dielektrizitätskonstante bei $25^{\circ} \mathrm{C}, \varepsilon$ $=9,14$ ) eingesetzt und zur Gewinnung der eher hydrophilen Phenolderivate (3.4.2.) das 
stärker polare Methanol $(\varepsilon=33,6)$. Beim DC-Screening (3.2.1.), d. h., zur summarischen Erfassung von Inhaltsstoffgruppen, die allgemein in Asteraceae und in Acmella-Arten im Besonderen vorkommen, wurden weitere Extraktionsmittel getestet (Tab. 1). Darüber hinaus gelangte in Anlehnung an das Fertigarzneimittel Spolera ${ }^{\circledR}$, das einen Gesamtpflanzenextrakt mit 60 \%igem 2-Propanol enthält, auch dieses Auszugsmittel (Tab. 1) zur Testung.

Zur Wirksamkeitsüberprüfung von Acmella-Extrakten gegenüber verschiedenen Enzymen (4.) wurden teilweise noch weitere Extraktionsmittel (Tab. 1) einbezogen, u. a. überkritisches Kohlendioxid (Destraktion). Unter diesen Bedingungen (10 MPa, $37^{\circ} \mathrm{C}$ bzw. $\left.15 \mathrm{MPa}, 34^{\circ} \mathrm{C}\right)$ werden vorrangig Kohlenwasserstoffe und organische, lipophile Verbindungen geringer Polarität extrahiert $\left(\varepsilon\left(\mathrm{CO}_{2}\right)=\right.$ ca. 1,25-1,5). Extrakte, die mit Dichlormethan bzw. dem Extraktionsmittel Aceton $(\varepsilon=20,7)$ gewonnen worden waren, dienten zur Entwicklung der galenischen Präformulierungen (5.).

Die Effektivität der verschiedenen Extraktionsmethoden zeigte sich u. a. beim nachfolgenden DC-Screening (3.2.). So erwies sich beispielsweise die Ultraschallbehandlung mit Extraktionsmittel $\mathrm{C}_{1}$ nach der halbquantitativen DC-Analytik als geeigneter gegenüber $\mathrm{C}_{2}$ (Rühren), indem eine größere Ausbeute erzielt und die Manipulation Zeit und Lösungsmittel sparend verlief. Zur Extraktion der Blüten, in denen ein besonders hoher Alkamidgehalt zu erwarten war, gelangte demzufolge die Ultraschallmethode zum Einsatz $\left(\mathrm{A}_{4}, \mathrm{C}_{3}, \mathrm{E}_{2}\right)$.

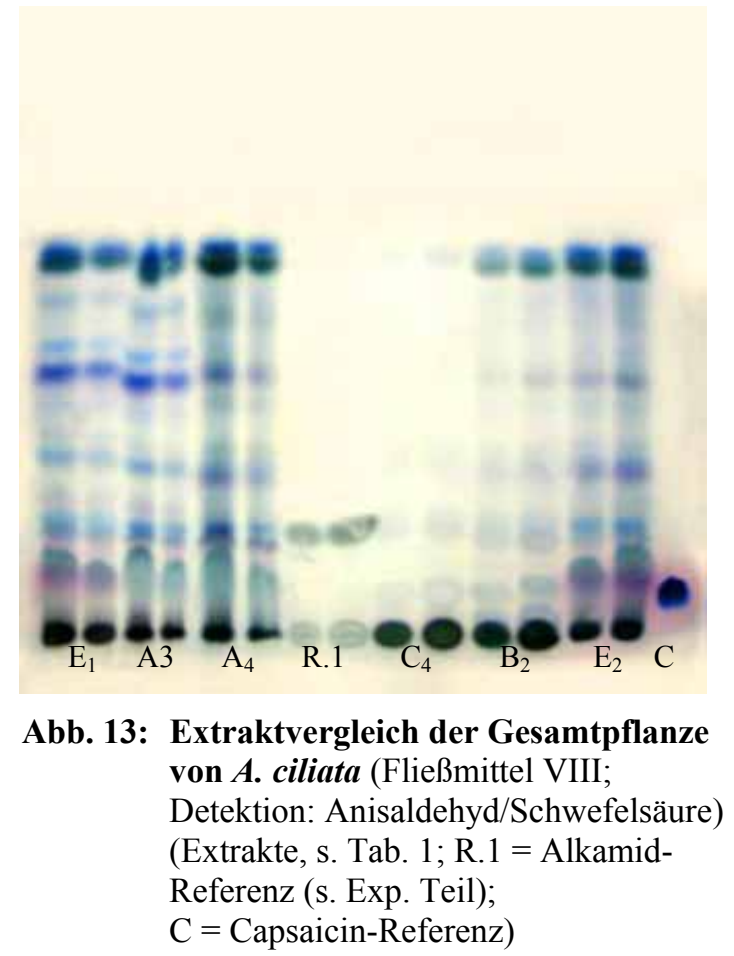

Das nebenstehende DC veranschaulicht die Extraktivkraft verschiedener Lösungsmittel (Ultraschall) hinsichtlich lipophiler Substanzen und weist die Extraktionsmittel Methanol $\left(\mathrm{B}_{2}\right)$ und besonders 2-Propanol $\left(\mathrm{C}_{4}\right)$ als wenig geeignet zur Isolierung der vorwiegend lipophilen Alkamide aus.

Die gewonnenen Extrakte wurden filtriert und unter schonenden Bedingungen im Vakuumrotationsverdampfer zur weitgehenden Trockne eingeengt und bis zur weiteren Verwendung im Kühlschrank oder, bei längerer 
Lagerung, im Gefrierschrank aufbewahrt (Exp. Teil).

Zur vergleichenden DC-Analytik von Acmella ciliata und den beiden Varietäten von Acmella oleracea (3.2.2.) wurden Frischpflanzenextakte herangezogen, gewonnen mit den folgenden Extraktionsmitteln: Dichlormethan (G), Methanol (H), Ethanol 45 \% (I) und Aceton (J).

Tabelle 1: Parameter zur Extraktion von Acmella ciliata - getrocknetes Pflanzenmaterial

\begin{tabular}{|c|c|c|c|c|}
\hline Extrakt & Extraktionsmittel & Methode & Pflanzenteile & $\begin{array}{l}\text { Verwen- } \\
\text { dung in }\end{array}$ \\
\hline$A_{1}$ & Dichlormethan & Ultraschall & oberirdische Gesamtpflanze & 3.2. \\
\hline $\mathbf{A}_{2}$ & Dichlormethan & Soxhlet & Gesamtpflanze & 3.3 .2 . \\
\hline $\mathbf{A}_{3}^{*}$ & Dichlormethan & Ultraschall & Gesamtpflanze & 4. \\
\hline $\mathbf{A}_{4}$ & Dichlormethan & Ultraschall & Blüten 2003 & 4. \\
\hline $\mathbf{A}_{5}$ & Dichlormethan & Ultraschall & Blüten 2004 & 4. \\
\hline$B_{1}$ & Methanol & Soxhlet & Gesamtpflanze & 3.4.2. \\
\hline $\mathbf{B}_{2}^{*}$ & Methanol & Ultraschall & Gesamtpflanze & 4. \\
\hline $\mathrm{C}_{1}$ & 2-Propanol 60\% (m/m) & Ultraschall & oberirdische Gesamtpflanze & 3.2. \\
\hline $\mathrm{C}_{2}$ & 2-Propanol 60\% (m/m) & Rühren & oberirdische Gesamtpflanze & 3.2. \\
\hline $\mathrm{C}_{3}$ & 2-Propanol 60\% (m/m) & Ultraschall & Blütenköpfe & 3.2. \\
\hline $\mathrm{C}_{4}{ }^{*}$ & 2-Propanol 60\% (m/m) & Ultraschall & Gesamtpflanze & 4. \\
\hline$D_{1}^{*}$ & Ethanol 45\% (m/m) & Ultraschall & Gesamtpflanze & 4. \\
\hline & Rohsaponingemisch & & & 3.2. \\
\hline & Etherphase & & & 3.2 . \\
\hline & wässerige Phase & & & 3.2. \\
\hline $\mathrm{E}_{1}^{*}$ & Aceton & Ultraschall & Gesamtpflanze & $4 ., 5$. \\
\hline $\mathbf{E}_{2}$ & Aceton & Ultraschall & Blüten & 4. \\
\hline$F_{1}$ & Kohlendioxid & $10 \mathrm{MPa}, 37^{\circ} \mathrm{C}$ & Gesamtpflanze & 5. \\
\hline $\mathbf{F}_{2}$ & Kohlendioxid & $15 \mathrm{MPa}, 34^{\circ} \mathrm{C}$ & Gesamtpflanze & 5. \\
\hline
\end{tabular}

*: Diese Extrakte wurden in analoger Weise auch aus Acmella oleracea hergestellt und in die enzymatischen Untersuchungen (4.) einbezogen. 
Die jeweils im Verhältnis 1:10 (Frischpflanze : Lösungsmittel) durchgeführte Extraktion mittels Ultraschall führte quantitativ zu unterschiedlichen Extraktausbeuten (Tab. 2). Demnach erwiesen die hydrophilen Extraktionsmittel H und I die größte Extraktivkraft (3- 5,6 \%), während Dichlormethan (G) weniger als $1 \%$ der Inhaltsstoffe herauslöste, was bereits eine Selektivität in Richtung lipophiler Verbindungen, repräsentiert vorrangig durch die Alkamide, erkennen lässt.

Tabelle 2: Prozentuale Ausbeute der Frischpflanzen-Extraktion

\begin{tabular}{|l|c|c|c|c|}
\hline & $\begin{array}{c}\text { Dichlormethan } \\
(\mathbf{G})\end{array}$ & $\begin{array}{c}\text { Methanol } \\
(\mathbf{H})\end{array}$ & $\begin{array}{c}\text { Ethanol 45\% } \\
(\mathbf{I})\end{array}$ & $\begin{array}{c}\text { Aceton } \\
(\mathbf{J})\end{array}$ \\
\cline { 2 - 5 } Acmella ciliata & 0,7 & 3,0 & 4,6 & 2,7 \\
Acmella oleracea (gelb) & 0,5 & 3,7 & 3,9 & 2,1 \\
Acmella oleracea (rot) & 0,2 & 4,9 & 5,6 & 1,7 \\
\hline
\end{tabular}

\section{Phytochemische Untersuchungen}

\subsection{Inhaltsstoffe allgemein}

Bisherige Untersuchungen zeigten, dass Acmella ciliata besonders reich an Säureamiden mit längerkettigen, teilweise mehrfach ein- und/oder zweifach ungesättigten Fettsäuren ist. Als Leitsubstanz wird das bereits bekannte Spilanthol (Synonym: Affinin) angesehen, zuerst isoliert aus „Spilanthes oleracea“ L. (Acmella oleracea, JANSEN) durch GERBER [42]. Insgesamt wurden bisher 20 verschiedene Säureamide (Alkamide bzw. Alkylamide) aus den Blütenköpfchen isoliert [110-113].

Strukturell ähnliche Alkamide wurden in fast allen Scharfstoffdrogen gefunden, wie auch im Schwarzen Pfeffer (Piper nigrum L., Piperaceae) [73, 156, 206] oder Cayennepfeffer (Capsicum frutescens L., Solanaceae) [73, 156, 206]. Große chemische Ähnlichkeit besteht mit den Amiden des Szechuanpfeffers (Zanthoxylum spec., Rutaceae) [4, 60, 63, 98, 186, 201, 206] (Tafel I, Teil 2 / Formel 12 unter 3.3.1.1.).

Besonders deutlich ist die chemische Verwandtschaft der Alkamide innerhalb der Pflanzengattung Acmella, auch wenn deren Arten teilweise unter veralteter Taxonomie 
geführt werden, sowie innerhalb desselben Subtribus, wie beispielsweise im Sonnenhut (Echinacea spec., Asteraceae). Interessanterweise konnten neben vielen ähnlichen Substanzen auch einige strukturell identische, u. a. Hauptalkamide von Echinacea-Species in Acmella ciliata nachgewiesen werden (s. 3.3.1.1.) $[6,13,16,30,35,50,53,124,132,133,134,140$, $144,148,173]$.

Außerdem wurden das Hydroxycumarin Scopoletin in den Wurzeln sowie die Sesquiterpene Humulen, Caryophyllen und Caryophyllenepoxid im ätherischen Öl des Sprosses gefunden $[110,112]$. Darüber hinaus waren zu Beginn dieser Arbeit die Flavonoide Isoquercitrin und Rutosid (beides Quercetinderivate) sowie die Apigeninderivate Apigenin-7-O-glucosid und Apigenin-7-O-neohesperidosid bekannt [177].

\subsection{DC-Screening}

Da über die Inhaltsstoffe von Acmella ciliata und auch Acmella oleracea, außer den Alkamiden und einigen anderen Substanzen (3.1.), wenig bekannt war, wurde zunächst ein DC-Screening durchgeführt, wobei auf häufige, in Asteraceae anzutreffende Inhaltsstoffe [64], einschließlich der Alkamide, geprüft wurde. Um ein möglichst breites Spektrum der potentiellen Hauptsubstanzklassen $\mathrm{zu}$ erfassen, gelangten mit Extraktionsmitteln unterschiedlicher Polarität und aus verschiedenen Pflanzenteilen sowie aus Frischpflanzen hergestellte Extrakte (Tab. 1 und G, H, I, J, s. 2.3.) zur Testung.

Die DC erfolgte auf Kieselgel-Fertigplatten mit Fluoreszenzindikator (s. Exp. Teil). Sofern vorhanden, wurden zu den einzelnen Inhaltsstoffgruppen entsprechende Referenzsubstanzen (s. Tab. 3a und b und Exp.Teil), in Methanol gelöst, aufgetragen, die Extrakte hingegen gelöst im jeweiligen Extraktionsmittel.

Die verwendeten Fließmittelsysteme (Tab. 33) orientieren sich weitgehend an der Standardund älteren Fachliteratur [165, 179], mussten aber teilweise den aktuellen Erfordernissen entsprechend modifiziert werden. Zur Detektion der Substanzen wurden neben der Betrachtung im UV-Licht die klassischen Gruppenreagenzien für die zu detektierenden Stoffklassen eingesetzt (s. Exp. Teil). 


\subsubsection{Acmella ciliata - Droge}

Die Ergebnisse des DC-Screenings sind in Tab. 3a und b dokumentiert. Z. T. resultierten lediglich unspezifische Anfärbungen, die nicht sicher auf das Vorliegen der betreffenden Substanzgruppe schließen ließen. Bereits vor der Detektion traten zuweilen gefärbte Banden auf, die von Chlorophyll und Blütenfarbstoffen stammten.

Für das Vorliegen von Alkamiden sprachen nach Besprühen mit dem Kaliumpermanganat/Schwefelsäure-Reagenz und Erhitzen bei $105^{\circ} \mathrm{C}$ mehrere violett-braune Banden, insbesondere bei Extrakt $A_{1}$ im Fließmittel I. Allerdings reagiert dieses Detergens mit allen oxidierbaren Verbindungen, wozu auch alle Alkamide, die keine vollständig konjugierte Kohlenwasserstoffkette besitzen, gehören [165].

Die gegenüber den Alkamiden weitaus hydrophileren phenolischen Komponenten, zu denen auch die Flavonoide zählen und die erwartungsgemäß eher in Extrakten mit hydrophileren Lösungsmitteln, wie 2-Propanol 60\%, zu finden sind, zeigten in den polareren Fließmitteln II und III gutes und fast identisches Laufverhalten. Im Fließmittel IV wanderten lediglich die Aglyka; die Glykoside verblieben auf der Startlinie. Die Bandentrennung war jedoch nicht befriedigend. Die Fließmittel I und V erwiesen sich als zu lipophil und eignen sich bestenfalls zur Trennung der auch in diesen Extrakten in geringerem Umfang vorhandenen Chlorophylle und Blütenfarbstoffe.

Als Referenzen dienten verschiedene Phenolcarbonsäurederivate sowie Flavonoide (s. Tab. 3a und Exp.Teil). So konnten bei den Extrakten $\mathrm{C}_{1}, \mathrm{C}_{2}$ und $\mathrm{C}_{3}$ in den Fließmitteln II und III $\left(\mathrm{C}_{1}\right)$ Hinweise auf die Anwesenheit von Chlorogen- bzw. Isochlorogensäuren und auf die Anwesenheit diverser Flavonoide, konkret auf Isoquercitrin und Rutosid, gewonnen werden, was sich im Laufe der Arbeit auch bestätigte (3.4.3.).

Vor dem Besprühen wurden die Platten zunächst unter UV-Licht bei $254 \mathrm{~nm}$ (Fluoreszenzminderungen) und $366 \mathrm{~nm}$ (stärkere Eigenfluoreszenz von Hydroxycumarinen gegenüber Phenolcarbonsäurederivaten) betrachtet, um zwischen Hydroxycumarinen und Phenolcarbonsäurederivaten zu differenzieren, die nach der Detektion mit Naturstoffreagenz A (Diphenylboryloxyethylamin) kaum noch zu unterscheiden sind. Die Detektion mit diesem Reagenz beruht auf Farbintensivierung durch Chromophorvergrößerung, zu betrachten im UV-Licht bei 366 nm, durch Bildung eines Komplexes an Keto-Enol-Strukturen [1]. 
Saponine sind schwierig zu detektieren, da zumeist chromophore Strukturen fehlen. Zur Detektion wurde hier das unspezifische Universalreagenz Anisaldehyd/Schwefelsäure mit anschließendem Erhitzen bis zur Farbausbildung bei ca. $105^{\circ} \mathrm{C}$ im Trockenschrank verwendet. Die mitgelaufenen Referenzen (s. Tab. 3b) zeigten zwar keine Übereinstimmungen in den $\mathrm{R}_{\mathrm{f}}$-Werten, jedoch in der Farbgebung: Saponin (Merck): oliv - dunkelgrüne Banden; Aescin: lila Hauptbande; Sonnenblumensaponingemisch: lila Banden.

Die in der Tab. $3 b$ angeführten Bandenfärbungen deuteten, zusammen mit einer entsprechenden Vorprobe (Schaumprobe, s. Exp. Teil), auf das Vorhandensein von Saponinen in dem mittels eines typischen Saponin-Extraktionsschemas gewonnenen „Rohsaponingemisch" und somit in Acmella ciliata hin [1].

In Extrakt $\mathrm{C}_{1}$ konnten nach Entwicklung im Fließmittel II und Detektion durch Echtblausalz B und nachfolgender Alkalisierung mit Ammoniak positive Hinweise auf Phenole und kupplungsfähige Amine erhalten werden [165].

Dragendorffs-Reagenz ergibt generell keinen spezifisch positiven Nachweis auf Alkaloide, sondern spricht bei allen tertiären Aminen mit Orange-Färbung an. Das Ergebnis war hier negativ [1].

Da Indolalkaloide nicht die Struktur tertiärer Amine aufweisen, war mittels Dragendorffs Reagenz diesbezüglich kein positiver Nachweis zu erwarten. Die positive Reaktion, die sich mit van Urk-Reagenz zeigte, ist spezifisch für in 2-Stellung unsubstituierte Indolderivate. Lediglich einige Aminosäuren ergeben falsch positive Nachweise, wie das Tryptophan, das einen biogenetischen Baustein dieser Alkaloide darstellt [1].

In den Extrakten $\mathrm{A}_{1}, \mathrm{C}_{1}$ und $\mathrm{C}_{2}$ fanden sich nach Detektion mit Antimon(III)-chlorid-Lösung und Eisessig unter UV-Licht bei $366 \mathrm{~nm}$ Hinweise auf das Vorhandensein pflanzlicher Cholesterolderivate, konkret auf Cholesterol und $\beta$-Sitosterol. 
Tabelle 3a: DC-Sreening von Acmella ciliata

\begin{tabular}{|c|c|c|c|c|c|c|c|}
\hline \multirow[t]{2}{*}{ Testgruppe } & \multirow[t]{2}{*}{ Referenzen } & \multirow[t]{2}{*}{ Extrakt } & \multirow[t]{2}{*}{ Fließmittel } & \multicolumn{2}{|c|}{ Detektion } & \multirow[t]{2}{*}{ Ergebnis } & \multirow[t]{2}{*}{ Anmerkung } \\
\hline & & & & Reagenz & UV [nm] & & \\
\hline \multirow[t]{6}{*}{ Alkamide } & Capsaicin & $\mathrm{A}_{1}, \mathrm{C}_{1}$ & II & $\mathrm{KMnO}_{4} / \mathrm{H}_{2} \mathrm{SO}_{4}$ & & positiv & mehrere braune Banden: Alkamide \\
\hline & & & & & & & Capsaicin mit gleicher Färbung, jedoch nie- \\
\hline & & & & & & & drigerer $\mathrm{R}_{\mathrm{f}}$-Wert, $\left(\mathrm{A}_{1}\right.$ : gutes Laufverhalten) \\
\hline & & & & & & & \\
\hline & & $\mathrm{A}_{1}, \mathrm{C}_{1}$ & $\mathrm{I}$ & $\mathrm{KMnO}_{4} / \mathrm{H}_{2} \mathrm{SO}_{4}$ & & positiv & polarerer Extrakt $\mathrm{C}_{1}$ verbleibt auf Startlinie; \\
\hline & & & & & & & eine braune Bande mit hohem $\mathrm{R}_{\mathrm{f}}$-Wert bei $\mathrm{A}_{1}$ \\
\hline |Phenolcarbonsäure- & & $\mathrm{C}_{1} \mathrm{C}_{2} \mathrm{C}_{2}$ & II & & & & gelb-orange Banden: Hinweis auf Flavonoide; \\
\hline \multirow{21}{*}{ derivate / Flavonoide } & s. Exp. Te1l & $C_{1}, C_{2}, c_{3}$ & & NaturstoII-Kgz. & 254,360 & positiv & blau-gefärbte Banden: Hinweis auf Phenol- \\
\hline & & & & & & & karbonsäurederivate \\
\hline & & & & & & & \\
\hline & & $\mathrm{C}_{1}$, Rohsap., & III & Naturstoff-Rgz. & 254,366 & positiv & Ergebnisse entsprachen in etwa denen mit \\
\hline & & Etherph., & & & & & FM II; beste Trennleistung dieser Stoff- \\
\hline & & $\mathrm{H}_{2} \mathrm{O}-\mathrm{Ph}$. & & & & & klassen \\
\hline & & & & & & & \\
\hline & & $\mathrm{C}_{1}$, Rohsap., & IV & Naturstoff-Rgz. & 254,366 & positiv & relativ lipohiles FM: gute Wanderung der \\
\hline & & Etherph., & & & & & Aglyka; Glykoside mehrheitlich auf Start- \\
\hline & & $\mathrm{H}_{2} \mathrm{O}-\mathrm{Ph}$. & & & & & linie; Bandentrennung insgesamt nicht gut \\
\hline & & & & & & & \\
\hline & & $\mathrm{C}_{1}$ & I & Naturstoff-Rgz. & 254,366 & negativ & lipohiles FM, keine Glykosidwanderung; \\
\hline & & & & & & & Trennung von Chlorophyllfraktionen: \\
\hline & & & & & & & mehrere grüne Banden nur bei Extrakt $\mathrm{C}_{1}$ \\
\hline & & & & & & & bereits vor Detektion (rot im UV $366 \mathrm{~nm}$ ) \\
\hline & & & & & & & \\
\hline & & $\mathrm{C}_{1}$, Rohsap., & V & Naturstoff-Rgz. & 254,366 & negativ & nur Trennung lipohiler Substanzen, wie \\
\hline & & Etherph., & & & & & Chlorophylle bei Extrakt $\mathrm{C}_{1}$ und Etherphase, \\
\hline & & $\mathrm{H}_{2} \mathrm{O}-\mathrm{Ph}$. & & & & & eine gelbe Bande (Blütenfarbstoff?) bei $\mathrm{C}_{1}$; \\
\hline & & & & & & & außerdem hier einige Banden nur im UV \\
\hline & & & & & & & $254 \mathrm{~nm}$ vor Detektion erkennbar \\
\hline
\end{tabular}


Tabelle 3b: DC-Screening von Acmella ciliata

\begin{tabular}{|c|c|c|c|c|c|c|c|}
\hline \multirow[t]{2}{*}{ Testgruppe } & \multirow[t]{2}{*}{ Referenzen } & \multirow[t]{2}{*}{ Extrakt } & \multirow[t]{2}{*}{ Fließmittel } & \multicolumn{2}{|c|}{ Detektion } & \multirow[t]{2}{*}{ Ergebnis } & \multirow[t]{2}{*}{ Anmerkung } \\
\hline & & & & Reagenz & $\mathbf{U V}[\mathbf{n m}]$ & & \\
\hline \multirow[t]{3}{*}{ Saponine } & s. Exp. Teil & Rohsapo- & VI & Anisaldehyd / & & positiv & mehrere grüne, gelbgrüne, graugüne, \\
\hline & & ningemisch & & $\mathrm{H}_{2} \mathrm{SO}_{4}$ & & & lila Banden; Farbgebung typisch, aber nicht \\
\hline & & & & & & & $\mathrm{R}_{\mathrm{f}}$-Wert-konform mit Referenzsubstanzen \\
\hline Phenole und kupplungs- & & $\mathrm{C}_{1}$ & II & Echtblausalz / $\mathrm{NH}_{3}$ & & positiv & fünf rote Banden sowie eine braune mit \\
\hline \multirow[t]{5}{*}{ fähige Amine } & & & & & & & hohem $\mathrm{R}_{\mathrm{f}}$-Wert \\
\hline & & & & & & & \\
\hline & & $\mathrm{A}_{1}, \mathrm{C}_{1}$ & I & Echtblausalz / $\mathrm{NH}_{3}$ & & negativ & FM zu lipohil, Trennung von Chlorophyllen \\
\hline & & & & & & & und Blütenfarbstoffen, grüne und gelbe \\
\hline & & & & & & & Banden (bereits vor Detektion erkennbar) \\
\hline & & & & & & & \\
\hline \multirow[t]{2}{*}{ Alkaloide } & & $\mathrm{C}_{1}$ & II & Dragendorffs-Rgz. & & negativ & keine orange-rot gefärbten Banden \\
\hline & & & & & & & \\
\hline \multirow{4}{*}{ Indolalkaloide } & & $\mathrm{C}_{1}$ & II & van Urk-Rgz. & & positiv & eine blaue Bande mit mittlerem $\mathrm{R}_{\mathrm{f}}$-Wert \\
\hline & & & & & & & \\
\hline & & $\mathrm{A}_{1}, \mathrm{C}_{1}$ & I & van Urk-Rgz. & & positiv & nur bei Extrakt $A_{1}$ einige blaue Banden mit \\
\hline & & & & & & & niedrigem $\mathrm{R}_{\mathrm{f}}$-Wert \\
\hline \multirow{6}{*}{ Cholesterolderivate } & Cholesterol. & $A_{1}, C_{1}$ & II & $\mathrm{Sb}_{2} \mathrm{Cl} /$ Eisessig & 366 & positiv & hei heiden Fytrakten · rosa-violett oefärhte \\
\hline & $\beta$-Sitosterol & $A_{1}, C_{1}$ & & $\mathrm{SO}_{3} \mathrm{Cl} / \mathrm{Clsessig}$ & 500 & positiv & $\begin{array}{l}\text { bei beiden Extrakten: rosa-violett getarbte } \\
\text { Banden mit hohem R-Wert auf Höhe der }\end{array}$ \\
\hline & & & & & & & Referenzsubstanzen sowie zwei weitere \\
\hline & & & & & & & Banden mit mittlerem bzw. niedrigem $\mathrm{R}_{\mathrm{f}}$-Wert \\
\hline & & & & & & & \\
\hline & & $\mathrm{C}_{1}, \mathrm{C}_{2}$ & $\mathrm{I}$ & $\mathrm{Sb}_{3} \mathrm{Cl} /$ Eisessig & 366 & positiv & wie bei FM II \\
\hline & & & & & & & \\
\hline Cumarine / Anthra- & s. Exp. Teil & $\mathrm{A}_{1}, \mathrm{C}_{1}, \mathrm{C}_{3}$ & I & KOH-Lsg. & 366 & negativ & kein Hinweis auf Cumarin - oder Anthranoid- \\
\hline chinone & & & & (methanol.) & & & aglyka \\
\hline & & & & & & & \\
\hline
\end{tabular}


Cumarine/Anthrachinone: In dem relativ lipophilen Fließmittelsystem I wäre mit der Detektion nur der Aglyka zu rechnen gewesen. Bei der Bornträgerreaktion (modifiziert) mittels 10 \%iger methanolischer Kalilauge kommt es zur Bildung eines vergrößerten Chromophors durch Entstehen eines Mesomerie stabilisierten Systems an Keto-EnolStrukturen, was zur Verschiebung in den längerwelligen Bereich des Lichts und dadurch meist zur Rotfärbung des Reaktionsprodukts führt. Dass diese Reaktion hier negativ ausfiel, später aber doch das Hydroxycumarin Aesculetin (3.4.3.4) isoliert wurde, erklärt sich mit den im Screening getesteten Extrakten $\mathrm{A}_{1}$ und $\mathrm{C}_{1}$, die aus der oberirdischen Gesamtpflanze gewonnen wurden, während zur Isolierung der phenolischen Verbindungen (3.4.2.) die Gesamtpflanze, einschließlich Wurzel, verwendet wurde. MARTIN [110] isolierte das Cumarinderivat Scopoletin auch aus der Wurzel. Daher ist es wahrscheinlich, dass in Acmella ciliata Cumarine bzw. deren Vorstufen nur in den Wurzeln akkumuliert werden. Außerdem hätten Hydroxycumarine bereits zuvor im UV-Licht bei $366 \mathrm{~nm}$ durch Eigenfluoreszenz auffallen müssen, was ebenfalls nicht der Fall war. Anthranoide sind offensichtlich nicht vorhanden und für einen Vertreter der Asteraceae ohnehin unwahrscheinlich [1].

\subsubsection{Acmella ciliata / Acmella oleracea- Vergleich - Frischpflanzen}

Abb. 14 stellt die Dünnschichtchromatogramme der Frischpflanzenextrakte mit Methanol $(\mathrm{H})$, Aceton (J) und Dichlormethan (G) von Acmella ciliata (1) und Acmella oleracea (gelb (2) und rot (3)) vergleichend gegenüber. Fließmittelsystem IX (Exp. Teil) und Anfärbung mit dem Anisaldehyd/Schwefelsäure-Reagenz dienten als geeignete DC-Parameter zur Auftrennung und Charakterisierung der vornehmlich lipophilen Alkamide. Die Extrakte wurden für die DC im jeweiligen Extraktionsmittel in einer Konzentration von $70 \mathrm{mg} / \mathrm{ml}$ gelöst, was halbquantitative Aussagen ermöglichte.
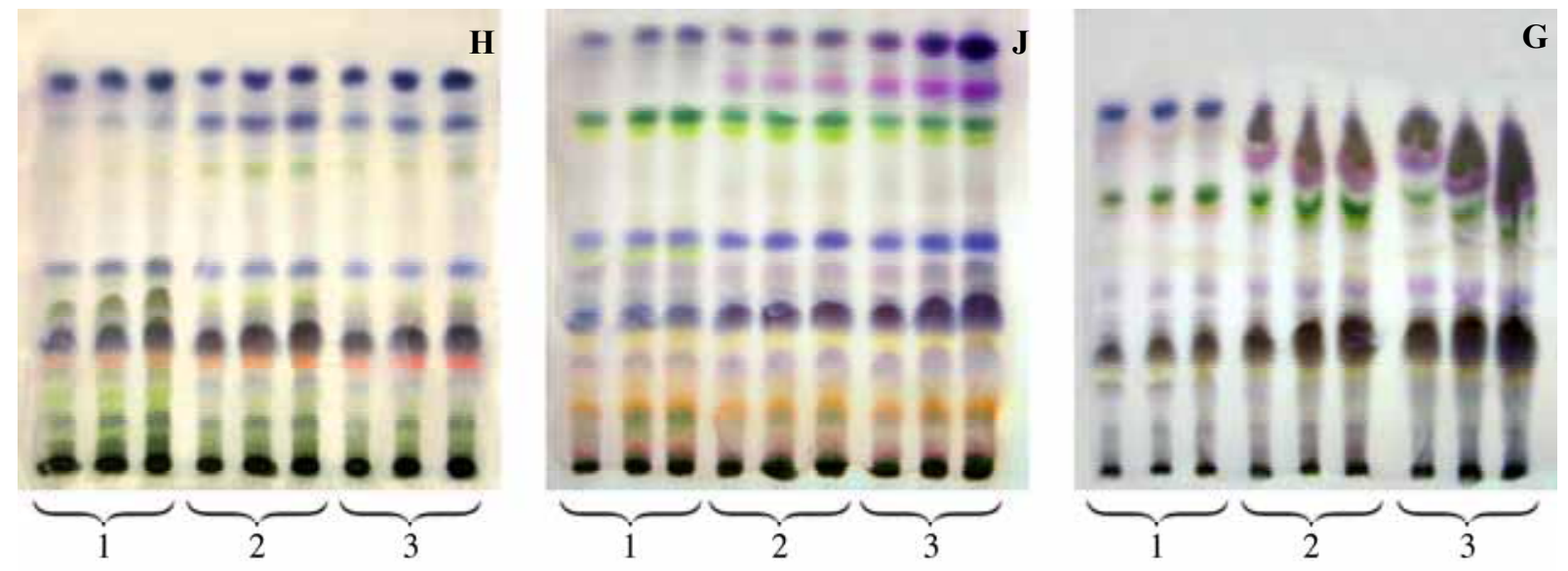

Abb. 14: Frischpflanzen-Extrakte von A. ciliata (1) und A. oleracea (gelb (2)) und (rot (3)), $\mathrm{H}=$ Methanol-, J=Aceton-, G=Dichlormethan-Extrakt; FM IX, Anisaldehyd/Schwefelsäure-Reagenz 
Alle drei Extrakte unterscheiden sich besonders im unteren Bereich (bis $\mathrm{R}_{\mathrm{f}}$ ca. 0,2) hinsichtlich Anzahl und Lage der Banden sowie deren Farbgebung. Mit steigender Lipophilie des Extraktionsmittels (Methanol $(\mathrm{H})<$ Aceton $(\mathrm{J})<$ Dichlormethan $(\mathrm{G})$ ) nimmt jedoch der Anteil der extrahierten lipophilen Substanzen zu: grüne Banden (Chlorophylle) und blau/violette bis braune Banden ab $R_{f}>0,7$. In der gleichen Reihenfolge $(H<J<G)$ ist im $\mathrm{R}_{\mathrm{f}}$-Bereich von 0,2 bis 0,7 [110] eine Zunahme der Alkamide zu ersehen (violett bis violettbraun: 2,4-Dienamide (vgl. [11]).

Eine gelbe Bande $\left(R_{f}\right.$ 0,32) in $J$ und $G$ lässt sich nach Bauer [11] als Monoenamid interpretieren. Bei der roten Bande $\left(\mathrm{R}_{\mathrm{f}} 0,27\right)$ in $\mathrm{H}$ könnte es sich um ein Lignan handeln (vgl. Abb. 49).

Die Unterschiede der drei Acmella-Arten/Varietäten sind eher quantitativer Natur. Eine grüne Bande fällt bei Acmella ciliata auf der Aceton-Platte und schwach auf der Methanol-Platte $\left(\mathrm{R}_{\mathrm{f}} 0,49\right)$ auf, die bei Acmella oleracea nicht detektiert wurde.

Eine zweite Versuchsreihe mit dem Fließmittelsystem VII und Detektion im UV-Licht bei $366 \mathrm{~nm}$ sowie Anfärbung mit dem Naturstoffreagenz eignete sich zur Darstellung der polareren phenolischen Verbindungen (Abb. 15 und 16). Hierbei erwies sich Methanol (H) als das eindeutig geeignetere Extraktionsmittel. Mit Aceton (J) hingegen sind nur stärker lipophile Substanzen extrahierbar, die in diesem Fließmittel mit mittlerem bis hohem $\mathrm{R}_{\mathrm{f}}$-Wert erscheinen.

Fließmittelsystem VII ist ein universelles System zum Screening phenolischer Bestandteile, die als blaue Eigenfluoreszenzen im UV-Licht bei $366 \mathrm{~nm}$ auffallen (außer Flavonoiden) (Abb. 15). Nach der Detektion mit Naturstoffreagenz (Abb. 16) färben sich Flavonoide gelb. Diese sind nur im Methanolextrakt $(\mathrm{H})$ erkennbar. Es fällt auf, dass Acmella oleracea mehr Flavonoide enthält als Acmella ciliata und hier wiederum die rote Varietät mehr als die gelbe. Andererseits zeigt Acmella ciliata intensivere blau-fluoreszierende Banden (Abb. 15 und 16), die für einen höheren Gehalt an Phenolcarbonsäuren (Zimt- bzw. Kaffeesäurederivate) sprechen.

Die detektierten Banden sind bei den drei Pflanzen qualitativ weitgehend identisch, mit einer Ausnahme. Im Gegensatz zu Acmella oleracea weist Acmella ciliata mit einem $\mathrm{R}_{\mathrm{f}}$-Wert von 
0,45 eine zusätzliche intensiv blaue Bande auf (Abb. $15(\mathrm{H})$ und $16(\mathrm{H})$, s. roter Pfeil). Wie sich im Verlauf der weiteren Arbeit herausstellte, handelt es sich hierbei um das cis-Isomer des Kaffeoyläpfelsäureesters (s. 3.4.3.2.2.). Dies scheint zumindest ein prägnanter qualitativer Unterschied zwischen den beiden Arten Acmella ciliata und Acmella oleracea zu sein, der als phytochemisches Unterscheidungsmerkmal herangezogen werden könnte.

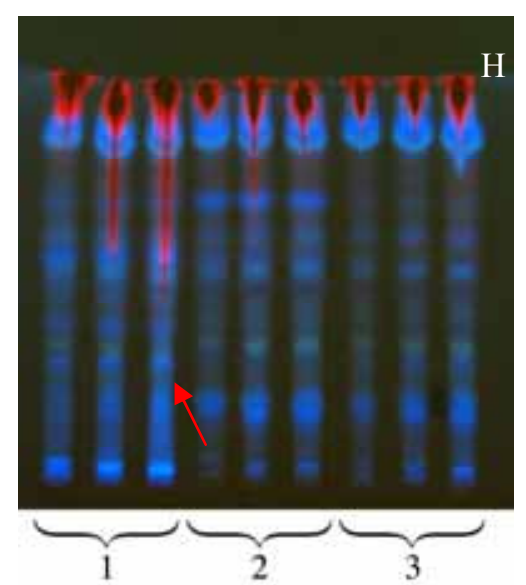

\section{Abb. 15:}

Extrakte von $A$. ciliata (1) und $A$. oleracea (gelb (2)) und (rot (3)), $\mathrm{H}=$ Methanol, $\mathrm{J}=$ Aceton; FM VII; UV= $366 \mathrm{~nm}$

Pfeil: cis-Kaffeoyläpfelsäureester

\section{Abb. 16}

Extrakte von $A$. ciliata (1) und A. oleracea (gelb (2)) und (rot (3)) $\mathrm{H}=$ Methanol, $\mathrm{J}=$ Aceton; FM VII, Naturstoff-Reagenz Pfeil: cis-Kaffeolyäpfelsäureester
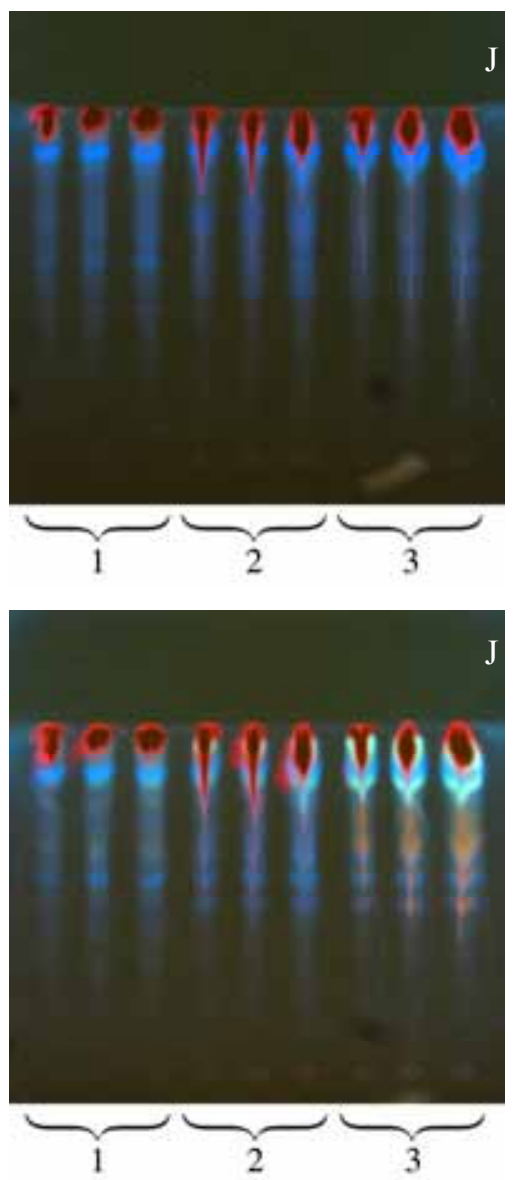

Der Ethanolextrakt (I), als typischer „Saponinextrakt“, diente zum Nachweis von Saponinen in den drei Acmella-Arten. Als Fließmittel wurde die nach Ausschüttelung abgetrennte Oberphase eines Standard-Saponin-Fließmittelgenischs (VI) verwendet.

Die Bandentrennung erfolgte jedoch nicht sauber, und Unterschiede zwischen den drei Pflanzen ließen sich ebenfalls nicht erkennen (nicht abgebildet). Das Vorhandensein von Saponinen ist aber an Hand der Detektion mehrerer Banden in diesem System zu vermuten und wird durch die ebenfalls positive Schaumprobe (Exp. Teil) untermauert. Außerdem gelang anderen Autoren [128] die Isolierung eines Saponins aus „Spilanthes acmella“ (Murr.), was die Präsenz von Saponinen auch in den untersuchten Acmella-Arten wahrscheinlich macht. 


\subsection{Alkamide}

\subsubsection{Literaturüberblick}

\subsubsection{Vorkommen / Strukturen}

Alkamide (= Alkylamide) treten gehäuft in den Familien der Asteraceae, Rutaceae und Piperaceae auf und mit einigen Vertretern auch in den Aristolochiaceae sowie in Phyllanthus fraternus ssp. togoensis Brunel \& Roux (Euphorbiaceae) und Capsicum frutescens L. (Solanaceae) [50, 110, 156, 162, 172, 188]. Letztere Pflanze ist als Cayennepfeffer oder Chili nicht nur als Gewürz gut bekannt, sondern durchaus auch von pharmazeutischer Relevanz.

Bezüglich des strukturellen Aufbaus der Alkamide und ihrer Biogenese bestehen auffällige Gemeinsamkeiten zwischen den Pflanzenfamilien der Asteraceae, Rutaceae und Aristolochiaceae [110]. Die Biogenese ihres Fettsäurerests geht aus von Linolen- bzw. Ölsäure, aus welcher erstere durch Oxidation (Dehydrierung) entsteht (s. u.). Wie von MARTIN und GREGER beschrieben [50, 110], finden diverse oxidative Prozesse, Kettenabbrüche (-verkürzungen) und Umlagerungen von Mehrfachbindungen, partiell anschließende Wasserabspaltung unter Bildung neuer ungesättigter Strukturen statt, teilweise enzymatisch katalysiert.

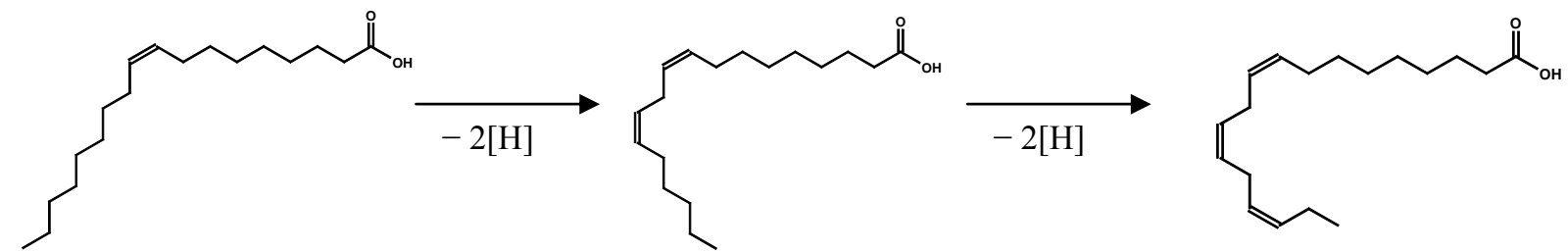

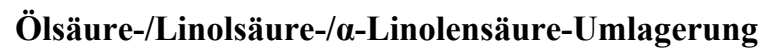

Demgegenüber erfolgt die Biogenese der Alkamidsäurereste der Piperaceae, z. B. der Piperinsäure im Piperin (Tafel I / Formel 8), durch Kondensation einer als Malonyl-Coenzym A aktivierten Acetateinheit an ein aktiviertes Zimtsäurederivat (Acetatweg). Der Fettsäurerest der Capsaicinoide von Capsicum spec. (Tafel I / Formel 10) wird aus Isobutyryl-Coenzym A und drei Acetyl-Gruppen zusammengesetzt [156].

Gemeinsam ist fast allen Alkamiden der Aufbau aus einem Carbonsäureteil mit einer meist aliphatischen und unverzweigten Alkylkette und einem Aminteil. Eine Ausnahme hiervon bilden einige Alkamide von Glycosmis-Species (Rutaceae), bei denen ein Kohlenstoffatom im 
Carbonylrest durch Schwefel ersetzt ist (Tafel I / Formel 1). Hier wird an Stelle einer Fettsäure von Cystein als Baustein ausgegangen [56].

Eine große Variationsbreite ist bezüglich des Aufbaus der Fettsäurereste anzutreffen. Es kommen rein „olefinische“ (Tafel I / Formel 2 und 12) sowie gemischte „olefinischacetylenische“ (Tafel I / Formel 3) Strukturen unterschiedlicher Kettenlänge und Isomerien vor. Allerdings werden Dreifachbindungen, also „acetylenische“ Strukturen, bisher lediglich bei Vertretern der Asteraceae beschrieben.

In der vorliegenden Arbeit wurden auch Amide aufgefunden, die sich von Dicarbonsäuren ableiten (3.5.2.), wobei eine dieser Verbindungen bereits in Piper hancei Maxim. (Piperaceae) nachgewiesen wurde (Tafel I / Formel 4) [171].

Im Fettsäurerest können darüber hinaus Hydroxyl- oder Hydroperoxy-Gruppen (Tafel I / Formel 5 und 15) sowie Epoxystrukturen (Tafel I / Formel 6) auftreten [7, 16, 50, 103, 110, 132, 160]. Wie GREGER [50] zeigte, geht die Bildung verschiedener Strukturen im Fettsäurerest durch unterschiedliche Oxidationsprozesse - verbunden oft mit Dehydratisierungen - sowie Reduktionsprozesse vonstatten. So können z. B. durch Oxidation Alkylamide mit OH-Gruppen entstehen und diese später durch Dehydratisierung in neue ungesättigte Strukturen übergehen, bis hin zu vollkommen ungesättigten (konjugierten) Systemen (Tafel I / Formel 13), was bei Achillea-Arten aus dem Tribus Anthemideae gefunden wurde [50, 54, 101]. Ein Dihydroxyderivat ist in Piper sylvaticum Roxb. (Piperaceae) (Tafel I / Formel 15) sowie in „Spilanthes callimorpha“ Moore (Acmella calva (DeCandolle in Wight) Jansen; Tafel I / Formel 16) nachgewiesen worden [50, 7, 103]. Diese Aussagen spielen auch bei der Strukturaufklärung der Acmella-Alkamide (3.3.3.) eine wichtige Rolle, da sich hier ebenfalls hydroxilierte Säurereste fanden.

Gelegentlich kommt auch ein Thiophen-Rest im Säureteil vor, der vermutlich durch Anlagerung von Schwefel aus $\mathrm{H}_{2} \mathrm{~S}$ an Dien-Strukturen entsteht. Eine solche Alkamid-Struktur wurde allerdings nur im Asteraceae-Tribus Anthemidae beschrieben [51], z. B. bei Matricaria pubescens (Desf.) Schultz Bip. (Tafel I / Formel 7).

Ausgangssubstanzen zur Biosynthese der Aminreste in den Alkamiden der betreffenden Pflanzenfamilien sind verschiedene biogene Aminosäuren. Als Aminteil fungiert zumeist der 
Isobutylamin-Rest (Tafel I / Formeln 2, 3, 4, 7, 12 und 15 sowie 3.3.3.) der aus Valin hervorgeht. Aus Isoleucin resultiert der Methylbutylamin-Rest (s. 3.3.3.), aus Phenylalanin der Phenylethylamin-Rest (Tafel I / Formeln 1, 5, 6 und 14 sowie 3.3.3.), aus Tyrosin der Hydroxyphenylethylamin-Rest, bisher nur für Anacyclus pyrethrum DC. beschrieben [22, 91], und aus Leucin entsteht der Isopentyl-Rest (Tafel I / Formel 13), der bisher nur in der Asteraceae-Gattung Achillea [54] gefunden wurde. Nach Decarboxylierung der entsprechenden Aminosäure kommt es zur Bildung dieser Aminreste.<smiles>CC(C)C(N)C(=O)O</smiles>

Valin<smiles>CCC(C)C(N)C(=O)O</smiles>

Isoleucin<smiles>NC(Cc1ccccc1)C(=O)O</smiles>

Phenylalanin<smiles>NC(Cc1ccc(O)cc1)C(=O)O</smiles>

Tyrosin<smiles>CC(C)CC(N)C(=O)O</smiles>

Leucin<smiles>NCCCCC(N)C(=O)O</smiles>

Lysin

In den Ecliptinae kommt neben den Isobutyl- sowie Phenylethylamin-Resten der Methylbutylamin-Rest vor (s. 3.3.3.). Das Dehydroderivat des Phenylethylamin-Rests, der Styrylamin-Rest, wurde ebenfalls nachgewiesen [16]. In der Gattung Echinacea scheint dagegen der Phenylethylamin-Rest zu fehlen.

Ein Piperidyl-Rest als Aminkomponente wurde neben den Piperaceae (Tafel I / Formel 8) auch bei den Asteraceae-Gattungen Achillea und Artemisia (Tafel I / Formel 9), Tribus Anthemideae, beschrieben. In Letzteren wurde außerdem das entsprechende 2,3Dehydroderivat entdeckt [14, 47-49, 52, 154, 156, 167, 172]. Der Piperidyl-Rest entsteht aus Lysin nach Zyklisierung und Decarboxylierung über die Vorstufe Piperideid (2,3Dehydropiperidin).

Ebenfalls in der Gattung Achillea und seltener bei den Piperaceae kommt der Pyrrolidinring vor, in Achillea auch das 2,3-Dehydroderivat [47-49, 52, 55, 156, 167, 172]. Ein 4-Hydroxy2,3-didehydropyrrolidinring wurde in Achillea falcata L. nachgewiesen [49]. Für Achillea ageratifolia (Sibth. \& Smith) Boiss. ssp. serbica (Nym.) Heimerl wird auch der Pyrrolring als Aminkomponente beschrieben [55].

Die meisten Alkamide der Piperaceae, die Capsaicinoide aus Capsicum (Solanaceae) und die Amide aus Phyllanthus fraternus ssp. togoensis Brunel \& Roux (Euphorbiaceae) weisen, wie bereits angedeutet, deutlich abweichende Strukturen von den Acmella- und insgesamt Ecliptinae-Alkamiden auf $[162,156]$. So stellt das Alkamid aus Phyllanthus fraternus ssp. togoensis Brunel \& Roux (Tafel I / Formel 11) ein einfaches, unsubstituiertes Säureamid dar, 
und im Capsaicin (Tafel I / Formel 10) wird der Aminrest, ein Benzylaminderivat, ebenfalls von Phenylalanin auf allerdings bislang ungeklärtem Wege gebildet.

Andererseits finden sich aber auch bei einigen Piperaceae mit Acmella-Alkamiden ähnliche bis identische Strukturen, z. B. das bereits erwähnte Fumarsäureamid (Tafel I / Formel 4) aus Piper hancei Maxim. sowie das 4,5-Dihydroxy-decen-isobutylamid (Sylvamid) [7, 171].

Tafel I: Allgemeine Alkamidbeispiele, Teil 1

$$
1
$$<smiles>CSC(=O)N(C)CCc1ccccc1</smiles>

Niranin (aus: Glycosmis spec.)

3

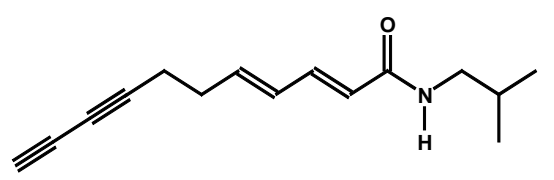

Undeca-2E,4E-dien-8,10-diinsäure-isobutylamid (aus: Acmella ciliata, Echinacea atrobubens, Achillea ptarmica, Achillea macrophylla)

5<smiles>C#CC#CCCC(O)C(O)C(=O)NCCc1ccccc1</smiles>

3-Hydroxyperoxy-2-hydroxy-nona-6,8-diinsäurephenylethylamid (aus: "Spilanthes acmella")

7<smiles>CC(C)CNC(=O)/C=C/C=C/Cc1cccs1</smiles>

6-(2-Thienyl)-Hexa-2E,4E-diensäure-isobutylamid (aus: Matricaria pubescens)<smiles>C/C=C\C=C/CC/C=C/C(=O)NCC(C)C</smiles>

Spilanthol (aus: Acmella ciliata, "Spilanthes oleracea", "Spilanthes acmella", Acmella caulirhiza, Acmella oppositifolia, Heliopsis longipes)

4<smiles>CC(C)CNC(=O)/C=C/C(=O)O</smiles>

(E)-4-[2-(methylpropyl)amino]-4-oxo-2butensäure (aus: Acmella ciliata, Piper hancei)

6<smiles>C#CC#CCCC1OC1C(=O)NCCc1ccccc1</smiles>

2,3(c)-Epoxy-6,8-diinsäure-phenylethylamid (aus: Acmella ciliata, "Spilanthes acmella")

8<smiles>O=C(/C=C/C=C/c1ccc2c(c1)OCO2)N1CCCCC1</smiles>

Piperin (aus: Piper spec.)

Ansonsten sind bei Zanthoxylum-Species Alkamide mit Zimtsäure als Baustein anzutreffen $[2,3,150]$, ebenso wie hydroxilierte Amide, wobei die Hydroxilierung meist am C-2' des 
Isobutyl-Rests stattfindet [4, 122, 186, 187]. Zimtsäureamide sind bei den Ecliptinae bisher lediglich bei „Spilanthes ocymifolia“ (Lamarck) Moore (Acmella alba (L’Heritier) Jansen var. alba Jansen) (Tafel I / Formel 14) nachgewiesen worden [19]. Interessanterweise konnte der Baustein Zimtsäure während dieser Arbeit auch in Acmella ciliata entdeckt werden (3.5.1.3.).

Tafel I: Allgemeine Alkamidbeispiele, Teil 2

9<smiles>CCCCC/C=C\C=C\C(=O)N1CCCCC1</smiles>

Deca-2E,4Z-diensäure-piperinamid (aus: Artemisia dranunculus)

11<smiles>CCC/C=C/C=C/C(N)=O</smiles>

Octa-2E,4E-diensäure-amid (aus: Phyllanthus fraternus)

13<smiles>C/C=C\C#CC#C/C=C/C=C/C=C/C(=O)NCCC(C)C</smiles>

Tetradeca-2E,4E,6E,12Z-tetraen-8,10-diinsäureisopentylamid (aus: Achillea wilhelmsii)

15<smiles>CCCCCC(O)C(O)C=CC(=O)NCC(C)C</smiles>

4,5-Dihydroxy-deca-2E-ensäure-isobutylamid (aus: Piper sylvaticum)
10<smiles>CC(C)/C=C/CCCCC(=O)NCc1ccc(O)c(O)c1</smiles>

Capsaicin (aus: Capsicum frutescens)

12<smiles>C/C=C/C=C/C=C\CC/C=C/C(=O)NCC(C)C</smiles>

$\alpha$-Sanshool (aus: Zanthoxylum spec.)

14<smiles>O=C(/C=C/c1ccccc1)NCCc1ccccc1</smiles>

N-2-Phenylethylcinnamoylamid (aus: „Spilanthes ocymifolia”)

16<smiles>CC(O)/C=C/C(O)CC/C=C/C=C/C(=O)NCC(C)C</smiles>

8,11-Dihydroxy-dodeca-2E,4E,9E-triensäureisobutylamid (aus: „Spilanthes callimorpha“)

Bei den Asteraceae kommen Säureamide nur im Subtribus Ecliptinae der Heliantheae und in den Tribus der Anthemideae (s. o.) und Senecioneae [15] vor. Zwischen den Amiden 
verschiedener Arten innerhalb einer Gattung besteht große strukturelle Ähnlichkeit. So kommt das Leitalkamid aus Acmella ciliata, das Spilanthol (Deca-2E,6Z,8E-triensäureisobutylamid), in mehreren Acmella-Arten, außerdem in den Gattungen Wedelia und Heliopsis [30, 53, 93, 124, 134, 148] vor. Sie alle gehören dem Subtribus Ecliptinae im Tribus Heliantheae an [208, 149]. Das Acmella ciliata-Alkamid Undeca-2E,4E-dien-8,10- diinsäureisobutylamid (A1, 3.3.3.2.; [110, 112]) wurde beispielsweise auch in Echinacea atrorubens Nutt. [35] gefunden. Vom Alkamid-Isomerenpaar Dodeca-2E,4E,8Z,10E/Z-tetraensäureisobutylamid, Hauptalkamid(e) in Echinacea purpurea Moench und Echinacea angustifolia DC. $[8,9,11,13]$, konnte in Acmella ciliata das 10E-Isomer(e) nachgewiesen werden ([110, 112] und 3.3.3.18.). Die Pflanzen der Gattung Echinacea stellen auch Vertreter der Ecliptinae dar.

In diesem Zusammenhang wäre die Untersuchung der Gattung Spilanthes, von der sich der Name der Verbindung Spilanthol ableitet, hinsichtlich ihres Alkamidgehalts interessant; denn bisherige Publikationen, die unter dem Gattungsnamen Spilanthes veröffentlicht sind, befassten sich nach der neuen Nomenklatur von JANSEN 1985 [81] eigentlich mit der Gattung Acmella.

\subsubsection{Anwendung / Wirkung}

\subsection{Traditionelle Anwendung}

Während die traditionelle Anwendung in Südamerika Frischpflanzenteile benutzt, kommt hier zu Lande die bei $40^{\circ} \mathrm{C}$ getrocknete Gesamtpflanze als Ausgangsdroge für die pharmazeutische Anwendung zum Einsatz [81].

Die traditionelle Anwendung der Pflanze geht auf südamerikanische Ureinwohner zurück, die Acmella ciliata bei Zahnfleischbluten einsetzten [163]. Die Blütenköpfe und Wurzeln werden in Südamerika auch heute noch bei Zahnschmerzen gekaut, womit man sich die analgetische, antiphlogistische und lokalanästhetische Wirkung zu Nutze macht. Ebenfalls wird Acmella ciliata zur Anregung des Speichelflusses gekaut und soll auch gegen Skorbut eine gewisse Bedeutung haben. Das Kauen der Pflanzenteile bewirkt zunächst ein Prickeln, gefolgt von leichter Taubheit im Mundraum (schwache Lokalanästhesie) [21, 57, 81, 110, 193, 210, 222]. Insektizide Wirkungen waren den Naturvölkern ebenfalls schon bekannt [81]. 
Auf Grund der enthaltenen „Scharfstoffe“ (Alkamide), die heute vorrangig für die medizinischen Wirkungen verantwortlich gemacht werden, erlangte die Pflanze auch als Gewürz Bedeutung, als welches sie beispielsweise Salaten, Suppen und Fleisch sowie Maniok, als einer der Hauptnahrungsquellen für die Menschen des Amazonasbeckens, zugesetzt wird [81, 206, 212]. Eine echte „Scharfwirkung“, wie z. B. beim Cayennepfeffer, dessen Hauptalkylamide sich in der Struktur auch von denen in Acmella ciliata nachgewiesenen (Tafel I, Teil 2 / Formel 10 unter 3.3.1.1.) deutlich unterscheiden, tritt jedoch nicht ein. Die aufgeführten Anwendungen beschränken sich nicht allein auf Acmella ciliata, sondern gelten auch für weitere verwandte Arten [81, 206].

Die heutige Anwendung und Zulassung als (traditionelles) Arzneimittel in der Bundesrepublik Deutschland orientiert sich an Überlieferungen der Volksmedizin und basiert auf anwendungsbezogenen klinischen Studien der Gesamtpflanzenextrakte (2.4.). Die registrierten Arzneiformen Salbe, Gel und Lösung unter dem Präparatenamen Spolera ${ }^{\circledR}$ sind zur topischen Anwendung zwecks Besserung des Befindens bei Prellungen und Stauchungen zugelassen $[75,164]$.

Bisher liegen keine Publikationen über in vitro-Studien (z. B. enzymatische Untersuchungen) an Acmella ciliata vor. Allerdings muss einschränkend hinzugefügt werden, dass es diverse Veröffentlichungen gibt, die einen nach JANSEN [81] als obsolet anzusehenden Pflanzennamen, z. B. Spilanthes acmella, benutzen, wobei es sich durchaus um ein Exemplar der Gattung Acmella handeln dürfte. Durch die im Kapitel 1.1. erwähnte Konfusion bezüglich der Taxonomie und Verwechslungen mit Artverwandten kann oft nicht exakt eruiert werden, auf welche Pflanze sich publizierte Untersuchungsergebnisse de facto beziehen.

Ausgehend von den beschriebenen Gemeinsamkeiten von Acmella ciliata mit ihren Verwandten, resultieren aus der Literatur insgesamt Hinweise auf folgende medizinische Wirkqualitäten: analgetisch, antiphlogistisch, lokalanästhetisch, antithrombotisch, fungizid, antibakteriell, antiviral, insektizid, larvizid und molluskizid, immunmodulatorisch, antihepatotoxisch und ichthyotoxisch $[5,23,24,39,40,81,93-95,110,125,137,138,141$, $151,152,163,164,169,193,210,223]$.

Darüber hinaus sind Anwendungen in der kosmetischen Industrie bekannt. Beispielsweise wird Spilanthol, vermutlich auf Grund seiner antibakteriellen Wirkung, Mundwässern und 
Zahnpasten zugesetzt [61, 84-86, 159, 168, 174, 175, 189]. Offensichtlich werden dem Spilanthol auch durchblutungsfördernde und geschmacksverbessernde Eigenschaften zugesprochen, was sich in der Verwendung als Badezusatz bzw. als Zusatz zu Süßungsmitteln widerspiegelt $[121,127]$.

\subsection{Potentielle Wirkungen / Wirkprinzip}

Von herausragendem Interesse hinsichtlich potentieller Anwendungsmöglichkeiten und zur Erklärung von traditionell mehr oder weniger empirisch erzielten Wirkungen dürfte die Wirkstoffgruppe der in Acmella ciliata gefundenen Alkamide sein, und insofern erscheint bezüglich des Wirkprinzips auch ein Blick auf die bisher untersuchten Pflanzen mit identischen und vergleichbaren Inhaltsstoffen (s. 3.1.) von Relevanz.

Das inzwischen gut bekannte Wirkprinzip der nichtsteroidalen Antiphlogistika und Antirheumatika basiert vornehmlich auf der Hemmung des Enzyms Cyclooxygenase-2 (COX2) $[105,142]$. COX-2 spielt eine entscheidende Rolle bei der Prostaglandin-Synthese [142]. Die Prostaglandine sind wiederum u. a. an Entzündungsreaktionen und der Schmerzwahrnehmung beteiligt [70]. Verschiedene Phytopharmaka-Studien versuchen nun, eine pharmakologische Erklärung für die beobachteten analgetischen und antiphlogistischen Wirkungen von Alkamiden bzw. alkamidreichen Extrakten und Fraktionen zu finden, indem sie ihnen ebenfalls eine Beeinflussung der COX-2 zusprechen. Untersuchungen hierzu wurden hauptsächlich an Echinacea-Alkamiden durchgeführt, die, wie unter 3.1. ausgeführt, Analogien zu den Alkamiden in Acmella ciliata aufweisen.

Beobachtet wurde einerseits eine Verringerung der Prostaglandin-Syntheseleistung an menschlichen Neurogliom-Zellen und andererseits eine Stimulierung der COX-2-Expression. Diese scheinbar paradoxe Wirkung war auch beim Einsatz höhermolekularer Konzentrationen nichtsteroidaler Antiphlogistika, wie Diclofenac, Indomethacin und Ibuprofen festzustellen [70]. Ebenso stellten WOELKART et al. [182] die Hemmung der COX-2-abhängigen Prostaglandin-Synthese durch Alkamide in bestimmten Zellkulturen fest, was mit Hilfe eines COX-2-Aktivitäts-Assays bestimmt wurde.

Für die Alkamide Dodeca-2E,4E,8Z,10Z/E-tetraensäure-isobutylamide - Hauptalkamide in Echinacea-Arten - wurden Hemmungen der Cyclooxygenase-1 (COX-1) sowie der 5- 
Lipoxygenase (5-LOX), also weiterer Enzyme der Entzündungskaskade, aufgezeigt [129]. Das 10E-Isomer wurde auch in Acmella ciliata nachgewiesen [110, 112 und 3.3.3.18.]. Ebenso hemmten Spilanthol, Leitsubstanz von Acmella ciliata und weiterer Acmella-Arten, die 5-LOX signifikant. Beim Vergleich eines mit Alkamiden angereicherten Extrakts von „Spilanthes oleracea“ L. (Acmella oleracea (L.) Jansen [81]) mit entsprechenden Extrakten von Echinacea purpurea (L.) Moench sowie Echinacea angustifolia DC. bewegte sich die Hemmaktivität von Echinacea purpurea im gleichen Bereich wie die von „Spilanthes oleracea“ (Acmella oleracea). Die Hemmstärke des Extrakts von Echinacea angustifolia war jedoch weit geringer [178].

Mittels eines ELISA-Testsytems am isolierten Reinenzym konnten verschieden starke Hemmungen der COX-1 und COX-2 mit oben erwähnten Amiden gezeigt werden [145]. Ebenso wiesen auch CLIFFORD et al. [29] unterschiedliche Inhibitionsraten für die COX-1 und -2 durch verschiedene Alkamide am isolierten Enzym aus Schafsamenblasen nach.

Die derzeitige Hauptanwendung von Echinacea-Präparaten beruht auf ihren immunstimulierenden Eigenschaften, wobei entzündungshemmende Effekte als ein Teil der immunstimulierenden Eigenschaften $\mathrm{zu}$ begreifen sind. Auch hier werden wiederum die Alkamide als mögliche Ursache zur Diskussion gestellt. Beispielsweise zeigten alkamidhaltige, lipophile Extrakte in vitro und in vivo eine Erhöhung der Phagozytoserate $[10,12]$. Außerdem konnten moderate entzündungshemmende NO-induzierende Wirkungen konstatiert werden [35].

Auffällig ist weiterhin die große Ähnlichkeit vieler Alkamide mit Anandamid (NArachidonyl-ethanolamin), einem endogenen Liganden der CB1- und CB2-Rezeptoren (Cannabinoidrezeptoren). Diese Rezeptoren gehören zur Familie der G-Protein-gekoppelten Rezeptoren. Den CB1-Rezeptor findet man hauptsächlich im Zentralnervensystem, während der CB2-Rezeptor in der Milz und in Immunzellen exprimiert wird [143, 182]. Exogene Cannabinoide, wie das Tetrahydrocannabinol (aus Cannabis sativa L.), weisen, vergleichbar mit körpereigenen Cannabinoiden, außer einer analgetischen Wirkung auch einen immunsuppressiven Effekt auf. Die dafür erforderliche Fähigkeit zur Bindung, besonders an den CB2-Rezeptor, besitzen auch viele Alkamide auf Grund ihrer Ähnlichkeit zu Anandamid [143, 182]. Durch Bindung an CB2-Rezeptoren wurde beispielsweise eine erhöhte Expression 
von Interleukin-6 (IL-6) im menschlichen Blut festgestellt, was große Bedeutung bei der Immunabwehr und speziell der Infektabwehr hat [35, 142, 143].

Erwähnenswert erscheint schließlich der neuere Befund, dass Alkamide in vivo resorbiert werden, nachgewiesen in nanomolarer Konzentration im menschlichen Blutplasma [34, 35, 114, 181, 183, 184]. Bereits in diesen niedrigen Konzentrationen beeinflussen Alkamide und Anandamid das Milieu der Zytokine im menschlichen Blut. Hier können sie nicht nur antiinflammatorisch, sondern auch pro-inflammatorisch wirken, was vom Stimulus, ihrer Konzentration und der Anzahl ihrer Doppelbindungen determiniert wird. Jedoch lassen sich nicht alle diese Effekte über eine Bindung am CB2-Rezeptor erklären, da auch ein dort nicht bindendes Alkamid ähnliche Effekte zeigte [143]. Hier bedarf es weiterer Untersuchungen.

Die dargestellten Forschungsergebnisse an alkamidhaltigen Pflanzen ermutigen durchaus dazu, auch in Acmella ciliata eine wesentlich potentere Pflanze zu sehen als ihrem derzeitigen Einsatzgebiet entspricht. In dem bisher zur Anwendung gelangten 2-PropanolGesamtpflanzenextrakt im Phytopharmakon Spolera ${ }^{\circledR}$ resultiert natürlich eine komplexe Wirkung (synergistischer Effekt) der verschiedenen Inhaltsstoffgruppen; für analgetische und antiphlogistische Wirkungen können beispielsweise auch phenolische Komponenten mitverantwortlich sein $[35,105]$. Deshalb kommt der weiteren Charakterisierung der Acmella ciliata-Inhaltsstoffe und der Untersuchung von Struktur-Wirkstoff-Beziehungen große Bedeutung zu.

\subsubsection{Isolierung}

Nach entsprechenden Vorversuchen (s. 2.3.) erwies sich das apolare Dichlormethan $\left(A_{2}, T a b\right.$. 1) als optimales Extraktionsmittel zur Gewinnung von Alkamid-reichen Extrakten aus der getrockneten und grob gepulverten Gesamtpflanze von Acmella ciliata.

Zur dc Charakterierung der Extrakte und späterer SC-Fraktionen war das Fließmittel Dichlormethan/Ethylacetat (80:20) geeignet, das bereits eine relativ differenzierte Trennung ergab. Die Detektion erfolgte visuell durch Fluoreszenzminderung im UV-Licht $(\lambda=254 \mathrm{~nm})$. $\mathrm{Da}$ es kein für Alkamide spezifisches Detergens gibt (Strukturvielfalt dieser Stoffklasse), wurde Kaliumpermanganat/Schwefelsäure-Reagenz (Nachtrocknung bei $105^{\circ} \mathrm{C}$ ) genutzt, mit 
dem allgemein organische, oxidierbare Substanzen detektiert werden. Es erschienen lilabraune Banden.

Im Ergebnis der Soxhlet-Extraktion mittels Dichlormethan (s. Exp. Teil) resultierten nach Filtration und Einengung im Vakuumrotationsverdampfer aus insgesamt $1 \mathrm{~kg}$ Droge 26,2 $\mathrm{g}$ Extrakt von intensiv grüner Farbe (Chlorophyll) und halbfester Konsistenz.

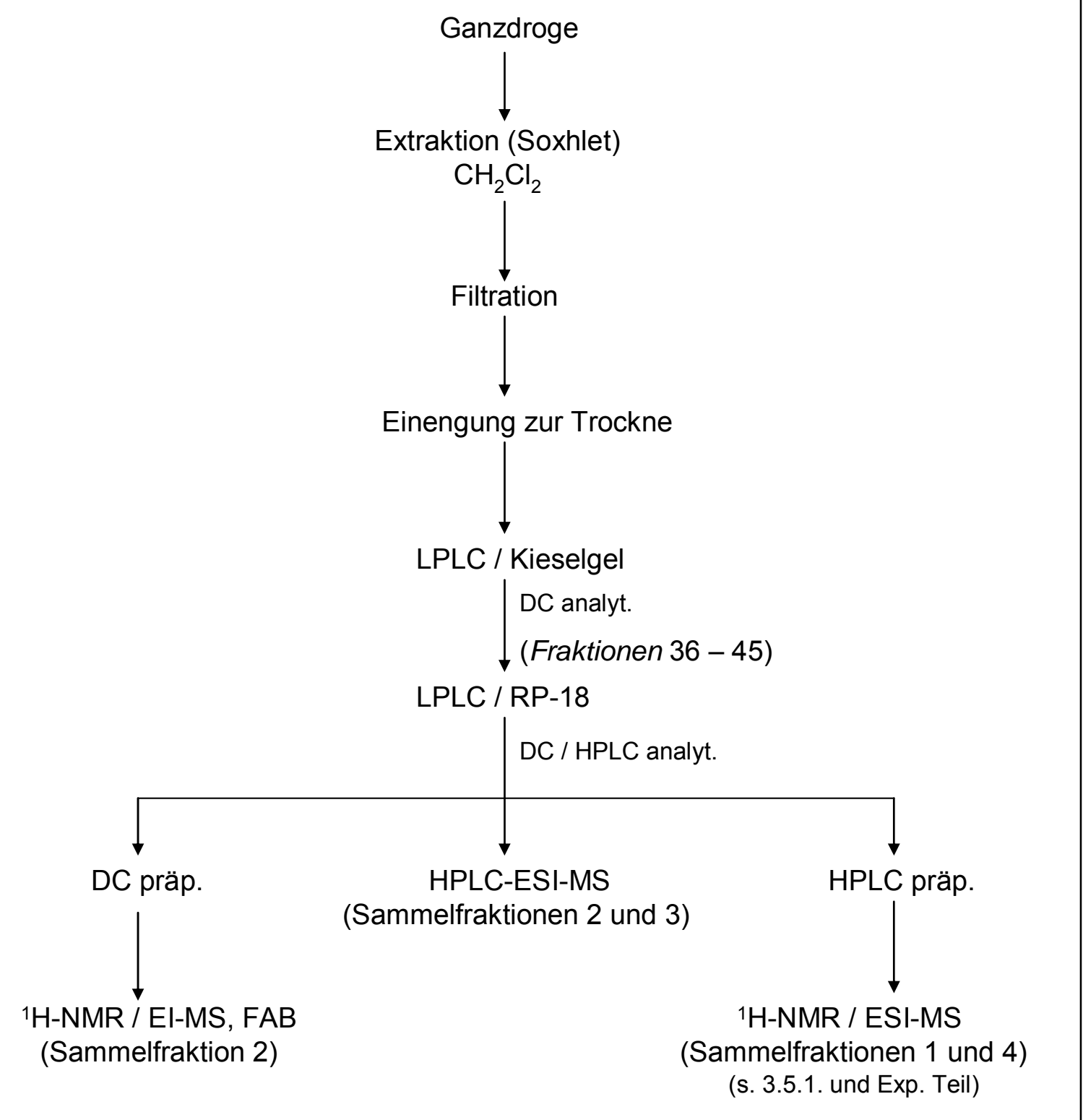

Abb. 17: Extraktion und Analytik von Alkamiden aus $A$. ciliata

Die weitere Aufarbeitung und Fraktionierung anteiliger Extraktmengen erfolgte entsprechend dem Schema in Abb. 17 zunächst durch Niederdruckchromatographie (LPLC) auf Kieselgel 
bei $1 \mathrm{MPa}$ mittels eines Stufengradienten von Dichlormethan/Ethylacetat (beginnend mit 90:10, s. Exp. Teil).

Die Kieselgelsäule diente zur groben Fraktionierung sowie der Abtrennung hydrophiler Begleitsubstanzen, die weitgehend auf der Säule verblieben. Relevante Sammelfraktionen aus dieser Vortrennung wurden durch LPLC an RP-18-Material (Stufengradient mit Methanol/Wasser, beginnend mit 100:0; 0,2 MPa, s. Exp. Teil)) weiter aufgetrennt. Die daraus resultierenden Sammelfraktionen 1, 2, 3 und 4 dienten der anschließenden Strukturaufklärung (s. Abb. 17).

\subsubsection{Strukturaufklärung}

Sammelfraktion 2 aus der RP-Trennung (s. Abb. 17) wurde analytisch weiter untersucht, nachdem mittels präparativer DC im Fließmittelgemisch Dichlormethan/Ethylacetat (90:10, s. Exp. Teil) eine Auftrennung in vier voneinander deutlich getrennte Banden erfolgt war. Bereits MARTIN [110] führte aus, dass die präparative DC durchaus Trennungen in Gemische mit z. T. nicht mehr als zwei Einzelkomponenten erzielen lässt.

Nach Auskratzen der Bande 1 (niedrigster $\mathrm{R}_{\mathrm{f}}$-Wert) und Elution mit dem Fließmittel (s. o.) wurde die isolierte Substanz mittels ${ }^{1} \mathrm{H}-\mathrm{NMR}$ sowie MS-FAB/EI untersucht. Es gelang die Strukturaufklärung zweier Hauptsubstanzen (A1 und $\boldsymbol{A 2}$ ), bei denen es sich um Alkamide handelte. Nach Vergleich mit den von MARTIN/Dietz/WoelKart [35, 110, 181] ermittelten Daten entsprach Substanz $\boldsymbol{A 1}$ dem Undeca-2E,4E-dien-8,10-diinsäure-isobutylamid und Substanz $\boldsymbol{A} 2$ dem Nona-2E-en-6,8-diinsäure-phenylethylamid. Durch die HPLC/ESI-MS-MS, mit der bei WOELKART [181] gute Erfahrungen bei der Charakterisierung teilweise identischer Amide aus Echinacea-Spezies vorliegen, war es im Folgenden möglich, an Hand der bei Alkamiden immer wiederkehrenden typischen Aufspaltungsmuster die mittels NMR erstellten Ergebnisse zu bestätigen und abzusichern (3.3.3.2. und 3.3.3.3.).

Bei den Banden 2 - 4 wurde auf Grund sehr geringer Substanzmengen und möglicher Probleme bei der Nebeneinanderbestimmung auf die NMR verzichtet und in Analogie zu der bereits untersuchten Bande 1 sowie durch Literaturvergleich [16, 35, 53, 110], hinsichtlich identischer Aufspaltungsmuster und entsprechender Molpeaks in der MS mittels zwei verschiedener Verfahren (EI-MS; +FAB-MS), auf folgende Substanzen geschlossen: 
Undeca-2E/Z, 4E/Z-dien-8,10-diinsäure-isobutylamid (A1-Isomer)

Nona-2Z-en-6,8-diinsäure-phenylethylamid $(\boldsymbol{A 9})$

Nona-2Z-en-6,8-diinsäure-isobutylamid ( $A$ 7)

2,3(c)-Epoxy-nona-6,8-diinsäure-isobutylamid ( $\boldsymbol{A} \boldsymbol{8})$

Da Substanzen mit denselben Molmassen in mehr als einer Bande vorkamen (A1 / $\boldsymbol{A} \mathbf{1}$-Isomer und $\boldsymbol{A 2}$ / $\boldsymbol{A 9}$ ), diese jedoch gut separiert voneinander zu sein schienen, liegen möglicherweise die entsprechenden E/Z-Isomere vor. Definitive Aussagen hierzu lassen sich allerdings nur über die als Gemisch per ${ }^{1} \mathrm{H}-\mathrm{NMR}$ bestimmten Verbindungen (Undeca-2E,4E-dien-8,10diinsäure-isobutylamid und Nona-2E-en-6,8-diinsäure-phenylethylamid) der Bande 1 treffen.

Weitere Molpeaks, als „Begleitsubstanzen“ entdeckt, waren nicht einfach zuzuordnen. Bei der Verbindung mit einer Molmasse von 264 könnte es sich um Acmellonat handeln, einem Ester der bisher in „Spilanthes acmella“ gefunden wurde [102].

Die Substanz mit $M_{r}$ 303, die, ebenso wie voranstehende, in der nachfolgenden Analytik als „Begleitsubstanz“ wieder auftaucht, hat, mittels Hochauflösung berechnet, eine wahrscheinliche Summenformel von $\mathrm{C}_{17} \mathrm{H}_{18} \mathrm{NO}_{2} \mathrm{Cl}$. Hier eine Struktur vorzuschlagen, erscheint allerdings zu spekulativ, zumal das Element Chlor zuvor nicht auftauchte.

Darüber hinaus gelangte Sammelfraktion 2 von der RP-18-Säule insgesamt, d. h., ohne vorherige präpartive DC, zur Strukturanalye mittels HPLC/ESI-MS. Oben erwähnte Substanzen $(\boldsymbol{A 1}, \boldsymbol{A 2}, \boldsymbol{A 7}, \boldsymbol{A 8})$ wurden dort als „Haupt“-Substanzen wiedergefunden und ihre Struktur bestätigt.

Zunächst wurde die Sammelfraktion 2 mittels HPLC/ESI-MS und SRM (Selected Reaction Monitoring) untersucht. Zur HPLC diente eine RP-18 Säule. Da es sich um eine kombinierte HPLC/MS-Anlage handelt, wird neben dem Chromatogramm (UV-Detektor; $\lambda=260 \mathrm{~nm}$ ) parallel dazu mittels massenselektiven Detektors ein Full-MS-Spektrum erhalten (Abb. 18 und 19 unten), das neben den Retentionszeiten die Molmassen der Substanzen (parent ions) im +ESI-Modus, also als $[\mathrm{M}+1]^{+}$, darstellt. Erfasst wurden hier Molmassen von m/z 150 600. Anschließend wurden die intensivsten Peaks (parent ions) in der Ionenfalle (ion trap) gespeichert. Mit diesen parent ions erfolgten Fragmentationsexperimente per Tandem-MS (MS-MS). Hierbei stoßen parent ions im Massenanalysator mit Hintergrundgas (Helium) zusammen und werden so zur Fragmentation angeregt. Entstehende Produkt(Tochter)-Ionen können ebenfalls in der Ionenfalle gespeichert werden, um sie anschließend wiederum 
fragmentieren $\mathrm{zu}$ lassen. Es resultierten entsprechende MS-MS-Spektren (MS ${ }^{\mathrm{n}}$; max. bis $\left.\mathrm{MS}^{4}\right)$. Als Scan-Modus fungierte das SRM [26, 153, 181].

In analoger Weise konnten Erkenntnisse über Inhaltsstoffe aus Sammelfraktion 3 (Abb. 19) gewonnen werden.

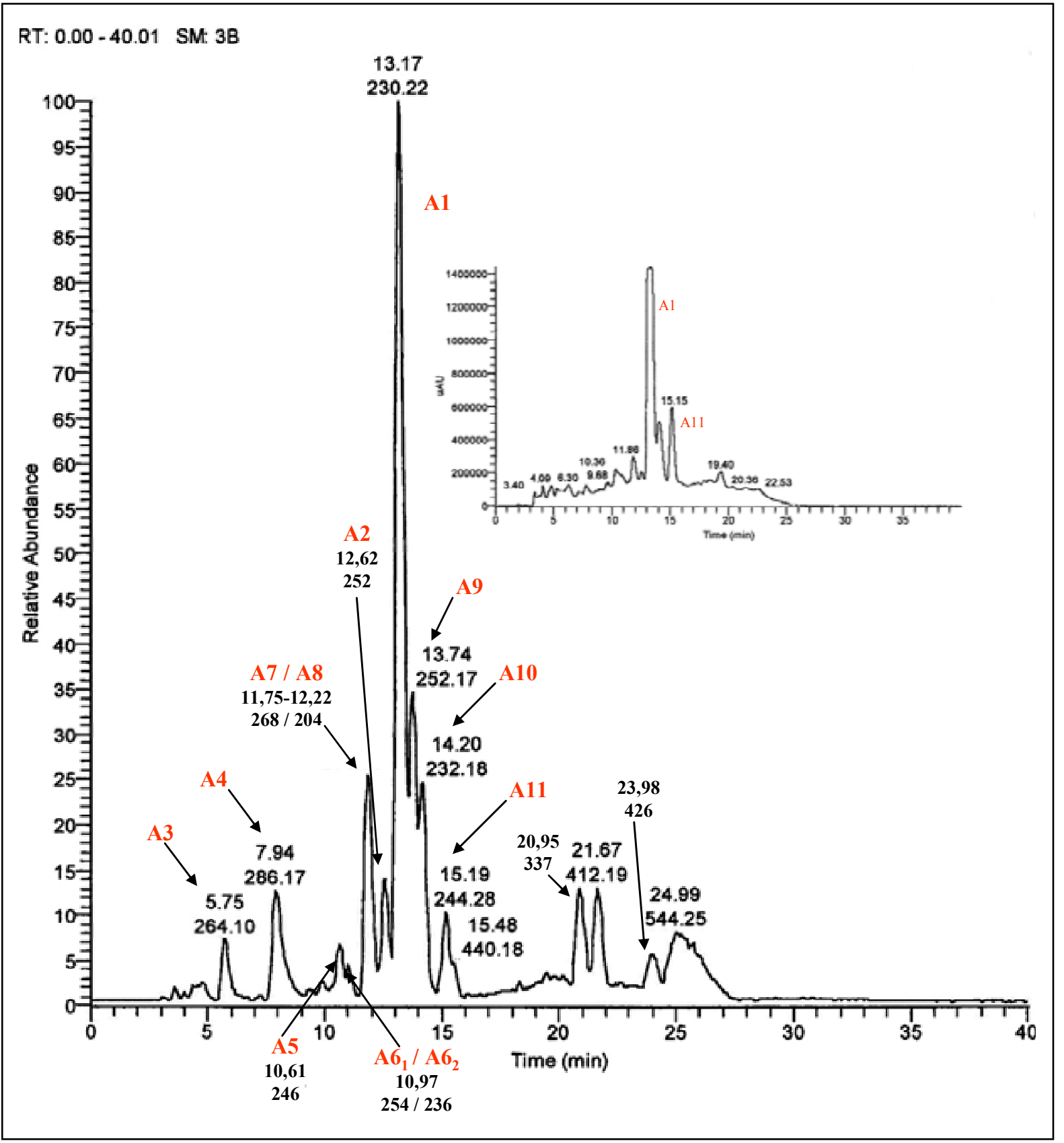

Abb. 18: oben rechts: HPLC-Trennung von Sammelfraktion 2 mit UV-Detektion bei $260 \mathrm{~nm}$ unten: Full-MS-Spektrum der Sammelfraktion 2, detektiert im +ESI-Modus (m/z 150-600)

Substanzen, die ein 2,4-Dienamidchromophor aufweisen, sind bei $260 \mathrm{~nm}$ (Abb. 18 oben) die Peaks mit den größten Intensitäten. In Sammelfraktion 3 treten derartige Strukturen nicht auf, was sich in der geringeren Intensität der Peaks bei dieser Wellenlänge äußert (Abb. 19 oben). 
Vergleicht man die Spektren (Abb. 18 und 19) miteinander, so sind bei Sammelfraktion 3 ein höheres „Grundrauschen“ sowie eine schlechtere Substanztrennung im Bereich bis 13 min gegenüber Sammelfraktion $2 \mathrm{zu}$ konstatieren. In beiden Spektren fällt jeweils eine Hauptsubstanz auf. In Sammelfraktion 3 ist es die Substanz mit $M_{r} 221$, bei der es sich wohl um das Hauptalkamid von Acmella ciliata, das Spilanthol (A13), handelt (s. 3.3.3.14.). In Sammelfraktion 2 dominiert die Substanz mit der Molmasse 229, die als Undeca-2E,4E-dien8,10-diinsäure-isobutylamid (A1) (s. 3.3.3.2.) zu identifizieren war. Diese Verbindung sowie deren Dihydroderivat Undeca-2E-en-8,10-diinsäure-isobutylamid (A10) (s. 3.3.3.10.) wurden quantitativ erfasst (s. Exp. Teil).

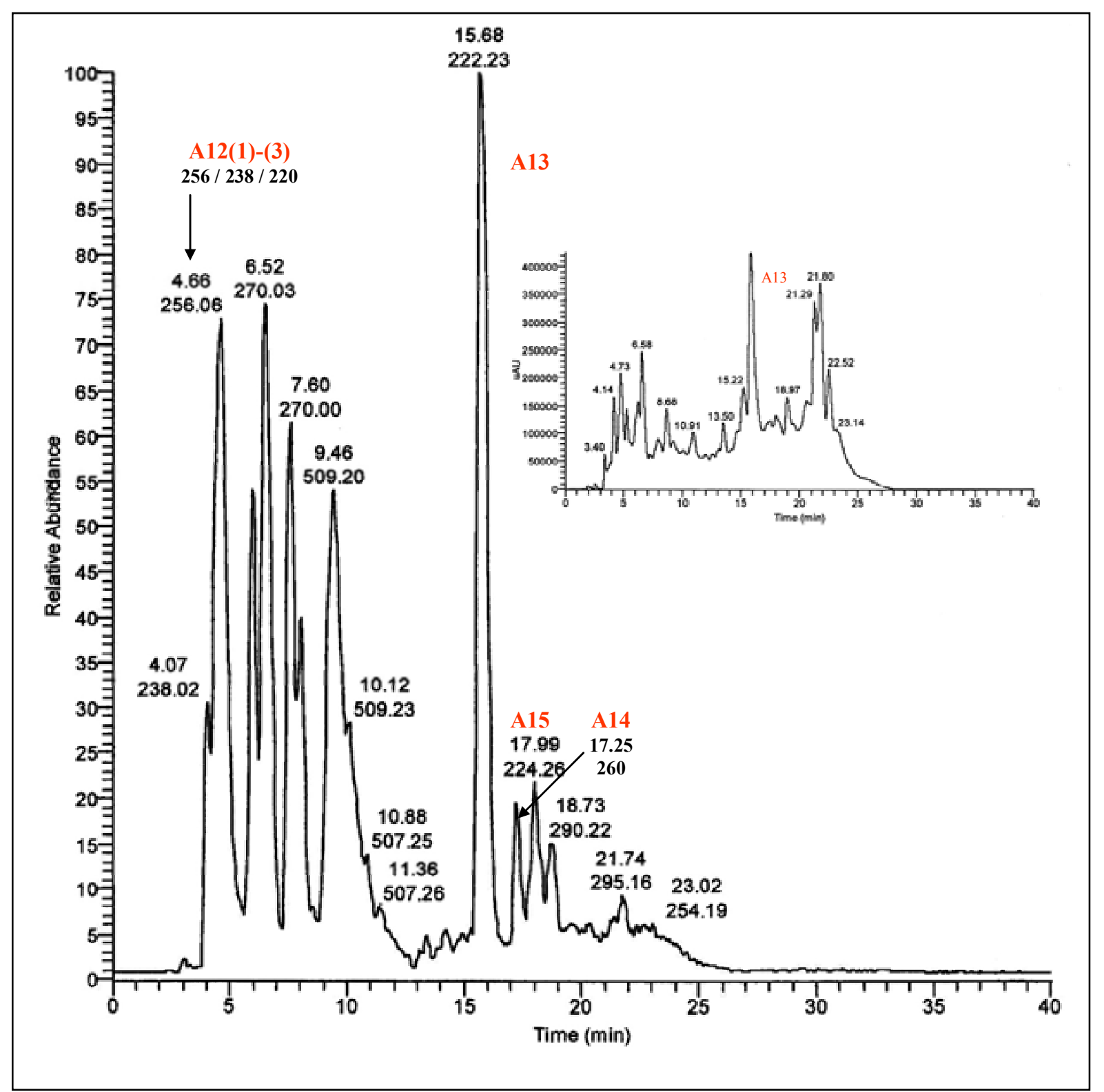

Abb. 19: oben: HPLC Trennung von Sammelfraktion 3 mit UV Detektion bei 260 nm unten: Full MS-Spektrum der Sammelfraktion 3, detektiert im +ESI-Modus 
Wie unter diesen Analysebedingungen $\mathrm{zu}$ erwarten, eluieren fast ausnahmslos die vermeintlich hydrophileren Substanzen (Hydroxy-Amide) mit den kürzeren Retentionszeiten von der lipophilen RP-Säule.

Zur Charakterisierung der einzelnen Alkamidstrukturen (3.3.3.2. - 3.3.3.15.) aus diesen Sammelfraktionen mit der gekoppelten HPLC-MS-Anlage wurde im +ESI (Elektronen-SprayIonisation)-Modus gearbeitet. Dieser Modus wurde gewählt, da Alkamide als Stickstoff enthaltende Verbindungen gut protonierbar sind.

Die hier nachgeschaltete Fragmentierung, teilweise bis $\mathrm{MS}^{4}$, hat die Charakterisierung von Substanzen, in diesem Fall Alkamiden, zum Ziel, die sich durch spezifische Fragmentierungsmuster auszeichnen, wodurch wiederkehrende Muster erkennbar und mit bekannten Strukturen vergleichbar werden. Auf diese Weise lässt sich in vielen Fällen auf die oft aufwändige präparative Gewinnung dieser Substanzen verzichten, und es können auch Alkamide identifiziert werden, die, wie im durchgeführten Screening, in sehr geringen Mengen vorliegen [26, 181].

Typische Alkamid-Fragmentierungsmuster liefern die Amidreste, von denen hier drei verschiedene angetroffen wurden: Isobutylamid-, Phenylethylamid- und MethylbutylamidRest. Deren Fragmentationsmuster werden in Kapitel 3.3.3.1. beschrieben. Außerdem sind oft typische Kettenbrüche des Säurerests bzw. des Kohlenwasserstoffrests nach Abspaltung des Amids erkennbar [26, 181].

Werden bei der Strukturdiskussion darüber hinaus energetische Aspekte und biogenetische Erkenntnisse berücksichtigt, so lassen sich auch (genauere) Aussagen zu den Positionen der Doppel- und Dreifachbindungen sowie zur Verknüpfung von Hydroxyl-Gruppen und zu den Isomerien an Doppelbindungen treffen. Beispielsweise ist die konjugierte Lage einer Doppelbindung in 2,3-Position zur Amidgruppe gegenüber einer 3,4-Position energetisch begünstigt, da es durch Mesomerieffekte zu einer größeren Ladungsverteilung hin zum C-O (Charakter einer $\alpha, \beta$-ungesättigten Carbonylverbindung) der Amidgruppe kommen kann, welche ihrerseits in Mesomerie steht zum benachbarten Stickstoff.

Zur Interpretationshilfe diente auch, dass bereits zwei Verbindungen (A1 und $\boldsymbol{A 2}$ ) durch präparative DC gewonnen, mittels FAB- sowie EI-MS untersucht und per ${ }^{1} \mathrm{H}-\mathrm{NMR}$ analysiert 
worden waren. Bei weiteren Substanzen konnte analog verfahren bzw. sogar auf die NMR verzichtet werden. Diese Substanzen (s. A3 - A16) ließen sich mit Hilfe der ESI-Messungen durch das Auffinden der entsprechenden Molpeaks sowie gleicher und weiterer typischer ESIFragmente an Hand der MS-MS-Spektren identifizieren. Schwierig war es jedoch, ohne die Verschiebungen und Kopplungskonstanten der NMR definitive Aussagen über die Isomerie an Doppelbindungen zu treffen. Hier werden Alkamide mit bestätigter Struktur, die bereits in Acmella ciliata bzw. andereren eng verwandten Arten nachgewiesen worden waren bzw. auf Basis von Vergleichsliteratur $[8,9,11,16,17,26,35,53,110-112,132,134,144,181]$ als wahrscheinlichste Variante angegeben. Handelt es sich um vermutlich neue Strukturen, werden nach Auswertung der Fragmentationsmuster bzw. der entsprechenden Bruchstücke, wie oben erwähnt, energetische und biogenetische Aspekte berücksichtigt und die sich daraus abzuleitende Struktur vorgeschlagen.

Ein Phänomen der Alkamide ist, dass sie häufig zur Dimerisierung neigen, was an entsprechenden Signalen mit doppelter Molmasse im Massenspektrum erkennbar ist [26, 181]. Neben der Dimerisierung wird auch eine Polymerisierung beschrieben [35, 181 und 5.3.3.] und war auch während dieser Arbeit zu beobachten. Diese äußert sich im Entstehen von schwarzen pigmentartigen „Substanzkörnchen“, die bei längerer oder unsachgemäßer Lagerung sowie erhöhter Temperatur auftreten können. Diese Polymerisate waren in keinem Lösungsmittel löslich und entzogen sich daher einer weiteren Analytik. Zu ihrer Minimierung wurde die Temperatur bei allen Arbeiten nicht höher als $40^{\circ} \mathrm{C}$ gewählt. Außerdem empfiehlt es sich, die betreffenden Substanzen zur Lagerung einzufrieren.

\subsubsection{1. +ESI-MS-Fragmentierung der Amidreste}

Der analytischen Betrachtung der Alkamide werden typische +ESI-MS-Fragmentierungsreaktionen ihrer Amid-Reste vorangestellt [181]. Die gebildeten Fragmente unterscheiden sich von den bei der EI-Messung gebildeten Fragmenten i. d. R. um die Masse eines Protons (vgl. Lit. [26, 181] mit Lit. [35, 110]). Allerdings treten nicht bei jedem Alkamid alle Spaltprodukte auf und deren Intensitäten können stark unterschiedlich sein, so dass substanzspezifische Spaltungen anzunehmen sind.

Beim Isobutylamid-Rest treten hauptsächlich vier Fragmente auf. Häufig erfolgt aber auch die Abspaltung des gesamten Rests, wobei es nicht zur Bildung des erwarteten Fragments M- 
100, sondern M-99 kommt, d. h., ein Proton wird zusätzlich vom Isobutylamid-Rest abgespalten, vermutlich durch Übertragung dieses Protons auf das Restmolekül (Kohlenwasserstoff-Rest), dessen Molmasse sich um 1 erhöht.
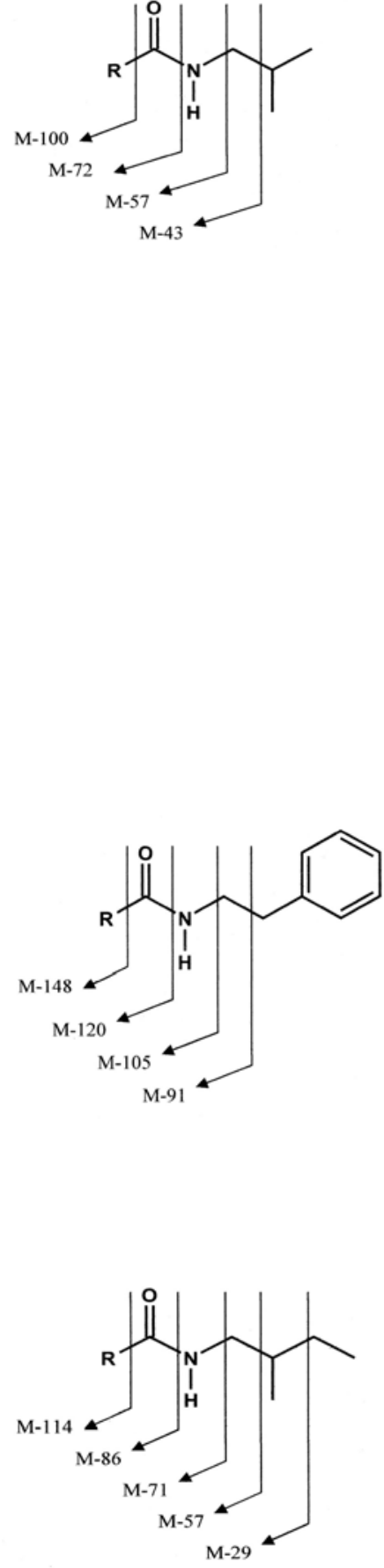

Das Gleiche ist $\mathrm{zu}$ beobachten bei den beiden typischen Spaltprodukten des Isobutylamin- bzw. Isobutyl-Rests M-57 bzw. M-43. Hier entstehen nach Übertragung eines Protons auf den linken Molekülteil die formal oxidierten Strukturen m/z 56 bzw. m/z 42 (vgl. [181]).

Anders verhält es sich dagegen nach Spaltung der Amidbindung. Der abgespaltene Isobutylamin-Rest „,verlässt“ das Molekül unter Aufnahme eines weiteren Protons, entweder aus dem anderen Molekülteil oder aus dem Lösungsmittel, und damit als Amin mit der Masse 73. Somit wird bei dieser Spaltung das Fragment M-73 detektierbar. Kleinere Massenfragmente als m/z 42 werden nur selten abgespalten.

Beim Phenylethylamid-Rest tritt das Fragment mit der Masse 104 - auch hier wird ein weiteres Proton abgespalten - stets auf, was auf seine relative Stabilität hindeutet. Das Fragment M-90 ist eher seltener anzutreffen. Die Abspaltung des gesamten Phenylethylamid-Rests (M-147) unter Protonenabgabe bzw. die Amidspaltung unter Aufnahme eines Protons (M-121) sind dagegen oft zu sehen.

Beim Methylbutylamid-Rest treten die nebenstehenden Spaltungen auf, wobei wiederum ein weiteres Proton abgegeben wird. Die Amidspaltung findet auch hier unter Protonenaufnahme durch den Stickstoff statt. Eine mögliche weitere Spaltung, die Abgabe von nur einer Methylgruppe, ist selten zu sehen. 


\subsubsection{Undeca- $2 E, 4 E$-dien-8,10-diinsäure-isobutylamid (Verbindung $A 1$ )}

Verbindung $\boldsymbol{A 1}$ wurde zunächst mittels präparativer DC (Bande 1, s. 3.3.3.) gewonnen. Dort trat sie zusammen mit Nona-2E-en-6,8-diinsäure-phenylethylamid (A2) auf. Die parallele NMR-Charakterisierung dieser beiden Substanzen war möglich, da entsprechende Literaturdaten $[35,110]$ als Vergleich zur Verfügung standen und sich die Überlagerung von Signalen in Grenzen hielt. Neben der ${ }^{1} \mathrm{H}-\mathrm{NMR}$ wurden EI- sowie FAB-MS-Messungen durchgeführt. Auch zur EI-MS-Messung lagen Vergleichsdaten vor [35, 110]. Im Verlauf der weiteren Arbeit wurde $\boldsymbol{A 1}$ auch mittels HPLC/ESI-MS-MS-Analytik in Sammelfraktion 2 identifiziert (Abb. 20), wo sie mit $\boldsymbol{A 2}$ als Nachbarpeak auftrat. Auf Basis dieser vier Analysenmethoden ist die Struktur dieser Substanz als bestätigt anzusehen.

In Bande 2 der präparativen DC (s. 3.3.3.) gab es Hinweise auf das Vorliegen eines Isomers von Undeca-2E,4E-dien-8,10-diinsäure-isobutylamid. Da aber in der mittels HPLC-MS untersuchten Sammelfraktion 2 kein weiterer Peak für ein solches Isomer spricht, ist zu vermuten, dass der detektierte Peak (RT 13,17 min) entweder als ein unter diesen HPLCBedingungen untrennbares Isomerengemisch vorliegt oder es zu einer Isomerisierung hin zur all-trans-Form kam. Da dies aber so nicht zu verifizieren ist, wird im Weiteren nur vom auch per NMR bestätigten 2E,4E-Isomer (s. Tab. 4) gesprochen.

Die Molmasse von $229\left[\mathrm{C}_{15} \mathrm{H}_{19} \mathrm{NO}\right]^{+}$ist im EI-MS bei $40^{\circ} \mathrm{C}$ sowie bei $80^{\circ} \mathrm{C}$ und im $+\mathrm{FAB}-$ MS sowie im +ESI-Modus als $\left[\mathrm{C}_{15} \mathrm{H}_{19} \mathrm{NO}+\mathrm{H}\right]^{+}$ersichtlich. Das EI-Fragment $\mathrm{m} / \mathrm{z} 157$ $\left[\mathrm{C}_{11} \mathrm{H}_{9} \mathrm{NO}\right]^{+}$stellt den Säurerest nach Spaltung der Amidbindung dar. Auch kommt es zur Spaltung zwischen C-6 und C-7 unter Beibehaltung der Amidbindung, dargestellt durch das Fragment m/z $166\left[\mathrm{C}_{10} \mathrm{H}_{16} \mathrm{NO}\right]^{+}$(s. auch $\left.[35,110]\right)$.

Die umfangreichsten Aussagen lieferte die ESI-MS-Fragmentation (Abb. 20). Zunächst ist festzuhalten, dass Verbindung $\boldsymbol{A} \mathbf{1}$ zur Dimerisierung neigt, zu sehen als $[2 \mathrm{M}+\mathrm{H}]^{+} \mathrm{m} / \mathrm{z} 459$. Das Fragment mit der Massenzahl 131 (b) stellt das Restmolekül nach Abspaltung des Isobutylamid-Rests $\left[\mathrm{M}-\mathrm{C}_{5} \mathrm{H}_{9} \mathrm{NO}+\mathrm{H}\right]^{+}$und eines weiteren Protons vom Ausgangsmolekül dar (230-99=131). Somit steht diese Masse für den Kohlenwasserstoffrest unter Aufnahme eines Protons. 
Undeca-2E,4E-dien-8,10-diinsäure-isobutylamid $\mathrm{C}_{15} \mathrm{H}_{19} \mathrm{NO}$; $\mathrm{M}_{\mathrm{r}} 229 ; \mathrm{RT} 13,17 \mathrm{~min}$

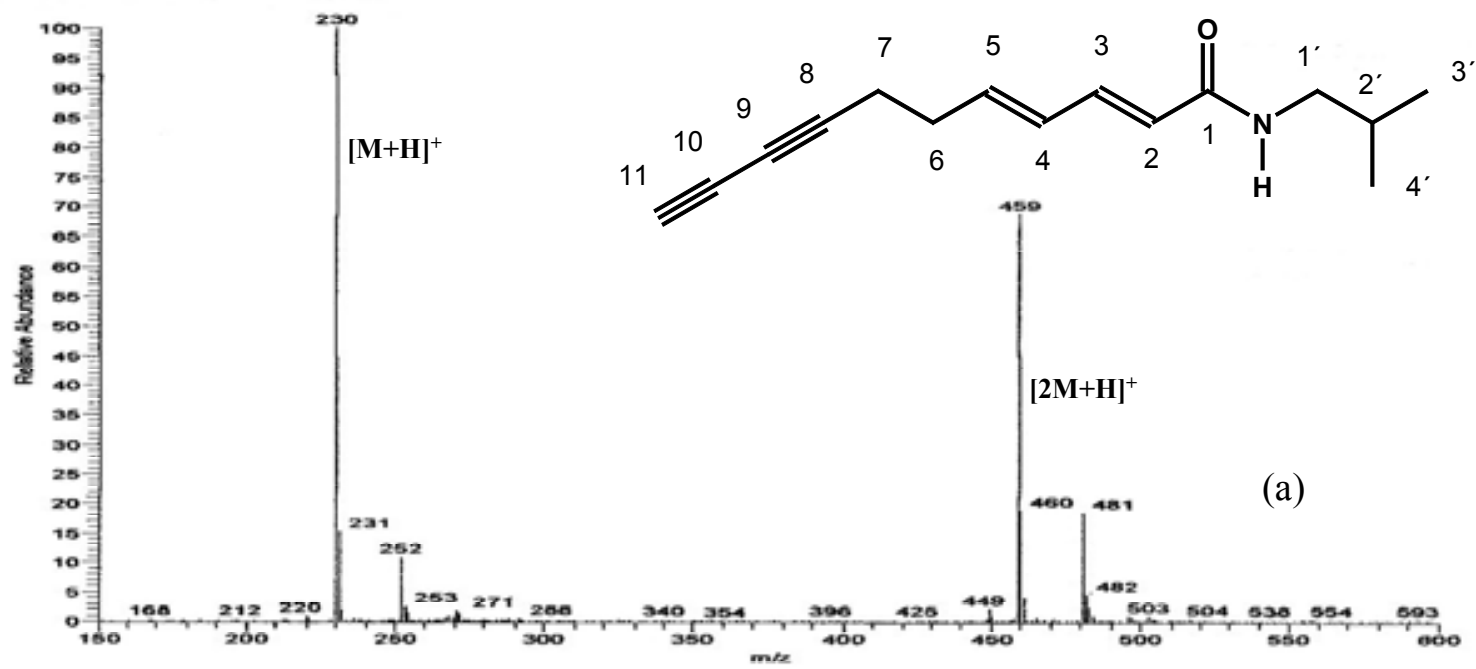

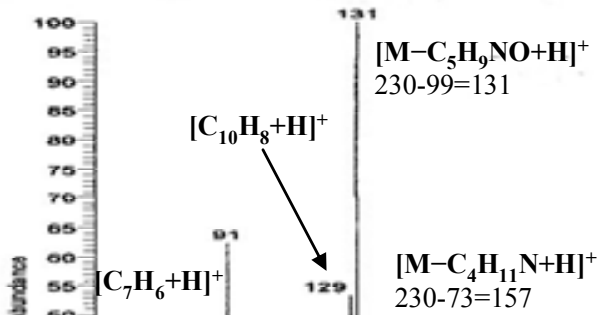
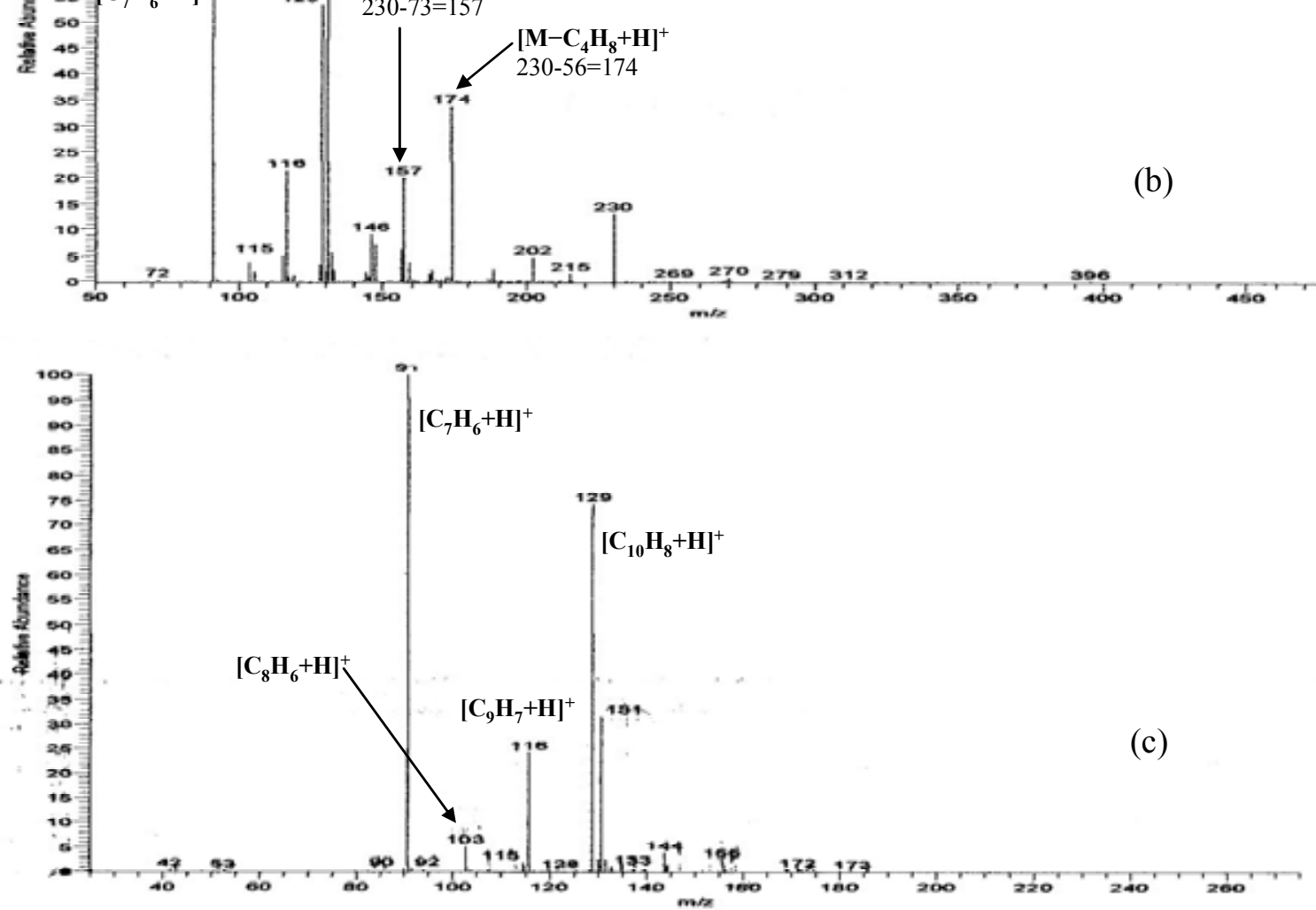

Abb. 20(a) - (c): +ESI-MS / $\mathrm{MS}^{2} / \mathrm{MS}^{3}$-Spektren von Verbindung $A 1$ 
Weitere hier zu beobachtende typische Spaltreaktionen des Isobutylamid-Rests (s. 3.3.3.1.) sind die Abspaltung des Isobutylamins nach Spaltung der Amidbindung unter Aufnahme eines weiteren Protons $\left[\mathrm{M}-\mathrm{C}_{4} \mathrm{H}_{11} \mathrm{~N}+\mathrm{H}\right]^{+}(230-73=157)$ sowie die Abspaltung des IsobutylRests unter Abgabe eines weiteren Protons $\left[\mathrm{M}-\mathrm{C}_{4} \mathrm{H}_{8}+\mathrm{H}\right]^{+}(230-56=174)$.

Innerhalb des Kohlenwasserstoffrests kommt es zu weiteren Spaltungen sowie Umlagerungen und Oxidationen in vermutlich energetisch günstigere Fragmente (Abb. 20(b) und (c)). Das Fragment $\left[\mathrm{C}_{10} \mathrm{H}_{8}+\mathrm{H}\right]^{+} \mathrm{m} / \mathrm{z} 129$ entsteht durch Abgabe zweier Protonen (Oxidation) aus dem ursprünglichen Kohlenwasserstoffrest und m/z $103\left(\left[\mathrm{C}_{8} \mathrm{H}_{6}+\mathrm{H}\right]^{+}\right)$durch Spaltung zwischen C-3 und C-4. Die Bildung des stabilen Fragments m/z $91\left(\left[\mathrm{C}_{7} \mathrm{H}_{6}+\mathrm{H}\right]^{+}\right)$sowie des Fragments m/z $116\left(\left[\mathrm{C}_{9} \mathrm{H}_{7}+\mathrm{H}\right]^{+}\right)$erscheint ungewöhnlich, da hier die Spaltung von Doppelbindungen Voraussetzung für deren Bildung $\mathrm{zu}$ sein scheint. Möglicherweise sind intramolekulare Bindungsumlagerungen vorausgegangen. Die Fragmentationsmuster sind mit denen in Literatur [181] identisch.

Tabelle 4: ${ }^{1} \mathrm{H}-\mathrm{NMR}$ Daten von Undeca-2E,4E-dien-8,10-diinsäure-isobutylamid in $\mathrm{CDCl}_{3}$

\begin{tabular}{|l|c|c|c|}
\hline H & $\begin{array}{c}\text { Verschiebung } \boldsymbol{\delta} \\
{[\mathrm{ppm}]}\end{array}$ & Signalform & $\begin{array}{c}\text { Kopplungskonst. J } \\
{[\mathrm{Hz}]}\end{array}$ \\
\hline 2 & 5,79 & $\mathrm{~d}$ & 15,0 \\
2 & 7,18 & $\mathrm{dd}$ & 15,$0 ; 10,0$ \\
3 & 6,19 & $\mathrm{dd}$ & 10,$0 ; 15,0$ \\
4 & 6,04 & $\mathrm{dt}$ & 15,$0 ; 6,5$ \\
5 & 2,38 & $\mathrm{~m} /(4 \mathrm{H})$ & - \\
6,7 & 1,95 & $\mathrm{t}$ & 1,0 \\
11 & 5,47 & $\mathrm{brs}$ & - \\
$\mathrm{N}-\mathrm{H}$ & 3,15 & $\mathrm{dd} /(2 \mathrm{H})$ & 7,$0 ; 6,5$ \\
$1^{\prime}$ & 1,81 & $\mathrm{~m}$ & - \\
$2^{\prime}$ & 0,91 & $\mathrm{dd} /(6 \mathrm{H})$ & 7,$0 ; 2,5$ \\
$3^{\prime}, 4^{\prime}$ & \multicolumn{3}{|c}{} \\
\hline
\end{tabular}

Die Isobutylamid-Struktur wurde neben den typischen Massefragmenten auch im ${ }^{1} \mathrm{H}-\mathrm{NMR}-$ Spektrum bestätigt (Tab. 4) durch die Signale H-1' (dd), H-2' (m) und H-3', H-4' (dd). Die Signale der Protonen am C-1' erscheinen als Triplett, was an gleichen Kopplungskonstanten zu H-2' und N-H liegt, mit denen es mit 7 Hz koppelt. Auf Grund der Nicht-Auflösung des Signals (N-H) bzw. des Multiplettcharakters von $\mathrm{H}-2^{\prime}$, bedingt durch acht Kopplungspartner, kann die zweite theoretische Kopplungskonstante mit $7 \mathrm{~Hz}$ nicht gefunden werden.

Gleiches gilt für die Kopplung von H-2' zu den Protonen am C-3'und C-4'. Hier lässt sich umgekehrt nur eine Kopplungskonstante dieser Protonen zu H-2'mit ebenfalls 7 Hz ermitteln. 
Diese beiden Methylgruppen am C-3'und C-4'müssten ein Doppeldublett als Signal ergeben. Da sie aber magnetisch äquivalent sind, fallen sie in einem Dublett zusammen, und somit kann hier keine weitere Kopplungskonstante ermittelt werden. Auf Grund von acht Kopplungspartnern des Protons am C-2' resultiert eine Aufspaltung höherer Ordnung, die am Signal nicht mehr zu verifizieren ist. Somit wird dieses Signal als Multiplett aufgefasst.

Die Doppelbindung des Säurerests zwischen C-2 und C-3 ist trans-konfiguriert, zu sehen an einer Kopplungskonstanten von $15 \mathrm{~Hz}$. Die gefundenen Verschiebungen stimmen mit den Literaturangaben $[35,110,112]$ überein. Die starke Tieffeldverschiebung von H-3 ist typisch für eine zur Carbonylfunktion $\alpha, \beta$-ständige Doppelbindung und resultiert aus dem Elektronen ziehenden Effekt des Carbonyls. H-3 koppelt mit H-4 mit einer Kopplungskonstanten von 11 Hz. H-4 stellt einerseits den Kopplungspartner für H-3 dar $(11 \mathrm{~Hz})$ und koppelt mit H-5 mit $15 \mathrm{~Hz}$, was für eine trans-Konfiguration der Doppelbindung zwischen H-4 und H-5 spricht.

Das Erscheinen der Signale von H-6 und H-7 im Hochfeld zeigt, dass hier kein Elektronen ziehender Effekt mehr wirkt. Das Signal für H-11 stellt ein Dublett mit der sehr kleinen Kopplungskonstanten von ca. $1 \mathrm{~Hz}$ dar, hervorgerufen durch eine longe-range-Kopplung über die beiden Dreifachbindungen mit den Protonen am C-7. Diese sind zwar magnetisch nicht äquivalent, weshalb eigentlich als Signal für H-11 ein Triplett resultieren müsste, das jedoch, bedingt durch die große Entfernung der Kopplungspartner, nicht ablesbar ist. Die Signale von H-6 und H-7 liegen direkt nebeneinander und lassen sich keinem eindeutigen Signalmuster zuordnen, weshalb sie als Multiplett betrachtet werden. Auf Grund der Signalvielfalt sowie einer Molmasse von 229 kam hier nur noch eine gesättigte Bindung mit Dimethylen-Struktur in Betracht.

Es erfolgte eine Quantifizierung von $\boldsymbol{A 1}$, wie auch von $\boldsymbol{A 1 0}$ (3.3.3.10. und Exp. Teil), über das Isomerenpaar (Dodeca-2E,4E,8Z,10E / 2E,4E,8Z,10Z-tetraensäure-isobutylamid (,Tetraen“)). Diese ergab für $\boldsymbol{A 1}$ einen Gehalt von 7,65\% in der Sammelfraktion 2, was 0,03\%, bezogen auf den gesamten Dichlormethan-Trockenextrakt, entspricht. 


\subsubsection{Nona-2E-en-6,8-diinsäure-phenylethylamid (Verbindung $A 2$ )}

Verbindung $\boldsymbol{A} \boldsymbol{2}$ wurde, wie oben erwähnt, zunächst per präparativer DC (Bande 1, s. 3.3.3.), zusammen mit Undeca-2E,4E-dien-8,10-diinsäure-isobutylamid (A1) gewonnen. Aus der + FAB-MS-Analyse ergab sich die Molmasse als $[\mathrm{M}+\mathrm{H}]^{+}$von 252 und im EI-MS bei $40^{\circ} \mathrm{C}$ war die Molmasse $[\mathrm{M}]^{+} 251$ ablesbar. Im EI-MS bei $80^{\circ} \mathrm{C}$ taucht die Molmasse nicht mehr auf; es erscheinen Fragmente, die auch schon bei $40^{\circ} \mathrm{C}$ zu konstatieren waren.

Alkamid $\boldsymbol{A} \boldsymbol{2}$ konnte an Hand der ESI-MS-Fragmentation ebenfalls in Sammelfraktion 2 nachgewiesen werden (Abb. 21). Auch hier ist die Molmasse als $[\mathrm{M}+\mathrm{H}]^{+} \mathrm{m} / \mathrm{z} 252$ ablesbar. Außerdem ist das Dimer zu finden als $[2 \mathrm{M}+\mathrm{H}]^{+} \mathrm{m} / \mathrm{z}$ 503. Aus den ebenfalls vorhandenen Molmassen von 267 (Verbindung $\boldsymbol{A 8}$ ) und 303 ist abzuleiten, dass Verbindung $\boldsymbol{A 2}$ nicht ganz rein erfasst wurde (s. 3.3.3.).

Die typischen Fragmente des Phenylethylamid-Rests sind bereits oben beschrieben worden (3.3.3.1.). Die Molmasse von 105 (als $\mathrm{M}+\mathrm{H})$ steht sowohl für den linken Molekülteil (Kohlenwasserstoffrest) nach Abspaltung des Phenylethylamid-Rests $\left[\mathrm{M}-\mathrm{C}_{9} \mathrm{H}_{9} \mathrm{NO}+\mathrm{H}\right]^{+}$und Protonenaufnahme als auch für das Phenylethyl-Fragment des rechten Molekülteils nach Abgabe eines weiteren Protons. Das Fragment m/z 131 entsteht nach Spaltung der Amidbindung $\left[\mathrm{M}-\mathrm{C}_{8} \mathrm{H}_{11} \mathrm{~N}+\mathrm{H}\right]^{+}$und das Fragment m/z 148 nach Abspaltung des Phenylethyls $\left[\mathrm{M}-\mathrm{C}_{8} \mathrm{H}_{8}+\mathrm{H}\right]^{+}$.

Das Fragment m/z $146\left[\mathrm{C}_{9} \mathrm{H}_{7} \mathrm{NO}+\mathrm{H}\right]^{+}$(Abb. 21(c)) stellt den Phenylethylamid-Rest in oxidierter Form nach Abgabe zweier weiterer Protonen dar, während das Fragment m/z 105 für den Kohlenwasserstoff-Rest steht. Dieser geht durch Protonenabgabe in das Fragment m/z $103\left[\mathrm{C}_{8} \mathrm{H}_{6}+\mathrm{H}\right]^{+}$über. An dieser Stelle zeigt sich auch, dass der „Diin-Rest“ des Moleküls über eine Methylengruppe mit dem Rest verbunden sein muss, da diese Umlagerung sonst nicht möglich wäre. Das bestätigt auch die Masse des Fragments $79\left[\mathrm{C}_{6} \mathrm{H}_{6}+\mathrm{H}\right]^{+}$, welches teilweise nach Protonenabgabe in das Fragment m/z $77\left[\mathrm{C}_{6} \mathrm{H}_{4}+\mathrm{H}\right]^{+}$übergeht. Dieses Fragment entsteht durch Bindungsbruch zwischen C-3 und C-4.

Die +ESI-Massenfragmentation und die aufgenommenen Daten bis $\mathrm{MS}^{3}$ als ein Teil der Strukturaufklärung werden hier erstmalig für Substanzen mit Phenylethylamid-Rest beschrieben. Da es sich bei Verbindung $\boldsymbol{A} \boldsymbol{2}$ um eine bekannte Substanz handelt, für die darüber hinaus die ${ }^{1}$ H-NMR-Daten vorliegen, kann in Analogie und unter Einbeziehung der 


\section{Nona-2E-en-6,8-diinsäure-phenylethylamid} $\mathrm{C}_{17} \mathrm{H}_{17} \mathrm{NO}$; $\mathrm{M}_{\mathrm{r}} 251$; RT 12,62 min

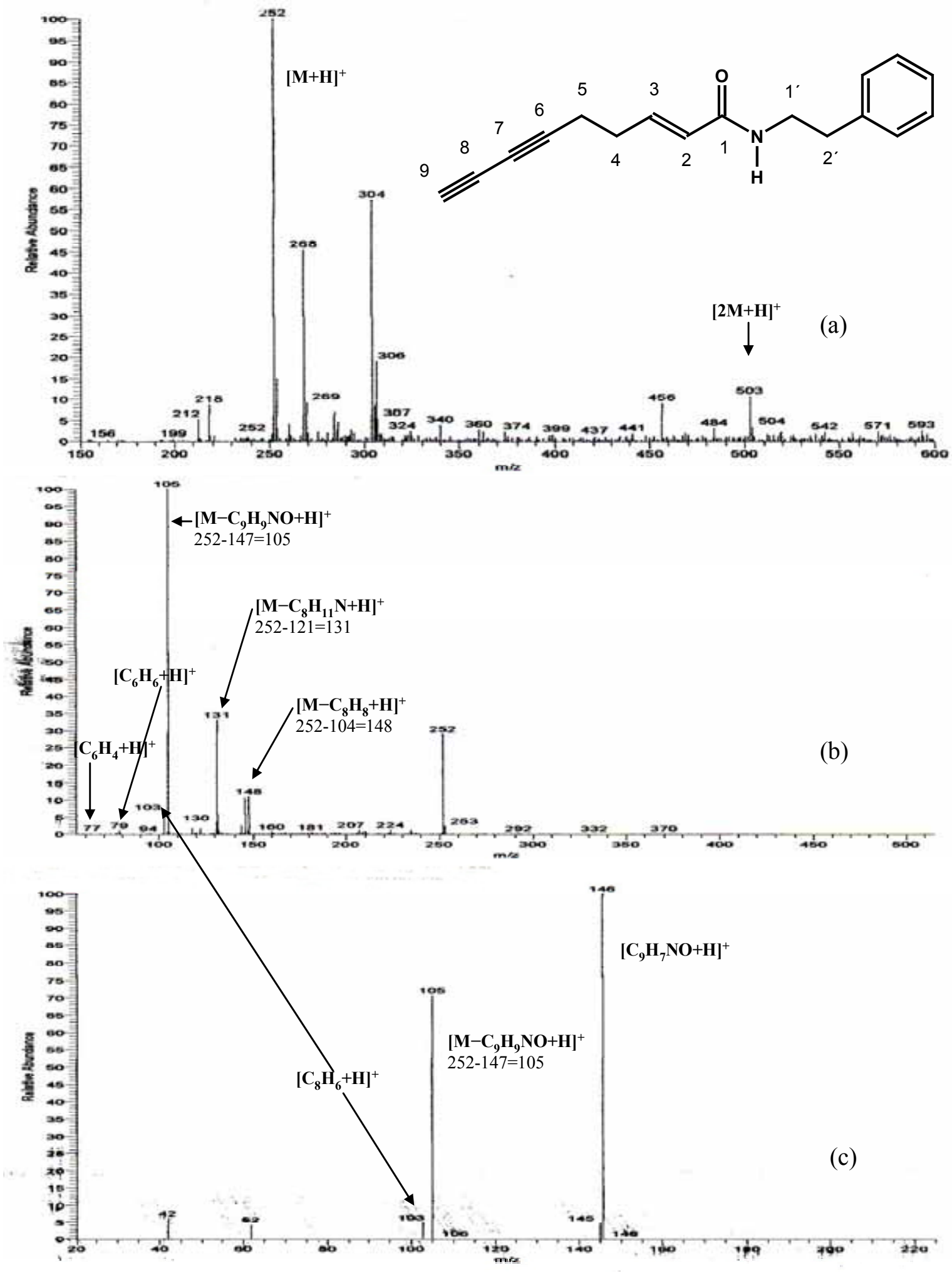

Abb. 21(a) - (c): +ESI-MS / $\mathrm{MS}^{2} / \mathrm{MS}^{3}$-Spektren von Verbindung $A 2$ 
Fragmentationsmuster der anderen beiden Amid-Reste (3.3.3.1.) diese Verbindung mit Phenylethylamid-Rest sicher beschrieben werden. Die Strukturaufklärung der weiteren Verbindungen mit einem Phenylethyl-Rest basiert auf den hier getroffenen Aussagen.

Tabelle 5: ${ }^{1} \mathrm{H}-N M R$ Daten von Nona-2E-en-6,8-diinsäure-phenylethylamid in $\mathrm{CDCl}_{3}$

\begin{tabular}{|l|c|c|c|}
\hline H & $\begin{array}{c}\text { Verschiebung } \boldsymbol{\delta} \\
{[\mathrm{ppm}]}\end{array}$ & Signalform & $\begin{array}{c}\text { Kopplungskonst. J } \\
{[\mathrm{Hz}]}\end{array}$ \\
\hline 2 & 5,69 & $\mathrm{~d}$ & 15,0 \\
3 & 6,80 & $\mathrm{dt}$ & 15,$0 ; 7,0$ \\
4,5 & 2,35 & $\mathrm{~m} /(2 \mathrm{H})$ & - \\
9 & 1,95 & $\mathrm{brs}$ & - \\
$\mathrm{N}-\mathrm{H}$ & 5,48 & $\mathrm{brs}$ & - \\
$1^{\prime}$ & 3,58 & $\mathrm{dt} /(2 \mathrm{H})$ & 6,$5 ; 6,5$ \\
$2^{\prime}$ & 2,84 & $\mathrm{t} /(2 \mathrm{H})$ & 6,5 \\
Phenyl- & 7,23 & $\mathrm{~m} /(5 \mathrm{H})$ & - \\
\hline
\end{tabular}

Im ${ }^{1} \mathrm{H}-\mathrm{NMR}$ erscheint der Phenylrest als Multiplett bei 7,23 ppm, ist jedoch teilweise überlagert durch das Signal des Lösungsmittels Deuterochloroform sowie durch das H-3 der Substanz $\mathrm{M}_{\mathrm{r}}$ 229. Lage, Signalform und Kopplungskonstanten von H-1' und H-2' sind aber identisch mit Literaturangaben $[110,112]$ (s. Tab. 5) und bestätigen somit den Phenylethylamid-Rest.

Das breite Singulett des N-H befindet sich bei 5,48 ppm und somit an derselben Stelle wie das der Substanz $\mathrm{M}_{\mathrm{r}} 229(\boldsymbol{A 1})$, was in der größeren Signalintensität zum Ausdruck kommt. Die entsprechende Kopplungskonstante von zu erwartenden 6,5 Hz der Kopplung zu H-1' lässt sich auf Grund der Nicht-Auflösung dieses Signals nicht finden.

Die Signale für H-2 bei 5,69 ppm und H-3 bei 6,8 ppm sind durch das benachbarte Carbonyl zum Tieffeld verschoben. H-2 und H-3 koppeln mit 15 Hz miteinander, was den transCharakter dieser Doppelbindung zeigt. Das Dublett von H-2 befindet sich bei der gleichen Verschiebung wie das des Dubletts von H-2 der Verbindung $\boldsymbol{A 1}\left(\mathrm{M}_{\mathrm{r}} 229\right)$, was in der doppelten Integralgröße beispielsweise gegenüber H-3 zum Ausdruck kommt.

H-3 koppelt außerdem mit den beiden Protonen am C-4 mit 7 Hz. Die Protonen H-4 und H-5 erscheinen dicht nebeneinander mit Signalaufspaltung höherer Ordnung und werden daher als Multiplett betrachtet. Somit lässt sich hier die Kopplungskonstante von ebenfalls $7 \mathrm{~Hz}$ aus der Kopplung mit H-3 nicht mehr ablesen. Das Signal für H-9 bei 1,98 ppm erscheint als 
Singulett und zeigt im Gegensatz zu H-11 bei $\mathrm{M}_{\mathrm{r}} 229$ keine weitere Aufspaltung. Die NMRDaten stimmen mit den Angaben von MARTIN [110, 112] zum Nona-2E-en-6,8-diinsäurephenylethylamid überein.

\subsubsection{7-Hydroxy-dodeca- $2 E, 4 E, 8 Z, 10 E$-tetraensäure-isobutylamid (Verbindung $A 3$ )}

Das Alkamid $\boldsymbol{A 3}$ konnte mittels +ESI-MS-MS-Messung in der Sammelfraktion 2 nachgewiesen werden. Die präparative DC erfasste $\boldsymbol{A} \mathbf{3}$, wie auch die hydroxilierten Amide $\boldsymbol{A} 4$ und $\boldsymbol{A 6}_{\boldsymbol{1}}$, nicht wegen ihrer in Bezug zum verwendeten Fließmittelsystem IX (s. Tab. 33) zu großen Hydrophilie; sie verblieben auf der Startlinie.

In Abb. 22(a) tritt lediglich der Molpeak deutlich hervor als $[\mathrm{M}+\mathrm{H}]^{+} \mathrm{m} / \mathrm{z} 264$, der unter Wasserabspaltung, wie im $\mathrm{MS}^{2}$-Spektrum (Abb. 22(b)) $\mathrm{zu}$ erkennen, in Dodeca2E,4E,6E,8Z,10E-pentaensäure-isobutylamid mit der Molmasse 245 (246 als $[\mathrm{M}+\mathrm{H}]^{+}$; 264-18=246) übergeht. Dieses Ion ist in Abbildung 22(b) als M(2) bezeichnet.

Die E/Z-Konfiguration ist gemäß $\boldsymbol{A 1 6}$ gewählt (s. 3.3.3.18.), wo das nicht hydroxilierte Analogon beschrieben wird.

Bei der Fragmentation des Moleküls M(2) entstehen die typischen Bindungsbrüche für den Isobutylamid-Rest mit den Fragmenten m/z 147, 173, 190 und 204 (Abb. 22(c)).

Die Bindungsbrüche im Kohlenwasserstoffteil des Moleküls sind in ihrer Intensität eher gering, was an der großen Stabilität des nach Wasserabspaltung entstandenen, voll konjugierten Systems liegen dürfte. Dies belegt auch das $\mathrm{MS}^{3}$-Spektrum (Abb. 22(c)), in dem der Molpeak M(2) weiterhin das intensivste Signal darstellt.

In geringerem Maße finden dennoch Spaltungen im Kohlenwasserstoffteil des Moleküls statt, begleitet von zusätzlicher Protonenabspaltung (Oxidationen) und Bindungsumlagerungen. Das Fragment $\left[\mathrm{C}_{8} \mathrm{H}_{8}+\mathrm{H}\right]^{+}$m/z 105 entsteht durch Spaltung zwischen C-9 und C-10 (C-2 bis C-9) und das Fragment $\left[\mathrm{C}_{7} \mathrm{H}_{6}+\mathrm{H}\right]^{+} \mathrm{m} / \mathrm{z} 91$ durch Spaltung zwischen C-5 und C-6. Aus der Spaltung zwischen C-3 und C-4 resultiert zunächst das Fragment $\left[\mathrm{C}_{9} \mathrm{H}_{10}+\mathrm{H}\right]^{+} \mathrm{m} / \mathrm{z} 119$ und nach Abspaltung weiterer zwei Protonen daraus das Fragment $\left[\mathrm{C}_{9} \mathrm{H}_{8}+\mathrm{H}\right]^{+} \mathrm{m} / \mathrm{z} 117$. 
7-Hydroxy-dodeca-2E,4E,8Z,10E-tetraensäure-isobutylamid $\mathrm{C}_{16} \mathrm{H}_{25} \mathrm{NO}_{2} ; \mathrm{M}_{\mathrm{r}} 263$; RT 5,75 min
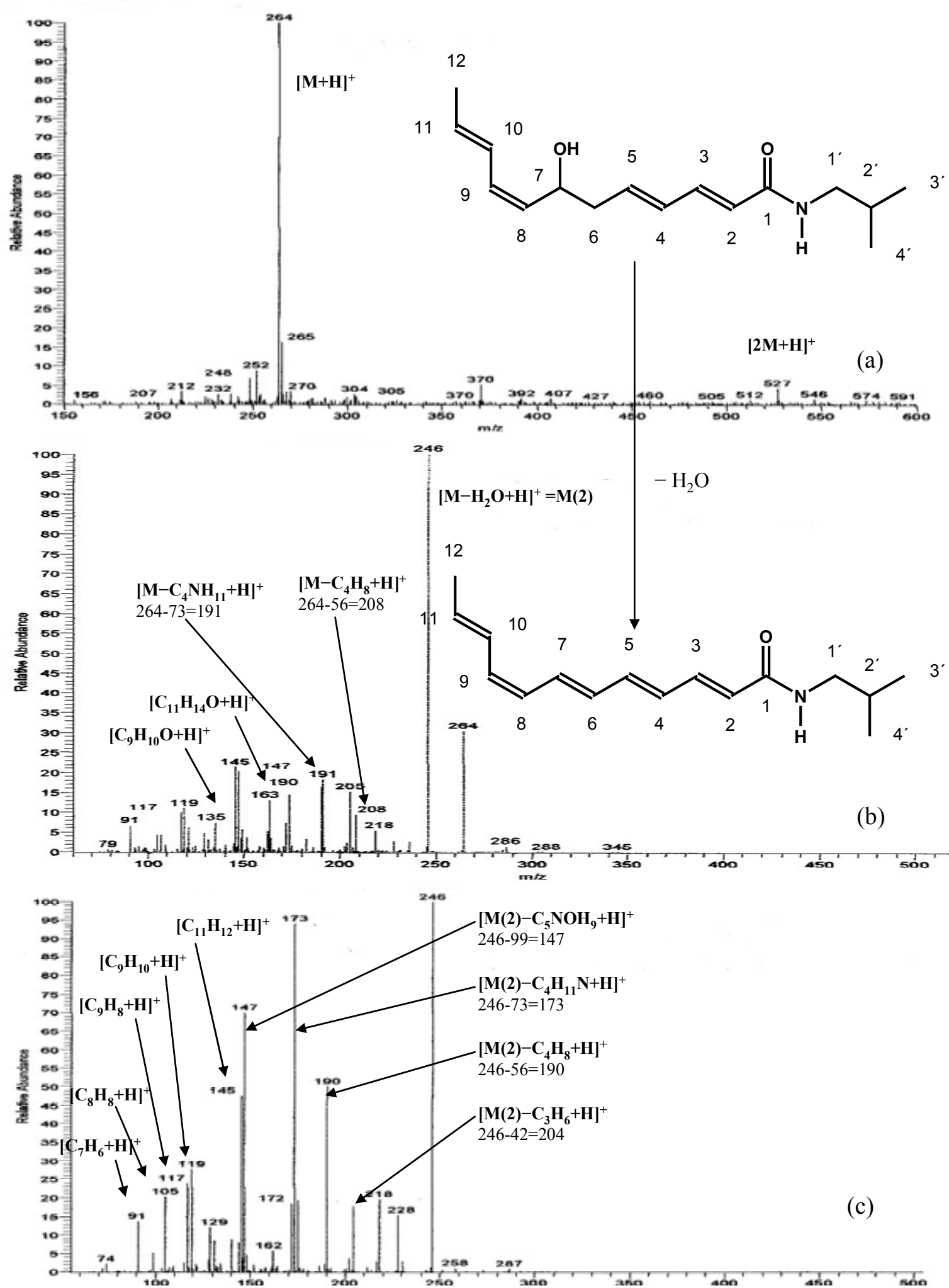

Abb. 22(a) - (c): +ESI-MS / $\mathrm{MS}^{2} / \mathrm{MS}^{3}$-Spektren von Verbindung $A 3$ 
Das Fragmentationsmuster von $\boldsymbol{A 3}$ entspricht nach Wasserabspaltung dem von WOELKART [181] beschriebenen Isomerenpaar Dodeca-2E,4E,8Z,10E/Z-tetraensäure-isobutylamid(e) mit $\mathrm{M}_{\mathrm{r}} 247$ aus Echinacea angustifolia, mit einer wichtigen Ausnahme: In der LiteraturVerbindung [181] erfolgt eine Spaltung zwischen C-6 und C-7, wo sich eine Einfachbindung befindet und im Prinzip eine Sollbruchstelle zwischen den beiden ungesättigten Enden des Fettsäurerests existiert, charakterisiert durch das Auftreten des Fragments m/z 81. Bei $\boldsymbol{A 3}$ kommt es dagegen an dieser Stelle nach Wasserabspaltung zur Ausbildung einer Doppelbindung, die gegenüber einer Einfachbindung eine weitaus größere Stabilität gegenüber Bindungsbrüchen aufweist. Aus diesem Grund tritt hier kein analoges Fragment in Erscheinung.

In Abb. 22(b) ist zu erkennen, dass das Ausgangsmolekül, unter Erhalt der Hydroxylfunktion, ebenfalls fragmentiert. Die Massenfragmente 191 und 208 gehen auf Spaltungen im IsobutylRest zurück. Das Fragment m/z $163\left[\mathrm{C}_{11} \mathrm{H}_{14}+\mathrm{H}\right]^{+}$stellt den Kohlenwasserstoffrest nach Abspaltung des gesamten Isobutylamid-Rests und zweier weiterer Protonen dar. Nach Spaltung zwischen C-3 und C-4 im Ausgangsmolekül entsteht das Fragment m/z 135 $\left[\mathrm{C}_{9} \mathrm{H}_{10} \mathrm{O}+\mathrm{H}\right]^{+}$. Die Hydroxylgruppe muss sich damit an einem der C-Atome C-4 bis C-12 befinden. Geht man als Basisstruktur von Dodeca-2E,4E,8Z,10E-tetraensäure oder einem ihrer Isomeren aus, die als „Begleitsubstanz(en)“ mit m/z 248 als $[\mathrm{M}+\mathrm{H}]^{+}$im Full-MSSpektrum (Abb. 22(a)) ebenfalls ersichtlich ist (sind), wäre sie am C-6 oder C-7 zu erwarten. Energetisch-räumliche Aspekte scheinen die C-7-Stellung zu präferieren, die folglich als Strukturvorschlag angegeben ist.

Somit ist $\boldsymbol{A 3}$ ein neuer Naturstoff.

\subsubsection{2,4-Dihydroxy-nona-6,8-diinsäure-phenylethylamid (Verbindung $A 4$ )}

Verbindung $\boldsymbol{A 4}$ aus Sammelfraktion 2 stellt ein zweifach hydroxiliertes Alkamid dar. In Abb. 23(a)-(c) sind drei Molpeaks erkennbar, die sich jeweils um die Massendifferenz 18 unterscheiden. Im Full-MS-Spektrum (Abb. 23(a)) ist zunächst das parent ion mit m/z 286 $\left([\mathrm{M}+\mathrm{H}]^{+}\right) \mathrm{zu}$ erkennen. Dieses geht durch Fragmentation unter Wasserabspaltung in das Ion $\mathrm{m} / \mathrm{z} 268\left([\mathrm{M}(2)+\mathrm{H}]^{+}\right)$(Abb. 23(b)) und dieses wiederum unter Wasserabspaltung in das Ion $\mathrm{m} / \mathrm{z} 250\left([\mathrm{M}(3)+\mathrm{H}]^{+}\right)(\mathrm{Abb} .23(\mathrm{~b})$ und (c)) über. 
2,4-Dihydroxy-nona-6,8-diinsäure-phenylethylamid $\mathrm{C}_{17} \mathrm{H}_{19} \mathrm{NO}_{3} ; \mathrm{M}_{\mathrm{r}} 285$; RT 7,94 min

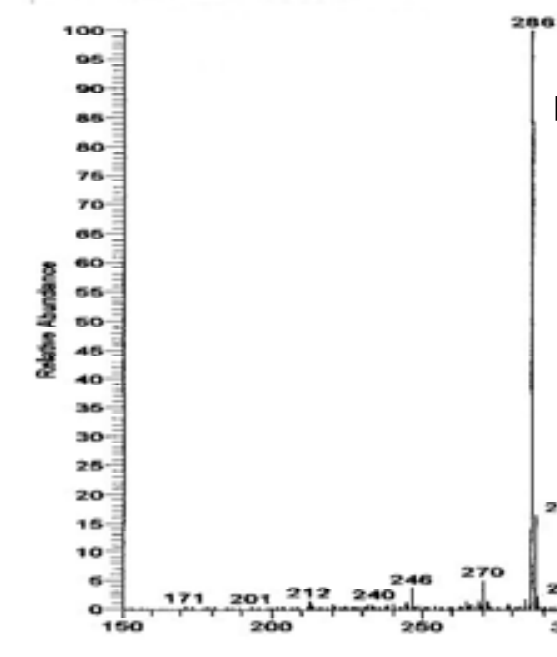

$[\mathbf{M}+\mathbf{H}]^{+}$

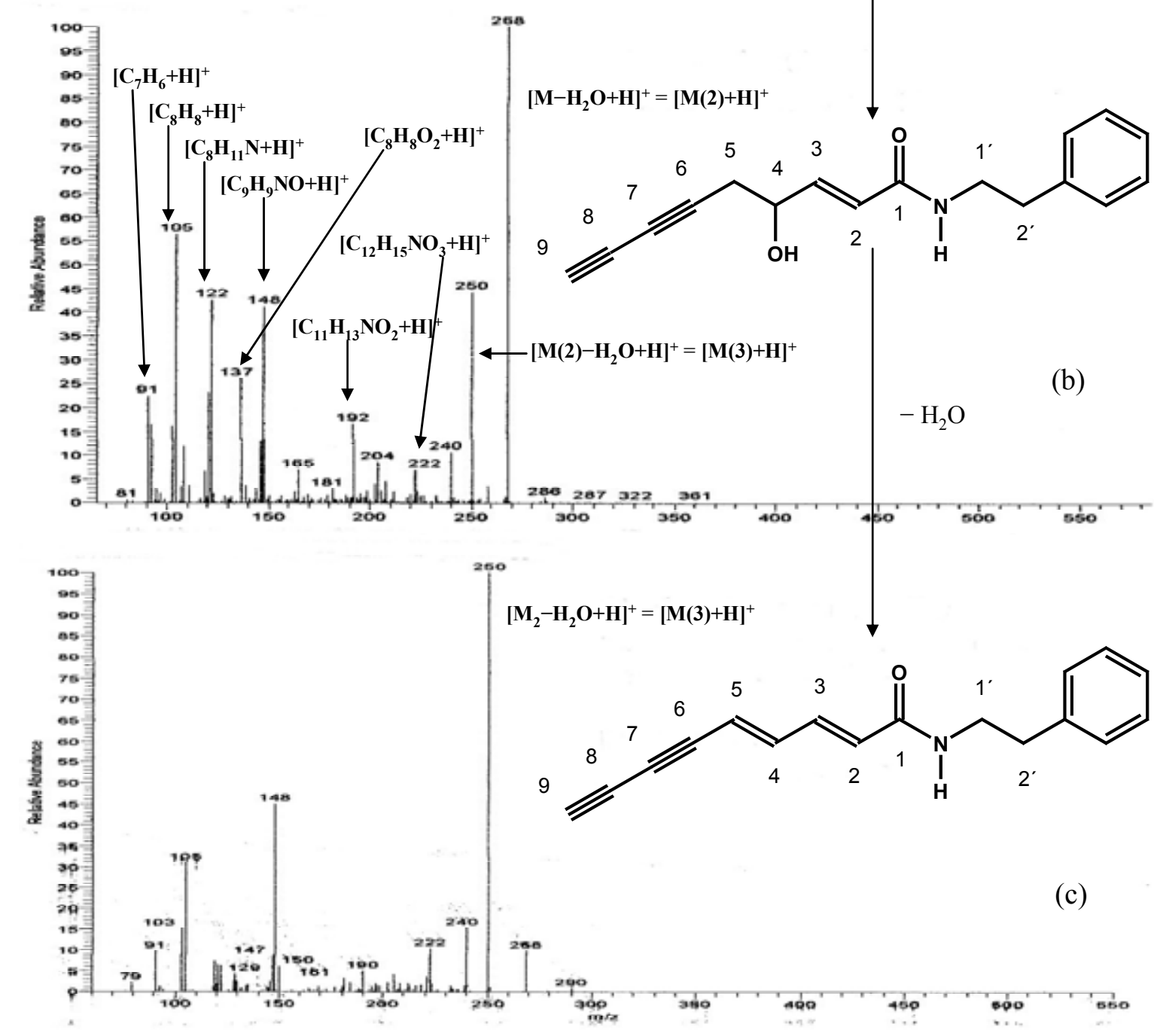

Abb. 23(a) - (c): +ESI-MS / MS ${ }^{2} / \mathrm{MS}^{3}$-Spektren von Verbindung $A 4$ 
Im $\mathrm{MS}^{3}$-Spektrum ist als intensivster Peak M(3) zu erkennen, dessen Fettsäurerest kaum noch fragmentiert, vermutlich wegen der großen Stabilität des vollständig ungesättigten Systems. Hier sind lediglich das Fragment, das den gesamten Kohlenwasserstoffrest beschreibt, als m/z 103, sowie die typischen Fragmente des Phenylethylamid-Rests sichtbar (m/z 91, 105, 122 , 148), wie bereits im $\mathrm{MS}^{2}$ Spektrum (Abb. 23(b)).

In Abb. 23(b) sind weiterhin Spaltungen des Ausgangsmoleküls zu beobachten. So könnte das Fragment m/z $137\left(\left[\mathrm{C}_{8} \mathrm{H}_{8} \mathrm{O}_{2}+\mathrm{H}\right]^{+}\right)$den Kohlenwasserstoffrest nach Spaltung zwischen $\mathrm{C}-1$ und C-2 unter Protonenabgabe darstellen. Das Fragment m/z $192\left(\left[\mathrm{C}_{11} \mathrm{H}_{13} \mathrm{NO}_{2}+\mathrm{H}\right]^{+}\right)$steht für eine Spaltung zwischen C-3 und C-4; es enthält eine Hydroxylgruppe. Nach der Spaltung wäre im Fragment der Masse 192 strukturell und rechnerisch die Bildung einer EpoxyStruktur möglich, bei Stellung der OH-Gruppe an C-2.

Das Fragment m/z $222\left(\left[\mathrm{C}_{12} \mathrm{H}_{15} \mathrm{NO}_{3}+\mathrm{H}\right]^{+}\right)$steht für eine Spaltung zwischen C-4 und C-5. Dieses Fragment enthält beide OH-Gruppen, was die Position der zweiten OH-Gruppe am C4 festlegt. m/z 222 kann ein weiteres Molekül Wasser anlagern $(\mathrm{m} / \mathrm{z} 240)$ oder abspalten $(\mathrm{m} / \mathrm{z}$ 204).

Das Alkamid $\boldsymbol{A} 4$ stellt einen neuen Naturstoff dar.

\subsubsection{Dodeca-2 $E$-en-8,10-diinsäure-isobutylamid (Verbindung $\boldsymbol{A 5}$ )}

Die in Abb. 24 vorgeschlagene Formel stellt die wahrscheinlichste Struktur für Verbindung A5, mit der Retentionszeit von 10,61 min, aus Sammelfraktion 2 dar. Diese Substanz wurde bisher in Acmella ciliata nicht nachgewiesen, konnte aber bereits isoliert werden aus den Wurzeln von Echinacea angustifolia DC. [11] und Echinacea atrorubens Nutt. [35].

Im +ESI-MS-MS ist wieder das typische Fragmentationsmuster des Isobutylamid-Rests (3.3.3.1.) zu sehen (Fragmente $\mathrm{m} / \mathrm{z}$ 147, 173, 190, entsprechend $\mathrm{M}-99, \mathrm{M}-73$ und $\mathrm{M}-56$ ).

Die Masse 145 repräsentiert den Kohlenwasserstoffrest nach Verlust weiterer zwei Protonen. Das Fragment $\left[\mathrm{C}_{8} \mathrm{H}_{13} \mathrm{NO}+\mathrm{H}\right]^{+} \mathrm{m} / \mathrm{z} 140$ steht für den rechten Molekülteil nach Bindungsbruch zwischen C-4 und C-5 unter Beibehaltung der Amidstruktur. Wie bei den vorangegangenen Alkamiden beschrieben, kommt es auch hier zu mehreren Bindungsbrüchen mit Protonen- 
abgabe (Oxidation) im restlichen Kohlenwasserstoffteil. $\left[\mathrm{C}_{9} \mathrm{H}_{10}+\mathrm{H}\right]^{+} \mathrm{m} / \mathrm{z} 119$ und daraus, unter Abgabe zweier Protonen, hervorgehend $\left[\mathrm{C}_{9} \mathrm{H}_{8}+\mathrm{H}\right]^{+} \mathrm{m} / \mathrm{z} 117$ entstehen nach Spaltung zwischen C-3 und C-4, bezogen auf das Ausgangsmolekül. Spaltung zwischen C-4 und C-5 führt $\mathrm{zu}\left[\mathrm{C}_{8} \mathrm{H}_{6}+\mathrm{H}\right]^{+} \mathrm{m} / \mathrm{z}$ 103. Schließlich führt die Spaltung zwischen C-5 und C-6 zu $\left[\mathrm{C}_{7} \mathrm{H}_{6}+\mathrm{H}\right]^{+}$mit der Masse 91 .

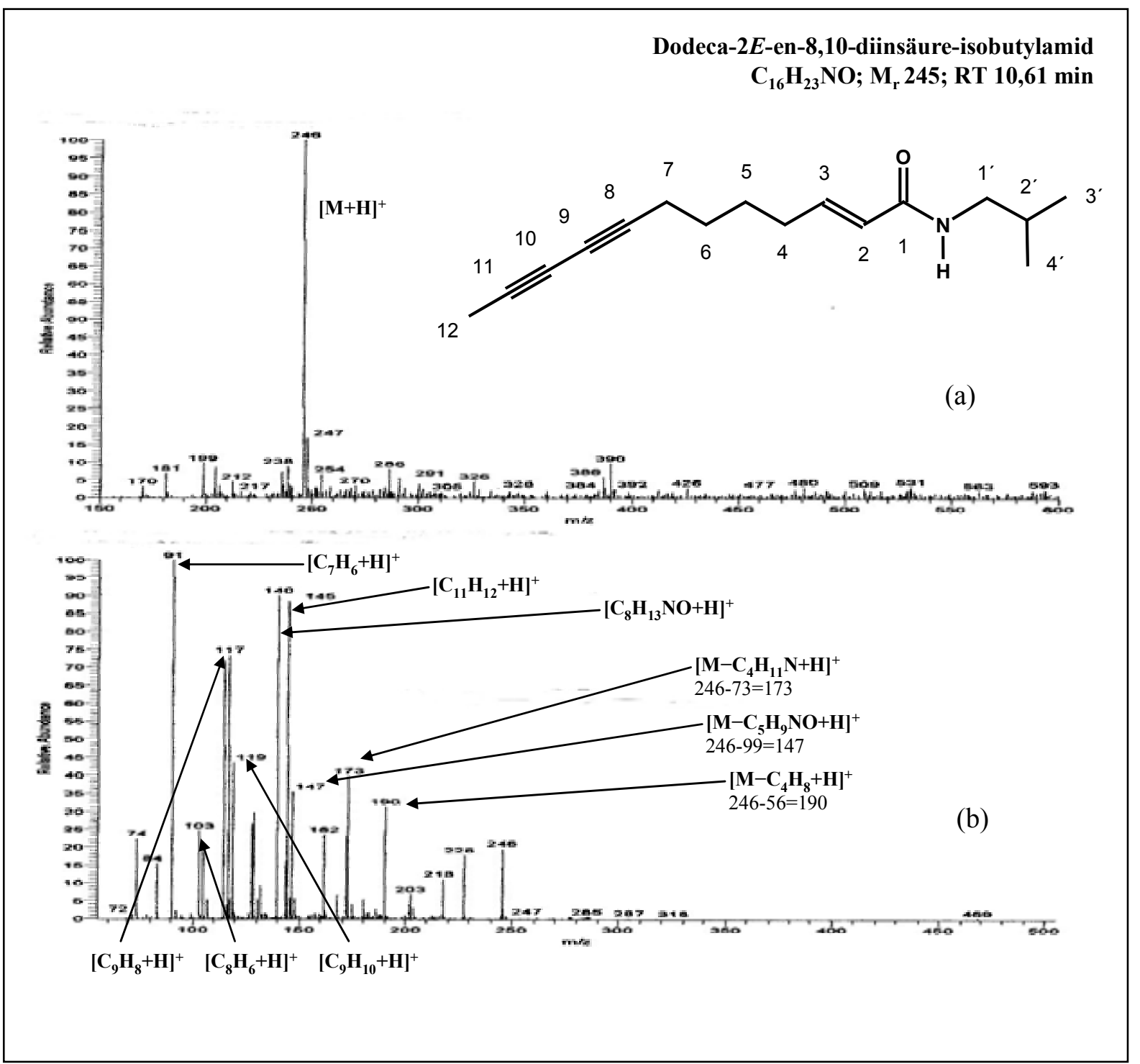

Abb. 24(a) - (b): +ESI-MS / MS $^{2} /$ MS $^{3}$-Spektren von Verbindung $A 5$

\subsubsection{2-Hydroxy-undeca-7Z,9E-diensäure-isobutylamid (Verbindung $\boldsymbol{A 6}_{1}$ ) Undeca-2E,7Z,9E-triensäure-isobutylamid (Verbindung $\boldsymbol{A 6}_{2}$ )}

Verbindung $\boldsymbol{A 6}_{\boldsymbol{1}}$ aus Sammelfraktion 2 stellt, ebenso wie $\boldsymbol{A 3}$ und $\boldsymbol{A 4}$, ein hydroxiliertes Alkamid mit der Molmasse $253\left(\mathrm{C}_{15} \mathrm{H}_{27} \mathrm{NO}_{2}\right)$ dar (Abb. 25). Die Stellung der $\mathrm{OH}-\mathrm{Gruppe}$ am 
2-Hydroxy-undeca-7Z,9E-diensäure-isobutylamid $\mathrm{C}_{15} \mathrm{H}_{27} \mathrm{NO}_{2} ; \mathrm{M}_{\mathrm{r}}$ 253; RT 10,97 min

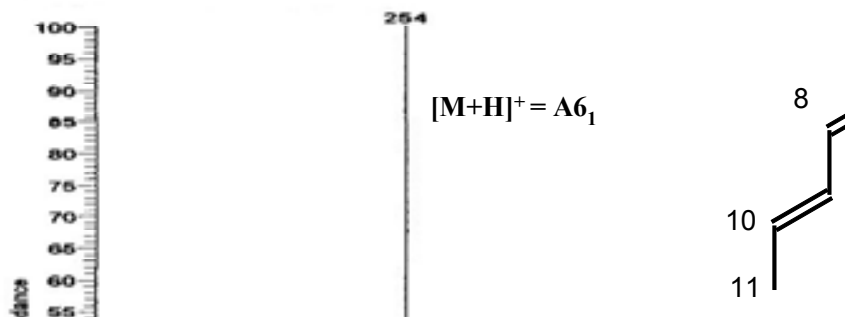<smiles>C/C=C\CCCCC(O)C(=O)NCC(C)C</smiles>
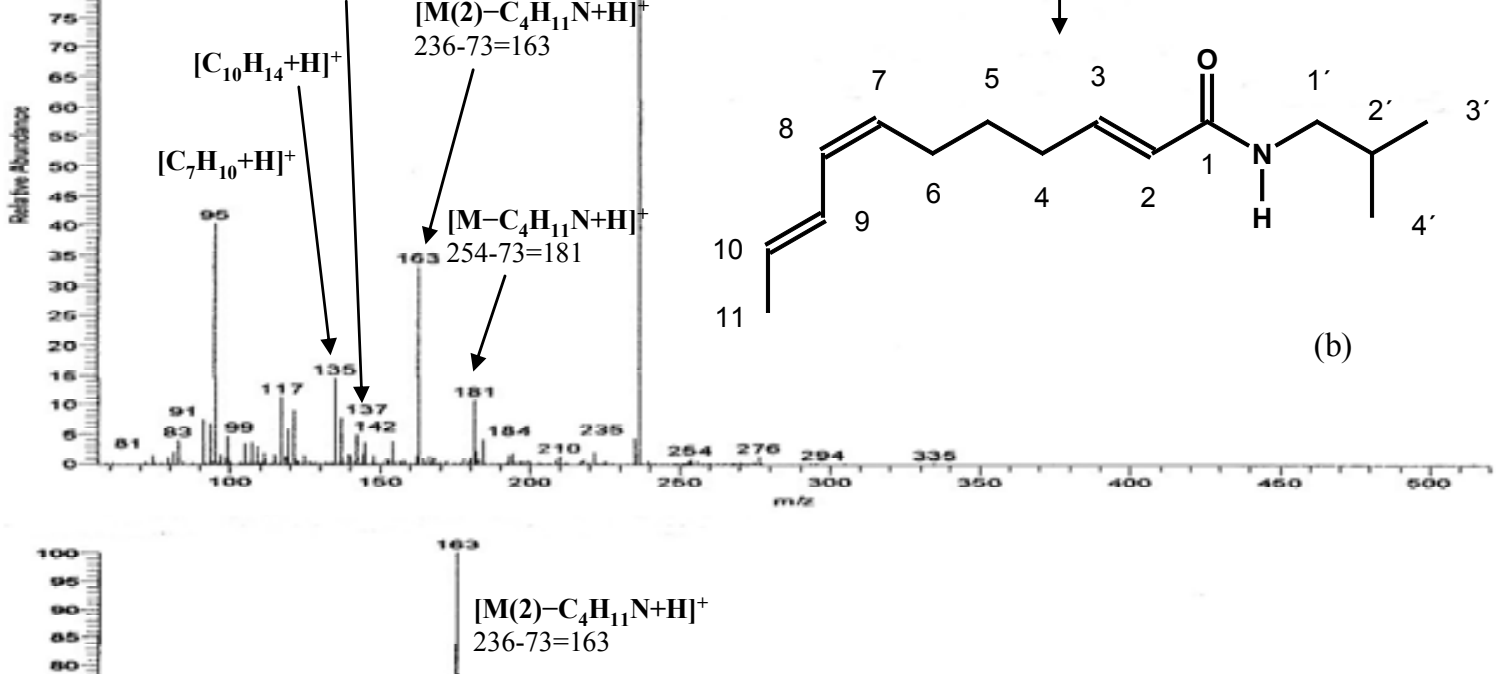

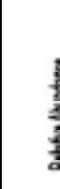

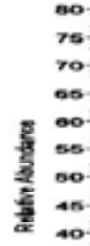
$\left[\mathrm{C}_{7} \mathrm{H}_{10}+\mathrm{H}\right]^{+}$ 236-73=163
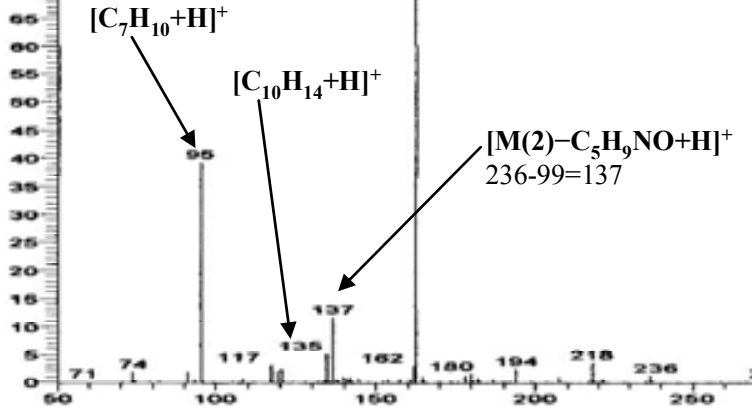

(c)

Abb. 25(a) - (c): +ESI-MS / MS ${ }^{2} / \mathrm{MS}^{3}$-Spektren der Verbindungen $A 6_{1}$ und $A 6_{2}$ 
C-2 erscheint aus energetischen Gründen sowie in Analogie zu den Verbindungen $\boldsymbol{A 4}, \boldsymbol{A 1 2}$ und $\mathrm{zu}$ Literatur [132] als sinnvoll, kann aber auf Grund des Fehlens entsprechender Fragmente nicht vollständig abgesichert werden.

Unter Wasserabspaltung wird aus $\boldsymbol{A 6}_{\boldsymbol{1}}$ das Undeca-2E,7Z,9E-triensäure-isobutylamid $\left(\boldsymbol{A 6}_{2}\right)$ mit $\mathrm{M}_{\mathrm{r}} 235\left(=\mathrm{M}(2): \mathrm{C}_{15} \mathrm{H}_{25} \mathrm{NO}\right)$. Dieses Molekül ist bereits für „Spilanthes acmella“ beschrieben worden [144].

Somit bleibt für die Position der $\mathrm{OH}-\mathrm{Gruppe}$ in Verbindung $\boldsymbol{A 6}_{\boldsymbol{1}}$ theoretisch lediglich die Stellung am C-3 als Alternative. Wie in Abb. 25(a) zu sehen, ist die Verbindung mit der Molmasse 235 bereits primär vorhanden und erhält daher die Bezeichnung $\boldsymbol{A 6}_{2}$.

Im $\mathrm{MS}^{3}$-Spektrum (Abb. 25(c)) fällt die Intensität des Fragments $\mathrm{m} / \mathrm{z} \quad 163$ $\left(\left[\mathrm{M}(2)-\mathrm{C}_{4} \mathrm{H}_{11} \mathrm{~N}+\mathrm{H}\right]^{+}\right)$auf, welches durch Amidspaltung entsteht. Diese Spaltung tritt auch in Verbindung $\boldsymbol{A 6}_{\boldsymbol{1}}$ auf, als Fragment m/z $181\left(\left[\mathrm{M}-\mathrm{C}_{4} \mathrm{H}_{11} \mathrm{~N}+\mathrm{H}\right]^{+}\right)$sichtbar.

Nur A6 $_{2}$ zeigt noch schwach die Abspaltung des gesamten Isobutylamids im Fragment m/z $137\left(\left[\mathrm{M}-\mathrm{C}_{5} \mathrm{H}_{9} \mathrm{NO}+\mathrm{H}\right]^{+}\right)$bzw. dessen Umlagerungsprodukt nach zusätzlicher Protonenabspaltung $\mathrm{m} / \mathrm{z} 135\left(\left[\mathrm{C}_{10} \mathrm{H}_{14}+\mathrm{H}\right]^{+}\right)$.

Das Fragment mit der Masse $95\left[\mathrm{C}_{7} \mathrm{H}_{10}+\mathrm{H}\right]^{+}$stellt den linken Teil des Kohlenwasserstoffrests dar, und es entsteht durch Bindungsbruch zwischen C-4 und C-5. Nach Abspaltung und Protonenabgabe dürfte dieses Fragment drei konjugierte Doppelbindungen enthalten, was große Stabilität zur Folge hat. Als einziges ist dieses Bruchstück im $\mathrm{MS}^{4}$-Spektrum noch vorhanden (nicht dargestellt) und fragmentiert offensichtlich nicht weiter.

\subsubsection{Nona-2Z-en-6,8-diinsäure-isobutylamid (Verbindung $A 7$ ) und 2,3(c)-Epoxy-nona-6,8-diinsäure-phenylethylamid (Verbindung $\boldsymbol{A B}$ )}

Die Verbindungen $\boldsymbol{A} 7$ und $\boldsymbol{A} \boldsymbol{8}$ werden gemeinsam besprochen, da sie durch den genutzten HPLC-Gradienten (s. Exp. Teil) nicht komplett voneinander getrennt und daher nebeneinander analysiert wurden. Sie resultieren ebenfalls aus Sammelfraktion 2 und wurden bereits durch präparative DC (Banden 2 - 4, s. 3.3.3.) isoliert. 
Nona-2Z-en-6,8-diinsäure-isobutylamid ( $A 7)$ $\mathrm{C}_{13} \mathrm{H}_{17} \mathrm{NO}$; $\mathrm{M}_{\mathrm{r}}$ 203; RT 11,75-12,22 min
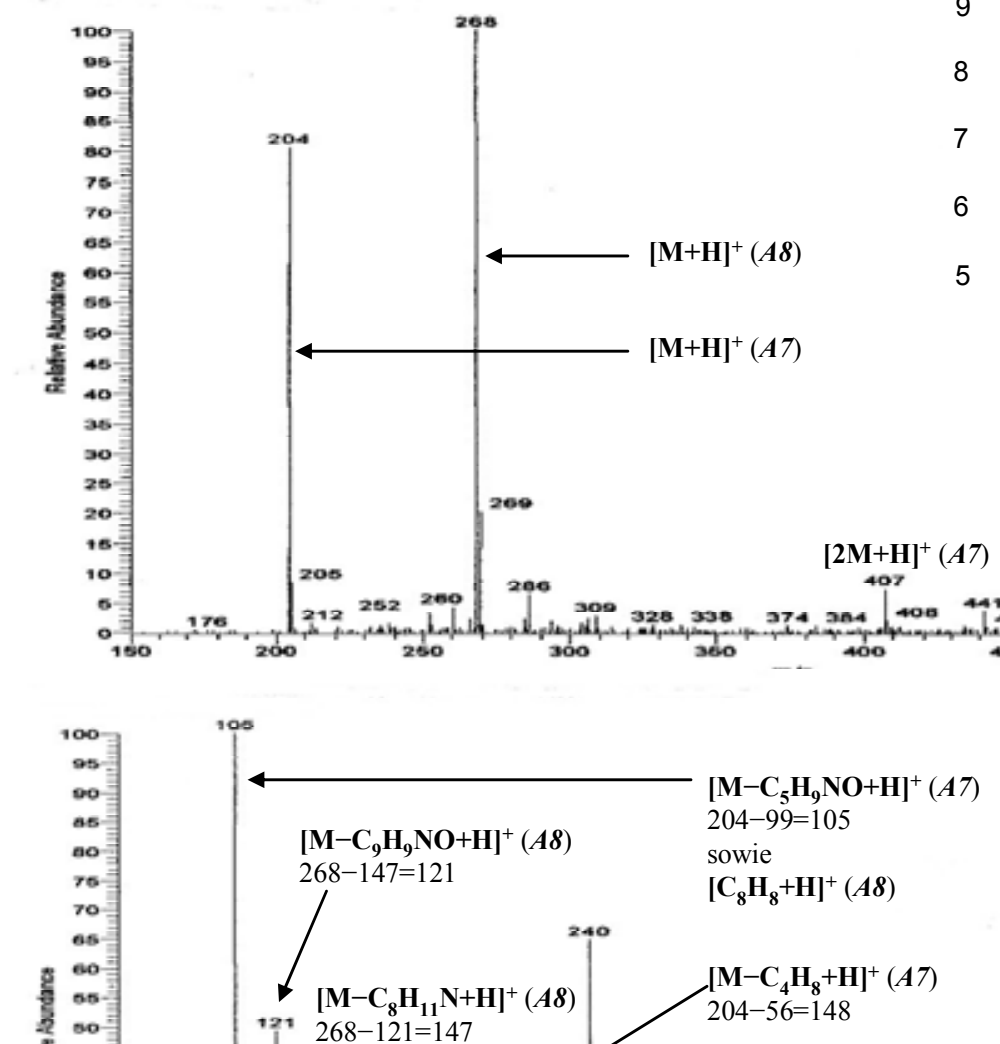

$\left[\mathrm{C}_{8} \mathrm{H}_{8}+\mathbf{H}\right]^{+}(\boldsymbol{A \delta})$

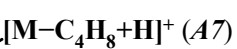

$204-56=148$
$\mathrm{C}_{17} \mathrm{H}_{17} \mathrm{NO}_{2} ; \mathrm{M}_{\mathrm{r}} 267$; RT 11,75-12,22 min

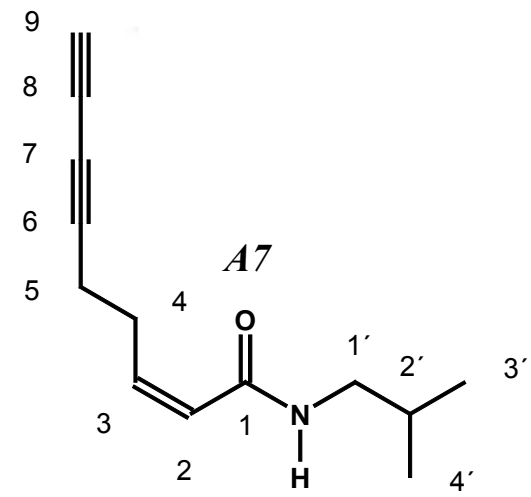

(a) A8
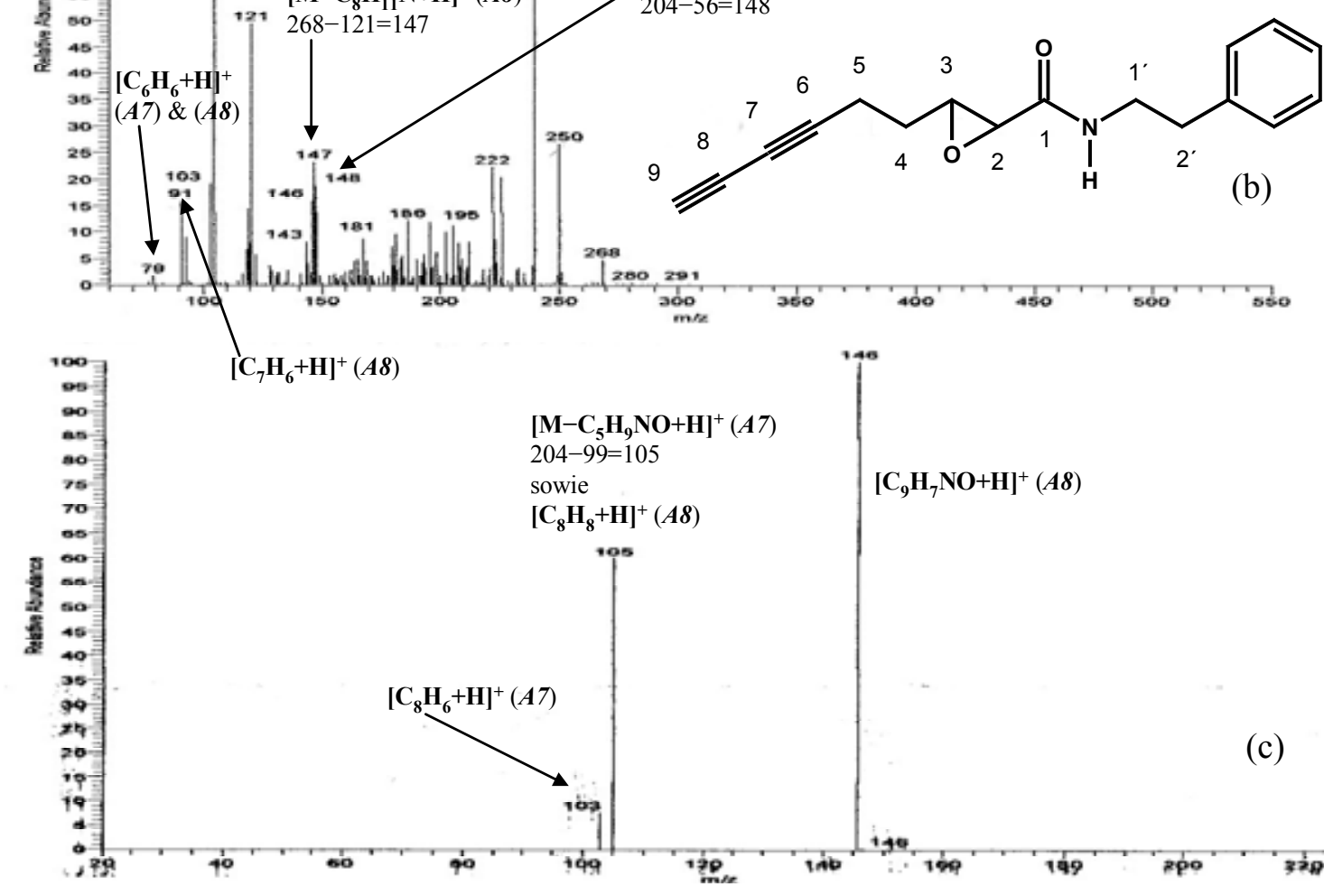

$\left[\mathrm{C}_{9} \mathrm{H}_{7} \mathrm{NO}+\mathrm{H}\right]^{+}(A 8)$

$[2 \mathrm{M}+\mathrm{H}]^{+}(A 8)$

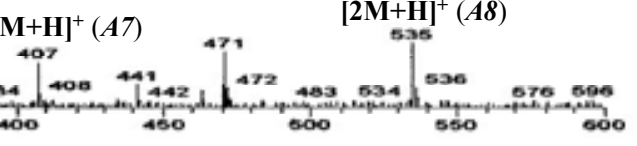


Bei Verbindung $\boldsymbol{A 8}$ handelt es sich um 2,3(c)-Epoxy-nona-6,8-diinsäure-phenylethylamid, das bereits in Acmella ciliata [110, 112] gefunden wurde. Das Fragment mit der Masse 147 (Abb. 26(b)) entspricht dem Restmolekül nach Spaltung der Amidbindung, und das Fragment 121 stellt den Kohlenwasserstoffrest nach Abspaltung des gesamten Phenylethylamids dar.

Hier unterbleibt jedoch die sonst stets beobachtete Bindungsumlagerung unter Protonenabgabe, in diesem Fall zum Fragment m/z 119, was auf Grund der Epoxidstruktur nicht möglich ist.

Im Gegensatz zu den hydroxilierten Verbindungen erfolgt bei $\boldsymbol{A} \boldsymbol{8}$ nur in geringerem Maße die Abspaltung von Wasser, zu erkennen an der schwachen Intensität des Signals m/z 250. Die Fragmente mit Massen zwischen 180 und 240 sind möglicherweise partiell unter intermediärer Anlagerung von Wasser entstanden. Diese sind jedoch in Abb. 26(c) ( $\mathrm{MS}^{3}-$ Spektrum) nicht mehr vorhanden.

Die Fragmente m/z 91, 105, 121, 148 treten als typische Bruchstücke des PhenylethylamidRests (s. 3.3.3.1.) bei $\boldsymbol{A} \boldsymbol{8}$ auf.

Bei dem Alkamid $\boldsymbol{A} 7$ mit der Molmasse von 203 dürfte es sich um Nona-2Z-en-6,8-diinsäureisobutylamid handeln. Diese Substanz wurde bereits aus der Verwandten Acmella oleracea (als „Spilanthes oleracea“) isoliert [53]. Das Fragmentationschema entspricht diesem Postulat. Es erfolgt eine Abspaltung des gesamten Amidteils $\left[\mathrm{M}-\mathrm{C}_{5} \mathrm{H}_{9} \mathrm{NO}+\mathrm{H}\right]^{+} \mathrm{m} / \mathrm{z} 105$ und daraus unter Protonenabgabe m/z 103 sowie die Abspaltung des Isobutyls $\left[\mathrm{M}-\mathrm{C}_{4} \mathrm{H}_{8}+\mathrm{H}\right]^{+} \mathrm{m} / \mathrm{z}$ 148 als typische Fragmentierung des Isobutylamid-Rests (s. 3.3.3.1.). Die Amidbindung wird dagegen nicht gespalten. Das Fragment mit der Massenzahl 79 entsteht durch Bindungsbruch zwischen C-3 und C-4 bei $\boldsymbol{A} 7$ und $\boldsymbol{A} 8$.

\subsubsection{Nona-2Z-en-6,8-diinsäure-phenylethylamid (Verbindung $A 9$ )}

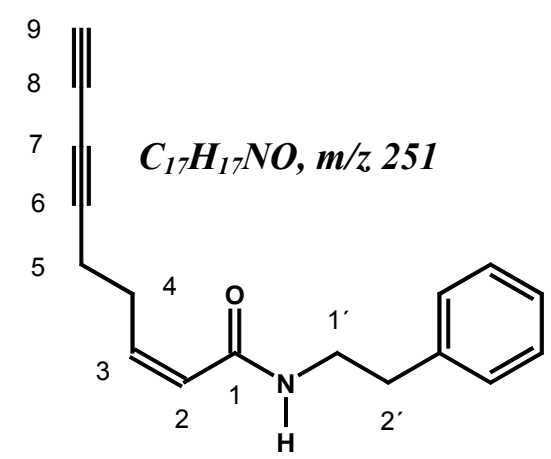

Verbindung $\quad \boldsymbol{A 9}$ aus Sammelfraktion $\mathbf{2}$ mit der Summenformel $\mathrm{C}_{17} \mathrm{H}_{17} \mathrm{NO}$ und der Molmasse von 251 zeigt dasselbe Fragmentationsmuster wie Verbindung $\boldsymbol{A 2}$ (3.3.3.3.). Die Retentionszeit von $\boldsymbol{A 9}$ beträgt 13,74 $\mathrm{min}$ 
gegenüber 12,62 min von $\boldsymbol{A} 2$ und stellt somit den einzigen Unterschied dar. Dieses Ergebnis bestärkt die Annahme, dass es sich um ein Isomer der Verbindung $\boldsymbol{A 2}$ handelt, zumal es bereits Hinweise darauf in der präparativen DC gab (s. 3.3.3.). Da das Fragmentationsmuster von $\boldsymbol{A} \mathbf{9}$ mit dem von $\boldsymbol{A 2}$ identisch ist, kann es sich nur um ein cis-/trans-Isomer bezüglich der Doppelbindung in Position 2,3 handeln. Somit wird für $\boldsymbol{A 9}$ das Z-Isomer von Nona-2-en-6,8diinsäure-phenylethylamid postuliert. Auf Grund der etwas anderen räumlichen Anordnung des Moleküls durch die cis-Bindung resultiert womöglich die etwas erhöhte Lipophilie von $\boldsymbol{A 9}$ gegenüber $\boldsymbol{A 2}$, was durch die längere Retentionszeit von $\boldsymbol{A 9}$ zum Ausdruck kommt.

Diese Substanz wurde bereits in den Gattungsverwandten Acmella oleracea (L.) Jansen (als „Spilanthes acmella“ L. var. oleracea Clarke) [132] und Acmella alba (L`Her.) Jansen (als „Spilanthes alba“ L'Heritier) [16] sowie in der Subtribus-Verwandten Salmea scandens L. DC. [17] nachgewiesen.

\subsubsection{Undeca-2 $E$-en-8,10-diinsäure-isobutylamid (Verbindung $\boldsymbol{A 1 0}$ )}

Verbindung A10, die in der vorliegenden Arbeit aus Sammelfraktion 2 hervorging, wurde bereits durch MARTIN $[110,112]$ in Acmella ciliata nachgewiesen. Darüber hinaus wurde sie in „Spilanthes acmella“ [144] gefunden sowie in zwei Echinacea-Arten [11, 35], also in Pflanzen desselben Subtribus.

Ebenso wie bei $\boldsymbol{A 1}\left(\mathrm{M}_{\mathrm{r}} 229\right)$, erfolgte eine Quantifizierung von $\boldsymbol{A 1 0}$ über „Tetraen“ (s. Exp. Teil) mit dem Ergebnis, dass der Gehalt in Sammelfraktion 2 2,29\% betrug, berechnet auf den Trockenextrakt der Dichlormethanextraktion jedoch nur 0,01\%.

Im Fragmentierungsmuster (Abb. 27) finden sich alle vier, für einen Isobutylamidrest typischen Fragmente m/z 133, 159, 176, 190. Diese Fragmente haben eine um 2 größere Masse, verglichen mit den entsprechenden Fragmenten von Verbindung A1. Daraus wird bereits deutlich, dass $\boldsymbol{A 1 0}$ das Dihydroderivat von $\boldsymbol{A} \mathbf{1}$ darstellt. Nach Amidspaltung lagert sich der Kohlenwasserstoffrest unter Abgabe von zwei Protonen um (Abb. 27(b) und (c)) und bildet das stabilere Fragment $\left[\mathrm{C}_{10} \mathrm{H}_{10}+\mathrm{H}\right]^{+} \mathrm{m} / \mathrm{z} 131$ (s. Abb. 27(c)). 


\section{Undeca-2E-en-8,10-diinsäure-isobutylamid $\mathrm{C}_{15} \mathrm{H}_{17} \mathrm{NO} ; \mathrm{M}_{\mathrm{r}} 231 ; \mathrm{RT} 14,15$ min}
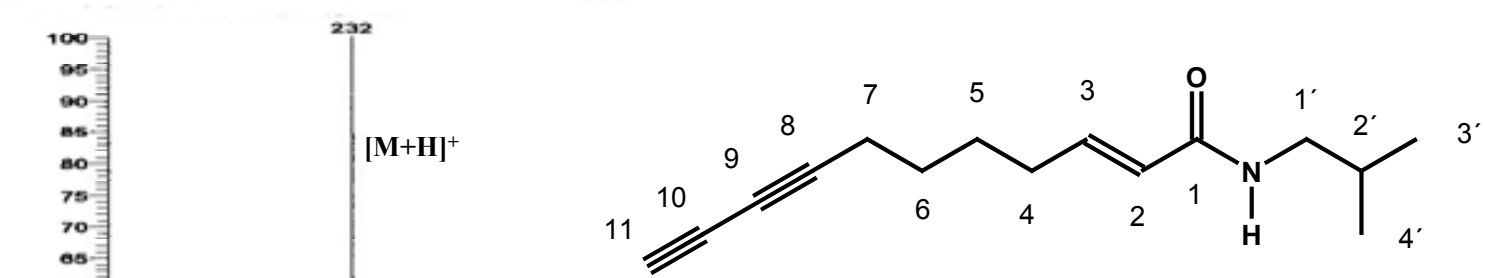

(a)
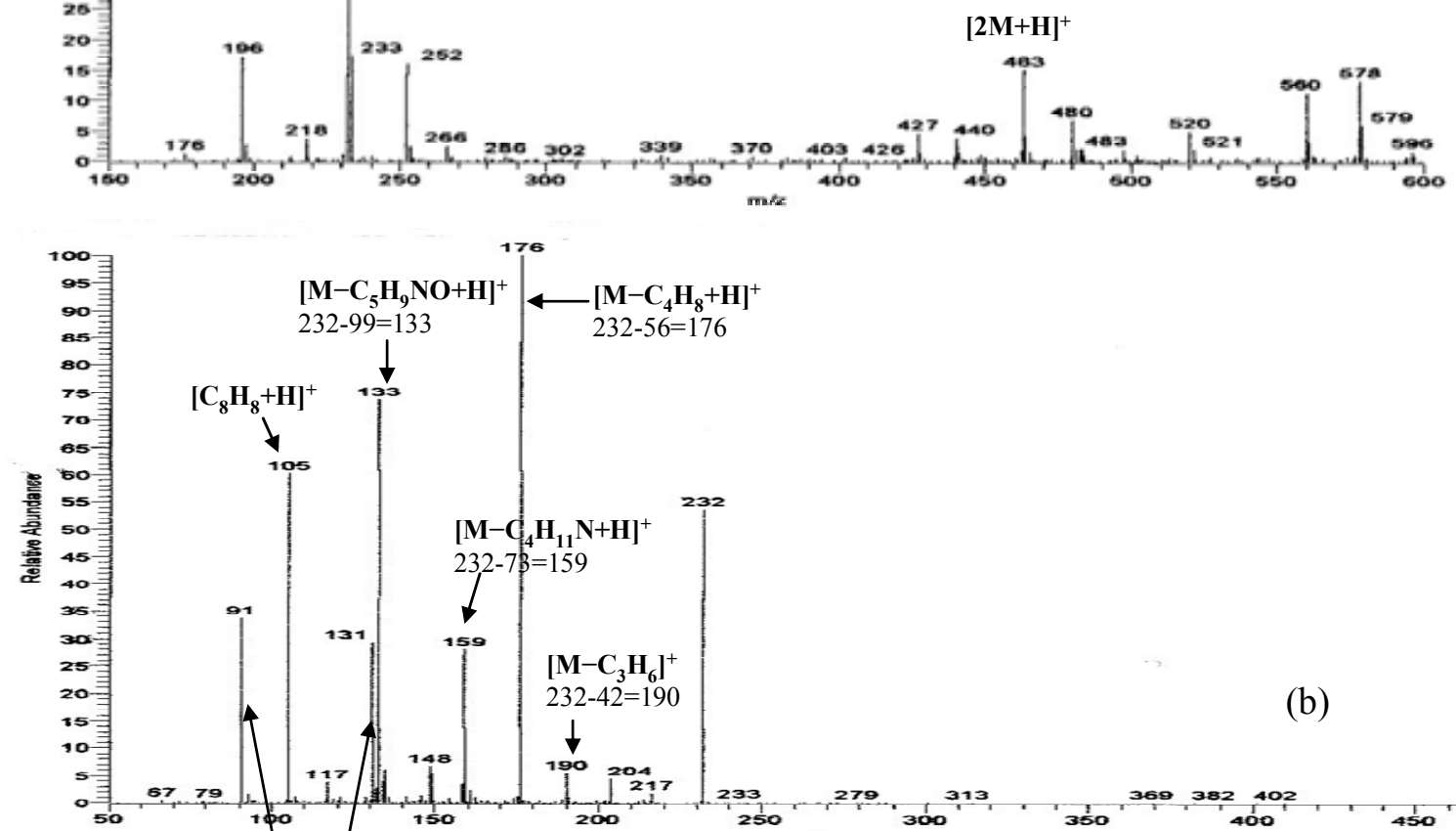

(b)

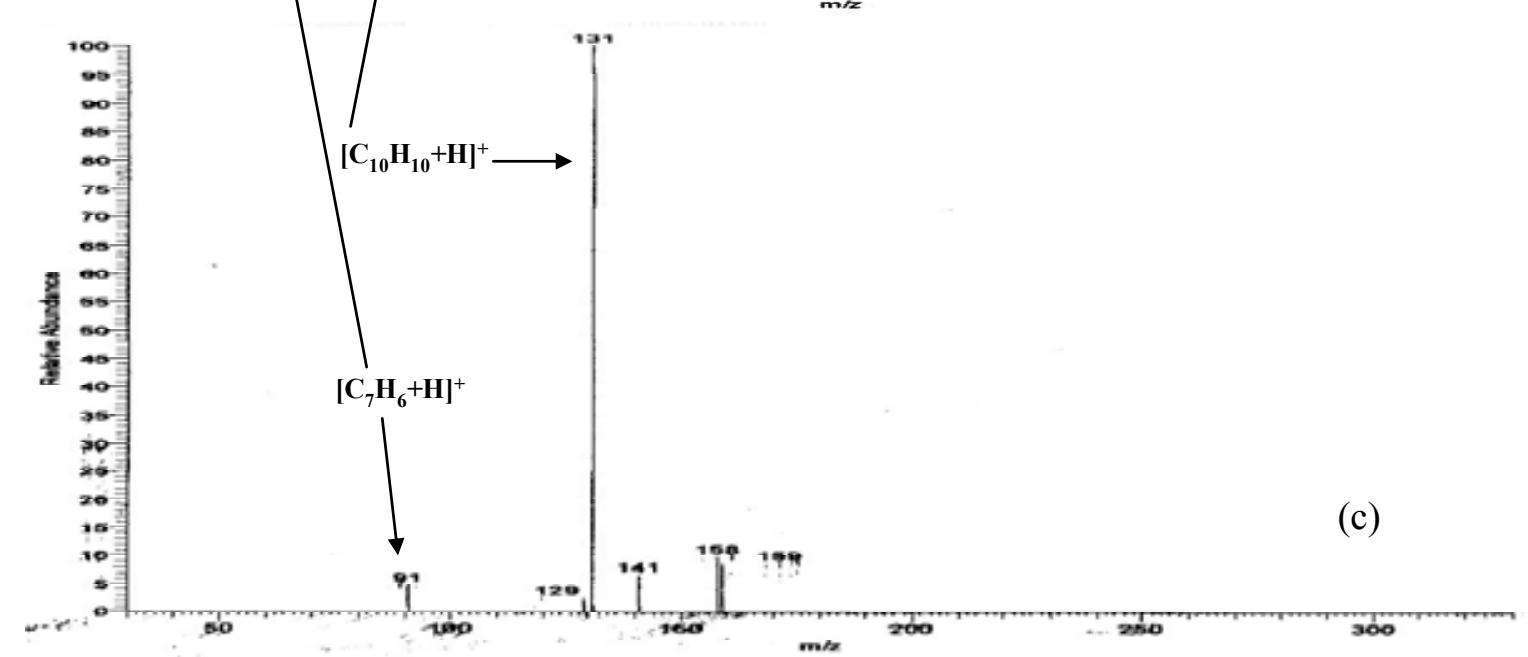

Abb. 27(a) - (c): +ESI-MS-Spektrum / $\mathrm{MS}^{2}$-/MS ${ }^{3}$-Spektren von Verbindung A10 
Wie bei den bereits beschriebenen Substanzen mit Diinstruktur und angrenzenden Methylengruppen $(\boldsymbol{A 1}, \boldsymbol{A 2}, \boldsymbol{A 5}, \boldsymbol{A 7}, \boldsymbol{A 8}, \boldsymbol{A 9})$ kommt es auch hier zu Bindungsbrüchen im „Methylenteil“, so zwischen C-4 und C-5 unter Entstehung des Fragments $\left[\mathrm{C}_{7} \mathrm{H}_{6}+\mathrm{H}\right]^{+} \mathrm{m} / \mathrm{z} 91$ sowie zwischen C-3 und C-4 unter Bildung des Fragmentions $\left[\mathrm{C}_{8} \mathrm{H}_{8}+\mathrm{H}\right]^{+} \mathrm{m} / \mathrm{z} 106$.

\subsubsection{Undeca-2E,4E-dien-8,10-diinsäure-methylbutylamid (Verbindung $A 11$ )}

Auch Verbindung $\boldsymbol{A 1 1}$ wurde aus Sammelfraktion 2 mittels HPLC-MS detektiert und ist identisch mit dem bereits von MARTIN $[110,112]$ aus den Blütenköpfchen von Acmella ciliata isolierten Undeca-2E,4E-dien-8,10-diinsäure-methylbutylamid. Es ist die einzige Substanz in den untersuchten Sammelfraktionen mit einem Methylbutylamid-Rest, dessen allgemeines Fragmentationsschema in 3.3.3.1. dargestellt ist. Diskutabel wäre auch das 4ZIsomer, das ebenfalls von MARTIN nachgewiesen wurde [110, 112]. Das Dimer mit der Masse 487 als $[2 \mathrm{M}+\mathrm{H}]^{+}$ist in Abb. 28(a) schwach zu erkennen.

$\left[\mathrm{M}-\mathrm{C}_{6} \mathrm{H}_{11} \mathrm{NO}+\mathrm{H}\right]^{+} \mathrm{m} / \mathrm{z} 131$ steht für den Säurerest nach Abspaltung des gesamten Amidteils

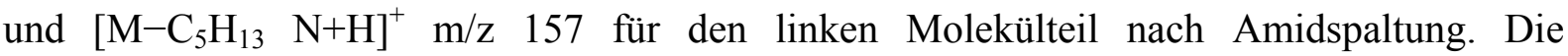
Abspaltung des Methylbutyls entspricht $\left[\mathrm{M}-\mathrm{C}_{5} \mathrm{H}_{10}+\mathrm{H}\right]^{+} \mathrm{m} / \mathrm{z}$ 174. Schließlich spaltet sich noch ein $\mathrm{C}_{2} \mathrm{H}_{4}$-Fragment aus dem rechten Molekülteil ab $\left(\left[\mathrm{M}-\mathrm{C}_{2} \mathrm{H}_{4}+\mathrm{H}\right]^{+}\right)$mit der Massenzahl des Restmoleküls von 216.

Weiterhin sind im Kohlenwasserstoffteil Spaltungen mit den entsprechenden Folgereaktionen zu sehen. Das Fragment mit der Masse 131 lagert sich, wie bei vielen anderen Verbindungen, unter Abgabe von zwei Protonen größtenteils in das Fragment $\left[\mathrm{C}_{10} \mathrm{H}_{8}+\mathrm{H}\right]^{+} \mathrm{m} / \mathrm{z} 129 \mathrm{um}$. Die Entstehung von Fragment $\left[\mathrm{C}_{7} \mathrm{H}_{6}+\mathrm{H}\right]^{+} \mathrm{m} / \mathrm{z} 91$ ist analog $\mathrm{zu} \boldsymbol{A 1}$ (s. 3.3.3.2.) vorstellbar, da die Fettsäurereste von $\boldsymbol{A 1 1}$ und $\boldsymbol{A 1}$ identisch sind.

In Abb. 28(a) sind zwei weitere Molmassen zu erkennen. Bei der Masse von 264, die bereits in der Analytik nach der präparativen DC, dort als „Begleitsubstanz“ bezeichnet (3.3.3.), auftauchte, könnte es sich um das Acmellonat, einen ungesättigten, langkettigen 2-Ketolester handeln, der aus „Spilanthes acmella“ isoliert wurde [102]. 


\section{Undeca-2E,4E-dien-8,10-diinsäure-methylbutylamid} $\mathrm{C}_{16} \mathrm{H}_{21} \mathrm{NO}$; $\mathrm{M}_{\mathrm{r}} 243$; RT 15,14 min

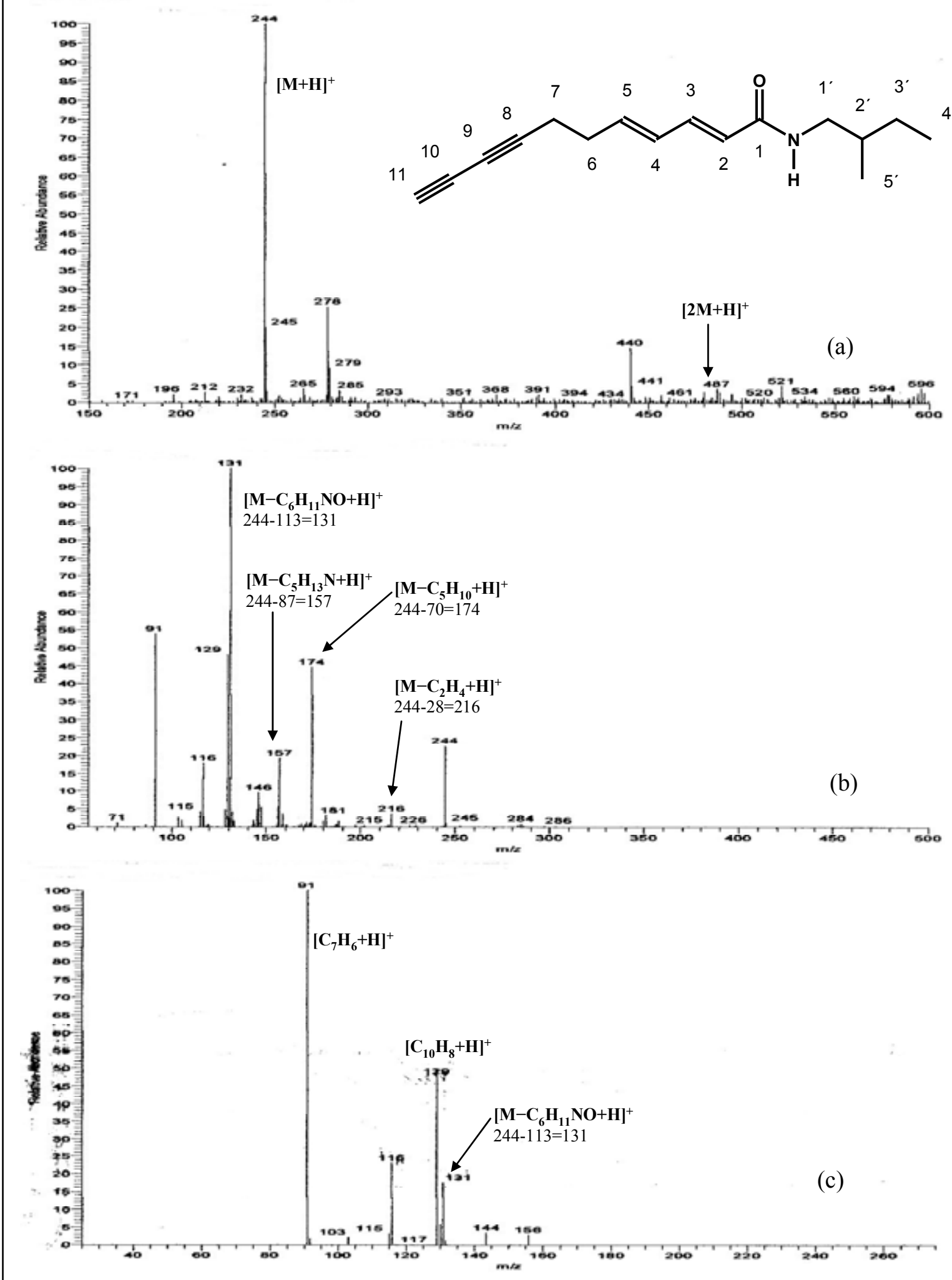

Abb. 28(a) - (c): +ESI-MS / $\mathrm{MS}^{2} / \mathrm{MS}^{3}$-Spektren von Verbindung $A 11$ 
Eine Verbindung mit der Molmasse 277 findet sich auch bei der MS der phenolischen Bestandteile aus dem Methanol-Extrakt, konnte bisher jedoch nicht zugeordnet werden.

Auffallend ist weiterhin eine Substanz mit der Molmasse 439 (Abb. 28(a)) ohne bisherige Zuordnung.

\subsubsection{Weitere Verbindungen aus Sammelfraktion 2 (Retentionszeiten 20,95, 21,67 und 23,98 min)}

Wie das HPLC-MS-Bild von Sammelfraktion 2 zeigt (Abb. 18), fallen außer den bereits zugeordneten Substanzen noch die Retentionszeiten von 20,95; 21,67 und 23,98 min ins Auge.

Die Substanz mit der Retentionszeit 20,95 min hat eine Molmasse von 336. Da diese Molmasse eine gerade bzw. das $[\mathrm{M}+\mathrm{H}]^{+}$-Ion im +ESI-MS mit m/z 337 eine ungerade Zahl ist, handelt es sich wahrscheinlich nicht um ein Alkamid. Das Fragmentationsmuster ist somit ohne Referenzen nicht auszuwerten. Da in den Spektren bis $\mathrm{MS}^{4}$ (nicht abgebildet) dreimal eine Abspaltung von Wasser $\mathrm{zu}$ beobachten war $(337-18=319-18=301-18=283)$, kann jedoch auf eine dreifach hydroxilierte Verbindung geschlossen werden.

Bei der Verbindung mit der Retentionszeit 21,67 min ist zunächst ein sog. parent ion mit der Masse 412 sichtbar, das unter Wasserabgabe in das Ion $\mathrm{m} / \mathrm{z} 394$ übergeht. Das im Ausgangsspektrum nur minimal erscheinende Ion m/z 286 tritt in größerer Intensität bei weiterer Fragmentierung auf. Hier ist auch wieder das Ausgangsion unter Wasserabspaltung in $\mathrm{m} / \mathrm{z} 394$ übergegangen (412-18=394). Das Ion $\mathrm{m} / \mathrm{z} 286$ geht unter Wasserabspaltung in $\mathrm{m} / \mathrm{z} 268$ und dieses ebenfalls unter Wasserabspaltung in $\mathrm{m} / \mathrm{z} 250$ über. Die beiden Letztgenannten treten im $\mathrm{MS}^{3}$-Spektrum (nicht gezeigt) nebeneinander auf. Im $\mathrm{MS}^{4}$-Spektrum zeigt sich nur noch das offensichtlich relativ stabile Ion m/z 250 mit großer Intensität, das wenig fragmentiert.

Dieser Prozess, ausgehend von der Verbindung mit der Molmasse 285, ebenso wie das Fragmentationschema der dehydratisierten Verbindung $\mathrm{m} / \mathrm{z} 249$, ist identisch mit der zuvor beschriebenen Verbindung $\boldsymbol{A 4}$ (3.3.3.5.), was auf ein Isomer von $\boldsymbol{A 4}$ schließen lässt. Allerdings verwundert die lange Retentionszeit für eine relativ hydrophile Verbindung mit 
zwei OH-Gruppen. Möglicherweise wird die Substanz m/z 285 erst durch Fragmentation aus einer größeren Verbindung freigesetzt, wobei hier ein Zusammenhang zur Verbindung mit der Molmasse 411 bestehen könnte.

Analoge Aussagen treffen auch für die Substanz mit der Retentionszeit 23,98 min zu, die das „Ausgangsion“ mit einer Masse von 426 und nach Wasserabspaltung von 408 aufweist.

3.3.3.13. 2,5-Dihydroxy-deca-6 $Z, 8 E$-diensäure-isobutylamid (2,5-Dihydroxyhydrospilanthol) (Verbindung $\boldsymbol{A 1 2}$ )

Verbindung $\boldsymbol{A 1 2}$ resultiert aus Sammelfraktion 3. Genau genommen handelt es sich um drei Verbindungen, hier bezeichnet als $A 1_{1}, A 12_{2}$ und $A_{12}$, die sich jeweils um die Massendifferenz 18, also um ein Molekül Wasser, unterscheiden. Alle drei Alkamide sind bereits im Ausgangsspektrum in unterschiedlichen Intensitäten vorhanden (s. Abb. 29(a)). Die Wasserabspaltung ist im $\mathrm{MS}^{2}$ - und $\mathrm{MS}^{3}$-Spektrum (Abb. 29(b) und (c)) sichtbar.

Verbindung $\boldsymbol{A 1 2}_{2}$ dürfte dem 2-Hydroxy-4E,6Z,8E-triensäure-isobutylamid entsprechen, wohingegen Verbindung $\boldsymbol{A 1 2}_{3}$ das voll konjugierte System von Deca-2E,4E,6Z,8Etetraensäure-isobutylamid darstellt.

Im $\mathrm{MS}^{3}$-Spektrum (Abb. 29(c)) können diverse Fragmente aller drei Moleküle beobachtet werden mit der auffallend großen Intensität der Amidspaltung im Isobutylamid-Rest. Hieraus resultieren die Fragmente m/z 183, 165 und 147. Die Abspaltung des gesamten Isobutylamids tritt hier nicht auf. In geringer Intensität ist die Abspaltung des Fragments m/z 42 aus dem Isobutylamid-Rest bei Verbindung $\boldsymbol{A 1 2}_{2}$ mit m/z 196 zu sehen.

Das Fragment m/z 137 entsteht nach Spaltung zwischen C-1 und C-2 in $\boldsymbol{A 1 2}_{2}$ und enthält eine OH-Gruppe. Die Stellung dieser OH-Gruppe in $\mathbf{A 1 2}_{2}$ an C-2 erscheint auf Grund des Fragmentions m/z $107\left(\left[\mathrm{C}_{8} \mathrm{H}_{10}+\mathrm{H}\right]^{+}\right)$als relativ sicher. Dieses Fragment symbolisiert den Bindungsbruch zwischen $\mathrm{C}-2$ und $\mathrm{C}-3$ und enthält keine OH-Gruppe, jedoch drei Doppelbindungen. Die zweite Hydroxy-Gruppe in A12 ${ }_{1}$ muss daher nach Abspaltung als Wasser zu einer der drei Doppelbindungen geführt haben. Angenommen wird die C-5Position (s. Abb. 29(a) - (c)). 


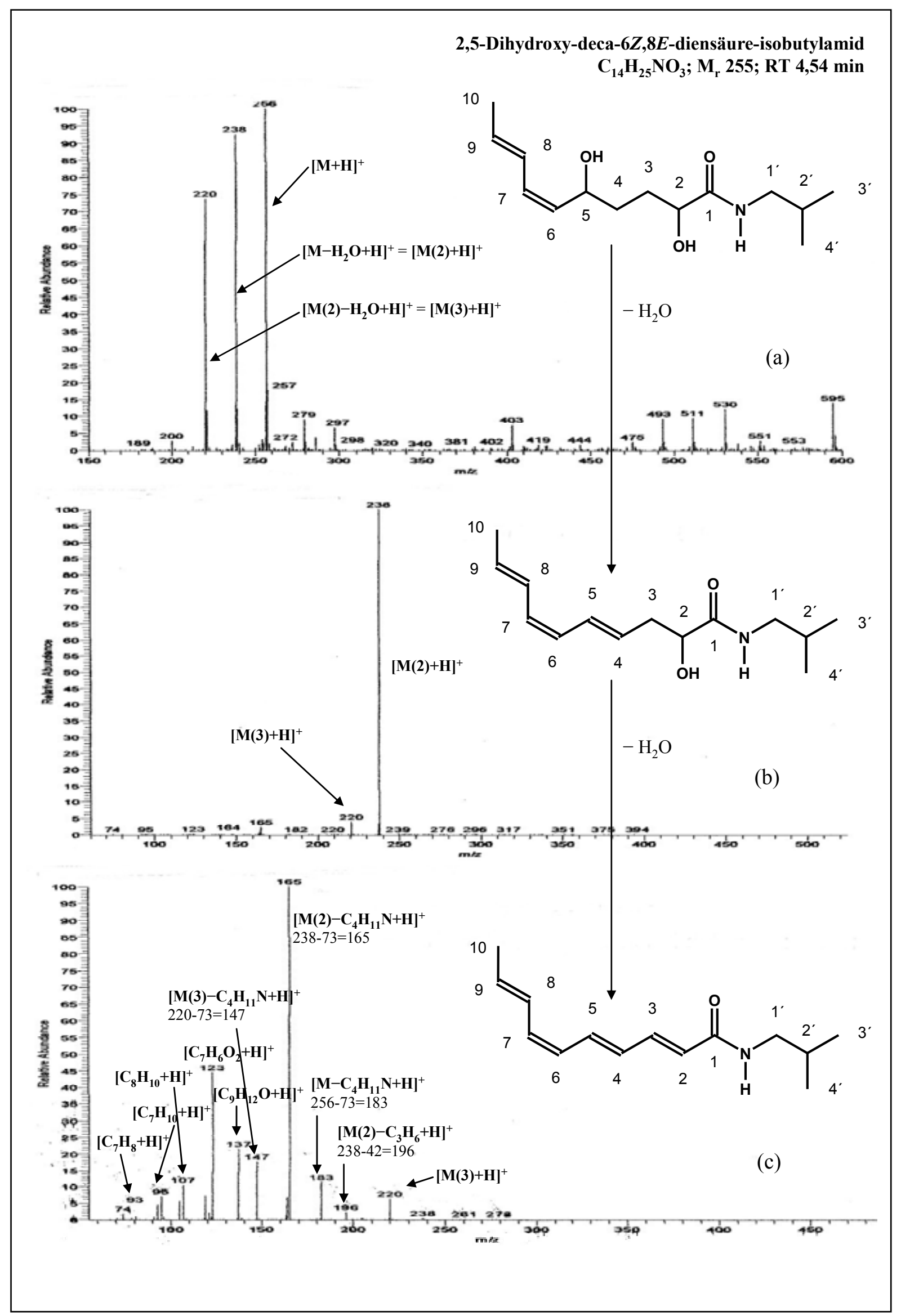

Abb. 29(a) - (c): +ESI-MS / $\mathrm{MS}^{2} / \mathrm{MS}^{3}$-Spektren der Verbindungen $A 12_{1}, A 12_{2}, A 12_{3}$ 
Weiterhin steht das Fragment m/z $137\left(\left[\mathrm{C}_{9} \mathrm{H}_{12} \mathrm{O}+\mathrm{H}\right]^{+}\right)$für den Bindungsbruch zwischen C-1 und $\mathrm{C}-2$ und m/z $123\left(\left[\mathrm{C}_{7} \mathrm{H}_{6} \mathrm{O}_{2}+\mathrm{H}\right]^{+}\right)$für das Fragment von $\mathrm{C}-1$ bis $\mathrm{C}-7$ nach Amidspaltung und Bindungsbruch zwischen C-7 und C-8. Zwei weitere Fragmente mit den Massen 93 und 95 (C-4 bis C-10) sind schließlich Spaltprodukte des Kohlenwasserstoffrests von Verbindung $\mathrm{Al2}_{2}$ bzw. $\mathrm{Al2}_{3}$.

Die Dimere der Verbindungen $\boldsymbol{A 1 2}_{\boldsymbol{1}}$ und $\boldsymbol{A 1 2}_{2}$ mit den Massen 511 bzw. 475 als $[\mathrm{M}+\mathrm{H}]^{+}$ treten hier ebenfalls auf.

Alle drei Verbindungen können als neue Naturstoffe betrachtet werden.

\subsubsection{Spilanthol (Deca-2E,6Z,8E-triensäure-isobutylamid) (Verbindung $\boldsymbol{A 1 3}$ )}

Verbindung $\boldsymbol{A 1 3}$ war in Sammelfraktion 3 zu identifizieren. Hierbei handelt es sich um das bereits durch MARTIN [110, 111] in Acmella ciliata nachgewiesene Spilanthol (Deca2E,6Z,8E-triensäure-isobutylamid). Es ist das erste beschriebene Alkamid, welches auch das Synonym Affinin trägt und das außerdem in weiteren verwandten Pflanzen (s. 3.3.1.1.) nachgewiesen wurde. Diese Substanz erscheint im HPLC-MS-Spektrum (Abb. 19) mit einer Retentionszeit von 15,68 min als intensivster Peak. Das Fragmentationsmuster wie auch die Molmasse von 221 sprechen für Spilanthol, das nach MARTIN [110] die Leitsubstanz der Stoffgruppe der Alkamide in Acmella ciliata darstellt.

Die typischen Fragmente, die durch Spaltungen im Isobutylamid-Rest entstehen, äußern sich in den Massen 123, 149 und 166. Der nach Amid-Abspaltung verbleibende Kohlenwasserstoffrest mit der Masse 123 geht auch hier, wie bei den meisten anderen Alkamiden zu beobachten, durch Protonenabgabe in das Fragment $\left[\mathrm{C}_{9} \mathrm{H}_{12}+\mathrm{H}\right]^{+}$mit der Masse 121 über. Die Fragmente mit den Massen 93, 81 und 67 entstehen analog wieder durch Spaltungen im Kohlenwasserstoffrest. Es kommt zu Spaltungen zwischen C-3 und C-4 $\left[\mathrm{C}_{7} \mathrm{H}_{8}+\mathrm{H}\right]^{+}, \mathrm{C}-4$ und C-5 $\left[\mathrm{C}_{6} \mathrm{H}_{8}+\mathrm{H}\right]^{+}$und zwischen C-5 und C-6 $\left[\mathrm{C}_{5} \mathrm{H}_{6}+\mathrm{H}\right]^{+}$. Fragment m/z 81 könnte auch die Atome C-2 bis C-7 umfassen und wäre damit analog dem Fragment m/z 83 beim Hydrospilanthol (s. Abb. 32(b) und (c)). Besonders auffällig ist in Abb. 30(a) das Dimer mit $\mathrm{m} / \mathrm{z} 443$, entsprechend $[2 \mathrm{M}+\mathrm{H}]^{+}$. 
Spilanthol (Deca-2E,6Z,8E-triensäure-isobutylamid) $\mathrm{C}_{14} \mathrm{H}_{23} \mathrm{NO}$; $\mathrm{M}_{\mathrm{r}} 221$; RT 15,76 min
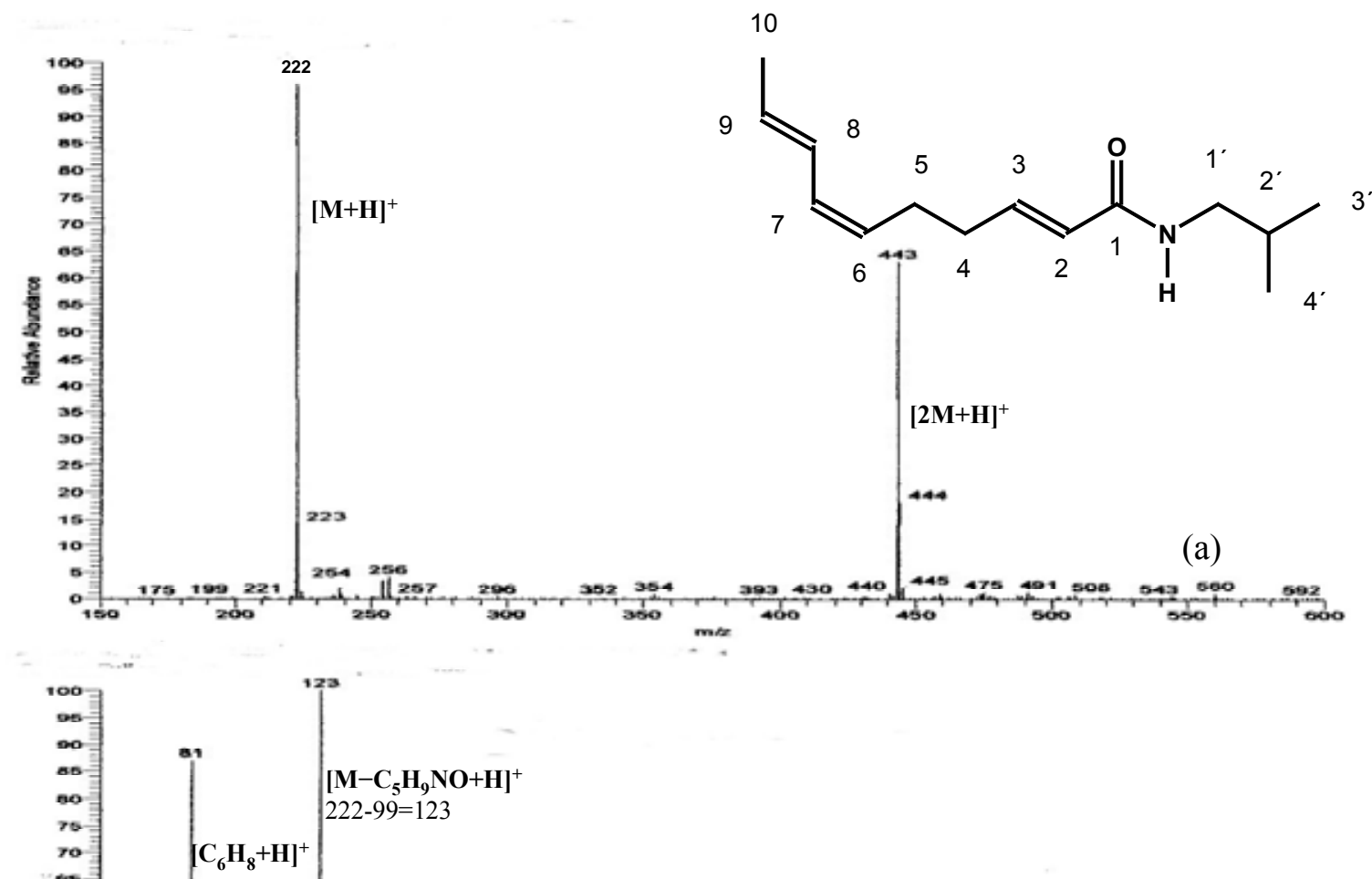

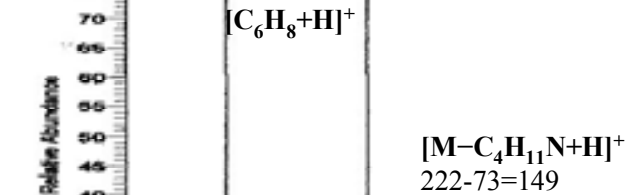

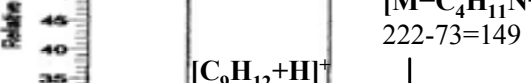

${ }_{25}{ }_{25}=\left[\mathrm{C}_{9} \mathrm{H}_{12}+\mathrm{H}\right]^{+} \quad \quad\left[\mathrm{M}-\mathrm{C}_{4} \mathrm{H}_{8}+\mathrm{H}\right]^{+}$
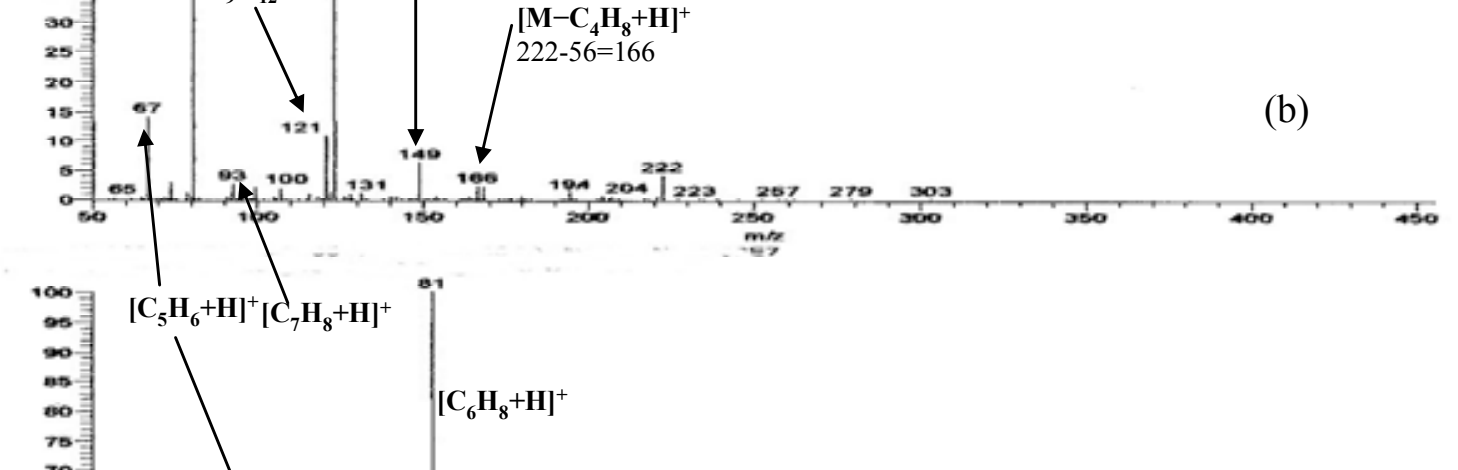

(c)

Abb. 30(a) - (c): +ESI-MS / $\mathrm{MS}^{2} / \mathrm{MS}^{3}$-Spektren von Verbindung $A 13$ 
3.3.3.15. Trideca-5Z-en-10,12-diinsäure-isobutylamid (Verbindung $A 14$ )

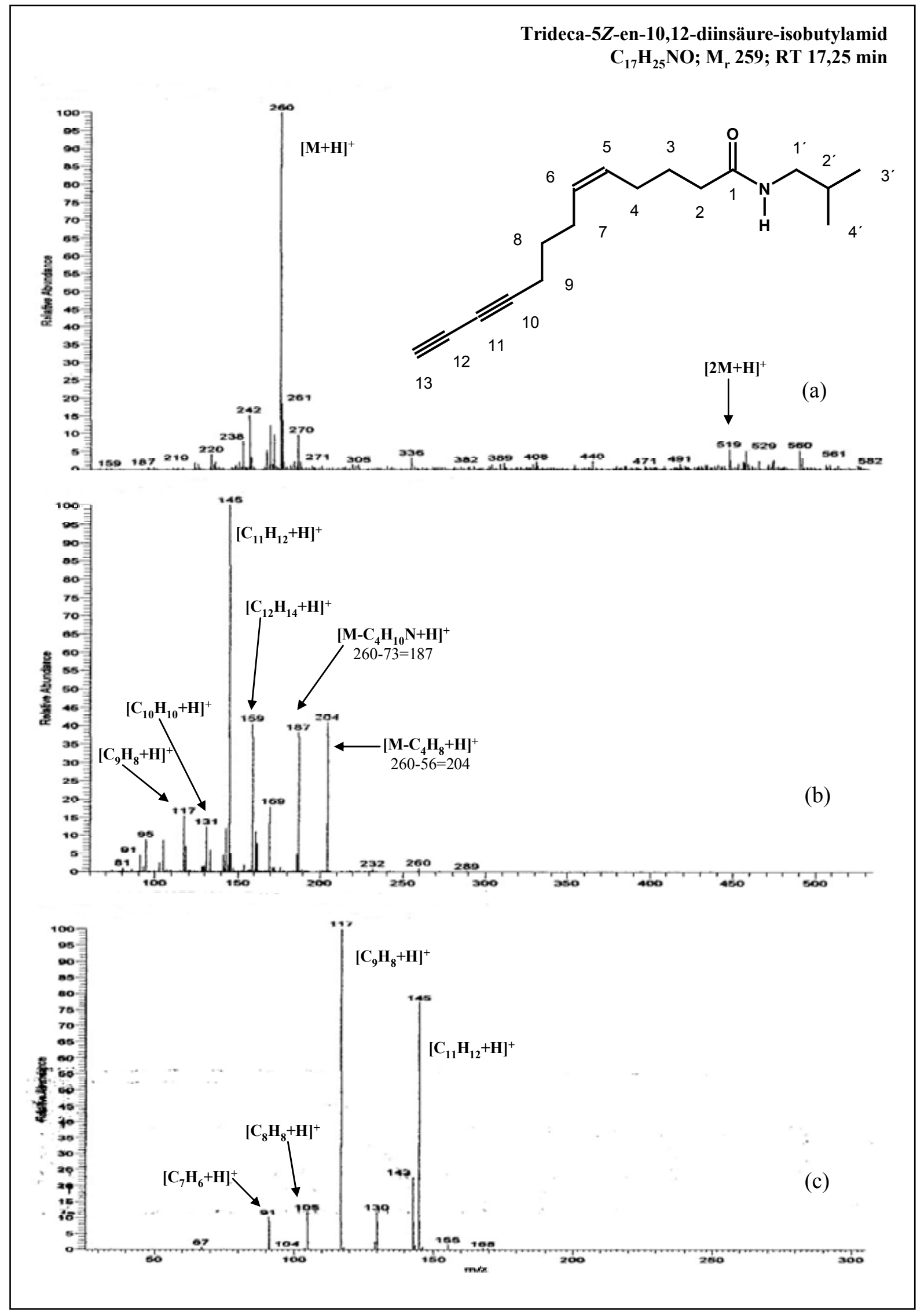

Abb. 31(a) - (c): +ESI-MS / MS $^{2} /$ MS $^{3}$-Spektren von Verbindung $A 14$ 
Verbindung $\boldsymbol{A 1 4}$ aus Sammelfraktion 3 weist große Gemeinsamkeiten auf mit der als Verbindung 4 (Trideca-7Z-en-10,12-diinsäure-isobutylamid) von NAKATANI und NAGASHIMA [134] beschriebenen Substanz, die aus „Spilanthes acmella L. var. oleracea“ isoliert wurde.

In Übereinstimmung mit den Literaturdaten (Messmethode aber EI-MS) fallen zunächst die Bruchstücke $\left[\mathrm{M}-\mathrm{C}_{4} \mathrm{H}_{10} \mathrm{~N}+\mathrm{H}\right]^{+} \mathrm{m} / \mathrm{z}$ 187, hervorgegangen aus der Amidspaltung, und $\left[\mathrm{M}-\mathrm{C}_{4} \mathrm{H}_{8}+\mathrm{H}\right]^{+} \mathrm{m} / \mathrm{z} 204$ (Abb. 31(b)) aus der Spaltung im Isobutylamid-Rest zwischen dem Stickstoff und C-1' auf. Der abgespaltene Kohlenwasserstoffrest ist als Fragment m/z 159 zu sehen. Innerhalb dieses Rests kommt es zur Abspaltung von je einer weiteren $-\mathrm{CH}_{2}$-Einheit, repräsentiert durch die Massen 145, 131, 117 (Abb. 31b)).

Die Massenzahl der nächsten beiden Fragmente 105 und 91 als $[\mathrm{M}+\mathrm{H}]^{+}$ist um 1 höher als für die Literaturverbindung, wo die Massen 103 und 89 auftraten. Auch das beschriebene Fragment m/z 63 [134] taucht hier nicht auf. Somit muss sich rein rechnerisch im vorliegenden Fall die Doppelbindung bereits zwischen C-5 und C-6 befinden, was für einen neuen Naturstoff spricht.

Die Formulierung als Z-Doppelbindung wurde in Anlehnung an die Literatur [134] vorgenommen. Mit Blick auf die Biogenese der Alkamide, die partiell aus der Fettsäure Linolensäure hervorgehen (s. 3.3.1.1.), scheint die Z-Bindung an dieser Stelle am wahrscheinlichsten.

Das Dimer mit der Masse 519 als $[\mathrm{M}+\mathrm{H}]^{+}$ist in Abb. 31(a) zu sehen.

\subsubsection{Hydrospilanthol (Deca-6Z,8E-diensäure-isobutylamid) (Verbindung $A 15$ )}

Bei Verbindung $\boldsymbol{A 1 5}$ aus Sammelfraktion 3 dürfte es sich um das bereits in Acmella ciliata nachgewiesene Hydrospilanthol (Deca-6Z,8E-diensäure-isobutylamid) [110, 111] handeln, wofür die Molmasse von 223 spricht.

Die Substanz erscheint als sehr stabil; denn ihre Fragmentation erweist sich als äußerst gering, und selbst im $\mathrm{MS}^{3}$-Spektrum (Abb. 32(c)) ist noch der Molpeak präsent. Ein Zusammenhang zwischen der langen Methylenbrücke und dem daher großen Abstand des ungesättigten Endes zur Amidgruppe, als verantwortlich für die große Stabilität, erscheint ungewöhnlich. 
Hydrospilanthol (Deca-6Z,8E-diensäure-isobutylamid) $\mathrm{C}_{14} \mathrm{H}_{25} \mathrm{NO} ; \mathrm{M}_{\mathrm{r}} 223$; RT 17,96 min
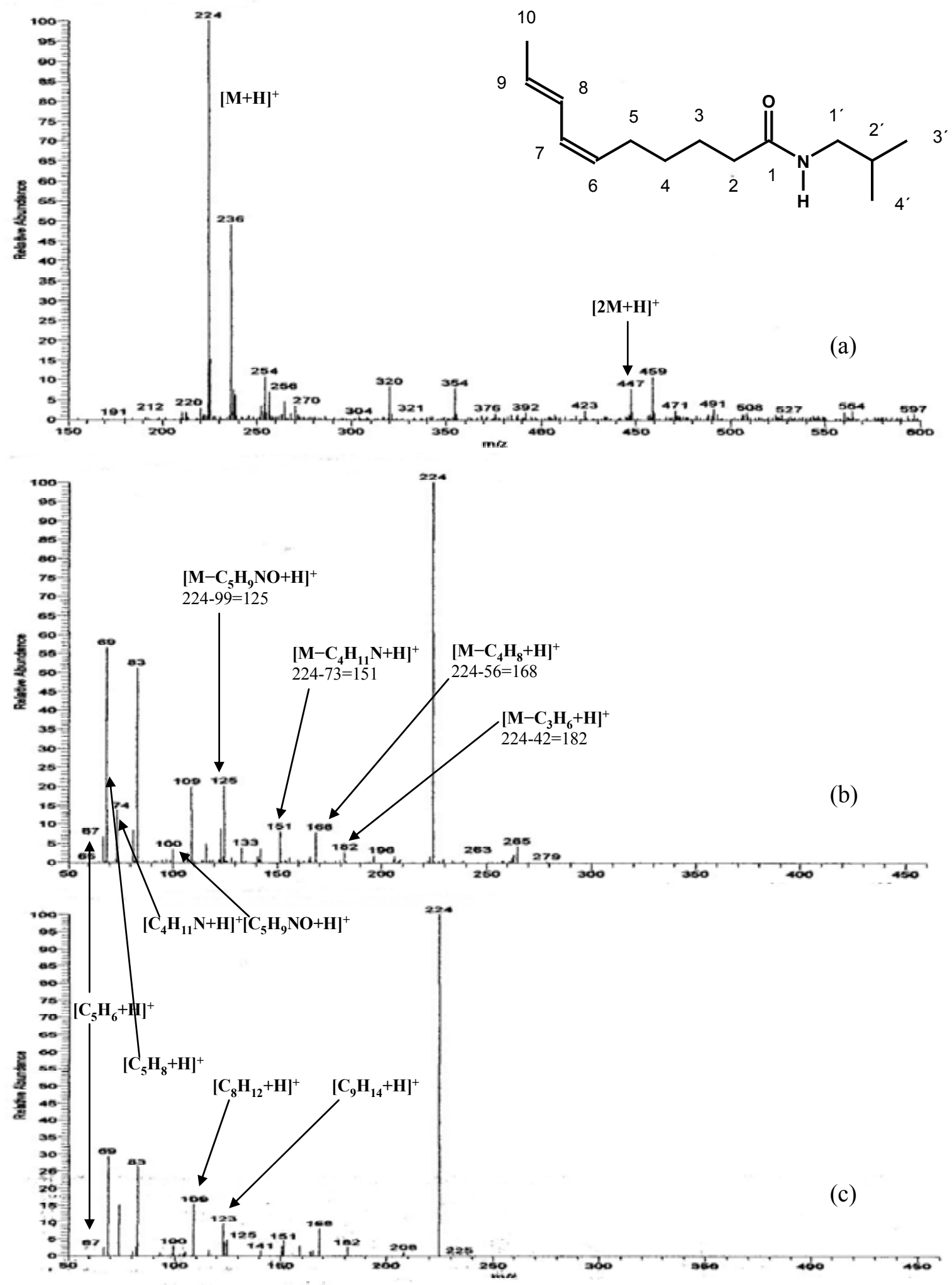

Abb. 32(a) - (c): +ESI-MS / MS ${ }^{2} / \mathrm{MS}^{3}$-Spektren von Verbindung $A 15$ 
Durch das weitgehende Fehlen der Möglichkeit zur Mesomerie-Stabilisierung wäre eher der umgekehrte Sachverhalt logisch. Allerdings spricht WOELKART [181] von einer geringeren Bereitschaft zum Fragmentieren bei zunehmender Lipophilie des Alkamids. Die diskutierte Verbindung Hydrospilanthol weist durch das Fehlen einer Doppelbindung in C-2/C-3Position relativ hohe Lipophilie auf.

Die dennoch erkennbaren Fragmente unterstützen die Strukturvermutung Hydrospilanthol. Der Isobutylamid-Rest wird durch die Fragmente mit den Massenzahlen 125, 151, 168 und 182 eindeutig definiert (Abb. 32b)).

Abb. 32(c) stellt Spaltfragmente im linken Molekülteil dar, die hier, ebenso wie im vorher beschriebenen Spilanthol (A13), allerdings mit einer um 2 größeren Massenzahl durch die zwei zusätzlichen Protonen am C-2 und C-3, auftreten. So repräsentiert das Fragment m/z 125 $\left[\mathrm{C}_{9} \mathrm{H}_{16}+\mathrm{H}\right]^{+}$den Kohlenwasserstoffrest nach Amid-Abspaltung und das Fragment m/z 123 $\left[\mathrm{C}_{9} \mathrm{H}_{14}+\mathrm{H}\right]^{+}$denselben Rest unter Abgabe von zwei weiteren Protonen.

Die Fragmente $\left[\mathrm{C}_{5} \mathrm{H}_{8}+\mathrm{H}\right]^{+}$m/z 69 bzw., nach Protonenabgabe, m/z $67\left(\left[\mathrm{C}_{5} \mathrm{H}_{6}+\mathrm{H}\right]^{+}\right)$dürften die Spaltung zwischen C-5 und C-6 repräsentieren. Fragment $\left[\mathrm{C}_{6} \mathrm{H}_{10}+\mathrm{H}\right]^{+} \mathrm{m} / \mathrm{z} 83$ umfasst die Moleküle C-2 bis C-7 und ist um die Massenzahl zwei größer als das analoge Fragment beim Spilanthol (s. Abb. 30(b) und (c)). Das Fragment m/z $109\left[\mathrm{C}_{8} \mathrm{H}_{12}+\mathrm{H}\right]^{+}$kann durch Spaltung zwischen C-2 und C-3 entstehen und kommt vermutlich beim Spilanthol deshalb nicht vor, weil sich dort an dieser Stelle die stabilere Doppelbindung befindet (s. 3.3.3.14.).

Auch beim Hydrospilanthol lässt sich das Dimer $[2 \mathrm{M}+\mathrm{H}]^{+} \mathrm{m} / \mathrm{z} 447$ erkennen (Abb. 32(a)). Außerdem erscheint in derselben Abb. ein weiterer intensiver Molpeak mit der Masse 235, wobei es sich um ein Isomer von Verbindung $\boldsymbol{A 6}_{2}$, nachgewiesen in Sammelfraktion 2 , handeln könnte.

\subsubsection{Weitere Verbindungen aus Sammelfraktion 3}

Mit Retentionszeiten von 6,03 - 7,99 min treten im HPLC-MS-Spektrum vier Peaks mit der Molmasse 269 auf (Abb. 19). Im Ausgangsspektrum ist weiterhin eine Verbindung mit der Molmasse $251 \mathrm{zu}$ erkennen, die mit erstgenannter offensichtlich über ein Mol Wasser $(\mathrm{m} / \mathrm{z}$ 18) im Zusammenhang steht. 
Daneben sind die beiden Verbindungen mit den Massen 237 und 219 präsent, die voranstehend als Verbindungen $\mathbf{A 1 2}_{2}$ und $\mathbf{A 1 2}_{3}$ bezeichnet wurden. Diese tauchen in allen Full-ESI-MS-Spektren neben den Molpeaks der Substanzen mit m/z 269 auf. Zwischen diesen scheint es allerdings keinen einfach erkennbaren Zusammenhang zu geben. Im Verlauf der Fragmentation spielen aber die Massen 269 und 251 offensichtlich keine Rolle; denn die $\mathrm{MS}^{3}$ - und $\mathrm{MS}^{4}$-Spektren stimmen mit denen unter 3.3.3.13. beschriebenen überein. Konkrete Aussagen lassen sich jedoch nicht treffen. Auch die nachfolgenden vier, nicht sauber getrennten Molpeaks mit den Massen 506 und 508 sind nicht zu konkretisieren.

Bei der Verbindung mit der Retentionszeit 18,73 min und der Molmasse 289 (Abb. 19) scheint es sich um eine hydroxilierte Verbindung $\mathrm{zu}$ handeln, die komplett unter Wasserabspaltung in die Verbindung mit der Molmasse 271 übergeht. Dass sie trotz einer hydrophilen $\mathrm{OH}-\mathrm{Gruppe}$ eine lange Retentionszeit aufweist, ist über einen langen gesättigten Fettsäurerest, der dem Molekül eine relativ hohe Lipophilie verleihen dürfte, vorstellbar. Da auch hier die Fragmentation gering ist, fehlen Ansätze zur Strukturdiskussion. Als sicher anzunehmen ist dagegen ein Phenylethylamid-Rest, charakterisiert durch die typischen Bruchstücke mit den Massen 148, 122, 105 und 91 als $[\mathrm{M}+\mathrm{H}]^{+}$.

\subsubsection{Dodeca- $2 E, 4 E, 8 Z, 10 E$-tetraensäure-isobutylamid (,Tetraen“)}

(Verbindung $\boldsymbol{A 1 6}$ )

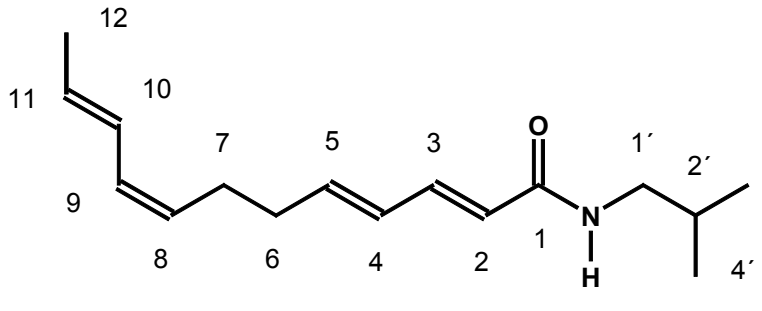

$\mathrm{C}_{16} \mathrm{H}_{25} \mathrm{NO} ; \mathrm{M}_{\mathrm{r}} 247$

Anders als bei MARTIN [110, 112], der außer dem 4E-Isomer auch das 4Z-Isomer dieser Verbindung nachwies, findet sich in Sammelfraktion 4 nur das $4 E$-Isomer. Das Hydroxyderivat dieser Verbindung (A3) wurde in Sammelfraktion 2 nachgewiesen (3.3.3.4.). Es eluierte eher von der RPSäule auf Grund seiner größeren Hydrophilie durch die OH-Gruppe.

Auch scheint bei Acmella ciliata nur das 10E-Isomer vorzuliegen, anders als bei Echinaceaund Asiasarum-Arten, wo stets das HPL-chromatographisch untrennbare 2E;4E,8Z,10E/ZIsomerengemisch gefunden wurde $[8,9,11,13,35,188]$. 
Die +ESI-MS ergab eine Molmasse von 248,2001 als [M+H] .

Tabelle 6: ${ }^{1} \mathrm{H}-\mathrm{NMR}$ von Dodeca-2E,4E,8Z,10E-tetraensäure-isobutylamid in $\mathrm{CD}_{3} \mathrm{OD}$

\begin{tabular}{|l|c|c|c|}
\hline H & $\begin{array}{c}\text { Verschiebung } \boldsymbol{\delta} \\
{[\mathrm{ppm}]}\end{array}$ & Signalform & $\begin{array}{c}\text { Kopplungskonst. J } \\
{[\mathrm{Hz}]}\end{array}$ \\
\hline 2 & & $\mathrm{~d}$ & 15,0 \\
2 & 5,93 & $\mathrm{dd}$ & 11,$0 ; 15,0$ \\
3 & 7,10 & $\mathrm{dd}$ & 11,$0 ; 15,0$ \\
4 & 6,22 & $\mathrm{dt}$ & 6,$0 ; 15,0$ \\
5 & 6,09 & $\mathrm{~m} /(2 \mathrm{H})$ & - \\
6 & 2,27 & $\mathrm{~m} /(2 \mathrm{H})$ & - \\
7 & 2,23 & $\mathrm{dt}$ & 11,$0 ; 7,0$ \\
8 & 5,25 & $\mathrm{dd}$ & 11,$0 ; *$ \\
9 & 5,95 & $\mathrm{dd}$ & 11,$0 ; 15,0$ \\
10 & 6,32 & $\mathrm{dq}$ & 15,$0 ; 7,0$ \\
11 & 5,67 & $\mathrm{~d} /(3 \mathrm{H})$ & 7,0 \\
12 & 1,76 & $\mathrm{~d}$ & 7,0 \\
$1^{\prime}$ & 3,06 & $\mathrm{~m}$ & - \\
$2^{\prime}$ & 1,78 & $\mathrm{~d} /(6 \mathrm{H})$ & 6,5 \\
$3^{\prime}, 4^{\prime}$ & 0,92 & \multicolumn{3}{c}{} \\
\hline
\end{tabular}

*: partiell überdeckt durch das Signal von $\mathrm{H}-2$

Das ${ }^{1} \mathrm{H}-\mathrm{NMR}-$ Spektrum (Tab. 6) zeigte gegenüber der in deuteriertem Chloroform gemessenen Referenz [35, 68, 112] geringe Verschiebungen, die jedoch die eindeutige Identifizierung dieser Substanz nicht verunsicherten. $\mathrm{Zu}$ beobachten ist im ${ }^{1} \mathrm{H}-\mathrm{NMR}$ wieder das typische Muster des Isobutyl-Rests.

H-2 hat mit H-3 nur einen Kopplungspartner und ergibt ein Dublett. H-3 ist, wie bei $\alpha-\beta-$ ungesättigten Carbonylfunktionen üblich, stärker zum Tieffeld verschoben. Neben H-2 koppelt es mit H-4 und stellt somit ein Dublettdublett dar.

H-3 und H-4 koppeln mit 11 Hz, während die Doppelbindungen zwischen C-2 und C-3 sowie C-4 und C-5 trans konfiguriert sind, was aus der Kopplungskonstanten von $15 \mathrm{~Hz}$ ersichtlich ist. Neben H-4 koppelt H-5 mit den zwei Protonen am C-6, und es ergibt sich als Signal ein Dubletttriplett. Auf Grund der Signalvielfalt der Protonen am C-6 und C-7 resultieren Kopplungsmuster höherer Ordnung. Daher werden diese Protonen als zwei Multipletts zusammengefasst.

Das Kopplungsmuster von H-8 ist identisch mit dem von H-5, wobei sich auch hier ein Dubletttriplett ergibt. Allerdings beträgt die Kopplungskonstante gegenüber H-9 nur $11 \mathrm{~Hz}$, was an dieser Stelle auf die cis-Doppelbindung verweist. H-9 und H-10 koppeln jeweils als 
Doppeldubletts mit $11 \mathrm{~Hz}$ miteinander, identisch mit H-3 und H-4. H-10 und H-11 koppeln wiederum mit $15 \mathrm{~Hz}$ miteinander, was auch hier die trans-Doppelbindung charakterisiert. Die drei Protonen der Methylgruppe am C-12 fallen in einem Signal zusammen und koppeln mit H-11 als Dublett mit 7 Hz.

\subsubsection{Resümee}

Entsprechend Tab. 7 wurde eine Reihe von Alkamiden $(\boldsymbol{A 1}$ - A16) im Dichlormethanextrakt der Gesamtpflanze von Acmella ciliata nachgewiesen und strukturanalytisch (HPLC/MS im +ESI-Modus, teilweise ${ }^{1} \mathrm{H}-\mathrm{NMR}$ ) untersucht. Die Kettenlänge der „olefinischen“ bzw. „acetylenisch-olefinischen“ Säurereste lag zwischen $\mathrm{C}_{9}$ und $\mathrm{C}_{13}$. Als Amidkomponenten traten dominant der Isobutylamid-, der Phenylethylamid-Rest in $\mathbf{A 2}, \boldsymbol{A 4}, \boldsymbol{A 8}$ und $\boldsymbol{A 9}$ und in A11 der Methylbutylamid-Rest auf.

$A 1,2,8,10,11,13,15$ und $\boldsymbol{A 1 6}$ konnten bereits von MARTIN [110 - 112] aus Blütenköpfchen von Acmella ciliata isoliert werden. $\mathbf{A 6}_{2}, \mathbf{7 ,} 9$ und 10 wurden in Acmella-Artverwandten nachgewiesen [144, 53, 132, 16, 17]. A1, 5, 10 und 16 wurden aus Echinacea-Arten [8, 9, 11, $35,181]$ isoliert.

Als neuer Naturstoff wurde in dieser Arbeit $A 14$ (Trideca-5Z-en-10,12-diinsäureisobutylamid) gefunden. $\boldsymbol{A 4}$ stellt ein neues Dihydroxyalkamid (2,4-Dihydroxy-nona-6,8diinsäure-isobutylamid) dar, das bei der +ESI-MS-Fragmentierung nach zweifacher Wasserabspaltung in Nona-2E/4E-en-diinsäure-isobutylamid übergeht.

Die Verbindungen $\boldsymbol{A 1 2}_{\boldsymbol{1}}$ (2,5-Dihydroxy-deca-6Z,8E-diensäure-isobutylamid) $\boldsymbol{A 1 2}_{2}$ (2Hydroxy-4E,6Z,8E-triensäure-isobutylamid) und $A_{12}$ (Deca-2E,4E,6Z,8E-tetraensäureisobutylamid) stehen ebenfalls über zwei Moleküle Wasser in Verbindung und wurden bisher als Naturstoffe nicht beschrieben.

$\boldsymbol{A 3}$ (7-Hydroxy-dodeca-2E,4E,8Z,10E-tetraensäure-isobutylamid) und $\boldsymbol{A 6}_{\boldsymbol{1}}$ (2-Hydroxyundeca-7Z,9E-diensäure-isobutylamid) stellen Monohydroxyverbindungen dar, die bei der +ESI-MS-Fragmentation relativ leicht Wasser abspalten und dabei zumindest intermediär vollständig ungesättigte Systeme bilden (vgl. Tafel II, Teil 2, Zusammenfassung), die bisher nur für Achillea spec. beschrieben wurden [54]. 
Tabelle 7: Acmella ciliata-Alkamide

\begin{tabular}{|c|c|c|c|c|c|c|c|}
\hline \multicolumn{4}{|c|}{ Säurerest } & \multirow[t]{2}{*}{ Aminrest } & \multirow[t]{2}{*}{ Verbindung } & \multirow{2}{*}{$\begin{array}{c}\text { Summen- } \\
\text { formel }\end{array}$} & \multirow[t]{2}{*}{ Molmasse } \\
\hline Kettenlänge & Doppel- & Dreifach- & Hydroxy- & & & & \\
\hline & bindungen & bindungen & Gruppen & & & & \\
\hline $\mathrm{C}=9$ & 1 & 2 & & Phenylethyl & $\mathrm{A} 2$ / A9 & $\mathrm{C}_{17} \mathrm{H}_{17} \mathrm{NO}$ & 251 \\
\hline $\mathrm{C}=9$ & & 2 & 2 & Phenylethyl & A4 & $\mathrm{C}_{17} \mathrm{H}_{19} \mathrm{NO}_{3}$ & 285 \\
\hline $\mathrm{C}=9$ & 1 & 2 & & Isobutyl & A7 & $\mathrm{C}_{13} \mathrm{H}_{17} \mathrm{NO}$ & 203 \\
\hline $\mathrm{C}=9$ & & 2 & Epoxy & Phenylethyl & A8 & $\mathrm{C}_{17} \mathrm{H}_{17} \mathrm{NO}_{2}$ & 267 \\
\hline$C=10$ & 2 & & 2 & Isobutyl & $\mathrm{A} 12(1)$ & $\mathrm{C}_{14} \mathrm{H}_{25} \mathrm{NO}_{3}$ & 255 \\
\hline $\mathrm{C}=10$ & 3 & & 1 & Isobutyl & $\mathrm{A} 12(2)$ & $\mathrm{C}_{14} \mathrm{H}_{23} \mathrm{NO}_{2}$ & 237 \\
\hline $\mathrm{C}=10$ & 4 & & & Isobutyl & A12(3) & $\mathrm{C}_{14} \mathrm{H}_{21} \mathrm{NO}$ & 219 \\
\hline$C=10$ & 3 & & & Isobutyl & A13 & $\mathrm{C}_{14} \mathrm{H}_{23} \mathrm{NO}$ & 221 \\
\hline $\mathrm{C}=10$ & 2 & & & Isobutyl & A15 & $\mathrm{C}_{14} \mathrm{H}_{25} \mathrm{NO}$ & 223 \\
\hline $\mathrm{C}=11$ & 2 & 2 & & Isobutyl & A1 & $\mathrm{C}_{15} \mathrm{H}_{19} \mathrm{NO}$ & 229 \\
\hline $\mathrm{C}=11$ & 2 & & 1 & Isobutyl & $\mathrm{A} 6(1)$ & $\mathrm{C}_{15} \mathrm{H}_{27} \mathrm{NO}_{2}$ & 253 \\
\hline $\mathrm{C}=11$ & 3 & & & Isobutyl & $\mathrm{A} 6(2)$ & $\mathrm{C}_{15} \mathrm{H}_{25} \mathrm{NO}$ & 235 \\
\hline $\mathrm{C}=11$ & 1 & 2 & & Isobutyl & A10 & $\mathrm{C}_{15} \mathrm{H}_{17} \mathrm{NO}$ & 231 \\
\hline $\mathrm{C}=11$ & 2 & 2 & & Methylbutyl & A11 & $\mathrm{C}_{16} \mathrm{H}_{21} \mathrm{NO}$ & 243 \\
\hline $\mathrm{C}=12$ & 4 & & 1 & Isobutyl & A3 & $\mathrm{C}_{16} \mathrm{H}_{25} \mathrm{NO}_{2}$ & 263 \\
\hline $\mathrm{C}=12$ & 1 & 2 & & Isobutyl & A5 & $\mathrm{C}_{16} \mathrm{H}_{23} \mathrm{NO}$ & 245 \\
\hline$C=12$ & 4 & & & Isobutyl & A16 & $\mathrm{C}_{16} \mathrm{H}_{25} \mathrm{NO}$ & 247 \\
\hline $\mathrm{C}=13$ & 1 & 2 & & Isobutyl & A14 & $\mathrm{C}_{17} \mathrm{H}_{25} \mathrm{NO}$ & 259 \\
\hline
\end{tabular}

Wie unter 3.3.3.12. und 3.3.3.16. dargestellt, zeigte das HPLC-MS-Spektrum darüber hinaus noch weitere Peaks, deren Strukturaufklärung jedoch nur partiell erfolgen konnte, da weder ausreichende Substanzproben noch Vergleichsdaten aus der Literatur zur Verfügung standen. Darüber hinaus fanden sich auch im Methanolextrakt von Acmella ciliata, der im Folgenden zur Charakterisierung der phenolischen Substanzen (3.4. ff) diente, noch alkamidische Verbindungen. Hierbei handelt es sich um Amide von Dicarbonsäuren (s. unter 3.5.2.), die eine wesentlich höhere Polarität aufweisen. 


\subsection{Phenolische Verbindungen}

\subsubsection{Allgemeines}

Die ubiquitär im Pflanzenreich vorkommenden phenolischen Verbindungen lassen sich in pharmazeutisch bedeutsame Inhaltsstoffklassen unterteilen. Hierzu zählen die Polyketide mit z. B. den Flavonoiden, die Gerbstoffe, Anthracenverbindungen, die Phenylpropanderivate sowie die sog. Abbauprodukte von Phenylpropanderivaten. Gemeinsam sind den Phenolen ihre desinfizierenden, d. h., antibakteriellen und z. T. virustatischen Eigenschaften. Im Folgenden soll kurz auf diejenigen Inhaltsstoffklassen näher eingegangen werden, aus denen einige Verbindungen in Acmella ciliata nachgewiesen wurden (3.4.3.).

Die Phenylpropanderivate entstehen biogenetisch auf dem Shikimat-Weg durch Verknüpfung von Erythrose-4-phosphat und Phosphoenolpyruvat und anschließende Zyklisierung. Nach Hinzutritt eines weiteren Moleküls Phosphoenolpyruvat und Decarboxylierung entstehen über verschiedene Zwischenstufen p-Hydroxy- und Phenylbrenztraubensäure, aus denen durch einen Aminogruppendonator die Aminosäuren L-Phenylalanin bzw. L-Tyrosin hervorgehen. Durch enzymatische Katalyse können sich daraus die Phenylacrylsäuren Zimtsäure bzw. pCumarsäure bilden, die durch Modifizierung der Seitenkette und des Benzenrings zu weiteren Phenylpropanderivaten umgewandelt werden [172].

Intermediate dieses Biogenesewegs sind Ausgangsverbindungen weiterer Sekundärstoffe. Chinasäure entsteht beispielsweise aus 5-Dehydrochinasäure. Die Chinasäure fungiert neben Kaffeesäure als Baustein der Kaffeesäureester mit dem Trivialnamen Chlorogensäure. Die Phenylacrylsäure-Derivate werden teilweise auch als Zimt- bzw. Hydroxyzimtsäure-Derivate bezeichnet [156]. Freie Zimtsäure selbst sowie deren Derivate p-Cumarsäure und Kaffeesäure konnten in Acmella ciliata im Verlauf dieser Arbeit nachgewiesen werden.

Noch häufiger im Pflanzenreich sind „gebundene“ Hydroxyzimtsäuren, die als Ester und/oder als Glykoside vorkommen. So wurden u. a. die 5-Kaffeoylchinasäure (Chlorogensäure) sowie einige Hydroxyzimtsäuren, verestert mit der aliphatischen Hydroxysäure Äpfelsäure, gefunden (3.4.3.2.). Chlorogensäure steigert die Magensaftsekretion und die Darmmotilität. Die meisten Kaffeesäurederivate wirken choleretisch und weisen virustatische und antibakterielle Eigenschaften auf. Auf Grund reduzierender und radikalfangender Eigenschaften wirken Kaffeesäurederivate antioxidativ und entzündungshemmend. So werden 
beispielsweise Rosmarinblätter, mit einem hohen Gehalt des Depsids Rosmarinsäure, ein Additionsprodukt aus zwei Mol Kaffeesäure, zum Konservieren von Fleisch und fetten Speisen verwendet. Vermutlich kommt der Chlorogensäure in der Pflanze die Bedeutung eines Resistenzfaktors gegen den Befall von pathogenen Mikroorganismen zu [156, 172].

Eine besondere Bedeutung bezüglich der Anwendung eines Acmella ciliata-Extrakts im Präparat Spolera ${ }^{\circledR}$ ist offensichtlich der entzündungshemmenden Wirkung der Kaffeesäurederivate zuzuschreiben. Vermutlich führt der antioxidative Effekt zu einer Hemmung der Lipoxygenasen und somit zur Hemmung der Leukotrienbiosynthese [172].

Die ebenfalls zu den Phenylpropanen gehörenden Hydroxycumarine entstehen aus Hydroxyzimtsäuren durch Hydroxylierung des Benzenrings und anschließende Lactonbildung. Diese können verschiedene pharmakologische Eigenschaften aufweisen, von spasmolytischen, choleretischen, diuretischen und antibiotischen bis $\mathrm{zu}$ antiexsudativen Wirkungen [172]. In Acmella ciliata konnte im Folgenden das Hydroxycumarinderivat Aesculetin nachgewiesen werden. Bisher bekannt war das Scopoletin $[110,112]$.

$\mathrm{Zu}$ den Abbauprodukten von Phenylpropanderivaten, die durch Verkürzung der Seitenkette gebildet werden, zählen z. B. die Benzoesäure- und Benzaldehyd-Abkömmlinge. Erstere entstehen aus Phenylacrylsäuren durch $\beta$-Oxidation, Benzaldehyd und Derivate durch Hydrierung zu Phenylpropansäuren und Abspaltung eines $\mathrm{C}_{2}$-Körpers. Die Benzaldehydderivate können auch durch Dehydrierung in Benzoesäuren überführt werden. Auf diesem Wege entsteht z. B. aus p-Cumarsäure p-Hydroxybenzoesäure und aus Kaffeesäure Protocatechusäure [172]. Diese letztgenannten vier Phenolcarbonsäuren konnten ebenfalls in Acmella ciliata nachgewiesen werden.

Die Benzaldehydderivate sind in ätherischen Ölen anzutreffen. Hiervon ist besonders Vanillin von pharmazeutischem Interesse, da es als antioxidativ wirksames Geruchs- und Geschmackskorrigens eingesetzt wird. Vanillin wird oft von weiteren Aromastoffen, wie pHydroxybenzaldehyd, begleitet. Aus einem Dichlormethanextrakt von Acmella ciliata konnte auch hier Vanillin neben p-Hydroxybenzaldehyd isoliert werden.

Die Flavonoide, die zu den Polyketiden gezählt werden, entstehen biogenetisch durch schrittweise Kondensation von drei Molekülen Malonyl-Coenzym A an p-Hydroxy- 
cinnamoyl-Coenzym A als Startermolekül über Zyklisierungen und Enolisierung. Aus dem primär gebildeten Isomerenpaar Chalkon/Flavanon gehen die anderen Flavonoidklassen durch Oxidation bzw. Reduktion hervor [156]. Die eigentlichen Flavonoide sind jedoch nur 4-Oxoflavanderivate, also Flavanone, Flavone oder Flavonole. Zur letztgenannten Klasse gehören auch die beiden isolierten Verbindungen Isoquercitrin und Rutosid.

Aus den vielfältigen chemischen Strukturen der Flavonoide ergibt sich eine Vielzahl von Wirkungen. Besonders antibiotische und antioxidative Eigenschaften spielen eine Rolle. Außerdem können verschiedene Enzymaktivitäten gehemmt werden, wie in vitro gezeigt wurde. Nicht alle Wirkungen ließen sich allerdings auf in vivo, insbesondere hinsichtlich systemischer Verfügbarkeit, übertragen, da die Bioverfügbarkeit der Flavonoide auf Grund des raschen Abbaus über Leber und Darm gering ist [172].

Flavonoide sind in der Lage, neben freien Radikalen auch zweiwertige Metallkationen unter Bildung von Chelatkomplexen abzufangen. Man spricht manchmal auch von Flavonoiden als Vitamin P. Dieser Vitamin-P-Effekt äußert sich in antiatherosklerotischen, antiexsudativen, entzündungshemmenden und radioprotektiven Eigenschaften sowie in einer Senkung von pathologisch erhöhter Permeabilität von Blutgefäßen. Als weitere Wirkungen einiger Flavonoide sind diuretische oder diaphoretische Effekte zu nennen oder auch die spezifische Wirkung des Rutosids und seiner Derivate bei chronischer Veneninsuffizienz (Anwendung als Venotonikum) [172].

Das breite Spektrum der beispielhaft aufgeführten Wirkqualitäten von phenolischen Verbindungen, die sich teilweise mit den traditionellen Anwendungen von Acmella ciliata decken [75, 81, 163, 164, 206], veranlasste in dieser Arbeit zur Untersuchung dieser Inhaltsstoffklasse neben den Alkamiden.

\subsubsection{Isolierung}

Zur Isolierung vorwiegend hydrophiler Inhaltsstoffe, insbesondere phenolischer Verbindungen, aus der getrockneten und grob gepulverten Gesamtpflanze von Acmella ciliata erwies sich Methanol als geeignetes Extraktionsmittel (Exp. Teil). Nach Soxhlet-Extraktion und Einengung der Auszüge im Vakuum resultierten insgesamt aus $1 \mathrm{~kg}$ Droge 91,5 g eines orange gefärbten, pulverförmigen Extrakts. 
Die DC auf Kieselgel-Platten $\mathrm{F}_{254}$ (Fließmittel: Ethylacetat/Ameisensäure/Eisessig/Wasser 100:11:11:27 (FM VII), Kammersättigung) dieses Extrakts und späterer Fraktionen aus der SC zeigte zahlreiche schwach bis intensiv blau - lila gefärbte eigenfluoreszierende Banden im UV-Licht bei $\lambda=366 \mathrm{~nm}$. Besprühen mit Naturstoffreagenz verstärkte die Fluoreszenz und ließ darüber hinaus einige gelb-orange gefärbte Banden erkennen.

Die weitere Aufarbeitung des Extrakts folgte dem Schema in Abb. 33. Zunächst wurden entsprechende Anteile des Extrakts mit einer Weinsäurelösung aufgenommen (Protonierung saurer und phenolischer Substanzen) und nacheinander mit Petrolether, Dichlormethan und Ethylacetat ausgeschüttelt (Exp.Teil). Während mit den ersten beiden Lösungsmitteln weitgehend Fette und andere apolare Bestandteile abgetrennt wurden, war im Ethylacetat mit einer Anreicherung phenolischer Substanzen zu rechnen. Die zur Trockne eingeengte Ethylacetat-Phase ergab einen Rückstand von $11 \mathrm{~g}$, der für den Trennungsgang zur Verfügung stand.

Zur weiteren Auftrennung wurden zwei verschiedene Wege eingeschlagen (Abb. 33). Weg 1 beinhaltete zunächst die Fraktionierung der Ethylacetat-Phase per SC an hängender Säule über Sephadex LH 20 [66] (Abb. 34), während Weg 2 auf die Vortrennungen per Schwerkraftsäule verzichtete und zur Auftrennung lediglich die Verfahren der HPLC anwendete (Abb. 65).

Das entsprechend Weg 1 zur SC eingesetzte Sephadex LH 20 ist ein hydroxypropyliertes Dextrangel, auf dem sowohl Adsorptions- und Verteilungsvorgänge als auch Fraktionierungen nach Molekülgrößen, vorwiegend polarer Substanzen, vonstattengehen. Dem Schema in Abb. 34 folgend, wurden vier Säulen nacheinander mit den jeweiligen, nach dc Kontrolle relevanten Fraktionen bzw. deren eingeengten Konzentraten beschickt und mit den Elutionsmitteln Methanol $>$ Aceton $>$ Ethylacetat (abnehmende Polarität: $\varepsilon_{25^{\circ} \mathrm{C}}=33,6>20,7>$ $6,02)$ fraktioniert.

In den Fraktionen 1-105 der Säule I (Tab. 35) waren dc zahlreiche, oft nur unzureichend getrennte orange und blau fluoreszierende Banden zu erkennen. Ab Fraktion 58 traten beim Stehen z. T. Niederschläge auf, die abgetrennt und vorerst nicht weiter untersucht wurden. Wegen des unbefriedigenden Trenneffekts gelangten die Filtrate des gesamten Eluats nach Einengen zur weiteren Fraktionierung auf Säule II (Aceton) und die ersten 41 Fraktionen 
davon anschließend auf Säule III (Ethylacetat). Interessant für die nachfolgende Strukturanalyse war ein durch Nachspülen von Säule I (s. Tab. 35) gewonnener „Gelber Nachlauf“, der zur Trockne einrotiert (Abtrennung sich bildender Niederschläge), anschließend DC-/ HPLC-analytisch untersucht und mittels präparativer HPLC aufgetrennt wurde.

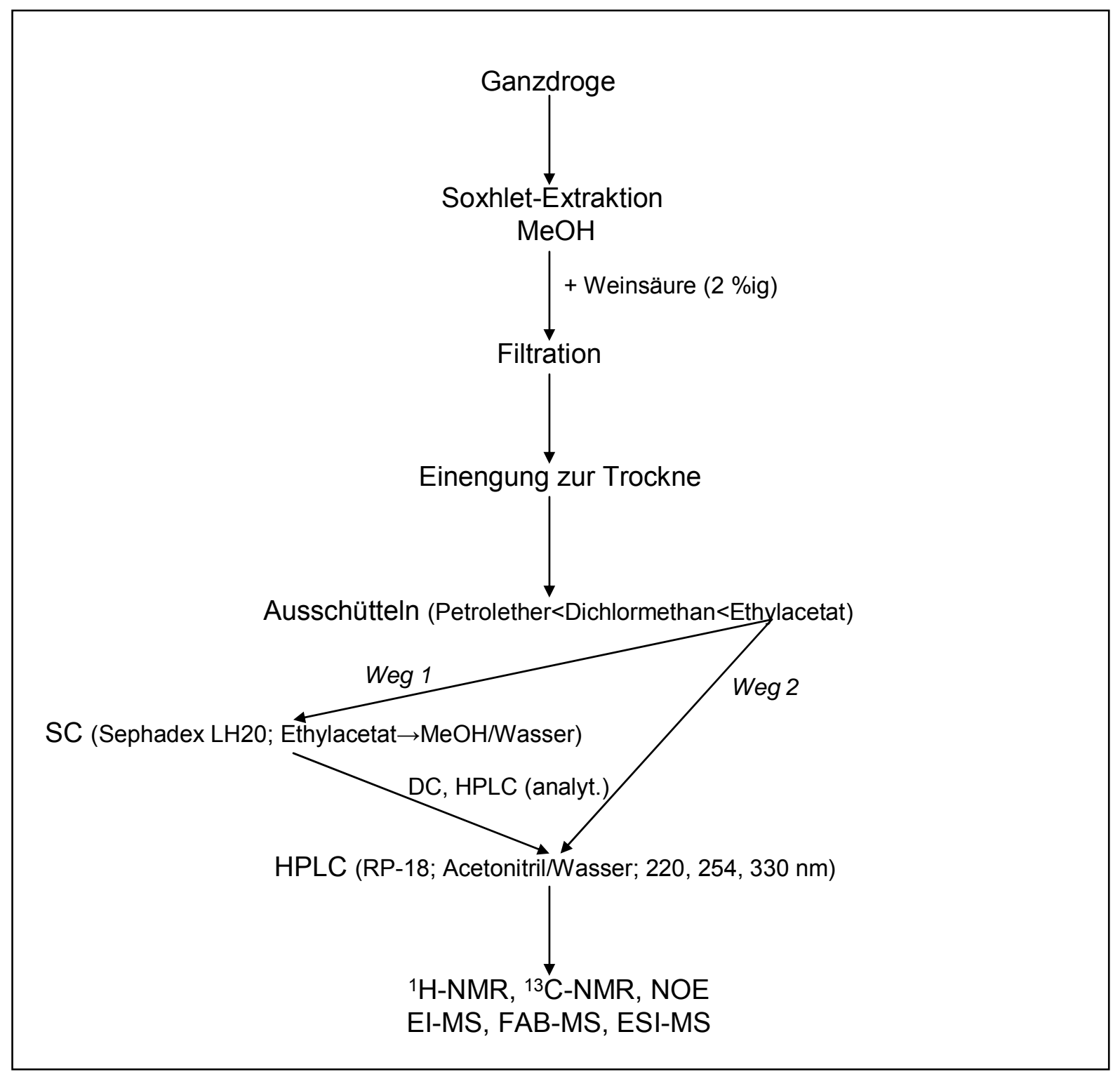

Abb. 33: Schema zur Isolierung und Strukturaufklärung phenolischer Verbindungen

Auf Säule III wurde nach den ersten 18 Fraktionen (Ethylacetat) 3mal das Elutionsmittel gewechselt (s. Tab. 35). Im Ergebnis paralleler DC-Auswertung erschien eine Vereinigung der nachfolgenden Fraktionen auf sechs Sammelfraktionen (III.1 - III.6, s. Abb. 34) als sinnvoll. Da jedoch in keiner dieser Sammelfraktionen Reinsubstanzen vorlagen, musste weiter aufgetrennt werden. Sammelfraktion III.1 wurde erneut sc fraktioniert (Säule IV) und anschließend per HPLC analysiert. 


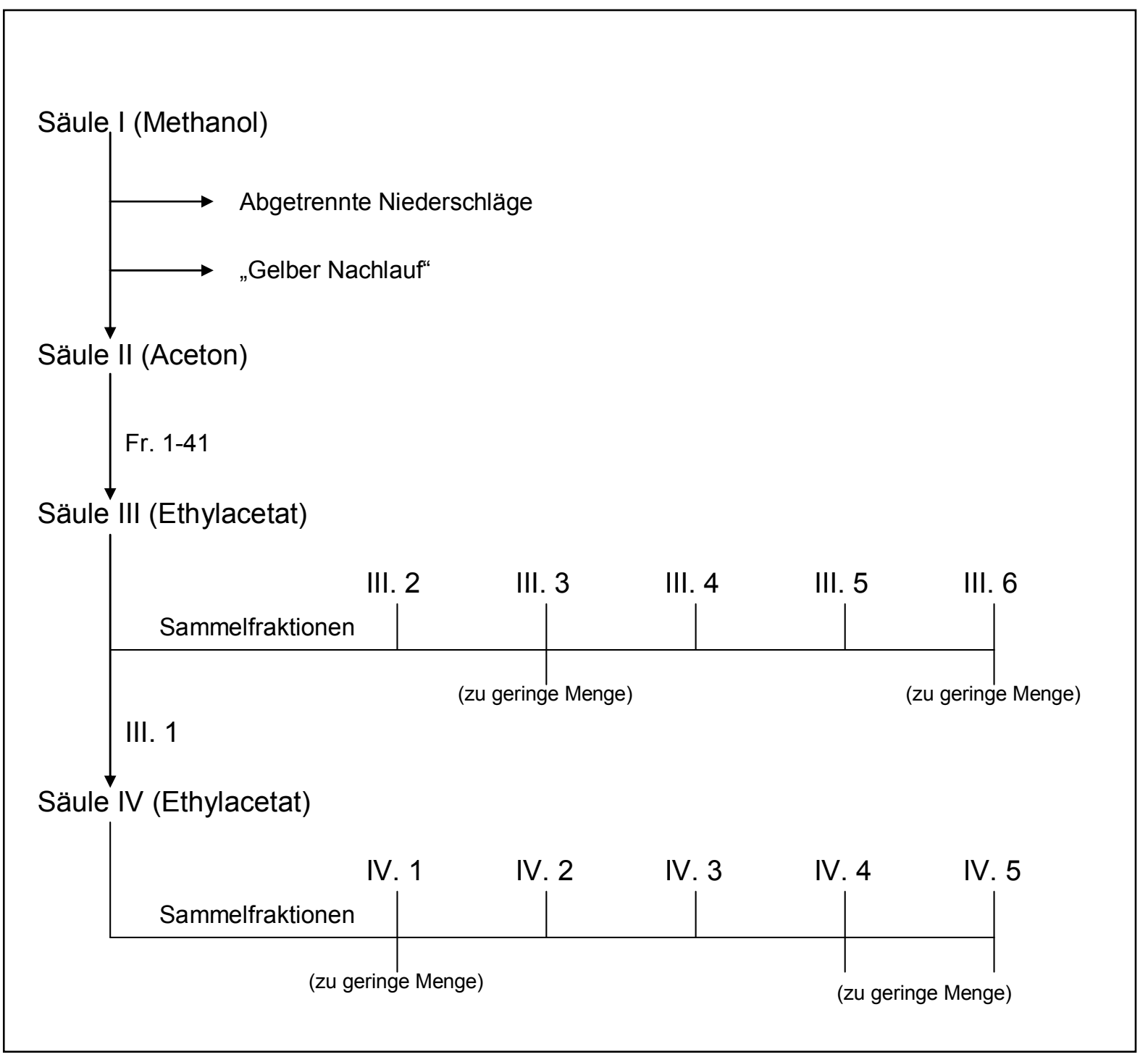

Abb. 34: Schema zur sc Fraktionierung der Ethylacetat-Phase (Weg 1)

Da jedoch auch Säule IV noch keine Reinsubstanzen erbrachte, wurde bei den Sammelfraktionen III.2 - III.5 (Säule III) von vornherein auf weitere Schwerkraftsäulen verzichtet. Vielmehr wurde hier, ebenso wie dann mit den Fraktionen der Säule IV (IV.2 und IV.3), direkt zum Einsatz der präparativen HPLC übergegangen (Abb. 62-64). Um die günstigsten Trennparameter festlegen zu können, erfolgte i. d. R. zuvor eine Untersuchung der Sammelfraktionen mittels analytischer HPLC. Durch den, im Gegensatz zur präparativen Anlage, in dieser Anlage vorhandenen DAD-Detektor, der die UV-Messung bei fünf verschiedenen Wellenlängen gleichzeitig ermöglichte (s. Exp. Teil), konnte die jeweils optimale Wellenlänge für die präparative Trennung ermittelt werden. Außerdem war es bereits möglich, aus den resultierenden Substanzpeaks und deren UV-Spektren auf Inhaltsstoff(e) bzw. -gruppen zu schließen. 
Die weitere Aufarbeitung der Ethylacetat-Phase entsprechend Weg 2 (vgl. Abb. 65) erfolgte direkt durch präparative HPLC.

\subsubsection{Strukturaufklärung}

Im Ergebnis der weiteren Auftrennung der aus Säule III und IV (Abb. 34) gewonnenen Sammelfraktionen erbrachte die präparative HPLC die in der nachfolgenden Übersicht (Abb. 35) aufgeführten phenolischen Verbindungen.

Eine spezifische Verteilung ist daraus nicht zu erkennen. Der überwiegende Anteil der Substanzen fiel bereits durch Elution mit Ethylacetat auf Säule III an und konnte von dort, nach z. T. weiterer HPLC-Fraktionierung (s. Schemata, Exp. Teil), isoliert und der Strukturaufklärung mittels UV-, ESI-MS- und NMR-Spektroskopie zugeführt werden.

Weiterhin benennt die Übersicht die aus dem sog. „Gelben Nachlauf“ (vgl. 3.4.2. und Abb. 66) und aus der Ethylacetatphase (Weg 2) durch präparative HPLC isolierten Verbindungen, wobei besonders die Ester P7, P8, P9, P10 anfielen. Das DC (Abb. 36) zeigt die chromatografische Verteilung der Verbindungen im Fließmittel VII.

\begin{tabular}{|c|c|c|c|c|}
\hline \multirow{2}{*}{$\begin{array}{l}\text { Säule III (Weg 1) } \\
\text { (Ethylacetat) } \\
\qquad \begin{array}{l}\text { III. } 1\end{array}\end{array}$} & III. 2 & III. 4 & III. 5 & \multirow{6}{*}{$\begin{array}{l}\text { P1: } \\
\text { P2:Hydroxybenzoesäure } \\
\text { P3: } \text { Protocatechusäure } \\
\text { P4: Kaffäure (cis/trans) } \\
\text { P5: Ferulääure } \\
\text { P6: Cumaroyläpfelsäureester (cis/trans) } \\
\text { P8: Feruloyläpfelsäureester (cis/trans) } \\
\text { P9: Kaffeoyläpfelsäure-1-methylester } \\
\text { P10: Kaffeoyläpfelsäure-4-methylester } \\
\text { P11: Chlorogensäure (cis/trans) } \\
\text { P12: 5-Kaffeoylepichinasäuremethylester } \\
\text { P13: Isoquercitrin } \\
\text { P14: Rutosid } \\
\text { P15: Aesculetin } \\
\text { P16: Matairesinol }\end{array}$} \\
\hline & $\begin{array}{l}\text { P2 } \\
\text { P8 } \\
\text { P15 }\end{array}$ & $\begin{array}{l}\text { P6 } \\
\text { P8 } \\
\text { P16 }\end{array}$ & $\begin{array}{l}\text { P11 } \\
\text { P13 } \\
\text { P14 }\end{array}$ & \\
\hline \multirow{2}{*}{\begin{tabular}{l}
\multicolumn{1}{c}{} \\
Säule IV \\
(Ethylacetat)
\end{tabular}} & IV. 2 & \multicolumn{2}{|l|}{ IV. 3} & \\
\hline & $\begin{array}{l}\text { P1 } \\
\text { P3 } \\
\text { P15 }\end{array}$ & \multicolumn{2}{|l|}{ P8 } & \\
\hline $\begin{array}{l}\text { Gelber Nachlauf } \\
\text { (von Säule I, } \\
\text { Methanol) }\end{array}$ & \multicolumn{3}{|c|}{$\begin{array}{c}\text { P5; P6; P7; P8; P11, P12, P13, } \\
\text { Flavonoide (s. 3.4.3.3.3.) }\end{array}$} & \\
\hline $\begin{array}{l}\text { Ethylacetat- } \\
\text { phase (Weg 2) }\end{array}$ & \multicolumn{3}{|c|}{ P4; P5; P7; P8; P9; P10, P11, P12 } & \\
\hline
\end{tabular}




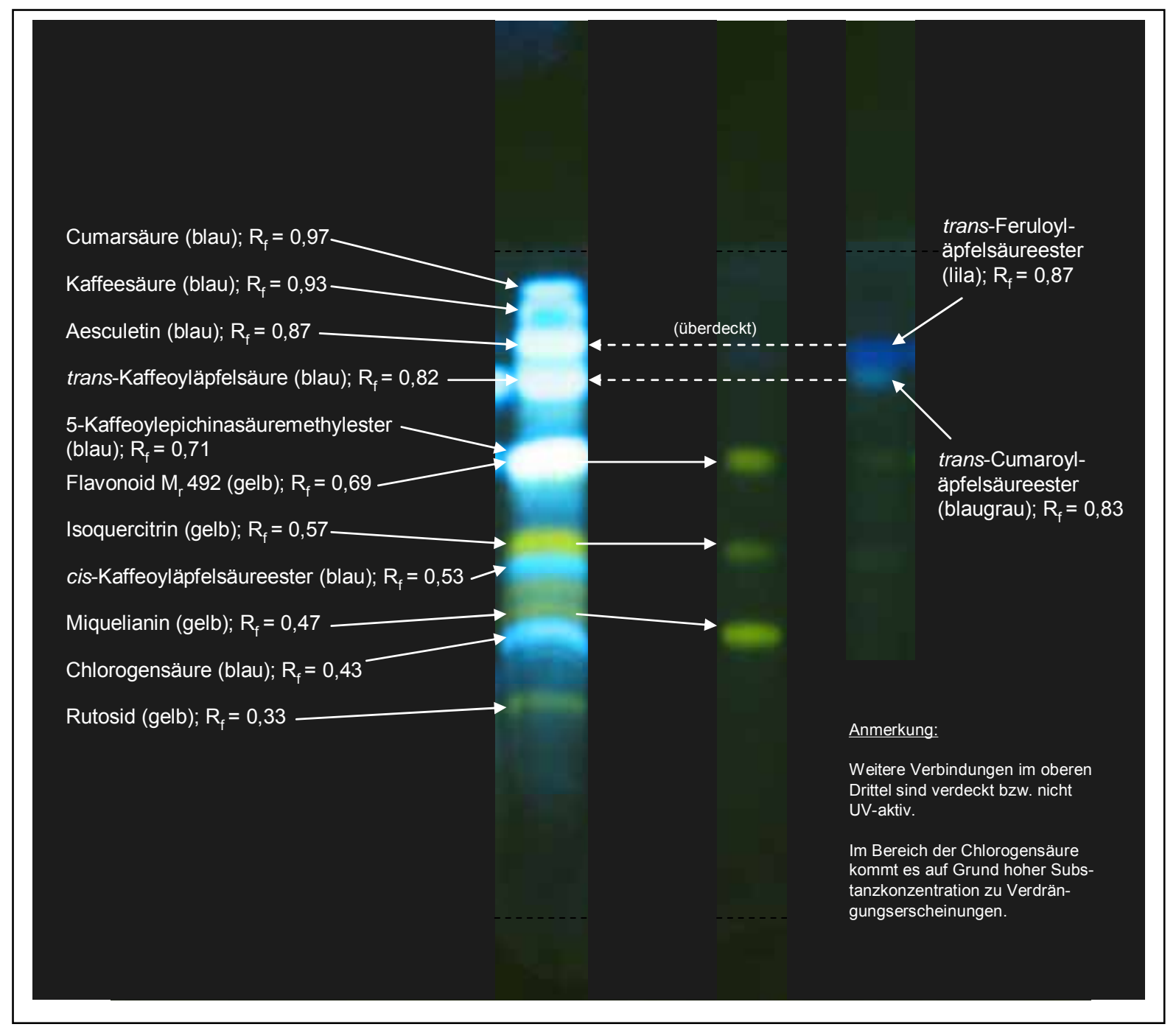

Abb. 36: DC-Verteilung der phenolischen Verbindungen (aus Ethylacetatphase, Abb. 33) im Fließmittel VII; Detektion: Naturstoff-Reagenz / UV 366 nm

Im Folgenden werden die einzelnen Verbindungen detailliert beschrieben.

\subsubsection{Phenol-Monocarbonsäuren}

\subsubsection{1. p-Hydroxybenzoesäure (Verbindung P1)}

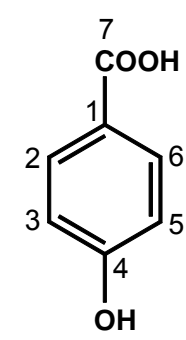

$\mathrm{C}_{7} \mathrm{H}_{6} \mathrm{O}_{3}$ $\mathrm{M}_{\mathbf{r}} 138$
Tabelle 8: ${ }^{1} \mathrm{H}$-NMR-Daten von p-Hydroxybenzoesäure in $\mathrm{CD}_{3} \mathrm{OD}$

\begin{tabular}{|l|c|c|c|}
\hline $\mathbf{H}$ & $\begin{array}{c}\text { Verschiebung } \boldsymbol{\delta} \\
{[\mathrm{ppm}]}\end{array}$ & Signalform & $\begin{array}{c}\text { Kopplungskonst. J } \\
{[\mathrm{Hz}]}\end{array}$ \\
\hline & & & 8,6 \\
2 & 7,87 & $\mathrm{~d}$ & 8,6 \\
3 & 6,81 & $\mathrm{~d}$ & 8,6 \\
5 & 6,81 & $\mathrm{~d}$ & 8,6 \\
6 & 7,87 & $\mathrm{~d}$ & \\
\hline
\end{tabular}


Das ${ }^{1}$ H-NMR (Tab. 8) dieser einfachsten Phenolcarbonsäure, die nach präparativer HPLC in den Fraktionen der Säule IV (Abb. 35) aufgefunden wurde, zeigt deutlich zwei Signale als Dubletts des 1,4-substituierten Aromaten. Zwei gegenüberliegende Signale fallen jeweils zusammen, da es sich hier um ein symmetrisch substituiertes Molekül handelt. Das Dublett für H-2/H-6 ist stärker zum Tieffeld verschoben als das Signal H-3/H-5, was durch den -MEffekt der benachbarten Carboxylfunktion zu erklären ist. Diese Daten sind mit Messungen in Deuterochloroform vergleichbar [72, 109].

Die -ESI-MS-Messung lieferte die Molmasse 137,0226 als $[\mathrm{M}-\mathrm{H}]^{-}$und die +ESI-Messung die Molmasse 161,0259 als $[\mathrm{M}+\mathrm{Na}]^{+}$.

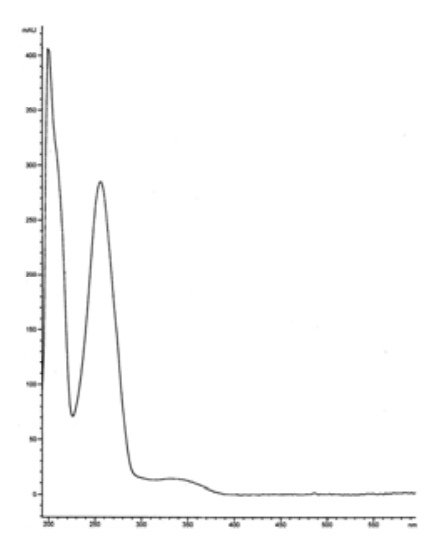

Abb. 37 zeigt das UV-Spektrum von p-Hydroxybenzoesäure mit einem Maximum bei $256 \mathrm{~nm}$.

Abb. 37: UV-Spektrum von p-Hydroxybenzoesäure

\subsection{Protocatechusäure (Verbindung P2)}<smiles>O=C(O)c1ccc(O)c(O)c1</smiles>

$\mathrm{C}_{7} \mathrm{H}_{6} \mathrm{O}_{4}$ $\mathrm{M}_{\mathbf{r}} 154$
Tabelle 9: ${ }^{1} \mathrm{H}-\mathrm{NMR}$ Daten von Protocatechusäure in $\mathrm{CD}_{3} \mathrm{OD}$

\begin{tabular}{|l|c|c|c|}
\hline H & $\begin{array}{c}\text { Verschiebung } \boldsymbol{\delta} \\
{[\mathrm{ppm}]}\end{array}$ & Signalform & $\begin{array}{c}\text { Kopplungskonst. J } \\
{[\mathrm{Hz}]}\end{array}$ \\
\hline & & & 2,0 \\
2 & 7,43 & $\mathrm{~d}$ & 8,5 \\
5 & 6,79 & $\mathrm{~d}$ & 2,$0 ; 8,5$ \\
6 & 7,42 & $\mathrm{dd}$ & \\
\hline
\end{tabular}

Die Protocatechusäure leitet sich von dem zweiwertigen Phenol Brenzcatechin ab. Ihre Struktur konnte mittels ${ }^{1} \mathrm{H}-\mathrm{NMR}$ bestätigt werden. Im NMR-Spektrum (Tab. 9) ist das sog. ABX-Sytem eines tri-substituierten Aromaten ersichtlich. Das Proton am C-6 koppelt mit C-5 mit einer Kopplungskonstanten von $8,5 \mathrm{~Hz}$ sowie mit dem gegenüberliegenden Proton am C2 mit 2,0 Hz. Das Signal stellt somit theoretisch ein Doppeldublett dar, aber nur ein Dublett 
ist zu erkennen. Hier ist zunächst die Kopplungkonstante von 2,0 Hz der Metakopplung zu H2 ersichtlich. Das Signal von H-2 überlagert einen Teil des Doppeldubletts von H-6, so dass die große Kopplungskonstante von 8,5 Hz mit dem Kopplungspartner H-5, also dem Dublett bei 6,79 ppm, zu entnehmen ist. Das Signal von H-2 seinerseits ist nicht aufgelöst und ergibt ein breites Singulett. Tatsächlich stellt dieses Signal den zweiten Kopplungspartner von H-6 dar, mit dem es theoretisch ebenfalls mit ca. $2 \mathrm{~Hz}$ koppelt. Damit wäre das Signal bei optimaler Auflösung als Dublett zu erkennen (s. Tab. 9). Diese Fakten stimmen mit der unter gleichen Bedingungen vermessenen Protocatechusäure-Referenz sowie mit Literaturangaben [44] überein.

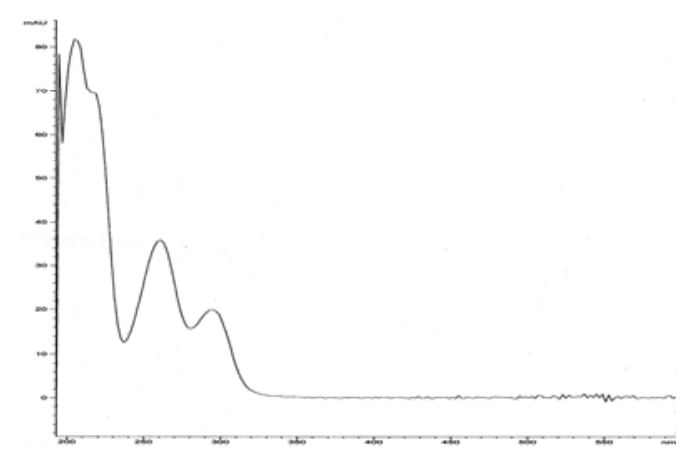

Die Molmasse von 153,0223 als $[\mathrm{M}-\mathrm{H}]^{-}$wurde durch die -ESI-MS-Messung bestätigt.

Die UV-Maxima (s. Abb. 38) liegen bei 262 und 294 nm. Des Weiteren ist eine Schulter bei $216 \mathrm{~nm}$ ersichtlich.

Abb. 38: UV-Spektrum der Protocatechusäure

\subsubsection{3. cis-/trans-p-Cumarsäure (Verbindung P3)}<smiles>CCCCCCCCCCCCC(=O)O</smiles>

Die p-Cumarsäure wurde in HPLC-Fraktionen der Säule IV sowie in der Ethylacetat-Phase (Weg 2) (Abb. 35) nachgewiesen und mittels ${ }^{1} \mathrm{H}-\mathrm{NMR}$ und ESI-MS analysiert.

Im NMR-Spektrum (Tab. 10) fallen auf Grund des symmetrischen Substitutionsmusters die Signale für die Protonen H-2 und H-6 sowie H-3 und H-5 zusammen. Diese Paare koppeln als Dublett jeweils mit den benachbarten Protonen mit 8,6 bzw. 8,8 Hz. Die Signalformen von cis- und trans-Isomer sind identisch. Die Werte [ppm] für die Verschiebungen weisen geringe 
Abweichungen auf (vgl. Tab. 10). Die NMR-Daten stimmen mit einer unter gleichen Bedingungen vermessenen authentischen Referenzsubstanz überein.

Tabelle 10: ${ }^{1} \mathrm{H}$-NMR Daten von cis- und trans-p-Cumarsäure in $\mathrm{CD}_{3} \mathrm{OD}$

\begin{tabular}{|l|c|c|c|c|c|}
\hline H & \multicolumn{2}{|c|}{$\begin{array}{c}\text { Verschiebung } \boldsymbol{\delta} \\
{[\mathrm{ppm}]}\end{array}$} & Signalform & \multicolumn{2}{|c|}{$\begin{array}{c}\text { Kopplungskonst. J } \\
{[\mathrm{Hz}]}\end{array}$} \\
\cline { 2 - 3 } \cline { 5 - 6 } & cis & trans & & & \multicolumn{2}{|c|}{ cis } & trans \\
\hline & & & & & \\
2 & 7,61 & 7,45 & $\mathrm{~d}$ & 8,8 & 8,6 \\
3 & 6,74 & 6,8 & $\mathrm{~d}$ & 8,6 & 8,6 \\
5 & 6,74 & 6,8 & $\mathrm{~d}$ & 8,6 & 8,6 \\
6 & 7,61 & 7,45 & $\mathrm{~d}$ & 8,8 & 8,6 \\
7 & 6,82 & 7,6 & $\mathrm{~d}$ & 12,8 & 15,9 \\
8 & 5,76 & 6,28 & $\mathrm{~d}$ & 12,8 & 15,9 \\
\hline
\end{tabular}

Bei der cis-Form koppeln H-7 und H-8 miteinander (Dublett) mit 12,8 Hz. Demgegenüber ist dieses Dublett bei der trans-Form mit $15,9 \mathrm{~Hz}$ ausgewiesen, was der typischen Kopplungskonstanten einer derartigen trans-Doppelbindung entspricht. Die Abweichungen von der trans-Form sind analog zum cis-Cumarsäurerest beim Cumaroyläpfelsäureester (s. 3.4.3.2.1.).

Die Molmasse ergab 163,0280 als [M-H] ${ }^{-}$im -ESI-Modus sowie 187,0370 als $[\mathrm{M}+\mathrm{Na}]^{+}$im + ESI-Modus.

Die UV-Spektren der cis- und trans-Form unterscheiden sich partiell in der relativen Intensität ihrer Maxima. Die Lage der Maxima ist weitgehend identisch (Maxima cis: 227, (303 sh), 308 nm; trans: 223, (298 sh), 308 nm; s. Abb. 39).

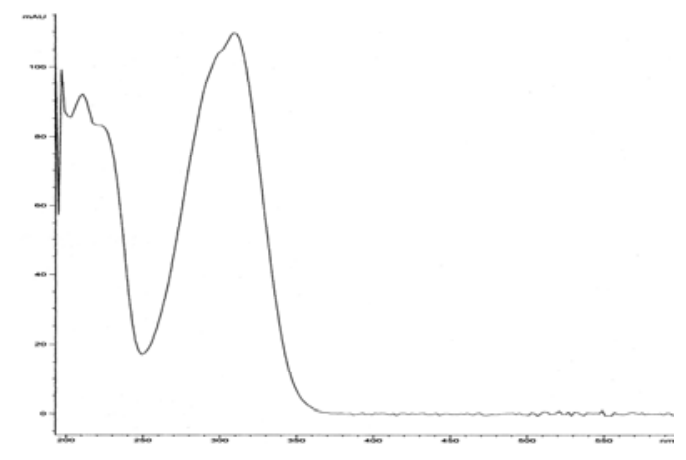

Abb. 39:

UV-Spektren von cis-und trans-pCumarsäure

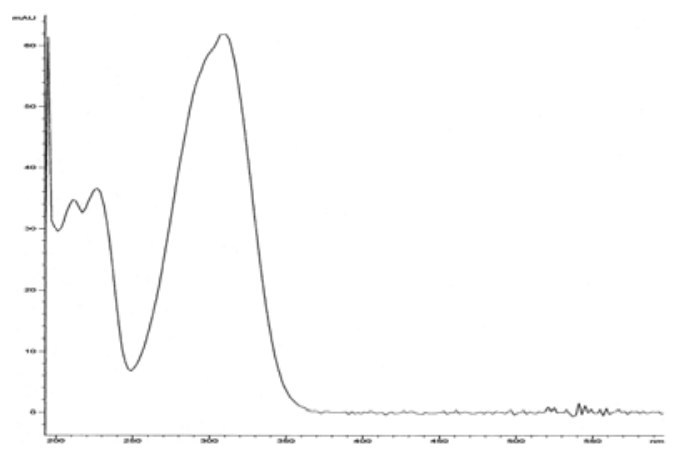


<smiles>O=C(O)C=Cc1ccc(O)c(O)c1</smiles>

Tabelle 11: ${ }^{1} \mathrm{H}-\mathrm{NMR}$ Daten von trans-Kaffeesäure in $\mathrm{CD}_{3} \mathrm{OD}$

\begin{tabular}{|l|c|c|c|}
\hline H & $\begin{array}{c}\text { Verschiebung } \boldsymbol{\delta} \\
{[\mathrm{ppm}]}\end{array}$ & Signalform & $\begin{array}{c}\text { Kopplungskonst. J } \\
{[\mathrm{Hz}]}\end{array}$ \\
\hline 2 & 7,03 & $\mathrm{~d}$ & 2,0 \\
5 & 6,77 & $\mathrm{~d}$ & 8,0 \\
6 & 6,93 & $\mathrm{dd}$ & 2,$0 ; 8,0$ \\
7 & 7,53 & $\mathrm{~d}$ & 16,0 \\
8 & 6,21 & $\mathrm{~d}$ & 16,0 \\
\hline
\end{tabular}

Kaffeesäure wurde aus der Ethylacetat-Phase (Weg 2) mittels präparativer HPLC isoliert, allerdings lediglich die trans-Form. Sie zeichnet sich strukturell gegenüber Cumarsäure durch eine zusätzliche Hydroxylgruppe in 3-Stellung am Aromaten aus.

Die Daten der ${ }^{1}$ H-NMR-Analyse dokumentiert Tab. 11. Dem NMR-Spektrum ist zu entnehmen, dass die Protonen am C-5 und C-6 miteinander mit 8 Hz koppeln. H-5 hat keinen weiteren Kopplungspartner und erscheint als Dublett. H-6 koppelt außerdem mit der kleinen Kopplungskonstanten von $2 \mathrm{~Hz}$ mit H-2 und erscheint daher als Doppeldublett. H-2 koppelt nur mit 2 Hz mit H-6 und zeigt daher ebenfalls ein Dublett. H-7 und H-8 koppeln als Dublett nur miteinander mit $16 \mathrm{~Hz}$, woraus der trans-Charakter der Doppelbindung ersichtlich wird.

Die zugehörige Masse lieferte die -ESI-MS mit 179,0402 als [M-H] ${ }^{-}$sowie die +ESI-MSMessung mit 203,0343 als [M+Na] $]^{+}$

Die ermittelten Daten stimmen mit der unter gleichen Bedingungen vermessenen Referenzsubstanz überein.

\subsection{Ferulasäure (Verbindung P5)}<smiles>COc1cc(/C=C/C(=O)O)ccc1O</smiles>

Ferulasäure tritt, ebenso wie Cumarsäure und Kaffeesäure, als Begleitsubstanz der nachfolgend besprochenen Äpfelsäureester (P6 - P8) auf. Letztere beiden waren aber bei der HPLCIsolierung auch allein $\mathrm{zu}$ finden, nicht nur unmittelbar 
zusammen mit ihren Estern, und offensichtlich in größerer Quantität als die Ferulasäure. Gemeinsam ist diesen drei Säuren, dass sie alle entweder Biosynthesebausteine für die nachfolgenden Ester oder deren Abbauprodukte darstellen.

Die -ESI-MS Messung lieferte den Molpeak der Ferulasäure von 193 als $[\mathrm{M}-\mathrm{H}]^{-}$neben dem des Esters (P8).

\subsubsection{Phenol-Monocarbonsäureester}

Von den oben beschriebenen Phenolcarbonsäuren Cumar-, Kaffee- und Ferulasäure wurden in Acmella ciliata Ester mit Äpfelsäure gefunden, in denen die Carboxylfunktion der betreffenden Phenolcarbonsäure mit der-OH-Gruppe der aliphatischen Dicarbonsäure verknüpft ist. Neben den Estern tritt auch die freie Äpfelsäure (Monohydroxydicarbonsäure; $S 8$; s. 3.5.2.4.) auf.

Äpfelsäureester sind bisher in der Familie der Asteraceae (Korbblütler) nicht beschrieben worden. Lediglich für vier Species wurde über deren Vorkommen berichtet [20, 58, 59, 104, 136]: Brassicaceae (Kreuzblütler: Raphanus sativus L., Brassica rapa L.), Fumariaceae (Erdrauchgewächse: Fumaria officinalis L.) sowie Papaveraceae (Mohngewächse: Chelidonium majus L. (hier nur Kaffeoyläpfelsäureester)). Hierzu existieren einige NMRDaten, auf die Bezug genommen werden konnte. Zusammen mit den eigenen Daten der ${ }^{1} \mathrm{H}-$ NMR und der hochauflösenden ESI-Massenspektrometrie, war es möglich, das Vorkommen dieser Ester in Acmella ciliata zu bestätigen.

\subsubsection{1. cis-/trans-p-Cumaroyläpfelsäureester (Verbindung P6)}<smiles>CCCCCCCCCCCCCCCCCC(=O)OC(=O)C=Cc1ccc(O)cc1</smiles><smiles>O=C(O)CC(CC(=O)O)OC(=O)/C=C/c1ccc(O)cc1</smiles>

Zur Strukturaufklärung dieser Verbindung, von der sowohl das cis- als auch das trans-Isomer per präparativer HPLC in III. 4 (Abb. 35) nachgewiesen wurde, ist auf die vorhandene 
Literaturangabe [104] zurückgegriffen worden, woraus sich Übereinstimmung mit den eigenen ermittelten ${ }^{1} \mathrm{H}-\mathrm{NMR}-\mathrm{Daten}$ (Tab. 12) ergab.

Tabelle 12: ${ }^{1} \mathrm{H}-\mathrm{NMR}$ Daten von cis- und trans-Cumaroyläpfelsäureester in $\mathrm{CD}_{3} \mathrm{OD}$

\begin{tabular}{|l|c|c|c|c|c|c|}
\hline H & \multicolumn{2}{|c|}{$\begin{array}{c}\text { Verschiebung } \boldsymbol{\delta} \\
{[\mathrm{ppm}]}\end{array}$} & \multicolumn{2}{c|}{ Signalform } & \multicolumn{2}{c|}{$\begin{array}{c}\text { Kopplungskonst. J } \\
\text { [Hz] }\end{array}$} \\
\cline { 2 - 7 } & cis & trans & cis & trans & cis & trans \\
\hline & & & & & & \\
$2^{\prime}$ & 7,67 & 7,48 & $\mathrm{~d}$ & $\mathrm{~d}$ & 8,8 & 8,7 \\
$3^{\prime}$ & 6,74 & 6,81 & $\mathrm{~d}$ & $\mathrm{~d}$ & 8,8 & 8,7 \\
$5^{\prime}$ & 6,74 & 6,81 & $\mathrm{~d}$ & $\mathrm{~d}$ & 8,8 & 8,7 \\
$6^{\prime}$ & 7,67 & 7,48 & $\mathrm{~d}$ & $\mathrm{~d}$ & 8,8 & 8,7 \\
$7^{\prime}$ & 6,92 & 7,66 & $\mathrm{~d}$ & $\mathrm{~d}$ & 12,8 & 15,9 \\
$8^{\prime}$ & 5,81 & 6,38 & $\mathrm{~d}$ & $\mathrm{~d}$ & 12,8 & 15,9 \\
$2^{\prime}$ & 5,44 & 5,48 & $\mathrm{dd}$ & $\mathrm{dd}$ & 4,$0 ; 9,0$ & 4,$2 ; 8,3$ \\
$3 \mathrm{a}$ & $\mathrm{x}$ & 2,83 & $\mathrm{x}$ & $\mathrm{dd}$ & $\mathrm{x}$ & 8,$3 ; 16,7$ \\
$3 \mathrm{~b}$ & $\mathrm{x}$ & 2,95 & $\mathrm{x}$ & $\mathrm{dd}$ & $\mathrm{x}$ & 4,$2 ; 16,7$ \\
\hline
\end{tabular}

Wie bei allen 1,4- und damit symmetrisch substituierten Aromaten fallen hier jeweils die Protonen von H-2' und H-6' sowie H-3'und H-5'zusammen zu Signalen doppelter Signalintensität. Diese Paare koppeln mit ihren Nachbarn nur als Dublett mit jeweils 8,7 bzw. 8,8 Hz.

Für das cis- und trans-Isomer resultierten bei gleichen Signalformen leicht unterschiedliche ppm-Werte und geringe Abweichungen der Kopplungskonstanten (s. Tab. 12; vgl. auch Cumarsäure, 3.4.3.1.3.).

Die trans-Doppelbindung wird durch die Kopplungskonstante von 15,9 Hz auf H-7' und H-8' festgelegt. Im cis-Isomer koppeln die Protonen $\mathrm{H}^{-} 7^{\prime}$ und $\mathrm{H}-8^{\prime}$ mit der typischen Kopplungskonstanten von $12,8 \mathrm{~Hz}$ miteinander. Die Signale für H-3a und H-3b des cisIsomers sind stärker durch das intensivere trans-Isomer überlagert, da hier die Verschiebungen beider Isomere fast identisch sind. Diese Werte fehlen daher in Tab. 12 und sind dort mit , $\mathrm{X}^{“}$ gekennzeichnet.

Die hier gefundenen Verschiebungen und Signale sind weitgehend auch mit denen des im Folgenden beschriebenen Kaffeoyl- sowie Feruloyläpfelsäureesters identisch.

Die ESI-MS-Messungen bestätigen die Strukturanalyse. Aus der -ESI-Messung geht m/z 279,0526 als $[\mathrm{M}-\mathrm{H}]^{-}$hervor; im +ESI-Modus zeigt sich ein schwaches Signal mit m/z 
303,0482 als $[\mathrm{M}+\mathrm{Na}]^{+}$. Für die massenspektrometrischen Untersuchungen wurde die folgende Vergleichsliteratur herangezogen: $[20,58,104,136]$.

\subsubsection{2. cis-/trans-Kaffeoyläpfelsäureester (Verbindung P7)}<smiles>O=C(O)CC(CC(=O)O)OC(=O)C=Cc1ccc(O)c(O)c1</smiles><smiles></smiles><smiles>O=C(O)CC(CC(=O)O)OC(=O)/C=C/c1ccc(O)c(O)c1</smiles>

Die Äpfelsäureester der Kaffeesäure fanden sich im „Gelben Nachlauf“ und der EthylacetatPhase (Weg 2) (s. Abb. 35 und Abb. 67, Exp. Teil).

Die Molmasse für Kaffeoyläpfelsäureester wurde massenspektrometrisch als m/z 295,0448 als $[\mathrm{M}-\mathrm{H}]^{-}$per - ESI-MS sowie als $\mathrm{m} / \mathrm{z} 319,0436$ als $[\mathrm{M}+\mathrm{Na}]^{+}$und 335,0100 als $[\mathrm{M}+\mathrm{K}]^{+}$per +ESI-MS ermittelt und mit der Literatur [20, 58, 59, 104, 136] verglichen.

Tabelle 13: ${ }^{1} \mathrm{H}-\mathrm{NMR}$-Daten von cis-/trans-Kaffeoyläpfelsäureester in $\mathrm{CD}_{3} \mathrm{OD}$

\begin{tabular}{|l|c|c|c|c|c|c|}
\hline \multirow{2}{*}{ H } & \multicolumn{2}{|c|}{$\begin{array}{c}\text { Verschiebung } \boldsymbol{C} \\
{[\mathrm{ppm}]}\end{array}$} & \multicolumn{2}{c|}{ Signalform } & \multicolumn{2}{c|}{$\begin{array}{c}\text { Kopplungskonst. J } \\
{[\mathrm{Hz}]}\end{array}$} \\
\cline { 2 - 7 } & cis & trans & cis & trans & cis & trans \\
\hline & & & & & & \\
$2^{\prime}$ & 7,45 & 7,05 & $\mathrm{~d}$ & $\mathrm{~d}$ & 2,0 & 2,0 \\
$5^{\prime}$ & 6,72 & 6,78 & $\mathrm{~d}$ & $\mathrm{~d}$ & 8,0 & 8,2 \\
$6^{\prime}$ & 7,04 & 6,96 & $\mathrm{dd}$ & $\mathrm{dd}$ & 2,$0 ; 8,0$ & 2,$0 ; 8,2$ \\
$7^{\prime}$ & 6,85 & 7,59 & $\mathrm{~d}$ & $\mathrm{~d}$ & 12,9 & 15,9 \\
$8^{\prime}$ & 5,75 & 6,31 & $\mathrm{~d}$ & $\mathrm{~d}$ & 12,9 & 15,9 \\
2 & 5,44 & 5,47 & $\mathrm{dd}$ & $\mathrm{dd}$ & 4,$2 ; 8,3$ & 4,$2 ; 8,3$ \\
$3 \mathrm{a}$ & $\mathrm{x}$ & 2,88 & $\mathrm{x}$ & $\mathrm{dd}$ & $\mathrm{x}$ & 8,$3 ; 16,7$ \\
$3 \mathrm{~b}$ & $\mathrm{x}$ & 2,96 & $\mathrm{x}$ & $\mathrm{dd}$ & $\mathrm{x}$ & 4,$2 ; 16,7$ \\
\hline
\end{tabular}

Bei der Strukturaufklärung mittels ${ }^{1} \mathrm{H}-\mathrm{NMR}$ (Tab. 13) ergaben sich Übereinstimmungen mit den Literaturdaten [104]. Das Kopplungsmuster im Aromatenteil entspricht dem aller 1,3,4substituierten Benzene. So koppeln zunächst H-5' und H-6' mit 8 bzw. 8,2 Hz miteinander. H-5' hat keinen weiteren Kopplungspartner und erscheint daher als Dublett. H-6' stellt ein Doppeldublett dar, da es außerdem mit dem am Ring in meta-Stellung befindlichen Proton H$2^{\prime}$ mit ca. 2 Hz koppelt. Diese kleine Kopplungskonstante ist oft ungenau aufgelöst und daher 
schlecht zu erkennen. H-2' hat ebenfalls nur H-6' als Kopplungspartner und erscheint folglich als Dublett. Die ppm-Werte für die beiden Isomeren zeigen auch hier wieder geringe Abweichungen (vgl. Tab. 13).

Die Kopplungskonstante von 15,9 Hz steht für die als Dublett koppelnden Protonen H-7' und H-8 in der trans-Form, während die cis-Form wiederum eine Konstante von $12,9 \mathrm{~Hz}$ aufweist. Die stärkere Tieffeldverschiebung von H-7' wird durch die an dieser Stelle vorhandene relative Elektronenarmut bedingt, hervorgerufen durch den -M-Effekt der Carbonylfunktion.

Die Protonen am C-3 des trans-Isomeren koppeln miteinander mit 16,7 Hz, jedoch mit unterschiedlichen Kopplungskonstanten $(4,2 \mathrm{~Hz}$ und 8,3 Hz) mit $\mathrm{H}-2$, welches wiederum nur mit den Protonen H-3a und H-3b koppelt. Somit erscheinen diese drei Protonen sämtlich als Doppeldublett (s. Tab. 13). Die Signale des cis-Isomeren für die Protonen am C-3 sind gegenüber dem trans-Isomer nur minimal verschoben, aber schwächer in der Intensität. Zur Erklärung der in Tab. 13 mit „,x“ gekennzeichneten Zellen siehe 3.4.3.2.1.

\subsubsection{3. cis-/trans-Feruloyläpfelsäureester (Verbindung P8)}<smiles>COc1cc(/C=C\C(=O)OC(CC(=O)O)C(=O)O)ccc1O</smiles><smiles>[14CH3]O[14CH3]</smiles><smiles>COc1cc(/C=C/C(=O)OC(CC(=O)O)C(=O)O)ccc1O</smiles>

Da es sich beim Feruloyläpfelsäureester ebenfalls um einen 1,3,4-substituierten Aromaten handelt und die NMR-Signale im Säureteil ohnehin identisch sind, ergibt sich ein dem Kaffeoyläpfelsäureester (P7) analoges Bild bezüglich der Signale und Kopplungskonstanten, mit kleinen Abweichungen in den ppm-Werten.

Als Charakteristikum für die Methoxygruppe erscheint bei 3,89 ppm ein Singulett, das für die drei Protonen dieser Gruppe in Position 3' des Aromaten steht. Die Position der Methoxygruppe an dieser Stelle des Moleküls konnte im NOE-Verfahren bewiesen werden (Abb. 40). 

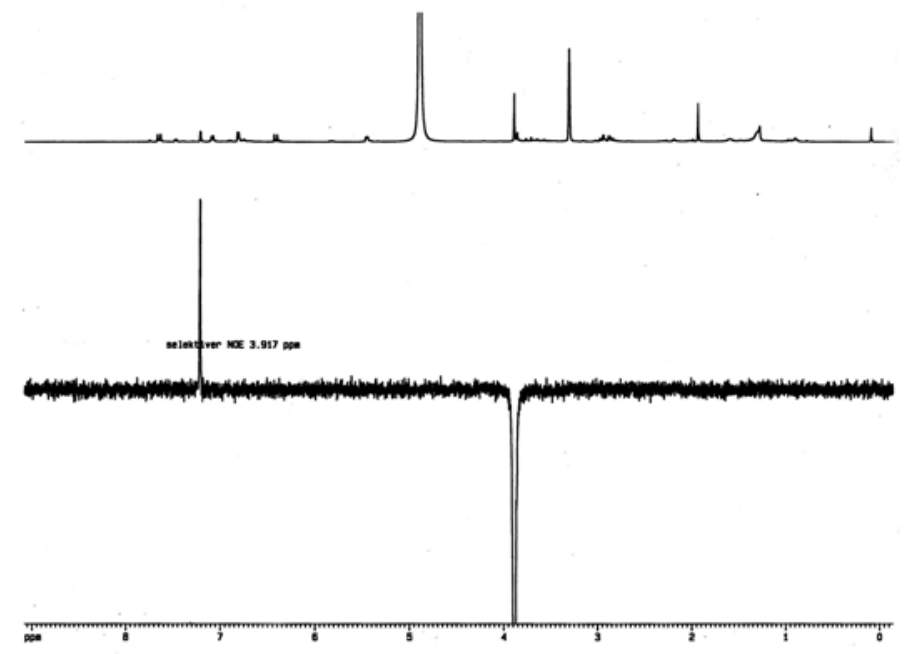

Abb. 40: Selektiver NOE 3,917 ppm von

Feruloyläpfelsäureester
Bei der NMR-Analyse des transIsomers in Aceton- $\mathrm{d}_{6}$ (Tab. 15) treten gegenüber dem Spektrum in $\mathrm{CD}_{3} \mathrm{OD}$ (Tab. 14) bezüglich der Signalformen keine Veränderungen auf, abgesehen von minimalen ppm-Wert-Unterschieden und kleinen Differenzen bei den Kopplungskonstanten.

Tabelle 14: ${ }^{1} \mathrm{H}$-NMR Daten des cis-/trans-Feruloyläpfelsäureesters in $\mathrm{CD}_{3} \mathrm{OD}$

\begin{tabular}{|l|l|c|c|c|c|c|}
\hline H & \multicolumn{2}{|c|}{$\begin{array}{c}\text { Verschiebung } \boldsymbol{\delta} \\
\text { [ppm] }\end{array}$} & \multicolumn{2}{c|}{ Signalform } & \multicolumn{2}{c|}{$\begin{array}{c}\text { Kopplungskonst. J } \\
{[\mathrm{Hz}]}\end{array}$} \\
\cline { 2 - 7 } & $\mathrm{cis}$ & trans & cis & trans & cis & trans \\
\hline $2^{\prime}$ & 7,76 & 7,21 & $\mathrm{~d}$ & $\mathrm{~d}$ & 2,0 & 2,0 \\
$3^{\prime}\left(-\mathrm{OCH}_{3}\right)$ & 3,86 & 3,89 & $\mathrm{~s}$ & $\mathrm{~d}$ & - & - \\
$5^{\prime}$ & 6,76 & 6,81 & $\mathrm{~d}$ & $\mathrm{~d}$ & 8,2 & 8,2 \\
$6^{\prime}$ & 7,11 & 7,09 & $\mathrm{dd}$ & $\mathrm{dd}$ & 2,$0 ; 8,2$ & 2,$0 ; 8,2$ \\
$7^{\prime}$ & 6,91 & 7,66 & $\mathrm{~d}$ & $\mathrm{~d}$ & 12,8 & 15,9 \\
$8^{\prime}$ & 5,83 & 6,43 & $\mathrm{~d}$ & $\mathrm{~d}$ & 12,8 & 15,9 \\
2 & 5,44 & 5,46 & $\mathrm{dd}$ & $\mathrm{dd}$ & 4,$2 ; 8,3$ & 4,$2 ; 8,3$ \\
$3 \mathrm{a}$ & $\mathrm{x}$ & 2,88 & $\mathrm{x}$ & $\mathrm{dd}$ & $\mathrm{x}$ & 8,$3 ; 16,7$ \\
$3 \mathrm{~b}$ & $\mathrm{x}$ & 2,96 & $\mathrm{x}$ & $\mathrm{dd}$ & $\mathrm{x}$ & 4,$2 ; 16,7$ \\
\hline
\end{tabular}

Tabelle 15: ${ }^{1} \mathrm{H}-\mathrm{NMR}$ Daten von trans-Feruloyläpfelsäureester in Aceton- $\mathrm{d}_{6}$

\begin{tabular}{|l|c|c|c|}
\hline H & $\begin{array}{c}\text { Verschiebung } \boldsymbol{\delta} \\
{[\mathrm{ppm}]}\end{array}$ & Signalform & $\begin{array}{c}\text { Kopplungskonst. J } \\
{[\mathrm{Hz}]}\end{array}$ \\
\hline $2^{\prime}$ & 7,4 & $\mathrm{~d}$ & 1,3 \\
$3^{\prime}\left(-\mathrm{OCH}_{3}\right)$ & 3,88 & $\mathrm{~s}$ & - \\
$5^{\prime}$ & 6,88 & $\mathrm{~d}$ & 8,0 \\
$6^{\prime}$ & 7,17 & $\mathrm{dd}$ & 1,$3 ; 8,0$ \\
$7^{\prime}$ & 7,66 & $\mathrm{~d}$ & 15,9 \\
$8^{\prime}$ & 6,46 & $\mathrm{~d}$ & 15,9 \\
$2^{\prime}$ & 5,54 & $\mathrm{dd}$ & 3,$9 ; 8,7$ \\
$3 \mathrm{a}$ & 2,91 & $\mathrm{dd}$ & 8,$7 ; 16,7$ \\
$3 \mathrm{~b}$ & 3,03 & $\mathrm{dd}$ & 3,$9 ; 16,7$ \\
\hline
\end{tabular}

Die vorhandenen Literaturdaten [104] beschreiben nur die Vermessung in deuteriertem Methanol. Da die Substanz aber zunächst in Aceton- $\mathrm{d}_{6}$ vermessen wurde, kann nunmehr ein 
Datenvergleich der Tab. 14 und 15 die Lösungsmittelabhängigkeit der Verschiebungen bezüglich der trans-Isomeren darstellen. Zu erkennen ist eine leichte Tieffeldverschiebung bei der Messung in Aceton- $\mathrm{d}_{6}$ gegenüber der Messung in $\mathrm{CD}_{3} \mathrm{OD}$.

Die UV-Spektren von trans- und cis-Isomer sind bezüglich der Lage ihrer Maxima identisch (Maxima: (217), 237, (300 sh), 327 nm; Abb. 41). Das cis-Isomer zeigt allerdings etwas weniger intensive Signale.

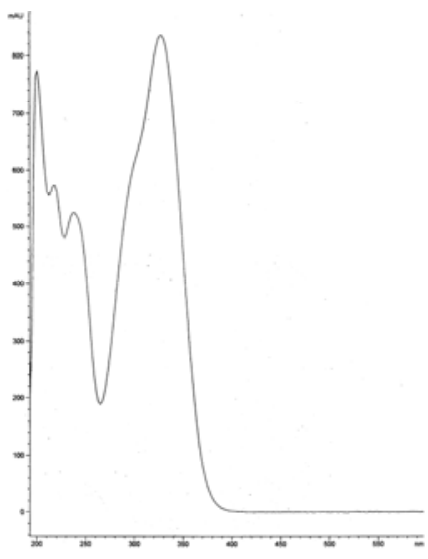

\author{
Abb. 41: \\ UV-Spektren von trans- (links) und \\ cis- (rechts) Feruloyläpfelsäureester
}

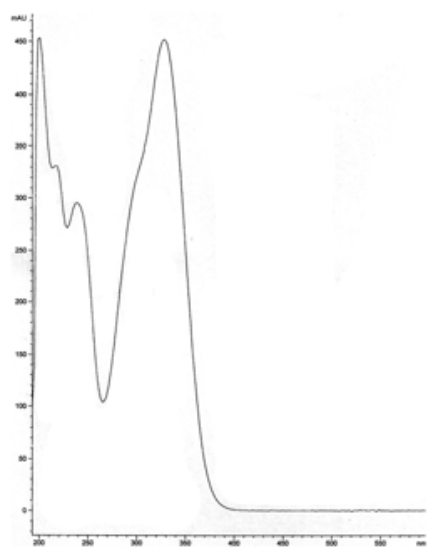

Neben der Hauptkomponenten trans-Feruloyläpfelsäureester tritt ebenfalls das cis-Isomer in geringerer Intensität auf. Da zum cis-Isomer ebenfalls NMR-Literaturdaten [104] vorhanden sind und im Spektrum lediglich geringe Überlappungen im Aromatenteil auftraten, war eine Parallelbestimmung möglich. Die Verschiebungen der cis-Form sind in Tab. 14 der transForm gegenübergestellt. Die Signalformen sind mit der trans-Form identisch, ebenso die meisten Kopplungskonstanten. Als cis-Isomer koppeln hier allerdings $\mathrm{H}^{-} \mathbf{7}^{\prime}$ und $\mathrm{H}-\mathrm{8}^{\prime}$ mit 12,8 $\mathrm{Hz}$.

Die Signale des cis-Isomers für die Protonen am C-3 sind gegenüber dem trans-Isomer nur minimal verschoben, aber in der Intensität schwächer. Es trifft der unter 3.4.3.2.1. beschriebene Sachverhalt zu. Dass es sich aber ebenfalls um den Ester handelt und nicht etwa nur um cis-Ferulasäure ist am zweifach vorhandenen Signal für H-2 zu erkennen. Hier wird die partielle Überlappung der Signale deutlich.

Letztendlich ergab die massenspektrometrische Untersuchung die Bestätigung der Struktur, wobei auch hier wieder Vergleichsliteratur herangezogen wurde [20, 58, 104, 136]. Die hochauflösende ESI-MS-Messung lieferte die Molmasse von 310 als [M-H] ${ }^{-}$mit 309,0624 im -ESI-Modus und eine Masse von 333,0580 als [M+Na] $]^{+}$im +ESI-Modus. 


\subsubsection{4. trans-Kaffeoyläpfelsäure-1-methylester / trans-Kaffeoyläpfelsäure-4-methylester} (Verbindungen $\boldsymbol{P 9}$ und $\boldsymbol{P 1 0}$ )
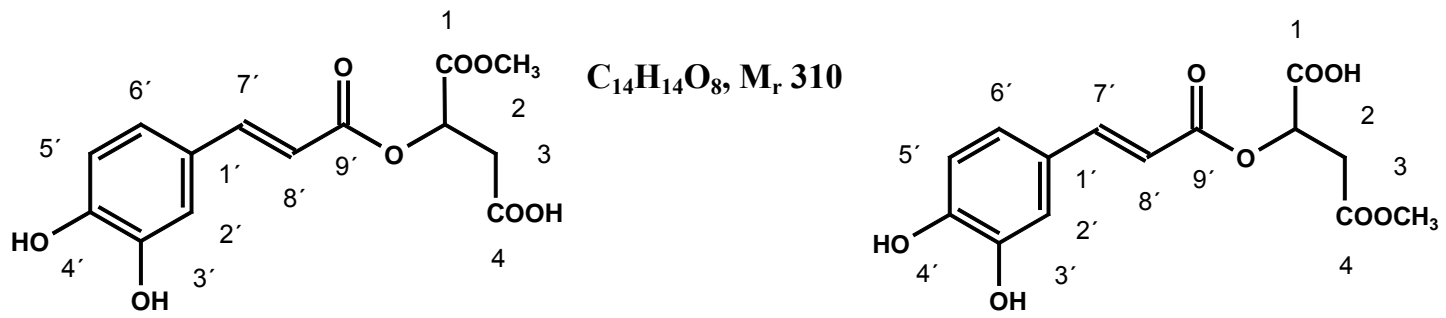

Die Verbindungen $\boldsymbol{P 9}$ und $\boldsymbol{P 1 0}$ sollen zusammen besprochen werden, da sie in einem Peak von der HPLC-Säule eluierten (s. Abb. 35). An Hand des ${ }^{1}$ H-NMR wurde ersichtlich, dass es sich aber um zwei Substanzen handeln musste, die ungefähr im Verhältnis 1:2 auftraten. Dies zeigte sich zunächst an den zwei Signalen der Methoxygruppe sowie an den ebenfalls doppelt vorhandenen Signalen für H-7'und H-8', die sich nur gering in ihren Verschiebungen unterschieden, während die Unterschiede bei der Verschiebung der jeweils drei Protonen der Methoxygruppe deutlich waren (3,72 ppm und 3,76 ppm; s. auch Tab. 16).

Tabelle 16: ${ }^{1} \mathrm{H}-\mathrm{NMR}$ der Kaffeoyläpfelsäuremethylester in $\mathrm{CD}_{3} \mathrm{OD}$

\begin{tabular}{|l|c|c|c|}
\hline H & $\begin{array}{c}\text { Verschiebung } \boldsymbol{\delta} \\
{[\mathrm{ppm}]}\end{array}$ & Signalform & $\begin{array}{c}\text { Kopplungskonst. J } \\
{[\mathrm{Hz}]}\end{array}$ \\
\hline $2^{\prime}$ & 7,05 & $\mathrm{~d}$ & 1,7 \\
$5^{\prime}$ & 6,78 & $\mathrm{~d}$ & 8,3 \\
$6^{\prime}$ & 6,96 & $\mathrm{~d}$ & 8,$3 ; 1,7$ \\
$7^{\prime}$ & $7,58 / 7,59$ & $\mathrm{~d}$ & 15,9 \\
$8^{\prime}$ & $6,29 / 6,30$ & $\mathrm{~d}$ & 15,9 \\
$2^{\prime}$ & 5,49 & $\mathrm{dd}$ & 4,$6 ; 8,0$ \\
$3 \mathrm{a}$ & 2,94 & $\mathrm{dd}$ & 8,$0 ; 16,9$ \\
$3 \mathrm{~b}$ & 3,0 & $\mathrm{dd}$ & 4,$6 ; 16,9$ \\
1 bzw. & $3,72 / 3,76$ & $\mathrm{~s}$ & - \\
$4\left(-\mathrm{OCH}_{3}\right)$ & & & \\
\hline
\end{tabular}

Auch bei den Protonen H-2 und H-3a/b zeigte sich eine Signalverdoppelung. Jedoch war auf Grund der Signalvielfalt an diesen Stellen eine Zuordnung und Bestimmung der Kopplungskonstanten lediglich des Signals der jeweils intensiveren Verbindung möglich. Die Verschiebungen für die Methoxygruppe und für H-7'und H-8' beider Verbindungen sind in Tab. 16, mit Schrägstrich voneinder getrennt, aufgeführt. 
Je weiter entfernt sich die Atome von der Methylestergruppierung befinden, desto weniger unterscheiden sich beide Verbindungen in ihren Signalverschiebungen voneinander. So überlagern sich die Signale beider Ester im Aromatenbereich vollständig.

Die -ESI-MS-Messung ergab eine Masse von 309 als [M-H] $]^{-}$Somit kam u. U. auch der Feruloyläpfelsäureester (P8) mit gleicher Molmasse in Betracht. Da aber die Signale der Methoxygruppen weiter zum Hochfeld verschoben waren als bei P8 (3,72 / 3,76 gegenüber 3,89; Tab. 16 und 14), deutete dies auf entsprechende Methylester hin.

Diese Hypothese bestätigte sich auch bei der DC dieser Estermischung neben Feruloyläpfelsäure im Fließmittelgemisch VII. Hier waren die Ester als zwei Banden mit etwas größeren $\mathrm{R}_{\mathrm{f}}$-Werten $(0,90 ; 0,95)$ und hellblauer Fluoreszenz $(\lambda=366 \mathrm{~nm})$ zu detektieren, während der trans-Feruloyläpfelsäureester einen $\mathrm{R}_{\mathrm{f}}$-Wert von 0,88 und lilafarbene Fluoreszenz zeigte. Im Übrigen eluierten die Ester $\boldsymbol{P 9}$ und $\boldsymbol{P 1 0}$ später von der RP-Säule bei der präparativen HPLC als Feruloyläpfelsäureester, was für größere Lipophilie ersterer spricht. Dieses Ergebnis korrelierte mit den Befunden der DC. Eine Trennung des Estergemischs mittels präparativer DC im Fließmittelgemisch VII erschien daher als möglich, wurde jedoch auf Grund zu geringer Substanzmengen nicht praktiziert.

Die ${ }^{1}$ H-NMR-Daten für den Kaffeesäureteil des Moleküls entsprechen denen des Kaffeoyläpfelsäureesters (s. Tab. 13). $\boldsymbol{P 9}$ und $\boldsymbol{P 1 0}$ liegen beide nur als trans-Isomer vor, was aus den Kopplungskonstanten der Signale für H-7'und $\mathrm{H}-8^{\prime}$ von $15,9 \mathrm{~Hz}$ deutlich wird. Geringe Abweichungen gegenüber den Daten des Äpfelsäureteils, beispielsweise bei P7, erklären sich aus der Methylierung der Äpfelsäure (s. Tab. 16 und 13).

Beide „Di““-Ester stellen neue Naturstoffe dar.

\subsubsection{5. cis-/trans-Chlorogensäure (cis-/trans-5-Kaffeoylchinasäure) (Verbindung P11)}
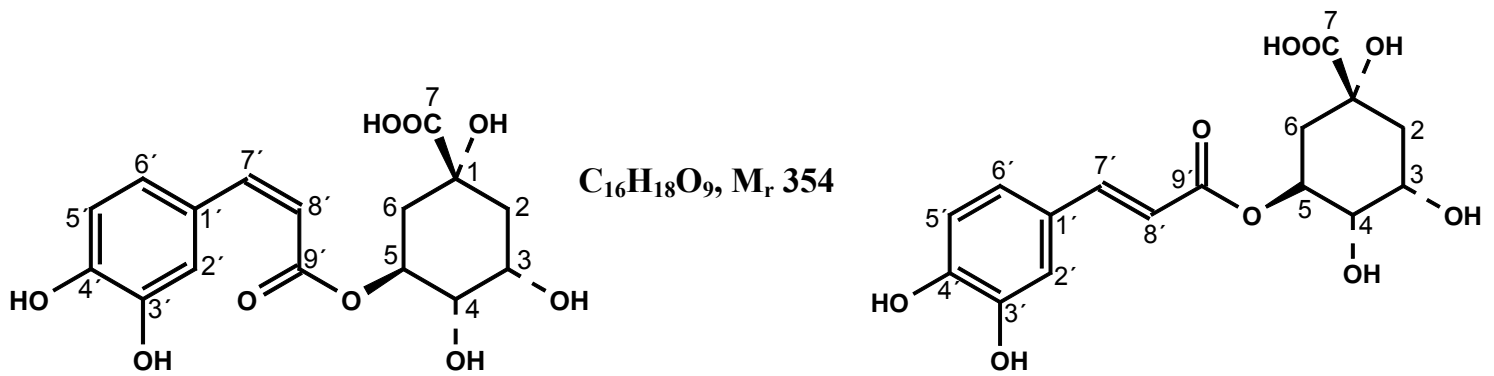
Chlorogensäure ist der Prototyp der Ester aromatischer Hydroxycarbonsäuren, die auch als Depside bezeichnet werden, und kommt in fast allen pflanzlichen Geweben, jedoch in relativ niedriger Konzentration (ca. 0,01 \%; Ausnahme: grüne Kaffeebohnen 3-8 \%) vor.

Tabelle 17: ${ }^{1} \mathrm{H}-\mathrm{NMR}-D a t e n$ von cis-/trans-5-Kaffeoylchinasäure in $\mathrm{CD}_{3} \mathrm{OD}$

\begin{tabular}{|c|c|c|c|c|c|}
\hline \multirow[t]{2}{*}{$\mathbf{H}$} & \multicolumn{2}{|c|}{$\begin{array}{c}\text { Verschiebung } \boldsymbol{\delta} \\
{[\mathrm{ppm}]}\end{array}$} & \multirow[t]{2}{*}{ Signalform } & \multicolumn{2}{|c|}{$\begin{array}{c}\text { Kopplungskonst. J } \\
{[\mathrm{Hz}]} \\
\end{array}$} \\
\hline & cis & trans & & cis & trans \\
\hline $2 \mathrm{ax}$ & $1,97-2,27$ & $1,95-2,25$ & $\mathrm{~m}$ & - & 一 \\
\hline $2 \mathrm{eq}$ & $1,97-2,27$ & $1,95-2,25$ & $\mathrm{~m}$ & - & 一 \\
\hline 3 & 5,33 & 5,32 & $\mathrm{dt}$ & 4,$5 ; 8,5$ & 4,$5 ; 8,5$ \\
\hline 4 & 3,73 & 3,72 & dd & 3,$5 ; 8,5$ & 3,$2 ; 8,5$ \\
\hline 5 & 4,13 & 4,16 & ddd & 3,$0 ; 3,5 ; 6,0$ & 2,$6 ; 3,2 ; 5,8$ \\
\hline $6 a x$ & $1,97-2,27$ & $1,95-2,25$ & $\mathrm{~m}$ & - & - \\
\hline $6 e q$ & $1,97-2,27$ & $1,95-2,25$ & $\mathrm{~m}$ & - & 一 \\
\hline $2^{\prime}$ & 7,39 & 7,04 & d & 2,0 & 2,0 \\
\hline $5^{\prime}$ & 6,72 & 6,77 & d & 8,0 & 8,2 \\
\hline $6^{\prime}$ & 7,06 & 6,94 & dd & 2,$0 ; 8,0$ & 2,$0 ; 8,2$ \\
\hline $7^{\prime}$ & 6,78 & 7,55 & d & 13,0 & 15,9 \\
\hline $8^{\prime}$ & 5,75 & 6,26 & d & 13,0 & 15,9 \\
\hline
\end{tabular}

Im NMR-Spektrum koppeln, wie bei den bereits besprochenen 1,3,4-substituierten Aromaten (s. v.), H-5' und H-6' miteinander als Dublett mit ca. 8 Hz. Da H-6', das mit H-2' mit ca. 2 Hz koppelt, noch einen weiteren Partner hat, ergibt sich als Signalform ein Doppeldublett. Die trans-Doppelbindung resultiert wiederum aus der Kopplungskonstanten von ca. $16 \mathrm{~Hz}$ für die beiden Protonen am C-7'und C-8'.

Für die selten in der Literatur erwähnte cis-Chlorogensäure, die im „Gelben Nachlauf“ neben der trans-Form vorlag, standen keine Referenzdaten zur Verfügung. Der Aromatenteil konnte aber mit dem Aromatenteil des cis-Kaffeoyläpfelsäureesters verglichen werden (P7), da in diesem Fall ebenfalls die cis-Kaffeesäure zu Grunde liegt, für die Vergleichsdaten existieren. Die Signalformen stimmen überein. Auch zeigen die Signalverschiebungen prinzipiell Übereinstimmung, ebenso mit dem trans-Isomer der Chlorogensäure (vgl. Tab. 17). Der cisCharakter der Doppelbindung war an Hand der Kopplungskonstanten von $13 \mathrm{~Hz}$ für die Protonen $\mathrm{H}-\mathrm{7}^{\prime}$ und $\mathrm{H}-\mathrm{8}^{\prime}$ festzulegen.

Die Signale für den Chinasäureteil stimmen bei beiden Isomeren, bis auf die auch bei den anderen cis-/trans-Isomeren gefundenen geringen Signalverschiebungen (vgl. Tab. 17), überein. Die insgesamt vier Protonen an C-2 und C-6 ergeben auf Grund der Signalvielfalt das 
Multiplett von 1,95 - 2,25 bzw. 1,97 - 2,27 ppm. H-3 koppelt mit H-4 mit 8,5 Hz sowie mit den beiden Protonen am C-2 ebenfalls mit 8,5 Hz. Diese beiden letztgenannten Protonen fallen in das eben erwähnte Multiplett. Aus diesen drei Kopplungen, von denen zwei eine identische Kopplungskonstante aufweisen, ergibt sich als Signalform ein Dubletttriplett. H-4 koppelt mit H-3 mit 8,5 Hz und mit H-5 mit 3,2 bzw. 3,5 Hz. Daraus resultiert das Doppeldublett. H-5 schließlich koppelt als Partner von H-4 mit diesem Proton mit 3,2 bzw. $3,5 \mathrm{~Hz}$.

Die vorliegenden Analysenergebnisse stimmen mit Literaturangaben [18, 158, 27] überein und sind mit der unter analogen Bedingungen vermessenen Referenzsubstanz transChlorogensäure konkruent. Schließlich lieferte die hochauflösende ESI-MS die entsprechende zugehörige Molmasse von 353,0858 als $[\mathrm{M}-\mathrm{H}]^{-}$bzw. 355,1037 als $[\mathrm{M}+\mathrm{H}]^{+}$sowie 377,0798 als $[\mathrm{M}+\mathrm{Na}]^{+}$.

In den Fraktionen der Chinasäureester wurde auch freie Chinasäure (1,3,4,5-Tetrahydroxy-1cyclohexen-1-carbonsäure) gefunden (3.5.2.5.).

Das UV-Spektrum der trans-Chlorogensäure (Abb. 42) zeigt die für Kaffeesäurederivate typi-

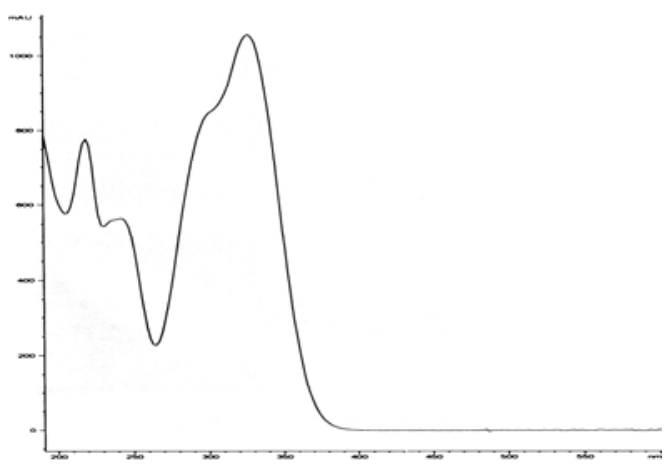

Abb. 42: UV-Spektrum der trans-Clorogensäure schen Maxima (218, 243, (298 sh), $326 \mathrm{~nm})$ und entspricht, mit geringen Abweichungen der Wellenlängen, auch den UV-Spektren von trans-Kaffeesäure (P4), 5-Kaffeoylepichinasäuremethylester (P12), transKaffeoyläpfelsäureester (P7) sowie der beiden Methylester (P9, P10). In allen diesen Fällen stellt trans-Kaffeesäure das UV-aktive Chromophor dar.

\subsubsection{6. $\quad$ trans-5-Kaffeoylepichinasäuremethylester (Verbindung P12)}<smiles>CCCCCCCOC(=O)C=Cc1ccc(O)c(O)c1</smiles>

Über das Vorkommen der Substanz mit der nebenstehend vorgeschlagenen Struktur ist bisher nicht berichtet worden. Bei der Extrakt- 
aufarbeitung von Acmella ciliata fiel sie in der Ethylacetat-Phase (Weg 2) und im „Gelben Nachlauf" an (s. Abb. 35).

Zur Strukturaufklärung diente zunächst die ESI-MS, die eine Molmasse von 367,0984 als $[\mathrm{M}-\mathrm{H}]^{-}$und 369,1192 als $[\mathrm{M}+\mathrm{H}]^{+}$sowie 391,1013 als $[\mathrm{M}+\mathrm{Na}]^{+}$ergab. Die Massendifferenz zur Chlorogensäure (3.4.3.2.5.) betrug somit 14, was der Masse einer Methylengruppierung entspricht. Somit waren eine Methoxygruppe am Aromaten oder der Methylester der Chinasäure am wahrscheinlichsten.

Tabelle 18: ${ }^{1}$ H-NMR-Daten von 5-Kaffeoylepichinasäuremethylester

\begin{tabular}{|l|c|c|c|}
\hline H & $\begin{array}{c}\text { Verschiebung } \boldsymbol{\delta} \\
{[\mathrm{ppm}]}\end{array}$ & Signalform & $\begin{array}{c}\text { Kopplungskonst. J } \\
{[\mathrm{Hz}]}\end{array}$ \\
\hline $2 \mathrm{ax}$ & $1,95-2,25$ & $\mathrm{~m}$ & - \\
$2 \mathrm{eq}$ & $1,95-2,25$ & $\mathrm{~m}$ & - \\
3 & 5,26 & $\mathrm{dt}$ & 7,$2 ; 4,8$ \\
4 & 3,73 & $\mathrm{dd}$ & 3,$0 ; 7,2$ \\
5 & 4,13 & $\mathrm{dt}$ & 3,$5 ; 6,9$ \\
$6 \mathrm{ax}$ & $1,95-2,25$ & $\mathrm{~m}$ & - \\
$6 \mathrm{eq}$ & $1,95-2,25$ & $\mathrm{~m}$ & - \\
$8\left(-\mathrm{OCH}_{3}\right)$ & 3,69 & $\mathrm{~s} /(3 \mathrm{H})$ & - \\
$2^{\prime}$ & 7,04 & $\mathrm{~d}$ & 2,0 \\
$5^{\prime}$ & 6,78 & $\mathrm{~d}$ & 8,2 \\
$6^{\prime}$ & 6,95 & $\mathrm{dd}$ & 2,$0 ; 8,2$ \\
$7^{\prime}$ & 7,52 & $\mathrm{~d}$ & 15,9 \\
$8^{\prime}$ & 6,21 & $\mathrm{~d}$ & 15,9 \\
\hline
\end{tabular}

Das ${ }^{1}$ H-NMR (Tab. 18) zeigte einen der Chlorogensäure identischen Kaffeesäurerest mit trans-Doppelbindung $(\mathrm{J}=15,9 \mathrm{~Hz})$ zwischen $\mathrm{C}-7^{\prime}$ und $\mathrm{C}-8^{\prime}$ (vgl. Tab. 17 und 18). Damit konnte die Ferulasäure als potentieller Baustein ausgeschlossen werden. Die Vermutung, dass es sich um den Methylester der Chinasäure handeln könnte, wurde durch einen einzigen Literaturhinweis [191], der den 5-Kaffeoylchinasäuremethylester als neuen Naturstoff beschreibt, gestützt. Die eigenen Daten der Signalmuster sowie der Kopplungskonstanten für die Protonen am C-3 und C-4 weichen jedoch von den Literaturangaben ab. Während in der vorliegenden Arbeit diese beiden Signale als dt in Erscheinung treten mit jeweils nur zwei verschiedenen Kopplungskonstanten (Tab. 18), beschreiben ZHU et al. [191] die Signalform dd für H-3 mit 3,5 Hz und ein ddd mit nur einer Kopplungskonstanten von 3,5 Hz für H-5. Eigentlich müssten bei einem Dublett zwei verschiedene Kopplungskonstanten existieren, bei einem dreifachen Dublett sogar drei. 
Zumindest lässt die Literaturangabe des dreifachen Dubletts für H-5 auf eine Substitution bzw. auf die Stellung der Carbonylfunktion analog der vorangehend beschriebenen Chlorogensäure schließen. Das Signal für das Proton an C-5 stellt bei der Chlorogensäure (Tab. 17) ebenfalls ein ddd dar, was mit dem stärker Elektronen ziehenden Effekt durch die axiale Position der Carbonylfunktion und damit die größere räumliche Nähe zu diesem Proton als zu dem Proton an C-3 zu erklären ist. Die größere räumliche Nähe zu H-5 kommt durch die axiale Position der Säurefunktion zu Stande.

Im Fall dieses Esters scheinen die räumliche Nähe und damit der Einfluss der Carbonylfunktion auf das Proton H-5 eine geringere Rolle zu spielen, da dieses Signal hier, ebenso wie das Signal für H-3, als dt in Erscheinung tritt, was nur durch eine äquatoriale Stellung der Carbonylfunktion zu erklären ist. Diese Unterschiede wären theoretisch noch genauer an den Protonen am C-2 und C-6 zu verdeutlichen. Jedoch fallen diese in einem Multiplett zusammen und ergeben somit keine auswertbaren Signale. Allerdings ist zu konstatieren, dass sich die Muster der Chlorogensäure und des hier beschriebenen Methylesters am Signal von H-5 deutlich voneinander unterscheiden, und auch das Signal für H-3 unterscheidet sich zumindest noch in den Kopplungskonstanten und an diesen Stellen auch prägnant von der Literatur [191] (vgl. Abb. 43).

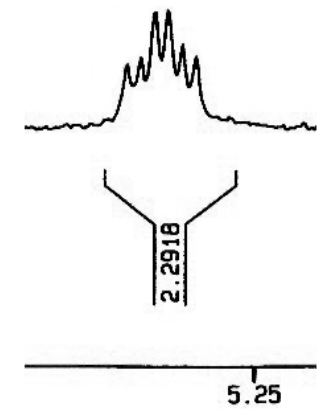

Signal für H-3 der trans-Chlorogensäure: $5,32 \mathrm{ppm} ; \mathrm{dt} ; 4,5 / 8,5 \mathrm{~Hz}$

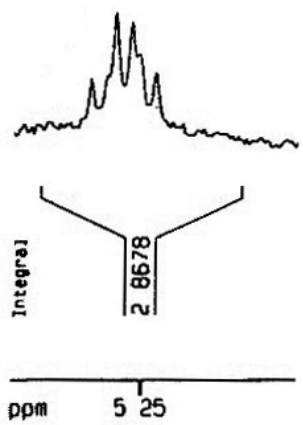

Signal für H-3 des trans-Kaffeoylepichinasäuremethylesters: 5,26 ppm; dt; 4,8 / 7,2 Hz

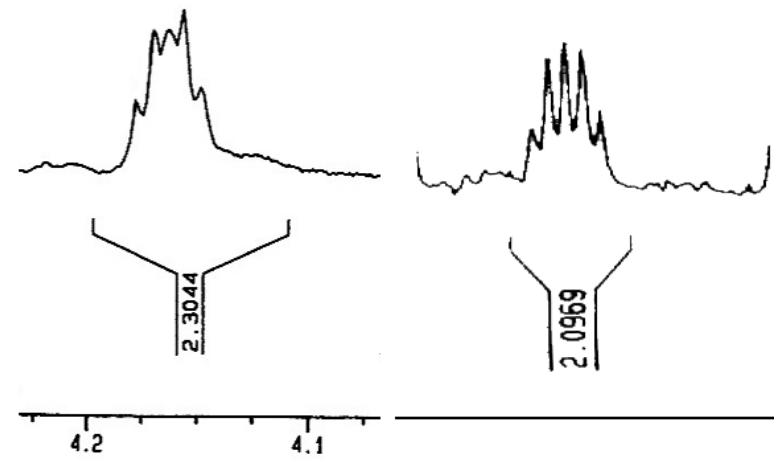

Signal für H-5 der

Signal für H-5 des transtrans-Chlorogensäure: Kaffeoylepichinasäure4,16 ppm; ddd; $2,6 / 3,2$ / methylesters: 4,13 ppm; $5,8 \mathrm{~Hz}$ $\mathrm{dt} ; 3,5 / 6,9 \mathrm{~Hz}$

Abb. 43: Vergleich der Signale für H-3 und H-5 der Verbindungen $P 11$ und $P 12$

Auf Grund der dargestellten Erkenntnisse kann für die isolierte Substanz die angenommene Strukur (s. Formel) als realistisch angesehen werden und der trans-5-Kaffeoylepichinasäuremethylester als neuer Naturstoff in Acmella ciliata gewertet werden. Das Präfix „epi“ im 
Trivialnamen wird analog zur 3,5-Dikaffeoylepichinasäure verwendet, bei welcher sich die Carbonylgruppe am C-1 der Chinasäure ebenfalls in äquatorialer Position befindet [74].

Das UV-Spektrum entspricht dem der trans-Chlorogen- bzw. trans-Kaffeesäure (s. 3.4.3.2.5.), da auch hier der Kaffeesäure-Teil das UV-aktive Element darstellt.

\subsubsection{Phenolglykoside}

\subsection{Isoquercitrin (Quercetin-3-O- $\boldsymbol{\beta}$-D-glucosid) (Verbindung P13)}

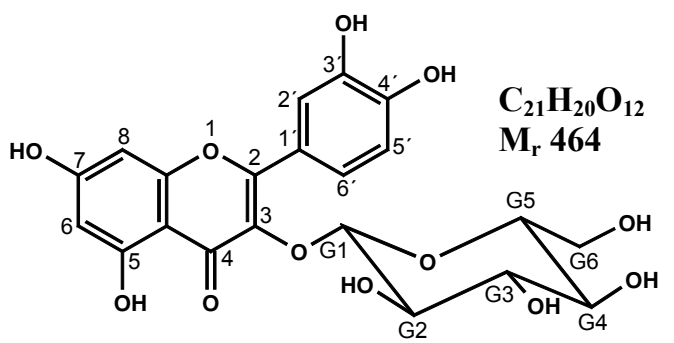

Dieses Phenolglykosid wurde von Säule III. 5 (s. Abb. 35), neben Rutosid (P14) und der oben beschriebenen Chlorogensäure (P11), zunächst mittels analytischer HPLC und paralleler DC, identifiziert. Für alle drei Verbindungen lagen die entsprechenden Referenzsubstanzen vor, so dass durch Cochromatographie und „Spiking“ per DC und HPLC (UV-Detektor) die Identität mit den Proben bestätigt werden konnte (identische Retentionszeiten und UV-Spektren).

Allerdings sind die UV-Spektren von Isoquercitrin und Rutosid auch konkruent (s. Abb. 44), da nur das Aglykon Quercetin UV-aktiv ist. $\mathrm{Zu}$ erkennen sind die für viele Flavonoide typischen zwei Maxima, die in diesem Fall bei 257, (266 sh, 294 sh) und 354 nm liegen (Abb. 44).
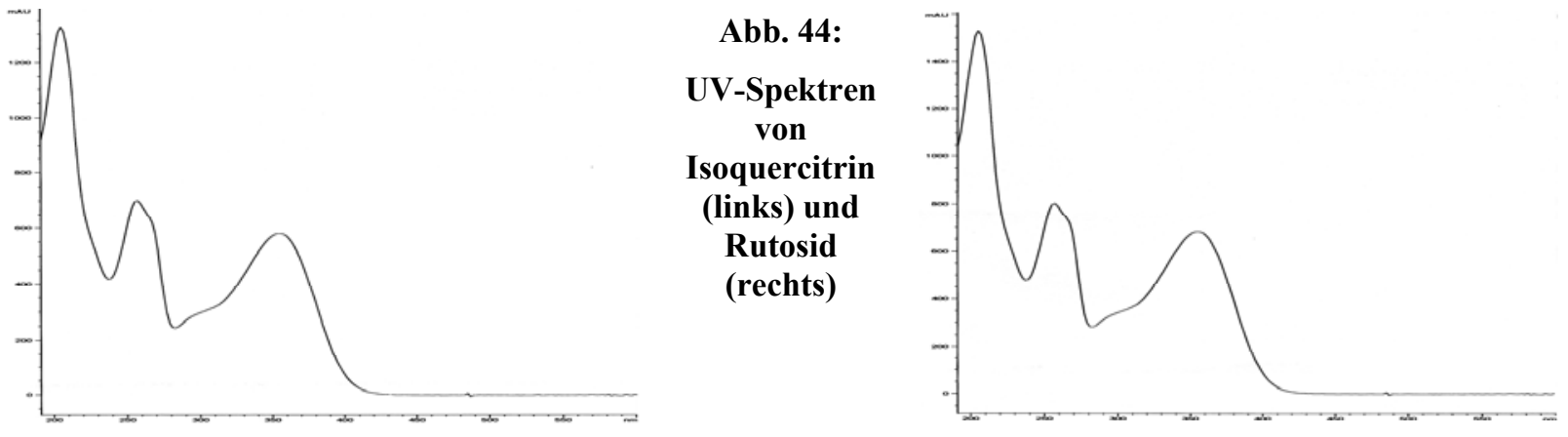

Isoquercitrin wurde anschließend per präparativer HPLC bei $330 \mathrm{~nm}$ (s. Abb. 45) isoliert. Da von Isoquercitrin etwas mehr Substanz als vom nachfolgend dargestellten Rutosid gewonnen 
werden konnte, wurde zur Strukturabsicherung darüber hinaus die ${ }^{1} \mathrm{H}-\mathrm{NMR}$ in $\mathrm{CD}_{3} \mathrm{OD}$ durchgeführt. Bei der Auswertung der NMR-Daten (Tab. 19) konnte Bezug genommen werden auf die unter gleichen Bedingungen vermessene Referenzsubstanz sowie auf entsprechende Literaturangaben [41, 107], was die Identität belegte.

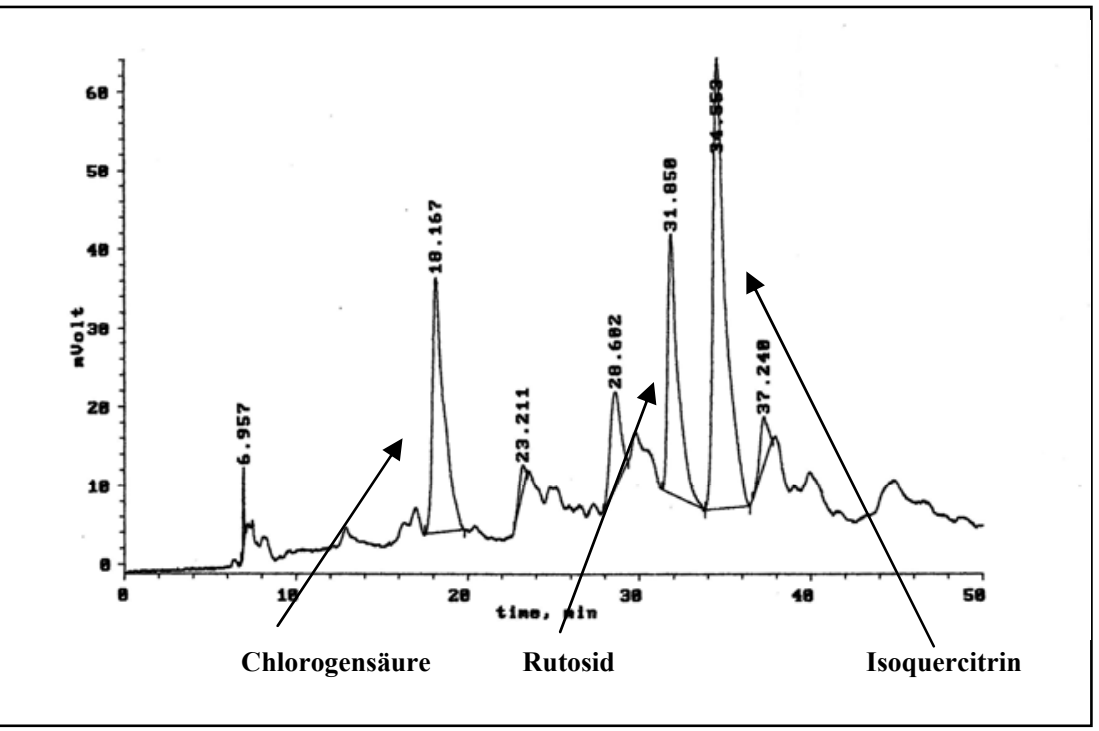

Abb. 45:

Chromatogramm der präparativen HPLC von Sammelfraktion III. 5 bei $330 \mathrm{~nm}$

(Parameter s. Exp. Teil)

Die Protonen am C-6 und C-8 koppeln nur untereinander als Dublett mit $2 \mathrm{~Hz}$. Das Kopplungsmuster der Protonen H-2', H-5' und H-6' entspricht dem der 1,3,4-trisubstituierten Aromaten (vgl. Tab. 9 unter 3.4.3.1.2.). Das anomere Proton der Glucose (G-1) zeigt sich bei 5,26 ppm als Dublett mit 7,5 der axial-axial-Kopplung mit G-2. Die Signale für G-2 und G-3 stellen schwer zu erkennende Doppeldubletts dar, da die Kopplungskonstanten für G-2 mit G$1(7,5 \mathrm{~Hz})$ und G-2 mit G-3 $(9,0 \mathrm{~Hz})$ sowie für G-3 mit G-4 $(8,5 \mathrm{~Hz})$ leicht differieren. Die Signale von G-4 und G-5 werden durch das Lösungsmittel Methanol (deuteriert) überdeckt.

Tabelle 19: ${ }^{1} \mathrm{H}-\mathrm{NMR}$ Daten von Isoquercitrin in $\mathrm{CD}_{3} \mathrm{OD}$

\begin{tabular}{|l|c|c|c|}
\hline H & $\begin{array}{c}\text { Verschiebung } \boldsymbol{\delta} \\
{[\mathrm{ppm}]}\end{array}$ & Signalfor m & $\begin{array}{c}\text { Kopplungskonst. J } \\
{[\mathrm{Hz}]}\end{array}$ \\
\hline 6 & 6,21 & $\mathrm{~d}$ & 2,0 \\
8 & 6,40 & $\mathrm{~d}$ & 2,0 \\
$2^{\prime}$ & 7,70 & $\mathrm{~d}$ & 2,0 \\
$5^{\prime}$ & 6,88 & $\mathrm{~d}$ & 8,5 \\
$6^{\prime}$ & 7,59 & $\mathrm{dd}$ & 2,$0 ; 8,5$ \\
G-1 & 5,26 & $\mathrm{~d}$ & 7,5 \\
G-2 & 3,48 & $\mathrm{dd}$ & 7,$5 ; 9,0$ \\
G-3 & 3,42 & $\mathrm{dd}$ & 8,$5 ; 9,0$ \\
G-4 & verdeckt & durch & Lösungsmittel \\
G-5 & $"$ & $"$ & $" 1$ \\
G-6a & 3,71 & $\mathrm{dd}$ & 2,$0 ; 12,0$ \\
G-6b & 3,57 & $\mathrm{dd}$ & 5,$0 ; 12,0$ \\
\hline
\end{tabular}

Die Kopplungskonstante der geminalen Kopplung der Protonen G-6a und G$6 \mathrm{~b}$ beträgt $12 \mathrm{~Hz}$. Diese Protonen koppeln einmal mit 2,0 Hz (G-6a) und mit 5,0 $\mathrm{Hz}$ (G-6b) mit dem axialen Proton G-5 (Tab. 19). 
Die -FAB-MS-Messung lieferte die entsprechende Masse von 463,2 als $[\mathrm{M}-\mathrm{H}]^{-}$und die +FAB-MS-Messung die Masse 487,1 als $[\mathrm{M}+\mathrm{Na}]^{+}$. Nachfolgende ESI-MS-Messungen erbrachten übereinstimmende Ergebnisse: 463,0884 [M-H] ; 487,0860 [M+Na] .

Isoquercitrin sowie Rutosid wurden bereits in Acmella ciliata, damals fälschlicherweise als „Spilanthes oleracea“ betrachtet, nachgewiesen. Jedoch wurde die ${ }^{1} \mathrm{H}-\mathrm{NMR}$-Analytik nicht zur Strukturaufklärung herangezogen [177]. Insgesamt kann die Struktur des Isoquercitrins durch die obige Analytik als bestätigt betrachtet werden.

3.4.3.3.2. Rutosid (Quercetin-3-O-(6-O- $\alpha-L-r h a m n o s y l)-\beta-D-g l u c o s i d)$ (Verbindung P14)

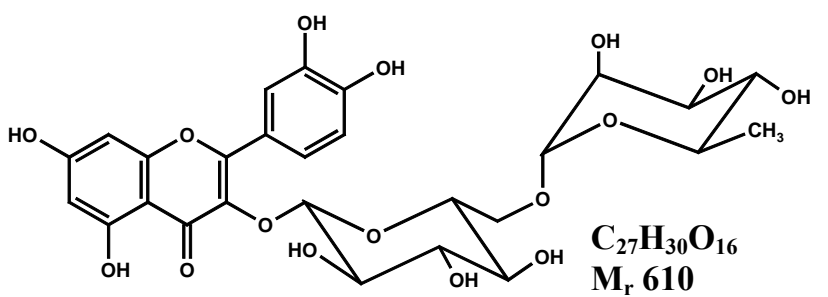

sind. Hinsichtlich der auf das mit Isoquercitrin gemeinsame Aglykon Quercetin bezogenen Analysendaten (Tab. 19) kann auf die obigen Aussagen Bezug genommen werden. Außerdem wurde auch hier die Identität durch Cochromatographie und „Spiking“ (DC, HPLC) mittels Referenzsubstanz bestätigt. Zuzüglich lieferte die +FAB-MS-Messung die Masse 633 als $[\mathrm{M}+\mathrm{Na}]^{+}$.

\subsection{Weitere Flavonoide}

Weitere Flavonoide konnten auf Grund zu geringer Mengen lediglich per DC detektiert (Abb. 36) und unter Einbeziehung der ESI-MS-Messungen teilweise zugeordnet werden. So ergab sich neben Isoquercitrin (P13) und Rutosid (P14) ein Hinweis auf das Vorliegen von Miquelianin (Quercetin-3-O- $\beta$-D-glucuronosid; 477,0675 [M-H] $; \mathrm{R}_{\mathrm{f}}=0,47$; Abb. 36), bei dem das Aglykon Quercetin mit Glukuronsäure $\beta$-glykosidisch verknüpft ist. Dafür spricht neben der entsprechenden Molmasse die Tatsache, dass diese Substanz nur im -ESI detektiert wurde und der $\mathrm{R}_{\mathrm{f}}$-Wert kleiner als der des Isoquercitrins ist, was auf die Säurefunktion hindeutet. 
Außerdem ist ein weiteres Flavonoid sichtbar $\left(\mathrm{R}_{\mathrm{f}}=0,69 ;\right.$ Abb. 36), dem die Molmasse 492 $\left(491,0832[\mathrm{M}-\mathrm{H}]^{-} ; 515,0813[\mathrm{M}+\mathrm{Na}]^{+}\right)$zugeordnet wurde. Die größere Lipophilie (höherer $\mathrm{R}_{\mathrm{f}}$-Wert) als Isoquercitrin, die Detektion auch im +ESI-Modus analog zu Isoquercitrin sowie eine Massendifferenz von 28 gegenüber Isoquercitrin führten zu der unten rechts dargestellten Strukturhypothese. Die Stellung der Methoxygruppen kann variieren.

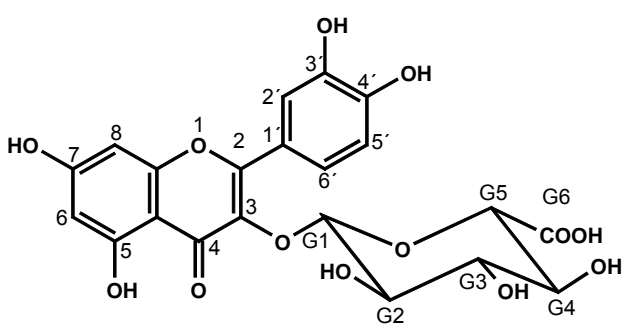

Miquelianin

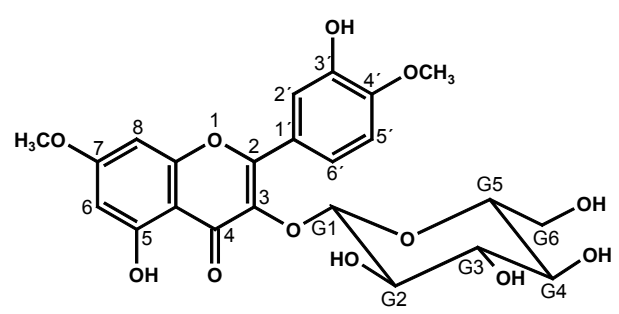

mögliche Struktur von Flavonoid $M_{\mathbf{r}} 492$

Die von VERYKOKIDOU-VITSAROPOUlOS und BECKER [177] beschriebenen Flavonoide Apigenin-7-O-glucosid und Apigenin-7-O-neohesperidosid (Rhoifolin) konnten nicht nachgewiesen werden.

\subsubsection{Aesculetin (Verbindung P15)}<smiles>O=c1ccc2cc(O)c(O)cc2o1</smiles>

$\mathrm{C}_{9} \mathrm{H}_{6} \mathrm{O}_{4}, \mathrm{M}_{\mathbf{r}} 178$
Tabelle 20: ${ }^{1} \mathrm{H}-\mathrm{NMR}$ Daten von Aesculetin in $\mathrm{CD}_{3} \mathrm{OD}$

\begin{tabular}{|l|c|c|c|}
\hline H & $\begin{array}{c}\text { Verschiebung } \boldsymbol{\delta} \\
{[\mathrm{ppm}]}\end{array}$ & Signalform & $\begin{array}{c}\text { Kopplungskonst. J } \\
{[\mathrm{Hz}]}\end{array}$ \\
\hline 3 & 7,78 & $\mathrm{~d}$ & 9,4 \\
4 & 6,16 & $\mathrm{~d}$ & 9,4 \\
5 & 6,93 & $\mathrm{~s}$ & - \\
8 & 6,75 & $\mathrm{~s}$ & - \\
\hline
\end{tabular}

Die Struktur des Aesculetins ergibt sich aus dem ${ }^{1}$ H-NMR, das vier Signale aufweist (Tab. 20). Die beiden Dubletts stehen für die Protonen der cis-Doppelbindung zwischen C-3 und C4 mit Kopplungskonstanten von 9,4 Hz. Durch den Elektronen ziehenden Effekt des benachbarten Carbonyls ist das Signal für H-3 stärker zum Tieffeld verschoben als für C-4. Die beiden Protonen an C-5 und C-8 sind isoliert und erscheinen somit als Singulett.

Die hochauflösende +ESI-MS bestätigte die Struktur und ergab ein Signal für den Molpeak von 179,0340 als $[\mathrm{M}+\mathrm{H}]^{+}$und 201,0159 als $[\mathrm{M}+\mathrm{Na}]^{+}$. Die -ESI-MS erbrachte 177,0181 als 
$[\mathrm{M}-\mathrm{H}]^{-}$. Schließlich bestätigte auch die EI-MS bei $200^{\circ}$ und $280^{\circ} \mathrm{C}$ die Molmasse von 178 als $[\mathrm{M}]^{+}$.

Die Identität der Substanz wurde außerdem durch Cochromatographie mit einer AesculetinReferenz bewiesen (s. Abb. 46).

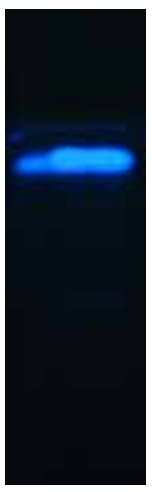

Abb. 46:

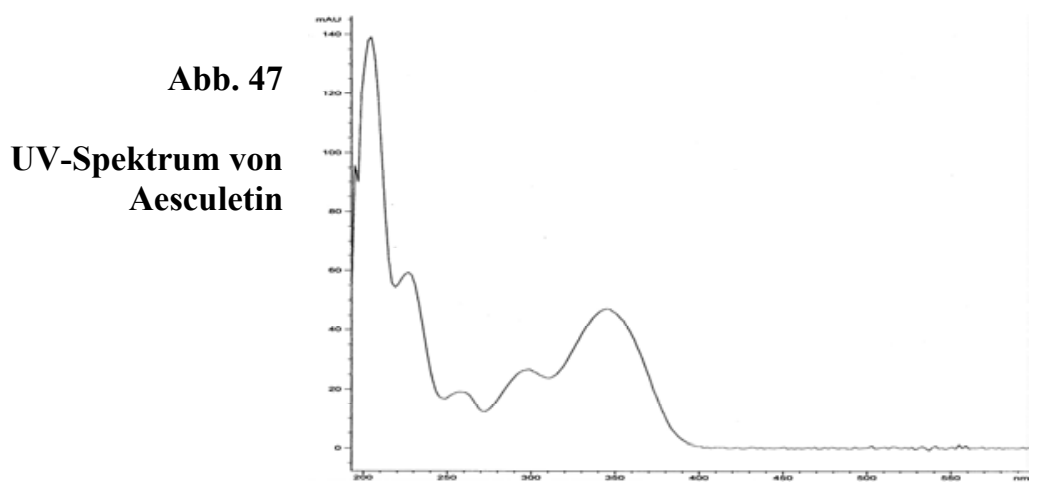

DC von Aesculetin von links nach rechts: Referenz, Probe, Probe/Referenz 1:1 (FM: VII; Det.: UV, $\lambda=366 \mathrm{~nm})$

Die $\mathrm{R}_{\mathrm{f}}$-Werte aller drei Banden (Abb. 46) waren identisch und zeigten die typische blaue Eigenfluoreszenz der Hydroxycumarin-Derivate im UV-Licht bei $366 \mathrm{~nm}$ mit Verstärkung nach Besprühen mit dem Naturstoffreagenz (s. Exp. Teil).

Im UV-Spektrum der Probensubstanz (Abb. 47) finden sich die für Hydroxycumarine typischen vier Maxima bei den Wellenlängen: $\lambda_{\max }=226,257,299,345 \mathrm{~nm}$.

\subsubsection{Matairesinol (Verbindung P16)}

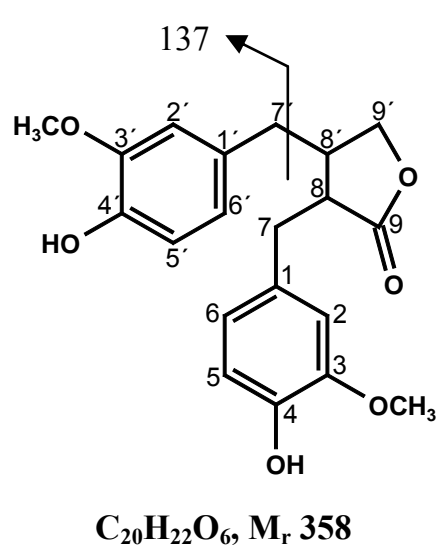

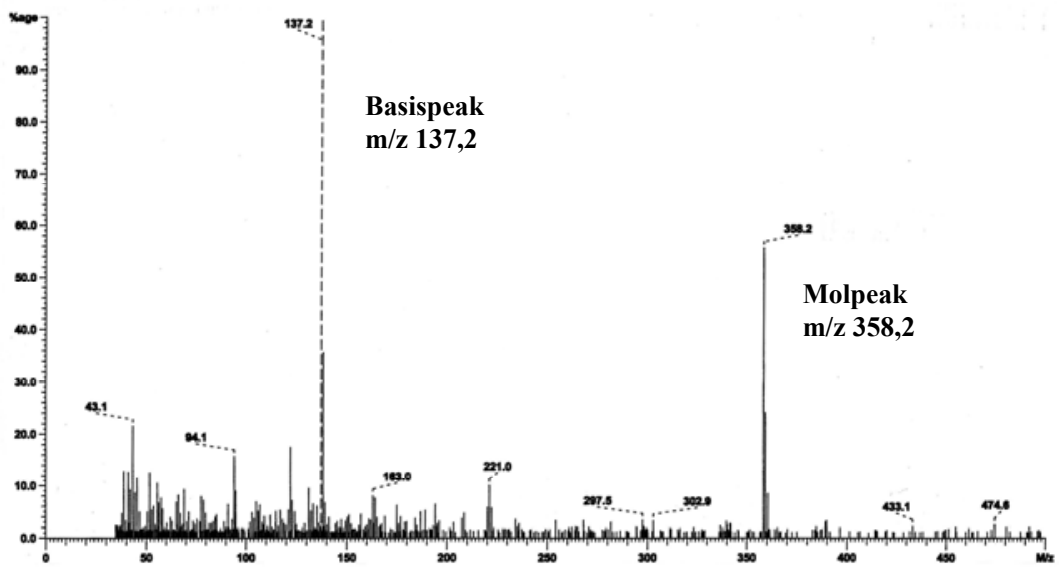

Abb. 48: EI-Massenspektrum von Matairesinol bei $170^{\circ} \mathrm{C}$ 
Matairesinol gehört zu den Lignanen, die biogenetisch Dimere von Phenylpropanderivaten darstellen. Lignane kommen häufig in den Familien der Cupressaceae, Piperaceae, Berberidaceae (pharmazeutisch relevant: Podophyllum peltatum L. = Maiapfel) und Zygophyllaceae [73] vor. In der Familie der Asteraceae sind Lignane eher selten anzutreffen, z. B. in Großer Klette (Arctium lappa L.) [180].

Das Vorkommen von Lignanen neben Alkamiden scheint eine Verstärkung der insektiziden Wirkung zu bedingen [46] und wurde in wenigen Asteraceae-Gattungen, z. B. in der Subtribus-Verwandten Heliopsis scabra, sowie in anderen „Alkamid-Familien“, den Piperaceae und Rutaceae, festgestellt [22]. Der Matairesinol-Nachweis in der Gattung Acmella konnte hier erstmalig erbracht werden.

Mittels vergleichender DC zwischen vorliegender Probe und einer Matairesinol-Referenz wurden nach Besprühen mit Anisaldehyd/Schwefelsäure zwei rot gefärbte Banden identischer $\mathrm{R}_{\mathrm{f}}$-Werte (0,31) sichtbar (Abb. 49). Auch das UV-Spektrum (Abb. 50) mit den für Lignane typischen Maxima $\left(\lambda_{\max }=230,283 \mathrm{~nm}\right)$ bewies die Identität durch Vergleich mit einem Referenzspektrum.

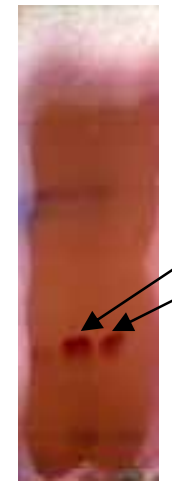

Abb. 49:

DC von Matairesinol Probe, Referenz (FM: IX; Det.: Anisaldehyd Schwefelsäure)
Abb. 50:

UV-Spektrum von Matairesinol in Methanol

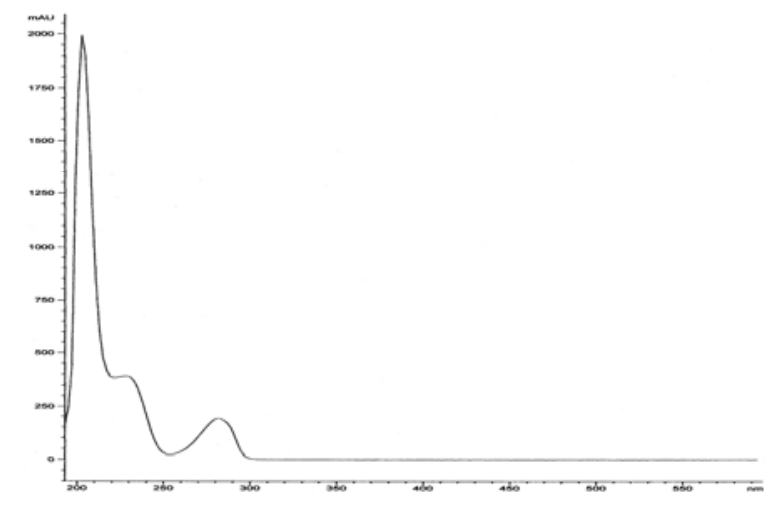

Die Molmasse resultiert aus der EI-MS mit 358,2. Im MS-Spektrum findet sich der Basispeak mit m/z 137,2, der dem Molekülteil mit der Summenformel $\mathrm{C}_{8} \mathrm{H}_{9} \mathrm{O}_{2}$ entspricht (s. Abb. 48 und Formel), entstanden nach Bindungsbruch zwischen C-7' und C-8' bzw. C-1 und C-7.

Das ${ }^{1}$ H-NMR-Spektrum der isolierten Substanz ist identisch mit der unter gleichen Bedingungen vermessenen Referenzsubstanz. Somit kann die Existenz von Matairesinol in Acmella ciliata als bewiesen angesehen werden. 
Hinsichtlich der NMR-Daten (Tab. 21) war auf entsprechende Literatur [45, 100, 123, 170] zurückzugreifen. Jedoch schien eine Präzisierung angeraten, da keine Daten für die Messung in Deutero-Methanol als Lösungsmittel vorlagen, in Deutero-Chloroform vermessene Proben zumeist älteren Datums sind und mit der damaligen Technik noch keine so präzisen Auflösungen zu erzielen waren. Die Messung in deuteriertem Methanol erschien hier logisch, da die Substanz in einem Methanol-Extrakt angefallen war und sie sich hervorragend in Methanol löste.

Durch Vergleich der ermittelten NMR-Daten mit den Angaben verschiedener Literatur, unter Berücksichtigung der teilweise etwas anderen Verschiebungen gegenüber jenen in DeuteroChloroform und in Kombination mit den oben erwähnten analytischen Methoden, kann die Identität als zweifelsfrei bewiesen angesehen werden.

Tabelle 21: ${ }^{1} \mathrm{H}-\mathrm{NMR}$ Daten von Matairesinol in $\mathrm{CD}_{3} \mathrm{OD}$

\begin{tabular}{|l|c|c|c|}
\hline $\mathbf{H}$ & $\begin{array}{c}\text { Verschiebung } \boldsymbol{\delta} \\
{[\mathrm{ppm}]}\end{array}$ & Signalform & $\begin{array}{c}\text { Kopplungskonst. J } \\
{[\mathrm{Hz}]}\end{array}$ \\
\hline 2 & 6,67 & $\mathrm{brs}$ & - \\
$3-\mathrm{OCH}_{3}$ & 3,79 & $\mathrm{~s}$ & - \\
5 & 6,70 & $\mathrm{~d}$ & 8,0 \\
6 & 6,58 & $\mathrm{dd}$ & 2,$0 ; 8,0$ \\
$7 \mathrm{a}$ & 2,89 & $\mathrm{dd}$ & 6,$0 ; 14,0$ \\
$7 \mathrm{~b}$ & 2,82 & $\mathrm{dd}$ & 7,$0 ; 14,0$ \\
8 & 2,66 & $\mathrm{ddd}$ & 6,$0 ; 7,0 ; 8,0$ \\
$2^{\prime}$ & 6,56 & $\mathrm{~d}$ & 2,0 \\
$3^{\prime}-\mathrm{OCH}_{3}$ & 3,78 & $\mathrm{~s}$ & - \\
$5^{\prime}$ & 6,68 & $\mathrm{~d}$ & 8,0 \\
$6^{\prime}$ & 6,51 & $\mathrm{dd}$ & 2,$0 ; 8,0$ \\
$7 \mathrm{a}^{\prime}$ & $2,46-2,55$ & $\mathrm{~m}$ & - \\
$7 \mathrm{~b}^{\prime}$ & $2,46-2,55$ & $\mathrm{~m}$ & - \\
$8^{\prime}$ & $2,46-2,55$ & $\mathrm{~m}$ & - \\
$9 \mathrm{a}^{\prime}$ & 4,16 & $\mathrm{dd}$ & 7,$5 ; 9,0$ \\
$9 \mathrm{~b}^{\prime}$ & 3,93 & $\mathrm{dd}$ & 7,$5 ; 9,0$ \\
\hline
\end{tabular}

Aus dem NMR-Spektrum (Tab. 21 und Abb. 51) ist zu entnehmen, dass H-6 und H5 zunächst untereinander mit $8 \mathrm{~Hz}$ koppeln, wobei sich für H-5 ein Dublett ergibt. H-6 besitzt in $\mathrm{H}-2$ einen weiteren Kopplungspartner, mit dem es mit $2 \mathrm{~Hz}$ koppelt und erscheint daher als Doppeldublett. Umgekehrt ist die kleine Kopplung von $\mathrm{H}-2$ mit H-6 nicht aufgelöst, so dass hier von einem breiten

Singulett zu sprechen ist. Dies liegt wohl an der Überlagerung des rechten Signalteils des Dubletts von H-5' mit dem Signal von H-2. Zu erkennen ist hier die Verbreiterung dieses Signals. Außerdem überlagern sich der linke Signalteil des Dubletts von H-5' und der rechte Signalteil des Dubletts von H-5, was ebenfalls in einer Verbreiterung des Signalteils gegenüber dem nicht überlagerten linken Teil von H-5 deutlich wird. Dadurch sind auch die Integrale der Signale $>1$. Das Doppeldublett von H-6 ist zudem teilweise durch das Dublett von H-2' überlagert, welches gegenüber den Literaturdaten (s. o.) für Messungen in Deutero- 
Chloroform offensichtlich durch das deuterierte Methanol stärker zum Tieffeld verschoben wird als alle anderen Signale (Tab. 21 und Abb. 51).

Im zweiten aromatischen Ring mit analogen Substituenten sind die Kopplungsmuster

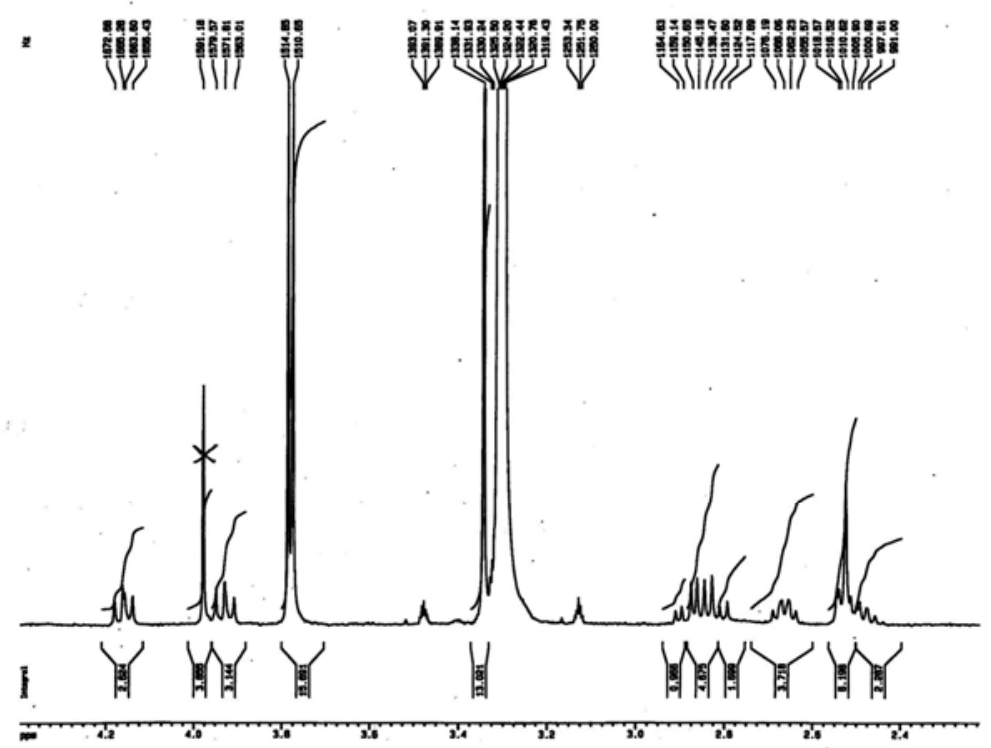

Abb. 51: ${ }^{1} \mathrm{H}-\mathrm{NMR}$ Spektrum von Matairesinol in $\mathrm{CD}_{3} \mathrm{OD}$ identisch, bis auf die Kopplung mit $2 \mathrm{~Hz}$ zwischen H-2 und H-6, die aufgelöst wird und als Dublett erscheint. Die beiden Methoxygruppen am C-3 und C-3' erscheinen als Singulett bei 3,79 bzw. $3,78 \mathrm{~Hz}$. Die insgesamt drei Protonen von C-7 und C-8 fallen in dem Multiplett mit 2,46-2,55 ppm zusammen. H-8 hat drei Kopplungspartner und erscheint daher als dreifaches Dublett mit drei Kopplungskonstanten, wobei der entsprechende Partner H-8' mit $8 \mathrm{~Hz}$ nicht wiederzufinden ist, da er in das zuvor erwähnte Dublett fällt. Die beiden Protonen am H-7 koppeln jeweils als Doppeldublett zunächst mit $14 \mathrm{~Hz}$ miteinander und dann mit 6 bzw. 7 Hz mit H-8.

Die Protonen am C-9' erscheinen unter dem Lacton-Einfluss etwas zum Tieffeld verschoben und koppeln mit $9 \mathrm{~Hz}$ untereinander und jeweils mit 7,5 Hz mit $\mathrm{H}-8^{\prime}$, wobei das Signal des Partners im bereits erwähnten Multiplett liegt. Auf Grund der gleichen Kopplungskonstanten der Protonen am C-9' kann deren Zuordnung als austauschbar betrachtet werden.

\subsubsection{Resümee}

Die phenolischen Verbindungen in Acmella ciliata konnten auf Grund ihres überwiegend hydrophilen Charakters im Methanolextrakt der Gesamtpflanze angereichert und durch Ethylacetat-Ausschüttelung und SC an Sephadex LH 20 fraktioniert und isoliert werden. Die Strukturaufklärung erfolgte nach HPLC mittels ${ }^{1} \mathrm{H}-\mathrm{NMR}$ und MS, zumeist unter Einbeziehung von entsprechenden Vergleichssubstanzen. 
Wie unter 3.4.1. aufgeführt, spielen die Phenolcarbonsäuren eine wichtige Rolle in der Biogenese und kommen ubiqitär im Pflanzenreich als Hydroxybenzoe- und Hydroxyzimtsäuren vor. Die erstere Kategorie ist in Acmella ciliata durch Hydroxybenzoesäure selbst und als Protocatechusäure vertreten. Dominanter sind jedoch die Hydroxyzimtsäuren Kaffee-, p-Cumar- und Ferulasäure. Diese aromatischen Hydroxycarbonsäuren treten auch als Depside, d. h., als Ester mit anderen Säuren auf. Es wurden die Äpfelsäureester aller drei Phenolcarbonsäuren gefunden sowie die bisher nicht nachgewiesenen Methylester des transKaffeoyläpfelsäureesters. Darüber hinaus lag die Kaffeesäure verestert mit Chinasäure als Chlorogensäure, dem Prototyp der Depside, vor. Mit dem trans-5-Kaffeoylepichinasäuremethylester wurde eine neue phenolische Verbindung nachgewiesen.

Weiterhin konnten das Hydroxycumarin Aesculetin und die zu den Lignanen gehörende symmetrische Verbindung Matairesinol identifiziert werden. Als Flavonoide wurden Isoquercitrin und Rutosid isoliert, Miquelianin und ein nicht weiter identifiziertes Flavonoid massenspektrometrisch erfasst. Weitere Flavonoide sowie Kaffeesäurederivate sind offenbar in Acmella ciliata enthalten und konnten parallel im Arbeitskreis Jenett-Siems [99] aus einem stärker hydrophilen Extrakt (Methanol 80\%) gewonnen werden.

An phenolischen Verbindungen waren aus Acmella ciliata bisher lediglich das Hydroxycumarin Scopoletin [110, 112] und vier Flavonoide [177] bekannt.

Das Vorliegen einer breiten Palette phenolischer Verbindungen erklärt zum Teil die unter 3.4.1. beschriebenen Wirkungen von Acmella ciliata und rechtfertigt wohl zumindest partiell die empfohlene Anwendung des Handelspräparats Spolera ${ }^{\circledR}$ bei Prellungen und Verstauchungen etc., für die bisher die Alkamide als maßgeblich verantwortlich angesehen werden, die jedoch in einem hydrophilen 2-Propanolextrakt lediglich in geringer Konzentration enthalten sein dürften (vgl. 2.3.). 


\subsection{Sonstige Verbindungen}

Sowohl bei der Aufarbeitung des Dichlormethanextrakts (s. Schema in Abb. 17) als auch des Methanolextrakts (s. Schema in Abb. 33) fielen neben den jeweiligen Hauptinhaltsstoffgruppen der dominant lipophilen Alkamide bzw. der vorwiegend hydrophilen phenolischen Verbindungen einige weitere Substanzen zumeist einfacherer Strukturen an. Die Unterteilung erfolgt nach den benannten Extraktionen.

\subsubsection{Verbindungen aus Dichlormethanextrakt}

Sammelfraktion 1 resultiert aus der Extraktion der Ganzdroge von Acmella ciliata mit Dichlormethan (s. Abb. 17 und Abb. 62, Exp. Teil). Nach DC-Analytik dieser Fraktion war auf das Vorliegen hydrophilerer Substanzen (z. T. Phenole) im Vergleich zu den Alkamiden zu schließen. Die weitere Auftrennung erfolgte durch präparative HPLC.

\subsubsection{1. p-Hydroxybenzaldehyd (Verbindung $\boldsymbol{S} 1$ )}

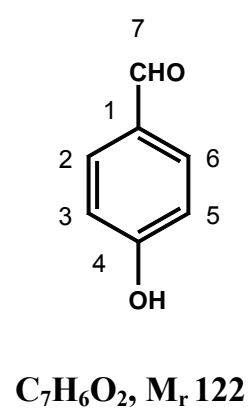

Tabelle 22: ${ }^{1} \mathrm{H}-\mathrm{NMR}$ Daten von p-Hydroxybenzaldehyd in $\mathrm{CD}_{3} \mathrm{OD}$

\begin{tabular}{|l|c|c|c|}
\hline H & $\begin{array}{c}\text { Verschiebung } \boldsymbol{\delta} \\
{[\mathrm{ppm}]}\end{array}$ & Signalform & $\begin{array}{c}\text { Kopplungskonst. J } \\
{[\mathrm{Hz}]}\end{array}$ \\
\hline 2 & 7,77 & $\mathrm{~d}$ & 8,6 \\
3 & 6,91 & $\mathrm{~d}$ & 8,6 \\
5 & 6,91 & $\mathrm{~d}$ & 8,6 \\
6 & 7,77 & $\mathrm{~d}$ & 8,6 \\
$7(\mathrm{CHO})$ & 9,76 & $\mathrm{~s}$ & - \\
\hline
\end{tabular}

Beim p-Hydroxybenzaldehyd handelt es sich um ein symmetrisch substituiertes Molekül, was zur Folge hat, dass die NMR-Signale der am Ring gegenüberliegenden Protonen $(2+6$ sowie $3+5)$ zusammenfallen. Diese ergeben ein Signal mit doppelter Intensität (2 H). In Tab. 22 sind jedoch alle Protonen einzeln aufgeführt. Das stark Tieffeld verschobene Signal bei 9,76 ppm ist typisch für das Aldehyd-Proton. Die Daten entsprechen mit geringfügigen Verschiebungen den Angaben in der Literatur (Messung in Aceton- $\mathrm{d}_{6}$ bzw. $\mathrm{CD}_{3} \mathrm{OD}$ ) [96, 109].

Die zugehörige Masse lieferte die -ESI-MS-Messung mit 121,0304 als [M-H] . 
3.5.1.2. Vanillin (Verbindung $\boldsymbol{S} 2$ )<smiles>COc1ccc(C=O)cc1O</smiles>

$\mathrm{C}_{8} \mathrm{H}_{8} \mathrm{O}_{3}, \mathrm{M}_{\mathrm{r}} 152$

Tabelle 23: ${ }^{1} \mathrm{H}-\mathrm{NMR}$ Daten von Vanillin in $\mathrm{CD}_{3} \mathrm{OD}$

\begin{tabular}{|l|c|c|c|}
\hline $\mathbf{H}$ & $\begin{array}{c}\text { Verschiebung } \boldsymbol{\delta} \\
{[\mathrm{ppm}]}\end{array}$ & Signalform & $\begin{array}{c}\text { Kopplungskonst. J } \\
{[\mathrm{Hz}]}\end{array}$ \\
\hline 2 & 7,45 & $\mathrm{~d}$ & 1,7 \\
5 & 6,94 & $\mathrm{~d}$ & 7,9 \\
6 & 7,43 & $\mathrm{dd}$ & 1,$7 ; 7,9$ \\
$7(\mathrm{CHO})$ & 9,77 & $\mathrm{~s}$ & - \\
$8\left(\mathrm{OCH}_{3)}\right.$ & 3,92 & $\mathrm{~s}$ & - \\
\hline
\end{tabular}

Das NMR-Signalmuster (Tab. 23) gleicht dem der ebenfalls 1,3,4-substituierten Protocatechusäure (P2). Die Kopplungskonstanten für Vanillin sind jedoch teilweise etwas kleiner. Charakteristisch für Vanillin sind außerdem das Signal für die drei Protonen der Methoxygruppe, die zum Singulett zusammenfallen, sowie das typische, stark zum Tieffeld verschobene Singulett der Aldehydfunktion. Diese Daten stimmen mit dem unter gleichen Bedingungen gemessenen NMR-Spektrum der Referenzsubstanz überein.

Bestätigt wurde die Struktur auch durch die hochauflösende ESI-MS-Messung. Diese ergab eine Masse von 151,0406 als [M-H] .

\subsubsection{Zimtsäure (Verbindung $\boldsymbol{S 3}$ )}

Tabelle 24: ${ }^{1} \mathrm{H}-\mathrm{NMR}$ Daten von trans-Zimtsäure in $\mathrm{CD}_{3} \mathrm{OD}$<smiles>O=C(O)C=Cc1ccccc1</smiles>

$\mathrm{C}_{9} \mathrm{H}_{8} \mathrm{O}_{2}, \mathrm{M}_{\mathrm{r}} 148$

\begin{tabular}{|l|c|c|c|}
\hline H & $\begin{array}{c}\text { Verschiebung } \boldsymbol{\delta} \\
{[\mathrm{ppm}]}\end{array}$ & Signalform & $\begin{array}{c}\text { Kopplungskonst. J } \\
{[\mathrm{Hz}]}\end{array}$ \\
\hline 2,6 & 7,59 & $\mathrm{~m}$ & - \\
$3,4,5$ & 7,4 & $\mathrm{~m}$ & - \\
7 & 7,67 & $\mathrm{~d}$ & 16,0 \\
8 & 6,48 & $\mathrm{~d}$ & 16,0 \\
\hline
\end{tabular}

Wie bei einfach substituierten Aromaten häufig, fallen auch hier Signale zusammen, so von H-2 und H-6 sowie von H-3, H-4 und H-5 (s. Tab. 24). Lediglich die Signale von H-7 und H8 lassen sich deutlich als Dublett erkennen. Die trans-Doppelbindung manifestiert sich in der großen Kopplungskonstanten von $16 \mathrm{~Hz}$. Die NMR-Daten wurden mit der Literatur [72] verglichen, und sie stimmen mit der unter gleichen Bedingungen gemessenen Referenzsubstanz überein. 
Dieses Ergebnis wird bestätigt durch die -ESI-MS-Messung. Es resultierte eine hochaufgelöste Masse von 147,0439 als [M-H] .

\subsubsection{Spilanthessäure (Deca-2E,6Z,8E-triensäure) (Verbindung $S 4$ )}

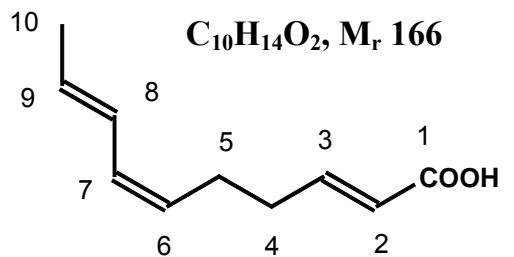

Die starke Tieffeldverschiebung des Signals für H-3, lässt sich durch den -M-Effekt der Carbonylfunktion erklären. Hier kommt der Charakter einer $\alpha, \beta$-ungesättigten Carbonylverbindung zum Tragen. Die weiteren Verschiebungen sind Tab. 25 zu entnehmen und entsprechen denen des Fettsäurerests von Spilanthol (A13).

Die Molmasse der resultierenden Säure beträgt damit 166. Diese Annahme wird durch die -ESI-MS-Messung gestützt, die eine hochaufgelöste Masse von 165,0914 als [M-H] ${ }^{-}$ergab.

Der Name Spilanthessäure wurde zuerst von MARTIN [110, 111] als Trivialname des Säurerests von Spilanthol geprägt. Nachgewiesen wurde die freie Säure allerdings nicht, so dass hier von einem neuen Naturstoff gesprochen werden kann. Leicht abweichende Signalverschiebungen in Bezug auf den Fettsäurerest des Spilanthols, welches laut Vergleichsliteratur in $\mathrm{CDCl}_{3}$ (s. o.) gemessen wurde, kommen durch das hier verwendete Lösungsmittel $\mathrm{CD}_{3} \mathrm{OD}$ zu Stande. Dieses Lösungsmittel erscheint jedoch für eine Säure auf

Tabelle 25: ${ }^{1} \mathrm{H}-\mathrm{NMR}$ Daten von Spilanthessäure in $\mathrm{CD}_{3} \mathrm{OD}$

\begin{tabular}{|l|c|c|c|}
\hline H & $\begin{array}{c}\text { Verschiebung } \boldsymbol{\delta} \\
{[\mathrm{ppm}]}\end{array}$ & Signalform & $\begin{array}{c}\text { Kopplungskonst. J } \\
{[\mathrm{Hz}]}\end{array}$ \\
\hline 2 & 5,82 & $\mathrm{brd}$ & 15,5 \\
3 & 6,95 & $\mathrm{dt}$ & 7,$0 ; 15,5$ \\
4 & $2,24-2,35$ & $\mathrm{~m}$ & - \\
5 & $2,24-2,35$ & $\mathrm{~m}$ & - \\
6 & 5,24 & $\mathrm{dt}$ & 11,$0 ; 7,5$ \\
7 & 5,97 & $\mathrm{dd}$ & 10,$0 ; 11,0$ \\
8 & 6,33 & $\mathrm{brdd}$ & 10,$0 ; 15,0$ \\
9 & 5,67 & $\mathrm{dq}$ & 7,$0 ; 15,0$ \\
10 & 1,76 & $\mathrm{~d}$ & 7,0 \\
\hline
\end{tabular}

Grund deren stärkerer Polarität als besser geeignet gegenüber dem in der Literatur für die apolareren Amide benutzten Chloroform.

$\mathrm{Zu}$ den weiteren NMRDaten: Die Protonen am C-2 
und C-3 koppeln miteinander mit 15,5 Hz, was den Charakter der trans-Doppelbindung unterstreicht. H-3 koppelt außerdem mit den beiden Protonen von H-4. Somit ergibt das Signal ein Dublett-Triplett. Die zugehörigen Kopplungspartner fallen in das Multiplett von H4/H-5. H-6 tritt mit derselben Signalform wie H-3 auf, mit ebenfalls drei Kopplungspartnern.

Auf Grund der Doppelbindung zwischen C-6 und C-7 sind die Kopplungskonstanten anders als am C-3. H-6 und H-7 koppeln mit 11 Hz, was hier die cis-Doppelbindung darstellt. H-7 koppelt außer mit H-6 auch mit dem Proton am C-8 mit $10 \mathrm{~Hz}$. Das Signal ist somit ein Doppeldublett.

H-8 koppelt neben H-7 auch mit H-9, hier jedoch mit 15 Hz. Somit befindet sich zwischen C8 und C-9 eine trans-Doppelbindung. H-9 koppelt neben H-8 auch mit den drei Protonen von H-9; damit ergibt das Signal ein Dublettquartett. Das Dublett bei 1,76 ppm schließlich steht für die drei Protonen am H-10, welche mit dem Proton H-9 koppeln. Bei fast allen Signalen sind weitere Feinstrukturen zu erkennen, ausgelöst durch Kopplungen über mehr als eine Bindung hinweg (long-range-Kopplung). Hierbei ergeben sich Signalmuster höherer Ordnung, die nicht mehr auswertbar sind.

\subsubsection{Verbindungen aus Methanolextrakt}

Diese Substanzen resultieren aus der Auftrennung der Säule IV (s. Schema in Abb. 63, Exp. Teil) bzw. des „Gelben Nachlaufs“ (s. Abb. 67, Exp. Teil) und wurden ebenfalls mittels präparativer HPLC erfasst und durch MS und NMR strukturanalytisch aufgeklärt. Hierbei wurden auch noch Alkamide höherer Polarität gefunden.

\subsubsection{Fumarsäure (trans-Butendisäure) (Verbindung $S 5$ )}

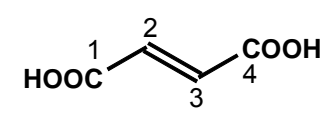

$\mathrm{C}_{4} \mathrm{H}_{4} \mathrm{O}_{4}, \mathrm{M}_{\mathrm{r}} 116$
Tabelle 26: ${ }^{13} \mathrm{C}-\mathrm{NMR}$ Daten für Fumarsäure in $\mathrm{CD}_{3} \mathrm{OD}$

\begin{tabular}{|l|c|c|}
\hline C & $\begin{array}{c}\text { Verschiebung } \boldsymbol{\delta} \\
{[\mathrm{ppm}]}\end{array}$ & Signalform \\
\hline $2,3(\mathrm{CH})$ & 135,2 & $\mathrm{~d}$ \\
$1,4(\mathrm{COOH})$ & 168,1 & $\mathrm{~s}$ \\
\hline
\end{tabular}


Die ESI-MS-Analytik erbrachte eine hochaufgelöste Molmasse von 115,0022 als [M-H]- Es wurde ebenfalls eine EI-MS-Messung bei $130^{\circ} \mathrm{C}$ durchgeführt. Diese ergab die Molmasse 116. Aus der anschließend durchgeführten -FAB-Messung mit Glycerol als Matrix resultierte m/z 115 als $[\mathrm{M}-\mathrm{H}]^{-}$. Außerdem kam es zur Clusterbildung mit der Matrix Glycerol (m/z 92). Das Signal m/z 207 steht für die Fumarsäure + Glycerol - 1 Proton als [M+Glyc] ${ }^{-}$.

Das ${ }^{1} \mathrm{H}-\mathrm{NMR}$ in $\mathrm{CD}_{3} \mathrm{OD}$ (nicht abgebildet) zeigt ein Singulett bei 6,76 mit hoher Intensität. Da es sich hier um ein symmetrisches Molekül handelt, fallen die theoretischen Dubletts für H-2 und H-3 zusammen. Eine Fumarsäure-Referenz, vermessen in Aceton- $\mathrm{d}_{6}$, zeigte identisches Verhalten im ${ }^{1}$ H-NMR. Parallel hierzu wurde das NMR-Spektrum einer Maleinsäure-Referenz (cis-Butendisäure) aufgenommen, das etwas abweichende Verschiebungen aufwies.

Im ${ }^{13} \mathrm{C}$-NMR fallen auf Grund der Symmetrie des Moleküls die beiden Methylgruppen sowie die beiden Carboxylgruppen zusammen. Diese Daten sind Tab. 26 zu entnehmen.

Fumarsäure fiel in größerer Quantität an als andere Inhaltsstoffe. Offensichtlich dient sie in der Pflanze als Baustein für höhermolekulare Inhaltsstoffe, z. B. für Verbindung $\boldsymbol{S 6}$.

\subsubsection{Fumaroylisobutylamid ((E)-4-[(2-methylpropyl)amino]-4-oxo-2-butensäure)} (Verbindung $\boldsymbol{S 6}$ )

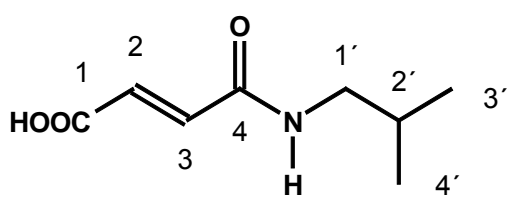

Im Vergleich zu TAKAO et al. [171], die diese Substanz in Piper hancei Maxim. fanden und eine ${ }^{1} \mathrm{H}-\mathrm{NMR}-\mathrm{Messung}$ im Lösungsmittel Aceton- $\mathrm{d}_{6}$ beschreiben, wurde hier $\mathrm{C}_{8} \mathrm{H}_{13} \mathrm{NO}_{3}, \mathrm{M}_{\mathrm{r}} 171$ deuteriertes Methanol benutzt, weshalb das breite Singulett bei ca. $7,7 \mathrm{~Hz}$ für das N-H nicht sichtbar wird. Somit erscheint das Signal für die beiden äquivalenten Protonen am C-1' als Dublett (Tab. 27). Dieses Verhalten war bei der Messung auch aller folgenden Amide, die in deuteriertem Methanol gemessen wurden, zu beobachten. Methanol wurde hier jedoch als Lösungsmittel für die NMR-Messungen präferiert, einesteils wegen der guten Methanollöslichkeit der Proben und zum anderen, da ein Methanolextrakt Ausgangsprodukt der Untersuchungen war. Ansonsten sind alle weiteren Verschiebungen mit den Literaturdaten [171] identisch und die typischen Signale des Isobutyl-Rests (3.3.3.1.) zu finden (s. Tab. 27), was zusammen mit der 
ESI-MS-Messung, die eine Molmasse von 171 bzw. hochaufgelöst eine Masse von 172,0984 als $[\mathrm{M}+\mathrm{H}]^{+}, 194,0807$ als $[\mathrm{M}+\mathrm{Na}]^{+}$und 170,0865 als $[\mathrm{M}-\mathrm{H}]^{-}$ergab, die Existenz dieser Substanz bestätigt.

Tabelle 27: ${ }^{1} \mathrm{H}-\mathrm{NMR}$ Daten von Fumaroylisobutylamid in $\mathrm{CD}_{3} \mathrm{OD}$

\begin{tabular}{|l|c|c|c|}
\hline H & $\begin{array}{r}\text { Verschiebung } \boldsymbol{\delta} \\
{[\mathrm{ppm}]}\end{array}$ & Signalform & $\begin{array}{c}\text { Kopplungskonst. J } \\
{[\mathrm{Hz}]}\end{array}$ \\
\hline 2,3 & 2,46 & $\mathrm{~m} /(4 \mathrm{H})$ & - \\
4 & 6,75 & $\mathrm{~m}$ & - \\
5 & 5,97 & $\mathrm{~d}$ & 15,3 \\
$1^{\prime}$ & 3,04 & $\mathrm{~d} /(2 \mathrm{H})$ & 6,9 \\
$2^{\prime}$ & 1,78 & $\mathrm{~m}$ & - \\
$3^{\prime}, 4^{\prime}$ & 0,91 & $\mathrm{~d}$ & 6,7 \\
\hline
\end{tabular}

Die große Kopplungskonstante von $15,4 \mathrm{~Hz}$ verdeutlicht das Vorliegen der transForm, also des „Fumarsäure“isobutylamids. Somit kommt hier die Maleinsäure als Säurekomponente nicht in Betracht.

Fumaroylisobutylamid beschreibt den Prototyp von Alkamiden, die offensichtlich aus einer Dicarbonsäure und einer Aminosäure gebildet werden. Bei S6 fungiert Fumarsäure (S5) als Baustein, die, wie bereits unter 3.5.3.2. angeführt, in größerer Menge aus Acmella ciliata isoliert wurde. Für die Familie der Asteraceae stellt $\boldsymbol{S} \boldsymbol{6}$ einen Vertreter einer neuen Stoffklasse dar.

\subsubsection{4E-Hexenoylisobutylamid ((E)-6-[(2-methylpropyl)amino]-6-oxo-4-hexen- säure) (Verbindung $S 7$ )}

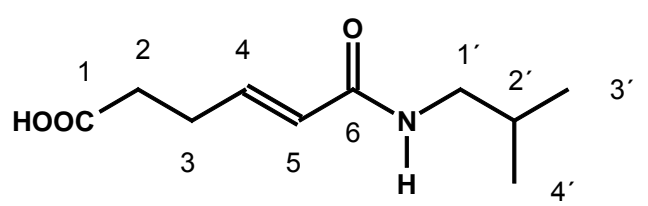

$\mathrm{C}_{10} \mathrm{H}_{17} \mathrm{NO}_{3}, \mathrm{M}_{\mathrm{r}} 199$

In Analogie zu Verbindung $\boldsymbol{S 6}$ leitet sich auch $\boldsymbol{S} 7$ von einer Dicarbonsäure ab.

Die -ESI-MS-Messung ergab eine Masse von 198,1010 als $[\mathrm{M}-\mathrm{H}]^{-}$, und aus der +ESI-MSMessung resultierte 222,1112 als $[\mathrm{M}+\mathrm{Na}]^{+}$. Dass hier die -ESI-MS-Messung ein Resultat erbrachte, liegt wie bei $\boldsymbol{S 6}$ an der Carboxyl-Gruppe als deprotonierbares Strukturelement. Die Massendifferenz zu $\boldsymbol{S 6}$ beträgt 28, was auf zwei zusätzliche Methylen-Gruppen schließen ließ. 
Für den rechten Verbindungsteil war auch hier analog zu S6 im NMR-Spektrum der IsobutylRest zu belegen (Tab. 28 und 27). Somit befinden sich die beiden zusätzlichen MethylenGruppen im linken Molekülteil.

Zur Bestimmung der Lage der Doppelbindung wurden die Signale für die Protonen der isolierten Doppelbindung von $\boldsymbol{S} 7$ zunächst mit der ebenfalls in $\mathrm{CD}_{3} \mathrm{OD}$ gemessenen

Spilanthessäure (S4; s. 3.5.1.4.) verglichen. Bei $\boldsymbol{S} 4$ befindet sich die isolierte Doppelbindung in Nachbarschaft zur Carboxylgruppe (zwischen C-2 und C-3). Die Verschiebungen für H-2 und H-3 liegen bei 5,82 und 6,95 ppm (s. Tab. 25) und stimmen damit nicht ganz mit den Verschiebungen der analogen Signale von $\boldsymbol{S} 7$ (5,97 und 6,75 ppm; s. Tab. 28) überein, was die Lage der Doppelbindung in Nachbarschaft zur Carboxylgruppe eher unwahrscheinlich macht.

Weiterhin wurde ein zweites ${ }^{1} \mathrm{H}-\mathrm{NMR}$ von $\boldsymbol{S} 7$ in $\mathrm{CDCl}_{3}$ aufgenommen, um mit Literaturdaten für Spilanthol, gemessen in $\mathrm{CDCl}_{3}$, vergleichen zu können. Es ergaben sich Verschiebungen von 5,85 und 6,85 ppm, die exakt mit Literaturwerten von Spilanthol [110,111], in dem sich die isolierte Doppelbindung in Nachbarschaft zur Amidgruppe (zwischen C-2 und C-3) befindet, korrespondieren. Damit ist die Stellung der trans-Doppelbindung von $\boldsymbol{S} 7$ in Nachbarschaft zur Amidgruppe (nach der Nomenklatur von S7, zwischen C-4 und C-5) bestätigt.

Tabelle 28: ${ }^{1} \mathrm{H}-\mathrm{NMR}$ Daten von $4 E$-Hexenoylisobutylamid in $\mathrm{CD}_{3} \mathrm{OD}$

\begin{tabular}{|l|c|c|c|}
\hline $\mathbf{H}$ & $\begin{array}{c}\text { Verschiebung } \boldsymbol{\delta} \\
{[\mathrm{ppm}]}\end{array}$ & Signalform & $\begin{array}{c}\text { Kopplungskonst. J } \\
{[\mathrm{Hz}]}\end{array}$ \\
\hline 2,3 & 2,46 & $\mathrm{~m} /(4 \mathrm{H})$ & - \\
4 & 6,75 & $\mathrm{~m}$ & - \\
5 & 5,97 & $\mathrm{~d}$ & 15,3 \\
$1^{\prime}$ & 3,04 & $\mathrm{~d} /(2 \mathrm{H})$ & 6,9 \\
$2^{\prime}$ & 1,78 & $\mathrm{~m}$ & - \\
$3^{\prime}, 4^{\prime}$ & 0,91 & $\mathrm{~d}$ & 6,7 \\
\hline
\end{tabular}

Somit koppelt H-5 nur mit H3 und ist daher als Dublett mit einer Verschiebung von 5,97 ppm zu erfassen. Die Kopplungskonstante von 15,3 $\mathrm{Hz}$ bezeichnet die transDoppelbindung analog zu S6 sowie zum Spilanthol [110, 111] bei Messung in $\mathrm{CDCl}_{3}$. $\mathrm{H}-4$ koppelt mit H-5 und mit den beiden Protonen am C-3, hinzu kommt die longe-range-Kopplung mit den Protonen an C-2, was eine Signalaufspaltung höherer Ordnung, gekennzeichnet durch das Multiplett bei 6,75 ppm in Tab. 28, ergibt. Die 
insgesamt vier Protonen der beiden Methylen-Gruppen fallen bei 2,46 ppm auf Grund der großen Signalvielfalt in einem Multiplett zusammen.

Bei der Messung in Deuterochloroform wandelt sich das Signal der beiden Protonen von C-1' in ein Triplett, da nun außerdem die Kopplung mit dem Proton des Stickstoffs sichtbar wird. Dies ist bei allen in $\mathrm{CDCl}_{3}$ gemessenen Alkamiden der Fall (vgl. Tab. 4 unter 3.3.3.2. (A1)).

$S 7$ stellt einen gänzlich neuen Naturstoff dar.

\subsubsection{4. Äpfelsäure (Monohydroxydicarbonsäure) (Verbindung $S \boldsymbol{S}$ )}<smiles>O=C(O)CC(O)C(=O)O</smiles>

$\mathrm{C}_{4} \mathrm{H}_{6} \mathrm{O}_{5}, \mathrm{M}_{\mathrm{r}} 134$

Auch die Äpfelsäure tritt als Begleitsubstanz, als Ausgangs- oder Abbauprodukt in geringer Quantität neben ihren Estern auf. Diese wurde nur durch die -ESI-MS Messung erfasst, die einen Molpeak von $\mathrm{m} / \mathrm{z} 133$ als $[\mathrm{M}-\mathrm{H}]^{-}$lieferte.

\subsubsection{Chinasäure (1,3,4,5-Tetrahydroxy-1-cyclohexen-1-carbonsäure)} (Verbindung $\boldsymbol{S 9}$ )<smiles>O=C(O)C1(O)CC(O)C(O)C(O)C1</smiles>

$\mathrm{C}_{7} \mathrm{H}_{12} \mathrm{O}_{6}, \mathrm{M}_{\mathrm{r}} 192$

Die freie Chinasäure trat neben Chlorogen- bzw. 5Kaffeoylepichinasäuremethylester auf, wie der Molpeak von m/z 191,0561 bei der -ESI-MS-Messung als [M-H] ${ }^{-}$zeigte. 


\section{Enzymatische Untersuchungen}

\subsection{Hemmung der humanen Neutrophilen-Elastase}

\subsubsection{Allgemeines}

Die humane Neutrophilen-Elastase (E.C. 3.4.21.37) wurde 1968 entdeckt. Sie ist eine Endopeptidase, die in den menschlichen neutrophilen Granulozyten neben den Proteinasen Cathepsin G und Proteinase 3, gespeichert in den azurophilen Granula, vorkommt, und besteht aus einer einzelnen Peptidkette, die sich aus 218 Aminosäuren mit vier Disulfidbrücken zusammensetzt. Als katalytisches Zentrum fungieren die Aminosäuren Serin, Histidin und Asparaginsäure in der sog. katalytischen Triade. Das pH-Optimum der Elastase liegt im Neutralbereich.

Die Granulozyten sind polymorphkernige Zellen, die zu den Leukozyten gehören, wo sie 60$70 \%$ der Blutleukozyten ausmachen. Sie werden entsprechend verschiedener Anfärbemethoden unterteilt in die oben erwähnte größte Gruppe der neutrophilen (>90\%), gefolgt von den eosinophilen (2-4 \%) und den basophilen (bis $1 \%$ ) Granulozyten. Als Zellen der Immunabwehr haben neutrophile Granulozyten die Aufgabe, opsonisierte Mikroorganismen, virusinfizierte Zellen, Tumorzellen und andere körperfremde Antigene zu phagozytieren. Hierzu besitzen sie, wie übrigens alle Granulozyten, die Fähigkeit zur Adhärenz an vaskuläres Endothel und zur Migration ins Gewebe. Außerdem enthalten die neutrophilen Granulozyten Leukozytenenzyme, wie die Serinprote(in)ase Elastase [116, 117, 118, 142].

Diese Elastase ist in der Lage, Elastin, das Strukturprotein der extrazellulären Matrix des elastischen Bindegewebes, zu spalten. Elastin, als Hauptprotein von z. B. Fasern, Sehnen, Bändern, Bronchien- und Arterienwänden, verleiht diesen Geweben eine hohe Elastizität. Hauptsächlich besteht es aus Polypetiden mit den Aminosäuren Glycin, Alanin, Prolin, Valin, Leucin und Isoleucin (chemische Strukturen s. unter 3.3.1.1. und unten). Eine Quervernetzung erfolgt durch die selten vorkommenden Aminosäuren Desmosin und Isodesmosin (s. u.). Elastin ist unlöslich in Wasser und Natronlauge und nicht denaturierbar durch Hitze.<smiles>NCC(=O)O</smiles>

Glycin

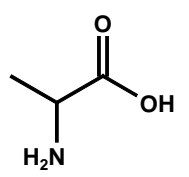

L-Alanin

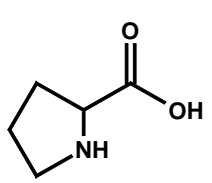

L-Prolin 
<smiles>NC(CCCC[n+]1cc(CCC(N)C(=O)O)c(CCC(N)C(=O)O)c(CCC(N)C(=O)O)c1)C(=O)O</smiles>

Desmosin<smiles>NC(CCCC[n+]1cc(CCC(N)C(=O)O)cc(CCC(N)C(=O)O)c1CCCC(N)C(=O)O)C(=O)O</smiles>

Isodesmosin

Die Elastase ist auf Grund ihrer geringen Substratspezifität auch in der Lage, weitere physiologisch bedeutsame Proteine zu spalten, beispielsweise verschiedene Kollagen-Typen und Plasmaproteine, wie Immunglobuline, Gerinnungs- und Komplementfaktoren. Die Fähigkeit der Proteinspaltung in Geweben ist von Bedeutung bei der Embryogenese, beim Um- und Neubau von Geweben sowie bei deren Abbau nach Schädigung durch Antigene [73, $115,116,142,146]$.

Eigentliche Aufgabe der Neutrophilen-Elastase ist die Proteolyse phagozytierter Partikeln (Bakterien (gramnegativ), Immunkomplexe) im Verlauf einer Entzündungreaktion. Die neutrophilen Granulozyten werden im frühen Stadium einer Entzündung aktiviert, „,gelockt“ durch verschiedene Zytokine und Chemoattractants (z. B. TNF- $\alpha$, IL-8, C5a, LPS, PAF, PMA, fMLP). Aus diesen werden die azurophilen Granula, Speicherort der Elastase, durch Exozytose ausgeschleust. Das Enzym wird anschließend in den Extrazellularraum freigesetzt und wandert zum Infektionsherd bzw. geschädigten Gewebe. Im Akutfall ist die Neutrophilen-Elastase notwendig zur Gewebereparatur (s. o.). Sie aktiviert weitere Proteinasen, die am Zusammenbruch von Matrixkomponenten beteiligt sind und inhibiert gleichzeitig körpereigene Proteinase-Inhibitoren.

Die Elastase ist auch in der Lage, die Bildung von IL-8, IL-6, GM-CSF zu induzieren und lockt neutrophile Granulozyten in entzündetes Gewebe. Darüber hinaus aktiviert Elastase die Metalloproteinase 9 (MMP-9), die ebenfalls an Entzündungsprozessen sowie an der Metastasierung von Tumoren beteiligt ist. Die Neutrophilen-Elastase stimuliert menschliche Nichtepithelzellen zur Produktion von entzündungsfördernden Zytokinen. Diese Zellen 
werden durch die Neutrophilen-Elastase zur Bildung von IL-8 und MCP-1 angeregt, vermittelt über den PAR-2-Weg (s. 4.2. und 4.3.). Das Auftreten von Elastase kann als Marker für (das Ausmaß von) Entzündungsreaktionen dienen [116, 118, 139, 142, 146, 176].

Der Abbau von infiziertem Gewebe, bei akuten Entzündungen ein sinnvoller Prozess, kann jedoch bei chronisch-entzündlichen Erkrankungen zu einem Problem werden. Normalerweise binden und inaktivieren endogene Serinproteinase-Inhibitoren (Alpha-1-Antitrypsin, Elafin, SLPI), (überschüssige) Elastase und verhindern damit die großflächige Zerstörung von extrazellulärem Bindegewebe. Jedoch kommt es besonders während chronischer Entzündungsprozesse, wie chronisch-obstruktive Lungenerkrankungen (COPD: chronische Bronchitis, Lungenemphysem), Mukoviszidose, rheumatoide Arthritis, aber auch bei Infektionskrankheiten, zur Störung des Elastase/Elastase-Inhibitor-Verhältnisses, beispielsweise durch Akkumulation großer Mengen an freigesetzten Proteinasen oder Oxidantien sowie durch sehr enge Bindung der Elastase an Extrazellulärmatrixzellen. Letzteres verhindert einen Inhibitorangriff. Die Folgen sind Verschlimmerung von Entzündungen und Unterdrückung von Heilungsprozessen. Extrem problematisch kann dieser Bindegewebszerstörungsprozess bei Patienten mit einem Alpha-1-Antitrypsin-Mangel werden. Es existieren Hinweise, dass der durch Alpha-1-Antitrypsin-Mangel bedingte Überschuss an Neutrophilen-Elastase mitverantwortlich für die Entwicklung und Metastasierung von Tumoren sein könnte. Außerdem hat die Elastase durch Bindegewebsabbau und damit verbundenem Verlust von Hautelastizität Anteil an frühzeitiger Hautalterung nach erhöhter Sonnenexposition $[116,117,118,161]$.

Im Falle dieser Erkrankungen erscheint es folglich als sinnvoll, den (extrazellulären) Gehalt an Neutrophilen-Elastase gering zu halten bzw. sogar zu verringern. Zu diesem Zweck befindet sich bereits ein synthetischer Inhibitor der Elastase (GW 311616A; GlaxoWellcome) in Erprobung [118]. Studien mit pflanzlichen Inhaltsstoffen etc. zeigten, dass auch Naturstoffe wirkungsvolle Inhibitoren der Neutrophilen-Elastase sein können [116 - 118, 146].

Ziel dieser Arbeit war es, verschiedene Extrakte von Acmella ciliata in vitro mittels eines Enzymassays hinsichtlich ihrer Elastase hemmenden Wirkung zu untersuchen und mit dem Handelspräparat Spolera ${ }^{\circledR}$ zu vergleichen, dessen Indikation auf entzündliche Prozesse zielt. Hieraus sollten Aussagen zu möglichen Wirkungssteigerungen, beispielweise durch Anwendung anderer Extrakte, resultieren. Von besonderem Interesse schien der Einfluss von 
Alkamiden bzw. Alkamide enthaltenden Extrakten. Derartige enzymatische Untersuchungen wurden bisher nicht durchgeführt. Darüber hinaus wurde ein Ausblick auf weitere potentielle Anwendungsmöglichkeiten von Acmella ciliata-Zubereitungen angestrebt. Dies steht im Zusammenhang mit der Entwicklung potentieller galenischer Formulierungen, besonders zur Anwendung im Mundbereich (s. 5.3.).

\subsubsection{Untersuchungen}

Der hier angewendete in vitro-Enzymassay soll zeigen, wie verschiedene pflanzliche Zubereitungen in unterschiedlichen Konzentrationen in der Lage sind, die Spaltungsaktivität der Elastase gegenüber einem bestimmten Substrat zu beeinflussen / zu hemmen. Ermittelt wird die jeweilige Restaktivität der Elastase. Die Differenz dieses Wertes zu 100 ergibt die prozentuale Hemmung der Aktivität des Enzyms durch die pflanzliche Zubereitung. Als Leitwert gilt der $\mathrm{IC}_{50}$-Wert (inhibitory concentration), also der Wert, bei welchem die Restaktivität bzw. die Hemmung der Elastase 50 \% beträgt. Als „Ersatz“ für das menschliche Bindegewebe fungiert als Substrat ein Peptid mit vier Aminosäuren, das mit einem Nitroaromaten gekoppelt ist (N-methoxysuccinyl-Ala-Ala-Pro-Val-p-Nitroanilid).

Zur Untersuchung gelangten die Acmella ciliata-Extrakte $\mathrm{A}_{3}, \mathrm{~B}_{2}, \mathrm{C}_{4}, \mathrm{D}_{1}$ und $\mathrm{E}_{1}$ (s. 2.3., Tab.1), ebenso die entsprechenden Extrakte von Acmella oleracea (gelb), in beiden Reihen aus getrocknetem Pflanzenmaterial (Dahlem, 2005). Acmella oleracea (rot) stand für diese Messungen nicht zur Verfügung. Neben der Aktivität dieser verschiedenen Extrakte konnten so auch vergleichende Aussagen zu den beiden Acmella-Arten getroffen werden. Darüber hinaus wurden Aceton- und Dichlormethanextrakte aus Blütenmaterial verschiedener Anbaujahre (getrocknet und teilweise frisch) in die Untersuchungen einbezogen. Außerdem kam das Präparat Spolera ${ }^{\circledR}$-flüssig, nach Entfernen des Lösungsmittels unter vermindertem Druck, sowie das in Acmella ciliata nachgewiesene Alkamid Dodeca-2,4,8,10 (E,E,Z,E / E,E,Z,Z)-tetraensäure-isobutylamid(e) $[110,112]$ als Reinsubstanz zur Testung.

Die Proben wurden in DMSO bzw. Pufferlösung gelöst bzw. entsprechend verdünnt (s. Exp. Teil), um die jeweiligen Konzentrationen zur Ermittlung der $\mathrm{IC}_{50} \mathrm{zu}$ erzielen, und nacheinander unter Schütteln in Vials pipettiert. Die anschließende Inkubation erfolgte im Wasserbad während einer Stunde bei $37^{\circ} \mathrm{C}$, um in vivo-Bedingungen zu simulieren. Dabei kommt es zur Abspaltung der Nitrogruppe des Peptids (Substrat) durch die Elastase. Die 
Reaktion wurde durch eine in vitro wirksame Trypsin-Inhibitorlösung aus Sojabohnen gestoppt und die Lösung UV/VIS-fotometrisch vermessen (Nitrogruppe $=$ Chromophor). Je mehr Nitroaromat abgespalten wird, desto höher ist der Wert für die gemessene Absorption als direktes Maß für die Restaktivität der Elastase.

Ein synthetischer, totaler Inhibitor der Elastase (GW 311616 A) als Positivkontrolle wurde stichprobenartig mit vermessen, um das „Funktionieren“ des Essays zu überprüfen (vgl. Exp. Teil).

\subsubsection{Ergebnisse und Diskussion}

Abb. 52 vergleicht zunächst die Extrakte $\mathrm{E}_{1}, \mathrm{~A}_{3}, \mathrm{~B}_{2}$ und $\mathrm{C}_{4}$ von Acmella ciliata und Acmella oleracea (ohne den schwach hemmenden Ethanol 45 \%-Extrakt $\mathrm{D}_{1}$ ) mit Spolera ${ }^{\circledR}$-flüssig und der Alkamidreferenz in der einheitlichen Konzentration von $1 \mu \mathrm{g} / \mathrm{ml}$. Es ist für Acmella ciliata zu erkennen, dass der Aceton-Extrakt $\left(E_{1}\right)$ in dieser geringen Konzentration noch eine Hemmaktivität von über $60 \%$ aufweist. Beim Dichlormethan $\left(\mathrm{A}_{3}\right)$ - und Methanol $\left(\mathrm{B}_{2}\right)$ Extrakt wird die $\mathrm{IC}_{50}$ erreicht bzw. knapp überschritten, während der Auszug mit 2-Propanol $60 \%$ sowie das Konzentrat von Spolera ${ }^{\circledR}$-flüssig bei Hemmwerten von 30 bzw. 40 \% liegen. Die Alkamid-Referenz in dieser Konzentration $\left(0,1 \mu \mathrm{g} / \mathrm{ml} \approx 4,0 \mu \mathrm{M} / \mathrm{M}_{\mathrm{r}} 247\right)$ hemmt das Enzym am stärksten mit über $90 \%$. Die Hemmung durch die Acmella oleracea-Extrakte tendiert jeweils zu etwas höheren Werten.

Zwischen 0,5 und $1 \mu \mathrm{g} / \mathrm{ml}$ erfolgte bei der Testsubstanz ein stärkerer Abfall der prozentualen Enzymaktivitätshemmung als bei den Extrakten, so dass die $\mathrm{IC}_{50}$ wenig oberhalb derjenigen der Aceton-Ganzpflanzen-Extrakte beider Pflanzen und des Dichlormethan-Extrakts von Acmella oleracea liegt (s. Tab. 29).

Abb. 53 zeigt den Vergleich der vier Extrakte im Konzentrationsbereich von (0,1)0,5-10 $\mu \mathrm{g} / \mathrm{ml}$. Obwohl sich signifikante Unterschiede nur in einzelnen Messpunkten ergaben, zeichnet sich bereits nach diesen Untersuchungen der Trend ab, dass eine Wirkungssteigerung eines Acmella ciliata-Präparats durch den Austausch des verwendeten Extraktionsmittels zu erwarten wäre. 


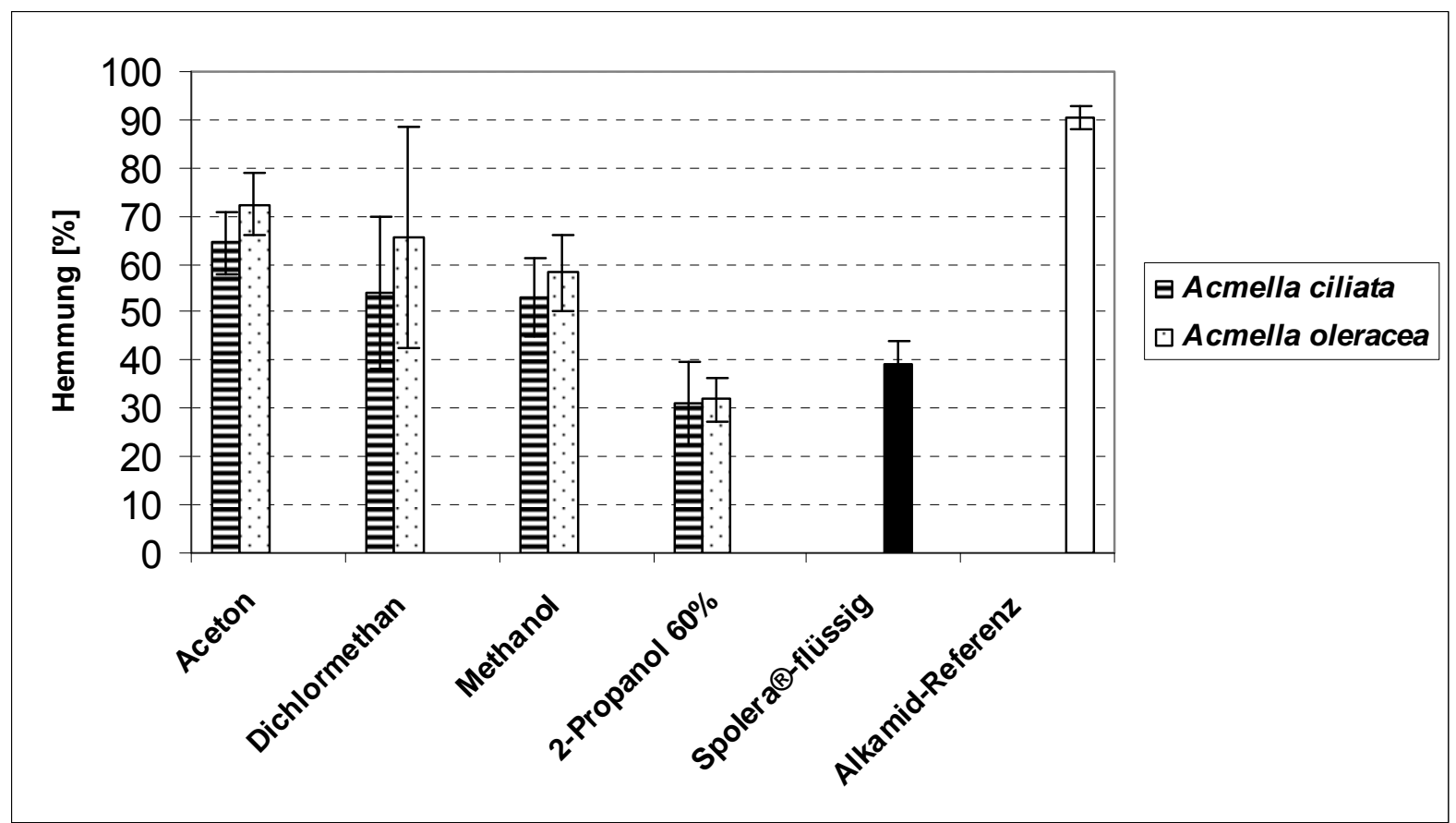

Abb. 52: Elastasehemmung von $\boldsymbol{A}$. ciliata- und $\boldsymbol{A}$. olercaea-Extrakten und Referenzen in der Konzentration $1 \mu \mathrm{g} / \mathrm{ml}$

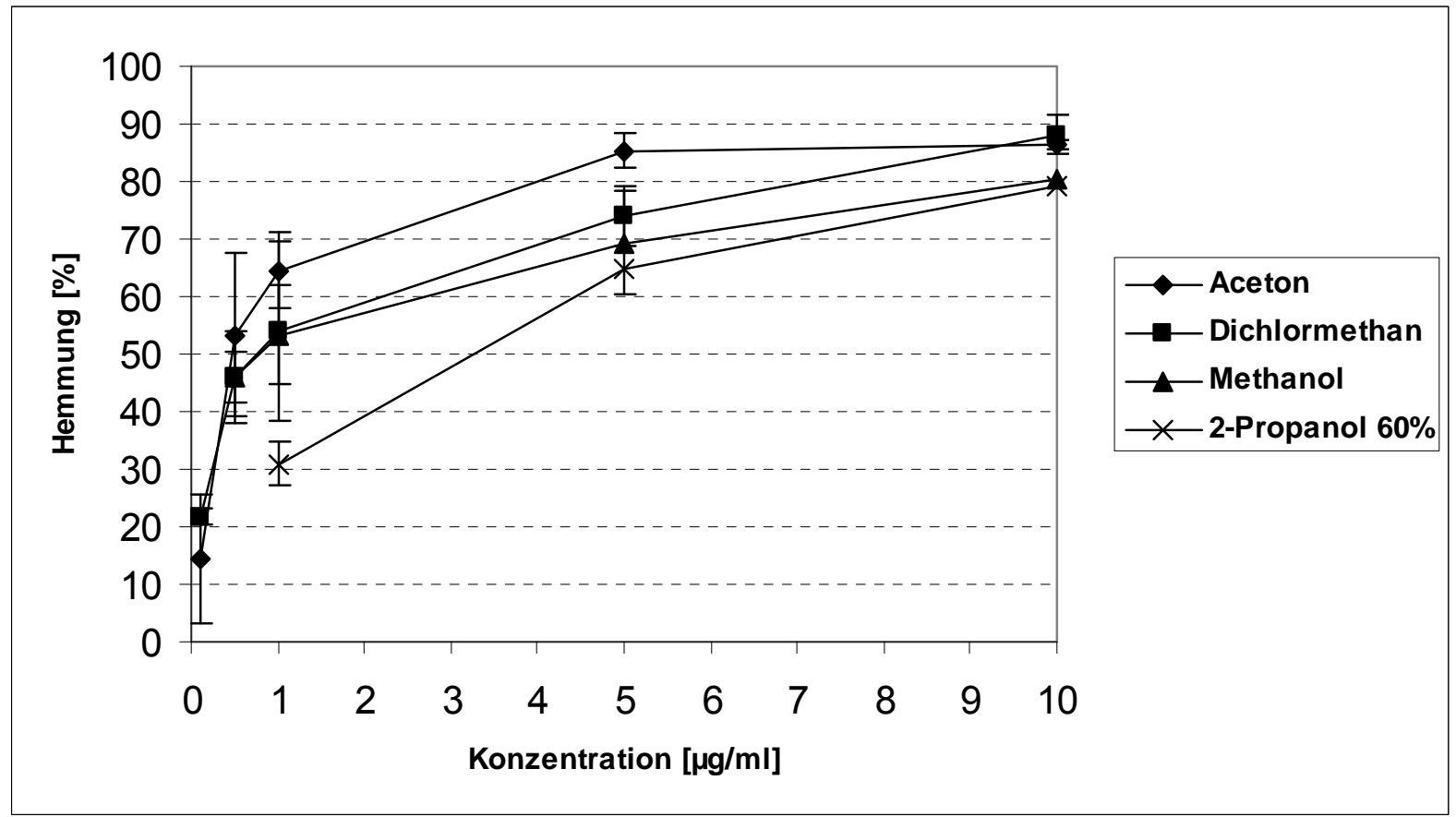

Abb. 53: Konzentrationsabhängige Elastasehemmung von A. ciliata-Extrakten im Bereich $0,1-10 \mu \mathrm{g} / \mathrm{ml}$

Mittels H-Test nach KRUSKAL und WALLIS (s. Exp. Teil), berechnet für die Hemmwerte bei der Extraktkonzentration von $1 \mu \mathrm{g} / \mathrm{ml}$ (s. o.), konnte bewiesen werden, dass das Extraktionsmittel sowohl bei Acmella ciliata als auch Acmella oleracea signifikanten Einfluss hat $(\mathrm{p}=0,0125$ bzw. 0,0171). Der anschließende multiple Vergleich nach DunN ergab 
allerdings bei beiden Pflanzen lediglich einen signifikanten Unterschied zwischen den Extraktionsmitteln Aceton und 2-Propanol. Einschränkend ist auf den relativ geringen Probenumfang hinzuweisen $(\mathrm{n} \geq 3)$.

Tabelle 29: IC $_{50}$-Werte der Extrakte (beide AcmellaDrogen) und Referenzen

\begin{tabular}{|l|c|c|}
\hline Extrakte/Referenzen & \multicolumn{2}{|c|}{$\begin{array}{c}\text { IC } \\
{[\mu \mathrm{g} / \mathrm{ml}]}\end{array}$} \\
& A. ciliata & A. oleracea \\
\hline Dichlormethan $\left(\mathrm{A}_{3}\right)$ & 0,8 & 0,4 \\
Methanol $\left(\mathrm{B}_{2}\right)$ & 0,8 & 0,7 \\
2-Propanol $60 \%\left(\mathrm{C}_{4}\right)$ & 3,3 & 2,8 \\
Ethanol 45\% $\left(\mathrm{D}_{1}\right)$ & 8,0 & 8,7 \\
Aceton $\left(\mathrm{E}_{1}\right)$ & 0,5 & 0,4 \\
Spolera ${ }^{\circledR}$-flüssig & & 1,9 \\
Alkamid-Referenz & \multicolumn{2}{|c|}{$0,6(2,4 \mu \mathrm{M})$} \\
\hline
\end{tabular}

In Tab. 29 sind die $\mathrm{IC}_{50}$-Werte aller Testpräparate zusammengefasst und die Ergebnisse für Acmella ciliata und Acmella oleracea gegenübergestellt. Die grafische Darstellung der prozentualen Hemmung als Funktion der Konzentration (Abb. 53) ergab, mit Ausnahme der Alkamid-Referenz (s. u.), einen logarithmischen Zusammenhang. Da jedoch die gemessenen Werte im Bereich der $\mathrm{IC}_{50} \quad \mathrm{zu}$ stark von der Regressionsgeraden abwichen, erfolgte die Berechnung der in Tab. 29 angegebenen Werte nicht durch lineare Regression, sondern durch lineare Interpolation. Hierbei wird zwischen den die $\mathrm{IC}_{50}$ einschließenden Werten ein linearer Zusammenhang angenommen (s. auch Exp. Teil).

Entsprechend den Daten in Tab. 29 ist der Aceton-Extrakt $\left(\mathrm{E}_{1}\right)$ mit einer $\mathrm{IC}_{50}$ von 0,5 bzw. 0,4 $\mu \mathrm{g} / \mathrm{ml}$ als der am stärksten hemmende Extrakt einzustufen. Die geringste Aktivität zeigte der Ethanol $45 \%$-Extrakt $\left(\mathrm{D}_{1}\right)$ mit einer $\mathrm{IC}_{50}$ von 8,0 resp. $8,7 \mu \mathrm{g} / \mathrm{ml}$.

Der auch im Präparat Spolera ${ }^{\circledR}$ verwendete 2-Propanol $60 \%$-Extrakt $\left(\mathrm{C}_{4}\right)$ schnitt mit einer $\mathrm{IC}_{50}$ von 3,3 / $2.8 \mu \mathrm{g} / \mathrm{ml}$ schlechter ab. Demgegenüber wurde für das Originalpräparat Spolera ${ }^{\circledR}$ selbst ein deutlich besserer Wert $\left(\mathrm{IC}_{50} 1,9 \mu \mathrm{g} / \mathrm{ml}\right)$ ermittelt, was auf andere Wachstums-bedingungen der Pflanze (Boden, Dünger, Klima (verschiedene Jahrgänge) etc.) und/oder industriell optimierte Extraktionsverfahren zurückzuführen sein könnte.

In diesem Zusammenhang ist eine vergleichende Untersuchung eines Aceton-Blütenextrakts von $2003\left(\mathrm{E}_{2}\right.$ mit $\mathrm{IC}_{50}$ 0,5) und zweier Dichlormethan-Blütenextrakte von Acmella ciliata aus 
unterschiedlichen Anbaujahren (2003 und 2004) mit sehr differenten klimatischen Bedingungen $\left(\mathrm{A}_{4}\right.$ und $\mathrm{A}_{5}$ mit $\mathrm{IC}_{50}$ jeweils 0,5$)$ sowie aus Frischpflanzenmaterial $\left(\mathrm{G}\right.$ mit $\mathrm{IC}_{50}$ 2,0), gewonnen durch Gewächshausaufzucht 2006, interessant. Signifikante Unterschiede konnten hier lediglich für den Frischpflanzenextrakt konstatiert werden, was wohl durch den höheren Wassergehalt zu erklären ist.

Wie aus Tab. 29 hervorgeht, wiesen der Methanol-Extrakt $\left(\mathrm{B}_{2}\right)$, der reich an phenolischen Verbindungen ist (s. Abb. 15 und 16) sowie der an Alkamiden reiche Dichlormethanextrakt $\left(\mathrm{A}_{3}\right)$ mit $\mathrm{IC}_{50}$-Werten von $0,8(0,7)$ bzw. $0,8(0,4) \mu \mathrm{g} / \mathrm{ml}$ starke und nahezu identische Hemmungen auf. Der niedrigste Acmella ciliata-IC $\mathrm{I}_{50}$-Wert für den Aceton-Extrakt ( $\left.\mathrm{E}_{1}\right)$ erklärt sich möglicherweise aus dem Vorhandensein sowohl von Alkamiden als auch Phenolen, zurückzuführen auf die gegenüber Dichlormethan (Extrakt $\mathrm{A}_{3}$ ) größere Polarität des Extraktionsmittels Aceton (vgl. aber Signifikanzberechnung oben!). Dagegen war Ethanol 45 \% $\left(\mathrm{D}_{1}\right)$, das Auszugsmittel mit der höchsten Polarität, offenbar nur begrenzt zur Extraktion von Elastase wirksamen Inhaltsstoffen geeignet - es extrahierte vorrangig hydrophile Bestandteile, wie Zucker und Saponine - und ist demzufolge hinsichtlich der Anwendungsorientierung nicht relevant.

Aus diesen Ergebnissen lässt sich schlussfolgern, dass hier nicht nur ein Wirkstoff bzw. eine Wirkstoffklasse für die Wirkung verantwortlich ist, sondern durch ein Zusammenspiel mehrerer Komponenten im Extrakt Synergieeffekte die Wirkung verstärken. Eine direkte Zuordnung der Elastasehemmung $\mathrm{zu}$ einer Inhaltsstoffgruppe oder bestimmten Einzelkomponenten in den Extrakten lässt sich demnach nicht treffen, und vergleichbare Untersuchungen als Interpretationshilfe liegen nicht vor.

Für einzelne phenolische Verbindungen (Reinsubstanzen), die auch in Acmella ciliata vorkommen und besonders im Methanolextrakt dominieren, wurden allerdings Messungen zur Aktivitätshemmung der Neutrophilen-Elastase durchgeführt [115, 116, 106]. Demnach hemmt Chlorogensäure $\left(\mathrm{IC}_{50}>400 \mu \mathrm{M}=141,6 \mu \mathrm{g} / \mathrm{ml}\right)$ schlecht, Kaffeoyläpfelsäureester $\left(\mathrm{IC}_{50} 33,7 \mu \mathrm{M}=10,0 \mu \mathrm{g} / \mathrm{ml}\right.$ ) und freie Kaffeesäure ( $\mathrm{IC}_{50} 93 \mu \mathrm{M}=16,7 \mu \mathrm{g} / \mathrm{ml}$ ) wiesen mittelstarke und die Flavonoide Rutosid ( IC $_{50} 0,8 \mu \mathrm{M}=6,0 \mu \mathrm{g} / \mathrm{ml}$ ) und Isoquercitrin ( $\mathrm{IC}_{50} 1,4$ $\mu \mathrm{M} \approx 0,65 \mu \mathrm{g} / \mathrm{ml})$ starke Hemmwirkungen auf. Da jedoch für die in Acmella ciliata nachgewiesenen Phenole keine Quantifizierungen vorgenommen wurden, ist eine vergleichbare Einstufung nicht möglich. Insgesamt kann aber dieser Wirkstoffklasse ein 
beträchtliches Hemmpotential gegenüber Elastase zugeschrieben werden, das offenbar durch die weiteren Wirkstoffe, vorrangig Alkamide, noch gesteigert wird.

Einschränkend hinzufügen ist, dass die obigen, im in vitro-Assay erstellten Aussagen der Abgleichung mit entsprechenden in vivo-Tests bedürfen. Ester der Kaffeesäure wurden z. B. im Blut nachgewiesen und kommen folglich als direkte Elastase-Inhibitoren in Betracht. Dagegen sind hydrophile Flavonoidglykoside, wie Rutosid und Isoquercitrin, auf Grund hoher Biotransformationsraten systemisch wenig verfügbar, und auch deren mögliche Abbauprodukte erwiesen sich kaum aktiv als Elastase-Hemmer [161]. Hinsichtlich der Bioverfügbarkeit scheint das eher lipophile Inhaltsstoffspektrum im Dichlormethan- und Acetonextrakt, die sich beide in vitro nicht signifikant unterschieden (s. o.), die bessere Wahl für einen möglichen in vivo-Einsatz zu sein. Zur Bioverfügbarkeit von Alkamiden liegen z. B Untersuchungen vor: sie waren im menschlichen Blut nachweisbar [34, 35, 114, 181, 183, $184]$.

$\mathrm{Zu}$ Alkamiden, die vor allem im Dichlormethanextrakt vorherrschen, oder anderen an Alkamiden reichen Pflanzenpräparaten gibt es bisher keine derartigen enzymatischen Untersuchungen. Die Elastasewirkung von Alkamiden betreffend, ist aber eine in vitro-Studie an freien Fettsäuren - partielle Bestandteile der Alkamide - interessant, deren Hemmaktivität sowohl mit der Anzahl der Doppelbindungen als auch mit der Kettenlänge zunahm [146]. Im Primärstoffwechsel stellen beispielsweise Öl-, Linol- und Linolensäure die Fettsäurebausteine der Alkamide dar (s. 3.3.1.1.). Für diese wurden $\mathrm{IC}_{50}$-Werte von 5-15 $\mu \mathrm{M}$ bestimmt.

Resümierend ergibt sich, dass auf Grund der komplexen Wirkung - hier die NeutrophilenElastase-Hemmung betreffend - die pharmazeutische Anwendung (zumindest extern) eines Acmella ciliata-Extrakts, entsprechende in vivo-Befunde vorausgesetzt, durchaus als sinnvoll $\mathrm{zu}$ beurteilen ist, nicht nur wegen der synergistischen Wirkung der Inhaltsstoffe, sondern letztlich auch aus ökonomischen Gründen, um eine kostenintensive Einzelstoffisolierung zu umgehen. Nach den vorliegenden Resultaten wäre hierfür der Aceton-Extrakt zu favorisieren. Für das Präparat Spolera ${ }^{\circledR}$ erscheint es nach diesen Untersuchungen als nicht realistisch, in erster Linie Alkamide für die Wirkung verantwortlich zu machen, da ein 2-Propanol 60 \%Extrakt Alkamide nur in geringer Quantität aufweist, allerdings, verglichen mit dem Methanolextrakt, auch nicht reich an Phenolen ist. 


\section{2. $\quad$ Trypsin-Hemmung}

\subsubsection{Allgemeines}

Auch das Enzym Trypsin stellt eine Proteinase (Endopeptidase) mit Serin, Histidin und Asparaginsäure im katalytisch aktiven Zentrum dar und wird, wie auch die NeutrophilenElastase (4.1.) und Thrombin (4.3.), den Serinproteasen bzw., nach neuerer Nomenklatur, den Serin-Peptidasen zugeordnet $[43,97,142]$.

In den exokrinen Acinuszellen des Pankreas wird zunächst die Vorstufe, das Proenzym Trypsinogen, gebildet und in den Dünndarm sezerniert. Während der Verdauung entsteht dort, in Anwesenheit von $\mathrm{Ca}^{2+}$-Ionen, durch Abspaltung eines Hexapeptids aus Trypsinogen Trypsin, katalysiert durch Enteropeptidase und Trypsin-Spuren (autokatalytisches Element).

Die eigentliche Aufgabe des Verdauungsenzyms Trypsin ist die Hydrolyse von Peptiden, wobei der Angriff substratspezifisch nach Arginin- und Lysin-Resten in der Mitte der Kette erfolgt. Auf Grund dieses Angriffspunkts zählt Trypsin auch zu den Endopeptidasen. Das pHOptimum von Trypsin liegt bei 7,5-8,5 [43, 97, 142].

Trypsin fungiert außerdem als Aktivator der Protease-aktivierten Rezeptoren, von denen bisher vier (PAR 1-4) bekannt sind und die erst Anfang der 1990er Jahre entdeckt wurden. Bei diesem Rezeptortyp handelt es sich um G-Protein-gekoppelte Rezeptoren mit sieben transmembranären Domänen [43, 166]. Zu ihrer Aktivierung muss ihr Ligand, der Teil der Rezeptorstruktur ist, durch proteolytische Abspaltung eines kurzen Peptids freigelegt werden. Die Abspaltung dieses Peptids erfolgt proteolytisch durch Angriff von Serin-Proteasen, wobei die Affinität der verschiedenen Proteasen an den einzelnen PAR-Rezeptoren unterschiedlich ist. Während Trypsin hohe Affinität an PAR-2 und PAR-4, aber geringe Affinität zu PAR-1 und PAR-3 aufweist, besitzt Thrombin eine hohe aktivierende Affinität gegenüber PAR-1, PAR-3 und PAR-4, wohingegen PAR-2 gegenüber Thrombin resistent ist [43, 71].

Neben physiologischen Vorgängen, wie Gewebereparatur nach Verletzung und Gewebeumbau sowie Prozessen während der Embryogenese, spielen die PARs eine wichtige Rolle bei Entzündungs- und Immunreaktionen. Auch einige Mediatoreffekte von Serinproteinasen bei der Zellkommunikation werden über PAR-Rezeptoren vermittelt. Zuweilen zeigen die PARs nach Aktivierung durch entsprechende Serinproteinasen 
proinflammatorische Eigenschaften durch Aktivierung entsprechender Entzündungsmediatoren und Zytokine. Mitunter werden PARs auch schützende, antientzündliche Eigenschaften zugesprochen [166].

Da PAR-Rezeptoren in vielen Geweben vorkommen, erscheint der Ansatz, über die Hemmung der die PARs aktivierenden entsprechenden Protease(n), in die Pathogenese von Krankheitsprozessen einzugreifen, als sinnvoll. Dies kann von Bedeutung sein, wenn das Verhältnis von Protease zu ihren natürlich vorkommenden Inhibitoren (z. B. Cathepsin G, Elastase) gestört ist, was Entzündungs- und übersteigerte Immunreaktionen provoziert. Speziell erscheint in manchen Fällen die Hemmung von Trypsin relevant, da eben der PAR 2Rezeptor, an dem - neben dem PAR-4-Rezeptor - die Trypsin-Aktivität am höchsten ist und der u. a. an den Bildungs- und Wirkorten (Pankreas/Dünndarm) des Trypsins vorkommt ${ }^{1}$, für inflammatorische Prozesse mitverantwortlich gemacht wird [43, 71]. Unter diesem Aspekt erschien auch die Überprüfung von Acmella ciliata-Extrakten hinsichtlich ihrer Trypsin hemmenden Potenz von Interesse.

\subsubsection{Untersuchungen}

Es sollten exemplarisch ausgewählte Acmella ciliata-Extrakte sowie die Alkamid-Referenz (s. 4.1.2.) auf eine mögliche Trypsinhemmung in einem in vitro-Enzymassay überprüft werden. Als Extrakte wurden der Aceton $\left(E_{1}\right)$ - und der Dichlormethan-Extrakt $\left(A_{3}\right)$, die bereits eine hohe Elastase-Hemmung gezeigt hatten (4.1.3.), in die Versuche einbezogen.

Die Versuchsdurchführung erfolgte im Wesentlichen ähnlich der Elastase-Bestimmung, simuliert auch hier in-vivo-Bedingungen und ist im Exp. Teil detailliert beschrieben. Ein Trypsin-Inhibitor (s. Exp. Teil, 1.1.5.) diente als Positivkontrolle.

Die Prüfung auf Signifikanz der Unterschiede der Hemmaktivitäten erfolgte mittels U-Test nach WiLCOXON, MANN und Whitney $(p<0,05)$ [43, 67].

\footnotetext{
1) Neben dem Gastrointestinaltrakt kommt der PAR-2-Rezeptor in den Immunzellen, Gelenken, in der Haut, den Atemwegen, im Gehirn und im peripheren Nervensystem sowie in der Vaskulatur vor und ist obendrein in Tumoren entdeckt worden [43].
} 
4.2.3. Ergebnisse und Diskussion

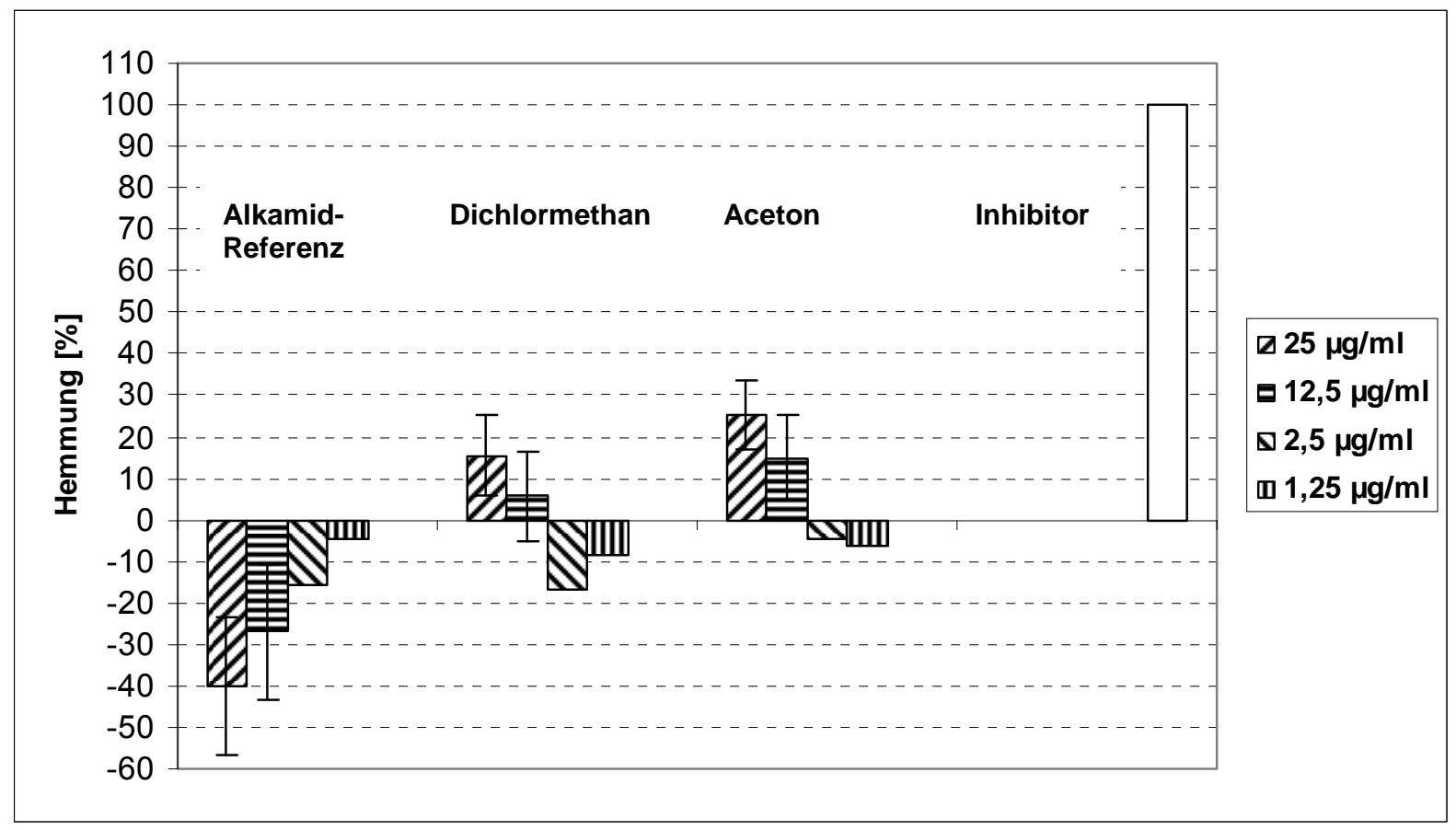

Abb. 54: Vergleich der Hemmaktivitäten [\%] des A. ciliata-Dichlormethan- und Acetonextrakts mit Alkamidreferenz und Inhibitor in abgestufter Konzentration

Aus Abb. 54 geht hervor, dass in einer Konzentration bis $\mathrm{zu} 25 \mu \mathrm{g} / \mathrm{ml}$ weder der Dichlormethan $\left(\mathrm{A}_{3}\right)$ - noch der Aceton $\left(\mathrm{E}_{1}\right)$-Extrakt die $\mathrm{IC}_{50}$ erreichen, wobei letzterer $\mathrm{zu}$ höheren Werten tendiert, maximal $25 \pm 8 \%$. Die Unterschiede zur unbehandelten Kontrolle zeigten allerdings Signifikanz. Eine Konzentrationserhöhung war aus Löslichkeitsgründen nicht möglich. Die Funktionsfähigkeit das Assays wurde durch den Inhibitorhemmwert von $100 \%$ bestätigt (Positivkontrolle).

Auffällig ist das Resultat mit dem lipophilen Alkamid-Isomerengemisch, dass in allen Konzentrationen anstatt einer Hemmung eine signifikante $(\mathrm{p}<0,05)$ Aktivierung der TrypsinAktivität zeigte, die bei einer Konzentration von $25 \mu \mathrm{g} / \mathrm{ml}(101 \mu \mathrm{M})$ bei $40 \pm 16 \%$ liegt. Eine Tendenz zur Trypsinaktivierung fällt gleichfalls in den niedrigen Konzentrationen der beiden Acmella-Extrakte $\left(\mathrm{A}_{3}>\mathrm{E}_{1}\right)$ auf. Für dieses Phänomen könnten die Alkamide verantwortlich zeichnen. So ist der bekannte Scharfstoff-Effekt des Alkamids Capsaicin bei der Verwendung von Capsicum-Arten als Nahrungsmittel und Gewürz hinsichtlich der verdauungsfördernden Wirkung möglicherweise nicht nur auf eine gesteigerte Sekretion von Magensäure, sondern auch auf eine Steigerung der Trypsin-Aktivität zurückzuführen. 
In den beiden, mit Lösungsmitteln unterschiedlicher Polarität gewonnenen Extrakten kommt es offenbar, entsprechend der quantitativen Inhaltsstoffverteilung, zu einer Konkurrenzreaktion zwischen eher Trypsin aktivierenden Alkamiden und vorrangig Trypsin hemmenden Phenolen und anderen hydrophilen Komponenten. Immerhin war, besonders für den hydrophileren Acetonextrakt in den höheren Konzentrationen, ein signifikanter TrypsinHemmeffekt gegenüber der Kontrolle zu konstatieren. In vivo könnte daraus eine reduzierte Aktivierung von PAR-2- und PAR-4-Rezeptoren und somit ggf. eine Verringerung von Entzündungsreaktionen, über den PAR-2-Rezeptor vermittelt, resultieren.

Das konträre Verhalten der geprüften Extrakte und des Testalkamids (konzentrationsabhängig) auf die Elastasehemmung (4.1.3.) einerseits und die Trypsinhemmung andererseits kann im Zusammenhang mit dem unterschiedlichen Einfluss der beiden Enzyme auf die PAR1- und PAR-2-Rezeptoren interpretiert werden: während Trypsin diese Rezeptoren aktiviert, wirkt Elastase als deren Inhibitor [43, 166].

Beispielhaft wurden einerseits die in Acmella ciliata vorkommenden phenolischen Verbindungen Kaffee- und Chlorogensäure sowie das Flavonoid Rutosid als Reinsubstanzen und andererseits die als partielle Alkamidvorstufen fungierenden Lipide Öl-, Linol- und Linolensäure hinsichtlich ihrer Trypsin hemmenden Potenz untersucht. In Tab. 30 werden die $\mathrm{IC}_{50}$-Werte bzw., sofern diese nicht erreicht wurden, die proz. Hemmwerte bei $1000 \mu \mathrm{g} / \mathrm{ml}$, mit den unter gleichen Bedingungen im Arbeitskreis gewonnenen Elastase- und ThrombinWerten $[43,67,115,116,119,146]$ verglichen.

Tabelle 30: Gegenüberstellung der Elastase- [115, 116, 106, 146], Trypsin- [43] und Thrombinhemmung $[67,119]$ durch ausgewählte Reinsubstanzen

\begin{tabular}{|c|c|c|c|c|}
\hline & Elastase & \multicolumn{2}{|c|}{ Trypsin } & \multirow{2}{*}{$\begin{array}{l}\text { Thrombin } \\
I C_{50}[\mu \mathrm{M}]\end{array}$} \\
\hline & $\mathrm{IC}_{50}[\mu \mathrm{M}]$ & $\mathrm{IC}_{50}[\mu \mathrm{M}]$ & $\begin{array}{c}\text { Hemmung }[\%] / \\
1000 \mu \mathrm{M}\end{array}$ & \\
\hline $\begin{array}{l}\text { Kaffeesäure } \\
\text { Chlorogensäure } \\
\text { Rutosid }\end{array}$ & $\begin{array}{c}93 \\
>400 \\
9,8\end{array}$ & $>200$ & $\begin{array}{l}10 \pm 8 \\
19 \pm 9\end{array}$ & $\begin{array}{l}\text { n. b. } \\
\text { n. b. } \\
>200\end{array}$ \\
\hline $\begin{array}{l}\text { Ölsäure } \\
\text { Linolsäure } \\
\text { Linolensäure }\end{array}$ & $\begin{array}{c}5 \\
10 \\
15\end{array}$ & 987 & $\begin{array}{c}29 \pm 8 \\
40 \pm 18\end{array}$ & $\begin{array}{c}7 \\
60 \\
124\end{array}$ \\
\hline
\end{tabular}


Generell ist die Elastasehemmung durch die Testsubstanzen wesentlich stärker als die Trypsinhemmung, was auch für die Acmella ciliata-Extrakte zutrifft. Messbare $\mathrm{IC}_{50}$-Werte für Trypsin waren lediglich für Rutosid und grenzwertig für Ölsäure zu erfassen. Gegenüber Elastase stellen die ungesättigten Fettsäuren starke Inhibitoren dar, ebenso das Rutosid, während der Hemmeffekt von Kaffeesäure als mittelstark und der von Chlorogensäure als relativ schwach einzuschätzen ist. Vergleicht man die Hemmeffekte der ungesättigten Fettsäuren (Tab. 30) gegenüber Elastase und Trypsin, so zeigt sich eine umgekehrter Trend: während im Elastase-Assay mit steigender Anzahl von Doppelbindungen der Hemmeffekt abnimmt (Linolensäure < Linolsäure), steigt er beim Trypsin (Linolensäure > Linolsäure).

\subsection{Thrombin-Hemmung}

\subsubsection{Allgemeines}

Das Kauen von Acmella ciliata und Art-Verwandten bei Zahnschmerzen ist in der Herkunftsgegend der Pflanzen auch heute noch gängige Praxis. Dabei macht man sich die analgetische, antiphlogistische und leicht anästhesierende Wirkung zu Nutze. Eine weitere Wirkqualität, über die berichtet wird, sind blutgerinnungshemmende Eigenschaften. Auch die Firma OTW spricht in der Fachinformation über ihr Präparat Spolera ${ }^{\circledR}$ von der blutgerinnungshemmenden Wirkung des in Acmella ciliata nachgewiesenen Leitalkamids Spilanthol [163].

Um diese Aussagen und Beobachtungen $\mathrm{zu}$ manifestieren, sollten einige ausgewählte lipophile Extrakte mit definitiv hohem Alkamid-Gehalt auf ihre Thrombin hemmende Wirkung überprüft werden.

Thrombin ist, ebenso wie die Neutrophilen-Elastase (4.1.) und Trypsin (4.2.), eine Prote(in)ase (Endopeptidase), die einen wesentlichen Faktor (IIa) der Blutgerinnungskaskade darstellt. Sie enthält in ihrem katalytischen Zentrum als wichtigste Aminosäure für die katalytische Wirkung Serin, weiterhin Histidin und Asparaginsäure. Daher zählt auch Thrombin zu den Serin-Prote(in)asen.

Thrombin entsteht durch Einwirkung des Prothrombinaktivators aus der im Blutplasma vorkommenden Vorstufe (Zymogen) Prothrombin. Thrombin wiederum bewirkt die Um- 
wandlung von Fibrinogen in Fibrin. Es löst (dadurch) die Thrombozytenaggregation und Freisetzung von Plättchenfaktoren aus [142, 97].

Als multifunktionelles Protein spielt Thrombin weiterhin eine Rolle bei Entzündungen, Allergien, Tumorwachstum und Metastasierung, Gewebeumbildung, Thrombosen und möglicherweise bei der Wundheilung. Viele der zellulären Aktivitäten des Thrombins werden über die G-Protein gekoppelten PAR-Rezeptoren (s. 4.2.1.) bewirkt. Thrombin ist in der Lage, als Ligand hoher Affinität PAR-1-, PAR-3- und PAR-4-Rezeptoren zu aktivieren. PAR-2 ist gegenüber Thrombin resistent (wird in erster Linie von Trypsin aktiviert). Während PAR-3 und PAR-4, gemeinsam Thrombin vermittelt, in dieselbe Signalkaskade involviert sind, die zur Aggregation der Blutplättchen führt, scheint die PAR-1-Aktivierung - ähnlich der PAR-2Aktivierung beim Trypsin - vorrangig eine proinflammatorische Rolle zu spielen. Da sich der PAR-1-Rezeptor in vielen humanen Geweben (z. B. Lungenwege, Blut (Thrombozyten), Blutgefäße, Herz-Kreislaufsystem, Immunsystem, Nervensystem, Haut) findet, kann eine Hemmung der proteolytischen Thrombinaktivität zu einer verminderten Aktivierung dieses Rezeptors und damit zur Beeinflussung entzündlicher Prozesse führen [43, 67, 119, 166].

\subsubsection{Untersuchungen}

Dieser Assay diente ebenfalls der in vitro-Bestimmung verschiedener Acmella ciliata-Proben in abgestufter Konzentration auf die Aktivität von Thrombin. Als Leitwert gilt wiederum die $\mathrm{IC}_{50}$. Zur Untersuchung gelangten der Dichlormethan-Ganzpflanzenextrakt $A_{3}$, der Dichlormethan-Blütenextrakt $\mathrm{A}_{4}$ sowie zwei mit überkritischem Kohlendioxid bei unterschiedlichen Parametern gewonnene Ganzdrogen-Extrakte $\left(\mathrm{F}_{1}: \mathrm{p}_{\mathrm{c}}=10 \mathrm{MPa} ; \mathrm{T}_{\mathrm{c}}=37^{\circ} \mathrm{C}\right.$ und $\left.\mathrm{F}_{2}: \mathrm{p}_{\mathrm{c}}=15 \mathrm{MPa} ; \mathrm{T}_{\mathrm{c}}=34^{\circ} \mathrm{C}\right)$.

Die genaue Versuchsdurchführung ist dem Exp. Teil zu entnehmen. Als Positivkontrolle diente der synthetische Inhibitor Pefabloc SC.

\subsubsection{Ergebnisse und Diskussion}

Die gemessenen Daten (Abb. 55) stammen aus zwei getrennten Messreihen mit jeweils drei Einzelmessungen. Auffallend bei allen vier Kurven ist die relative Konstanz der Hemmung 
über einen großen Konzentrationsbereich, mit einer Depression bei $250 \mu \mathrm{g} / \mathrm{ml}$, die hier nicht erklärt werden kann.

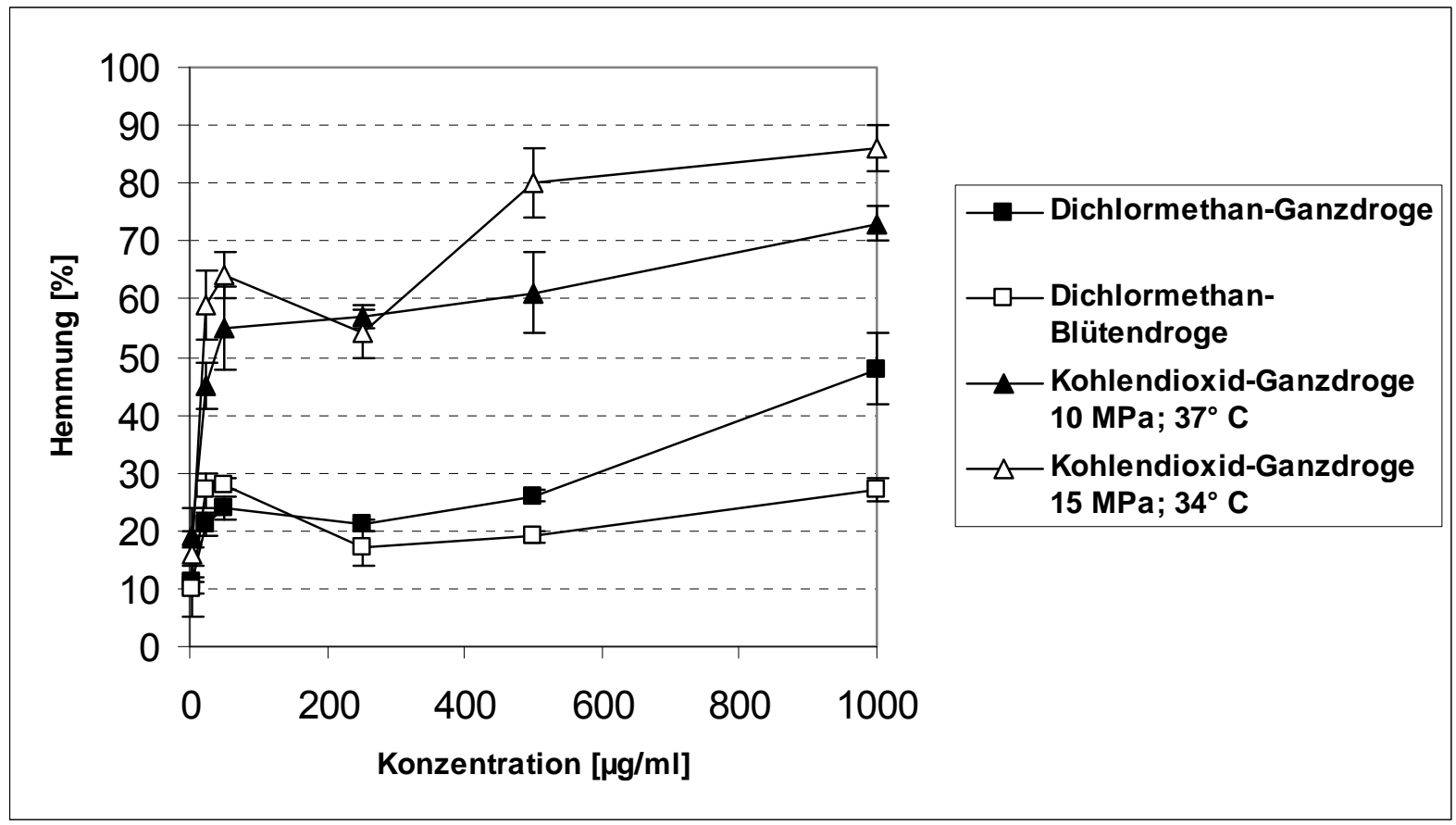

Abb. 55: Prozentuale Thrombinhemmung durch Dichlormethan- und $\mathrm{CO}_{2}$-Extrakte von $\mathrm{A}$. ciliata

Die Kurven (Auftragung der Hemmung [\%] gegen die Konzentration $[\mu \mathrm{g} / \mathrm{ml}]$ ) lassen keinen einheitlichen mathematischen Zusammenhang erkennen, so dass keine Linearisierung und Ermittlung der $\mathrm{IC}_{50}$ durch lineare Regression möglich waren. Daher kann lediglich ihr Bereich angegeben werden.

Daraus resultiert, dass die $\mathrm{IC}_{50}$ des Dichlormethan-Blütenextrakts $\left(\mathrm{A}_{4}\right)>1000 \mu \mathrm{g} / \mathrm{ml}$ sein muss bzw. auf Grund zu geringer Hemmwirkung dieses Präparats gar nicht erreicht wird. Der Dichlormethan-Ganzpflanzenextrakt $\left(\mathrm{A}_{3}\right)$ erreicht bei $1000 \mu \mathrm{l} / \mathrm{ml}$ mit $48 \%$ knapp die $\mathrm{IC}_{50}$. Eine weitere Konzentrationserhöhung war analytisch wegen ungenügender Löslichkeit des Extrakts im Puffer nicht möglich. Zudem dürfte wohl auch kaum eine so hohe Konzentration von pharmakologischer Relevanz sein.

Dagegen erscheinen die beiden $\mathrm{CO}_{2}$-Extrakte durchaus von pharmazeutischem Interesse. Bei $\mathrm{F}_{1}$ liegt die $\mathrm{IC}_{50}$ zwischen 25 und $50 \mu \mathrm{g} / \mathrm{ml}$ und bei $\mathrm{F}_{2}$ sogar nur zwischen 5 und $25 \mu \mathrm{g} / \mathrm{ml}$. Da $\mathrm{CO}_{2}(\varepsilon=$ zwischen 1,25 - 1,5) gegenüber Dichlormethan $(\varepsilon=9,14)$ das lipophilere Lösungsmittel darstellt, könnten lipophile Inhaltsstoffe, möglicherweise Alkamide, für die relativ 
große Hemmwirkung verantwortlich sein. Durch Druckvariation könnte womöglich die Ausbeute lipophiler Bestandteile noch gesteigert werden. Außerdem ermöglicht $\mathrm{CO}_{2}$ prinzipiell die selektive Isolierung von Inhaltsstoffen und ist prädestiniert für die Extraktion von Kohlenwasserstoffen und organischen Verbindungen geringer Polarität, so dass ggf. besonders Alkamide gewonnen werden können. Eine Weiterarbeit auf diesem Gebiet könnte durchaus erfolgversprechend sein.

Vergleicht man jedoch die Ergebnisinterpretation ,zunehmende Lipophilie = steigender Hemmeffekt" mit Untersuchungen an freien Fettsäuren [119], findet sich keine Bestätigung dieser Hypothese. Z. B. zeigte die Ölsäure mit einer Doppelbindung gegenüber Stearinsäure mit der gleichen Kettenlänge, aber ohne Doppelbindung (Ölsäure = hydrophiler als Stearinsäure), eine weitaus stärkere Thrombin-Hemmung. Andererseits wurde bei den ungesättigten Fettsäuren mit steigender Anzahl an Doppelbindungen bei gleicher C-Anzahl (entspricht Zunahme der Hydrophilie) eine drastische Verringerung der Hemmwirkung gefunden: Ölsäure (18:1): $\mathrm{IC}_{50}=7 \mu \mathrm{M}$; Linolsäure (18:2): $\mathrm{IC}_{50}=60 \mu \mathrm{M}$; Linolensäure (18:3): $\mathrm{IC}_{50}=124 \mu \mathrm{M}$. Somit scheint kein einfacher Zusammenhang zwischen Lipophilie und Thrombinhemmung zu bestehen. Von Bedeutung für den Grad der Hemmung dürften demnach auch weitere spezifische Strukturelemente sein [119].

Beim Vergleich des Dichlormethanextrakts $\mathrm{A}_{3}$ im Thrombin- und Elastase-Assay (4.1.3.) wird ebenfalls deutlich, dass kein einfacher Zusammenhang zwischen Hemmwirkung und Lipophilie oder Strukturelementen der Probe besteht. Obwohl es sich bei Thrombin und Elastase um strukturell verwandte Serin-Prote(in)asen handelt, zeigte $\mathrm{A}_{3}$ eine bemerkenswerte Hemmung gegenüber der Elastase-Aktivität $\left(\mathrm{IC}_{50}=0,8 \mu \mathrm{g} / \mathrm{ml}\right)$, während dieser Extrakt die Thrombin-Aktivität nur gering beeinflusste $\left(\mathrm{IC}_{50}>1000 \mu \mathrm{g} / \mathrm{ml}\right)$. Ähnliche Aussagen ließen sich bereits zur Hemmung der Trypsin-Aktivität treffen (s. 4.2.3.).

Pharmazeutisch relevant wären hier möglicherweise $\mathrm{CO}_{2}$-Extrakte, die, auf Grund des obigen Datenmaterials, als medizinisch einsetzbare Hemmer der Thrombinaktivität fungieren und durch eine verminderte Aktivierung (durch Proteolyse), besonders des PAR-1-Rezeptors, positiv auf den Verlauf von Entzündungsreaktionen einwirken könnten. 


\section{Galenische Präformulierungen}

Als Präformulierungen werden im Folgenden potentielle galenische Formulierungsvarianten bezeichnet, mit denen zum einen die Einarbeitbarkeit von Acmella-Extrakten prinzipiell geprüft und zum anderen geeignete Applikationsmöglichkeiten zur orientierenden in vivoTestung gefunden werden sollten. Das vorrangige Interesse bestand in der Wirksamkeitsüberprüfung von ausgewähltem Acmella ciliata-Extrakt nach Anwendung auf Haut und Schleimhaut. Wie aus dem DC in Abb. 13 ersichtlich, zeigte der Acetonextrakt $\left(E_{1}\right)$ aus der getrockneten Gesamtpflanze, ebenso wie der Dichlormethan-Blütenextrakt $\left(\mathrm{A}_{4}\right)$, ein breites Spektrum an Alkamiden, die als hauptverantwortlich für die Wirkung anzusehen waren. Als Voraussetzung für die Testung im Selbst- und „Kollegenversuch“ wurden die nachfolgenden Formulierungen hergestellt bzw. entwickelt.

\subsection{Hautsalbe}

Zunächst sollte ein Acmella-Extrakt in eine Salbengrundlage inkorporiert und nach Applikation am Unterarm der Versuchspersonen vergleichend mit dem Handelspräparat Spolera ${ }^{\circledR}$-Salbe nach organoleptischen Kriterien bewertet werden.

Hierzu wurden 1 und $10 \%$ Dichlormethan-Blütenextrakt (Extrakt $\mathrm{A}_{4}$, Tab. 1) in DACBasiscreme [33], eine ambiphile Salbengrundlage (s. Exp. Teil), eingearbeitet, was herstellungstechnologisch problemlos möglich war. Die Präparate zeigten je nach Extraktkonzentration helle bis dunkle gelb-grüne Färbung und waren von streichfähiger Konsistenz.

Die kutane Testung nach topischer Applikation am ventralen Unterarm erbrachte bei vier Probanden nach zweistündiger Einwirkung unter Okklusionsbedingungen (s. Exp. Teil) keine makroskopisch sichtbaren Veränderungen oder subjektive Empfindungen, allenfalls eine kurzzeitige, konzentrationsunabhängige Rötung nach Versuchsende und nach Applikation bei zwei Probanden einen leichten Hautreiz (leichtes Prickeln).

Die unter analogen Bedingungen getestete Spolera ${ }^{\circledR}$-Salbe, die sich auf Grund von gewisser Inhomogenität schlecht auf der Haut verteilen ließ, zeigte keinerlei Reaktion. 


\subsection{Nanoemulsion}

Als zweite mögliche Formulierungsvariante wurden Nanoemulsionen in Betracht gezogen. Mit einer solchen innovativen Formulierung wäre eine höhere Penetretionsrate der Wirkstoffe zu erwarten. Nanoemulsionen eignen sich sowohl zur Applikation per se als auch zur Einarbeitung in Salben- oder Gelgrundlagen. Im pharmazeutischen Bereich haben sie bisher vor allem bei der parenteralen Ernährung Bedeutung [108].

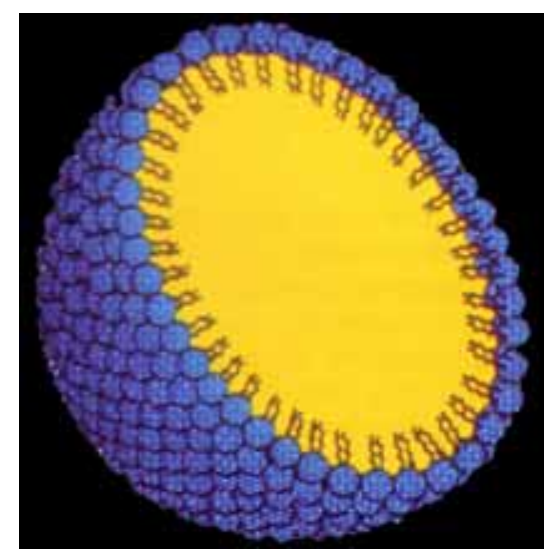

Abb. 56: Struktur einer Nanoemulsion

Unter Nano- oder Submikronemulsionen werden allgemein $\mathrm{O} / \mathrm{W}$-Emulsionen verstanden, bei denen die Tropfen der dispergierten, flüssigen Lipidphase einen Durchmesser von ca. 50-100 nm aufweisen. Die mittlere Tropfengröße liegt üblicherweise im Bereich von 100$500 \mathrm{~nm}$ [31]. Die mit einem Ölgehalt von 10-20\% und zumeist durch Phospholipide stabilisierten Partikeln unterscheiden sich deutlich von den Liposomen. Während bei letzteren der hydrophile Innenraum durch eine Phospholipidmembran (-bilayer) umgeben ist, wird bei den Nanoemulsionen der flüssige, lipophile Kern durch eine Monoschicht des Phospholipids bzw. anderen Tensids gegenüber der umgebenden Wasserphase begrenzt (Abb. 56).

Im vorliegenden Fall wurde als Tensid Poloxamer und als flüssiges Lipid Miglyol (Neutralöl) benutzt. Die Herstellung erfolgte mittels Hochdruckhomogenisierung bei $5 \cdot 10^{7} \mathrm{~Pa}$ während 150 s (s. Exp. T.). In die lipophile Phase wurden 0,1\% eines Dichlormethanextrakts der Blütenköpfchen ( $\left.\mathrm{A}_{4}, \mathrm{Tab} .1\right)$ eingearbeitet. Da sich der vorher mit Chloroform aufgenommene Extrakt nur unvollständig in Miglyol löste, musste eine Suspension mit der Poloxamerlösung vermischt und homogenisiert werden. Für das schwach gelbliche, milchig trübe Präparat resultierten nach Messung im Photonenkorrelationsspektrometer durchschnittliche Partikeldurchmesser von $165 \mathrm{~nm}$ und ein Polydispersitätsindex von 0,165 .

Hinsichtlich Hautverträglichkeit ergab die Prüfung im Selbstversuch weder subjektive Empfindungen noch objektive Erscheinungen, wie Schwellung oder Rötung. Über die Wirkstofffreigabe, besonders der lipophilen Alkamide, aus dieser Nanoemulsion können keine Aussagen getroffen werden. Da jedoch die Extrakteinarbeitung schwierig war und noch 
nicht zu einer optimalen Formulierung führte, was sich auch an veränderlicher Partikelgröße (Laserdiffraktometer) verdeutlichte, wurden zunächst keine weiteren Versuche durchgeführt.

\subsection{Mukoadhäsive Arzneiformen}

\subsubsection{Allgemeines}

Der übergeordnete Begriff Bioadhäsion kann definiert werden als die Fähigkeit des Anhaftens von synthetischem oder biologischem Material an biologische Gewebe über eine bestimmte Zeitperiode [139]. Zur Verlängerung der Verweildauer von Arzneiformen an ihrem Applikationsort und dadurch der Verbesserung der Bioverfügbarkeit entstand zu Beginn der 1980er Jahre die Idee, bestimmte Arzneiformen zu entwickeln, die sich an die betreffenden Gewebe „ankleben“ ließen. Da es sich bei diesen Geweben um mukosale Epithelien (Mund, Auge, Nase, Gastrointestinaltrakt, Vagina, Lunge) handelte, wurde für diese Art von Bioadhäsion der Begriff Mukoadhäsion geprägt. Die den Schleimhautzellen aufliegende Mukus-Gelschicht dient dabei als Verbindungsglied zwischen Arzneiform und Gewebe. Das Haften am mukosalen Gewebe verlängert nicht nur die Kontaktzeit, sondern auch die Intensität der Wirkstoffabsorption. Da Schleimhäute zudem gut durchblutet sind, kann u. U. eine rasche systemische Verfügbarkeit des Wirkstoffs resultieren.
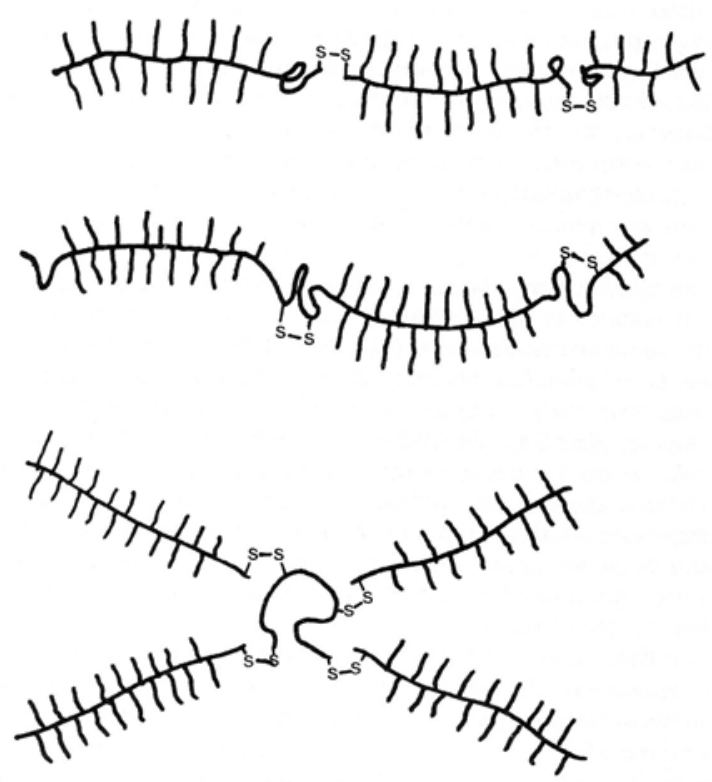

Abb. 57 : Glykoproteinmolekülstruktur
Der vorwiegend von den Gobletzellen sezernierte hochviskose Mukus bedeckt die Mukosa und schützt sie vor mechanischen, chemischen, bakteriellen und viralen Aggressionen. Der wässerige Schleim besteht je nach Lokalisation zu 0,5-5\% aus Glykoproteinen und enthält etwa $1 \%$ Mineralsalze, $0,5-1 \%$ freie Proteine und geringe Lipidmengen. Die Glykoproteine determinieren die Viskosität sowie die adhäsiven und kohäsiven Eigenschaften des Mukus. 
Abb. 57 zeigt schematisch die Struktur eines Glykoproteinmoleküls, und Abb. 58 demonstriert den Aufbau der Oralmukosa.

Voraussetzung für die Mukoadhäsion ist ein intensiver Kontakt zwischen dem Bioadhäsivum und dem Rezeptorgewebe, wofür gute Benetzbarkeit und/oder Quellung des Bioadhäsivums erforderlich sind. In der zweiten Phase erfolgt dann eine Adsorption an der Gewebeoberfläche und Interpenetration der bioadhäsiven Ketten mit denen des Mukus (s. Abb. 59, $60)$.

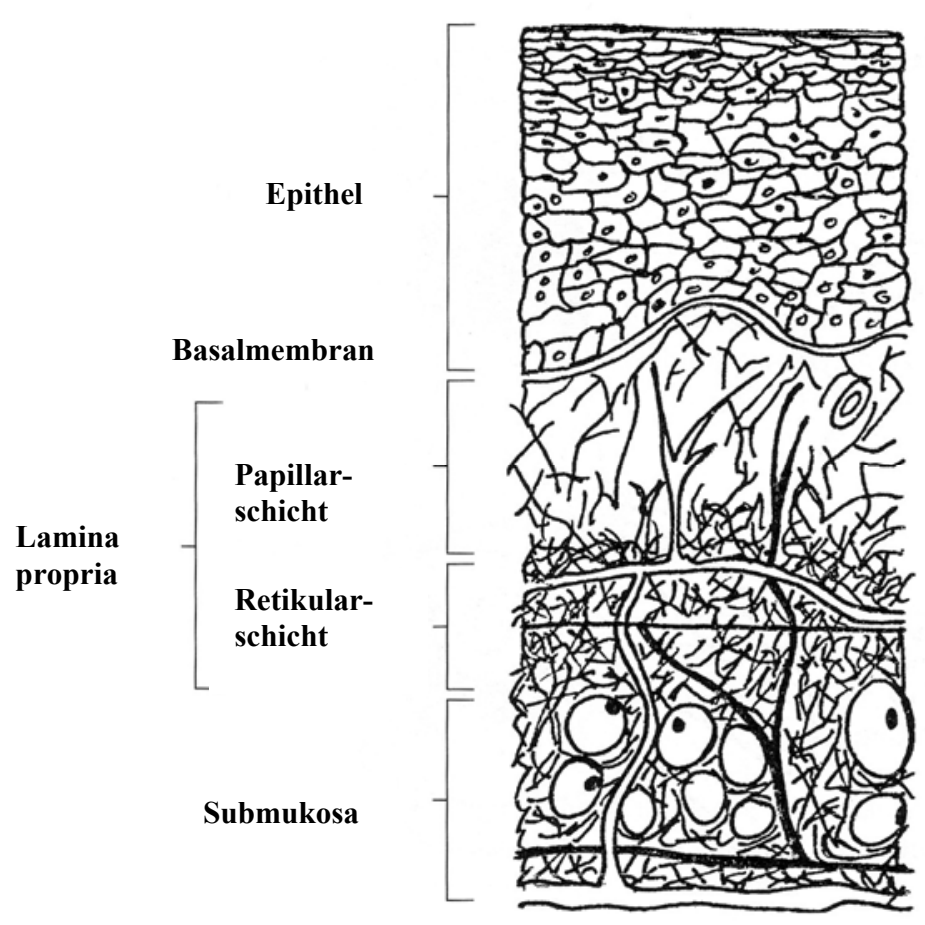

Abb. 58: Schematische Darstellung der Oralmukosa-Struktur

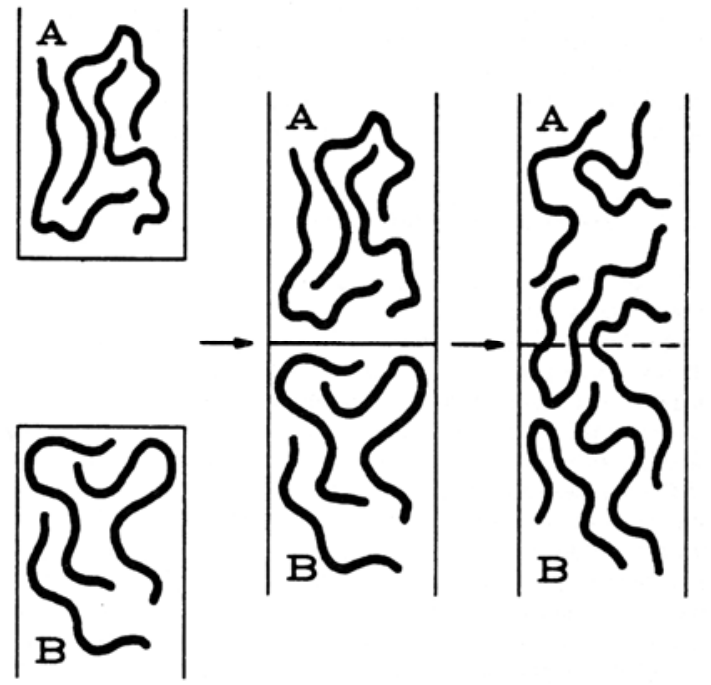

Abb. 59: Ketteninterpenetration zwischen Polymer und Mukus

(A: Polymer; B: Glykoproteinkette)

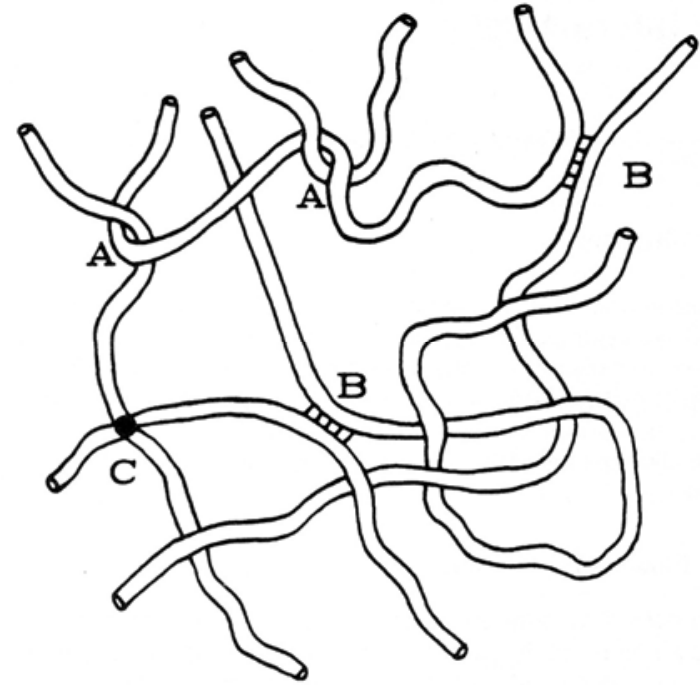

Abb. 60: Schema der Mukusquervernetzung (A: physikalische Verknäulung; B: molekulare Assoziationen; C: chemische Quervernetzung)

Darüber hinaus spielen Sekundärbindungskräfte, wie Wasserstoffbrücken, hydrophobe Wechselwirkungen und van der Waals'sche Kräfte, eine Rolle. Für bioadhäsive Substanzen mit geladenen Gruppen sind elektrostatische Wechselwirkungen zu erwarten. Die detaillierte 
Kenntnis der komplexen Bioadhäsion steht noch aus; es werden verschiedene Mechanismen, wie die elektronische, die Benetzungs-, die Bruch-, Adsorptions-Interpenetrations-, Diffusions-, Kapillar- sowie die mechanische Theorie, diskutiert. Am wahrscheinlichsten ist ein Wechselspiel der Kräfte in Abhängigkeit von den physiologischen Bedingungen einerseits und den chemischen und physikalischen Eigenschaften des Bioadhäsivums andererseits.

Ausgehend von den aus der traditionellen Anwendung von Acmella ciliata bekannten Effekten im Mundraum, die als prickelnd, brennend, anästhesierend, Salivation fördernd usw. beschrieben werden (s. 3.3.1.2.1.), erschien die orale Testung der Extrakte als geeignet und deren Applikation als mukoadhäsive Formulierung prädestiniert. Als solche potentielle Arzneiformen wurden Adhäsiv-Tabletten, Gelatine-Haftplättchen und eine SchleimhautHaftsalbe entwickelt (5.3.2. - 5.3.4.).

Bei der bukkalen oder sublingualen Administration muss generell zwischen systemischen und lokalen Effekten diffenziert werden. Die z. T. gute Resorption aus Subligual- oder BukkalTabletten ist vor allem für gastrointestinal instabile (z. B. Insulin und andere Peptide) oder nebenwirkungsintensive Pharmaka oder auch zur Erzielung rascher Wirkung, wie bei Nitroglycerin, relevant, da sie auf diesem Wege unter Umgehung der Darm- und Leberpassage (first pass-Effekt) schnell in den Blutkreislauf gelangen.

Andererseits sind die verlängerten Kontaktzeiten im Mundraum auch zur Behandlung lokaler Erkrankungen, wie Entzündungen, Infektionen, Läsionen, als auch zur Protektion, z. B. im stomatologischen Bereich, gefragt, da ansonsten die Verweildauer von Arzneiformen in der Mundhöhle durch die Salivation sowie durch das Schlucken, Sprechen und Kauen nur außerordentlich kurz ist.

Bereits 1985 publizierte NAGAI [131] die Entwicklung einer bioadhäsiven Tablette mit lokaler Aktivität gegen Stomatitis aphthosa mit dem Wirkstoff Triamcinololacetonid, kommerziell unter dem Namen „Aftach“ - in der aktuellen Roten Liste [224] als Aftab ${ }^{\circledR}$ Hafttabletten registiert - bekannt. (Ein Salbenpräparat mit demselben Wirkstoff war bereits vorher verfügbar.) Die kleine Doppelschichttablette (Durchmesser $7 \mathrm{~mm}$, Dicke 1,1 mm) besteht aus einer gefärbten Laktoseschicht zum Schutz der aktiven Ingredienzien sowie zur einfachen Applikation und aus einer Adhäsivschicht von Hydroxypropylcellulose und Carbopol 934, den Wirkstoff enthaltend. 
Tabelle 31: Beispiele mukoadhäsiver Topika zur bukkalen Applikation

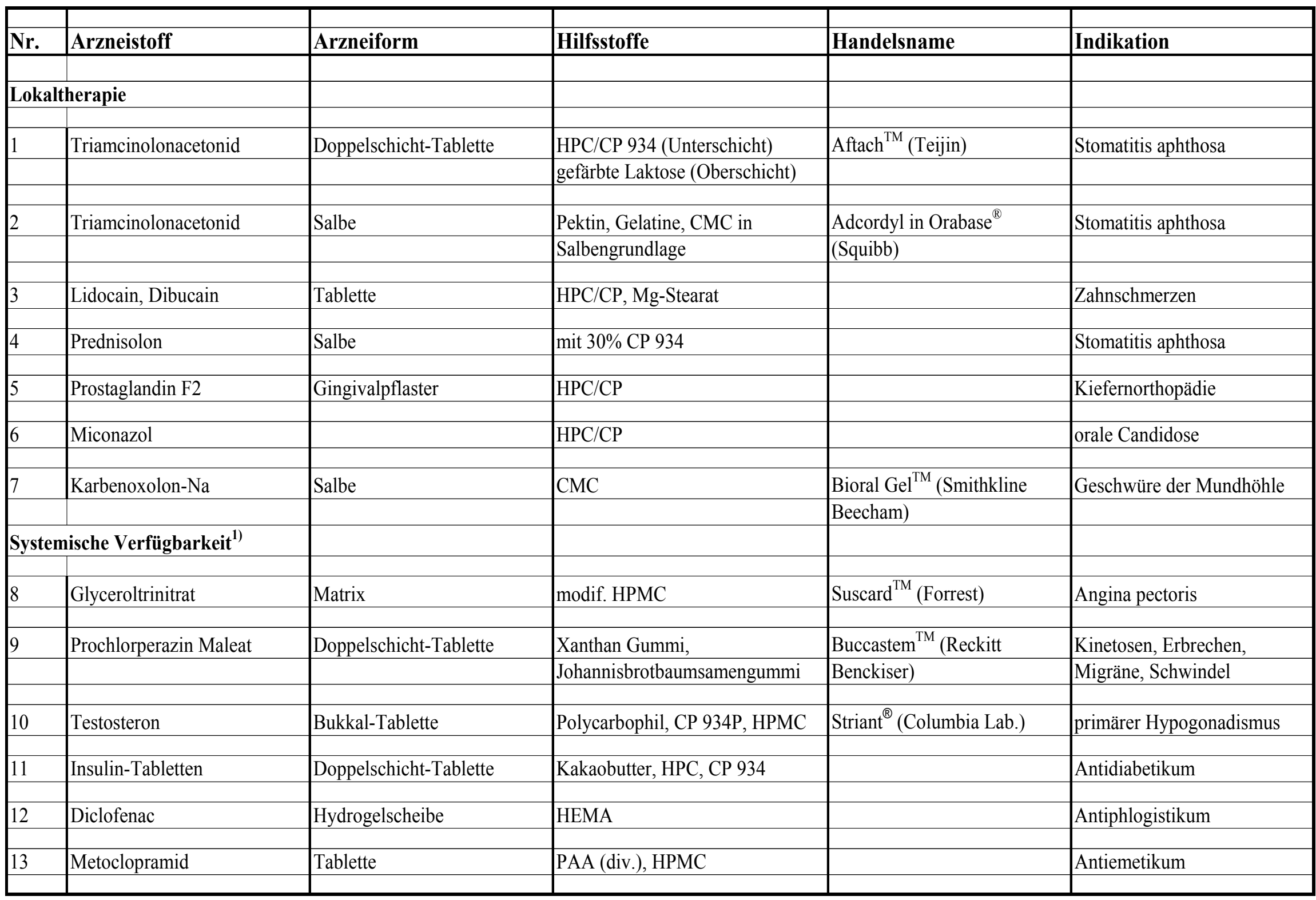

$\mathrm{HPC}=$ Hydroxypropylcellulose $\mathrm{CP}=$ Carbopol $^{\circledR} ; \mathrm{CMC}=$ Carboxymethylcellulose; HPMC = Hydroxypropylmethylcellulose; HEMA = Hydroxyethylmethacrylat; PAA = Polyacrylsäure ${ }^{1)}$ Weitere Beispiele s. [36]. 
Die obere Schicht zerfällt bald nach Applikation, während die untere Schicht, die die kranke Stelle bedeckt, unter gradueller (kontrollierter) Quellung den Arzneistoff freisetzt. Im Arbeitskreis NAGAI wurden nachfolgend weitere bioadhäsive Formulierungen zur Applikation in der Mundhöhle entwickelt, z. B. eine Prednisolon-Salbe [78] und lokalanästhetisch gegen Zahnschmerzen wirkende Tabletten mit Lidocain und alternativ mit Dibucain [77, 131]. Tetracain wurde in Filme eingearbeitet [190].

In der Folgezeit resultierte aus zahlreichen Forschungsarbeiten eine Reihe von Patenten für verschiedene orale, bioadhäsive Arzneiformen, z. B. Tabletten [83, 130, 79], Pulver [130], Granulate [130], Mikrokapseln [157], Filme und Tapes [87 - 90], überwiegend bestehend aus einer aktiven und einer neutralen Schicht.

Die Anzahl der definitiv auf dem Pharmamarkt etablierten Präparate zur Applikation in der oralen Kavität, prädestiniert Gele und Salben, ist allerdings überschaubar und ihre Anwendung auf wenige Indikationen beschränkt (s. auch Tab. 31). Als Wirkstoffe zur Lokaltherapie (s. auch Rote Liste [224]) dominieren dabei Glukokortikoide (Triamcinolonacetonid, Prednisolon-acetat) und Lokalanästhetika (Lidocain), vereinzelt Antimykotika (Nystatin) und Desinfizienzien (Chlorhexidin), und als bioadhäsive Hilfsstoffe Polyacrylsäure $\left(\right.$ Carbopol $^{\circledR}$, Carbomer $^{\circledR}$ ) und Cellulosederivate, daneben Pektin, Gelatine, Natriumalginat, Guar-Gummi und Guargalactomannan u. a. Die Beispiele 8-13 in Tab. 31 (Beispiele aus Forschung und Handel ohne Anspruch auf Vollständigkeit) repräsentieren Präparate mit Wirkstoffen zur systemischen Verfügbarkeit, deren Auswahl zumeist auf kleine, lipophile Moleküle beschränkt ist, die in der Lage sind, die bukkale Mukosa schnell zu passieren.

Wie aus Tab. 31 und den obigen Ausführungen hervorgeht, gelangen als mukoadhäsive Hilfsstoffe hydrophile, biokompatible Makromoleküle natürlichen, synthetischen und halbsynthetischen Ursprungs zur Anwendung. Ihre Auswahl richtet sich u. a. nach der Verträglichkeit mit den inkorporierten Arzneistoffen sowie nach der angestrebten Adhäsivkraft. Hinsichtlich letzterer erweisen sich vorrangig ionische Polymere, zu nennen sind hier besonders Polyacrylsäure, Polycarbophil und Natriumcarboxymethylcellulose, als am stärksten adhäsiv. Neben dem Ionencharakter des Makromoleküls nehmen dessen Konzentration sowie seine Molekülmasse und -struktur, d. h. verzweigter oder kettenförmiger 
Aufbau, Einfluss auf die Bioadhäsion. Davon und vom umgebenden Milieu (Wasserpräsenz, pH-Wert usw.) wird letztendlich auch das Quellvermögen determiniert.

\subsubsection{Haftpaste}

Verschiedene Typen von mukoadhäsiven, halbfesten Formulierungen hydrophilen und hydrophoben Charakters zur Behandlung von Mucositis, Stomatitis, Aphthen und anderen Affektionen im Mundraum waren bisher relativ häufig Gegenstand der Forschung und führten z. T. zur Etablierung marktreifer Salben, Gele und Pasten (vgl. auch Tab. 31). In der Roten Liste [224] findet sich z. B. die Mundheilpaste Dontisolon ${ }^{\circledR}-$ Prednisolon als Wirkstoff - mit der Indikation Gingivitis, Stomatitis und Perikoronitis. Gegen schmerzhafte, entzündliche Erkrankungen der Mundschleimhaut und gegen Prothesendruckstellen wird die Solcoseryl ${ }^{\circledR}$ Dental Adhäsivpaste empfohlen, die ein proteinfreies Hämolysat aus Kälberblut und Macrogollaurylether als arzneilich wirksame Bestandteile und als adhäsive Hilfsstoffe Carmellose-Natrium, Gelatine und Pektin enthält. Interessant erscheint im Hinblick auf die eigenen Untersuchungen das Präparat Kamillostad ${ }^{\circledR}$ Gel, dass neben Lidocain einen Kamillenblütenextrakt enthält und auf Basis von Polyacrylsäure konzipiert ist.

Das Neue Rezeptur-Formularium (NRF, [135]) gibt Rezepturhinweise für einige Schleimhaut-Haftgele und unterteilt diese in:

- wasserfreie Haftpasten auf lipophiler Basis mit hydrophilem Quellstoff

- Hydrogele, z. T. mit emulgierendem Lipidanteil (Haftcremes)

- Tensidgele (Poloxamergele)

Zur Einarbeitung des Acetonextrakts von Acmella ciliata $\left(\mathrm{E}_{1}\right)$ zwecks Anwendung in der bukkalen Kavität wurde die nichtionische Hypromellose-Haftpaste 40 \% NRF 7.8 ausgewählt. Sie stellt ein wasserfreies Paraffingel dar, in dem ein hydrophiles Quellmittel suspendiert vorliegt und ist zusammengesetzt aus 40 \% Hypromellose 2000 oder Methylhydroxypropylcellulose 2000, ein wasserlöslicher Celluloseether, und $60 \%$ Hydrophobem Basisgel DAC [33] (s. Exp. Teil). Bei Kontakt mit der feuchten Schleimhaut quellen die Hypromellose-Partikeln, der Wasserfilm wird rasch aufgenommen, und die Paste haftet stark auf dem Gewebe. Dabei verzögert die lipophile Paraffin-Matrix die Quellung der dispergierten Hypromellose-Teilchen, was zu einer langen Verweildauer in der Mundhöhle führt. 
Zur Herstellung und Extrakteinarbeitung s. Exp. Teil. Die Konzentration an Extrakt betrug 5 \%. Eine Unverträglichkeit auf Grund der im Extrakt enthaltenen phenolischen Verbindungen wurde nicht beobachtet.

Die in vivo-Testung an drei freiwilligen Probanden ergab eine sehr gute Haftfähigkeit der wirkstoffhaltigen Präparation am Zahnfleisch des Oberkiefers bzw. zwischen Backentasche und Oberkiefer, die über den Testzeitraum von zwei Stunden andauerte. Die Platzierung an einer bestimmten Stelle in der Mundhöhle war jedoch schwierig und nicht präzise. Der Geschmack wurde von zwei Testern als kräuter- oder heuartig charakterisiert. Ansonsten waren keine Empfindungen wahrzunehmen, weder Kribbeln, Brennen noch Anästhesie.

\subsubsection{Gelatineplättchen}

Eine weitere Idee war die Testung von Gelatineplättchen. Hierzu wurde ein Gel mit der Zusammensetzung Gelatine/Wasser/Glycerol im Verhältnis 1:5:5, mit einem Zusatz von 0,1 \% Chitosan, gelöst in 5 \%iger Zitronensäurelösung, im Ultraschallbad bei $55-60^{\circ} \mathrm{C}$ hergestellt (s. Exp. Teil). Zur Inkorporierung des Acetonextrakts $\left(E_{1}\right)$ in $5 \%$ iger Konzentration wurde dieser, gelöst in Ethanol, vorgelegt und die Gelatinelösung unter Erwärmen im Ultraschallbad homogen und möglichst luftblasenfrei eingearbeitet. Nach Ausgießen in Petrischalen und Erstarren wurden aus dem dünnen Film Plättchen ausgestochen.

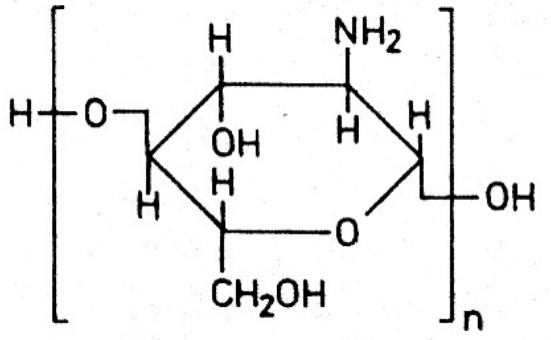

Chitosan

Die Gelatine als offizineller, amphoterer Hilfsstoff (Eur. Pharmakopoe) zeigt zwar lediglich eine als befriedigend eingeschätzte Bioadhäsivität (115\% in Relation zum Pektin $=100 \%$ ), erschien jedoch durch ihre erweichende, elastische Konsistenz für das Anheften an die Bukkalmukosa als geeignet. Zur Verbesserung der Adhäsivität erfolgte eine Zugabe von Chitosan (Formel), einem kationischen Polymer, das durch N-Deacetylierung aus Chitin gewonnen wird [92]. Chitosan selbst weist wundheilende und antiulcerative Effekte auf, weshalb es in verschiedenen Formulierungen zur oralen Applikation gelangte [62, 65, 76, 120, 155].

Die Testung der Gelatineplättchen erfolgte im Selbstversuch durch bukkale Applikation. Es waren eine gute Akzeptanz dieser Präformulierung und eine schwache Lokalanästhesie zu 
konstatieren, jedoch nur ein geringes Haftvermögen. Da aber die Herstellung der Rezeptur, insbesondere die Inkorporierung des Extrakts, wegen der zur Gelatinelöslichkeit erforderlichen Mindesttemperatur von $55^{\circ} \mathrm{C}$ einerseits und der Temperaturempfindlichkeit der Naturstoffe - es bildeten sich schwarze „Pünktchen“, ggf. Polymerisationsprodukte der Alkamide (vgl. 3.3.3.), - andererseits große Schwierigkeiten bereitete, wurde diese Formulierung nicht weiter verfolgt.

\subsubsection{Adhäsivtabletten}

Während einige Präparate in Form von mukoadhäsiven Bukkaltabletten mit chemisch definierten Einzelwirkstoffen existieren (vgl. auch Tab. 31), sind bisher noch keine derartigen Formulierungen mit Pflanzenextrakten bekannt. Zum Zweck der orientierenden in vivoTestung wurden einfache, biplane Monolayer-Tabletten mit Facettenrand konzipiert (Durchmesser $9 \mathrm{~mm}$, Dicke 1,5 mm), in die sich 5, 10 und $20 \mathrm{mg}$ des AcetonGesamtpflanzenextrakts $\left(\mathrm{E}_{1}\right)$, gelöst in Ethanol, inkorporieren ließen (s. Exp. Teil). Der Acetonextrakt wurde auf Gund seiner höchsten Elastasehemmaktivität (s. 4.1.3.) ausgewählt. Als Adhäsivum gelangte die nichtionische Hydroxypropylmethylcellulose (HPMC, Eur. Pharmakopoe, s. Formel) zum Einsatz, die hinsichtlich ihrer Adhäsivkraft als gut bis sehr gut einzustufen ist. Ihre Konzentration betrug $70 \%$ (weitere Tablettenhilfsstoffe: Avicel und Aerosil).

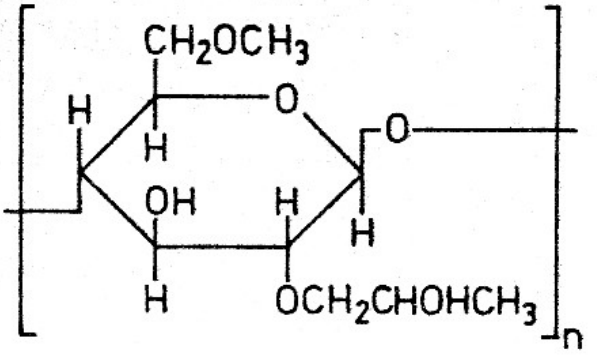

Hydroxypropylmethylcellulose

Es resultierten gleichmäßig grün gefärbte Tabletten (konzentrationsbedingte Farbintensität), die vergleichend mit entsprechenden Placebo-Tabletten zur Wirksamkeitsüberprüfung in der oralen Kavität (bukkale/sublinguale Applikation) im Selbstversuch an drei freiwilligen Testpersonen gelangten (Prüfschema s. Exp. Teil). Daneben wurden in den Blindversuch 2 und 4 \%ige Benzocain-Tabletten gleicher Hilfstoffzusammensetzung einbezogen. Die Auswahl dieses Vergleichspräparats orientierte sich an dem u. a. zu erwartenden anästhesierenden Effekt durch die Extrakt-Tabletten und dessen Wirkstoffkonzentration in handelsüblichen Lokalanästhetika-Präparaten.

Im Ergebnis der in vivo-Testung resultierte eine gute Applizierbarkeit und Haftfähigkeit für die Placebotablette, die sich nach $2 \mathrm{~h}$ in toto entfernen ließ. Der Geschmack war indifferent. 
Eine Testperson vermerkte eine leichte Erwärmung und pelzige Empfindung, eine zweite eine kurze brennende Schärfe.

Die 2 mg Benzocain enthaltende Testtablette verhielt sich ähnlich, zeigte aber gegen Ende der Versuchszeit Auflösungstendenz, was zu einer gelatinösen Oberfläche führte. Eine eindeutige Anästhesie war nicht zu spüren; in einem Fall zeigte sich eine geringe Erwärmung, im anderen bei der Berührung mit der Zungenspitze eine schwach anästhesierende Empfindung. Für die 5 mg-Tablette werden eine schlechtere Applizier- und Haftfähigkeit angegeben, ein neutraler Geschmack und von zwei Testern eine geringe Erwärmung bzw. leichte Schärfe an der Zungenspitze.

Bei den Extrakttabletten konstatierten die Testpersonen eine mit der Extraktkonzentration (5, 10 und $20 \mathrm{mg}$ ) zunehmende Haftfähigkeit und Erweichung bis Zerfall im Mundraum. Der Geschmack wurde von einer Person als unauffällig, von den beiden anderen als zunehmend heu- bzw. kräuterartig beschrieben. Eine eindeutige Anästhesie wurde auch hier nicht empfunden (konzentrationsunabhängig). Partiell traten eine leichte Schärfe bis Prickeln und schwache Anästhesie an der Zungenspitze auf, z. T. nach Herausnahme anhaltend. Die Akzeptanz und Verträglichkeit dieser Tabletten wurde insgesamt als gut beschrieben.

Diese Tabletten könnten nach entsprechender Prüfung am Patienten zur Behandlung von Läsionen am Zahnfleisch sowie von Entzündungen, Affektionen und Schmerzen im Mundund Rachenraum als weitgehend nebenwirkungsfreie pflanzliche Alternative $\mathrm{zu}$ chemischen Fabrikaten dienen. Als solche wären sie patientenfreundlicher als z. B. Salben und Gele (s. auch 5.3.4.) und könnten entsprechend ihrer mukoadhäsiven Zusammensetzung eine kontrollierte Verweilzeit aufweisen.

Eine umfangreiche in vivo-Testung vorausgesetzt, macht sich jedoch eine galenische Optimierung erforderlich, ggf. in Form und Größe. Weiterhin empfiehlt sich eine zweifarbige Bilayer-Tablette mit einer Haft- und wirkstoffhaltigen Seite und einer neutralen Oberseite (Schutzschicht). Auf diese Weise verlässt der Wirkstoff die Arzneiform lediglich in eine Richtung durch direkten Kontakt mit dem erkrankten Gewebe, und ein Wirkstoffverlust in Richtung Mundhöhle $\rightarrow$ Systemikum wird eingeschränkt. 


\section{Experimenteller Teil}

\section{Material}

\subsection{Chemikalien}

\subsubsection{Lösungs-/Extraktions-/Fließmittel}

\section{Extraktion / HPLC / DC}

- Methanol

- Dichlormethan

- 2-Propanol

- Ethanol absolut

- Essigsäureethylester

(Ethylacetat)

- n-Butanol

- Hexan

- Petroleumbenzin (Petrolether)

- Aceton

- Acetonitril

- Diethylether

- Trichlormethan

- Essigsäure

- Ameisensäure

- Trifluoressigsäure

- Wasser

\section{HPLC-MS}

- Acetonitril

- Ameisensäure

- Wasser

NMR

- $\mathrm{CD}_{3} \mathrm{OD}$

- $\mathrm{CDCl}_{3}$

- Aceton-d 6

- Tetramethylsilan (TMS)

\section{MS (EI / FAB / ESI)}

- Methanol

- Acetonitril

- Ameisensäure

- 3-Nitrophenol

- Glycerol
HiPerSolv HPLC-Gradient Grade Chromanorm zur Analyse zur Analyse zur Analyse zur Analyse

$99 \%$ reinst

zur Analyse

zur Analyse

zur Analyse

Baker HPLC analyzed

ROTIPURAN $^{\circledR} \geq 99 \%$ p.a.

zur Analyse

mind. 99,8\%

98-100\% zur Analyse

zur Synthese

entmin. (Ionenaustausch)

ROTISOLV $^{\circledR}$, HPLC grade

bidestilliert

Roth

Roth

Barnstead Easy pure RF

Deutero

Deutero

Deutero

Cambridge Isotope

Laboratories

Optigrade $^{\circledR}$ für die HPLC

LGC Promochem

Optigrade ${ }^{\circledR}$ HPLC Gradient Grade LGC Promochem

98-100\% pro analysi

zur Synthese

Merck

etwa $87 \%$ zur Analyse
Merck

Merck
Leuven, B

Lohmar, D

Lohmar, D

Lohmar, D

Lohmar, D

Lohmar, D

Lohmar, D

Renningen, D

Lohmar, D

Deventer, NL

Karlsruhe, D

Lohmar, D

Lohmar, D

Lohmar, D

Hohenbrunn, D

Karlsruhe, D

Karlsruhe, D

Kastellaun, D

Kastellaun, D

Kastellaun, D

Andover, USA

Wesel, D

Wesel, D

Darmstadt, D

Darmstadt, D

Darmstadt, D 


\subsubsection{Referenzsubstanzen}

Alkamide

- Dodeca-2,4,8,10

(E,E,Z,E / E,E,Z,E)-tetraen-

säure-isobutylamid(e)

- Capsaicin

$\underline{\text { Flavonoide }}$

- Apigenin

- Apigenin-7-glucosid

- Apiin

- Astragalin

- Chrysin

- Datiscetin

- Dihydrofisetin

- Hesperetin

- Hyperosid

- Isorhamnetin

- Isoquercitrin

- Kampferid

- Kämpferol

- Kämpferol-7-rhamnosid

- Luteolin

- Luteolin-7-glucosid

- Myricetin

- Myricitrin

- Naringenin

- Naringin

- Orientin

- Pentaacetylquercetin

- Phloretin

- Phloridzin

- Quercetin (Dihydrat)

- Quercetin-3-arabinosid

- Quercitrin

- Rhamnetin

- Rhoifolin

- Rutosid (Rutin)

- Taxifolin

- Vitexin

- Vitexin-O-rhamnosid

Phenolcarbonsäuren

- Benzoesäure

- Chlorogensäure

- o-Cumarsäure

- m-Cumarsäure

- p-Cumarsäure

- $\mathrm{p}$-Cumarsäureacetat

- p-Cumarsäuremethylether

- Cumarsäuremethoxymethylether

- Dihydrokaffeesäure

98\% Aldrich $^{\circledR}$

- Ferulasäure

- p-Hydroxyphenylessigsäure

- Isochlorogensäure(n)

- Isoferulasäure

- Kaffeesäure

- Neochlorogensäure

Isolat

purum

Reag. Ph. Eur.

Isolat

puriss.

Isolat

$>90 \%$

rein

ca. $99 \%$

depur

Isolat

Isolat

Isolat

reinst

techn.

Isolat

Reagenz

zur Synthese
Art.-Nr.: 89187

Charge-Nr.: 1075

Phytolab

BioChemika; $\geq 97 \%$ (HPLC)

Sigma-Aldrich

Vestenbergsgreuth, D

Taufkirchen, D

ROTICHROM $^{\circledR}$

Roth

Roth

ROTICHROM $^{\circledR}$

Roth

Karlsruhe, D

Karlsruhe, D

Karlsruhe, D

Roth

Karlsruhe, D

Merck

Darmstadt, D

Fluka

Buchs, $\mathrm{CH}$

Roth

Roth

Serva

ROTICHROM $^{\circledR}$

Serva

Sigma

Roth

Roth

ROTICHROM $^{\circledR}$

ROTICHROM $^{\circledR}$

Roth

Roth

Roth

Roth

Merck

Darmstadt, D

Schuchardt

Roth

Merck-Schuchardt

Sigma-Aldrich Sigma Chemical Co. Fluka

Roth

Fluka
Karlsruhe, D

Karlsruhe, D

Heidelberg, D

Berlin, D

Karlsruhe, D

Karlsruhe, D

Heidelberg, D

St. Louis, USA

Karlsruhe, D

Karlsruhe, D

Karlsruhe, D

Karlsruhe, D
München, D

Karlsruhe, D

Hohenbrunn, D
Steinheim, D

St. Louis, USA

Buchs, CH

Karlsruhe, D

Buchs, $\mathrm{CH}$ 
- Protocatechusäure

- Rosmarinsäure

- Veratrumsäure

- Zimtsäure

Cumarine

- Aesculetin

- Aesculin

- Cumarin

- Herniarin

- $\beta$-Methylumbelliferon

- Umbelliferon

- Scopoletin

- Scopolin

Sonstige

- B-Aescin

- Äpfelsäure

- (-)-Chinasäure

- Cholesterol

- Fumarsäure

- Maleinsäure

- Matairesinol

- Rohsaponingemisch

- Saponin

- ß-Sitosterin

- Vanillin $\begin{array}{ll}\text { purum }>99 \% \text { (UV) } & \text { Fluka } \\ \text { purum } & \text { Fluka }\end{array}$

$\begin{array}{ll}\text { purum }>99 \%(\mathrm{UV}) & \text { Fluka } \\ \text { purum } & \text { Fluka }\end{array}$

zur Synthese

Isolat aus Arctium lappa

Isolat aus Helianthus annuus L.

weiß rein

rein

Lebensmittelqualität
Schönert

Merck-Schuchardt

Fluka

Roth

Merck-Schuchardt

Roth

Sojuzchimexport

Arcochemie

Merck

Schuchardt

Merck-Schuchardt

DHZ Chemie

Merck-Schuchardt

Leipzig, D

Hohenbrunn, D

Buchs, $\mathrm{CH}$

Karlsruhe, D

Hohenbrunn, D

Karlsruhe, D

Moskau, RU

Berlin, D

Buchs, $\mathrm{CH}$

Buchs, $\mathrm{CH}$

Darmstadt, D

München, D

Hohenbrunn, D

Berlin, D

Hohenbrunn, D

Merck

Arcochemie

Merck

Darmstadt, D

Berlin, D

Darmstadt, D

\subsubsection{Adsorbentien}

- Kieselgel zur Chromatographie unter erhöhtem Druck

- Lichroprep RP-18 für die

Flüssigkeits-Chromatographie

- Sephadex ${ }^{\circledR}$ LH-20

- Celite ${ }^{\circledR} 545$
Korngröße 0-0,63 $\mu \mathrm{m}$ Schüttdichte etwa $0,3 \mathrm{~g} / \mathrm{ml}$ Korngröße 25-40 $\mu \mathrm{m}$ (od.: 40-63 $\mu \mathrm{m}$ )

\subsubsection{Reagenzien DC}

- Naturstoffreagenz A (Diphenylboroxyethylamin)

- 4-Methoxybenzaldehyd (Anisaldehyd)

- Antimon(III)-chlorid

- Kaliumiodid

- 4-(Dimethylamino)benzaldehyd

- Wismut(III)-nitrat

- Kaliumpermanganat

- Schwefelsäure

- Kaliumhydroxid

- Polyethylenglykol 4000

- Echtblausalz B

- Natriumhydroxid

$$
\geq 98 \% \text {, p.a. }
$$

zur Synthese

zur Analyse

reinst

zur Synthese

$\geq 71 \%$, p.a., basisch

zur Analyse

95-98\% reinst

$85 \%$ reinst DAC Pellets

ROTIPURAN $^{\circledR} \mathrm{Ph}$. Eur.

Plätzchen rein
Woelm Pharma

Merck

Fluka BioChemika Fluka Chemika
Eschwege, D

Darmstadt, D

Buchs, $\mathrm{CH}$

Buchs, $\mathrm{CH}$
Roth

Merck-Schuchardt

Merck

Merck

Merck-Schuchardt

Roth

Merck

Merck

KMF

Roth

Riedel-de Haen

Merck
Karlsruhe, D

Hohenbrunn, D

Darmstadt, D

Darmstadt, D

Hohenbrunn, D

Karlsruhe, D

Darmstadt, D

Darmstadt, D

Lohmar, D

Karlsruhe, D

Seelze, D

Darmstadt, D 


\subsubsection{Reagenzien Enzymatik}

- Humane Neutrophilen-Elastase Aktivität: 20 U/mg Protein

- N-Methoxysuccinyl-Ala-AlaPro-Val-p-Nitroanilid

- Trypsin-Inhibitor

- TRIS

- Inhibitor (synthet.)

- Pufferlösung

- Pufferlösung

- Pufferlösung

- Trypsin

- N-Bz-DL-Arg-p-Nitroanilid. $\mathrm{HCl}$

- $\mathrm{NaCl}$

- $\mathrm{CaCl}_{2}$

- DMSO

- Thrombin

- Pefabloc SC

- H-D-Phe-homoPro-Arg-p-NADiacetat
ICN Biomedicals Inc.

Sigma-Aldrich

Sigma-Aldrich

Merck

Tris(hydroxymethyl)-aminomethan z. A., Puffersubstanz GW 311616A; $\geq 97 \%$ (HPLC)

CertiPUR $® \mathrm{pH}\left(20^{\circ}\right)=2,01$

pH 9,00 $\pm 0,02$, blau $\mathrm{pH} 7,00 \pm 0,02\left(20^{\circ}\right)$, grün

TPCK treated, from bovine pankreas

$\mathrm{m} / \mathrm{z} 552,7$

Uvasol $^{\circledR}$

vom Rind

4,2-(Aminoethyl)-benzensulfonylfluorid $\cdot \mathrm{HCl}$
Sigma-Aldrich

Merck

Roth

Roth

Sigma-Aldrich

Bachem

Merck

Merck

Merck

Sigma-Aldrich

Roche

Bachem
Aurora, USA

Taufkirchen, D

Taufkirchen, D

Darmstadt, D

Taufkirchen, D

Darmstadt, D

Karlsruhe, D

Karlsruhe, D

Taufkirchen, D

Weil a. Rhein,

D

Darmstadt, D

Darmstadt, D

Darmstadt, D

Taufkirchen, D

Mannheim, D

Weil a. Rhein, D

\subsubsection{Galenische Hilfsstoffe}

- Hydroxypropylmethylcellulose Premium EP (HPMC) Methocel E 50-LV

- Mikrokristalline Cellulose Avicel PH 103

- Aerosil 200

- Gelatine Platin

- Glycerol

- Chitosan

- Citronensäure

- DAC-Basiscreme

- Hypromellose 2000

- Hydrophobes Basisgel DAC

- Mygliol

- Poloxamer 188
Colorcon Ltd.

FMC Europe N.V

Caesar \& Loretz

Roth

Roth

Roth

Merck-Schuchardt

Caesar \& Loretz

Caesar \& Loretz

Caesar \& Loretz

Caesar \& Loretz

BASF

\section{Dartford, UK}

Brüssel, B

Hilden, D

Karlsruhe, D

Karlsruhe, D

Karlsruhe, D

Hohenbrunn, D

Hilden, D

Hilden, D

Hilden, D

Hilden, D

Ludwigshafen, D

\subsubsection{Sonstige Chemikalien}

- L(+)-Weinsäure, $\geq 99,5 \%$

- Natriumsulfat wasserfrei

- Benzocain

- Ammoniak(lösung) 25\%

- Salzsäure rauchend 37\%

- Zierpflanzenspray zur Analyse

Ph. Eur.

reinst

reinst

Lizetan ${ }^{\circledR}$ plus
Roth

KMF optiChem

Synopharm

Merck

Merck

Bayer
Karlsruhe, D

Lohmar, D

Barsbüttel, D

Darmstadt, D

Darmstadt, D

Leverkusen, D 


\subsection{Geräte und sonstiges Zubehör}

- Rührer

- (Licht-) Mikroskop

- System-Mikroskop*1

- Digital Camera

kombiniert mit $\left(*^{1}\right)$

- Trockenschrank

- Hochleistungsschneidmühle

+ Sieb, Maschenweite 28

- Vakuumrotationsverdampfer

- Membran-Vakuumpumpe ${ }^{2}$

- Vakuum-Controller kombiniert mit $\left(*^{2}\right)$ im

Chemiepumpstand 5

- Ultraschallwasserbad

- Waage

- Analysenwaage

- Analysenwaage

- pH-Meter

- Wasserbad

- Magnetrührer / Heiz-

Platte

- Schüttler

- Schüttler

- Pipetten

- UV-Fotometer

- Microplate autoreader

- Kapillarröhrchen für Schmelzpunkt-Bestimmung

- UV-Lampe

- Analysenlampe mit

Durchlicht

- Dokumentationsauf-

satz zur CabUVIS

- Kamera

- Trockenschrank

- 96-Well-Platten

- Korkbohrer

- Tablettenpresse

- Gelbtafeln

- Raubmilben

- Ultra-Turrax

- Hochdruckhomogenisator

- Photonenkorrelationsspektrometer/Zetasizer

- Laserdiffraktometer
RZR 0 Typ 740

Model SE

Vergrößerung 10x40x100

BX 41TF

DXM 1200

ULE 800

Typ SM 2000

Rotavapor R und R-114

Vacuubrand MZ 2C/1,7

CVC 2

Bandelin Sonorex

RK 100 und TK 52

2254

CP 224S-OCE

BP 211D

Labor-pH-Meter 766

GFL $^{\circledR}$ Inkubations-/ Inaktivierungsbad Typ 1002

TYP RCT

KMS1 Minishaker

Vortex-Genie 2;

Modell G-560 E

Research-Pipetten

UV-VIS-Scanning

Spectrophotometer

Spectra Fluor

beidseitig offen

Superior

CAMAG; Cat. No.

29230, Ser. No. 940913

Cab-UVIS; Nr. 131305,

Serie: 04620

Nr. 131312

PowerShot G5 Digital

Camera

Wärmeschrank

Baureihe 6000

transparent, F-Boden,

unbeschichtet

Aluminium

EK 0; Stempel $9 \mathrm{~mm}$

Neudorff ${ }^{\circledR}$

EmulsiFlex C5

Nano-ZS

LS 230
Heidolph Instruments

Nikon Corporation

Olympus Optical

Nikon Corporation

Memmert

Retsch

Büchi Labortechnik

Vacuubrand

Vacuubrand

Bandelin electronic

Sartorius-Werke

Sartorius

Sartorius

Knick

$\mathrm{GFL}^{\circledR}$

Janke \& Kunkel /

IKA ${ }^{\circledR}$-Labortechnik

IKA $^{\circledR}$ Works

Scientific Industries

Eppendorf

Shimadzu Scientific;

Instruments

Tecan

Marienfeld Laboratory

Glassware

CAMAG

DESAGA, Sarstedt-

Gruppe

DESAGA, Sarstedt-

Gruppe

Canon (Europa)

Heraeus Instruments

Roth

bzw. Greiner

Wepa

Korsch

Neudorff

Neudorf

IKA-Werke

Avestin

Malvern

Beckman Coulter
Schwabach, D

Tokyo, JP

Tokyo, JP

Tokyo, JP

Schwabach, D

Haan, D

Flawil, $\mathrm{CH}$

Wertheim, D

Wertheim, D

Berlin, D

Göttingen, D

Göttingen, D

Göttingen, D

Berlin, D

Burgwedel, D

Staufen i. Br., D

Wilmington,

USA

Bohemia, USA

Hamburg, D

Columbia,

USA

Grödig, A

Lauda-Königs-

hofen, D

Berlin, D

Wiesloch, D

Wiesloch, D

Amsterdam, NL

Hanau, D

Karlsruhe, D

Frickenhausen, D

Hillscheid, D

Berlin, D

Emmerthal, D

Emmerthal, D

Staufen, D

Mannheim, D

Herrenberg, D

Krefeld, D 


\section{Methoden}

\subsection{Pflanzen-/Drogengewinnung}

Das zur makroskopischen Beschreibung und mikroskopischen Untersuchung sowie zur Extraktgewinnung genutzte Pflanzenmaterial von Acmella ciliata und der gelben und roten Varietät von Acmella oleracea wurde ausschließlich durch Eigenanbau in Feld- und/oder Gewächshauskulturen gewonnen (s. hierzu Allgem. Teil, Kapitel 2.1.).

Der Erntezeitraum im Freiland erstreckte sich vom Zeitpunkt des Erblühens der Blütenköpfchen (ca. Juni/Juli bei Auspflanzung im Mai nach den letzten Spätfrösten) bis zum ersten Frost. Geerntet wurde hauptsächlich in den Monaten September bis November. Im Gewächshaus war der Erntezeitpunkt weitgehend witterungsunabhängig.

Die Frischpflanzen wurden nach der Ernte sofort von anhaftender Erde befreit, in für die weitere Aufarbeitung vorgesehene Pflanzenteile selektiert, grob zerkleinert und zur Trocknung bereit gestellt. Die Trocknung erfolgte auf Blechen im Trockenschrank (s. 1.2.) bei $40^{\circ}$ bzw. $60^{\circ} \mathrm{C}$ während durchschnittlich vier Tagen bis zur Durchknickbarkeit des Stängels, als dem Pflanzenteil mit dem höchsten Wassergehalt (s. Tab. 32). Die Restfeuchte wurde nicht bestimmt. Um jedoch eine Orientierung zum Verhältnis Frischpflanze(nteile) zu Trockenpflanze(nteilen) $\mathrm{zu}$ gewinnen, wurde mit einer Ernte vom 15.07.2003 der Trocknungsverlust von Blüten, Triebspitzen, Blättern, Stängeln und der oberirdischen Gesamtpflanze in Abhängigkeit von der Temperatur $\left(40^{\circ}\right.$ bzw. $\left.60^{\circ} \mathrm{C}\right)$ ermittelt (s. Tab. 32$)$.

Das getrocknete Pflanzenmaterial wurde in entsprechenden Drogenvorratsgefäßen oder Papiersäcken bei Zimmertemperatur trocken gelagert. Vor der Herstellung der entsprechenden Extrakte erfolgte eine Zerkleinerung der Droge mittels Hochleistungsschneidmühle (s. 1.2.) mit eingebautem Sieb der Maschenweite 28.

Im Freilandanbau wurde partiell eine Stickstoffdüngung mit handelsüblichem Kalkammonsalpeter in zwei Düngestufen (60 und 120 kg/ha Stickstoff) im Jahr 2004 erprobt, indem der Dünger vor dem Auspflanzen gleichmäßig unter die Erde gemischt wurde, was zu einem gesteigerten Größenwachstum sämtlicher Organe im Vergleich zu den benachbart wachsenden, ungedüngten Exemplaren führte. Eine weitere Steigerung dieses Wachstums mittels Düngestufe 2 gegenüber Stufe 1 konnte nicht beobachtet werden. 
Tabelle 32: Trocknungsverluste verschiedener Pflanzenorgane von $\boldsymbol{A}$. ciliata

\begin{tabular}{|l|c|c|c|}
\hline Pflanzenorgan & $\begin{array}{c}\text { Trocknungstemperatur } \\
\left.{ }^{\circ} \mathrm{C}\right]\end{array}$ & $\begin{array}{c}\text { Verhältnis } \\
\text { Droge : Frischpflanze }\end{array}$ & $\begin{array}{c}\text { Trocknungsverlust } \\
{[\%]}\end{array}$ \\
\hline Blüten & 40 & $1: 4,5$ & 78,1 \\
Triebspitzen & 40 & $1: 5,3$ & 81,2 \\
& 60 & $1: 5,2$ & 80,8 \\
Blätter & 40 & $1: 5,4$ & 81,3 \\
& 60 & $1: 4,9$ & 79,6 \\
Stängel & 40 & $1: 6,4$ & 84,4 \\
& 60 & $1: 6,9$ & 85,6 \\
Gesamtpflanze & 40 & $1: 5,1$ & 80,2 \\
(ohne Wurzel) & 60 & $1: 5,9$ & 83,1 \\
\hline
\end{tabular}

Im Gewächshausanbau musste zur Schädlingsbekämpfung (Weiße Fliege, Spinnmilbe), neben Raubmilben (s. Allg. Teil und 1.2.) und an Stäben befestigten und gleichmäßig über das Feld verteilten Gelbtafeln (s. 1.2.), bei starkem Befall gelegentlich Zierpflanzenspray (s. 1.1.7.) eingesetzt werden. Hiermit wurden die Pflanzen kurz aus ca. $40 \mathrm{~cm}$ Entfernung angesprüht. Diese Manipulation musste innerhalb weniger Tage ca. 2 mal wiederholt werden.

\subsection{Extraktionsverfahren}

Zur Extraktion des Drogenmaterials gelangten verschiedene Extraktionsmittel zur Erprobung. Ihre Auswahl richtete sich einmal nach dem zu extrahierenden Gut (Gesamtpflanze oder Blüten) und zum anderen nach dem determinierenden Charakter der zu isolierenden Inhaltsstoffe (Alkamide: dominant lipophil; phenolische Verbindungen: vorherrschend hydrophil). Tab. 1, Allgem. Teil, 2.4., zeigt eine Übersicht.

Die Extraktion erfolgte zunächst durch Rühren, später durch Ultraschallextraktion, die sich als effektiver erwies (s. v.). Die Extrakte zur Isolierung der Alkamide und phenolischen Verbindungen wurden mittels Soxhletierung gewonnen. 
Beim Rühren wurde das zerkleinerte Pflanzenmaterial im Verhältnis Droge : Lösungsmittel wie 1+10 3mal $4 \mathrm{~h}$ mittels stufenlos regelbarem Rührer (s. 1.2.) behandelt. Das Lösungsmittel wurde jeweils nach $3 \mathrm{~h}$ gewechselt.

Die Extrakt-Lösungen wurden unter vermindertem Druck ,abgenutscht“ (Porzellanfiltertrichter) und anschließend vereinigt im Vakuumrotationsverdampfer bei einer Wasserbadtemperatur von $40^{\circ} \mathrm{C}$ bis zur Trockne des Rückstands eingeengt. Sofern keine unmittelbare Weiterverarbeitung der Extrakte erfolgte, wurden diese im verschlossenen Rundkolben, speziell mit Parafilm abgedichtet, im Gefrierschrank bei $-20^{\circ} \mathrm{C}$ gelagert; kürzere Zwischenlagerung erfolgte im Kühlschrank bei 2-8 ${ }^{\circ} \mathrm{C}$.

Zur Ultraschallextraktion dienten Ultraschallwasserbäder (s. 1.2.) bei einer Maximaltemperatur von $40^{\circ} \mathrm{C}$ unter Temperaturkontrolle. Ab einer Extraktionsdauer von mehr als 30 min war eine Kühlung des Wasserbads mittels Wassereis erforderlich. Das Droge/Lösungsmittel-Verhältnis betrug ebenfalls $1+10$, die Extraktionszeit $1 \mathrm{~h}$, wobei das Lösungsmittel nicht gewechselt wurde. Die weitere Aufarbeitung erfolgte analog zur Rührmethode.

Zur Extraktion mittels Soxhlet-Apparatur, wiederum im Verhältnis Droge zu Extraktionsmittel von 1+10, wurde $1 \mathrm{~kg}$ Droge, in 10 Ansätzen pro $100 \mathrm{~g}$, bei der Siedetemperatur des Lösungsmittels (Dichlormethan bzw. Methanol) $3 \mathrm{~h}$ extrahiert. Die Extraktlösungen wurden vereinigt, „abgenutscht" und im Vakuumrotationsverdampfer portionsweise bei einer Wasserbadtemperatur von $40^{\circ} \mathrm{C}$ schonend eingeengt. Die Aufbewahrung erfolgte analog, wie unter Rühren beschrieben.

Im Falle der Isolierung phenolischer Inhaltsstoffe wurde der Extrakt mit 10 Teilen 2 \%iger wässeriger Weinsäurelösung aufgenommen, bis zum Verhältnis 1:30 mit Wasser weiter verdünnt und anschließend fraktioniert ausgeschüttelt: 4mal mit Petrolether (1:1), 3mal mit Dichlormethan (1:1), 3mal mit Ethylacetat (1:1). Lediglich die Ethylacetat-Phase gelangte in dieser Arbeit nach Einengen zur Trockne (Vakuumrotationsverdampfer) zur weiteren Auftrennung durch Säulenchromatographie (2.4.).

Bei den enzymatischen Untersuchungen (4.3.) wurden u. a. Acmella ciliata-GesamtpflanzenAuszüge getestet, die mittels Extraktion mit überkritischem Kohlendioxid gewonnen worden waren (Abteilung Pharmazeutische Technologie der Christian-Albrechts-Universität 
Kiel): $\mathrm{F}_{1}: \mathrm{p}_{\mathrm{C}}\left(\mathrm{CO}_{2}\right)=10 \mathrm{MPa} ; \mathrm{T}_{\mathrm{C}}\left(\mathrm{CO}_{2}\right)=37^{\circ} \mathrm{C} ; \mathrm{F}_{2}: \mathrm{p}_{\mathrm{C}}\left(\mathrm{CO}_{2}\right)=15 \mathrm{MPa} ; \mathrm{T}_{\mathrm{C}}\left(\mathrm{CO}_{2}\right)=34^{\circ} \mathrm{C}$. Hierzu wurden jeweils $10 \mathrm{~g}$ Acmella ciliata-Gesamtdroge in einer ASES-Anlage (Aerosol Spray Extraction System) $1 \mathrm{~h}$ statisch und $2 \mathrm{~h}$ dynamisch extrahiert und die Extrakte aus dem Separator in Aceton (insgesamt je $40 \mathrm{ml}$ ) aufgenommen. Dabei zeigte der Extrakt $\mathrm{F}_{1}$ goldgelbe und $\mathrm{F}_{2}$ dunkelgrüne Farbe.

\section{Saponingewinnung:}

1 Teil grob gepulverte Gesamtdroge wurde mit 10 Teilen Ethanol 45\% (m/m) versetzt, 30 min stehen gelassen, anschließend 1,5 h im Ultraschallwasserbad unter Eiskühlung extrahiert und danach nochmals 30 min stehen gelassen. Das Lösungsmittel Ethanol wurde anschließend am Vakuumrotationsverdampfer entfernt.

Die verbleibende wässerige Phase wurde mehrfach mit n-Butanol ausgeschüttelt und die wässerige Phase (s. Tab. 1) abgetrennt. Die mit Wasser gesättigte n-Butanol-Phase wurde am Vakuumrotationsverdampfer zur Trockne eingeengt, der Rückstand mit Diethylether versetzt und der entstandene Niederschlag nach mehrfachem Waschen mit Ether von der Etherphase (s. Tab. 1) abgetrennt. Der hier gewonnene Niederschlag stellt das Rohsaponingemisch (Tab. 1) dar, dessen Ausbeute, bezogen auf die eingesetzte Droge, $0,8 \%$ betrug.

\subsection{Dünnschichtchromatographie}

Zur analytischen DC wurden Kieselgel-Fertiglatten der Firma Merck; Darmstadt, D (DCAluminiumfolien Kieselgel $60 \mathrm{~F}_{254}, 20 \times 20 \mathrm{~cm}$ ) verwendet. Entsprechend verschiedener Anforderungen wurden die Platten ggf. auf passende Maße zurecht geschnitten.

Die Auftragung erfolgte bandenförmig mittels Schmelzpunktkapillaren (s. 1.2.). Zur Entwicklung dienten Vertikalkammern mit Kammersättigung. Die Laufstrecke betrug in der Regel $13 \mathrm{~cm}$, wobei diese unterschiedlichen Fragestellungen angepasst wurde. Die verwendeten Fließmittelsysteme und deren Einsatz sind in Tab. 33 zusammengefasst.

Zur Detektion von Acmella-Inhaltsstoffen allgemein (vgl. DC-Screening, Allgem. Teil, 3.2.) und zur Sichtbarmachung der Alkamide bzw. phenolischen Verbindungen im Besonderen gelangten neben der Betrachtung im UV-Licht (Eigenfluoreszenz, Fluoreszenzminderung) die folgenden Farbreagenzien zur Anwendung (Tab. 34): 
Tabelle 33: Fließmittel zur DC

\begin{tabular}{|l|l|l|l|}
\hline Nr. & Fließmittelgemisch & Mischungsverhältnis & Literatur \\
\hline III & Ethylacetat / Methanol & $95: 5$ & [165] \\
III & Ethylacetat / Ameisensäure / Wasser & $80: 10: 10$ & [165] \\
IV & Ethylacetat / Ameisensäure / Wasser & $45: 12,5: 15$ & [165] (modif.) \\
V & Chloroform / Methanol & $50: 5: 20$ & [165] (modif.) \\
VI & n-Butanol / Eisessig / Wasser (Oberphase) & $95: 5$ & [179] \\
VII & Ethylacetat / Eisessig / Ameisensäure / Wasser & $100: 11: 11: 27$ & [179] \\
VIII & Dichlormethan / Ethylacetat & $80: 10: 40$ & [110] (modif.) \\
IX & Dichlormethan / Ethylacetat & $90: 10$ & [110]
\end{tabular}

Tabelle 34: DC-Sprühreagenzien

\begin{tabular}{|c|c|c|c|}
\hline Nr. & Reagenz & Spezifität & Literatur \\
\hline 1 & Anisaldehyd - Schwefelsäure & unspezifisch & [38] \\
\hline 2 & Anisaldehyd - Schwefelsäure & unspezifisch & [165] \\
\hline 3 & Antimon(III)-chlorid - Eisessig & Steroide / Diterpene & [165] \\
\hline 4 & Kaliumhydroxid-Lösung (methanolisch) & Cumarine / Anthranoide & [179] \\
\hline 5 & $\begin{array}{l}\text { Dragendorffs-Reagenz nach Munier und } \\
\text { Macheboeuf }\end{array}$ & $\begin{array}{l}\text { tertiäre Amine } \\
\text { (Alkaloide) }\end{array}$ & [165] \\
\hline 6 & Kaliumpermanganat - Schwefelsäure & $\begin{array}{l}\text { oxidierbare Verbind. } \\
\text { (unspezifisch) }\end{array}$ & [165] \\
\hline 7 & Naturstoffreagenz nach $\mathrm{Neu}$ & $\begin{array}{l}\text { Hydroxyflavonole } \\
\text { (Flavonoide) }\end{array}$ & [165] \\
\hline 8 & Naturstoff - Polyethylenglykol & $\begin{array}{l}\text { Hydroxyflavonole } \\
\text { (Flavonoide) }\end{array}$ & {$[179]$} \\
\hline 9 & van Urk-Reagenz (modifiziert nach Stahl) & Indol-Derivate & [165] \\
\hline 10 & Echtblausalz B (Diazo-Reagenz) & $\begin{array}{l}\text { Phenole / kupplungs- } \\
\text { fähige Amine }\end{array}$ & {$[165]$} \\
\hline
\end{tabular}


1: Sprühlösung: 0,5 ml Anisaldehyd werden mit $10 \mathrm{ml}$ Eisessig, $85 \mathrm{ml}$ Methanol und $5 \mathrm{ml}$ Schwefelsäure in der angegebenen Reihenfolge gemischt.

Nachbehandlung: Trockenschrank: $105^{\circ} \mathrm{C}, 5 \mathrm{~min}$

2: Sprühlösung: Frisch bereitete Lösung von 0,5 $\mathrm{ml}$ Anisaldehyd in $50 \mathrm{ml}$ Eisessig unter Zusatz von $1 \mathrm{ml}$ Schwefelsäure (konz.)

Nachbehandlung: Trockenschrank: $100-105^{\circ} \mathrm{C}$, bis zur max. Farbintensität der Flecken

3: Sprühlösung: $20 \mathrm{~g}$ Antimon(III)-chlorid, gelöst in einem Gemisch aus $20 \mathrm{ml}$ Eisessig und $60 \mathrm{ml}$ Chloroform Nachbehandlung: Trockenschrank: $100^{\circ} \mathrm{C}, 5 \mathrm{~min}$

Auswertung: langwelliges UV-Licht

4: Sprühlösung: 10 \%ige ethanolische (modif.: methanolische) Kaliumhydroxid-Lösung Auswertung: VIS und UV-Licht $366 \mathrm{~nm}$

5: Lösung a: 0,85 g bas. Wismutnitrat, gelöst in $10 \mathrm{ml}$ Eisessig und $40 \mathrm{ml}$ Wasser Lösung b: 8 g Kaliumiodid, gelöst in $20 \mathrm{ml}$ Wasser Vorratslösung: Gleiche Volumina der Lösungen a und b werden gemischt.

Sprühlösung: Vor Gebrauch wird $1 \mathrm{ml}$ Vorratslösung mit $2 \mathrm{ml}$ Eisessig und $10 \mathrm{ml}$ Wasser gemischt.

6: Sprühlösung: 0,5 g Kaliumpermanganat, gelöst in $15 \mathrm{ml} \mathrm{Schwefelsäure} \mathrm{(konz.)}$

Nachbehandlung: Trockenschrank: $105-110^{\circ} \mathrm{C}$, ca. 5 min

7: Sprühlösung: 1 \%ige Lösung ( $\mathrm{m} / \mathrm{V})$ von Diphenylboryloxyethylamin R in Methanol R Auswertung: UV-Licht $366 \mathrm{~nm}$

8: Sprühlösung: nacheinander: 1 \%ige methanolische Lösung von Diphenylborsäure- $\beta$-ethylaminoester nach NEU (=Diphenylboryloxyethylamin) und 5 \%ige ethanolische Polyethylenglykol-4000-Lösung

9: Sprühlösung: $1 \mathrm{~g}$ 4-Dimethylaminobenzaldehyd, gelöst in $50 \mathrm{ml}$ Salzsäure (36\%), plus $50 \mathrm{ml}$ Ethanol

10: Sprühlösung I: frisch bereitete Echtblausalz-B-Lösung (0,5 \% in Wasser)

Sprühlösung II: 0,1 N Natronlauge; nacheinander mit I und II besprühen

Mittels Cochromatographie mit entsprechenden Referenzsubstanzen (s. 1.1.2.) und Errechnung der $\mathrm{R}_{\mathrm{f}}$-Werte konnte in einigen Fällen die Identität isolierter Substanzen verifiziert werden.

Zur präparativen DC, die u. a. der Alkamidisolierung diente (s. Allgem. Teil., 3.3.2./3.3.3.), wurden Kieselgelplatten ohne Fluoreszenzindikator genutzt:

- DC-Aluminiumfolien 20x20 cm mit KG 60 (= 60 Å Porenweite); Merck, Darmstadt, D

- Glasplatten 20x20 cm mit SIL G-50 (=KG mit Gipsbinder, Porenweite 50 Å);

Macherey-Nagel, Düren, D

Das Auftragen erfolgte als Bande, abgesehen von $1 \mathrm{~cm}$ Rand rechts und links, über die gesamte Plattenbreite mit Schmelzpunktkapillaren (s. o.). Um die Lage der einzelnen Banden zu identifizieren, musste ein schmaler Randstreifen mit Sprühreagenz (AnisaldehydSchwefelsäure, 1, Tab. 34) markiert werden. 
Nach Abdunsten der Lösungsmittel, beschleunigt durch Fönen, erfolgte das Abkratzen der Banden von der Aluminiumfolie bzw. Glasplatte. Anschließend wurde die KieselgelSubstanz-Mischung durch wiederholte, jeweils 10minütige Ultraschallextraktion mit dem Fließmittelgemisch IX (s. Tab. 33) aus dem Kieselgel extrahiert und mittels Filtration per Papier- und anschließend Membranfilter (Porenweite 0,25 $\mu \mathrm{m}$ ) vom Adsorbens getrennt. Das Lösungsmittel wurde evaporiert und der Rückstand der Strukturanalyse zugeführt.

\section{Schaumprobe:}

Eine definierte Menge der gepulverten Droge (0,5 g) wird in einem Reagenzglas mit $10 \mathrm{ml}$ heißem Wasser übergossen und nach dem Abkühlen $10 \mathrm{~s}$ geschüttelt. Sind Saponine vorhanden, entsteht ein 1-10 cm hoher Schaum [1].

\subsection{Säulenchromatographie}

Die SC gelangte als Gelpermeationschromatographie (GPC) zur Fraktionierung der Ethylacetat-Ausschüttelung (s. 2.2.) und Isolierung der phenolischen Verbindungen zum Einsatz. Die Auftrennung erfolgte an Schwerkraftsäulen mit dem Füllmaterial Sephadex LH20, das am Vortage der Säulenbeschickung zur Quellung in dem entsprechenden Eluens suspendiert wurde.

Das Analysenmaterial (6 g Extrakt) wurde in $24 \mathrm{ml} \mathrm{Methanol/Wasser} \mathrm{(5 \% )} \mathrm{gelöst} \mathrm{und} \mathrm{per}$ „Einsicker-Methode“ auf die Säule I appliziert. Die Details sind Tab. 35 zu entnehmen.

Tab. 35 verdeutlicht, dass nacheinander mit dem Gesamtextrakt bzw. ausgewählten Fraktionen (DC-Kontrolle) vier Säulen (Säule I-IV) beschickt und mit den Hauptlösungsmitteln Methanol - Aceton - Ethylacetat - Ethylacetat eluiert wurden (vgl. auch das Schema in Abb. 34). Nach DC bzw. analytischer HPLC (2.6.) wurden schließlich relevante Fraktionen zur weiteren Auftrennung und Strukturaufklärung der präparativen HPLC (2.6.) unterzogen.

Aus Säule I (Tab. 35) wurden durch Nachwaschen mit Methanol die in der Startzone verbliebenen gelb gefärbten Substanzen als sog. Gelber Nachlauf gewonnen, zur Trockne eingeengt, mit Methanol wieder aufgenommen, vom anfallenden Niederschlag durch Filtration getrennt und per präparativer HPLC aufgearbeitet (s. 2.6.). 
Tabelle 35: Säulen-Chromatographie an Sephadex LH-20

\begin{tabular}{|c|c|c|c|c|}
\hline & Säule I & Säule II & Säule III & Säule IV \\
\hline & (Methanol) & (Aceton) & (Ethylacetat) & (Ethylacetat) \\
\hline Gelbetthöhe [mm] & 780 & 685 & 710 & 380 \\
\hline \multirow[t]{4}{*}{ Beschickung } & Ethylacetat-Phase $(6 \mathrm{~g})$ & eingeengte und von Nieder- & eingeengte Fraktionen 1 - 41 & eingeengte Fraktionen 1 - 17 \\
\hline & gelöst in 24 ml Methanol / & schlägen befreite Fraktionen & $(5,5 \mathrm{~g})$ von Säule II, gelöst in & von Säule III, gelöst in \\
\hline & Wasser 95:5 & 1 - 105 von Säule I, gelöst in & $36 \mathrm{ml}$ Aceton / Methanol 1:1 & $6 \mathrm{ml}$ Ethylacetat / Aceton 1:1 \\
\hline & & $60 \mathrm{ml}$ Methanol / Wasser $80: 20$ & & \\
\hline \multirow[t]{3}{*}{ Mobile Phase } & 1. Methanol / Wasser & 1. Aceton & 1. Ethylacetat & 1. Ethylacetat \\
\hline & $90: 10 \rightarrow 70: 30$ & 2. Methanol & 2. Aceton & 2. Ethylacetat / Aceton 1:1 \\
\hline & 2. Methanol (Spülung) & 3. Methanol / Wasser $80: 20$ & 3. Aceton / Methanol $80: 20$ & 3. Aceton \\
\hline Flussrate [ml/min] & 2 & 2 & 2 & 2 \\
\hline Fraktionsgröße [m/] & 5 & 10 & 5 & 10 \\
\hline weiter bearbeitete & 1 - 105 nach Abtrennung der & 1 - 41 (Aceton) $\rightarrow$ Säule III & 1 - 18 (= III. 1) $\rightarrow$ Säule IV; & 1 - 17, Rest-Eluat $\rightarrow$ DC / HPLC \\
\hline \multirow[t]{4}{*}{ Fraktionen } & Niederschläge $\rightarrow$ Säule II, & & III. 2, III 4., III. $5 \rightarrow$ DC / HPLC & \\
\hline & "Gelber Nachlauf" der Methanol- & & & \\
\hline & spülung nach Abtrennung des & & & \\
\hline & Niederschlags & & & \\
\hline \multirow[t]{3}{*}{ Niederschläge } & nach kurzem Stehenlassen & - & - & - \\
\hline & aus den Fraktionen 58 - 90 und & & & \\
\hline & aus dem "Gelben Nachlauf" & & & \\
\hline & & & & \\
\hline
\end{tabular}




\subsection{Niederdruckflüssigchromatographie}

Die LPLC erwies sich als Methode der Wahl zur Charakterisierung der Alkamide aus dem Dichlormethanextrakt $\left(\mathrm{A}_{2}\right)$. Zunächst wurde auf einer Kieselgelsäule (KG-Säule) durch Normalphasenchromatographie eine gewisse Vortrennung der Substanzen erzielt. Die nach DC-Auswahl relevanten Fraktionen 36-45 (eingeengt $=0,59 \mathrm{~g}$ ) gelangten anschließend zur weiteren Auftrennung durch LPLC auf eine Säule mit dem Umkehrphasenmaterial RP-18 (RP-Säule) (s. Abb. 17). Die LPLC-Parameter beider Säulen gibt Tab. 36 wieder.

Tabelle 36: LPLC der Alkamide

\begin{tabular}{|c|c|c|}
\hline & KG-Säule & RP-Säule \\
\hline Pumpe & $\begin{array}{l}\text { Chromatographiepumpe, } \\
\text { Typ: B 681, Büchi Labortechnik } \mathrm{GmbH} \text {; } \\
\text { Flawil, } \mathrm{CH}\end{array}$ & $\begin{array}{l}\text { pulsationsgedämpfte Dosierpumpe, } \\
\text { Typ: Duramat }^{(B)} 80 \text {, CfG ProMinent; } \\
\text { Heidelberg, D }\end{array}$ \\
\hline Druck [kPa] & 10 & 2 \\
\hline Trennsäule Ø / h [mm] & $\begin{array}{l}52 \text { / } 540 \\
\text { Kronwald Separationstechnik; } \\
\text { Sinsheim, D }\end{array}$ & $40 / 250$ \\
\hline Stationäre Phase & $\begin{array}{l}\text { Kieselgel zur Chromatographie unter } \\
\text { erhöhtem Druck, Woelm Pharma; } \\
\text { Eschwege, D } \\
\text { Korngröße: } 0 \text { - } 0,63 \mu \mathrm{m} \\
\text { Schüttdichte: ca. } 0,3 \mathrm{~g} / \mathrm{ml} \\
150 \mathrm{~g} \text { vorgequollen }\end{array}$ & $\begin{array}{l}\text { Lichroprep RP-18 für die Flüssigkeits- } \\
\text { chromatographie, Merck; } \\
\text { Darmstadt, D } \\
\text { Korngröße: } 25 \text { - } 40 \mu \mathrm{m} \\
40 \mathrm{~g}\end{array}$ \\
\hline Beschickung & $\begin{array}{l}\text { Dichlormethanextrakt }(10 \mathrm{~g}) \text {, gelöst } \\
\text { in } 30 \mathrm{ml} \text { Dichlormethan }\end{array}$ & $\begin{array}{l}\text { Fraktionen } 36 \text { - } 45(0,59 \mathrm{~g}) \text { von } \\
\text { KG-Säule, gelöst in } 10 \mathrm{ml} \text { Methanol } \\
\text { plus } 2 \mathrm{~g} \text { Celite }{ }^{\circledR} 545 \text {, Fluka Chemika; } \\
\text { Buchs, CH }\end{array}$ \\
\hline Mobile Phase & Dichlormethan / Ethylacetat & Methanol / Wasser \\
\hline Stufengradient & $9: 1 \rightarrow 1: 1 ;$ je $300 \mathrm{ml}$ & $\begin{array}{l}1: 1 \rightarrow 10: 0 ; \text { je } 200 \mathrm{ml} \text {, letzte Stufe } \\
600 \mathrm{ml}\end{array}$ \\
\hline Flussrate [ml/min] & 1,5 & 1,5 \\
\hline Fraktionsgröße [ml] & 20 & 40 \\
\hline
\end{tabular}


Die aus der RP-Säule resultierenden Fraktionen wurden zu Sammelfraktionen zusammengefasst, von denen 1 - 4 entsprechend den Angaben im Schema der Abb.17 der weiteren Auftrennung und Strukturanalyse unterzogen wurden:

Sammelfraktion 1 = Fraktionen (1-12): Das nachfolgende Schema (Abb. 61) zeigt die weitere Auftrennung mittels der unter 2.6. beschriebenen präparativen HPLC bei $254 \mathrm{~nm}$ (Flussrate: 5,5 ml/min, Gradienten s. im Schema), woraus durch anschließende NMR die vorwiegend hydrophilen Verbindungen $\boldsymbol{S} 1$ bis $\boldsymbol{S} 4$ (s. Allgem. Teil, 3.5.1.) identifiziert werden konnten.

Sammelfraktion $2=$ Fraktionen $(13-17) \rightarrow$ präparative DC/ ${ }^{1} \mathrm{H}-\mathrm{NMR}$ und HPLC/ESI-MS (Alkamid-Strukturaufklärung (s. Allgem. Teil, 3.3.3.ff.))

Sammelfraktion 3 = Fraktionen $(18-20) \rightarrow$ HPLC/ESI-MS (Alkamid-Strukturaufklärung (s. Allgem. Teil, 3.3.3.ff.))

Sammelfraktion $\mathbf{4}=$ Fraktionen (21-22) $\rightarrow$ Die weitere Auftrennung erfolgte per präparativer HPLC (s. 2.6.) (Gradient: 80:20 - 90:10, $90 \mathrm{~min}, 260 \mathrm{~nm}, 5,5 \mathrm{ml} / \mathrm{min}$.). Es resultierten zwei Peaks, von denen lediglich der zweite per ${ }^{1} \mathrm{H}-\mathrm{NMR}$ strukturanalytisch aufklärbar war. Dieser ergab Verbindung A16 (s. Allgem. Teil, 3.3.3.17.).

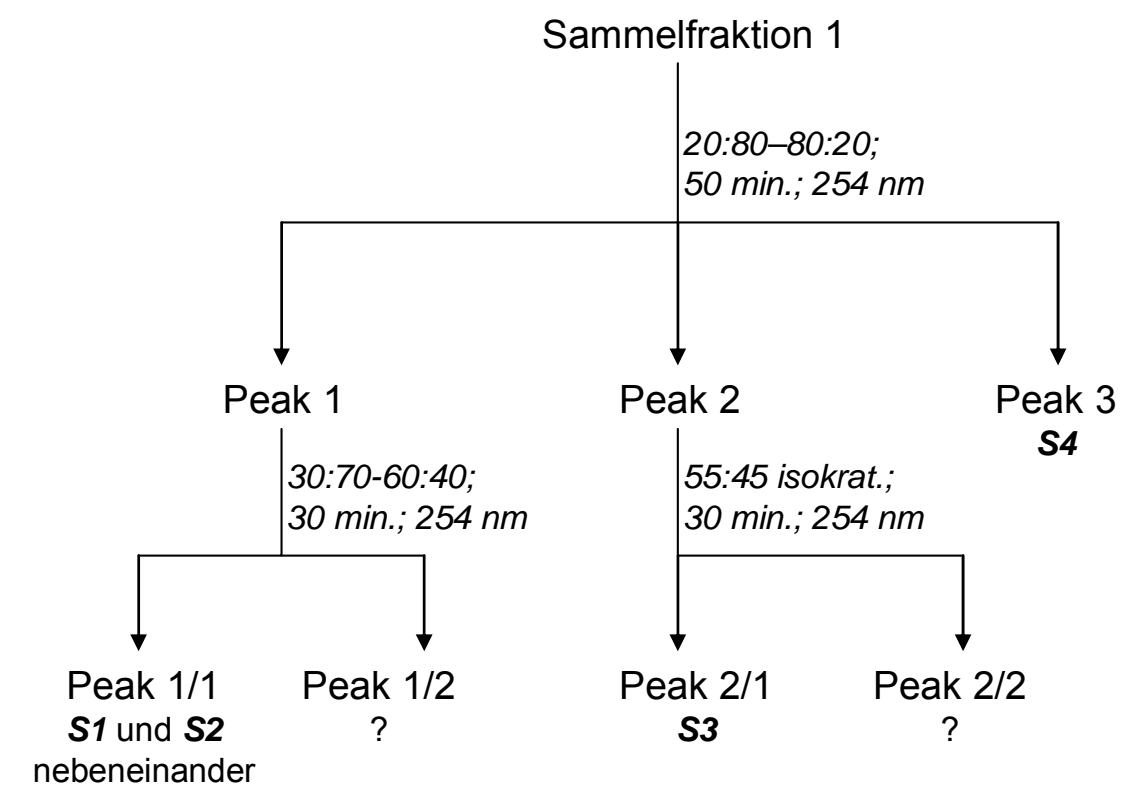

Abb. 61: HPLC-Trennung von Sammelfraktion 1 


\subsection{Hochleistungsflüssigchromatographie}

Die HPLC gelangte sowohl zur Charakterisierung und Identifizierung von Verbindungen durch deren unter festgelegten chromatographischen Bedingungen typische RT (retention time) als auch mittels Cochromatographie mit Referenzsubstanzen zum Einsatz sowie zur Fraktionierung von Substanzgemischen auf präparativem Wege. Aus Tab. 37 gehen die Geräte- und Versuchsbedingungen für die analytische HPLC hervor, und Tab. 38 dokumentiert die entsprechenden Parameter für die präparative HPLC.

Tabelle 37: Geräte- und Versuchsparameter der analytischen HPLC

\begin{tabular}{|c|c|}
\hline Gerät & HP 1090 Series II HPLC System, Hewlett Packard (D); Waldbronn, D \\
\hline Software & Berit’s LC (online) \\
\hline Injektionsvolumen [ $\mu \mathrm{l}]$ & $20 \mu \mathrm{l}$ \\
\hline Stationäre Phase & 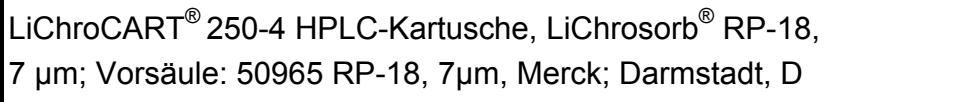 \\
\hline Mobile Phase & $\begin{array}{l}\text { Acetonitril / Wasser (wechselnde Zusammensetzung und Gradienten) } \\
\text { jeweils als } 0,1 \% \text { ige Lösungen von Trifluoressigsäure }\end{array}$ \\
\hline Flussrate $[\mathrm{ml} / \mathrm{min}]$ & $1-1,5$ \\
\hline Wellenlänge [nm] & $\begin{array}{l}\text { wechselnd: } 200,210,220,230,254,260,330,365 \\
\text { max. } 5 \text { Wellenlängen parallel }\end{array}$ \\
\hline
\end{tabular}

Tabelle 38: Geräte- und Versuchsparameter der präparativen HPLC

\begin{tabular}{|c|c|}
\hline Pumpe & Knauer Smartline Pump 1000, Knauer; Berlin, D \\
\hline Detektor & Knauer Variable Wavelength Monitor \\
\hline Interface & Knauer Smartline Manager 5000 \\
\hline Software & Eurochrom 2000 \\
\hline Injektion & max. $500 \mu \mathrm{l}$; Rheodyne-Ventil; Spritze: Microsyringe Knauer $500 \mu \mathrm{l}$ \\
\hline Stationäre Phase & LiChrospher 100 RP-18 endcapp. 5 m, Knauer; Berlin, D \\
\hline Mobile Phase & $\begin{array}{l}\text { Acetonitril / Wasser (wechselnde Zusammensetzung und Gradienten) } \\
\text { jeweils als 0,1\%ige Lösungen von Trifluoressigsäure }\end{array}$ \\
\hline Flussrate [ml/min] & 5,5 \\
\hline Wellenlänge [nm] & wechselnd: 220, 254, 260, 330 \\
\hline
\end{tabular}


Da nach sc Fraktionierung auf Sephadex (2.4.) zur Gewinnung phenolischer Verbindungen noch keine Reinsubstanzen anfielen, wurden relevante Fraktionen der Säulen III und IV (Abb. 34) mittels HPLC weiter aufgetrennt. Zunächst wurden die Proben bei verschiedenen Wellenlängen mittels analytischer HPLC untersucht (Tab. 37), um für die präparative HPLC die geeignete Wellenlänge $\mathrm{zu}$ eruieren. In den folgenden vier Schemata sind diese aufwändigen Manipulationen wiedergegeben und die isolierten Substanzen eingetragen.

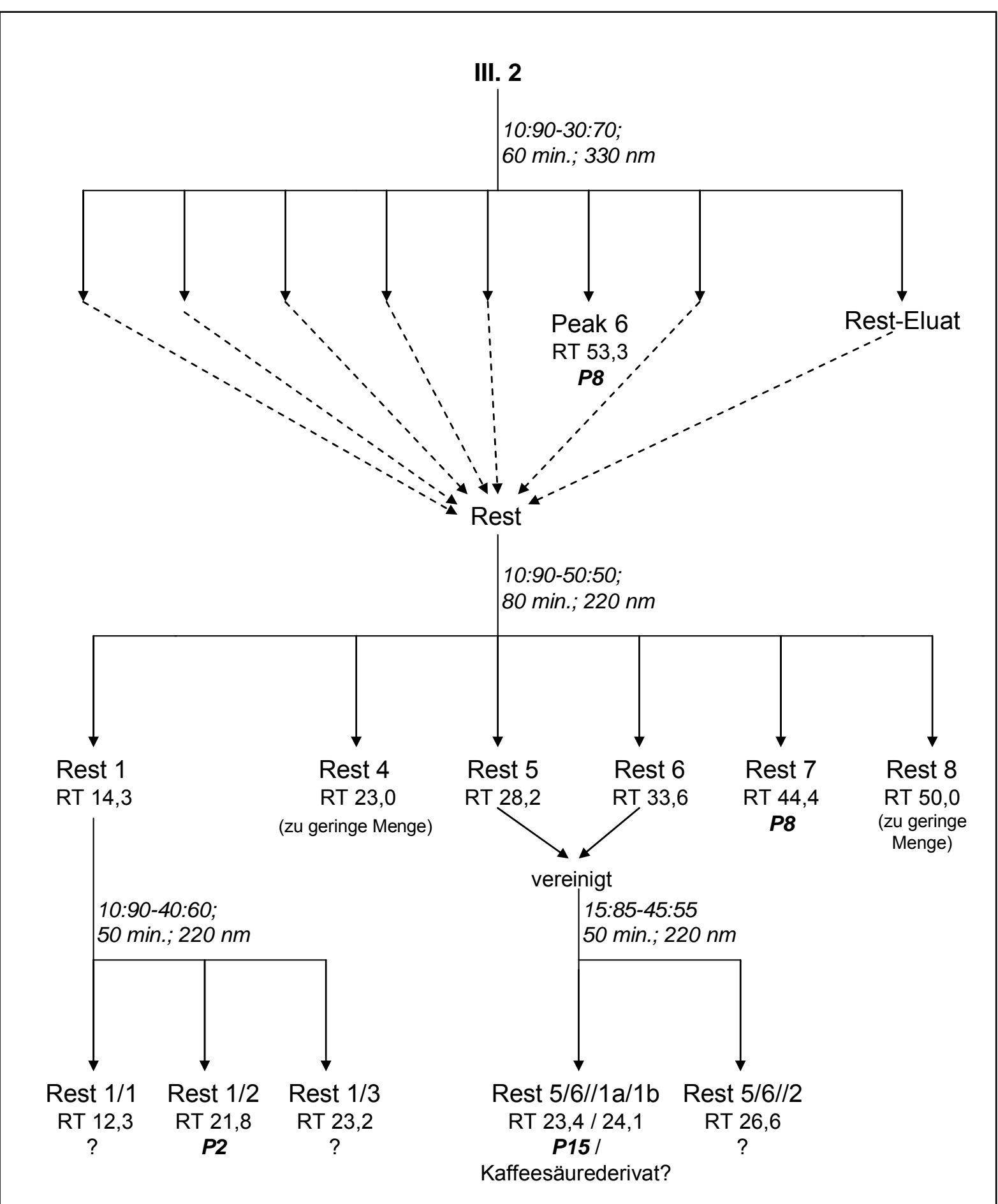

Abb. 62: HPLC-Trennung von Sammelfraktion III. 2 (s. Abb. 34) 


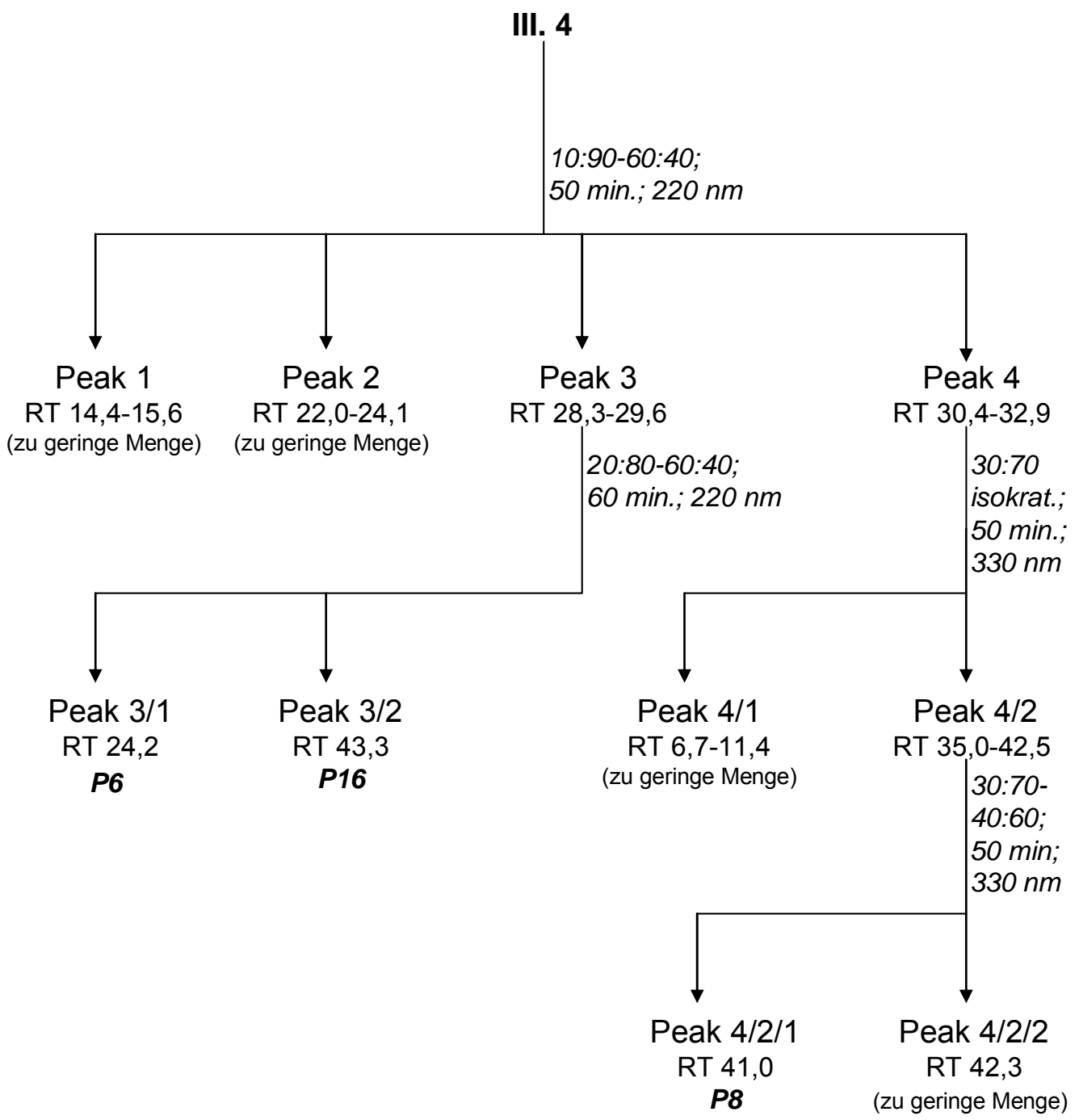

Abb. 63: HPLC-Trennung von Sammelfraktion III. 4 (s. Abb. 34) 


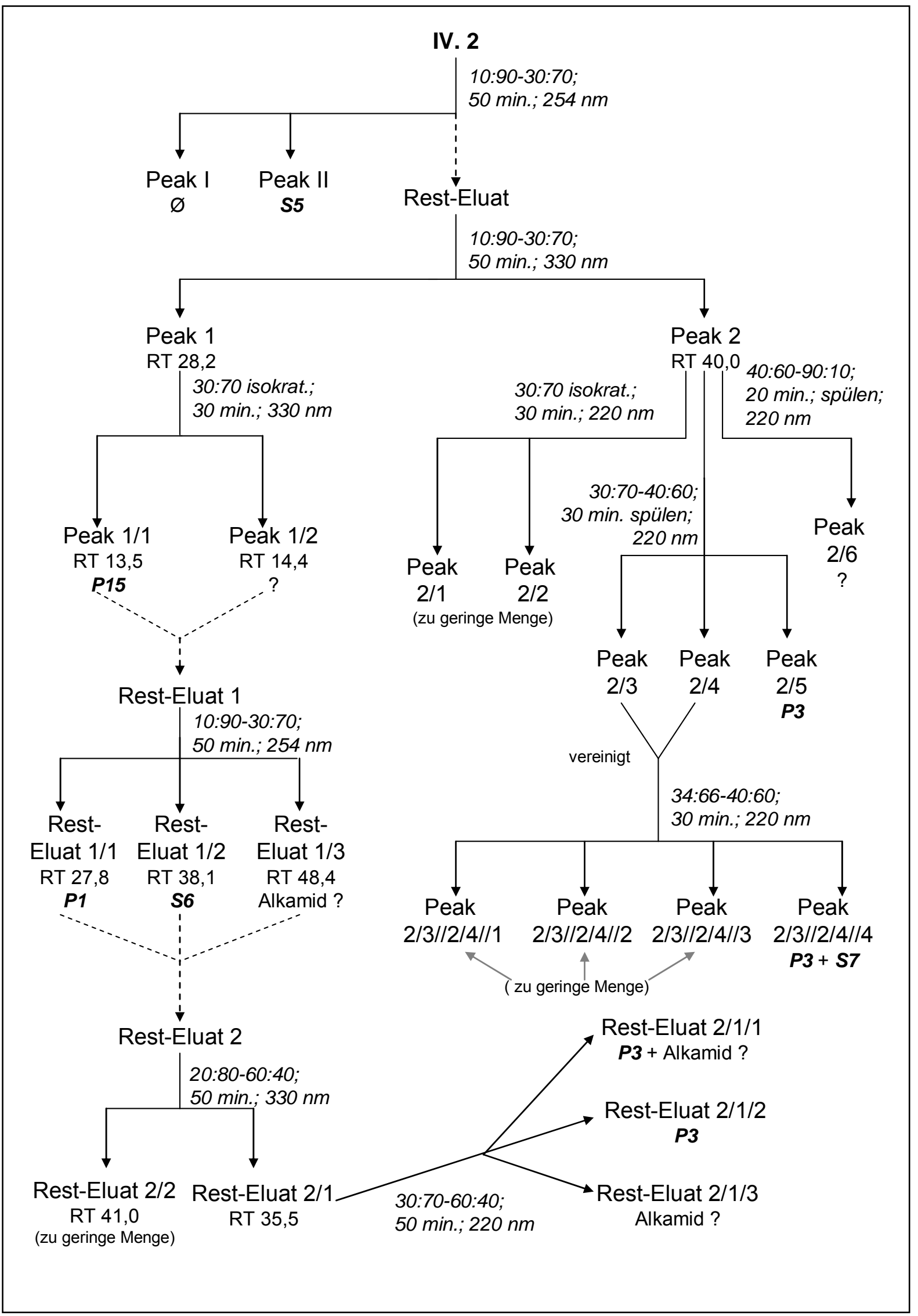

Abb. 64: HPLC-Trennung von Sammelfraktion IV. 2 (s. Abb. 34) 


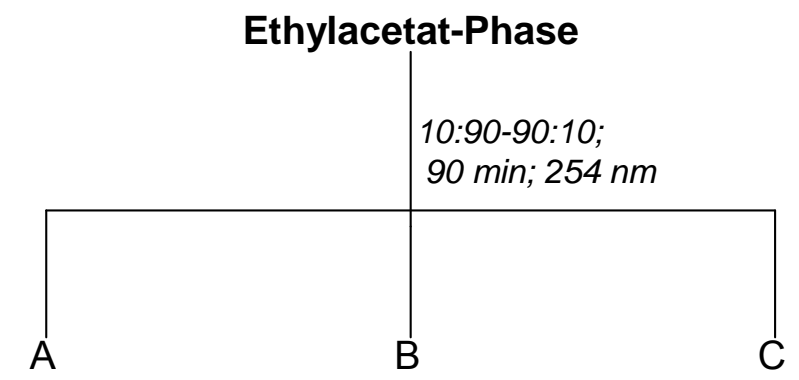

RT 49-58

RT 60-70

30:70-60:40;

30:70-60:40;

RT 70-79

50 min.; $220 \mathrm{~nm}$

$50 \mathrm{~min} ; 254 \mathrm{~nm}$

10:90-60:40;

50 min.; $220 \mathrm{~nm}$

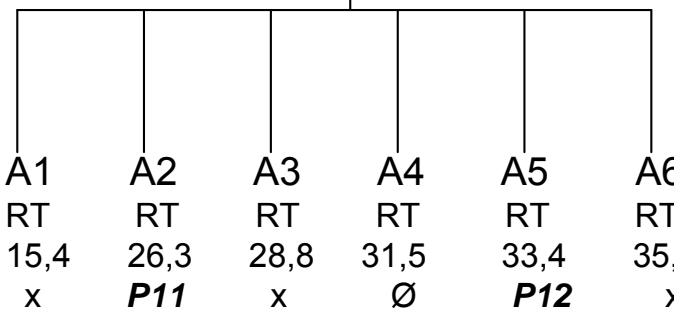

B1

RT 5,0 -7,6

30:70-40:60; 30 min.; 30 min.; $254 \mathrm{~nm}+$ spülen: 40:60-50:50; 30 min.; 254 nm

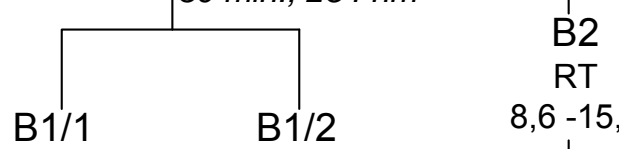

RT $7,3 \quad 16,8$

$6-15,7$

C1

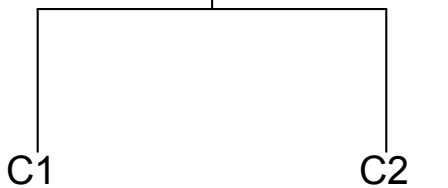

$x$

$\mathrm{x}$

10:90-60:40;

50 min.; $220 \mathrm{~nm}$

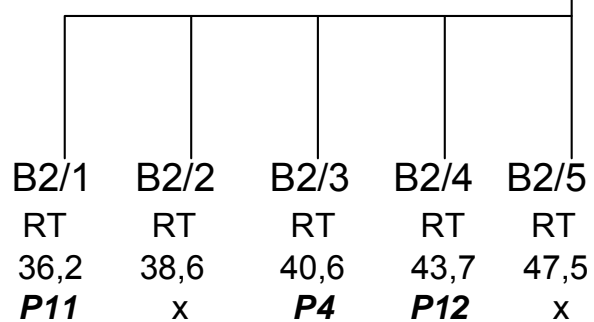

RT 4,4 -7,7

10:90-40:60

50 min.; $220 \mathrm{~nm}$

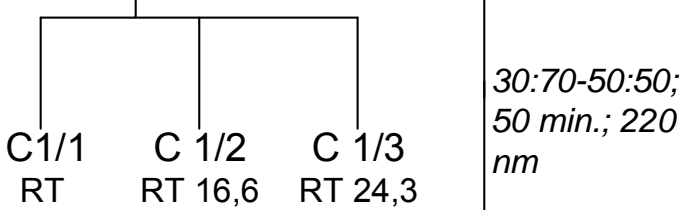

50 min. 220
RT 6,2 -10,7<smiles>[TeH][TeH]</smiles><smiles>C[Al]</smiles>
10:90-40:60;

$\mathrm{C} 2 / 2$

RT $11,4-30,7$

Abb. 65: HPLC-Trennung der Ethylacetat-Phase (Weg 2; s. Abb. 33)

$\mathrm{x}$ : zu geringe Menge; Ø: nicht weiter bearbeitet 
Die präparative HPLC (220 nm; Acetonitril/Wasser = 1:9 - 1:1; Flussrate 5,5 ml/min; $50 \mathrm{~min})$ diente darüber hinaus zur Aufarbeitung des sog. Gelben Nachlaufs (s. 2.4.), der, wie Abb. 66 zeigt, eine Reihe von Substanzen enthielt. Die gekennzeichneten Peaks wurden strukturanalytisch untersucht und als phenolische Verbindungen bzw. als Fumarsäure (S5) (s. auch Übersicht unter 3.4.3., Allgem. Teil) identifiziert.

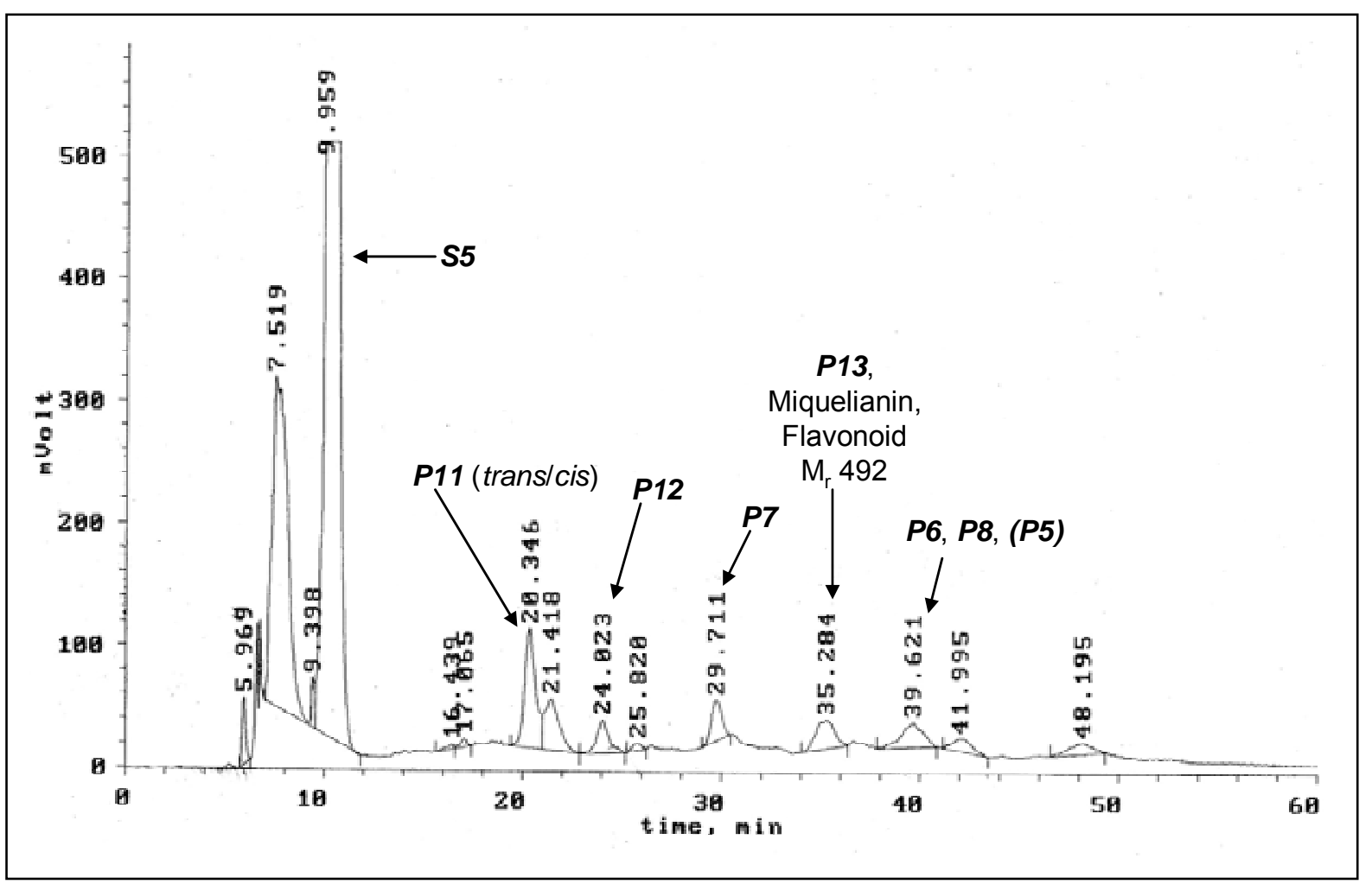

Abb. 66: HPLC Gelber Nachlauf

\subsection{Spektroskopische/- metrische Methoden}

\subsubsection{UV-Spektroskopie}

UV-Spektren wurden teilweise zur Charakterisierung von phenolischen Verbindungen herangezogen und dienten u. a. zur Substanzidentifizierung im Abgleich mit Referenzsubstanzen. Die Spektren wurden online aufgenommen mit Hilfe des DAD-UVDetektors des HPLC-Systems (s. Tab. 37). 


\subsubsection{NMR-Spektroskopie}

Die ${ }^{1} \boldsymbol{H}$-NMR wurde unter folgenden Bedingungen durchgeführt:

Gerät: Bruker DPX 400 (400 MHz); Bruker BioSpin GmbH, Bruker Corporation; Rheinstetten, D

Lösungsmittel: $\mathrm{CD}_{3} \mathrm{OD}$, Aceton- $\mathrm{d}_{6}, \mathrm{CDCl}_{3}$

interner Standard:

TMS

Die ${ }^{13} \boldsymbol{C}$-NMR wurde unter folgenden Bedingungen durchgeführt:

Gerät: Bruker DPX 400 (100,6 MHz); Bruker BioSpin GmbH, Bruker Corporation; Rheinstetten, D

Lösungsmittel: $\mathrm{CD}_{3} \mathrm{OD}$, Aceton- $\mathrm{d}_{6}$

interner Standard:

TMS

\subsubsection{Massenspektrometrie}

EI-MS: $\quad$ Gerät: MAT 711, Varian MAT; Bremen, D

FAB-MS: $\quad$ Gerät: CH-5, Varian MAT; Bremen, D

ESI-MS: $\quad$ Agilent 6210 system mit ESI-MSD-TOF-Massenspektrometer; Agilent; Darmstadt, D

(HP)LC-MS:

Die Kombination der HPLC mit der Massenspektrometrie (HPLC/MS-Kopplung, s. 3.3.3.ff., Allgem. Teil) unter Nutzung der Elektronenspray-Ionisation (ESI-MS, s. Tab. 39) ließ sich als geeignetes Verfahren zur Identifizierung und Charakterisierung von Substanzen, insbesondere der Alkamide aus Gemischen, anwenden. 
Tabelle 39: Geräte und Versuchsparameter der HPLC-(+ESI)MS

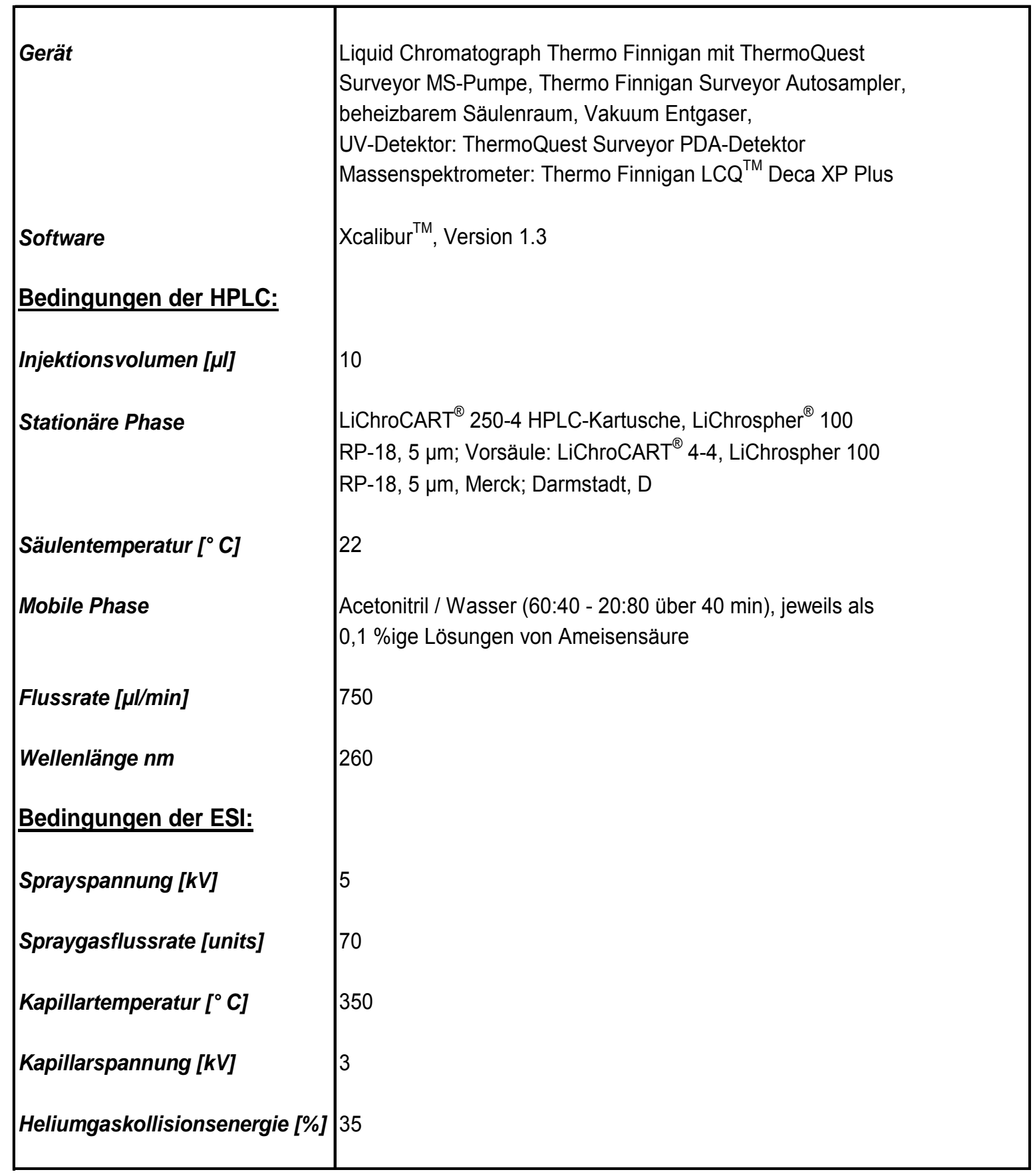

\subsection{Enzymatische Methoden}

2.8.1. Hemmung der Aktivität der humanen Neutrophilen-Elastase

\subsubsection{Lösungen}

Entsprechend der Literatur [115] wurden zunächst Puffer-, Substrat-, Enzym-, Inhibitor- und Proben-Lösungen hergestellt: 
Puffer: Tris (Tris-(hydroxymethyl)-aminomethan)-HCl-Puffer $\mathrm{pH}=7,5$ (60 mM)

Vorratslösung: $\quad 3,63 \mathrm{~g}$ Tris, gelöst in $125 \mathrm{ml}$ Reinstwasser $\rightarrow \mathrm{pH}-$ Einstellung mit $\mathrm{HCl}(\mathrm{pH} 7,5) \rightarrow$ Auffüllen auf $500 \mathrm{ml}$

(Reinstwasser); aufbewahrt bei $2-8^{\circ} \mathrm{C}$

Substrat: N-Methoxysuccinyl-Ala-Ala-Pro-Val-p-Nitroanilid

Substratstammlösung (10 mM):

Substratverdünnung $(1,4 \mathrm{mM})$ :

Humane Neutrophilen-Elastase: Aktivität: 2,1 U/100 $\mu \mathrm{g}$

Enzymstammlösung:

Enzymverdünnung:

Aktivitätsbestimmung:
$29,53 \mathrm{mg}$ Substrat mit $250 \mu 1 \mathrm{DMSO}$ und $4750 \mu 1$ TrisHCl-Pufferlösung gemischt (,,vorgetext"); aliquotiert in Vials; aufbewahrt bei $-20^{\circ} \mathrm{C}$

$140 \mu 1+1000 \mu 1$ Pufferlösung; $125 \mu 1$ pro Vial
$100 \mu \mathrm{g}$ Elastase, gelöst in $1000 \mu 1$ Reinstwasser; aliquotiert in Vials; aufbewahrt bei $2-8^{\circ} \mathrm{C}$

z. B. 1+9 mit Puffer (entsprechend Aktivität), weiter 1:20 vor Versuch mit Puffer

$125 \mu 1$ Substratverdünnung $+1 \mu 1$ Enzymstammlösung $+474 \mu 1$ Puffer $\rightarrow$ vermischt $\rightarrow 1 \mathrm{~h}$ bei $37^{\circ} \mathrm{C}$ inkubiert (Wasserbad) $\rightarrow$ Absorptionsmessung bei $405 \mathrm{~nm}$ (Absorption muss zwischen 0,2 und 0,8 liegen!)

Trypsin-Inhibitor aus Sojabohne, Typ I-S (Aktivität: $1 \mathrm{mg}$ inhibiert 1,4 mg Trypsin der Aktivität 10000 BAEE U/mg Protein)

Inhibitorlösung: $10 \mathrm{mg}$ in $50 \mathrm{ml}$ Puffer gelöst; aufbewahrt bei $2-8^{\circ} \mathrm{C}$

Proben: Acmella ciliata-Extrakte $\mathrm{A}_{3}, \mathrm{~B}_{2}, \mathrm{C}_{4}, \mathrm{D}_{1}, \mathrm{E}_{1}, \mathrm{E}_{2}, \mathrm{~A}_{4}, \mathrm{~A}_{5}$, G; Spolera ${ }^{\circledR}$-flüssig; Referenz-Alkamid(e) (Dodeca-2,4,8,10 (E,E,Z,E / $E, E, Z, Z)$-tetraensäure-isobutylamid(e) sowie die mit den Extrakten $\mathrm{A}_{3}-\mathrm{E}_{1}$ korrelierenden Extrakte von Acmella oleracea (gelb)

Probenstammlösung: 1:100 in DMSO (100\%-Werte)

Probenverdünnungen:

z. B. $100 \mu 1$ Probenstammlösung $+900 \mu 1$ Puffer usw. bis zum Überschreiten der $\mathrm{IC}_{50}$

Anmerkung: Auf Grund schlechter Löslichkeit von lipophilen Proben musste Puffer partiell durch DMSO substituiert werden Die Verdünnung der letzten Stufe musste jedoch in jedem Fall mit Puffer erfolgen, da der Assay und die UV-VIS-Messung nur im wässerigen Milieu funktionieren (Probenleerwerte analog!). 


\subsubsection{Versuchsdurchführung}

\section{Probenschema:}

Pufferleerwert:

$600 \mu 1$ Pufferlösung

Substratleerwert:

$125 \mu 1$ der Substratverdünnung $+475 \mu 1$ Puffer

Probenleerwert 100\%:

$60 \mu 1$ Probenstammlösung $+540 \mu 1$ Puffer

Probenwerte $100 \%(n=3-4)$ :

$60 \mu 1$ Probenstammlösung $+395 \mu 1$ Puffer $+125 \mu 1$

Substratverdünnung $+20 \mu 1$ Enzymverdünnung

Anmerkung: Enzymlösung stets zum Schluss zufügen, da dadurch die Reaktion gestartet wird.

Probenleerwert(e) verdünnt: $\quad 60 \mu 1$ Probenverdünnung $+540 \mu 1$ Puffer

Probenwert(e) verdünnt ( $n=3): \quad 60 \mu$ l Probenverdünnung

Es wurden zu jeder Probe Konzentrationsreihen in 3 bis 5 Stufen im Bereich von 0,1 bis 10 $\mu \mathrm{g} / \mathrm{ml}$ bestimmt.

Die Reaktion verläuft im temperierbaren Wasserbad bei $37^{\circ} \mathrm{C}$. Die Vials befinden sich in entsprechenden Haltevorrichtungen (Ständern) und werden gegen ein Aufschwimmen gesichert. Die Reaktionsdauer beträgt $1 \mathrm{~h}$ (s. auch Aktivitätsbestimmung oben). Danach werden die Vials aus dem Wasserbad entfernt, in jedes Vial wird $500 \mu$ l TrypsinInhibitorlösung (s. o.) pipettiert, geschüttelt (KMS1 Minishaker, s. 1.2.) und damit die Reaktion gestoppt. Anschließend erfolgt die Absorptionsmessung am UVZweistrahlfotometer (s. 1.2.) bei $\lambda=405 \mathrm{~nm}$ in Quarzküvetten gegen Reinstwasser (jeweils drei Messungen).

\subsubsection{Auswertung}

Es wurden die drei Messwerte pro Vial und die 3-4 Probenwerte jeder Konzentration gemittelt und der Probenleerwert sowie der Minuent aus Substratleerwert und Pufferleerwert von diesen Mittelwerten subtrahiert. Die Restaktivität [\%] einer Probe mit einer bestimmten Konzentration bezieht sich auf die Probenwerte (100\%) und errechnet sich mittels Prozentrechnung (Dreisatz). Schließlich wird die Hemmung, also 100 - Restaktivität, angegeben. 
Die Probenergebnisse stammen aus mindestens drei unabhängigen Experimenten, jedes mit drei Proben gleicher Konzentration, die jeweils dreimal vermessen wurden.

Der stichprobenartig mit vermessene totale synthetische Elastase-Inhibitor GW 311616A ergab im Konzentrationsbereich von $100 \mu \mathrm{g} / \mathrm{ml}$ Hemmungen $>99 \%$, was die Funktionsfähigkeit des Assays anzeigte.

Zur grafischen Auswertung der Versuche und zur Berrechnung der $\mathrm{IC}_{50}$ s. 2.11.2.

\subsubsection{Hemmung der Trypsin-Aktivität}

Trypsin: aus Rinderpankreas; Aktivität 5,03 U ( $\mu \mathrm{M} / \mathrm{min})$

Durch vorherige Behandlung mit dem Chymotrypsin-Inhibitor Tosyl-L-phenylalaninchlormethylketon (TPCK) wird die Aktivität der in Spuren vorhandenen Serinproteinase Chymotrypsin gehemmt, ohne die Trypsin-Aktivität zu beeinträchtigen. Da die optimale Enzymaktivität des Trypsins in einer Konzentration von $218 \mathrm{nM}$ erreicht war, wurden eine Stammlösung in einer Konzentration von $0,1 \mathrm{mg} / \mathrm{ml}$ mit Tris-Puffer, entsprechend 4,37 $\mu \mathrm{M}$, hergestellt und davon jeweils $10 \mu 1$ pro Well (s. 1.2.) pipettiert.

Substrat: N-Benzoyl-DL-Arg-p-Nitroanilid·HCl

Stammlösung:

in DMSO; $1,1 \mathrm{mM}$

Puffer: Tris (Tris-(hydroxymethyl)-aminomethan)-HCl-Puffer $\mathrm{pH}=8,0$ (50 mM)

Stammlösung:

Tris $50 \mathrm{mM}, 150 \mathrm{mM} \mathrm{NaCl}, 1 \mathrm{mM} \mathrm{CaCl} 2 ; \mathrm{pH}=8,0$ ( $\mathrm{HCl}$ ); autoklaviertes Wasser. Der $\mathrm{CaCl}_{2}$-Zusatz dient der Stabilisierung des Trypsins in wässeriger Lösung.

Trypsin-Inhibitor aus Sojabohne:

Stammlösung:

in Tris-Puffer; $2,5 \mu \mathrm{M}$

Proben: Acmella ciliata-Extrakte $\mathrm{A}_{3}, \mathrm{E}_{1}$; Referenz-Alkamid(e) (Dodeca-2,4,8,10 (E,E,Z,E / E,E,Z,Z)-tetraensäure-isobutylamide); gelöst in DMSO unter Zuhilfenahme eines Schüttlers (Vortex-Genie 2, s. 1.2.), verdünnt mit Puffer zu 4 verschiedenen Konzentrationen $(1,25 ; 2,5$; $12,5 ; 25 \mu \mathrm{g} / \mathrm{ml})$. 
Über die genaue Zusammensetzung der im Versuch eingesetzten Proben-, Vergleichs- und Blindlösungen gibt Tab. 40 Auskunft.

\section{Versuchsdurchführung:}

Für die Versuchsansätze wurden die Lösungen in folgender Reihenfolge in die Wells pipettiert: Tris-Puffer $\rightarrow$ Testsubstanz (Probe) bzw. LM bzw. Inhibitor $\rightarrow$ Trypsin. Nach 5minütiger Vorinkubation bei $37^{\circ} \mathrm{C}$ im Messgerät (Microplate Autotreader, s. 1.2.) erfolgten die Substratzugabe und der Start der Messungen im UV/VIS bei $405 \mathrm{~nm}$ (aller $3 \mathrm{~min}$; insgesamt $21 \mathrm{~min}=8$ Messwerte).

Mit entsprechenden Blindproben von Probe und Vergleich wurde analog verfahren. Die Blindproben dienten der Erfassung möglicher Eigenabsorptionen von Proben, Substrat und Inhibitor. Ein Ansatz mit Trypsin-Inhibitor fungierte als Positivkontrolle, die das Funktionieren des Assays anzeigt (s. Tab. 40).

Tabelle 40: Pipettierschema im Trypsin-Assay

\begin{tabular}{|c|c|c|c|c|}
\hline \multirow[t]{2}{*}{ Lösung } & \multicolumn{4}{|c|}{ Zusammensetzung (je $10 \mu \mathrm{l}) ;$ Tris-Puffer pH 8,0 ad $200 \mu \mathrm{l}$} \\
\hline & Testsubstanz & $\begin{array}{l}\text { Enzym } \\
\text { (Trypsin) } \\
\end{array}$ & Substrat & Inhibitor \\
\hline $\begin{array}{l}\text { Probe } \\
\text { Probe - Blindwert }\end{array}$ & $\begin{array}{l}x \\
x\end{array}$ & $x$ & $\begin{array}{l}x \\
x\end{array}$ & \\
\hline $\begin{array}{l}\text { Vergleich } \\
\text { Vergleich - Blindwert }\end{array}$ & $\begin{array}{l}\text { (nur Lösungsmittel) } \\
\text { (nur Lösungsmittel) }\end{array}$ & $\mathrm{x}$ & $\begin{array}{l}x \\
x\end{array}$ & \\
\hline $\begin{array}{l}\text { Inhibitor } \\
\text { Inhibitor - Blindwert }\end{array}$ & & $\mathrm{x}$ & $\begin{array}{l}x \\
x\end{array}$ & $\begin{array}{l}x \\
x\end{array}$ \\
\hline
\end{tabular}

Nach Abzug der entsprechenden Blindwert-Absorptionen wurde der Mittelwert $(n=3)$ des Anstiegs der Ausgleichsgeraden einer bestimmten Konzentration, bezogen auf den Vergleich (=100\%-Wert), berechnet. Dieser Wert entspricht der prozentualen Restaktivität des Enzyms bei der jeweiligen Probenkonzentration. Die Hemmung ergibt sich somit als die Restaktivität subtrahiert von 100. Nach Auftragung der ermittelten Konzentrationen (Abszisse) und ihrer 
dazugehörigen Restaktivitäten (Ordinate) wird die $\mathrm{IC}_{50}$ mittels linearer Regression aus der sich ergebenden Ausgleichsgeraden errechnet.

\section{Auswertung:}

s. Allgem. Teil, 4.2.2.

\subsubsection{Hemmung der Thrombin-Aktivität}

Thrombin: vom Rind; Ist-Aktivität $=34,8 \mathrm{NIH}$ units/mg

Stammlösung:

$29 \mathrm{mg}$ Enzym $+2 \mathrm{ml}$ Tris-Puffer (Aktivität $=504,6 \mathrm{NIH}$ units/ml)

Verdünnung:

$1 \mu 1$ Stammlösung $+770 \mu 1$ Tris-Puffer (Aktivität $=1,31$ $\mathrm{NIH}$ units/ml)

Anmerkung: täglich frisch bereiten!

Puffer: Tris (Tris-(hydroxymethyl)-aminomethan)-HCl-Puffer ; $\mathrm{pH}=7,5 ; 50 \mathrm{mM}$

Lösung:

$3,0289 \mathrm{~g}$ Tris, gelöst in $500 \mathrm{ml}$ autoklaviertem Wasser $\rightarrow$ pH-Einstellung $(7,5$; konz. $\mathrm{HCl})+2,85 \mathrm{~g}(0,1 \mathrm{M}) \mathrm{NaCl}$

Substrat: H-D-Phe-Homopro-Arg-p-Nitroanilid Diacetat

Lösung (2,475 mM):

$1,368 \mathrm{~g}$, gelöst in $1 \mathrm{ml}$ Tris-Puffer $=2,475 \mathrm{mM}$

Proben:

Lösungen:
Acmella ciliata-Extrakte $\mathrm{A}_{3}, \mathrm{~A}_{4}, \mathrm{~F}_{1}$ und $\mathrm{F}_{2}$

in 6 Konzentrationen $(5 ; 25 ; 50 ; 250 ; 500 ; 1000 \mu \mathrm{g} / \mathrm{ml})$ in Tris-Puffer unter Zuhilfenahme eines Schüttlers (Vortex-Genie 2, s. 1.2.)

Inhibitor: Pefabloc SC (s. 1.1.5.)

\section{Versuchsdurchführung:}

$100 \mu 1$ Pufferlösung wurden vorgelegt, dann $50 \mu$ l jeweilige Proben- und schließlich $20 \mu 1$ Thrombin-Lösung pro Well pipettiert, vermischt und $5 \mathrm{~min}$ bei $37^{\circ} \mathrm{C}$ per Heizplatte vorinkubiert. Nach Zugabe von $20 \mu 1$ Substratlösung erfolgte der Start der Reaktion und gleichzeitig wurde die kinetische UV/VIS-Messung bei $405 \mathrm{~nm}$ über einen Zeitraum von 36 min (alle 3 min) begonnen. 
Auch sind entsprechende Vergleiche sowie Leerwerte zu Vergleich und Probe mit vermessen worden. Das Pipettierschema entspricht Tab. 41. Die Messungen erfolgten dreimal, jeweils als Doppelbestimmung.

Die mathematischen Berechnungen erfolgten analog zu denen des Trypsin-Assays (s. 2.8.2.).

Tabelle 41: Pipettierschema im Thrombin-Assay

\begin{tabular}{|l|c|c|c|}
\hline \multirow{2}{*}{ Lösung } & \multicolumn{3}{|c|}{ Tris-Puffer pH 7,5 ad 200 $\mu \mathrm{l}$} \\
\cline { 2 - 4 } & $\begin{array}{c}\text { Testsubstanz } \\
(50 \mu \mathrm{l})\end{array}$ & $\begin{array}{c}\text { Enzym } \\
(20 \mu \mathrm{l})\end{array}$ & $\begin{array}{c}\text { Substrat } \\
(20 \mu \mathrm{l})\end{array}$ \\
\hline Probe & $\mathrm{x}$ & $\mathrm{x}$ & $\mathrm{x}$ \\
Probe-Blindwert & $\mathrm{x}$ & & $\mathrm{x}$ \\
& & $\mathrm{x}$ & $\mathrm{x}$ \\
Vergleich & & & $\mathrm{x}$ \\
Vergleich-Blindwert & & & \\
\hline
\end{tabular}

\section{Auswertung:}

s. Allgem. Teil, 4.3.3.

\subsection{Galenische Methoden}

\subsubsection{Herstellung einer Hautsalbe}

Als Salbengrundlage diente die DAC-Basiscreme, eine im Handel erhältliche hydrophile Creme folgender Zusammensetzung:

$\begin{array}{lr}\text { Glycerolmonostearat } 60 & 4,0 \mathrm{~g} \\ \text { Cetylalkohol } & 6,0 \mathrm{~g} \\ \text { Mittelkettige Triglyceride } & 7,5 \mathrm{~g} \\ \text { Weißes Vaselin } & 25,5 \mathrm{~g} \\ \text { Macrogol-20-glycerolmonostearat } & 7,0 \mathrm{~g} \\ \text { Propylenglycol } & 10,0 \mathrm{~g} \\ \text { Gereinigtes Wasser } & 40,0 \mathrm{~g}\end{array}$

Wenig Basiscreme wurde in einer Fantaschale vorgelegt, dann der gesamte (halbfeste) Extrakt $\left(\mathrm{A}_{4} ; 0,1\right.$ bzw. 1,0 g) hinzugefügt, mittels Pistill verrieben, anschließend die restliche Salben- 
grundlage ad 10,0 $\mathrm{g}$ in Anteilen eingearbeitet, manuell homogenisiert und die Creme in Salbenkruken abgefüllt.

\subsubsection{Herstellung einer Nanoemulsion}

Die Nanoemulsion hat folgende Grundzusammensetzung:

$\begin{array}{ll}\text { Miglyol } & 1,00 \mathrm{~g}(10 \%) \\ \text { Poloxamer } 188 & 0,25 \mathrm{~g}(2,5 \%) \\ \text { Aqua bidest. } & 8,75 \mathrm{~g}(\mathrm{ad} 100 \%)\end{array}$

$250 \mathrm{mg}$ des Dichlormethanblüten-Extrakts $\left(\mathrm{A}_{4}\right)$ wurden in $2 \mathrm{ml}$ Chloroform gelöst. Davon wurden $0,8 \mathrm{ml}$ in ein Rollrandglas überführt und das Chloroform abgedampft. Die nunmehr $100 \mathrm{mg}$ Extrakt wurden in $1 \mathrm{~g}$ Miglyol (Neutralöl) suspendiert (Extrakt nicht vollständig löslich in Miglyol). Eine Tensidlösung aus 0,25 g Poloxamer und 8,75 g Wasser (Aqua bidest.) wurde mit der Miglyolsuspension vereinigt und anschließend mit dem Ultra-Turrax (s. 1.2.) $30 \mathrm{~s}$ bei $8500 \mathrm{U} / \mathrm{min}$ voremulgiert. Danach erfolgte die Hochdruckhomogenisierung (s. 1.2.) dieser Emulsion bei $5 \mathrm{MPa}$ für $150 \mathrm{~s}$.

Mittels PCS (Photonenkorrelationsspektroskopie; s. 1.2.) wurden für die milchig-trübe Nanoemulsion ein mittlerer Partikeldurchmesser von 165 nm und ein Polydispersitätsindex von 0,165 ermittelt.

\subsubsection{Herstellung einer Haftpaste}

Zur Herstellung der Hypromellose-Haftpaste 40\% (NRF 7.8.) wurden zunächst $2 \mathrm{~g}$ Hypromellose 2000 in der Fantaschale vorgelegt, danach der gesamte Extrakt (0,25 g) hinzugefügt und homogenisiert. Dann wurde mit Hydrophobem Basisgel DAC, bestehend aus 95 Masseteilen Dickflüssigem Paraffin und 5 Masseteilen Hochdruckpolyethylen, ad 5,0 g verrieben und die fertige Zubereitung, die 5 \% Extrakt enthielt, in eine Salbenkruke abgefüllt. 


\subsubsection{Herstellung von Glycerol-Gelatine-Plättchen}

Diese Plättchen bestehen aus einer Mischung von Gelatine / Wasser / Glycerol = 1: $5: 5$ und enthalten einen Zusatz von 0,1\% Chitosan (bezogen auf die Gesamtformulierung) in 5 \%iger Zitronensäurelösung und wurden folgendermaßen hergestellt:

1. 0,011 g Chitosan wurden auf 5,0 g einer 5 \%igen wässerigen Zitronensäurelösung aufgestreut und unter Rühren gelöst.

2. 1,0 g Gelatine wurde in diese Lösung eingearbeitet und 20 min vorgequollen.

3. 5,0 g Glycerol wurden hinzugefügt, und der Ansatz wird ca. 15 min im Ultraschallbad bei $55-60^{\circ} \mathrm{C}$ homogenisiert.

4. $0,55 \mathrm{~g}$ Acetonextrakt $\left(\mathrm{E}_{1}\right)$ wurden vorgelegt und die Gelatinelösung unter Erwärmen $\left(55-60^{\circ} \mathrm{C}\right)$ und Rühren inkorporiert.

5. Diese Gelatinezubereitung wurde in Petrischalen (Durchmesser $70 \mathrm{~mm}$ ) in einer Schichtdicke von $<1 \mathrm{~mm}$ ausgegossen, und nach dem Erstarren wurden mittels Korkbohrer runde Plättchen mit einem Durchmesser von 10 mm ausgestochen.

\subsubsection{Herstellung von Adhäsivtabletten}

Die Hafttabletten wiesen folgende Grundzusammensetzung auf:

$\begin{array}{lr}\text { HPMC } & 70 \% \\ \text { Avicel PH 103 } & 28 \% \\ \text { Aerosil 200 } & 2 \%\end{array}$

Wirkstofffreie, biplane Tabletten (Placebo) wiesen eine Steghöhe von 1,5 mm, einen Durchmesser von $9 \mathrm{~mm}$ und eine durchschnittliche Masse von 135,2 mg auf. Für einen Ansatz mit $5 \%$ Extrakt wurden beispielsweise auf eine Hilfsstoffmischung von 3,6 g HPMC, 1,5 g Avicel (mikrokristalline Cellulose) und 0,1 g Aerosil $200 \mathrm{mg}$ Acetonextrakt ( $\mathrm{E}_{1}$ ), gelöst in $1 \mathrm{ml}$ Ethanol, aufgetropft, intensiv manuell verrieben bzw. homogenisiert, getrocknet und mit der Exzenterpresse verpresst.

Neben diesem Probeansatz wurden weitere Ansätze nach demselben Schema hergestellt:

1. $100 \mathrm{mg}$ Acetonextrakt, gelöst in $500 \mu \mathrm{l}$ Ethanol, auf 2,6 g Pulvermischung $\rightarrow \mathbf{5} \mathbf{~ m g}$ Extrakt / Tablette (Doppelansatz)

2. $100 \mathrm{mg}$ Acetonextakt, gelöst in $500 \mu \mathrm{g}$ Ethanol, auf $1,3 \mathrm{~g}$ Pulvermischung $\rightarrow \mathbf{1 0} \mathbf{~ m g}$ Extrakt /Tablette (Doppelansatz) 
3. $200 \mathrm{mg}$ Acetonextrakt, gelöst in $1000 \mu \mathrm{l}$ Ethanol, auf 1,3 g Pulvermischung $\rightarrow \mathbf{2 0} \mathbf{~ m g}$ Extrakt /Tablette

4. 2 und $5 \mathrm{mg}$ Benzocain enthaltende Vergleichstabletten

5. Placebo-Tabletten (ohne Wirkstoff) der obigen Zusammensetzung

\subsection{In vivo-Methoden}

Ausgewählte galenische Präformulierungen (s. o.) wurden in orientierenden Versuchen an wenigen freiwilligen Probanden nach topischer Applikation unter den folgenden Kriterien getestet:

\section{Hautsalbe (DAC-Basiscreme) zur Anwendung auf der Oberhaut:}

Testpersonen: $\quad 4$ hautgesunde Pers. (2 §, 2 + $)$, Durchschnittsalter 52,5 Jahre

Applikation: $\quad$ Ort: Innenseite Unterarm; Größe: 1 Euro-Areal

Dosierung: 1 Augenspatelspitze (Glas), leicht einmassiert, abgedeckt mit Mull und Alufolie (Okklusion)

Testzeit: $\quad 2 \mathrm{~h}$

Bewertung: $\quad$ Sensorische Empfindungen, z. B. Kribbeln, Brennen, Anästhesie Makroskopische Erscheinungen, z. B. Hautrötung, -quellung

\section{Adhäsivtabletten zur Anwendung in der oralen Kavität:}

Zur Testung gelangten hier im Blindversuch neben den Extrakt enthaltenden Tabletten in 3 Konzentrationen noch Placebo- sowie Benzocain (2 Wirkstärken) enthaltende Tabletten.

Testpersonen: $\quad 3$ mundgesunde Pers. $(2 \hat{\jmath}, 1$ ) $)$, Durchschnittsalter 54 Jahre

Applikation: $\quad$ Vorbereitung: mind. $1 \mathrm{~h}$ nach der letzten, leichten und wenig gewürzten Mahlzeit; Mund gespült

Ort: zwischen Backentasche und Kiefer (bukkal) oder unter der Zunge (sublingual)

Testzeit: $\quad 2 \mathrm{~h}$ bzw. bis Zerfall oder Auflösen der Tablette

Bewertung: $\quad$ 1. Applikationsfähigkeit und mechanischer Effekt im Mundraum

2. Haftvermögen der Tablette (Mukoadhäsion)

3. Geschmack

4. Sensorische Wahrnehmungen, z. B. brennend, anästhesierend usw. 
5. sonstige Beobachtungen

\section{Schleimhaut-Haftpaste zur oralen Anwendung:}

Testpersonen: $\quad 3$ mundgesunde Probanden $(2 \hat{\jmath}, 1$ ) $)$, Durchschnittsalter 54 Jahre

Applikation: $\quad$ s. Adhäsivtablette

Testzeit: $\quad 2 \mathrm{~h}$

Bewertung: $\quad$ Haftfähigkeit, Geschmack, Empfindungen (s. o.), Sonstiges

\subsection{Mathematische / Statistische Methoden}

\subsubsection{Gehaltsbestimmung von Alkamiden}

Das Hauptamid der Sammelfraktion 2 (s. 3.3.3., Allg. Teil), das Undeca-2E,4E-dien-6,8diinsäure-isobutylamid (A1), sowie dessen Dihydroderivat Undeca-2E-en-6,8-diinsäureisobutylamid (A10) wurden mittels Full-MS im +ESI-Modus, mittels Xcalibur ${ }^{\circledR}$ Software über Dodeca-2E,4E,8Z,10E/Z-tetraen-isobutylamide („Tetraen“) quantifiziert (Abb. 68).

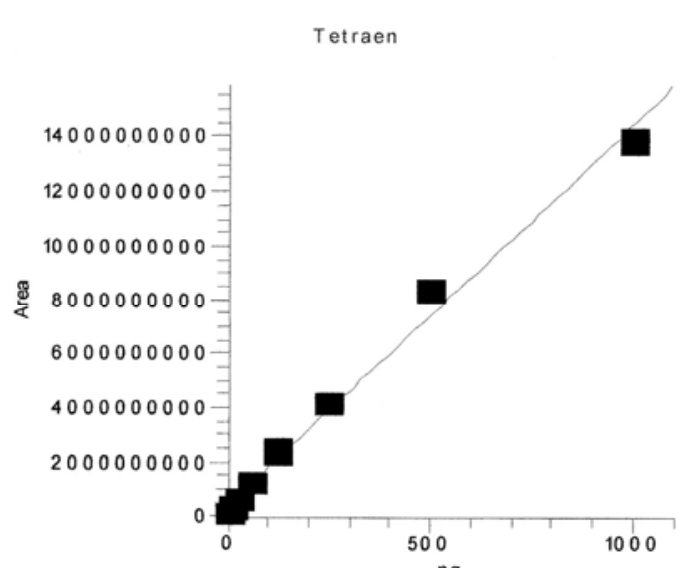

Abb. 67: Kalibriergerade für ,Tetraen“6

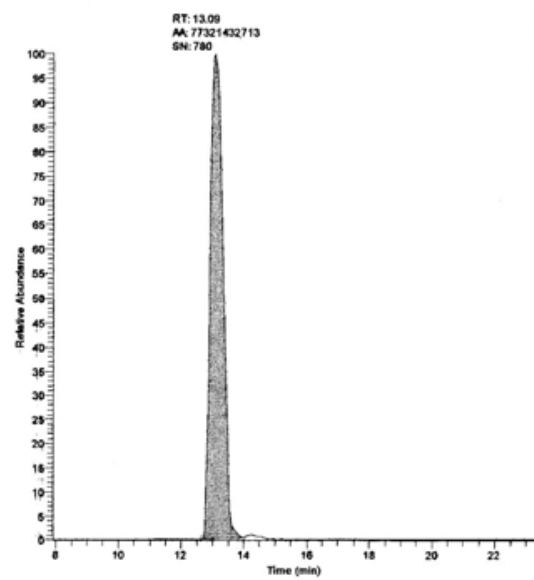

Aus acht Proben zunehmender Konzentrationen des Bezugsalkamids „Tetraen“ ergab sich mittels Computer gestützter Berechnung (Xcalibur ${ }^{\mathrm{TM}}$ Software) der lineare Zusammenhang (s. Abb. 67) $\mathrm{y}=1,40272 \cdot 10^{7} \cdot \mathrm{x}+$ $4,10903 \cdot 10^{8}$ mit einem Korrelationskoeffizienten $\mathrm{R}^{2}=0,9903$.

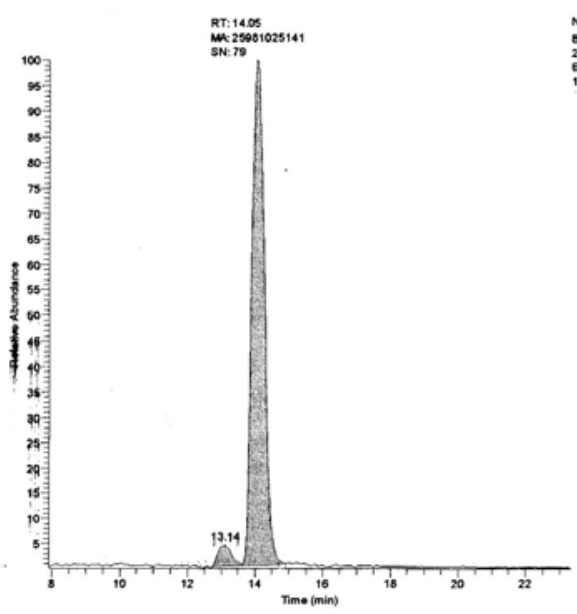


Eingespritzt wurden $10 \mu \mathrm{l}$, was bei der Berechnung der Masse pro Milliliter berücksichtigt werden muss.

Es resultierte ein absoluter Wert von 8,69 mg für $\boldsymbol{A 1}$ in der Sammelfraktion 2 (insgesamt 113,6 mg), entsprechend einem prozentualen Anteil von 7,65 \%. Für $\boldsymbol{A 1 0}$ betrug der absolute Wert lediglich 2,6 mg bzw. 2,29\% der Sammelfraktion 2.

Bezogen auf den gesamten Dichlormethan-Trockenextrakt von 26,2 g, der aus einem $\mathrm{kg}$ Droge gewonnen worden war (s. 3.3.2., Allgem. Teil), errechnete sich für die Substanz A1 allerdings nur ein prozentualer Anteil von $0,03 \%$ und für $\boldsymbol{A 1 0}$ von $0,01 \%$.

\subsubsection{Auswertung der Enzymversuche}

Die Ermittlung der $\mathrm{IC}_{50}$ für Elastase erfolgte rechnerisch, indem die, durch lineare Regression ermittelte Geradengleichung der Ausgleichsgeraden $(\mathrm{y}=\mathrm{mx}+\mathrm{b})$ nach $\mathrm{x}$ umgestellt und $\mathrm{y}=$ 50 gesetzt wurde. Hierbei wurden die Werte von $0,1-10 \mu \mathrm{g} / \mathrm{ml}$ berücksichtigt. Es ergab sich bei allen Proben, mit einer Ausnahme, ein logarithmischer Zusammenhang zwischen Probenkonzentration und prozentualer Enzymhemmung, so dass durch Auftragen des dekadischen Logarithmus der Probenkonzentrationen gegen die Hemmung $(y=m(\log x)+b)$ eine Linearisierung resultierte. Tatsächlich wurde allerdings die $\mathrm{IC}_{50}$ durch lineare Interpolation zwischen den beiden, die $\mathrm{IC}_{50}$ begrenzenden Konzentrationen ermittelt (s. 4.1.3., Allgem. Teil).

Der H-Test nach KRUSKAL und WALLIS diente zum Vergleich mehrerer unabhängiger Stichproben hinsichtlich des Einflusses des Extraktionsmittels auf den Hemmeffekt $(\mathrm{p} \leq$ 0,05). Anschließend wurde ein multipler Vergleich nach DuNN durchgeführt $(p \leq 0,05)$. Die Berechnungen erfolgten mit dem Auswertungsprogramm Graph Pad Prism (Graph Pad Software, La Jolla, USA).

Zur grafischen Auswertung der Trypsin- und Thrombin-Versuche s. im Allgem. Teil unter 4.2.2. bzw. 4.3.3. 


\section{Zusammenfassung}

Acmella ciliata (H.B.K.) Cassini ist eine zum Subtribus Ecliptinae gehörende Asteraceae, beheimatet in Südamerika, inzwischen aber global verbreitet, vor allem in tropischen und subtropischen Regionen. Bisher kam es häufig zu Verwechslungen mit der bekannteren Acmella oleracea, die bis zur eindeutigen botanischen Zuordnung durch JANSEN [81] 1985 fälschlicherweise der Gattung Spilanthes, mit verschiedenen Synonymen, zugeschrieben wurde.

Um fernerhin solche Arten- und Namenskonfusion weitgehend zu vermeiden, wurden in der vorliegenden Arbeit makroskopische und mikroskopische vergleichende Studien an Acmella ciliata und Acmella oleracea, die in einer gelben und einer roten Varietät vorlag, unternommen. Die Unterscheidungsmerkmale sind jedoch gering und beschränken sich weitgehend auf die Form und Farbe der Blütenköpfchen und vor allem auf die lediglich bei Acmella ciliata vorhandenen Zungenblüten.

Auch ein DC-Screening verschiedener Extrakte aus getrocknetem und frischem Pflanzenmaterial aller drei Acmella-Arten/Varietäten hinsichtlich ihrer Inhaltsstoffklassen erbrachte keine wesentlichen Unterschiede. In quantitativer Hinsicht ergaben sich Differenzen bei den phenolischen Inhaltsstoffen. Hier wies Acmella ciliata einen höheren Gehalt an Phenolcarbonsäuren auf, während Acmella oleracea ( $\operatorname{rot}>$ gelb) einen höheren FlavonoidGehalt zeigte.

Die nachfolgenden phytochemischen Untersuchungen befassen sich ausschließlich mit Acmella ciliata, da für diese Pflanze trotz ihrer weiten Verbreitung und vielfältigen Anwendungen in der Volksmedizin der Ursprungsländer und mittlerweile auch bei uns mit dem registrierten, traditionellen Externum Spolera ${ }^{\circledR}$ weder erschöpfende Untersuchungen zu den Inhaltsstoffen noch pharmakologische Studien vorliegen. Für die bisher beschriebenen Wirkungen, vor allem analgetisch, antiphlogistisch, lokalanästhetisch, insektizid u. a., werden die reichlich enthaltenen Alkamide als verantwortlich angesehen, von denen MARTIN [110 112] 20 Verbindungen aus den Blütenköpfchen isolierte.

Für die eigenen Untersuchungen standen einerseits die Alkamide der Gesamtpflanze sowie die bisher kaum untersuchten phenolischen Verbindungen im Mittelpunkt des Interesses. Die 
Alkamide wurden aus einem apolaren Dichlormethan-Extrakt nach sc Vortrennung (Kieselgel, RP 18) durch präparative DC und z. T. direkt durch HPLC-MS-Analytik aus entsprechenden Sammelfraktionen gewonnen und charakterisiert. Die MS-MS erfolgte im +ESI-Modus. Partiell wurde die ${ }^{1}$ H-NMR herangezogen.

Insgesamt wurden in einem Acmella ciliata-Gesamtpflanzenextrakt die Alkamide $\boldsymbol{A 1}$ - $\boldsymbol{A 1 6}$ identifiziert (s. Tafel II), die im Säurerest „olefinische“ und/oder „acetylenische“ Strukturen aufweisen und als Aminkomponente dominant den Isobutylamid-Rest tragen; $\boldsymbol{A 8}$ und $\boldsymbol{A 9}$ sind Phenylethylamide und nur $\boldsymbol{A 1 1}$ ist ein Methylbutylamidderivat.

Die Verbindungen $\boldsymbol{A 1}, \mathbf{2}, \mathbf{8}, \mathbf{1 0}, \mathbf{1 1}, \mathbf{1 3}, 15$ und $\boldsymbol{A 1 6}$ konnte MARTIN [110 - 112] auch in den Blütenköpfchen identifizieren. $\boldsymbol{A 6}_{2}, \boldsymbol{A 7}, \boldsymbol{A 9}$ und $\boldsymbol{A 1 0}$ wurden in Acmella-Artverwandten nachgewiesen [16, 53, 132, 144] und $\boldsymbol{A 1}, \boldsymbol{A 5}, \boldsymbol{A 1 0}$ und $\boldsymbol{A 1 6}$ für Echinacea-Arten beschrieben $[8,9,11,35]$.

$\boldsymbol{A 1 4}$ (Trideca-5Z-en-10,12-diinsäure-isobutylamid) stellt einen neuen Naturstoff dar. Auch $\boldsymbol{A 4}$ ist ein neues Alkamid, ein Dihydroxydiinsäure-Derivat des Isobutylamins. Ebenso tritt $\boldsymbol{A 1 2}_{\boldsymbol{1}}$ als Dihydroxyalkamid auf; $\boldsymbol{A 3}$ und $\boldsymbol{A 6}_{\boldsymbol{1}}$ stellen Monohydroxyalkamide dar, und alle gehen durch ms Fragmentierung unter stufenweiser Wasserabspaltung in die entsprechenden dehydratisierten Verbindungen (vgl. Tafel II, Teil 2) über. Bei $\boldsymbol{A 6}$ und $\boldsymbol{A 1 2}$ waren die dehydratisierten Strukturen bereits primär (Full-MS-Spektrum) präsent.

Außer den abgebildeten Alkamiden wurden noch weitere auf Grund ihrer Retentionszeiten im HPLC-Spektrum und partiell durch ihre Molmasse identifiziert, konnten jedoch mangels ausreichender Probesubstanz und/oder Vergleichsdaten nicht eindeutig strukturell aufgeklärt werden (3.3.3.12. und 3.3.3.16).

Anders verhält es sich bei zwei weiteren Alkamiden (Fumarsäureisobutylamid (S6) und 4EHexenoylisobutylamid (S7)), die sich von Dicarbonsäuren ableiten und deshalb in dem hydrophileren Methanolextrakt aufgefunden wurden. Als Prototyp hierfür und gleichzeitig als neuer Asteraceen-Inhaltsstoff wurde $\boldsymbol{S 6}$ isoliert sowie $\boldsymbol{S} 7$ als gänzlich neuer Naturstoff identifiziert. Interessant ist weiterhin der Nachweis der Spilanthessäure $(\boldsymbol{S} 4)$, die den Säurerest des Spilanthols (A13) darstellt und in ihrer isolierten Form ebenfalls ein neuer Naturstoff ist. 
Tafel II: Alkamide, Teil 1

\section{$\underline{\text { Amidrest }}$}

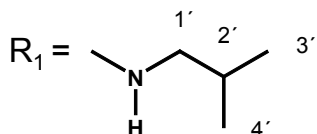

\section{Säurerest}

$\mathrm{C}_{9}$

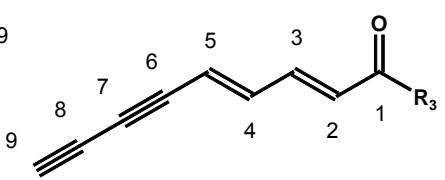

Nona-2E-en-6,8-diinsäure-phenylethylamid (A2)

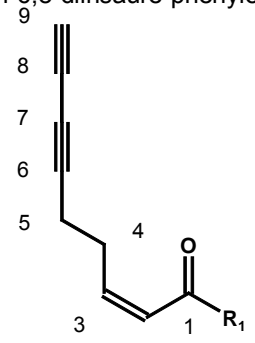

Nona-2Z-en-6,8-diinsäure-isobutylamid (A7)

$\mathrm{C}_{10}$

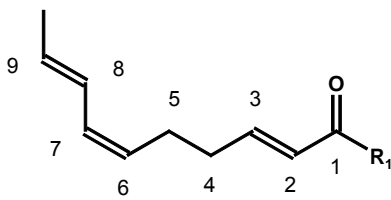

Deca-2E,6Z,8E-triensäure-isobutylamid (A13) (Spilanthol)

$\mathrm{C}_{11}$

11

10

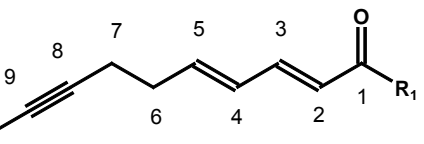

Undeca-2E,4E-dien-8,10-diinsäure-isobutylamid (A1)
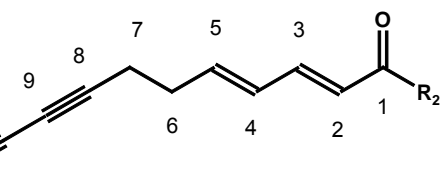

Undeca-2E,4E-dien-8,10-diinsäure-methylbutylamid (A11)

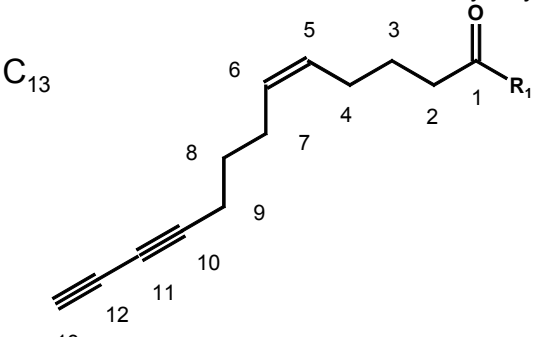

13

Trideca-5Z-en-10,12,-diinsäure-isobutylamid (A14)<smiles></smiles>

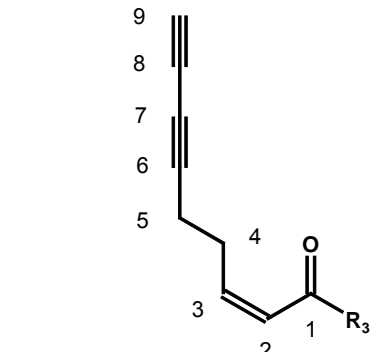

Nona-2Z-en-6,8-diinsäure-phenylethylamid ( $\boldsymbol{A 9}$

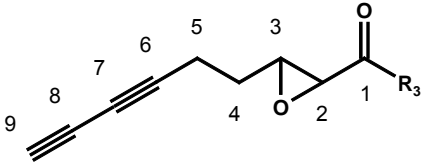

2,3(c)-Epoxy-nona-6,8-diinsäure-phenylethylamid (A8)

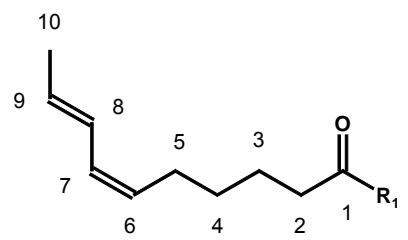

Deca-6Z,8E-diensäure-isobutylamid (A15) (Hydrospilanthol)

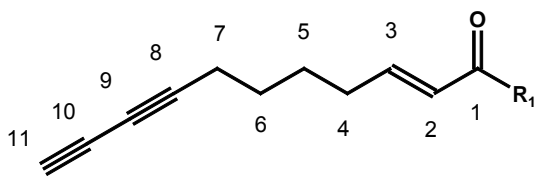

Undeca-2E-en-8,10-diinsäure-isobutylamid (A10)

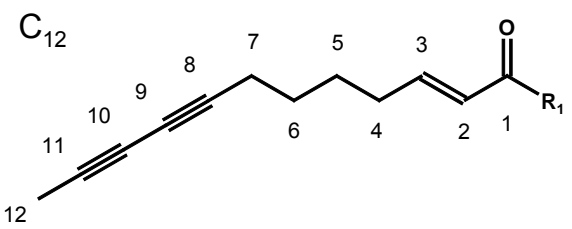

Dodeca-2E-en-8,10-diinsäure-isobutylamid (A5)

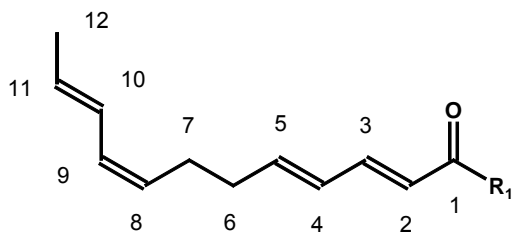

Dodeca-2E,4E,8Z,10E-tetraensäure-isobutylamid (A16) 
Tafel II: Alkamide, Teil 2

Monohydroxyalkamide

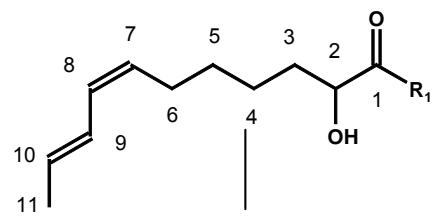

2-Hydroxy-undeca-7Z,9E-diensäureisobutylamid $\left(\boldsymbol{A \boldsymbol { G } _ { 1 }}\right)$

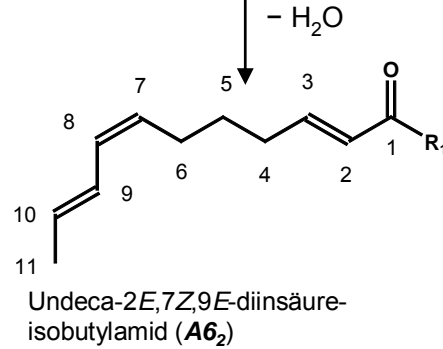

Dihydroxyalkamide

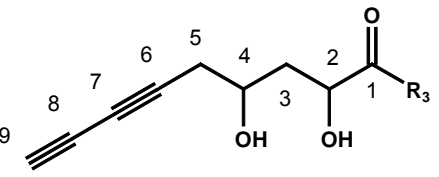

2,4-Dihydroxy-nona-6,8-diinsäurephenylethylamid $\left(\boldsymbol{A} \mathbf{4}_{1}\right)$

西

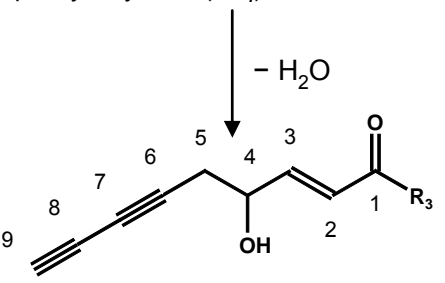

4-Hydroxy-nona-2E-en-6,8-diinsäurephenylethylamid $\left(\boldsymbol{A 4}_{2}\right)$

西

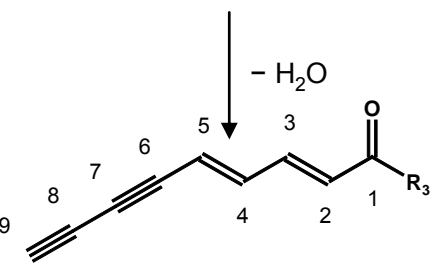

Nona-2E,4E-en-6,8-diinsäurephenylethylamid $\left(\mathrm{A4}_{3}\right)$

\section{Dicarboxyalkamide}<smiles>CC(C)CNC(=O)/C=C/C(=O)O</smiles>

Fumaroylisobutylamid (S6)

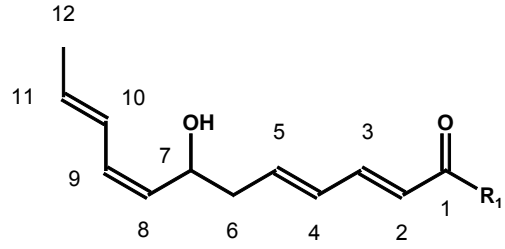

7-Hydroxy-dodeca-2E,4E,8Z,10E-tetraensäure -isobutylamid $\left(A 3_{1}\right)$

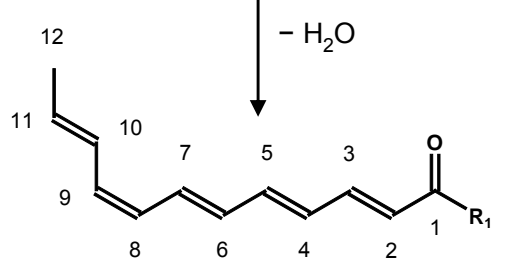

Dodeca-2E,4E,6E,8Z,10E-pentaensäure -isobutylamid $\left(\boldsymbol{A 3}_{\mathbf{2}}\right)$

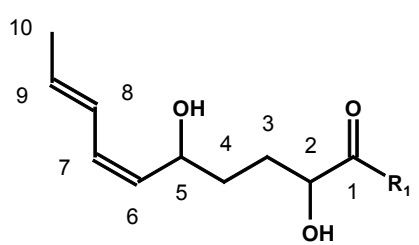

2,5-Dihydroxy-deca-6Z,8E-diensäureisobutylamid $\left(\boldsymbol{A} 12_{1}\right)$

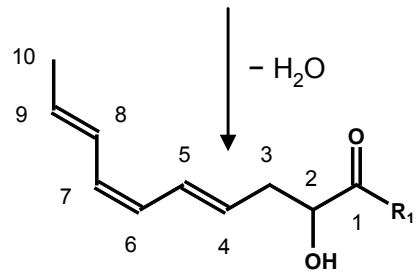

2-Hydroxy-deca-4E,6Z,8E-triensäureisobutylamid $\left(\boldsymbol{A 1 2}_{\mathbf{2}}\right)$

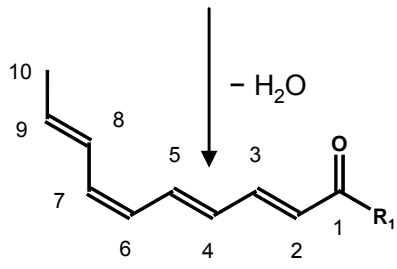

Deca-2E,4E,6Z,8E-tetraensäureisobutylamid $\left(\boldsymbol{A 1 2}_{\mathbf{3}}\right)$

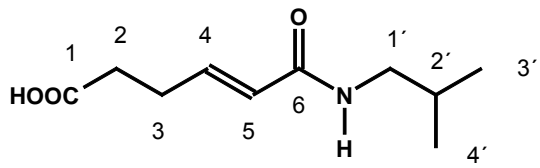

4E-Hexenoylisobutylamid (S7) 
Aus dem Methanolextrakt und durch nachfolgende SC an Sephadex LH 20 sowie weitere HPLC-Fraktionierung und Strukturaufklärung mittels UV-, ESI-MS- und NMRSpektroskopie resultieren, inklusive cis/trans-Isomeren, 22 phenolische Verbindungen. Neben den biogenetisch relevanten einfachen Monocarbonsäuren p-Hydroxybenzoesäure (P1) und Protocatechusäure (P2) traten cis/trans-p-Cumar- $(\boldsymbol{P 3})$, trans-Kaffee-(P4) und Ferulasäure (P5) auf. Darüber hinaus wurden für die drei letzteren die seltenen und in der Familie der Asteraceae zuvor nicht beschriebenen Äpfelsäureester (P6 - P8) sowie des Weiteren die trans-Chlorogensäure (P11) und deren selten auftretendes cis-Isomer gefunden. Als neue Verbindungen konnten die trans-Kaffeoyläpfelsäure-1/4-methylester (P9/10) sowie der trans5-Kaffeoylepichinasäuremethylester (P12) beschrieben werden.

Das ebenfalls nachgewiesene Aesculetin (P15) zählt zu den Dihydroxycumarinen und Matairesinol (P16) zu den Lignanen. An Flavonoiden wurden unter den gewählten Bedingungen der Extraktgewinnung und -aufarbeitung lediglich das Isoquercitrin (P13) und Rutosid (P14) sowie Miquelianin gefunden. Für ein weiteres, bisher unbekanntes Flavonoid wurde ein Strukturvorschlag unterbreitet.

Bei der Aufarbeitung der Extrakte fiel eine Reihe weiterer Verbindungen an, die als biogenetische Bausteine fungieren können und partiell als Geruchsstoffe Anteil am aromatischen Aroma der Extrakte haben dürften: p-Hydroxybenzaldehyd (S1), Vanillin $(\boldsymbol{S} 2)$, Zimt- (S3), Fumar- (S5), Äpfel- (S8) und Chinasäure (S9). Die Vielzahl der Inhaltsstoffe, allein phenolischer und alkamidischer Natur, macht eine Differenzierung in Haupt-, Nebenund Begleitstoffe nahezu unmöglich. Deshalb erschien es als opportun, für die nachfolgenden enzymatischen Untersuchungen von der komplexen Wirkung der Gesamtextrakte auszugehen und hiervon eine Auswahl unterschiedlicher Polarität zu untersuchen.

Gegenüber der humanen Neutrophilen-Elastase, ein Enzym, das u. a. akut am Entzündungsgeschehen im Organismus beteiligt ist, wiesen alle Testextrakte aus Acmella ciliataGanzdroge eine starke Hemmaktivität mit $\mathrm{IC}_{50}$-Werten unter $10 \mu \mathrm{g} / \mathrm{ml}$ auf. Bezogen auf den Aceton-Extrakt mit dem größten Hemmeffekt $(0,5 \mu \mathrm{g} / \mathrm{ml}=1)$ ergab sich tendenziell eine Reihenfolge der Extrakte wie 1:1,6 (Dichlormethan) $=1: 1,6$ (Methanol) $>1: 3,8$ (Spolera ${ }^{\circledR}$ flüssig) $>1: 6,6$ (2-Propanol $60 \%$ ) > 1:16,0 (Ethanol $45 \%$ ). Für ein AlkamidisomerenReferenzgemisch (Dodeca-2E,4E,8Z,10E/Z-tetraensäure-isobutylamid(e)) resultierte eine $\mathrm{IC}_{50}$ 
von $0,6 \mu \mathrm{g} / \mathrm{ml} \quad(2,4 \mu \mathrm{M})$. Entsprechende Acmella oleracea (gelb)-Extrakte zeigten Hemmeffekte in der gleichen Größenordnung.

Die erhaltenen Ergebnisse sprechen für eine Abnahme der Hemmwirkung gegenüber Elastase mit zunehmender Polarität des eingesetzten Extraktionsmittels, lassen jedoch keine dominante Abhängigkeit von einzelnen Inhaltsstoff(en) bzw. -gruppen erkennen. Eine relative Ausgewogenheit von alkamidischen und phenolischen sowie weiteren Komponenten, wie sie hier am ehesten im Acetonextrakt vorlag, scheint offenbar günstig zu sein (Synergieeffekt). Es ist zu schlussfolgern, dass sich die komplexe entzündungshemmende Wirkung des Handelspräparats Spolera ${ }^{\circledR}$ insofern durch optimierte Extraktionsmittel und -verfahren steigern ließe.

Weiterhin war die Untersuchung ausgewählter Acmella ciliata-Extrakte hinsichtlich ihrer Wirkung gegenüber dem Enzym Trypsin, das als Aktivator vor allem des PAR-2-Rezeptors mitverantwortlich für inflammatorische Prozesse ist, von Interesse. Jedoch konnten weder durch den Aceton- noch durch den Dichlormethan-Extrakt im untersuchten Konzentrationsbereich relevante Hemmeffekte erzielt werden. Dagegen deutete sich im niederen Konzentrationsbereich der umgekehrte Effekt an. Die Alkamid-Testsubstanz (s. v.) zeigte sogar ausschließlich eine signifikante Trypsinaktivierung.

Auch gegenüber Thrombin, ebenfalls eine Serinprotease mit hoher Affinität zu den PAR-1-, PAR-3- und PAR-4-Rezeptoren, erwiesen sich die Dichlormethanextrakte aus Ganz- und Blütendroge von Acmella ciliata als uneffektiv. Dagegen ergaben zwei Testpräparate, die mit überkritischem Kohlendioxid gewonnen worden waren, durchaus Thrombin-Hemmeffekte, die von pharmakologischem Interesse sein könnten ( $\mathrm{IC}_{50}$ zwischen 5 und $50 \mu \mathrm{g} / \mathrm{ml}$, in Abhängigkeit von Druck und Temperatur). Auch dieser Befund verdeutlicht wiederum die Bedeutung des Extraktionsverfahrens zur Optimierung hinsichtlich spezieller Wirkprofile.

Dichlormethan- und Acetonextrakte wurden in verschiedene galenische Präformulierungen inkorporiert, in DAC-Basis-Creme, in eine Nanoemulsion, bestehend aus dem flüssigen Lipid Miglyol und dem Tensid Poloxamer 188, weiterhin in die mukoadhäsiven Formulierungen Gelatineplättchen (Chitosan enthaltend), Adhäsivtabletten auf Hydroxypropylmethylcellulose-Basis sowie in Hypromellose-Haftpaste $40 \%$ (NRF 7.8). Von der anschließenden orientierenden in vivo-Testung wurden vorerst die Nanoemulsion sowie die Gelatineplättchen ausgenommen; erstere wegen nicht befriedigender Beschaffenheit und letztere wegen der zu 
ihrer Herstellung erforderlichen Temperatur $\left(\mathrm{ca} .55^{\circ} \mathrm{C}\right)$, die sich auf die Extrakte negativ auswirkte.

In die verbleibenden Präparate waren die Extrakte gut einzuarbeiten und konnten nach topischer Applikation getestet werden. Im Ergebnis der Anwendung der Hautsalbe am ventralen Unterarm von vier freiwilligen Probanden zeigte sich während zweistündiger Okklusion lediglich eine kurze Reaktion zu Testbeginn (leichtes Brennen) und allenfalls eine schwache Hautrötung nach Versuchsende, unabhängig von der Extraktkonzentration in der Salbe (1 bzw. $10 \%$ Dichlormethan-Blütenextrakt).

Die oral getestete Haftpaste (5\% Aceton-Extrakt enthaltend) wird als schwierig applizierbar, aber als sehr gut haftend charakterisiert und wies partiell einen drogenartigen Geschmack auf, jedoch keinerlei sonstige organoleptische Besonderheiten.

Die Adhäsivtabletten, enthaltend 5, 10 bzw. 20 mg Extrakt, wurden im Blindversuch, vergleichend mit Placebo sowie mit Benzocain-Tabletten (2 und $5 \mathrm{mg}$ ), an drei Testpersonen in der oralen Kavität (Applikation bukkal oder sublingual) getestet. Sie zeigten eine mit der Konzentration zunehmende Haftfähigkeit und gute Verträglichkeit und wiesen einen heu- bis kräuterartigen Geschmack auf. Lediglich geringe subjektive Empfindungen, wie leichtes Brennen oder Prickeln und schwache Anästhesie an der Zungenspitze, wurden beschrieben. Weiterführende Studien und ggf. galenische Optimierungen sind hier - die weitere Wirksamkeitsüberprüfung der Acmella ciliata-Extrakte vorausgesetzt - erfolgversprechend, zumal bisher keine Pflanzenextrakte als mukoadhäsive Bukkaltabletten formuliert wurden und es sich demnach um innovative Formulierungen handelt. 


\section{Kurzzusammenfassung}

Die im tropischen Südamerika beheimatete und bisher wenig untersuchte Heil- und Gewürzpflanze Acmella ciliata (H.B.K.) Cass. (Asteraceae) ist Gegenstand dieser Arbeit. Zunächst waren taxonomische Fragen $\mathrm{zu}$ beantworten, da es in der Literatur ständig zu Irritationen bezüglich der Nomenklatur und $\mathrm{zu}$ Verwechslungen mit der bekannteren Artverwandten Acmella oleracea (L.) Jansen kam/kommt. Aus vergleichenden makroskopischen und erstmals durchgeführten mikroskopischen Untersuchungen sowie aus einem phytochemischen Screening ging hervor, dass allein die makroskopischen Merkmale (insbesondere am Blütenkopf) eine eindeutige Unterscheidung zulassen.

Die nachfolgenden umfangreichen phytochemischen sowie enzymatischen Untersuchungen beschränkten sich vornehmlich auf Acmella ciliata mit dem Ziel der Strukturaufklärung von bevorzugt alkamidischen und phenolischen Verbindungen und ihrer Wirksamkeitserfassung, vorrangig im Hinblick auf die u. a. beschriebenen antiinflammatorischen Effekte. Hierbei wurde auch auf das traditionell zugelassene Arzneimittel Spolera ${ }^{\circledR}$ (enthält Propanolextrakt von Acmella ciliata) Bezug genommen. Weiterhin wurden Arzneiformen (Hautsalbe, Nanoemulsion, Haftpaste, Gelatineplättchen, Adhäsivtabletten) konzipiert, die neue oder verbesserte Darreichungsformen darstellen, insbesondere zur potentiellen Anwendung im Mundbereich.

Bei den phytochemischen Untersuchungen konnten zahlreiche Alkamide (Isobutyl-, Methylbutyl- und Phenylethylamide von zwei- und dreifach ungesättigten Mono- und Dicarbonsäuren mit neun bis 13 bzw. vier bis sechs C-Atomen) sowie eine Reihe phenolischer Substanzen (einfache Phenole und Phenolcarbonsäuren sowie -ester, Flavonoide, ein Hydroxycumarin und ein Lignan) mittels chromatographischer Methoden (SC, DC, HPLC, (HP)LC-MS) erfasst und deren Strukturen durch spektroskopische Verfahren (UV, NMR, MS, (HP)LC-MS) aufgeklärt werden. Die meisten der identifizierten Verbindungen, waren entweder in Acmella ciliata bisher nicht nachgewiesen worden, sind neu für die Familie der Asteraceae oder stellen völlig neue Naturstoffe dar.

Anwendungsbezogen ließ sich, wie in vitro-Enzymassays (Elastase-, Trypsin- und ThrombinEnzym-Inhibition) zeigten, besonders im Bereich der Entzündungshemmung, eine Wirkungsverstärkung durch Anwendung von bevorzugt Aceton-Extrakten im Gegensatz zum 2-Propanolextrakt $\left(\right.$ Spolera $\left.^{\circledR}\right)$ erzielen. Diese enzymatisch ermittelte Wirkung lässt sich nicht einer bestimmten Inhaltsstoffgruppe bzw. deren vorwiegend lipophilen oder hydrophilen Eigenschaften zuordnen, sondern ist eher als Synergieeffekt zu interpretieren, was sowohl aus 
pharmakologischen als auch aus ökonomischen Erwägungen den potentiellen Einsatz von Extrakten gegenüber Reinsubstanzen präferieren würde.

Hinsichtlich der galenischen Präformulierungen erscheinen mukoadhäsive Tabletten prädestiniert, um eine therapeutische Anwendung im Mundbereich zu ermöglichen und ein standardisiertes Präparat zu entwickeln. Um diese und ggf. weitere Anwendungen von Acmella ciliata-Zubereitungen $\mathrm{zu}$ etablieren, sind weitere entsprechende Studien zur Wirksamkeit und Verträglichkeit etc. Voraussetzung. 


\section{Summary}

Acmella ciliata (H.B.K.) Cass., a medicinal and spice plant which is native to the South American tropics, has been the subject of these investigations. There are permanent irritations concerning its nomenclature and it has often been confused with the better known and closely related species Acmella oleracea (L.) Jansen. That is why a taxonomic review was carried out first. Comparing these two species (Acmella oleracea in two varieties) along their microscopic and macroscopic features as well as in a phytochemical screening it turned out that the macroscopic features especially the differences in the flower heads seem to be the best way to separate the species precisely.

The following phytochemical and enzymatic investigations were almost restricted to Acmella ciliata and were carried out with special regard to the structural elucidation of mainly alkylamides and phenolic derivatives as well as to the effectiveness of Acmella ciliata plant extracts in reducing of inflammatory conditions and diseases. Last mentioned point was checked by enzymatic in-vitro tests as described below also with attention to Spolera ${ }^{\circledR}$, a traditionally approved phytopharmacon on the German pharmaceutical market which contains a 2-propanole extract of Acmella ciliata. Novel or improved drug delivery systems (topic ointment, nanoemulsion, adhesive paste, jelly platelets, bioadhesive tablets) were developed mainly for an oral application.

The phytochemical investigations resulted in the finding of a couple of alkylamides (isobutyl, methylbutyl- and phenylethylamides of double and triple-unsaturated mono- and dicarboxylic acids with nine to thirteen or four to six carbonic-atoms respectively). A series of phenolic substances (simple phenols and phenolcarboxylic acids- and esters, flavonoids, a hydroxycumarine and a lignane) could be detected and elucidated by the means of chromatographic (CC, TLC, HPLC, (HP)LC-MS) and spectroscopic (UV, NMR, MS, (HP)LC-MS) methods. Most of the identified compounds have not been reported for Acmella ciliata or not even for any member of the Asteraceae family yet. Some of some turned out new natural substances.

In-vitro enzymatic assays (elastase-, trypsine- and thrombine-enzymatic inhibition) showed that application of acetone extracts instead of 2-propanole extracts (Spolera ${ }^{\circledR}$ ) may improve the anti-inflammatory effect. This enzymatically determined effect can not be assigned neigher to mainly lipophilic nor to mainly hydrophilic compounds but rather to a synergistic effect. Therefore pharmacological as well as economical reasons would prefer the use of extracts to isolated substances. Concerning the technological pre-formulations the 
mucoadhesive tablets should be the drug delivery system of choice for oral application to develop a standardized preparation. To establish this or other forms of application for Acmella ciliata preparations pharmacological and medicinal in-vivo studies need to be carried out. 


\section{Literatur}

[1] Adam P, Becker H: Analytik biogener Arzneistoffe. Pharmazeutische Biologie, Band 4. Wissenschaftliche Verlagsgesellschaft mbH Stuttgart; 1. Auflage 2000.

[2] Adesina S K, Reisch J: Amides from Zanthoxylum rubescens. Phytochemistry 1989; 28(3): 839-842.

[3] Adesina S K, Olugbade T A, Akinwusi D D, Willert D J: New amides from Zanthoxylum lemairie pericarps. Planta Med 1997; 63(3): 286-287.

[4] Adesina S K: The Nigerian Zanthoxylum; chemical and biological values. Afr J Trad Compl Altern Med. 2005; 2(3): 282-301.

[5] Ansari A H, Mukharya D K, Saxena V K: Analgesic study on N-isobutyl-4,5 decadien-amide isolated from the flowers of Spilanthes acmella Murr. Indian J Pharm Sci 1988; 50(2): 106.

[6] Asano M, Kanematsu T: Constituents of Spilanthes acmella L. f. fusca Makino. Yakugaku Zasshi 1927; 544: 521-525.

[7] Banerji A, Pal S C: A new alkamide from Piper sylvaticum. Phytochemistry 1982; 21(6): 1321-1323.

[8] Bauer R, Khan I A, Wagner H: TLC and HPLC analysis of Echinacea pallida and Echinacea angustifolia roots. Planta Med 1988; 54(5): 426-430.

[9] Bauer R, Remiger P, Wagner H: Echinacea. Vergleichende DC- und HPLC-Analyse der Herba-Drogen von Echinacea purpurea, E. pallida und E. angustifolia. (3. Mitt.) Dtsch Apoth Ztg 1988; 128(4): 174-180.

[10] Bauer R, Jurcic K, Puhlmann J, Wagner H: Immunologische In-vivo und in-vitroUntersuchungen mit Echinacea Extrakten. Arzneim Forsch/Drug Res 1988; 38: 276281.

[11] Bauer R, Remiger P: TLC and HPLC analysis of alkamides in Echinacea drugs. Planta Med 1989; 55(4): 367-371.

[12] Bauer R, Remiger P, Jurcic K, Wagner H: Beeinflussung der Phagozytose-Aktivität durch Echinacea-Extrakte. Z Phytother 1989; 10: 43-48.

[13] Bohlmann F, Grenz M: Über die Inhaltsstoffe aus Echinacea-Arten. Chem Ber 1966; 99: 3197-3200.

[14] Bohlmann F, Zdero C: Neue Inhaltsstoffe aus Achillea-Arten. Chem Ber 1973; 106: 1328-1336.

[15] Bohlmann F, Zdero C: Neue $\mathrm{C}_{10}$-Säureamide, Furanoeremophilane und andere Inhaltsstoffe aus Bolivianischen Senecio-Arten. Phytochemistry 1979; 18: 125-128. 
[16] Bohlmann F, Ziesche J, Robinson H, King RM: Neue Amide aus Spilanthes alba. Phytochemistry 1980; 19: 1535-1537.

[17] Bohlmann F, Hartono L, Jakupovic J: Highly unsaturated amides from Salmea scandens. Phytochemistry 1985; 24(3): 595-596.

[18] Bonnländer B: Isolierung, Charakterisierung und Synthese von Aromavorläufern aus Wein, Dillkraut und Lindenblüten. [Dissertation]. Gemeinsame

Naturwissenschaftliche Fakultät der Technischen Universität Carolo-Wilhelmina zu Braunschweig; 2002.

[19] Borges-del-Castillo J, Vazquez-Bueno P, Secundino-Lucas M, Martinez-Martir A I, Joseph-Nathan P: The N-2-phenylethylcinnamamide from Spilanthes ocymifolia. Phytochemistry 1984; 23(11): 2671-2672.

[20] Brandl W, Herrmann K: Hydroxycinnamoyl esters of malic acid in small radish (Raphanus sativus L. var. sativus). Z Naturforsch 1984; 39c: 515-520.

[21] Bryant B, Mezine I: Pungency and tingling: sensations and mechanisms of trigeminal chemical sensitivity. ACS Symp Ser 2002; 825(Chemistry of Taste): 202-212.

[22] Burden R S, Crombie L: Amides of vegetable origin. Part XII. A new series of alka2,4-dienoic tyramine-amides from Anacyclus pyrethrum D.C. (Compositae). J Chem Soc (C) 1969: 2477-2481.

[23] Calle M, Caballero J D: Anti-herpes and anti-aphtha effects of Spilanthes Americana (Chisaca). Rev Fed Odontol Colomb 1977; 24 (120): 59-66

[24] Carle R: Pflanzliche Antiphlogistika und Spasmolytika. Spilanthes oleracea Parakresse. Z Phytother 1988: 67-76(9): 70-71.

[25] Cassini H: Spilanthes. In: Dictionaire des sciences naturelles, von H. Cassini. 1822; 24: 328-331. Paris: Le Normant. Zit. n. [82].

[26] Cech N B, Eleazer M S, Shoffner L T, Crosswhite M R, Davis A C, Mortenson A M: High performance liquid chromatography/electrospray ionization mass spectrometry for simultaneous analysis of alkamides and caffeic acid derivatives from Echinacea purpurea extracts. J Chromatogr A 2006; 1103: 219-228.

[27] Cheminat A, Zawatzky R, Becker H, Brouillard R: Caffeoyl conjugates from Echinacea species: structures and biological activity. Phytochemistry 1988; 27(9): 2787-2794.

[28] Chung K-F, Kono Y, Wang C-M, Peng C-I: Notes on Acmella (Asteraceae: Heliantheae) in Taiwan. Bot Stud 2008; 49: 73-82.

[29] Clifford L J, Nair M G, Rana J, Dewitt D L: Bioactivity of alkamides isolated from Echinacea purpurea (L.) Moench. Phytomedicine 2002; 9: 249-253. 
[30] Crouch N R, Langlois A, Mulholland D A, Nair J J: A novel alkylamide from the leaves of Acmella caulirhiza (Asteraceae), a traditional surface analgesic. S Afr J Bot 2005; 71(2): 228-230.

[31] Daniels R: Moderne Grundlagen und ihre Wirksamkeit. Pharm Ztg 1998; 143(35): 38.

[32] DeCandolle A P: Spilanthes. In: Prodromus systematics naturalis regni vegetablis, ed. A. P. DeCandolle. 1836; 5: 620-626. Paris: Treuttel und Würtz. Zit. n. [82].

[33] Deutscher Arzneimittel-Codex (DAC). Ergänzungsbuch zum Arzneibuch. GoviVerlag Pharmazeutischer Verlag GmbH, Eschborn; Deutscher Apotheker-Verlag, Stuttgart; 2007.

[34] Dietz B, Heilmann J, Bauer R: Absorption of Dodeca-2E,4E,8Z,10E/Z-tetraenoic acid isobutylamides after oral application of Echinacea purpurea tincture. Planta Med 2001; 67(9): 863-864.

[35] Dietz B: Untersuchungen zu den Inhaltsstoffen von Echinacea atrorubens sowie zur Wirkung und Bioverfügbarkeit von Alkamiden. [Dissertation]. Heinrich-HeineUniversität Düsseldorf; 2002.

[36] Dittgen M: Arzneistoffzufuhr über die Mundschleimhaut - Moderne orale Haftarzneiformen. In: Müller R H, Hildebrand G E: Pharmazeutische Technologie: Moderne Arzneiformen. Wissenschaftliche Verlagsgesellschaft mbH Stuttgart; 2. Auflage; 1998.

[37] Duchêne D, Touchard F, Peppas N A: Pharmaceutical and medical aspects of bioadhesive systems for drug administration. Drug Dev Ind Pharm 1988; 14 (2\&3): 283-318.

[38] Europäisches Arzneibuch (Ph. Eur.); Band 1 Allgemeiner Teil, Monographiegruppen. Deutscher Apotheker Verlag Stuttgart, Govi-Verlag-Pharmazeutischer Verlag GmbH Eschborn; 5. Ausgabe Grundwerk; 2005.

[39] Fabry W, Okemo P, Ansorg R: Fungistatic and fungicidal activity of East African medicinal plants. Mycoses 1996; 39(1-2): 67-70.

[40] Fabry W, Okemo P O, Ansorg R: Antibacterial activity of East African medicinal plants. J Ethnopharmacol 1998; 60(1): 79-84.

[41] Fernandez J, Reyes R, Ponce H, Oropeza M, VanClasteren M-R, Jankowski C, Campos M G: Isoquercitrin from Argemone platyceras inhibits carbachol and leukotriene D4-induced contraction in guinea-pig airways. Eur J Pharmacol 2005; 522: 108-115.

[42] Gerber E: Über die chemischen Bestandteile der Parakresse. Arch Pharm 1903; 241: 270-286. 
[43] Gollesch F: Untersuchungen zur Hemmung der Trypsin-Aktivität durch ausgewählte Naturstoffe. [Diplomarbeit]. Ernst-Moritz-Arndt-Universität Greifswald; 2007.

[44] Gödecke T: Phytochemische und pharmakologische Untersuchungen an Pelargonium sidoides DC. [Dissertation]. Freie Universität Berlin; 2005.

[45] Gözler B, Arar G, Gözler T, Hesse M: Isodaurinol, an arylnaphthalene lignan from Haplophyllum cappadocicum. Phytochemistry 1992; 31(7): 2473-2475.

[46] Greger H: Sesamin-type lignans as chemical markers within Artemisia. Biochem Syst Ecol 1981; 9(2/3): 165-169.

[47] Greger H, Grenz M, Bohlmann F: Amides from Achillea species and Leucocyclus formosus. Phytochemistry 1981; 20(11): 2579-2581.

[48] Greger H, Grenz M, Bohlmann F: Piperidides and other amides from Achillea species. Phytochemistry 1982; 21(5): 1071-1074.

[49] Greger H, Zdero C, Bohlmann F: Weitere ungesättigte Amide aus Achillea-Arten. Liebigs Ann Chem 1983: 1194-1201.

[50] Greger H: Alkamides: structural relationship, distribution and biological activity. Planta Med 1984; 50(5): 366-375.

[51] Greger H, Hofer O: On the pungent principle of Matricaria pubescens. Phytochemistry 1984; 23(5): 1173-1174.

[52] Greger H, Zdero C, Bohlmann F: Pyrrolidine and piperidine amides from Achillea. Phytochemistry 1984; 23(7): 1503-1505.

[53] Greger H, Hofer O, Werner A: New amides from Spilanthes oleracea. Monatsh Chem 1985; 116(2): 273-277.

[54] Greger H: Highly unsaturated isopentyl amides from Achillea wilhelmsii. J Nat Prod 1987; 50(6): 1100-1107.

[55] Greger H, Zdero C, Bohlmann F: Pyrrole amides from Achillea ageratifolia. Phytochemistry 1987; 26(8): 2289-2291.

[56] Greger H, Zechner G, Hofer O, Vajrodaya S: Bioactive amides from Glycosmis species. J Nat Prod 1996; 59: 1163-1168.

[57] Greiner K, Weber A: Kräuter. Gräfe und Unzer Verlag GmbH München; 2006.

[58] Hahn R, Nahrstedt A: High content of hydroxycinnamic acids esterified with (+)-Dmalic acid in the upper parts of Fumaria officinalis. Planta Med 1993; 59: 189-190.

[59] Hahn R, Nahrstedt A: Hydroxycinnamic acid derivatives, caffeoylmalic and new caffeoylaldonic acid esters, from Chelidonium majus. Planta Med 1993; 59: 71-75. 
[60] Hashimoto K, Satoh K, Kase Y, Ishige A, Kubo M, Sasaki H, Nishikawa S, Kurosawa S, Yakabi K, Nakamura T: Modulatory effect of aliphatic acid amides from Zanthoxylum piperitum on isolated gastrointestinal tract. Planta Med 2001; 67: 179181.

[61] Hatasa S, Iioka I: Spilanthol-containing compositions for oral use. (Lion Dentifrice Co., Ltd.). U.S. 1973, 4 pp. CODEN: USXXAM US 372076219730313 [Patent].

[62] He P, Davis S S, Illum L: Chitosan microspheres prepared by spray drying. Int J Pharm 1999; 187(1): 53-65.

[63] He W, Puyvelde van L, Kimpe de N, Verbruggen L, Anthonissen K, Flaas van der M, Bosselaers J, Mathenge S G, Mudida F P: Chemical constituents and biological activities of Zanthoxylum usambarense. Phytother Res 2002; 16: 66-70.

[64] Hegnauer R: Chemotaxonomie der Pflanzen. Eine Übersicht über die Verbreitung und die systematische Bedeutung der Pflanzenstoffe. Band 3; Birkhäuser Verlag Basel und Stuttgart; 1964.

[65] Hejazi R, Amiji M: Stomach-specific anti-H. pylori therapy. I: preparation and characterization of tetracycline-loaded chitosan. Int J Pharm 2002; 235(1-2): 87-94.

[66] Henke H: Präparative Gelchromatographie an Sephadex LH 20.; Alpha Druck GmbH Goldbach, 1994.

[67] Henke K: Etablierung eines Thrombin-Assays und Testung phenolischer Naturstoffe auf Thrombin-inhibitorische Aktivität. [Diplomarbeit]. Ernst-Moritz-Arndt-Universität Greifswald; 2004.

[68] Herz W, Kulanthaivel P: An amide from Salmea scandens. Phytochemistry 1985; 24(1): 173-174.

[69] Hiller K, Melzig M F: Lexikon der Arzneipflanzen und Drogen. Spektrum Akademischer Verlag, Heidelberg, Berlin; 2003.

[70] Hinz B, Woelkart K, Bauer R: Alkamides from Echinacea inhibit cyclooxygenase-2 activity in Human neuroglioma cells. Biochem Bioph Res Co 2007; 360: 441-446.

[71] Hollenberg M D, Compton S J: International union of pharmacology. XXVIII. Proteinase-activated receptors. Pharmacol Rev 2002; 54: 203-217.

[72] Hsieh T-J, Su C-C, Chen C-Y, Liou C-H, Lu L-H: Using experimental studies and theoretical calculations to analyze the molecular mechanism of coumarin, p-hydroxybenzoic acid and cinnamic acid. J Mol Struct 2005; 741: 193-199.

[73] Hunnius Pharmazeutisches Wörterbuch von Burger A und Wachter H; Verlag Walter de Gruyter, Berlin, New York; 7. Auflage; 1993.

[74] Hyoung K J, Yong L S: Identification of new dicaffeoylquinic acids from Chrysanthemum morifolium and their antioxidant activities. Planta Med 2005; 71: 871-876. 
[75] Indikationsliste nach $\S 109$ a Absatz 3 Arzneimittelgesetz (AMG); Stand 23.07.2001; Lfd.Nr. 274: Acmella-ciliata-Ganzpflanze.

[76] Inoyatov N, Celebi N, Acarturk F: Preparation and evaluation of a prolonged release pentoxifylline tablet with chitosan. Pharm Ind 1998; 60(5): 472-475.

[77] Ishida M, Nambu N, Nagai T: Mucosal dosage form of lidocaine for toothache using hydroxypropyl cellulose and carbopol. Chem Pharm Bull 1982; 30(3): 980-984.

[78] Ishida M, Nambu N, Nagai T: Highly viscous gel ointment containing carbopol for application to the oral mucosa. Chem Pharm Bull 1983; 31(12): 4561-4564.

[79] Iwayama Y (Tanpei Seiyaku K. K.), Japanisches Patent 60237018 (85 237 018), 25 November 1985. Zit. n. [37].

[80] Jansen R K, Stuessy T F: Chromosome counts of compositae from Latin America. Am J Bot 1980; 67: 585-594. Zit. n. [82].

[81] Jansen R K: The systematics of Acmella (Asteraceae-Heliantheae). Syst Bot Mon $1985 ; 8$.

[82] Jansen R K: Systematics of Spilanthes. Syst Bot 1981; 6(3): 231-257.

[83] Japanisches Patent: Daiichi Seiyaku Co. Ltd., 59181218 (84 181 218), 15 Oktober 1984. Zit. n. [37].

[84] Japanisches Patent: Flavor for dentifrices. (Lion Corp., Japan). Jpn. Tokkyo Koho 1982, 3 pp. CODEN: JAXXAD JP 57036891 B4 198220806 Showa.

[85] Japanisches Patent: Dentifrices containing silica and spilanthol abrasives. (Lion Corp., Japan). Jpn. Kokai Tokkyo Koho 1985, 9 pp. CODEN: JKXXAF JP 60041606 A2 19850305 Showa.

[86] Japanisches Patent: Dentifrices containing spilanthols and spice extracts. (Lion Corp., Japan). Jpn. Kokai Tokkyo Koho 1985, 8 pp. CODEN: JKXXAF JP 60075424 A2 19850427 Showa.

[87] Japanisches Patent: Nitto Electric Co. Ltd., Sunstar Inc., Japanese Patent 59232553 (84 232 553), 27 Dezember 1984; Zit. n. [37].

[88] Japanisches Patent: Nitto Electric Industrial Co. Ltd., Japanese Patent 60116630 (85 11- 630), 24 Juni 1985; Zit. n. [37].

[89] Japanisches Patent: Nitto Electric Industrial Co. Ltd., Japanese Patent 60116631 (85 116 631), 24 Juni 1985; Zit. n. [37].

[90] Japanisches Patent: Teikoku Seiyaku Co. Ltd., Nippon Kayaku Co. Ltd., Kaken Pharma-ceutical Co. Ltd., Japanese Patent 59186913 (84 186 913), 23 Oktober 1984. Zit. n. [37]. 
[91] Jente R, Bonnet P-H, Bohlmann F: Über die Inhaltsstoffe von Anacyclus pyrethrum DC. Chem Ber 1972; 105: 1694-1700.

[92] Jess K, Steckel H: The extrusion and spheronization of Chitosan. Pharm Techn Eur 2007; 19(7): 21-24, 27-30.

[93] Johns T, Graham K, Towers G H N: Molluscicidal activity of affinin and other isobutylamides from the Asteraceae. Phytochemistry 1982; 21(11): 2737-2738.

[94] Jondiko I J O: A mosquito larvicide in Spilanthes mauritania. Phytochemistry 1986; 25(10): 2289-2290.

[95] Kadir H A, Zakaria M B, Kechil A A, Azirun M S: Toxicity and electrophysiological effects of Spilanthes acmella Murr. extracts on Periplaneta americana L. Pestic Sci 1989: 25(4): 329-335.

[96] Kametani S, Kojima-Yuasa A, Kikuzaki H, Kennedy D O, Honzawa M, Matsui-Yuasa I: Chemical constituents of Cape Aloe and their synergistic growth-inhibiting effect on Ehrlich ascites tumor cells. Biosci Biotech Biochem 2007; 71(5): 1220-1229.

[97] Karlson P, Doenecke D, Koolman J: Kurzes Lehrbuch der Biochemie für Mediziner und Naturwissenschaftler. Georg Thieme Verlag Stuttgart, New York; 14. Auflage, 1994.

[98] Kashiwada Y, Ito C, Katagiri H, Mase I, Komatsu K, Namba T, Ikeshiro Y: Amides of the fruit of Zanthoxylum spp. Phytochemistry 1997; 44(6): 1125-1127.

[99] Kasper J: Phytochemische Untersuchungen an Acmella ciliata. [Diplomarbeit]. ErnstMoritz-Arndt-Universität Greifswald und Freie Universität Berlin; 2008.

[100] Kayser C: Phytochemische Untersuchungen an pantropischen Arten der Gattung Ipomoea als Beitrag zur Chemotaxonomie der Convolvulaceae. [Dissertation]. Freie Universität Berlin; 1994.

[101] Kuropka G, Koch M, Glombitza K-W: Säureamide aus Achillea ptarmica. Planta Med 1986; 3: 244-245.

[102] Ley J P, Blings M, Krammer G, Reinders G, Schmidt C-O, Bertram H-J: Isolation and synthesis of acmellonate, a new unsaturated long chain 2-ketol ester from Spilanthes acmella. Nat Prod Res 2006; 20(9): 798-804.

[103] Li G-P, Shen B-C, Zhao J-F, Yang X-D, Li L: Two new alkamides from Spilanthes callimorpha. J Integr Plant Biol 2007; 49(11): 1608-1610.

[104] Liang Y-S, Lefeber A W M, Erkelens C, Choi Y H, Verpoorte R: Identification of phenylpropanoids in methyl jasmonate treated Brassica rapa leaves using twodimensional nuclear magnetic resonance spectroscopy. J Chromatogr A 2006; 1112 : 148-155.

[105] Lischka-Güntzel H, Melzig M F: Epilobium angustifolium L. - Schmalblättriges Weidenröschen. Z Phytother 2007; 28: 201-206. 
[106] Löser B, Kruse S O, Melzig M F, Nahrstedt A: Inhibition of neutrophil elastase activity by cinnamic acid derivatives from Cimicifuga racemosa. Planta Med 2000; 66: 751-753.

[107] Lommen A, Godejohann M, Venema D P, Hollman P C H, Spraul M: Application of directly coupled HPLC-NMR-MS to the identification and confirmation of quercetin glycosides and phloretin glycosides in apple peel. Anal Chem 2000; 72: 1793-1797.

[108] Lucks J-S, Müller B W: Parenterale Fettemulsionen. Krankenhauspharmazie 1994: 15(2): 51-57.

[109] Machida K, Kikuchi M: Norisoprenoids from Viburnum dilatatum. Phytochemistry 1996; 41(5): 1333-1336.

[110] Martin R.: Säureamide und andere lipophile Inhaltsstoffe aus Acmella ciliata (H.B.K.) Cass. [Dissertation]. Ruprecht-Karls-Universität Heidelberg; 1985.

[111] Martin R, Becker H: Spilanthol-related amides from Acmella ciliata. Phytochemistry 1984; 23: 1781-1783.

[112] Martin R, Becker H: Amides and other constituents from Acmella ciliata. Phytochemistry 1985; 24(10): 2295-2300.

[113] Martin R, Becker H: Isolierung strukturverwandter Säureamide durch Kombination von Nieder- und Hochdruck-Flüssig-Chromatographie. Fresenius Z Anal Chem 1984; 318: $247-248$.

[114] Matthias A, Addison R S, Penman K G, Dickinson R G, Bone K M, Lehmann R P: Echinacea alkamide disposition and pharmacokinetics in humans after tablet ingestion. Life Sci 2005; 77: 2018-2029.

[115] Melzig M F, Löser B, Lobitz G O, Tamayo-Castillo, Merfort I: Inhibition of granulocyte elastase activity by caffeic acid derivatives. Pharmazie 1999; 54 (9): 712.

[116] Melzig M F, Löser B, Ciesielski S: Inhibition of neutrophil elastase activity by phenolic compounds from plants. Pharmazie 2001; 56: 967-970.

[117] Melzig M F, Tran G D, Henke K, Selassie C D, Verma R P: Inhibition of neutrophil elastase and thrombin activity by caffeic acid esters. Pharmazie 2005; 60: 869-873.

[118] Melzig M F: Hemmung der neutrophilen Elastase durch ätherische Öle. Z Phytother 2006; 27: 227-230.

[119] Melzig M F, Henke K: Inhibition of thrombin activity by selected natural products in comparison to neutrophil elastase. Planta Med 2005; 71: 787-789.

[120] Mindermann-Nogly F: Herstellung von Casein- und Chitosanpellets im Zweischnecken-extruder und ihre Charakterisierung. [Dissertation]. ChristianAlbrechts-Universität Kiel; 2003. Zit. n. [93]. 
[121] Miyazawa T., Matsuda T., Muranishi S., Miyake K.: Taste-improving agent for sweetener having high sweetness. (Ogawa \& Co., Ltd., Japan). PCT Int. Appl. 2006, 26 pp. CODEN: PIXXD2 WO 2006087991 A1 20060824. [Patent].

[122] Mizutani K, Fukunaga Y, Tanaka O, Takasugi N, Saruwatari Y-I, Fuwa T, Yamauchi T, Wang J, Jia M-R, Li F-Y, Ling Y-K: Amides from Huajiao, pericarps of Zanthoxylum bungeanum Maxim. Chem Pharm Bull 1988; 36(7): 2362-2365.

[123] Modonova L D, Voronov V K, Leont'eva V G, Tyukavkina N A: Lignan compounds from Picea obovata. Him Prir Soedin 1971; 2: 165-170.

[124] Molinatorres J, Salgado-Garciglia R, Ramierz-Chavez E, Del Rio R E: Purely olefinic alkamides in Heliopsis longipes and Acmella (Spilanthes) oppositifolia. Biochem Syst Ecol 1996; 24(1): 43-47.

[125] Molina-Torres J, Garcia-Chavez A, Ramirez-Chavez E: Antimicrobial properties of alkamides present in flavouring plants traditionally used in Mesoamerica: affinin and capsaicin. J Ethnopharmacol 1999; 64(3): 241-248.

[126] Moore A H: Revision of the genus Spilanthes. Am Acad Arts 1907; 42: 521-569. Zit. n. [82].

[127] Moroe M., Hosoda F.: Bath preparations containing spilanthol. (Takasago Perfumery Co., Ltd., Japan). Jpn. Kokai Tokkyo Koho 1985, 4 pp. CODEN: JKXXAF JP 60215610 A2 19851029 Showa. [Patent].

[128] Mukharya D K, Ansari A H, Singh H: Olean-12-ene-3-O-ß-D-galactopyranosyl(1 $\rightarrow$ 4)-O- $\alpha$-L-rhamnopyranoside: A new triterpenoidal saponin from the roots of Spilanthes acmella (Murr.). Indian J Chem 1987; 26B(1): 87.

[129] Müller-Jakic B, Breu W, Probstle A, Redl K, Greger H, Bauer R: In vitro inhibition of cyclooxygenase and 5-lipoxygenase by alkamides from Echinacea and Achillea species. Planta Med 1994; 60(1): 37-40.

[130] Nagai T, Machida Y, Suzuki Y, Ikura H: Teijin Ltd., Patent GB 2042 888, 27 Februar 1979. Zit. n. [37].

[131] Nagai T, Machida Y: Pharm Int 1985; 6: 196. Zit. n. [37].

[132] Nagashima M, Nakatani N: A new alkamide from Spilanthes acmella L. Chem Expr 1992; 7(2): 153-156.

[133] Nagashima M, Nakatani N: LC-MS analysis and structure determination of pungent alkamides from Spilanthes acmella L. flowers. Lebensm Wiss Technol 1992; 25(5): 417-421.

[134] Nakatani N, Nagashima M: Pungent alkamides from Spilanthes acmella L. var. oleracea Clarke. Biosci Biotech Biochem 1992; 56(5): 759-762.

[135] Neues Rezeptur-Formularium (NRF). Pharmazeutisches Laboratorium, Govi-Verlag Pharmazeutischer Verlag, Eschborn; 2006. 
[136] Nielsen J K, Olsen O, Pedersen L H, Sørensen H: 2-O-(p-coumaroyl)-L-malate, 2-Ocaffeoyl-L-malate and 2-O-feruloyl-L-malate in Raphanus sativus. Phytochemistry 1984; 23(8): 1741-1743.

[137] Oya T, Tsukada H: Plant oils as insecticides. (Natural Products Co., Japan; Echigo Yakusou K. K.). Jpn. Kokai Tokkyo Koho 2002, 6 pp. CODEN: JKXXAF JP 2002363012 A2 20021218. [Patent].

[138] Pendse G S, Gokhale V G, Phalnikar N L, Bhide B V: Investigation of new plant larvicides with special reference to Spilanthes acmella. J Univ Bombay, Sci: Phys Sci, Math, Biol Sci Med 1946; 15(20): 26-30.

[139] Peppas N A, Burim P A: Surface, interfacial molecular aspects of polymer bioadhesion on soft tissues. J Control Rel 1985; 2: 257-275.

[140] Perry N B, Klink van J W, Burgess E J, Parmenter G A: Alkamide levels in Echinacea purpurea: a rapid analytical method revealing differences among roots, rhizomes, stems, leaves and flowers. Planta Med 1997; 63: 58-62.

[141] Pitasawat B, Choochote W, Kanjanapothi D, Panthong A, Jitpakdi A, Chaithong U: Screening for larvicidal activity of ten carminative plants. Southeast Asian J Trop Med Public Health 1998; 29(3): 660-662.

[142] Pschyrembel Klinisches Wörterbuch. Verlag: Walter de Gruyter Berlin, New York; 259. Auflage, 2002.

[143] Raduner S, Majewska A, Chen J-Z, Xie X-Q, Hamon J, Faller B, Altmann K-H, Gertsch J: Alkylamides from Echinacea are a new class of cannabinomimetics. J Biol Chem 2006; 281(20): 14192-14206.

[144] Ramsewak R S, Erickson A J, Nair M G: Bioactive N-isobutylamides from the flower buds of Spilanthes acmella. Phytochemistry 1999; 51: 729-732.

[145] Reininger E: Vergleichende phytochemische und pharmakologische Untersuchungen zur Hemmung der Prostaglandin-H-Synthase Isoenzyme mit Arzneidrogen der chinesischen Medizin, insbesondere Platycodi radix und Chaenomelis fructus. [Dissertation]. Heinrich-Heine-Universität Düsseldorf; 2001. Zit. n. [35].

[146] Rennert B, Melzig M F: Free fatty acids inhibit the activity of Clostridium histolyticum collagenase and human neutrophil elastase. Planta Med 2002; 68: 767769.

[147] Richard L C: Acmella. In: Synopsis plantarum von C. Persoon. 1807: 472-473. Paris. Zit. n. [82].

[148] Rios-Chavez P, Ramirez-Chavez E, Armenta-Salinas C, Molina-Torres J: Acmella radicans var. radicans: in vitro culture establishment and alkamide content. In Vitro Cell Dev Biol-Plant 2003; 39: 37-41. 
[149] Robinson H E: A revision of the tribal and subtribal limits of the Heliantheae (Asteraceae). In: Smithonian Contributions to Botany 1981: 51. Zit. n. [208].

[150] Ross S A, Al-Azeib M A, Krishnaveni K S, Fronczek F R, Burandt Ch L: Alkamides from the leaves of Zanthoxylum syncarpum. J Nat Prod 2005; 68: 1297-1299.

[151] Rote Liste 1949.

[152] Rote Liste 2007.

[153] Rücker G, Neugebauer M, Willems G G: Instrumentelle pharmazeutische Analytik. Lehrbuch zu spektroskopischen, chromatographischen, elektrochemischen und thermischen Analysenmethoden. Wissenschaftliche Verlagsgesellschaft $\mathrm{mbH}$ Stuttgart; 3. Auflage, 2001.

[154] Saadali B, Boriky D, Blaghen M, Vanhaelen M, Talbi M: Alkamides from Artemisia dranunculus. Phytochemistry 2001; 58: 1083-1086.

[155] Säkkinen M, Seppala U, Heinanen P, Marvola M: In vitro evaluation of microcrystalline chitosan (MCCh) as gel-forming exipient in matrix granules. Eur $\mathrm{J}$ Pharm Biopharm 2002; 54(1): 33-40. Zit. n. [93].

[156] Schneider G, Hiller K: Arzneidrogen. Spektrum Akademischer Verlag Heidelberg, Berlin; 4. Auflage, 1999.

[157] Sealer R P, Japanisches Patent 60120811 (85 120 811), 28 Juni 1985. Zit. n. [37].

[158] Sefkow M, Kelling A, Schilde U: First efficient syntheses of 1-, 4-, and 5caffeoylquinic acid. Eur J Org Chem 2001: 2735-2742.

[159] Shimada T, Gomi T: Spilanthol-rich essential oils for manufacturing toothpastes or other oral compositions. (Lion Corp., Japan). Jpn. Kokai Tokkyo Koho 1995, 6 pp. CODEN: JKXXAF JP 07090294 A2 19950404 Heisei. [Patent].

[160] Sieck R, Siems K, Odenthal K P, Witthohn K: New substances detected in the pressed juice $[1.7-2.5: 1]$ from Echinacea purpurea herbs. Poster auf dem 48. Kongress der Gesellschaft für Arzneipflanzenforschung; Zürich 2000.

[161] Siedle B, Hrenn A, Merfort I: Natural compounds as inhibitors of human neutrophil elastase. Planta Med 2007; 73: 401-420.

[162] Sittie A A, Lemmich E, Olsen C E, Hviid L, Brøegger Christensen S: Alkamides from Phyllanthus fraternus. Planta Med 1998; 64: 192-193.

[163] Spolera $^{\circledR}$ - Fachinformation/Botanik Organotherapeutische Werke GmbH, Ettlingen.

[164] Spolera ${ }^{\circledR}$ flüssig und Spolera ${ }^{\circledR}$ Salbe Gebrauchsinformation Organotherapeutische Werke GmbH, Ettlingen.

[165] Stahl E: Dünnschichtchromatographie. Ein Laboratoriumshandbuch. Springer-Verlag Berlin, Heidelberg, New York; 2. Auflage, 1967. 
[166] Steinhoff M, Buddenkotte J, Shpacovitch V, Rattenholl A, Moormann C, Vergnolle N, Luger T A, Hollenberg M D: Proteinase-activated receptors: transducers of proteinasemediated signaling in inflammation and immune response. Endocr Rev 2005; 26(1): 143.

[167] Stöhr J R, Xiao P-G, Bauer R: Constituents of Chinese Piper species and their inhibitory activity on prostaglandin and leukotriene biosynthesis in vitro. $\mathrm{J}$ Ethnopharmacol 2001; 75: 133-139.

[168] Sugano H, Yoshida F, Watanabe Y, Tokumoto N: Dentifrices containing organic acids and flavours. (Lion Corp., Japan). Jpn. Kokai Tokkyo Koho 1987; 7 pp. CODEN: JKXXAF JP 62198611 A2 19870902 Showa. [Patent].

[169] Suja S R, Latha P G, Rajasekharan S, Pushpangadan P: Antihepatotoxic activity of Spilanthes ciliata. Pharm Biol 2003; 41(7): 536-541.

[170] Takaku N, Choi D-H, Mikame K, Okunishi T, Suzuki S, Ohashi H, Umezawa T, Shimada M: Lignans of Chamaecyparis obtusa. J Wood Sci 2001; 47: 476-482.

[171] Takao N, Mikinao T, Reiko I, Toshimasa I, Toru O: Studies on the constituents of Piper hancei of spice from Okinawa. Nat Med 1995; 49(4): 438-441.

[172] Teuscher E, Melzig M F, Lindequist U: Biogene Arzneimittel. Ein Lehrbuch der Pharmazeutischen Biologie. Wissenschaftliche Verlagsgesellschaft mbH Stuttgart; 6. Auflage, 2004.

[173] Tiwari H P, Kakkar A: Phytochemical examination of Spilanthus acemella (Murr.). J Indian Chem Soc 1990; 76(9): 784-785.

[174] Tokumoto N, Watabe M, Honma Y: Xylitol with spilanthol for improving taste of food and dentifrice. (Lion Corp., Japan). Jpn. Kokai Tokkyo Koho 2001, 8 pp. CODEN: JKXXAF JP 20011178395 A2 20010703. [Patent].

[175] Tsuchiya S, Hatasa S, Hoka I: Dentifrice composition. (Lion Dentifrice Co., Ltd., Japan). Brit. 1976, 5 pp. CODEN: BRXXAA GB 1438205.

[176] Uehara A, Murmoto K, Takada H, Sugawara S: Neutophil serine proteinases activate Human nonepithelial cells to produce inflammatory cytokines through proteaseactivated rezeptor 2 . J Immunol 2003; 170: 5690-5696.

[177] Verykokidou-Vitsaropoulos E, Becker H: Flavonoide aus Spilanthes oleracea Jacq. Arch Pharm (Weinheim) 1983; 316: 815-816.

[178] Wagner H, Breu W, Willer F, Wierer M, Remiger P, Schwenker G: In vitro inhibition of arachidonate metabolism by some alkamides and prenylated phenols. Planta Med 1989; 55(6): 566-567.

[179] Wagner H, Bladt S, Zgainski E M: Drogenanalyse: dünnschichtchromatographische Analyse von Arzneidrogen. Springer-Verlag Berlin, Heidelberg, New York; 1983. 
[180] Wang H Y, Yang J S: Chemical constituents of Arctium lappa L. Yao Xue Xue Bao 1993; 28(12): 911-917.

[181] Woelkart K: Über phytochemische und pharmakologische Untersuchungen von Echinacea angustifolia DC. sowie klinische Studien und neue Erkenntnisse zu molekularen Wirkmechanismen. [Dissertation]. Karl-Franzens-Universität Graz; 2005.

[182] Woelkart K, Xu W, Pei Y, Makriyannis A, Picone RP, Bauer R: The endocannabinoid system as a target for alkamides from Echinacea angustifolia roots. Planta Med 2005; 71: 701-705.

[183] Woelkart K, Koidl C, Grisold A, Gangemi J D, Turner R B, Marth E, Bauer R: Bioavailability and pharmacokinetics of alkamides from the roots of Echinacea angustifolia in Humans. J Clin Pharmacol 2005; 45: 683-689; Zit. n. [185].

[184] Woelkart K, Marth E, Raggam R, Suter A, Schoop R, Koidl C et al.: Bioavailability and pharmacokinetic studies on Echinacea purpurea preparations and their interaction with the immune system. Int J Clin Pharm Ther 2006; 44: 401-408; Zit. n. [185].

[185] Woelkart K, Bauer R: The role of alkamides as an active principle of Echinacea. Planta Med 2007; 73: 615-623.

[186] Xiong Q, Shi D, Yamamoto H, Mizuno M: Alkylamides from pericarps of Zanthoxylum bungeanum. Phytochemistry 1997; 46(6): 1123-1126.

[187] Yang X: Aroma constituents and alkylamides of red and green Huajiao (Zanthoxylum bungeanum and Zanthoxylum schinifolium). J Agric Food Chem 2008; 56(5): 16891696.

[188] Yasuda I, Takeya K, Itokawa H: Structures of amides from Asiasarum heterotropoides MAEK. var. mandshuricum MAEK. Chem Pharm Bull 1981; 29(2): 564-566.

[189] Yoshida F, Yamagishi K, Tokumoto N, Sugano H: Dentifrices containing cineole. (Lion Corp., Japan). Jpn. Kokai Tokkyo Koho 1986, 7 pp. CODEN: JKXXAF JP 61155315 A2 19860715 Showa. [Patent].

[190] Yotsuyanagi T, Yamamura K, Akao Y: Mucosa-adhesive film containing local analgesic. Lancet 1985; 2(8455): 613. Zit. n. [37].

[191] Zhu X, Dong X, Wang Y, Ju P, Kuo S: Phenolic compounds from Virburnum cylindricum. Helv Chim Acta 2005; 88: 339-342.

$\underline{\text { Internet-Recherchen: }}$

[192] Acmella in Bolivia checklist @ efloras.org. http://www.efloras.org/florataxon.aspx?flora_id=40\&taxon id=242433510

[193] Arthur Lee Jacobson. Plant of the Month: August 2003. Toothache Plant. http://www.arthurleej.com/p-o-m-Aug03.html 
[194] Asteraceae-Fotos; thail.

http://forum.ctu.edu.vn/viewtopic.php? $\mathrm{t}=3800 \&$ sid $=895$

Acmella ciliata als Spilanthes oleracea (Bild); thailändisch.

[195] Checklist das plantas do nordeste Brasileiro: Angiospermae e Gymnospermae. http://www.chip.org.br/bdpn/checklistNE.pdf

[196] Discover Life - Asteraceae: Acmella ciliata (Kunth) Cass. - Spilanthes ciliata. http://pick4.pick.uga.edu/mp/20q?search=Acmella+ciliata\&guide=Guianas flora

[197] Discover Life / Global Mapper. Acmella ciliata.

http://pick4.pick.uga.edu/mp/20m?kind=Acmella+ciliata

[198] Discover Life - Asteraceae: Acmella oleracea (L.) R. K. Jansen. http://www.discoverlife.org/mp/20q?search=Acmella+oleracea

[199] Discover Life / Global Mapper. Acmella oleracea. http://www.discoverlife.org/mp/20m?kind=Acmella+oleracea

[200] ElFlowers.exblog.jp. http://elflowers.exblog.jp/m2006-11-01/

Acmella ciliata als Spilanthes acmella (Toothache plant; Bild).

[201] Essential oils.

http://www.bojensen.net/EssentialOilsEng/EssentialOils27/EssentialOils27.htm

[202] Estudio Etnobotanico. Proyecto Tambopata Inambari. www.ibcperu.org/doc/isis/5931.pdf

[203] Fieldmuseum.org. Neotropical Herbarium Specimens. http://fm1.fieldmuseum.org/vrrc/?page=view\&id $=59198$

[204] Fitway Thailand. Thai Nutrition \& Herbs Center. http://www.thaifitway.com/Education/Ndata/N2db/question.asp?QID=91 Acmella ciliata als Spilanthes acmella (Linn.) Murr. Compositae, Para Cress, Toothache plant, Spot flower.

[205] Fundena.org.ve. http://200.74.218.204/fudena/contenido.asp? $\mathrm{SC}=50 \& \mathrm{SSC}=31 \& \mathrm{CN}=383$

[206] Gernot Katzers Gewürzseiten: Parakresse. http://www.uni-graz.at/ katzer/germ/Spil acm.html

[207] Global Compendium of Weeds. Acmella ciliata (Asteraceae). http://www.hear.org/gcw/species/acmella_cilata/

[208] Heliantheae aus Wikipedia, der freien Enzyklopädie. http://de.wikipedia.org/wiki/Heliantheae 
[209] Herbalistics. Spilanthes oleracea / Spilanthes acmella - Toothache plant (seed). http://www.herbalistics.com.au/shop/advanced_search_result.php?keywords= $\underline{\text { Spilanthes }}$

[210] Herbs are Special. Free herb information - Toothache plant. http://www.herbsarespecial.com.au/free-herb-information/toothache-plant.html Acmella oleracea als Spilanthes acmella syn. Spilanthes oleracea F.

[211] Instituto de Biologia - Plantas aquáticas e palustres do Estado de São Paulo. http://www.ib.unicamp.br/plant-aq-SP/img/plantas/Spilanthes acmella.html Acmella ciliata als Spilanthes acmella (Bild), gefunden in der Nähe von São Paulo.

[212] Jambú aus Wikipedia, der freien Enzyklopädie. http://de.wikipedia.org/wiki/Jamb\%C3\%BA

[213] Listagem de Material Herborizado por Gênero. http://herbario.iac.sp.gov.br/Relatorios/listagens/lgenero.asp?Tarefa=Acmella

[214] Lohmüller FA: The botanical system of the plants/Das botanische System der Pflanzen. http://www.f-lohmueller.de/botany/phyta $0 . h t m$

[215] Missouri Botanical Garden-w ${ }^{3}$-TROPICOS. Nomenclatural Data Base. http://mobot.mobot.org/cgi-bin/search_vast?name=Acmella\%20ciliata\&cl=Ecuador. Literature-based distribution maps.

[216] Missouri Botanical Garden-w $w^{3}$-TROPICOS. Nomenclatural Data Base. http://mobot.mobot.org/cgi-bin/search_vast?name=Acmella $\% 20$ oleracea\&cl=Ecuador Literature-based distribution maps.

[217] Missouri Botanical Garden-w $w^{3}$-TROPICOS. Nomenclatural Data Base. http://mobot.mobot.org/cgi/-bin/search_vast?name=Acmella\%20ciliata\&cl=Ecuador Specimen list and maps.

[218] Missouri Botanical Garden-w ${ }^{3}$-TROPICOS. Nomenclatural Data Base. http://mobot.mobot.org/cgi-bin/search_vast?name=Acmella\%20oleracea\&cl=Ecuador Specimen list and maps.

[219] Multilingual Multiscript Plant Name Database. Sorting Acmella names. http://www.plantnames.unimelb.edu.au/Sorting/Acmella.html

[220] Multilingual Multiscript Plant Name Database: Sorting Blainvillea names. http://www.plantnames.unimelb.edu.au/Sorting/Blainvillea.html

[221] Multilingual Multiscript Plant Name Database: Sorting Spilanthes names. http://www.plantnames.unimelb.edu.au/Sorting/Spilanthes.html

[222] Plant of the week 12/05/2005: Toothache plant (Spilanthes acmella): http://www.killerplants.com/plant-of-the-week/20051205.asp Acmella oleracea als Spilanthes acmella (L.) Murray 
[223] Protabase record display.

http://database.prota.org/

Published species (English). Acmella oleracea.

[224] Rote Liste online; 2008.

http://www.rote-liste.de

[225] Royal Botanic Gardens, Kew. Herbarium Catalogue. http://apps.kew.org/herbcat/navigator.do Search: Acmella ciliata.

[226] Royal Botanic Gardens, Kew. Herbarium Catalogue. http://apps.kew.org/herbcat/navigator.do Search: Acmella oleracea.

[227] The New York Botanical Garden. Virtual Herbarium. http://sweetgum.nybg.org/vh/specimen_list.php Search. Basic Search: Acmella ciliata.

[228] The New York Botanical Garden. Virtual Herbarium. http://sweetgum.nybg.org/vh/specimen list.php Search. Basic Search: Acmella oleracea. 


\section{Publikationen}

Keipert R, Melzig M F: Acmella ciliata: Phytochemisches Screening und Untersuchungen zur Hemmung der Aktivität der humanen Leukozyten-Elastase. Poster auf der Veranstaltung: Der wissenschaftliche Nachwuchs stellt sich vor der Landesgruppe Berlin-Brandenburg der Deutschen Pharmazeutischen Gesellschaft, Freie Universität Berlin, Institut für Pharmazie; 2004.

Keipert R, Melzig M F: Acmella ciliata: Phytochemical screening and investigations on inhibition of the activity of the human neutrophil elastase. Poster auf dem 53. Kongress der Gesellschaft für Arzneipflanzenforschung, Florenz, Italien; 2005.

Keipert R, Melzig M F: Acmella ciliata: Phytochemical screening and investigations on inhibition of the activity of the human neutrophil elastase. Poster auf der Veranstaltung: Der wissenschaftliche Nachwuchs stellt sich vor der Landesgruppe Berlin-Brandenburg der Deutschen Pharmazeutischen Gesellschaft, Freie Universität Berlin, Institut für Pharmazie; 2006.

Keipert R, Melzig M F: Acmella ciliata (H.B.K.) Cassini, Portrait einer Arzneipflanze. Zeitschrift für Phytotherapie 2009; 30: 44-50. 